\title{
Knowledge Acquisition Methods and Their Role in Producer-Driven Collaborative Innovation Performance: A Dynamic Capabilities Perspective
}

\author{
by \\ Robert Helal, Ph.D. Candidate \\ Sprott School of Business, Carleton University
}

\begin{abstract}
A thesis submitted to the
Faculty of Graduate and Postdoctoral Affairs

in partial fulfillment of the requirements for the degree of

Doctor of Philosophy

In

Management
\end{abstract}

Sprott School of Business

Carleton, University

Ottawa, Ontario

(C) 2017 Robert Helal 
"No company can act alone with the hope to innovate beyond the competition without external sources as part of their overall strategy."

$\sim$ Quinn (2000) 


\begin{abstract}
Innovation remains the lifeblood of today's organizations. With the increasing pace of business and shorter product lifecycles, organizations are turning to new open innovation models and knowledge acquisition methods (KAMs) to access rich sources of expert knowledge and new resources beyond the traditional boundaries of the R\&D functions. Firms look to develop and use KAMs and new capabilities that will contribute to their innovation objectives and competitive differentiation.

While earlier research demonstrated the benefits of external market knowledge and collaboration on firm performance, studies have focused largely on the firm-level dynamics of co-creation of innovation activity. To date, little research has explored the elements of knowledge acquisition methods, the complexities of inter-firm and intra-firm co-creation interaction and processes, and the role that knowledge and firm capabilities play in collaborative innovation performance. There have been calls for new research towards filling this gap.
\end{abstract}

This study examines the role of knowledge acquisition methods on co-creation of innovation and its impact on collaborative innovation performance. Through a crossdomain theoretical lens (i.e., open innovation, co-creation of innovation, dynamic capabilities, knowledge management), an exploratory comparative cross-case analysis was conducted examining a First-of-a-Kind (FoaK) collaborative innovation program and performance across five co-creation of analytics innovation initiatives. This study is the first to apply a dynamic capabilities perspective, examining the role of knowledge capacities across the collaborative innovation process, and investigating the role of the KAM as a higher-order integrative dynamic capability. 
Findings from this research answer the call for greater insight into the inner workings and complexity of co-creation of innovation, detailing the observed indirect impact of the KAM on innovation performance. New initiative-level evidence describes the role of the KAM, stakeholder engagement, and the interaction of collaboration processes within and across the boundaries of the firm. In addition to highlighting the role of knowledge-based dynamic capabilities, the findings suggest that the development of knowledge capacities is multi-tiered across levels of the KAM program, initiatives, and the resource pool. The study presents first evidence that the knowledge acquisition method serves as a higher-order dynamic capability with its primary role being that of co-creation "orchestration."

Keywords: analytics, co-creation of innovation, collaborative innovation, dynamic capabilities, First-of-a-Kind (FoaK), higher-order dynamic capabilities, knowledge acquisition methods, knowledge management, knowledge capacity framework, open innovation, orchestration 


\section{ACKNOWLEDGMENTS}

\section{Dedicated to my parents George and Hind Helal}

Completing a doctorate was always part of the plan. A personal goal, without a clear path in the early days. An ever-present thought, but wondering what voice I wanted to bring to the work, and what contribution I wanted to make. The opportunity for contribution became clear as my career and our young family grew, but not the path. I remember the decision to enroll in a business marketing Ph.D. seminar at Carleton University in the Winter. That was it. Whether it was the excellence and openness of my professor, the forum for new thought, or the level of challenge, it was clear to me that it was time to begin the journey. I knew it would involve sacrifice, hard work, dedication and the support of many along the way. This is my thanks to all of them.

My gratitude cannot be overstated to my supervisor and committee. The journey is long and sometimes treacherous, and it is the quality of your team that helps to get you through. Dr. Michel Rod, my supervisor. He was new to the university and Louise suggested, "You should meet this guy," and the partnership began. From the start, he understood the balance needed for a Ph.D. candidate in industry, and we set a course that was both pragmatic and fulfilling. His passion and support for my work, guidance, and perpetual positive disposition has played a big part in completing this dissertation process. Dr. Louise Heslop was "that first seminar" professor, and my first supervisor. Her generosity of time and experience helped me set the vision and foundation for my journey.

She was the spark. Dr. Gerald Grant represented the potential of what my chosen field of research could be. His guidance helped chart my course - always available, thoughtful and interested. Thank you all for your commitment and investment in my efforts. 
Thanks to the IBM Corporation and the First-of-a-Kind program for the commitment to this study and support of its goal in helping the advancement of collaborative innovation research. A special thanks to the FoaK leadership team, in particular to Alexis Bantel and Jae-Eun Park for their valued support throughout. My thanks as well to all the participants in the study for sharing their time and experience, from already busy schedules. I recognize my good fortune in spending my days within this innovation charged environment and with its talented people.

Thanks to Carleton University and the Sprott School of Business. The foresight in providing the option for practitioners to pursue their Ph.D. while in industry presented the opportunity, the channel, and the exceptional environment. Thanks to the many faculty and colleagues for sharing throughout this journey.

To my circle of trusted advisors who are equally passionate and curious about new ideas. Tom Fazal, there at the start and who said, "I get it. You need to pursue this." He served as a valued and trusted voice throughout. David Marmer who embraced my passion in spirit and in action. Erick Brethenoux and Brad Jeffers, both always open to the work in progress, and never shy to give me the straight goods. There are too many others to mention - thanks for hearing, challenging and strengthening my work.

To extended family and friends, many thanks for your support. Missing Sunday dinners, events, get togethers because "you are working on the thesis" could have gone much worse without your understanding. To my father-in-law, Philippe DuPerron who shared his ear and time in reviewing early drafts. To the Meadows Board of Directors, who always checked in on my progress as part of the agenda. 
Above all, thanks to my family. Balancing career, study and life ... this journey could not have been possible without their love and support. To my wife and love of my life, Chantal. Always there in providing me the time, space, and nudge needed to get to the next milestone. To my children, pride, joy and inspiration - Alexander and Gabrielle. It was a rare blessing to be working on my thesis while they were both completing their own Master's theses. A memory I cherish.

I dedicate this thesis to my parents George and Hind. Their boundless love and commitment to all of us represent the best part of me. Love you.

A Final Thought - The impact and sacrifice that this level of undertaking has on an individual cannot be overstated, and likely can only be truly understood by those who have walked the path to the end. This has been one of the most fulfilling experiences of my life, and I am better for it. 


\section{TABLE OF CONTENT}

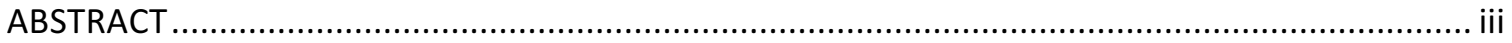

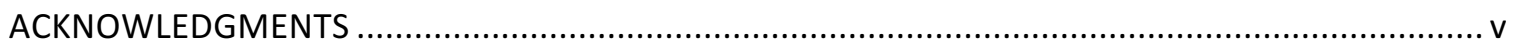

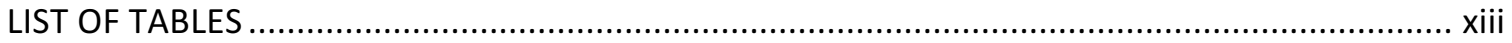

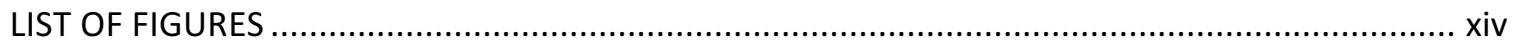

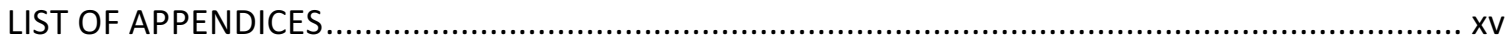

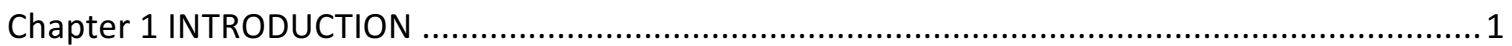

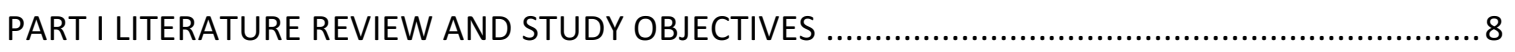

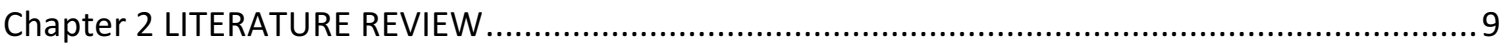

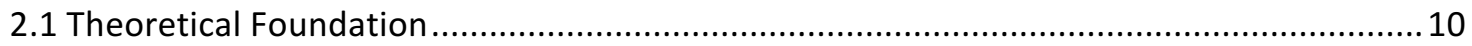

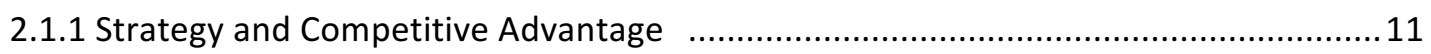

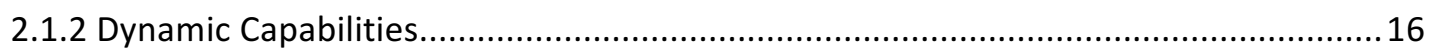

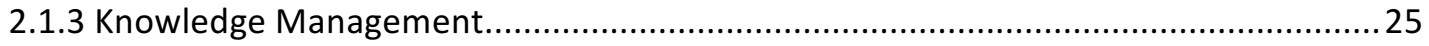

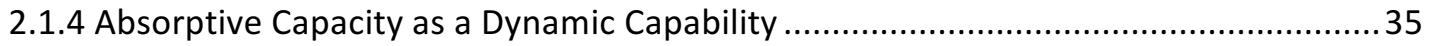

2.2 Co-Creation of Innovation and the Methods of Knowledge Acquisition ............................38

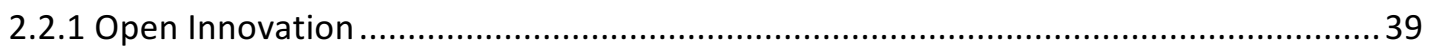

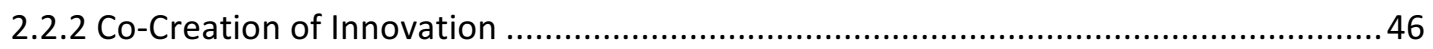

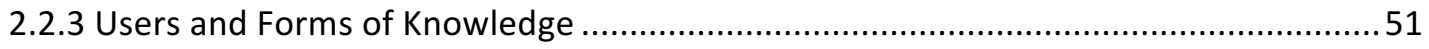

2.2.4 Co-Creation and the Methods of Knowledge Acquisition ..........................................55

2.2.5 The Nature of Knowledge Acquisition Methods.......................................................59

2.3 Knowledge Management, Co-Creation and Impact on Performance ................................62

Chapter 3 STUDY OBJECTIVES, RESEARCH QUESTIONS AND THE CONCEPTUAL FRAMEWORK ... 74

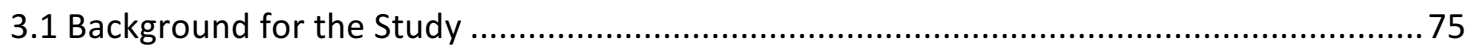

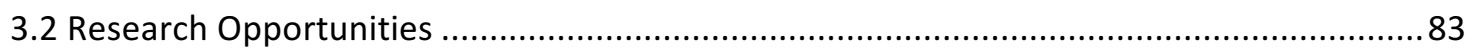

3.2.1 Interaction Between Activities in Open Innovation Initiatives .................................... 83

3.2.2 Higher-Order Dynamic Capabilities, Capability Interaction and Performance .............84

3.2.3 Relationship Between Context, Capabilities, and Performance .................................. 86

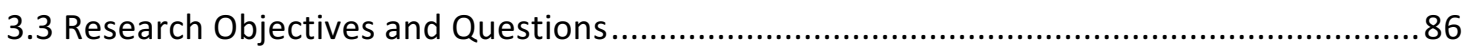

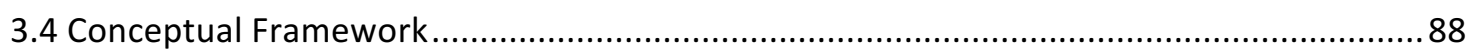

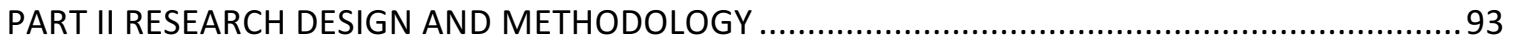

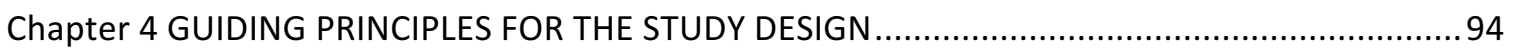

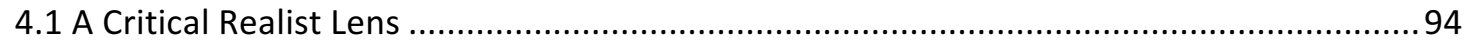

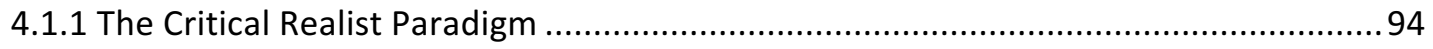




\section{TABLE OF CONTENT (cont'd)}

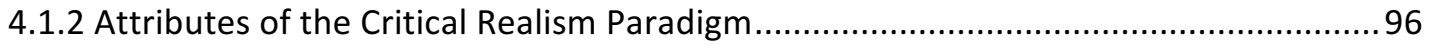

4.1.3 Critical Realism: Research Strategies and the Case Study Method ...........................98

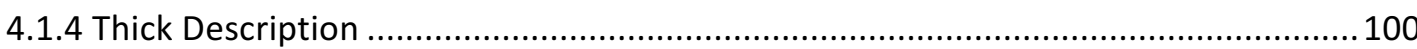

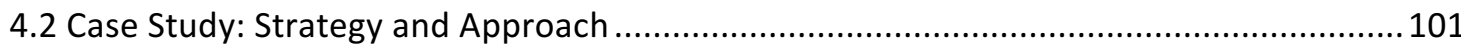

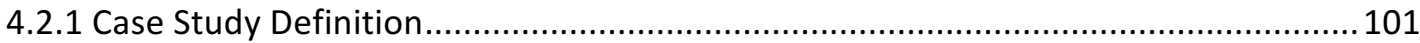

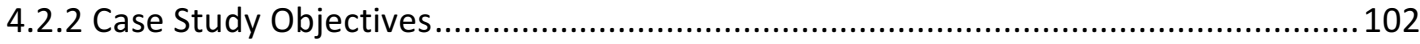

4.2.3 Case Study Reasoning - Deductive, Inductive, Abductive, Retroductive Theory ........104

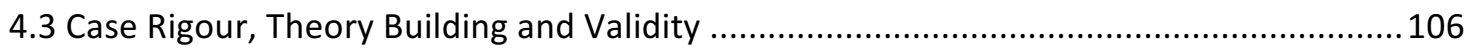

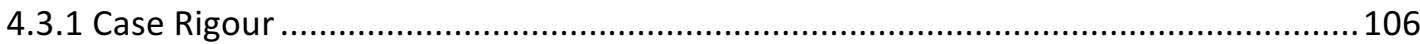

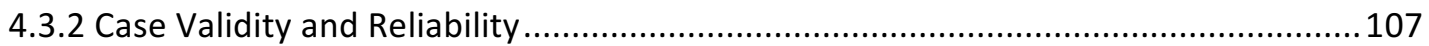

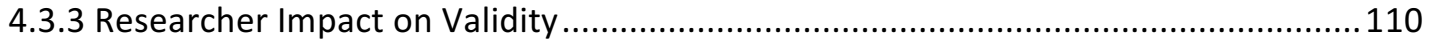

4.3.4 Triangulation and Multi-Methods in Case Research................................................111

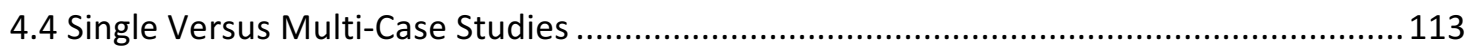

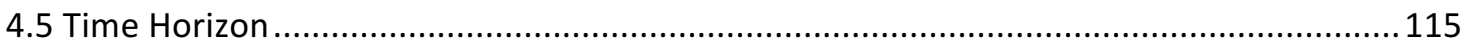

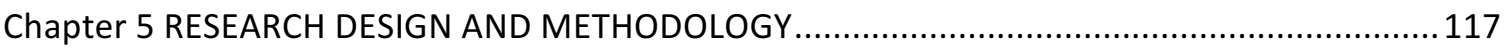

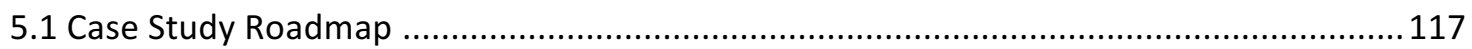

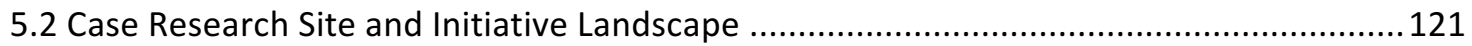

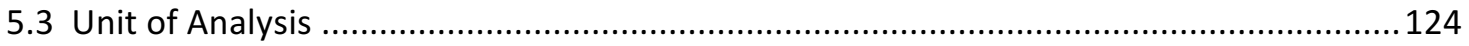

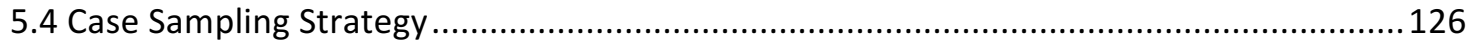

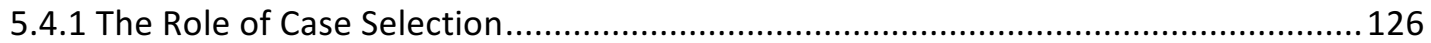

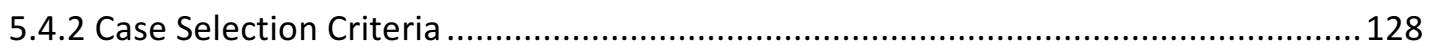

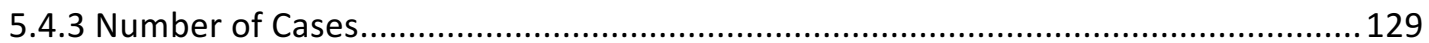

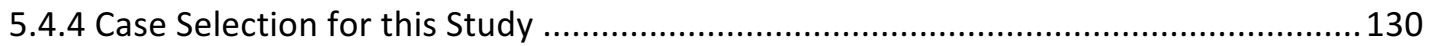

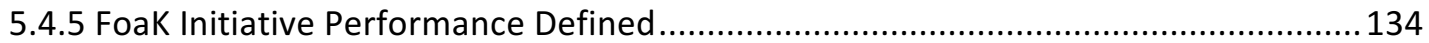

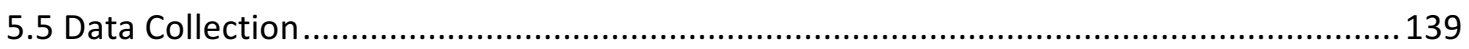

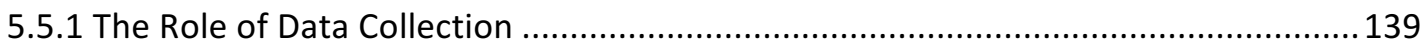

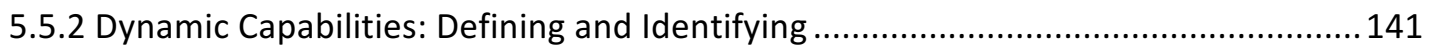

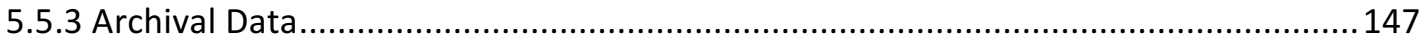

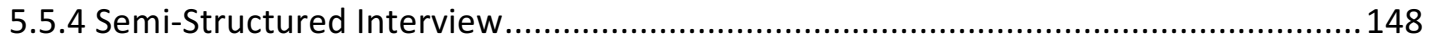

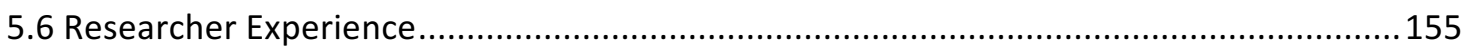

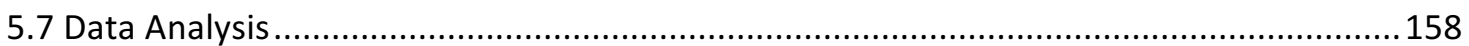

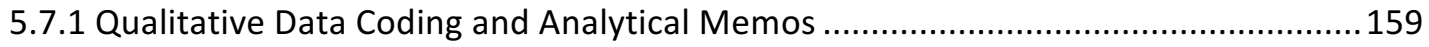

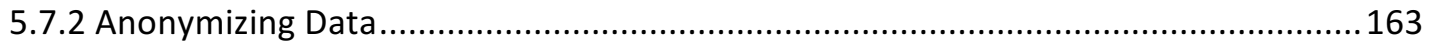

5.7.3 Context, Mechanisms and Outcomes (CMO) Mapping …......................................... 


\section{TABLE OF CONTENT (cont'd)}

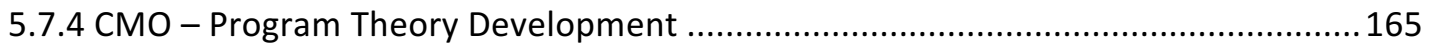

5.7.5 Formulating Alternatives, Interpretation and Presentation ..................................... 169

PART III OVERVIEW OF THE CASE STUDIES AND FOAK PROGRAM THEORY ............................... 171

Chapter 6 AN INTRODUCTION TO THE FIRST-OF-A-KIND PROGRAM AND CASE STUDIES ......... 172

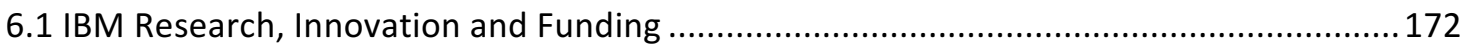

6.2 Knowledge Acquisition Method: The First-of-A Kind (FoaK) Program ............................. 175

6.2.1 Research Funding and the FoaK Program ............................................................. 180

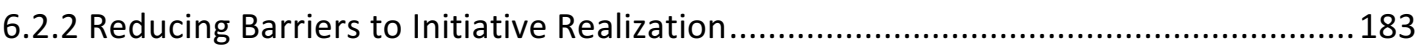

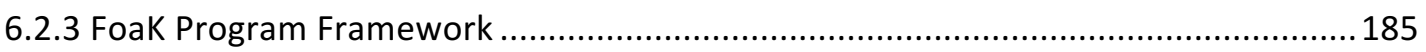

6.2.4 FoaK Initiative Investment Criteria, Funding, and Governance ............................... 190

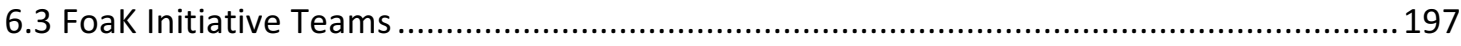

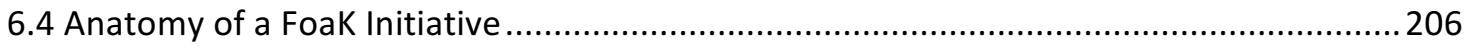



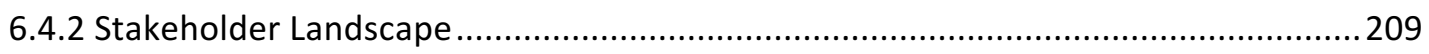

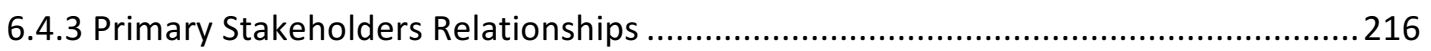

6.5 Case Studies: Initiatives Profile, Informants and Program Context .................................226

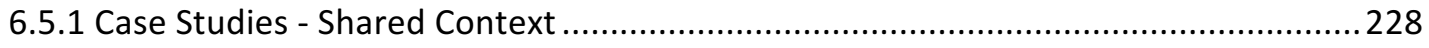

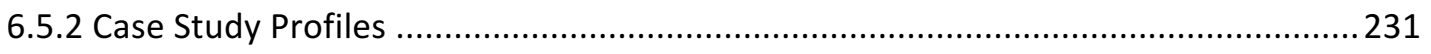

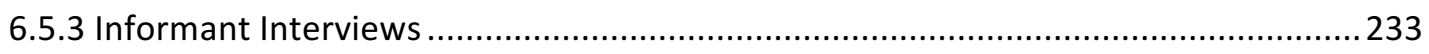

Chapter 7 DEFINING THE FOAK PROGRAM THEORY AND MECHANISMS ..................................236

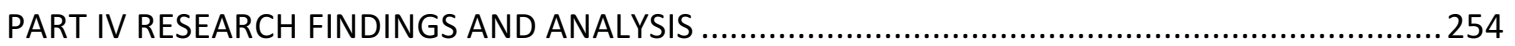

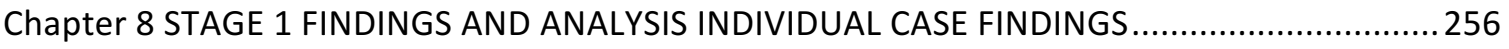

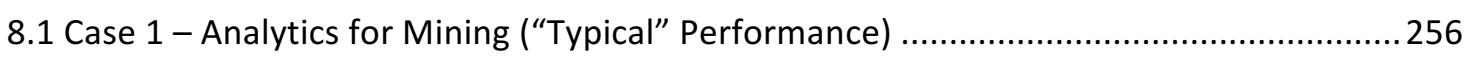

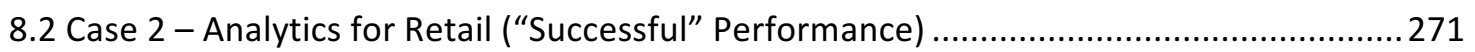

8.3 Case 3 - Analytics for Automotive ("Successful" Performance) ......................................288

8.4 Case 4 - Analytics for Travel ("Unsuccessful” Performance) ............................................302

8.5 Case 5 - Analytics for Healthcare (“Unsuccessful” Performance) ....................................316

Chapter 9 STAGE 2 FINDINGS AND ANALYSIS COMPARATIVE CROSS-CASE FINDINGS ................331

9.1 Program Theory: Additional Case Mechanisms (M14-M18) .......................................... 331

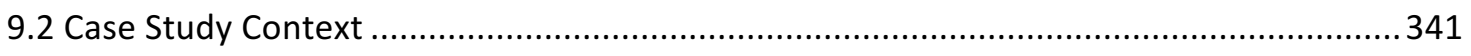

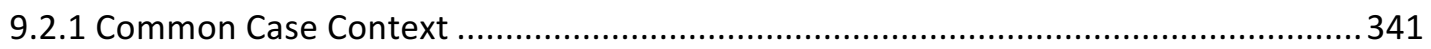

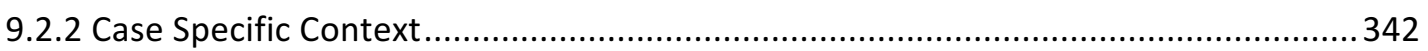

9.3 Case Study Performance - Foak Initiative Profiles ...................................................... 345 


\section{TABLE OF CONTENT (cont'd)}

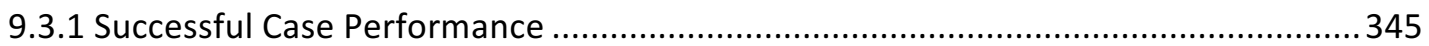

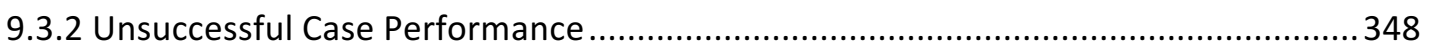

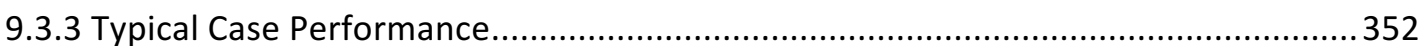

9.4 FoaK Firm and Program Level Mechanism Findings (M1-M5, M13) ................................ 354

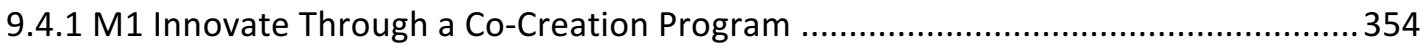

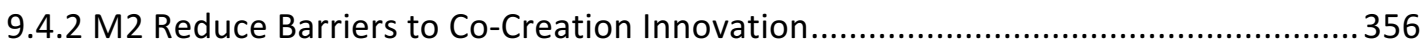

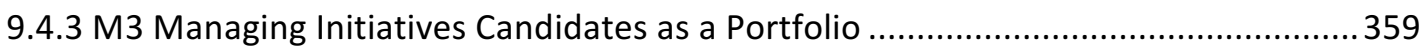

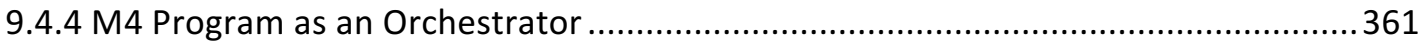

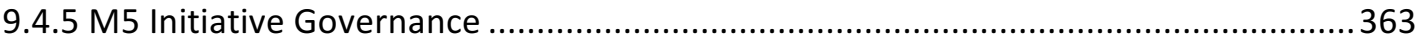

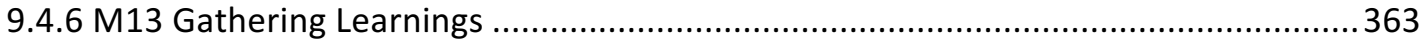

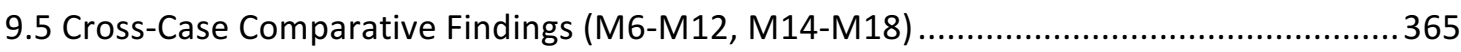

9.5.1 M6 Conditions to Participate for Initiative Candidates ..............................................366

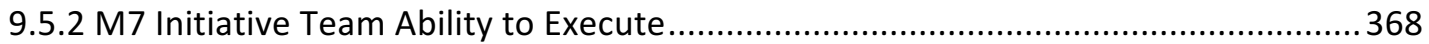

9.5.3 M8/M9 Program Guidance (Directed, Experiential) to Initiatives Teams................... 371

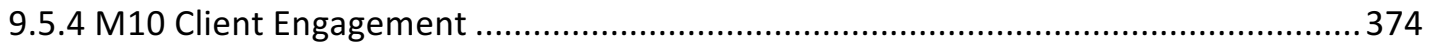

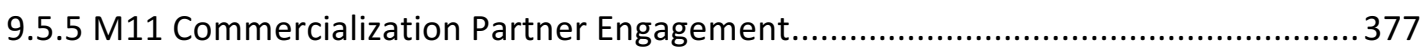

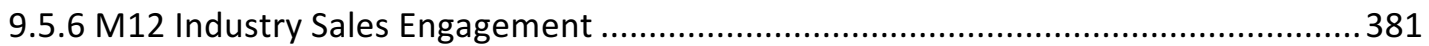

9.5.7 M14 - M18 Stakeholder Focus, Motivation, Expectation, and Investment

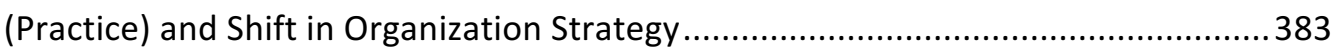

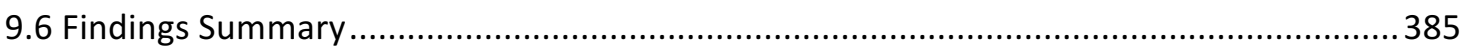

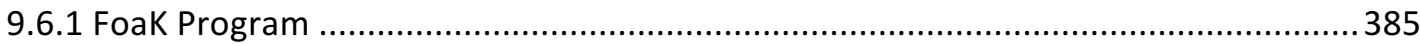

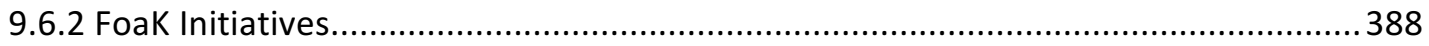



Chapter 10 FOAK PROGRAM THEORY AND THE KNOWLEDGE CAPACITY FRAMEWORK .............390

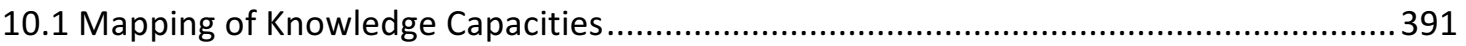

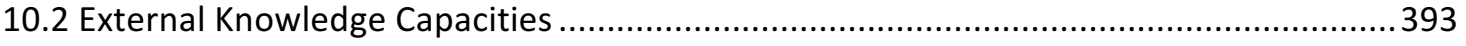

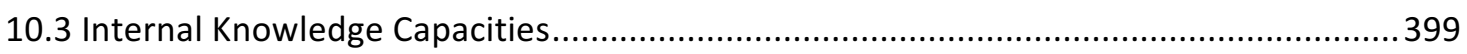

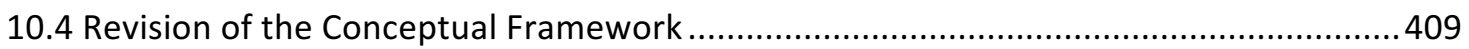

10.4.1 Portfolio Management Capacity (internal knowledge; optimization) ......................409

10.4.2 FoaK as a Higher-Order Dynamic Capability - "Orchestration" ..............................412

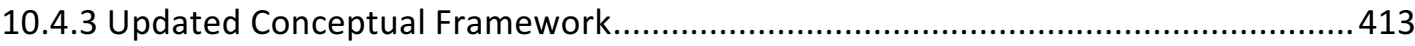

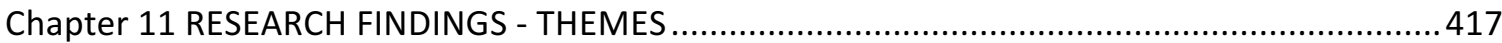

11.1 Knowledge in Co-Creation of Innovation Initiatives ..................................................... 417

11.2 Knowledge Capacities - Initiative-Level Perspective ..................................................423 


\section{TABLE OF CONTENT (cont'd)}

11.3 Knowledge Acquisition Method as a Co-Creation Orchestrator (HoDC) ........................430

11.4 Knowledge Acquisition Method and Portfolio Management Capacity (KCi) ...................433

11.5 Knowledge Capacities in Co-Creation Initiative Interactions ....................................... 435

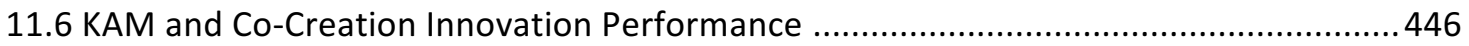



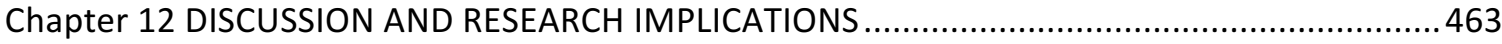

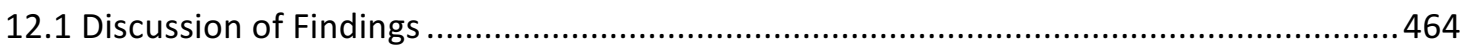

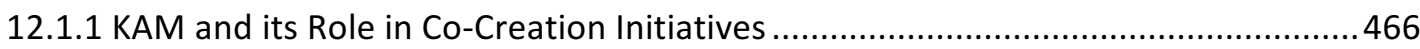

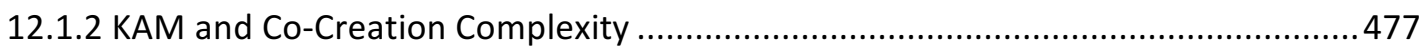

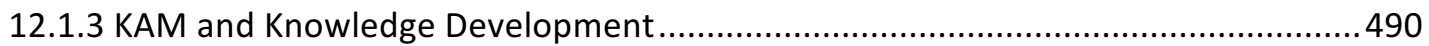

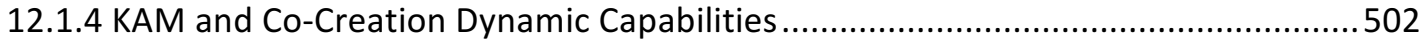

12.1.5 KAM Role in Co-Creation Innovation Performance ...............................................514

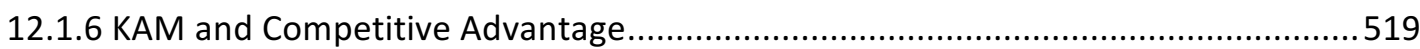

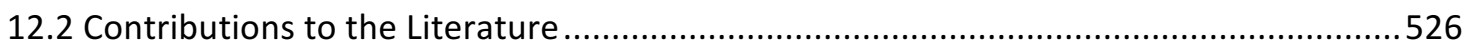

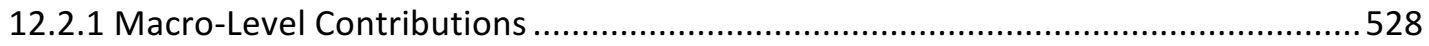

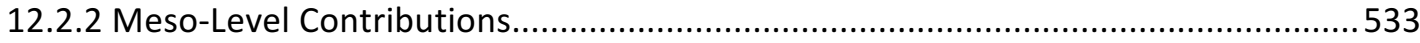

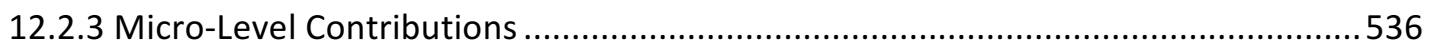

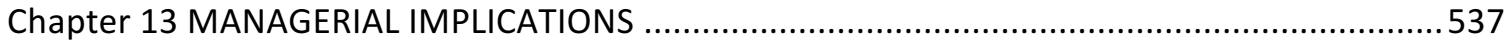

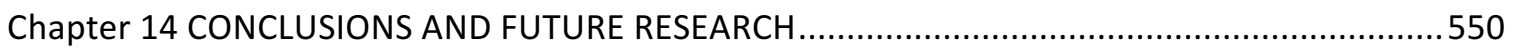

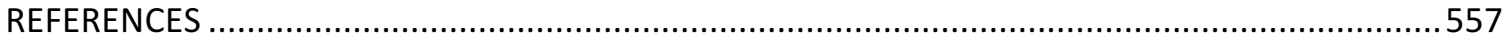

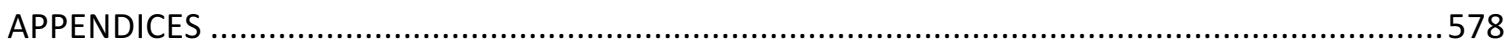




\section{LIST OF TABLES}

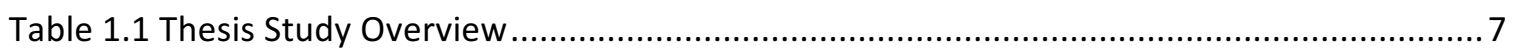

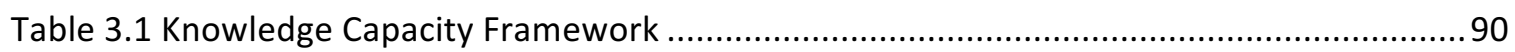

Table 4.1 Eight Designs Relevant to Critical Realist-Informed Research ....................................99

Table 4.2 Validity Typology for Critical Realism-Based Case Research ..................................... 110

Table 5.1 Methodological Principles of Critical Realism......................................................... 120

Table 5.2 Eisenhardt Process for Building Theory from Case Study Research .......................... 121

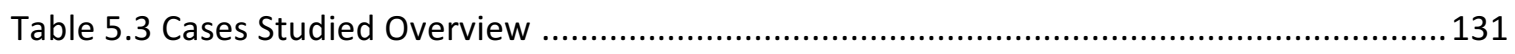

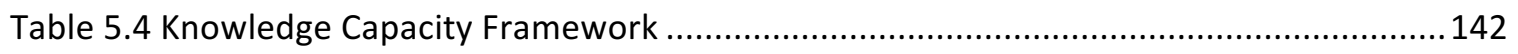

Table 5.5 Informant Grid by Informant Type (Practitioner/Subject/Evaluator) ......................... 151

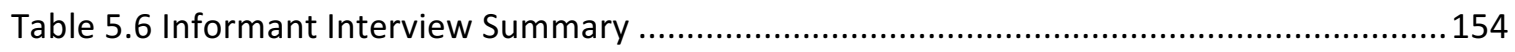

Table 6.1 Foak Initiative Selection Criteria Categories............................................................. 194

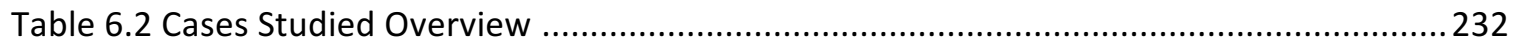

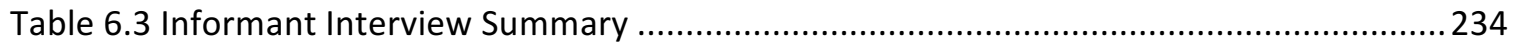

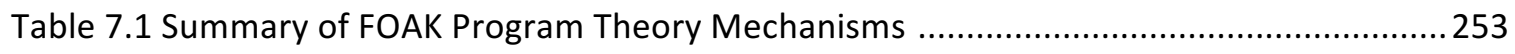

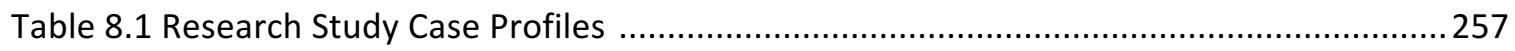

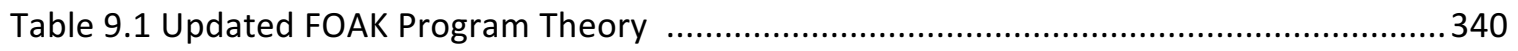

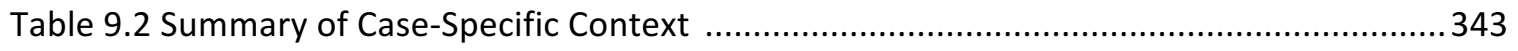

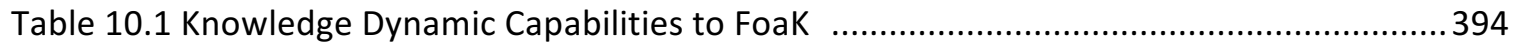

Table 11.1 Mapping Dynamic Capabilities to FoaK Initiative Knowledge Types ........................422

Table 11.2 Comparative Dynamic Capability Development (KAM versus Cross-Case) .............427

Table 11.3 Mapping of Dynamic Capability Role across Co-Creation Relationships

Knowledge capacity Framework ....................................................................437

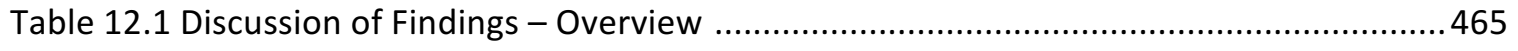




\section{LIST OF FIGURES}

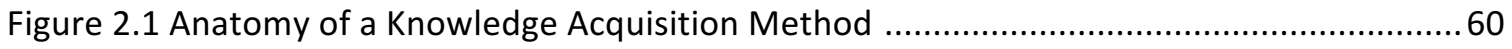

Figure 3.1 Knowledge Acquisition Methods Framework …....................................................... 79

Figure 3.2 Co-Creation of Innovation Conceptual Framework …...............................................92

Figure 5.1 Research Multi-Case Study Roadmap …................................................................. 122

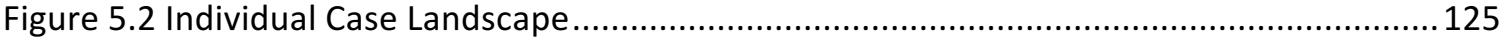

Figure 5.3 Study Informant Types (Initiative-Level) .............................................................. 150

Figure 5.4 Data Collection - Process for Creating Questions ................................................... 153

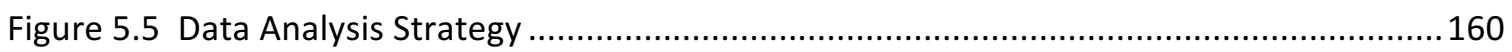

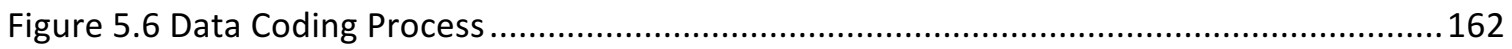

Figure 5.7 Bygstad and Munkvold Stepwise Framework for Critical Realist Data Analysis ........167

Figure 6.1 IBM FoaK Program Collaborative Innovation Process ................................................178

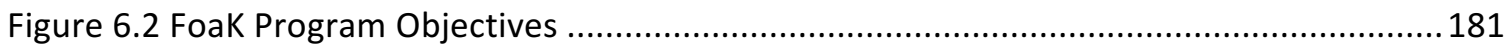

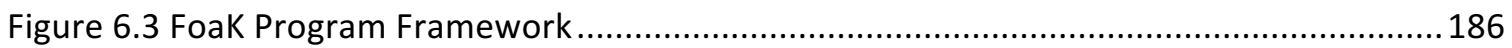

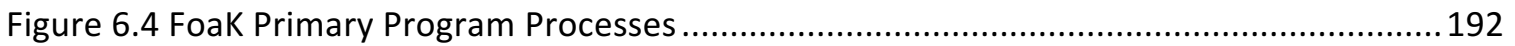

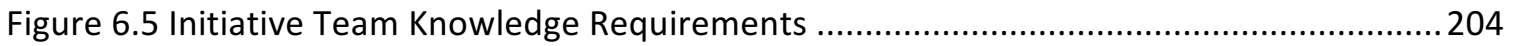

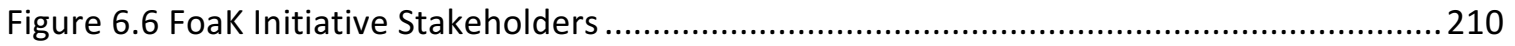

Figure 6.7 FoaK Initiative Primary Stakeholder Relationships (Inter/Intra-firm Interlock Points)

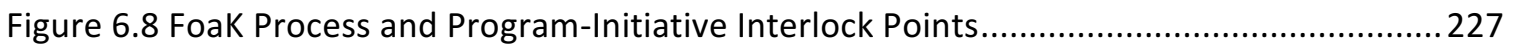

Figure 7.1 FoaK Process and Program-Initiative Mechanisms................................................237

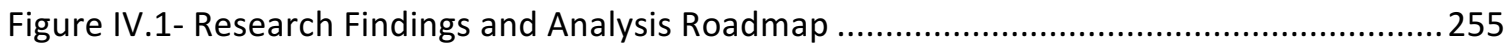

Figure 8.1 Comparative FoaK Initiative and Stakeholder Interlock Profiles ...............................330

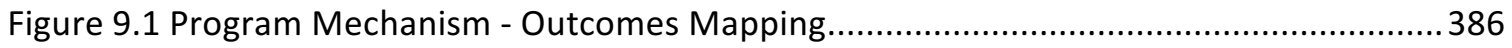

Figure 10.1 Updated - Co-Creation of Innovation Conceptual Framework ...............................416

Figure 11.1 Cross-Case Comparative Knowledge Type Profiles .............................................. 419

Figure 11.2 Co-Creation of Innovation Tiers (Strategy, KAM, Initiative) …...............................425

Figure 11.3 Knowledge Acquisition Method as an "Orchestrator" (Higher-Order DC) ...............432

Figure 11.4 Dynamic Capability Relationships in Co-Creation Innovation Activity .....................446

Figure 11.5 Orchestration, Alignment and Comparative Cross-Case Performance ...................454

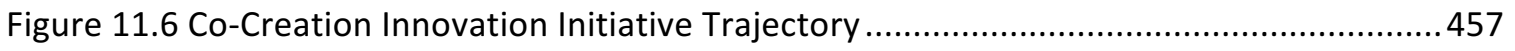

Figure 11.7 Knowledge Acquisition Method Boundaries and Chasms ......................................459

Figure 12.1 Knowledge Acquisition Method System - Dynamic Capabilities Perspective ..........521

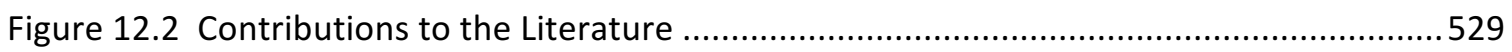




\section{LIST OF APPENDICES}

Appendix 4.1 Comparison of Critical Realist, Positivist and Interpretivist Philosophical Paradigms 579

Appendix 5.1 Semi-Structured Interview Guide...... 580

Appendix 5.2 Informant Recruitment Communication.... 588

Appendix 5.3 Pre-Interview Informant Consent Form 589

Appendix 6.1 FoaK Board Membership Profile (between 2010 and 2014).... 591 


\section{Chapter 1 INTRODUCTION}

Business strategy research has long examined the question of how organizations establish and maintain a sustainable competitive advantage towards achieving superior firm performance. Since Penrose's (1959) work on the theory of the firm, a number of schools of thought have emerged providing necessary insight into this essential question, both for the academic community and practitioners alike. It can be argued that the understanding of competitive advantage and its contribution to the survival and growth of the firm sits at the heart of management research. If understanding competitive advantage is the heart, then research exploring the mechanisms governing firm innovation is the lifeblood.

A firm's ability to innovate ensures its potential to remain relevant in the marketplace. Research in the areas of strategic management, innovation management, and new product development (NPD) have been consistent in the message that firms can no longer depend solely on their own resources for the expertise needed in the continual development of superior offerings (Quinn 2000). Organizations must draw upon external sources of knowledge to fill the gaps in their resource base and expertise. Chesbrough's (2006) work on open innovation (OI) also spawned a stream of research examining strategies that move beyond traditional closed innovation to models that open the borders of the firm to its external environment. Over time, open innovation research has overlapped with other areas of study, including co-creation of innovation (CCOI) and new product development, in examining the dynamics of incorporating various sources of external knowledge into the firm's innovation process (Lichtenthaler \& Lichtenthaler 2009). These 
areas of research have also been consistent in their expression of the complexities involved in understanding the dynamics of these new business models and the factors that contribute to firm performance (Kang \& Kang 2009; Wang \& Ahmed 2007).

Co-creation of innovation research has stressed the importance of knowledge management $(\mathrm{KM})$ and knowledge-based skill development in the firm (Cohen \& Levinthal 1990; Piller et al. 2011; Wang \& Ahmed 2007). From the perspective of a knowledge-based view of the firm (KBV), integrating knowledge is central to the role of the organization and its management team (Grant 1996). KBV argues that organizations must develop knowledge-based skills that in turn support activities pursuing sustainable competitive advantage. From the perspective of a resource-based view of the firm (RBV), knowledge is an essential asset that is only strategic to an organization to the extent that it is inimitable (Barney 1996). This is a consideration when firms seek the desired tacit knowledge from external sources for their innovation process. However, while RBV helps to explain the role of strategic resources in creating competitive advantage, it does not answer the question as to why some firms fail where others succeed, when comparing similar firms in dynamic markets (Cheng \& Shiu 2015). It is a dynamic capabilities view (DCV) of the firm that helps better describe the strategic skills that an organization needs to help it respond and adapt to a changing environment (Teece et al. 1997). Furthermore, in the context of innovation and competitive advantage, DCV helps explain what skills are required for these firms to most effectively co-create with external stakeholders, source the necessary knowledge, and exploit that knowledge through the commercialization of new offerings (Lichtenthaler \& Lichtenthaler 2009; Piller et al. 2011). 
There is a growing body of research examining open co-creation of innovation strategies, the role of knowledge management, insight into the development of dynamic capabilities (DC), and how these elements come together as part of a firm's strategy. Studies are now drawing upon theory from across these multiple domains to inform research exploring a range of complex multifaceted questions. However, while a review of the literature reveals there is considerable interest in the questions emerging in this domain, there remain significant gaps in theory development across open innovation, knowledge management, and dynamic capabilities both within and across these streams of study. Research findings have been clear in their call for more study providing insight into understanding the relationships between resources, skills, and activity that contribute to emerging co-creation of innovation models. Also, more exploratory insight is required to help explain interactions between inter-firm and intra-firm processes, which are fundamental elements of co-creation initiatives (Schleimer \& Faems 2015). While the literature argues the importance of seeking external stakeholder knowledge, and for investing in the necessary skills needed to effectively engage sources of expertise, there is still little understanding into the "how" and "why" of these models (Wynn \& Williams 2008). Insight into a firm's approach to sourcing knowledge from external sources using knowledge acquisition methods is a critical component in understanding these questions.

The study presented in this thesis examines the role of the knowledge acquisition method, and the dynamic capabilities contributing to the method, on its co-creation of innovation initiatives' performance. Specifically, this study examines the question of "How does a knowledge acquisition method impact the performance of a collaborative new-to-the-firm innovation initiative?" It explores the knowledge acquisition method as a 
higher-order integrative dynamic capability, and examines the relationship of the method to new-to-the-firm innovation performance. This study is informed by research in the domains of open innovation, co-creation of innovation, dynamic capabilities and knowledge management.

The research design includes an exploratory case study method, and is informed by the critical realist tradition. Using a cross-sectional, two-tailed comparative multi-case design, this study examines five First-of-a-Kind (FoaK) program directed co-creation of innovation initiatives, and the factors that contribute to successful and unsuccessful initiative performance. The study uses a multi-methods model, which includes a stratified semi-structured interview approach.

Findings from this research answer the call for greater insight into the inner workings of co-creation of innovation initiatives, highlighting the role of the knowledge acquisition method, stakeholder engagement, and the interaction of collaborative processes within and across the boundaries of the firm. In addition to highlighting the role of knowledge-based dynamic capabilities, the research findings suggest that the development of knowledge capacities is multi-tiered across levels of the program, the initiative, and the resource pool. The study presents evidence that the knowledge acquisition method serves as a higher-order dynamic capability with its primary role being that of co-creation "orchestration."

This study pushes forward research in co-creation of innovation by contributing new initiative-level insight into the dynamics of co-creation endeavours. The findings highlight the role of the knowledge acquisition method (KAM), stakeholder engagement, and the interaction of collaborative processes in the co-creation of new-to-the-firm 
innovation. The study highlights that a KAM influences co-creation of innovation performance indirectly through its influence on an initiative team's ability to execute. The research findings also describe that the development of knowledge capacities is multitiered across levels of the KAM co-creation system. Evidence is presented characterizing the knowledge acquisition method as a higher-order dynamic capability with its primary role being that of co-creation "orchestration." In addition to these contributions, this study also presents first empirical support and revision for the Lichtenthaler and Lichtenthaler (2009) open innovation knowledge capacity framework, and introduces the First-of-a-Kind knowledge acquisition method to the literature. Finally, researchers are also presented with a new conceptual framework for co-creation of innovation study, and a KAM multi-tiered framework supporting dynamic capability development research in this domain.

The chapters in this thesis are divided into five parts. Part I presents the study objectives, beginning with a review of the literature (Chapter 2), followed by a discussion of the study objectives, research questions, and a presentation of the conceptual framework used in this research (Chapter 3). Part II presents the research design and methodology. This begins with the guiding principles used to shape this study (Chapter 4), followed by the details of the research design and the methodology used (Chapter 5). Part III prepares for the research analysis by providing an overview of the case studies examined (Chapter 6) and the definition of the program theory developed for the study. Part IV presents the research analysis. This section begins with the Stage 1 individual case analysis findings (Chapter 8), followed by the results of the Stage 2 comparative cross-case analysis (Chapter 9). Chapter 10 presents a mapping of the updated program theory to the knowledge capacity framework, and the linkage between the knowledge acquisition method to the dynamic 
capabilities being studied. The section on analysis then concludes with a discussion of the primary research findings themes (Chapter 11). Part V presents the discussion of findings and their implications. This section begins with a discussion of the research findings, and their contribution to the literature (Chapter 12). The section then concludes with a discussion of the managerial implications that the study presents (Chapter 13). The final chapter of the thesis presents conclusions and an overview of the research limitations and opportunities for future research (Chapter 14). Table 1.1 provides a bird's-eye view of this thesis and the study. 


\section{Table 1.1 Thesis Study Overview}

\begin{tabular}{|c|c|c|}
\hline Research Design Elements & Study Summary & $\begin{array}{l}\text { Research } \\
\text { Roadmap } \\
\text { (Section) }\end{array}$ \\
\hline Philosophy & Critical Realism & 4.1 \\
\hline Approach & Abductive/Reductive Reasoning & 4.2 \\
\hline Theoretical Streams & $\begin{array}{l}\text { Dynamic Capabilities } \\
\text { Open Innovation and Co-Creation of Value Collaboration } \\
\text { Knowledge Management } \\
\text { New Product Development }\end{array}$ & Chapter 2 \\
\hline Primary Research Question & $\begin{array}{l}\text { How does a knowledge acquisition method impact the performance of a } \\
\text { co-creation collaborative new-to-the-firm innovation? }\end{array}$ & Chapter 3 \\
\hline \multirow{3}{*}{ Research Strategy } & Guiding Principles & Chapter 4 \\
\hline & $\begin{array}{l}\text { Case Study Method } \\
\text { Exploratory }\end{array}$ & 4.2 \\
\hline & $\begin{array}{l}\text { Comparative Multi-Case Study } \\
\text { Two-Tailed, Nested Design }\end{array}$ & 4.4 \\
\hline Time Horizon & Cross-Sectional, Retrospective & 4.5 \\
\hline Sources of Evidence & Multi-Methods & 4.3 \\
\hline \multicolumn{3}{|l|}{ Techniques and Procedures } \\
\hline Subject Environment & $\begin{array}{l}\text { Single Company (i.e., IBM Corporation) } \\
\text { Single Program (i.e., FoaK Program) } \\
\text { Multi-Initiative (IBM FoaK Initiative) }\end{array}$ & 5.2 \\
\hline Unit of Analysis & FoaK Initiative & 5.3 \\
\hline Case Selection Strategy & $\begin{array}{l}\text { Theoretical/Purposeful Selection (two-tailed comparative) } \\
5 \text { cases: "typical" case (1), "successful" case (2), "unsuccessful" case (2) }\end{array}$ & 5.4 \\
\hline Data Collection & $\begin{array}{l}\text { Multi-Tiered Semi-Structured Interview ( } 25-30 \text { interviews) } \\
\text { Program Archives } \\
\text { Initiative Archives } \\
\text { Documentation }\end{array}$ & 5.5 \\
\hline Data Analysis & $\begin{array}{l}\text { Analysis: } \\
\text { Iterative Qualitative Data-Coding } \\
\text { (cycle 1: evaluation, causation coding/cycle 2: axial, pattern coding) } \\
\text { Analytical Memo Writing } \\
\text { CMO Mapping and Program Theory Development }\end{array}$ & 5.7 \\
\hline \multirow{2}{*}{ Case Studies Background } & Introduction to the First-of-a-Kind Program and Case Studies & Chapter 6 \\
\hline & Defining the FoaK Program Theory and Mechanisms & Chapter 7 \\
\hline \multirow{3}{*}{ Findings } & Stage 1 Analysis - Individual Case Findings & Chapter 8 \\
\hline & Stage 2 Analysis - Cross-Case Findings & Chapter 9 \\
\hline & Mapping Program Theory to Knowledge Capacities (Dynamic Capabilities) & Chapter 10 \\
\hline \multirow{3}{*}{$\begin{array}{l}\text { Discussion \& } \\
\text { Conclusions }\end{array}$} & Discussion of Findings and Contributions to Theory & Chapter 12 \\
\hline & Managerial Implications & Chapter 13 \\
\hline & Conclusions, Research Opportunities and Limitations & Chapter 14 \\
\hline
\end{tabular}




\section{PART I \\ LITERATURE REVIEW AND STUDY OBJECTIVES}

This study examines the role of a knowledge acquisition method on producerdriven collaborative innovation performance. Its contributions build on multidisciplinary research in the areas of open innovation, co-creation of innovation, dynamic capabilities and knowledge management. Part I presents a foundation of the relevant literature and how it has informed the objectives of this research. This section includes chapters presenting a review of the literature, a discussion of the study objectives, the research questions guiding the research, and the conceptual framework developed for this investigation.

\section{Chapter 2 Literature Review}

Chapter 3 Study Objectives, Research Questions and the Conceptual Framework 


\section{Chapter 2 LITERATURE REVIEW}

A firm's survival is dependent upon its ability to develop and sustain competitive advantage that drives superior performance for the enterprise. The strategic question of how organizations achieve competitive advantage is complex due to considerations regarding the role of managerial decision-making, multifunctional interaction at the level of execution, the external environment and context. Many domains of study have focused on building theory to illuminate the question of developing and sustaining competitive advantage.

Open innovation (Chesbrough 2006) and co-creation of value (Piller et al. 2011) research has examined strategy models that expand the traditional closed boundaries of the firm to engage external sources of knowledge and expertise in developing new innovation. The knowledge-based view of the firm (Grant 1996) and research in knowledge management have examined how knowledge and learning, both inter-firm and intra-firm, and knowledge capabilities can differentiate an organization. Various branches of the theory of the firm examine strategic decisions from different perspectives, including transaction governance and cost optimization (i.e., transaction cost view), achieving competitive advantage through the management of strategic resources (i.e., resource-based view (Barney 1996)), and the view that a firm's knowledge is central to its competitive advantage (i.e., knowledge-based view (Grant 1996)). Dynamic capability theory extends the resource-based view of the firm, by providing insight into how organizations adapt to dynamic environments as an explanation of why some firms succeed where others fail (Teece et al. 1997). With attention mounting about sustainable competitive advantage, 
many of these streams of literature continue to grow and converge on developing the theoretical base. However, many of these streams are still in their formative stages and present a significant opportunity for contributions to theory development.

This chapter provides an overview of the literature in the areas of open innovation and co-creation of value, knowledge management, and dynamic capabilities as they relate to collaboration with external partners in a business-to-business (B2B) context (i.e., customers and users). Specifically, this review focuses on knowledge acquisition methods used by firms in acquiring external knowledge for internal innovation processes. As these streams of literature converge on the topic of this study, this chapter is structured to illustrate the logical linkage between high-level theories of the firm and practice-based theories in the areas of open innovation and co-creation of value. The chapter begins with a review of higher-level theories as they relate to firm strategy and competitive advantage, including an overview of the dynamic capabilities literature. The knowledge management literature is then examined, including a discussion on the concept of absorptive capacity and its role in leveraging external knowledge for use in new innovation. The chapter then bridges over to the practice-centric literature examining open innovation theory, cocreation of innovation, and the role of users as a source of external knowledge for the firm. Finally, the chapter discusses new product development (NPD) research and knowledge acquisition methods, which provide a foundation for this study.

\subsection{Theoretical Foundation}

This study is informed by a cross-section of literature including higher-level theories of the firm and more practice-oriented research. This section begins with an 
overview of the literature that forms the first part of the theoretical foundation for this study, presenting a brief discussion of the varied theories of the firm and competitive advantage (i.e., Porter's competitive forces, transaction cost economics and the resourcebased view of the firm). This is followed by a more detailed examination of dynamic capabilities, knowledge management, and absorptive capacity research.

\subsubsection{Strategy and Competitive Advantage}

Management research has long examined the link between strategic decisions in changing environments and their impact on firm performance (Barreto 2010). Firms look to drive sustainable growth by establishing competitive advantages in the market. Building on Porter's (1980) competitive forces view of organizational strategy, other theoretical perspectives have evolved to help explain strategic decision-making and the link to execution within the firm. Transaction cost economics (TCE) and the resource-based view (RBV) of the firm have sought to explain organizational decision-making processes from the perspective of achieving competitive advantage through the management of transaction-level exchanges and the assembly of strategic resources (Porter 1980; Teece et al. 1997; Williamson 1981). While TCE views competitiveness as a function of how firms manage decisions based on limited information, the bounded rationality of decision makers and the purposeful use of governance instruments (Williamson 1989), RBV argues that competitiveness comes from a firm's ability to assemble a unique set of resources that are valuable, rare, inimitable and non-substitutable (i.e., VRIN resources) (Barney 1996). When considering resources as a source of competitiveness, either through the outcomes of TCE build-buy-partner decisions or resource-based strategies of RBV, these 
complimentary theories on competitive advantage remain insufficient in explaining why firms with a similar resource base perform differently. This is the question that dynamic capabilities (DC) theory aims to address.

\section{Competitive Forces and Corporate Strategy}

The competitive forces approach to strategy and competitive advantage, highlighted by the seminal work of Porter (1980; 1985), focuses on the dimensions of developing firm-level competitive strategy in response to the industry-specific environment in which they compete. Porter's competitive forces model argues that a firm's potential profitability is based on its position relative to five industry forces: the nature of barriers to entry, the threats of substitution for the firm's offering, the extent of buyer bargaining power, the extent of supplier bargaining power, and the degree of rivalry amongst incumbents in an industry (Porter 1980). This industry-level view of strategy focuses on how a firm can increase rents by minimizing market competition through its strategic decision-making in the context of these five competitive forces (Porter 1980). However, it is argued that this view of strategy limits the role of a firm's resources and the ability to scale in response to its target markets. Specifically, research has challenged that when using a five forces assessment of industry attractiveness, the firm will begin decisions by determining which industries provide the greatest opportunity for success, and will subsequently determine the required resources (i.e., owned or acquired) necessary to compete (Teece et al. 1997). 


\section{The Transaction Cost Economics (TCE) View}

Transaction cost economics has been broadly used in academic research examining organizational strategy and the development of competitive advantage through organizational efficiency and governance in an information imperfect world (Joshi \& Stump 1999). TCE examines the dynamics of economic exchanges made by the firm, and governed through contracts that are characterized by incomplete information regarding the transaction partner and the options available (Williamson 1981). Transaction uncertainty is characterized by information asymmetry, and introduces the risk of opportunism from exchange partners (Rindfleisch \& Heide 1997) and the bounded rationality of firm decision makers (Rindfleisch \& Heide 1997). Both opportunism and bounded decision-making mechanisms have been used to examine make-buy-partner strategic decisions (Jacobides \& Billinger 2006), and in developing theory regarding the dynamics of customer collaboration within the firm (Rothwell 1977).

Fundamental to TCE is the assumption that there exists an industry-level homogeneity of firms and their resources. Proponents of organizational competencies and dynamic capabilities (DC) theory has been critical of the TCE focus on opportunism without recognizing the heterogeneous nature of firm capabilities (Kogut \& Zander 1992; Winter 2003). They argue that the difference between firm capabilities is what allows for some organizations to execute activities in a more production cost efficient way, as well as influencing their make-buy-partner decisions. 


\section{The Resource-Based View (RBV) of the Firm}

A key to firm success is the ability to create competencies that are distinctive in the markets where they compete (Learned et al. 1969). Contrary to TCE, the resource-based view of the firm argues that competitive advantage is gained when firms differentiate themselves through their heterogeneous and "sticky bundles" of resources (Teece et al. 1997). It is the collection of these resources (i.e., all assets, capabilities, processes, firm attributes, information, and knowledge) and how they are developed and maintained that impact the firm's performance efficiency and effectiveness (Dahlander \& Wallin 2006). From an RBV perspective, firms compete by investing in systems and assets that deliver either lower costs or superior products, versus investing in Porter-style deterrence to market entry by competitors (Teece et al. 1997).

Resources that contribute to competitive advantage are described as VRIN, meaning they are valuable, rare, inimitable and non-substitutable (Barney 1991). While strategy theory states that firms reach a level of resource equilibrium over time due to the mobility of those resources (Porter 1980), RBV argues that firms are in fact heterogeneous, and that the strategic resources they control are not mobile (Barney 1991). This view of "resource immobility" supports the argument that the differences that exist between competing firms can be preserved over time (Barney 1991). The literature highlights that a resource-based perspective focuses on how strategies leverage existing firm-specific assets. In response, researchers have called for more consideration to be given to managerial strategies needed for the acquisition of new skills, and the management of knowledge and expertise (Teece et al. 1997). 
The resource-based view of the firm also builds on the Porter-inspired competitive advantage research (Porter 1980; Porter 1985), arguing that firms achieve sustainable competitive advantage by recognizing their organizational strengths and weaknesses and then designing strategies based on a thorough understanding of their environment (Barney 1991; Brettel \& Cleven 2011). Counter to the competitive forces view (Porter 1985), where strategy begins by identifying and choosing attractive target industries and then determining which resources are required to execute, RBV argues that firms must start by identifying their unique resources that determine which industries present the greatest opportunity for returns (Teece 1980; Teece 1982). In addition to the complexities associated with business development, and the capacity limitations of firms quickly developing new competencies (Dierickx \& Cool 1989), a distinct difference between the RBV and Porter competitive views on strategy is RBV's recognition that certain assets are not readily tradable (e.g., tacit knowledge and experience) (Teece 1980).

\section{Criticisms of $R B V$}

$\mathrm{RBV}$ is not without its challenges from academics. A review of the various theories of competitive decision-making highlights the importance of understanding the strategic perspective adopted by the firm, industry-in or resource-out views. The role that resources play across these theories requires attention towards an understanding of the nature of competitive decisions by the firm.

While a resource-based strategy argues for achieving competitive advantage through the accumulation of valuable assets (e.g., knowledge, intellectual property) (Barney 1991), others have argued that assembling a unique bundle of resources is 
insufficient in explaining the mechanisms that are active in achieving that advantage (Teece et al. 1997). For instance, while firms may have collected many technology assets, they may still lack the necessary capabilities needed to fully benefit from these assets, and to compete (Teece et al. 1997).

RBV is a static theory described through an equilibrium-based view of the environment (Barney 1991; Barney 1996). As such, it has been challenged for providing a limited and inadequate perspective when explaining how firms maintain competitive advantage within changing and dynamic environments (Teece \& Pisano 1994). It is argued that the resource-based advantages of RBV can become limiting when faced with a changing external environment. What initially is viewed as resource-based advantages and core competencies for a firm, can soon become rigidities in the face of changing environmental conditions (Leonard-Barton 1992). It is from these recognized shortcomings of RBV that Teece and Pisano's dynamic capabilities (DC) theory (Teece \& Pisano 1994) emerged to address the gap (Barreto 2010).

Dynamic capabilities research is an extension of the resource-based view of the firm (Barreto 2010; Teece \& Pisano 1994). DC theory picks up where RBV ends by answering the static equilibrium-based model gap with theory describing an adaptive dynamic perspective of the environment and how firms respond to change (Teece 2007). It is the dynamic nature of DC theory that distinguishes its appeal from RBV (Smith 2009).

\subsubsection{Dynamic Capabilities}

Dynamic capabilities represent the firm's ability to adapt to a changing environment through the reassembly of its resources in response to the evolving needs of 
the organization. This section will discuss what dynamic capabilities are, the hierarchical nature of dynamic and organizational capabilities, and the relationship of context to their development. As dynamic capabilities are closely related to research in strategy and competitive advantage, dynamic capabilities and their impact on performance is a central question in the literature, which will also be briefly discussed in this section.

\section{What are Dynamic Capabilities?}

Dynamic capabilities view (DCV) theory is an area of study that continues to gain interest and attention in management research (Barreto 2010; Easterby et al. 2009). Based on the seminal work of Teece et al. (1997), DCV builds on the resource-based view of the firm (Barney 1991; Barney 1996) by shifting from a focus on how organizations assemble strategic resources in static environments towards how these resources are used in establishing competitive advantage for the firm in ever-changing environments. Dynamic capabilities theory focuses on the specific differences between resources as available assets solely controlled by the firm, and the organization's "capabilities" which refer to its capacity to deploy those resources using processes that achieve desired outcomes (Amit \& Schoemaker 1993). This perspective on organizational capabilities contributing to competitive advantage has resulted in dynamic capabilities research being focused on issues of strategic growth, renewal, and adaptation of the firm (Smith 2009). 


\section{Dynamic Capabilities - Definition}

Teece et al. (1997) first defined dynamic capabilities generally as:

"the firm's ability to integrate, build, and reconfigure internal and external competencies to address rapidly changing environments."

It is likely that this initial broad statement describing dynamic capabilities was intended to be general by design, providing room for contribution from the research community. As a result, it has served as a seed for many definitions to follow in the literature. However, a byproduct from the initial simplicity of the definition is the absence of a single predominant definition of dynamic capabilities today (Barreto 2010).

Eisenhardt and Martin (2000) extended Teece et al.'s (1997) definition from a focus on firm processes and the use of resources towards "achieving new resource configurations as markets emerge, collide, split, evolve, and die." Teece (2000) further emphasized the intention of dynamic capabilities in seizing opportunities "quickly and proficiently." Zollo and Winter (2002) added that dynamic capabilities are "learned and stable" patterns of activity that modify operating routines towards improved effectiveness. Winter (2003) alternatively describes dynamic capabilities as operating to "extend, modify, or create ordinary capabilities" in the firm. Other definitions of dynamic capabilities further described the link between firm actions to the desired outcomes envisioned by principal decision makers (Barreto 2010; Zahra et al. 2006).

Teece (2007) revisited and revised the definition of dynamic capabilities to include a more robust description associating the firm's external strategic sensing capabilities to competitive action: 
"dynamic capabilities can be disaggregated into the capacity (a) to sense and shape opportunities and threats (b) to seize opportunities, and (c) to maintain competitiveness through enhancing, combining, protecting, and when necessary, reconfiguring the business enterprises in tangible and intangible assets.

Teece's (2007) definition of dynamic capabilities will be used in this study, as it reflects the key considerations of the phenomenon related to co-creation of innovation.

\section{Dynamic Capabilities - Purpose and Role}

Dynamic capabilities describe the nature of firms that constantly explore how to best integrate, reconfigure, renew and re-create their resources and capabilities, and endeavour to reconstruct their core capabilities towards achieving competitive advantage (Wang \& Ahmed 2007). Dynamic capabilities can represent processes that are either standalone or embedded within other processes (Wang \& Ahmed 2007). In this context, the term "capabilities" refers to the firm's effectiveness in combining and deploying resources-based on both their explicit and tacit knowledge (Amit \& Schoemaker 1993). Knowledge management theory would suggest that when considering the nature of tacit knowledge that dynamic capabilities are firm specific and are developed over time (Amit \& Schoemaker 1993).

Along with the broad range of definitions, there also exists some variation on the perceived role of dynamic capabilities in the firm. Teece et al. (1997) initially describe the primary purpose of dynamic capabilities as helping organizations to "address rapidly changing environments." Others expand the purpose of dynamic capabilities to include a firm's objectives of growing proficiency in creating or addressing market change (Eisenhardt 1989), improving organizational effectiveness (Zollo et al. 2002), and as a way 
to achieve competitive advantage through purposeful changes made in the combination, distribution, and use of firm resources (Helfat \& Winter 2011).

Dynamic capabilities have been described in many ways in the literature. Dynamic capabilities have been referred to as capacities, liabilities, processes, or routines (Teece 2000; Winter 2003; Zahra \& George 2002). They have also been described as specific and identifiable processes (Eisenhardt \& Martin 2000), that can be conducted in both an acceptable and repeatable manner (Barreto 2010). Dynamic capabilities are described as being learned and stable patterns of aggregated activity (Zollo et al. 2002), which are expected to evolve with changes in market dynamics (Eisenhardt \& Martin 2000). This evolution of dynamic capabilities can range from the simple acquisition of new knowledge for basic context specific processes, to developing advanced analytical and repeatable routines (Eisenhardt \& Martin 2000). The discussion on what dynamic capabilities are is further supported by a framework provided by Teece et al. (1997) which presents a common approach in defining these types of capabilities. The framework states that dynamic capabilities can be described along seven dimensions: their purpose, their nature, the specific role they play, the relevant context in which they operate, the capability creation mechanisms, the related assumptions on the heterogeneity of capabilities, and the resulting outcomes.

Overall, dynamic capabilities focus primarily on the change of components that are internal to the firm (Barreto 2010), ranging across various elements of the organization including its resources (Zahra et al. 2006), operating routines (Zollo \& Winter 2002), and other dynamic capabilities (Eisenhardt \& Martin 2000; Teece et al. 1997). The hierarchical 
position of the various types of dynamic capabilities also provides insight into the role they play towards achieving competitive advantage.

\section{Dynamic Capabilities and the Hierarchy of Capabilities}

Firms possess capabilities that are described as either organizational or dynamic in nature. The literature suggests that dynamic capabilities not only vary in the role they play within the firm relative to organizational capabilities, but that capabilities coexist within a hierarchy based on the level at which they contribute to competitiveness. There is debate as to whether dynamic capabilities are a specific type organizational capability. Wang and Ahmed (2007) state that dynamic capabilities represent the ultimate set of firm capabilities that drive competitive advantage, as opposed to being a subset of the total group of capabilities that a firm possesses, as suggested by Teece et al. (1997). In general, the literature supports dynamic capabilities as being distinctly different from the concept of organizational capabilities.

Winter (2003) introduces the concept of organizational capabilities based on the broader concept of organizational routines. Organizational capabilities are described as "high-level routine(s) (or collection of routines) that, together with implementing input flows, confers upon an organization's management a set of decision options for producing significant outputs of a particular type" (Winter 2003). This refers to routines that come through learned behaviour, patterns, repetitious activities based on tacit knowledge, and that are focused on specific objectives (Winter 2003). Ad hoc problem-solving or generalpurpose processes do not qualify as routines in this context (Winter 2003). Based on this 
definition, dynamic capabilities contrast organizational capabilities primarily through their focus on change for the firm (Collis 1994).

Dynamic capabilities and firm resources have been described as having a hierarchical order, as they relate to a firm's competitive advantage (Wang \& Ahmed 2007; Winter 2003). "Zero-order" elements in the hierarchy refer to resources as a potential source of competitive advantage based on their VRIN attributes (Wang \& Ahmed 2007). Winter (2003) describes zero-order elements as locally defined capabilities that a firm possesses when at equilibrium, representing what is needed to "how (they) earn a living now." As firm attributes and environment are not persistent over time, zero-order attributes cannot provide sustainable competitive advantage for the organization on their own.

Capabilities that are described as "first-order" elements, represent the firm's ability to distribute resources towards achieving specific outcomes (Wang \& Ahmed 2007). These are described as capabilities that make changes to products, the production process, the scaleability of an organization, or to the customers/markets which are not served by zeroorder capabilities (Winter 2003).

"Second-order" or "higher-order" capabilities represent strategic capabilities of the firm at a specific point in time, representing the ability to integrate resources and capabilities in support of a firm's strategic direction (Wang \& Ahmed 2007). Higher-order capabilities have been described as those that are used to modify operating routines (Zollo et al. 2002). These higher-order capabilities represent strategic activity that often involves investments in organizational learning supporting strategic processes that facilitate the creation and modification of dynamic capabilities (e.g., alliances, collaboration, acquisitions), and are often involved in changing the game for the firm (Winter 2003). 
Higher-order capabilities have also been described as holding the potential to integrate and coordinate individual capabilities of the firm that in actuality, do not operate as distinctively standalone (Lichtenthaler \& Lichtenthaler 2009; Wang \& Ahmed 2007). As the environment changes, these capabilities are no longer strategic and present the potential of becoming "core rigidities" (Leonard-Barton 1992). This results in a "competency trap" that can result in firms maintaining an ever-decreasing list of relevance or competencies (Teece et al. 1997).

The notion of organizational and dynamic capabilities as part of a hierarchy suggests that firms must develop capabilities of various types and at various levels of the organization. These capabilities in turn support both the execution of operational activity and facilitate effective change in dynamic environments.

\section{Dynamic Capabilities and Context}

Another common theme in the dynamic capabilities literature is the discussion of how capabilities are created and the role that context plays. Learning processes and the mechanisms for the creation of dynamic capabilities has been central to this discussion. While some researchers have examined the importance of developing learning processes and the mechanisms that enable organizations to evolve in changing environments (Zollo \& Winter 2002), others have suggested that research must also focus on the mechanisms captured through the repeated practice of processes, learning from past mistakes, and developing ways to accelerate new learning (Eisenhardt \& Martin 2000). Discussions on the creation of capabilities have also included the role of evolving dynamic capabilities 
through processes such as trial and error, improvisation, and imitation of others (Zahra et al. 2006).

The relevance of context and environments as they relate to dynamic capabilities are also debated. One group of researchers argue that dynamic capabilities are best suited in highly dynamic environments (Teece et al. 1997), while others argue that dynamic capabilities are relevant across a broader range of environmental conditions (Eisenhardt \& Martin 2000). Similarly, there are varying perspectives on whether dynamic capabilities are firm specific in nature. Teece et al. (1997), extending on the resource-based view the firm, believe that dynamic capabilities are restricted and unique to a specific organization. Eisenhardt and Martin (2000) argue that dynamic capabilities can be viewed as best practices, and as such, they can exist across organizations. However, the notion of best practices is disputed by some research. It is argued that while there are some dynamic capabilities that may demonstrate commonality across organizations, they are not exact, and are more firm specific when the details of their execution are considered (Barreto 2010).

\section{Dynamic Capabilities and Performance}

Dynamic capabilities are intended to help drive competitive differentiation, with a resulting impact on firm performance. There are varying views in the literature on how and to what degree dynamic capabilities influence performance.

Early research expected a direct relationship between a firm's dynamic capabilities and their impact on performance (Teece et al. 1997). To some degree, this connection to performance was further extended to include the impact on a firm's overall survival (Zollo 
$\&$ Winter 2002). Others argue that dynamic capabilities in and of themselves are insufficient to explain long-term competitive advantage. Improved strategic position comes from how resources are ultimately reconfigured to compete versus simply the existence of the dynamic capability (Eisenhardt \& Martin 2000). Firms that can use their dynamic capabilities towards change, sooner and more effectively than their competition, will benefit from a better competitive position in the market (Eisenhardt \& Martin 2000). Wang and Ahmed (2007) argue that firm performance is indirectly impacted by dynamic capabilities through their relationship to firm strategy and the ability to create capabilities in the firm. This is further supported by Zahra et al. (2006), who argue that the indirect influence of dynamic capabilities on performance is based on the degree to which these capabilities can drive change in organizational routines and lower-level capabilities.

\subsubsection{Knowledge Management}

Inbound open innovation (iOI) and co-creation of value activities are dependent upon the firm's capacity to establish channels to external sources of new knowledge and expertise, and convert knowledge that has been successfully collected into assets supporting the innovation process. The knowledge management and learning literature continue to play a significant role in understanding the relationship between strategic engagements and knowledge management in the firm, and the function of knowledgebased capabilities in establishing sustainable competitive advantage. This section will discuss the knowledge-based view of the firm, the role of knowledge management in the organization, and the link between knowledge management and dynamic capability 
theories. This will include a discussion on the concept of absorptive capacity, and its role as a dynamic capability connecting the firm to external sources of knowledge.

\section{Knowledge-Based View of the Firm}

Penrose's theory of the firm (Penrose 1959) argues that the growth of an organization is dependent on its ability to reorganize its resources, including its tangible assets and people, to improve both productivity and managerial capacity (Macpherson et al. 2004). While the theory of the firm has been referenced throughout research, the literature does not contain a single multipurpose theory to be drawn upon (Grant 1996). By example, the resource-based view of the firm builds on Penrose by explaining how firms can manage their VRIN resources towards achieving sustainable competitive advantage, and a goal of superior firm performance (Barney 1996).

Grant (1996) presents the knowledge-based view of the firm as another lens to explain organizational behaviour. KBV states that knowledge is at the centre of establishing a firm's competitive advantage, and as such, the primary purpose of the organization is to integrate knowledge. In this theory, the production and integration of all knowledge is the core of organizational excellence (Macpherson et al. 2004). Competitiveness of the firm is dependent upon its ability to develop core competencies for sourcing and exploiting the knowledge needed by the organization when it is needed (Macpherson et al. 2004). From this perspective, the primary role of management is to build organizations that can create, manage and integrate knowledge (Nonaka \& Takeuchi 1995). Managers are expected to define processes for knowledge integration and the organization of labour within the firm (Macpherson et al. 2004). The success of the firm 
using the created knowledge is dependent upon its ability to ensure that the right information, is available at the right time, in the right form, for the users that need it (Nielsen 2013).

The knowledge-based view also examines the boundaries of the firm as they relate to sources of knowledge. A central argument of the theory is that the existence of the firm is required because it can more efficiently perform transactions than the marketplace

(Penrose 1959). With the emergence of open innovation strategies (Chesbrough 2003) and recognition that inter-organizational collaboration is an important source of new knowledge in the innovation process (Nonaka \& Takeuchi 1995), KBV raises a question as to what the boundaries of the firm must be if the need is to access specialized knowledge that does not exist within the firm (Grant 1996).

\section{Knowledge Management Within the Organization}

Grant's (1996) representation of the knowledge-based view of the firm highlights the attributes that are needed by an organization in its management of knowledge when seeking to build and maintain its competitive advantage. This includes developing capabilities that support the firm's effective transfer of knowledge, skill in establishing collaborative relationships for acquiring knowledge, and the ability to realize value from that new knowledge.

The knowledge-based view of the firm highlights the importance of transferability of knowledge both within the organization and between firms, and must possess the capacity to aggregate knowledge. Knowledge aggregation refers to the efficiency with which knowledge can be shared within the organization, which in turn is limited by the 
firm's ability to receive information. This is consistent with the seminal work of Cohen and Levinthal (1990) on absorptive capacity. The ability to efficiently transfer knowledge suggests that organizations must develop a level of specialization in the creation and management of knowledge for the firm, with individuals developing expertise in specific areas of the knowledge management landscape (e.g., knowledge creation, acquisition, storage) (Grant 1996).

The knowledge-based view of the firm encourages organizations to consider the role of collaboration processes both inside and outside the firm (Grant 1996). This is particularly relevant in open innovation strategies involving the co-creation of innovation (Prahalad \& Ramaswamy 2004). Choices for collaboration outside the boundaries of the firm are managerial decisions, and require the development of mechanisms that support the integration of specialized knowledge between individuals and organizations, and across firms (Chesbrough 2007; Grant 1996).

Finally, KBV emphasizes the importance of the appropriability of knowledge, which is described as the ability of the owner of a resource to receive a return of equal value for its exchange (Grant 1996). Both explicit and tacit knowledge presents the firm with questions as to whether there exists value that can be extracted from knowledge available in the market, and whether transactions of this knowledge can be made in a form where the value can be realized (Enkel et al. 2005). Aside from legal protections provided by patents and copyrights, Grant (1996) argues that knowledge lacks clear property rights, which introduces ambiguity on the question of its ownership.

At its essence, knowledge management focuses on the building and evolution of a knowledge store for the organization, and all the activities related to exploiting that 
information effectively (Dierickx \& Cool 1989). This involves the flow of knowledge in and out of the firm, including new and existing organizational knowledge (Nielsen 2013). Knowledge management activities include all the processes needed from the creation of knowledge to its exploitation (Nielsen 2013). Based on a review of the knowledge management literature (Alavi \& Leidner 2001; O'Dell \& Grayson 1998; Teece 1998), Neilsen (2013) presents a framework of eight types of core knowledge management activities: knowledge creation, acquisition, capture and articulation, knowledge assembly, knowledge sharing, knowledge integration and recombination, the leveraging of knowledge, and the application of knowledge in the firm.

For each of these activity types, there are distinct considerations across firms when acquiring external knowledge through collaboration with stakeholders, such as users and customers. Collaboration and co-creation of innovation with external stakeholders look to create new knowledge for the firm. To this end, knowledge creation activity promotes the importance of formalized knowledge development (e.g., formal projects and programs) (Nielsen 2013). While the value in creating knowledge through external relationships is recognized (Chesbrough 2006), acquisition of knowledge from external partners can be a challenge due to the nature of knowledge (Gils \& Zwart 2004), the dynamics of relationships between the firm and its external stakeholders (Szulanski 1996), and the firm's readiness and ability to receive, internalize, and act on the information (Cohen \& Levinthal 1990; Zahra \& George 2002).

When acquiring knowledge through a co-creation of innovation model, the richest external expert knowledge is often tacit in nature, presenting challenges for the firm trying to access this information for its innovation process (von Hippel 1978). While tacit 
knowledge is described as offering significant potential as a source of new knowledge for the innovation process, its value can only be realized through the capture and articulation of this knowledge in a form that the firm can use (Zollo \& Winter 2002). Articulating knowledge for the organization requires some form of codification to allow for the sharing of information more readily (Boisot 1998). However, with an increased ease in knowledge articulation, it follows that knowledge codification then presents the risk of information being more easily transferable between firms. This potentially impacts the competitive advantage it was initially intended to provide (Boisot 1998). Knowledge management creation activities also require the development of meta-knowledge, that is used to track and identify what sources of knowledge are available and where they reside in the firm (Nielsen 2013). As an example, this can include a mapping of skilled resources within the organization that are best suited for a collaboration project with an external stakeholder, and in building knowledge across the organization (Hansen 1999).

Collaborative co-creation of innovation initiatives requires the firm to bring together varied and appropriate skill sets. From the perspective of knowledge management activities, this refers to the firm's effectiveness in assembling knowledge, which reflects their ability to bring together the right collection of knowledge-based resources towards delivering a specific objective (Nielsen 2013). Similarly, in the context of co-creation activity, this relates to the firm's ability to assemble complementary skill sets towards collaborative innovation activity and the commercialization of new products (Teece 1986). From a dynamic capabilities perspective, knowledge assembly is key in the development of organizational capabilities that bring together knowledge resources for creating new knowledge-based capabilities, increasing awareness across the firm, identifying knowledge 
gaps and triggering new knowledge to be produced (Nielsen 2013). However, it is insufficient to simply bring knowledge resources together. Knowledge management activities must also focus on the integration and recombination of specific resources towards a more synergistic and coordinated set of activities and flow of information (Zahra \& George 2002). These synergy creating activities are central in the development of the firm by leveraging its pool of integrative resources (Teece et al. 1997; Winter 2003), establishing new organizational capabilities (Grant 1996) or through the introduction of platforms in new competitive areas (Teece et al. 1997). This level of integration can be seen as a new source of knowledge for the firm based on co-creation activities driven by experimentation and the combination of different resources for the first time (Nielsen 2013). Collaborative innovation methods of knowledge acquisition often use these practices as the firm explores new sources of knowledge with experts external to the organization.

Effective co-creation of innovation activities are dependent upon the firm's ability to share new knowledge across the units of the organization that need the insight (Nielsen 2013). This includes processes for capturing knowledge across the collaboration process, clearly and efficiently transferring it within the organization, and the relevant units of the organization being ready and able to receive the knowledge (Szulanski 1996). This sharing of information can be limited due to the "sticky" nature of knowledge (von Hippel 1994). Szulanski (1996) explains that there are three major issues that typically impact effective knowledge sharing: the lack of absorptive capacity of the recipient, causal ambiguity regarding the knowledge being shared, and the relationship dynamics between the sender and the recipient. In general, the primary responsibility of a knowledge-based firm is to 
help facilitate effective knowledge sharing in the organization, which can be achieved through development of the capabilities and culture needed to support this objective (Nielsen 2013). The development of knowledge acquisition methods and related dynamic capabilities has the potential to contribute to this end.

Finally, it is the firm's ability to leverage, apply and exploit the knowledge that it collects, creates, and shares, that will determine whether the knowledge contributes to its performance and competitive advantage. Grant (1996) reminds us the knowledge-based view of the firm argues that competitiveness rests in the firm's ability to apply knowledge, not simply the ability to create new knowledge in and of itself. From a knowledge management perspective, the firm must look for ways to exploit integrated knowledgebased resources as broadly as possible (Hamel \& Prahalad 1994; Wang \& Ahmed 2004). This can include the focus on innovations for new markets as well as in the creation of strategic alliances where the firm combines its resources with external sources (Gils \& Zwart 2004)

\section{The Knowledge-Based View and Dynamic Capabilities}

A knowledge-based view of the firm describes dynamic capabilities as an integrated set of knowledge management activities that change, renew, and exploit the firm's knowledge-based assets (Nielsen 2013). Competitive advantage is dependent upon a firm's dynamic capabilities used in the configuration of knowledge within the organization (Macpherson et al. 2004). While it has been expressed that the literature is vague on the nature of dynamic capabilities (Wang \& Ahmed 2007), studies have identified their 
importance in areas such as product development routines, strategic decision-making, resource allocation, and acquisitions (Eisenhardt \& Martin 2000).

Arguments in favour of the relationship between dynamic capabilities and knowledge mechanisms are well-established (Nielsen 2013; Zollo \& Winter 2002), as is the importance of the link to inter-firm relations (Macpherson et al. 2004; Rothwell \& Dodgson 1991). When examining these knowledge relationships and interactions, both within and outside firm boundaries, an understanding of how knowledge is transferred is necessary (Macpherson et al. 2004). Central to knowledge relationships is the focus on the knowledge-based firm's approach to encouraging knowledge sharing routines between entities (Dyer \& Singh 1998; Nonaka \& Takeuchi 1995).

When considering the relationship between dynamic capabilities and knowledge management, one must consider the role that dynamic capabilities play in developing core activities such as organizational learning (Winter 2003; Zollo \& Winter 2002), processes for exploration and exploitation of knowledge (Benner et al. 2003), and activities involving knowledge transfer and developing absorptive capacity (Zahra \& George 2002). These are all capabilities necessary in the co-creation of innovation and the methods of knowledge acquisition.

Research has shown that a firm's ability to build strong working relationships with external stakeholders (e.g., customers, suppliers) beyond standard market transactions, strengthen their strategic core (Nesheim 2001). A firm's ability to improve the depth, quality, and range of inter-organizational relationships and networks may be key to their ability to access new knowledge (Conway \& Steward 1998). In addition, with the increasing specialization of knowledge in the marketplace, there is an even greater need 
for firms to establish external relationships that provide access to new knowledge (Brusoni et al. 2001). The potential for a firm to create competitive advantage extends beyond an ability to develop knowledge resources, towards a dependency upon their ability to establish the necessary strategic relationships with key organizations (Dyer \& Singh 1998). The argument in favour of establishing unique collaborative relationships provides support for the development of knowledge resources and dynamic capabilities. These knowledge resources are achieved through the creation of new product knowledge and integrated communication structures specific to establishing these strategic relationships (Macpherson et al. 2004).

Collaboration and co-creation of innovation are dependent upon well-established dynamic capabilities within the firm, designed to achieve high levels of integration and to fully exploit strategic relationships (Croom 2001). In turn, organizational capabilities are both created and renewed through the influence of different dynamic capabilities (Winter 2000; Zollo \& Winter 2002). Applying a firm's knowledge management and dynamic capabilities towards co-creation of innovation requires that managers develop ways of acquiring new knowledge through strategic relationships with external sources that provide access to additional resources for the innovation process (Chandler \& Hanks 1998; Tsoukas 1996). Developing capabilities, such as absorptive capacity, ensures that the firm not only has the ability to encourage information sharing across the organization, but also its exploitation in the creation of new innovation-based value (Macpherson et al. 2004). Firms must develop practices that use knowledge more effectively as part of organizational learning and in the creation of the necessary core competencies (Powell et al. 1996). 
It is apparent that theory regarding knowledge mechanisms and dynamic capabilities are intertwined. In the context of co-creation of innovation, a firm develops dynamic capabilities based on its previous experience in managing projects from past interorganizational relationships, joint ventures, and acquisitions (Zollo \& Winter 2002). Nielsen (2006) presents KM-based dynamic capabilities, which encapsulate the primary knowledge management activities: knowledge development, knowledge (re)combination, and knowledge use. These three dynamic capabilities cover the range of knowledge management activities associated with the development of strong inter-organizational relationships focused on external knowledge acquisition and the co-creation of innovation. The "knowledge development" dynamic capability consists of activities associated with knowledge creation, knowledge acquisition, and knowledge capture. The "knowledge (re) combination" dynamic capability includes the activities of knowledge assembly, knowledge sharing, and knowledge integration. Finally, the "knowledge use" dynamic capability focuses on the activities needed to benefit from the information collected and includes knowledge leverage and knowledge exploitation activities.

\subsubsection{Absorptive Capacity as a Dynamic Capability}

Knowledge management and organizational learning play an important role in the innovation process (Lichtenthaler \& Lichtenthaler 2009). However, the effectiveness of acquisition of new knowledge through co-creation activity is insufficient to explain the performance of inbound open innovation strategies. It is necessary to understand the firm's ability to receive and exploit the knowledge that is acquired, and the skills and mechanisms required to do so on an ongoing basis. Absorptive capacity (ACAP) has been well 
documented in the literature as an important aspect contributing to the success of open innovation (Newey 2010), and has been applied in a range of research fields including organizational learning, strategic management, innovation management (Zahra \& George 2002), and open innovation (Lichtenthaler \& Lichtenthaler 2009; Vanhaverbeke et al. 2008). Absorptive capacity has been conceptualized as a set of dynamic capabilities that firms require to effectively exploit the knowledge acquired from external sources (Lichtenthaler \& Lichtenthaler 2009; Zahra \& George 2002).

The concept of absorptive capacity was first introduced in the seminal work of Cohen and Levinthal (1990), who describe ACAP as a capability that enables organizations to innovate. They argue that the firm's innovative capability is dependent upon its ability to identify, acquire, assimilate, and apply external knowledge into their new offerings (Cohen \& Levinthal 1990). A firm's absorptive capacity is dependent upon prior knowledge and the diversity of its previous experience (Cohen \& Levinthal 1990). The most widely used definition of absorptive capacity is the one first presented by Cohen and Levinthal (1990), describing ACAP as "the ability to value, assimilate, and apply new knowledge." Emphasized is the cumulative nature of ACAP, specifying that ongoing investment in developing absorptive capacity makes it easier for future development of absorptive capacity. Cohen and Levinthal (1990) argue that organizational investment in $R \& D$ is necessary to the development of their absorptive capacity. They state that without this form of investment, firms may be faced with an inability to realize the value of external information in areas where they have not previously invested in developing skills (Cohen \& Levinthal 1990). 
Other researchers have also introduced definitions of absorptive capacity building on Cohen and Levinthal (1990). Alternate versions of ACAP have emphasized the need for a broad array of skills in the firm to access external tacit knowledge, and the need for developing learning capabilities and problem-solving skills (Kim 1998). The various definitions of absorptive capacity shared a common view of the multi-dimensional nature of the concept.

Zahra and George (2002) further contributed to the development of absorptive capacity by re-conceptualizing the concept to distinguish between two forms of absorptive capacity, "potential" and "realized" capacities. In their definition of absorptive capacity, they link the concept to organizational dynamic capabilities. Zahra and George (2002) define absorptive capacity as "a set of organizational routines and processes by which firms acquire, assimilate, transform, and exploit knowledge to produce a dynamic organizational capability." In their view, absorptive capacity is a single dynamic capability that is embedded within the firm's routines and processes, with four sub-capabilities that build upon each other forming ACAP. It is a combination of potential absorptive capacities (i.e., acquisition, assimilation) and realized absorptive capacities (i.e., transformation, exploitation) that drive competitive advantage by reinforcing a firm's strategic flexibility, innovation, and performance (Zahra \& George 2002). These two branches of absorptive capacity address the capabilities required in acquiring new knowledge and subsequently exploiting it in the form of commercialized new offerings. Similarly, Lane et al. (2006) also treat absorptive capacity as a single dynamic capability, and provide an alternate conceptualization of ACAP in which they differentiate between exploratory, transformative, and exploitative learning processes. However, while Zahra and George 
(2002) have represented absorptive capacity as a multidimensional construct, it is argued that empirical studies have not yet developed or validated this position (Wang \& Ahmed 2007).

Lichtenthaler and Lichtenthaler (2009) further develop the linkage between absorptive capacity, dynamic capabilities, and open innovation. They present a dynamic capabilities-based framework representing the critical capacities needed by the firm for managing internal and external knowledge within an open innovation context. They identify absorptive capacity as a necessary dynamic capability needed for knowledge exploration along with the processes involving the acquisition of external inter-firm knowledge. For this study, absorptive capacity will follow the approach used in the Lichtenthaler and Lichtenthaler (2009) framework, which treats absorptive capacity as a single dynamic capability.

\subsection{Co-Creation of Innovation and the Methods of Knowledge Acquisition}

This section discusses the literature examining strategic and innovation management, and the topic of co-creation of innovation. There is significant research examining open innovation strategy, its role in establishing sustainable competitive advantage and enhancing firm performance. The research landscape in this area is broad, with open innovation strategies touching all levels of management research. As has been established, the knowledge management and dynamic capabilities literature have been consistent in expressing the importance of sourcing external knowledge in the innovation process, and the need for requisite skills and capabilities to realize the benefit from this knowledge. 
Research in the areas of open innovation and co-creation of innovation will be examined. Central to both research themes are the topics of user types, knowledge types, and the knowledge acquisition methods used in engaging with external sources of expertise. Research in each of these areas will be discussed. This section will close by framing the anatomy of knowledge acquisition methods, which is used in forming the conceptual framework that guides this study (see Chapter 3, Figure 3.2).

\subsubsection{Open Innovation}

Open innovation (OI)research examines the emerging innovation strategy model that promotes the importance of firms reaching beyond the traditional boundaries of closed innovation practices, to their environment, in pursuit of expertise and resources needed by the organization for new innovation. This section will discuss the role of open innovation strategies, adoption of OI practices, and the impact of knowledge gaps on the firm. Inbound open innovation practices will be examined as the primary area of OI research to date, which includes co-creation of innovation initiatives within its scope.

\section{Knowledge Gaps in the Firm}

Innovation continues to be at the centre of a firm's ability to compete in the marketplace (Bruce et al. 1995). The objective of the firm is to innovate and become a leader in delivering a clear and compelling customer value proposition (Porter 1980). It is the firm's ability to continue to develop new ideas that create value for the marketplace that ensures its ability to maintain its competitive position, drive growth, and deliver on 
stakeholder expectations (Chesbrough 2007). This requires the ability to identify, capture, and translate market needs into commercialized offerings (Cooper 1980).

Markets have seen a shift in the customer-supplier balance of power. Through the unprecedented availability of information to customers, there has been a resulting increase in the demand for offerings that have a closer fit to their business needs (IDC 2013). Users are also looking to become more involved in the innovation process working closely with their product and service providers (IDC 2013).

When considering capabilities of market-driven organizations from a resourcebased perspective, it is argued that firm resources and capabilities become fixed due to an inside-out perspective (Day 1994). This limits a firm's ability to respond to customer and market needs and in developing new capabilities (Day 1994). Often, this results in firms being limited in the markets they can serve effectively when responding to new competitive threats (Christensen et al. 2001). Firms realize that they cannot possess all the expertise needed to drive new innovation, and that they must find new strategies to bridge their information gaps with new thinking (Day 1994).

Many firms are challenged in sourcing the necessary insight needed to drive new innovation using traditional methods (Brockhoff 2003; Leonard-Barton 1990; von Hippel 1978). It is argued that firms that adopt a market orientation are found to be more innovative than those that do not (Bergen et al. 1992). This is in part due to the unifying focus that a market orientation provides across the firm (Atuahene-Gima 1995; Kohli \& Jaworski 1990). Market-oriented organizations must look to gather information about customer needs and have the processes in place to share that information across the firm in a way that the various functions respond to the signals collected (Kohli \& Jaworski 1990). 
The ability of an organization to connect its market orientation with a customer collaboration strategy will help guide them to a greater firm-level understanding of customer needs (Kohli \& Jaworski 1990).

To address the innovation-knowledge gap, an organization must look outside their traditional boundaries to identify opportunities where expertise can be brought into the firm, and the firm's expertise can be shared with the market through partnership (Chesbrough 2006). Open innovation strategies look to address these information needs through updated business models that expand firm boundaries to their environment with the goal of enhancing innovation effectiveness (Chesbrough 2006).

\section{Open Innovation Strategy}

An often cited definition of open innovation describes "the use of purposive inflows and outflows of knowledge to accelerate internal innovation, and to expand the markets for external use of innovation, respectively" (Chesbrough 2006b).

The concept of open innovation has grown from the seminal work of Chesbrough (2007). At its essence, open innovation argues that firms must adopt strategies that open up the traditionally closed boundaries of the firm to allow for the flow of knowledge, resources and innovation with their external environment (Huizingh 2011; Rigby \& Zook 2002), as a means of expanding value creation for the organization (Chesbrough 2007). Open innovation looks for new sources of innovation and paths to market (Dahlander \& Wallin 2006), expanding beyond closed innovation models where the firm is a source of all innovation and maintains itself as the locus of innovation control (Piller et al. 2011). 
An open innovation strategy is considered to be the third level of innovation management, with a focus towards achieving a systematic approach to innovation within an open system (Van der Meer 2007). Adopting such a strategy requires a fundamental shift in the firm's view towards engaging with its environment, and its concepts on the control of innovation (Chesbrough \& Appleyard 2007). The state of the market is such that the question of adoption of an open innovation strategy has become less about whether to, but rather how to best leverage this innovation model to contribute to a firm's competitive advantage (Van der Meer 2007).

Similarly, research has also examined strategies related to knowledge acquisition as part of a firm's R\&D practices (Cohen \& Levinthal 1990; Zahra \& George 2002). Findings have emphasized that before embarking into collaboration with a goal of acquiring new knowledge, firms must ensure that they decide upon an open or closed innovation strategy and a desired governance model (Pisano \& Verganti 2008). Others have presented collaboration models as a systematic progression into new markets based on the development of both offensive and defensive strategies that depend on establishing deep working relationships with the target partners (Selden \& MacMillan 2006). Finally, collaboration models have also been presented with a risk management focus, proposing strategies based on varying degrees of customer involvement in the innovation process (Ogawa \& Piller 2006).

The literature has presented various taxonomies representing open innovation strategies. These have included strategies that map methods of innovation to classes of innovation (Chesbrough \& Crowther 2006), classifying degrees of openness based on the nature of open innovation and the role of pecuniary elements (Dahlander \& Gann 2010), 
and a model which maps open innovation strategies based on sources of information and the firm objectives (Luthje et al. 2003).

Open innovation addresses both outbound open innovation (oOI), which speaks to the outflow of ideas to the market, and inbound open innovation (iOI) which focuses on the inflow of knowledge in support of the firm's strategy (Chesbrough 2006). Studies examining the adoption of open innovation strategies have found that inbound strategies dominate, with firms looking to address gaps in their portfolios by searching for external knowledge in support of radical innovation objectives (Chesbrough \& Crowther 2006; Huizingh 2011).

\section{Adoption of Open Innovation Strategies}

With open innovation theory still in the early stages of its development (Trott \& Hartmann 2009), there has been an increasing number of studies reporting the rise in the adoption of open innovation models in practice (Van der Meer 2007). Firm innovation strategies are not limited to an either-or decision when selecting open or closed innovation models. Firms often consider a blended form of closed and open innovation as part of their overall innovation strategy (Chesbrough 2003; Jaworski et al. 2000). Chesbrough and Appleyard (2007) stress that open innovation initiatives must be informed by traditional business strategy for the new strategies to be successful.

Adoption of open innovation models is being reported across industries (Christensen \& Bower 1996; Christensen et al. 2001; Van der Meer 2007), with varying rates of uptake (Chesbrough \& Crowther 2006). Industries favouring open innovation models are characterized by higher levels of innovation intensity, lower economies of 
innovation, a higher need for cumulative innovations, and in markets with significance volatility (Rigby \& Zook 2002)

Finally, adoption of open innovation has shown that even with studies reporting high levels of perceived value seen in OI models, a majority of firms remain focused on closed innovation models in practice with an intention to move towards open innovation. This suggests there is a middle-ground that organizations will pass through, where they look to maintain control associated with their traditional models while opening the boundaries of the firm beyond what their traditional models have allowed.

\section{Inbound Open Innovation and Customer Collaboration}

Inbound open innovation (iOI) represents a subclass of open innovation scenarios where organizations use external knowledge and assets towards advancing their offerings (Chesbrough 2003). This includes activities such as acquiring new knowledge and expertise from users, collaborative relationships, licensing technologies, and buying the required expertise from outside the firm (Dahlander \& Gann 2010). Co-creation of value initiatives and other models of user collaboration within the innovation process are considered part of an inbound open innovation strategy (Chesbrough 2003).

Acquisition of specialized knowledge through collaboration with external experts is considered to be a modern form of innovation that aims to produce a positive innovation climate, through a systematic methodology, and an open system approach that engages untapped sources of information (Chesbrough 2003; Chesbrough \& Crowther 2006; Van der Meer 2007). In such relationships, the firm strives to commercialize both internal and 
external ideas by creating new pathways for knowledge exchange (Chesbrough \& Appleyard 2007).

By engaging with external sources of knowledge, it is the intention of the firm to access sources of expertise that are missing within their organization, and to increase the number of new idea "building blocks of innovation" needed to stimulate the NPD process (Rigby \& Zook 2002). At the heart of inbound open innovation activity, are knowledge acquisition methods and engagement with the right resources. Chesbrough and Appleyard (2007) stress that knowledgeable users should be treated as rare resources, for which firms must be prepared to compete to secure access to their valued expertise. As a result, broadening the net of knowledge beyond the traditionally closed and controlled boundaries of the firm is expected to enhance both the quality and variety of offerings produced by the firm (Chesbrough 2007). In turn, those innovations are further improved through "the network effect" produced by increased user demand and ecosystem development in support of the resultant superior offerings (Chesbrough 2007).

Common to inbound open innovation are methods of firm collaboration with external users as the primary sources of new expertise. This includes strategies for engaging users through both existing and potential customers in deep boundary spanning activities, all towards gaining access to knowledge and generating innovative ideas (Gales \& Mansour-Cole 1991). Firms look to engage users in a variety of ways, across processes, by defining different and new potential areas of contribution by users that will most benefit from their experience and knowledge (Brockhoff 2003; Grabher et al. 2008).

The competitiveness of a firm's offering portfolio depends on an accurate understanding of user needs (von Hippel 1976). Accessing the knowledge of the right users 
in the market can provide the rich pool of ideas and opportunity (Berthon et al. 2007), with the potential to present unique knowledge generating innovations outside the traditional arenas used by a firm's typical R\&D practices (Chatterji \& Fabrizio 2007). Engaging external users and their new-to-the-firm knowledge in the innovation process stimulates the creation of new ideas and innovations by combining and reconfiguring these insights within new offerings (Kristensson et al. 2004). Studies in NPD (Baldwin et al. 2006; Kristensson et al. 2008; Ramakrishnan et al. 2004) also support arguments for accessing external user expertise in the delivery of new offerings. They have found that offerings incorporating external knowledge are more closely aligned with market needs and user expectations, are higher quality products, and benefit from reduced time-to-market.

For this study, existing and potential customer users are defined as the external source of new knowledge and innovation with which firms engage in co-creation of innovation.

\subsubsection{Co-Creation of Innovation}

Co-creation of innovation is the process of engaging external partners in a systematic model for creating innovation based on a shared objective. This method of inbound open innovation provides a specific form of user engagement and access to external expert knowledge. This section will discuss research on the topic of co-creation of innovation, and the reported benefits and risks associated with this model. 


\section{Co-Creation of Innovation Research}

Co-creation of innovation appears in the marketing literature along with the discussion of the shifting role of the customer from traditional transactions to more collaborative relationship development with the producer firm (Prahalad \& Ramaswamy 2004). Organizations no longer focus simply on buying goods, but rather, look for offerings that deliver specific value and experience in use (Macdonald et al. 2011). Customer-firm relationships have evolved from a perspective focusing primarily on the exchange of products and tangible goods in the marketplace, to nurturing the intangible aspects of valuable relationships, with knowledge being at the centre of this new thinking (Vargo \& Lusch 2004).

In the literature, co-creation of innovation has been referred to in different terms, including "customer co-creation" (Grönroos 2013), "co-innovation" (Thomke \& von Hippel 2002), "co-production" (Rice 2002) and "co-development" (Thomke \& von Hippel 2002). Co-creation of innovation refers to the collaboration between customers and the firm in the production of valuable products and services (Anbardan \& Raeyat 2014). Roser (2013) defines co-creation as an "interactive creative and social process between stakeholders that is initiated by the firm at different stages of the value creation process." Sawhney et al. (2005) describes co-creation as a route for organizations to expand their innovation capabilities while continuing to maintain strong connections with their customers, in addition to an impact on decreasing the time and cost to market with new offerings. Co-creation of innovation has also been conceptualized as the interaction between internal and external stakeholders working towards a common goal, through 
relationships that require proper alignment, synchronization, clear boundaries and governance, and commitment (Owen et al. 2008).

It is important to note that co-creation of innovation refers specifically to innovation partners which occur during the offering consumption process, where the firm and the customer collaborate towards a product or service that is closer to what the customer desires (Anbardan \& Raeyat 2014). As a result, value is often attributed to the partnering customer's response to the delivered innovation (Kristensson et al. 2008). For this study, the definition presented by Piller et al. (2011) of co-creation of innovation in the context of inbound open innovation will be used. They describe the activity of co-creation as being centred on an idea and an active, creative, and cooperative process between the producer and its partner customers.

Co-creation of innovation remains an emerging research discipline and managerial paradigm (Piller et al. 2011) that is not yet sufficiently understood (Hoyer et al. 2010). Cocreation of value has been discussed in a broad range of related streams of literature including marketing and management theory, group decision-making, innovation and knowledge management literature (Roser et al. 2009). Co-creation in the literature is applicable both in the $\mathrm{B} 2 \mathrm{~B}$ and $\mathrm{B} 2 \mathrm{C}$ contexts, working with customers or consumers, using engagement methods that are distinctly different between these two perspectives (Roser et al. 2013). B2B co-creation is also found in the open innovation (Roser et al. 2013) and strategic alliances literature (Doz \& Hamel 1998), with studies focused primarily on cocreation as co-innovation relationships. The literature on user engagement and innovation processes also falls under the co-creation of innovation literature (Anbardan \& Raeyat 
2014). Much of the research to date in this area has been towards the shaping of the concept and defining a coherent research agenda (Bogers 2013).

The strategy literature argues that firms must engage in co-creation to renew their value propositions (Lusch et al. 1992) and to remain connected with the market and trends (Witell et al. 2011). Engaging customers through co-creation relationships provides a way to develop offerings that better fit user needs (Kristensson et al. 2004). Research has reported the adoption of co-creation processes by organizations towards developing future products and strengthening their business models in pursuit of realizing a competitive advantage in the market (Prahalad \& Ramaswamy 2004). Co-creation of innovation occurs when external stakeholders, such as customers, interact directly with the producing firm in their product development processes, which includes playing an active role in defining the value of what is being delivered in the offering (Sheth \& Uslay 2007). Depending on the customer's attributes, their involvement will vary across a range of activities including ideation, design, production, promotion and distribution (Sheth \& Uslay 2007). When discussing co-creation in the context of $\mathrm{B} 2 \mathrm{~B}$ collaborative innovation, it is characterized as a relationship initiated by the producing firm (Roser 2009), with processes that focus specifically on offering concept design with customers. A broader view of co-creation of value also defines stakeholders as extending beyond customers, to include employees, suppliers, partners, and competitors (Roser et al. 2013).

\section{Co-Creation of Innovation: Benefits and Risks}

While the literature is found to promote collaboration and co-creation of value processes, it also emphasizes that with the significant benefits that come from engaging 
external sources of knowledge, firms must weigh these benefits against the related tradeoffs and risks facing the firm (Pisano \& Verganti 2008).

Co-creation of value and user collaboration presents an opportunity for R\&D to bridge their gaps in knowledge and resources (Kang \& Kang 2009). Working with users in the innovation process is argued to deliver better product quality (Füller \& Matzler 2007), greater customer satisfaction (Nambisan \& Baron 2007) and an overall reduction in risk for the firm (Maklan et al. 2008). Reaching beyond the traditional boundaries of the firm provides the benefits of access to new investment, knowledge, and skills that are needed to fuel innovation strategy and development within the firm (Quinn 2000), as well as reducing uncertainty in the innovation process (Becker \& Dietz 2004). In addition to accessing new sources of knowledge (Gassmann et al. 2010), co-creation with users helps to clarify user needs, reduce the costs associated with late-stage iterations, and presents potentially motivated early buyers for the offering (Atuahene-Gima 1995; Gassmann et al. 2010; Murphy \& Kumar 1996).

Along with the benefits of open strategies, there are also risks and trade-offs recognized in the literature. In general, the processes of innovation development and collaboration, by their nature, present new uncertainty (Enkel \& Gassmann 2005). This can include uncertainty related to the firm's experience with the new offering domain, the organizational readiness and absorptive capacity for the external knowledge needed, and the risk associated with market acceptance and new offering performance (Cohen \& Levinthal 1990; Deszca et al. 1999; Leonard \& Rayport 1997). There are also the risks associated with incorporating customers into the firm's NPD process, including the user selection process, relationship building, knowledge sharing processes, and expectation 
setting (Becker \& Dietz 2004; Enkel \& Gassmann 2005; Gassmann et al. 2010; Pisano \& Verganti 2008). As much of the valuable knowledge possessed by users is tacit and "sticky" in nature (von Hippel 1978; von Hippel 1994), new complexities are introduced into the process of establishing strong inter-firm linkages, developing new knowledge acquisition competencies within the firm, and the cost of coordinating the knowledge acquisition process (Deszca et al. 1999; Leonard 2002; von Hippel 1986). As such, customer engagement in the innovation process can be complex, time-consuming, and costly (Becker et al. 2014; Enkel \& Gassmann 2005; Kang \& Kang 2009). Christensen \& Bower (1996) argue that beyond incremental innovation, the value of customer integration may be marginal due to the users' limited view of the potential offering horizon, which in turn may hinder rather than enhance the innovation process.

Researchers have been clear that there is a trade-off that firms must consider when deciding on the role that external stakeholders and users can play in the co-creation process. However, they are also clear that firms cannot expect to innovate beyond their competition without opening the boundaries of their firm to new expertise (Quinn 2000). It has been stated that any innovation strategy should be balanced for greater customer integration (Enkel et al. 2005; Rigby \& Zook 2002). The key to managing co-creation risk is in how the organization approaches the process of engaging users and accessing knowledge (Gassmann et al. 2010; Rigby \& Zook 2002).

\subsubsection{Users and Forms of Knowledge}

Co-creation of innovation and knowledge acquisition methods are dependent upon an understanding of the nature of external sources of knowledge and the role they play in 
the innovation process. This section provides a brief overview of the research discussion on stakeholder knowledge and the different forms of knowledge types.

\section{External Stakeholder Knowledge}

Fundamentally, co-creation of innovation emphasizes reaching outside the traditional boundaries of the firm in search of knowledge and expertise that will support innovation objectives. Central to this is the role of the user and the various forms of knowledge that the firm is searching for. Users present a potential wealth of ideas and opportunity for new offerings (Berthon et al. 2007). Organizations that look to engage users in a co-creation of value context will integrate these participants in a range of activities determined by their level of expertise, skills, and motivations (Brockhoff 2003; Grabher et al. 2008; von Hippel 1986). Organizations look to user engagement as a way to enhance firm performance (Gales \& Mansour-Cole 1991) by delivering offerings that more closely meet the needs of the market (Baldwin et al. 2006; Kristensson et al. 2008; Ramakrishnan et al. 2004). However, this open model also requires that the firm changes its traditional approach to the innovation process (Chesbrough 2010; Cook 2008).

User knowledge provides a unique asset in the generation of innovation that provides a different perspective and experience than is traditionally found in corporate R\&D (Chatterji \& Fabrizio 2007). The process of co-creation of innovation with users presents new knowledge and creates additional knowledge assets and ideas through the recombination of information based on new understanding (Kristensson et al. 2004). Many studies have presented support for the value of user knowledge in the innovation process. Research has shown that user innovation across a range of innovation types was judged to 
provide significant gains in functional utility (von Hippel 1976). Stakeholder participation early in the innovation process has also been found to present an increase in the potential sources of new technology, which improve the offering's fit to market (Douthwaite et al. 2001). In their study examining the range of user types, Kristensson et al. (2004) found that engaging users in the co-creation process resulted in generating new product ideas, ideas of greater value and improvements in offering alignment with customer needs. Chatterji and Fabrizio (2007) examined professional user inventions, finding that inventions from these types of users possessed deeper solution knowledge and better anticipated potential paths for innovation over time.

\section{Knowledge Types}

The identification, evaluation, and integration of knowledge about solutions and needs for new products are what the firm is trying to acquire through co-creation activity (Cohen \& Levinthal 1990). It is necessary for firms planning to engage in co-creation relationships that they are clear on their expectations of participant users and the type of knowledge they are looking to source. They need to understand whether their interest is in understanding user needs or solution requirements (von Hippel 1994) and the degree to which the knowledge to be acquired is tacit or explicit in nature (Sánchez-González et al. 2009; von Hippel 1994).

Von Hippel (1994) describes two forms of information that an organization looks for when entering collaborative and co-creation relationships, "needs information" and "solution information." Needs information refers to knowledge from the customer about specific requirements and preference options for a given product (von Hippel 1994). This 
type of information is often used towards incremental innovations and can also contribute to new radical offerings. In the case where the information sought is focused on user needs, customer engagement in the innovation process is often limited (Zogaj \& Bretschneider 2012)

Solution information goes beyond product related needs with a focus on understanding the problems that users are facing and developing offerings towards addressing those challenges (von Hippel 1994). This form of collaboration provides an opportunity for the customer to participate directly in the innovation process more actively (Zogaj \& Bretschneider 2012). The degree to which customers can provide sufficient insight in delivering radical innovations will vary based on the type of users being engaged (Brockhoff 2003). As an example, research on lead users identifies them as a small and particular class of forward thinking users that provide leading-edge insights for new offerings that will meet the needs of the general market in the future (Schreier et al. 2007; Urban \& von Hippel 1988; von Hippel 1986). Similar research on user types is seen in the work of Rogers' Adoption Lifecycle (Roger 1995) and Moore's Technology Adoption Lifecycle (Moore 2002).

Accessing knowledge from users requires that the firm and its resources have the necessary capabilities to effectively source what is needed (Leonard 2002; Leonard \& Sensiper 1998). The degree to which this is possible varies based on the type of information being sought and the ability of the customer to express that information clearly and accurately to the firm. While customers may be willing to share their knowledge, they often have needs and preferences they are not aware of (Nonaka 1994). Much of the valued knowledge of users in co-creation is tacit in nature (Afuah 2001; Day 1994; Grabher et al. 
2008; Leonard \& Sensiper 1998), making it difficult to duplicate as it is related to personal user experience (Nonaka 1994). Firms must make the investment necessary to capture this tacit knowledge and codify it so that it can be shared explicitly across the innovation process (Nonaka 1994; Un et al. 2010). Von Hippel (1994) explains that "sticky information" represents a deeper level of tacit knowledge that requires more effort to access. However, the point is also made that users with the tacit knowledge are often motivated to innovate.

With the organization's varying information needs and the challenges faced in accessing some forms of information, co-creation relationships require that firms have a variety of knowledge acquisition methods to support the specific task at hand (Zogaj \& Bretschneider 2012). Not all techniques are effective in acquiring information equally well across a variety of information types (Brockhoff 2003; Leonard 2002). Organizations adopting co-creation activities need to ensure that they have knowledge acquisition methods that are effective in extracting tacit user knowledge (Leonard \& Rayport 1997; Leonard \& Sensiper 1998) and that their "absorptive capacity" is sufficient to receive, integrate and exploit the new knowledge (Cohen \& Levinthal 1990).

\subsubsection{Co-Creation and the Methods of Knowledge Acquisition}

The primary objective of co-creation relationships is the acquisition of user knowledge into the firm's innovation process. The dynamics of these relationships, including the nature of knowledge, the role of users and the innovation process and how users are engaged by the firm is studied extensively in the knowledge management literature (Buur \& Matthews 2008; Corso et al. 2001; Gales \& Mansour-Cole 1991; 
Leonard \& Rayport 1997; Piller et al. 2011). Studies examining how users and firms can engage in co-creation of innovation have focused either on the intensity of customer involvement (Bitner 1997) or on specific contexts (O’Hern \& Rindfleisch 2010). Studies have explored questions ranging from where specific classes of users engage in the innovation process (Sofianti et al. 2010; Swink 2006; Voss 1985), where the skills of the various classes of users provide potential for the greatest impact in the process (Brockhoff 2003; Grabher et al. 2008) and what the associated benefits and risks are of these various forms of engagement (Enkel et al. 2005).

A consistent finding in the literature regarding user involvement in the innovation process has been that users can play a variety of roles and multiple roles across all stages of NPD (Enkel et al. 2005; Piller et al. 2011; Swink 2006; Voss et al. 2002; von Hippel 1978). The degree to which customers participate in the co-creation of products or services will vary (Anbardan \& Raeyat 2014). Involvement may limit user input to incremental innovation development (Brockhoff 2003; Zogaj \& Bretschneider 2012) or may involve broader involvement of the firm with customer invention (Piller et al. 2011; Prahalad \& Ramaswamy 2004). Some studies have presented taxonomies examining this intersection between user type, knowledge requirements, and the stage of engagement in the co-creation of innovation process. Studies have examined the processes used for the sourcing of various types of knowledge (e.g., internal and external to the firm (Kang \& Kang 2009)), the systems used by firms in the creation, management and sharing of knowledge (Corso et al. 2001), and the flow of new knowledge between customers and the firm (Sofianti et al. 2010). What is clear from the literature is that firms need to recognize that not all users are equal, and that how users can contribute will differ based on their varying levels of 
experience, knowledge, skills, and motivation to participate in the innovation process (Brockhoff 2003). It is suggested that while deep user engagement should provide benefits, specific user types are more conducive to innovation than others (Brockhoff 2003; von Hippel 1986).

Another stream within the literature examines the knowledge acquisition methods available to firms to engage with users across the innovation process. These studies have examined methods used by firms in accessing tacit knowledge both through observation and direct engagement with users (Brockhoff 2003; Buur \& Matthews 2008; Leonard \& Rayport 1997; Luthje et al. 2003; Olson \& Bakke 2001; Piller et al. 2011). This has included study of both traditional knowledge acquisition methods (Piller et al. 2011; Zogaj \& Bretschneider 2012), and more advanced methods such as lead user theory (Schreier \& Prügl 2008; Urban \& von Hippel 1986; von Hippel 1988), participatory design (Buur \& Matthews 2008; Pals et al. 2008; Sanders 2002), and empathic design (Leonard \& Rayport 1997). The common theme across this literature is the complexity associated with accessing tacit knowledge and the need for methods that are well suited to both effectively and efficiently source and code user experience in a form usable by the firm.

In the co-creation of value, customers can provide a rich source of product knowledge, experience, and expertise (Anbardan \& Raeyat 2014; Prahalad \& Ramaswamy 2004; Zogaj \& Bretschneider 2012). Co-creation and customization of offerings are different in the degree to which customers are active in the process, in that co-creation requires a greater level of involvement from users than is required in the customization process of existing offering (Kristensson et al. 2008). Depending on the level of user motivation, the nature of the expertise desired, and the access available to that expertise, 
will determine which methods are best used in engaging users and acquiring the desired knowledge for the firm's innovation process (Zogaj \& Bretschneider 2012).

Piller et al. (2011) argue that there are three modes in which organizations engage with customers in the innovation process: "listening into" the customer domain, "asking" customers, and "building" with customers. Each of these modes represents different levels of engagement investment and engagement intensity between the firm and the users. They propose a conceptual topology based on dimensions presented by Diener and Piller (2010) which include the innovation process stage, the degree of collaboration, and the degrees of user freedom within the engagement. Their model illustrates that each method of knowledge acquisition is distinct in what it is, where it is best used, and the knowledge management skills and investment needed by the firm in managing the engagement (Piller et al. 2011). Similarly, in a recent study, Cui and Wu (2016) argue that there are three forms of customer involvement in the innovation process: the user as an information source, customer involvement as a co-developer, and the customer as an innovator. They found that these forms of customer involvement would use different approaches to leverage customer knowledge, and in turn are influenced by the nature of the knowledge being accessed, the firm's knowledge management strategy, and the organization's support for the knowledge acquisition methods. Zogaj and Bretschneider (2012) examined a range of fifteen passive and active customer collaboration methods. They found that knowledge acquisition methods that were more active in integrating customers into the innovation process were also more impactful when acquiring customer knowledge in the development of new innovations (Zogaj \& Bretschneider 2012). 


\subsubsection{The Nature of Knowledge Acquisition Methods}

Knowledge acquisition methods represent the processes and techniques used by a firm to engage external sources of knowledge and expertise for identifying, gathering and integrating valued knowledge into the innovation process. From the literature on cocreation of value with users, it is evident that while knowledge acquisition methods are distinct in their specific goals and execution, they also share a set of common traits. As expressed by Roser et al. (2009), all B2B co-creation approaches share three characteristics: 1) they expand organizational boundaries, 2) they involve engagement with external customers and users as co-creators (i.e., the focus on collaborative innovation involves co-conception and co-design), which in turn involves 3) a focus on co-creating new value with users that are initiated by the firm (see Figure 2.1).

Value co-creation can only be achieved through proactive interaction between the firm and customers/users, without which no new value can be realized (Anbardan \& Raeyat 2014). Knowledge acquisition methods, from a learning perspective, involve processes that are iterative in the construction and reconstruction of knowledge and experience that in turn feed both the firm and the customer learning processes (Payne et al. 2008). Co-creation and knowledge acquisition methods often involve providing a platform for users to experiment in an open and free way (Anbardan \& Raeyat 2014). This flow of knowledge ultimately impacts the organization's learning with the potential to trigger business transformations (Owen et al. 2008), which is a central argument in dynamic capabilities theory (Wang \& Ahmed 2007).

Knowledge acquisition methods must provide the setting for firms and their customers to engage effectively across the co-creation process. Prahalad and Ramaswamy 
(2004) state that co-creation methods, or knowledge acquisition methods, must provide the building blocks to establish the necessary relationships provided for value creation. This includes enabling dialogue, access to knowledge, transparency in the relationship, and risk management (Prahalad and Ramaswamy 2004). Firms must recognize that they may adopt and manage many knowledge acquisition methods depending on their strategic orientation and the co-creation aims they are pursuing (Roser et al. 2013).

Figure 2.1 Anatomy of a Knowledge Acquisition Method

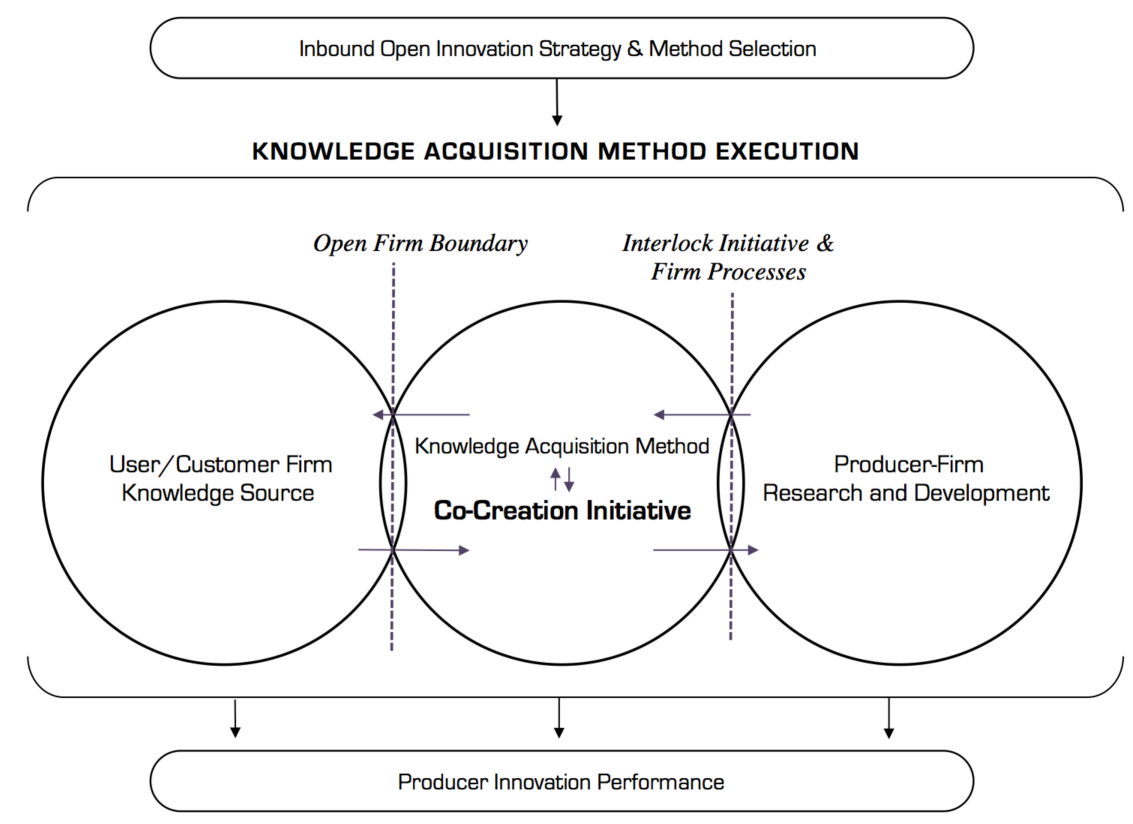

Roser et al. (2013) provide a series of questions to help guide in co-creation ventures: 1) What is the co-creator type that the firm will be engaged with? 2) What is the purpose of the engagement? 3) Where is the locus of innovation? 4) What is the degree of engagement between the firm and the user? 5) How much time is the co-creator required in the process? and 6) What incentives are in place to motivate user participation and innovation process? Roser et al. (2013) guidance translates to the concept of knowledge 
acquisition methods and helps in determining which methods best suit the innovation objectives they are intended to serve. However, firm-customer interaction in and of itself is not sufficient to create value (Grönroos 2013). Firms must also be committed to collaborative innovation and towards transforming the organization to create a collaborative culture which includes new capabilities in leadership, communication and reinforcement, performance tracking, institutionalized learning and knowledge management (Owen et al. 2008).

Owen et al. (2008) explain that collaborative innovation requires that there is proper inter- and intra-organizational alignment and synchronization by the firm with the customer. This alignment is achieved by establishing a shared vision and innovation goals (Owen et al. 2008). In part, this includes a recognition of the changing boundaries of the firm and clarity on the governance mechanisms to manage what may be new forms of engagement (Owen et al. 2008).

Co-creation of innovation activities open the traditional boundaries of the firm to allow for a greater level of engagement with external users in the innovation process. This extension of the firm's boundaries requires different forms of governance to help manage and optimize co-creation relationships (Roser et al. 2013). Governance mechanisms can take the form of market, hierarchical, or relational governance methods, as described by Heide (1994). Market governance is associated with the discrete exchange between the firm and the customer, whereas hierarchical governance focuses more on one party having the right to impose conditions on another in the bilateral relationship (Heide 1994). In hierarchical governance models, the governing authority may be in the form of knowledge acquisition methods governance or program, of which the managing team provides the 
necessary oversight (Heide 1994). Intensive B2B co-creation methods are more likely to adopt hierarchical forms of co-creation governance with the primary role being the alignment of parties (Roser et al. 2009). Relational governance depends on trust and social norms that help govern behaviour between the parties involved (Heide 1994). Due to the nature of co-creation of innovation activities, it is expected that relational governance will always play a role along with either of the other two forms (Roser et al. 2013). In this study, the method of knowledge acquisition being explored uses a program team to provide a hierarchical governance mechanism to manage initiatives that use the method.

Finally, the context in which the knowledge acquisition method exists, and the role that it plays, must also be well understood. Co-creation context is diverse by nature. Roser et al. (2013) explain that managing co-creation initiatives requires combining knowledge acquisition method activity along with organizational processes, which establish an interlocking behaviour between the various actors and the organization. This is supported by Lichtenthaler and Lichtenthaler (2009) who argue that when considering the development of dynamic capabilities in an open innovation context with a goal of fully exploiting sourced knowledge, one must consider the link between user engagement and the absorptive capacity of the firm's R\&D organization. Firms require frameworks that will help guide their knowledge acquisition methods and co-creation ventures across distinctive contexts (Roser et al. 2013).

\subsection{Knowledge Management, Co-Creation and Impact on Performance}

A firm's adoption of innovation strategies is aimed at achieving competitive advantage and delivering superior performance. Innovation strategy decisions are made in 
the face of increasing R\&D costs, reduced budgets, growing competition, and shorter production cycles (Fjeldstad et al. 2012). In response, firms are turning to open innovation strategies, knowledge acquisition methods and developing new capabilities that are expected to strengthen their innovation capacity (Zhou \& Li 2012).

While the OI literature has reported much support for the positive impact of open innovation on firm performance, there remains considerable inconsistency, uncertainty and debate on the factors which determine successful innovation in this model (Nitzche et al. 2016; Vanhaverbeke et al. 2008; West \& Bogers 2014). Similarly, dynamic capability theory proposes to explain the "why" of observed cross-firm performance variance in fastchanging markets (Lin \& Wu 2014; Teece et al. 1997). Dynamic capabilities have been found to deliver long-term firm performance (Wang \& Ahmed 2007; Zahra \& George 2002). However, the relationship between dynamic capabilities and firm performance is complex, having both a direct and indirect impact (Lin \& Wu 2014; Wang \& Ahmed 2007). The literature in these areas continues to see a debate on what relationships drive firm performance, calling for more research to provide deeper insight into understanding the mechanisms involved (Ahn et al. 2016; Cheng \& Shiu 2015; Dahlander \& Gann 2010; Lin $\& \mathrm{Wu}$ 2014). This section discusses the literature examining open innovation strategy and practices, dynamic capabilities, and their impact on firm performance.

\section{Open Innovation and Performance}

There is significant research supporting the role of a firm's market orientation and the benefits to both product innovation and firm performance. It is argued that a deeper understanding of the market, results in unique and superior products through better 
understanding of user needs (Chesbrough 2006; Cooper \& Kleinschmidt 1987; MontoyaWeiss \& Calantone 1994). Research has shown that open innovation can be a winning strategy for firm performance (Ahn et al. 2016). Studies in the area of open innovation agree that the relationship between open innovation activities and innovation performance should exist, but that the relationship is more complicated than initially believed (Cheng \& Shiu 2015). Open innovation research has reported diverging findings on the relationship between open innovation practices and firm performance. There has been significant research reporting the positive relationship between open innovation activities and innovation performance across measures such as profitability (Chiang \& Hung 2010), R\&D performance (Chiesa et al. 2009) and new product success (Rohrbeck et al. 2009). Contrary to these findings, other researchers have reported the negative effects of open innovation on performance (Christensen \& Bower 1996; Vega-Jurado et al. 2009).

It is argued that the existing studies on open innovation only partially explain what is being observed in the relationship between OI activities and innovation performance (Cheng \& Shiu 2015). Overall, firm performance is affected by the openness of the firm due to the impact of activities that touch every aspect of the firm's strategy and innovation practices (Ahn et al. 2016). Firms that take a positive and active attitude towards openness can develop and transform the range of OI-related innovation capacities (Ahn et al. 2016). It has also been noted that while previous research has provided evidence supporting advantages of open innovation strategies, the implementation of open innovation does not automatically imply improved performance (Cheng \& Huizingh 2014). 


\section{External Sources of Knowledge}

Inbound open innovation is dependent upon the firm's ability to establish relationships with external sources of knowledge. Much of the research examining inbound open innovation activities has focused on the positive impact of forming external partnerships and accessing new knowledge on overall innovation performance (Lichtenthaler 2008). Many studies have examined the value of establishing relationships with external knowledge sources and the impact on firm performance (Lilien et al. 2002; von Hippel 1986; Witell et al. 2011). Similarly, strategic alliance and innovation research also report a positive relationship between alliances and firm performance (Ahuja \& Katila 2001; Stuart 2000).

Sourcing external knowledge through partnership provides an opportunity to integrate specific proprietary "know-how" that can result in larger returns from developing new competitive products (Lin \& Wu 2014). Chen (2015) found that firms could benefit from collaborations with a variety of partner types external to the organization (e.g., lead users, major users), but that not all external sources of knowledge equally impact innovation performance. Laursen and Salter (2006) in their seminal work examine the link between the firm's openness and performance by examining openness as both the breadth and depth of the search for new knowledge. They found that working with external partners through structured search processes had a positive relationship to the sales of new or improved products (Laursen \& Salter 2006). This was further supported by Chiang and Hung (2010) who found that the breadth of a firm's search for external information positively affected incremental innovation, whereas radical innovation was more closely linked with the depth of the search for external partners. Chen et al. (2015) warn that the 
breadth of search perspective potentially dilutes their view that not all partner types will have an equal impact on firm performance. They also stress that delivering innovation not only depends on combining internal R\&D activities with external knowledge, but that creating the right mix of external partners is important in achieving a complementary blending of resources (Chen et al. 2015).

The nature of the relationship between the firm and users in a co-creation model has also been found to affect innovation performance. Keil et al. (2008) suggests that the relationship between external sources of information and innovative performance is moderated by the level of relatedness between the firm and the external partner. When the knowledge of the focal firm and the external partner are too close, there is a risk that initiatives will present little new knowledge resulting in less opportunity to learn (Sapienza et al. 2004) and fewer new innovations (Ahuja \& Katila 2001).

\section{Knowledge Management and Firm Capabilities}

The resource-based view of the firm (Barney 1991), the knowledge-based theory of the firm (Grant 1996), and the dynamic capabilities view (Eisenhardt \& Martin 2000; Teece et al. 1997) all support the position that organizational capabilities and knowledge learning form the foundation of sustainable competitive advantage, which in turn promotes superior firm performance. Cheng and Shiu (2015) further argue that organizational capabilities and knowledge learning are the fundamental capabilities that form bridges between open innovation activities (i.e., inbound and outbound) and innovation performance. Ahn et al. (2016) further support this view through their findings on the 
relationships between openness, open innovation capacities, and firm performance, where they reported significant positive interrelationships between these three dimensions.

There has also been a number of longitudinal studies examining the impact of dynamic capabilities on long-term firm performance, furthering arguments supporting the role of dynamic capabilities in firm survival and success (Helfat 1997; Pisano 2000; Zahra \& George 2002). As with open innovation activities, research has demonstrated the direct and indirect impact of dynamic capabilities on firm performance. Wang and Ahmed (2007) argue that while dynamic capabilities are conducive to long-term firm performance, the relationship is not a direct one. They argue that the dynamic capabilities-performance relationship is mediated by the firm's ability to develop dynamic capabilities and in turn, this relationship is further mediated by the degree of alignment of capabilities with firm strategy (Wang \& Ahmed 2007). What this suggests is that dynamic capabilities are more likely to lead to better firm performance when specific capabilities, such as knowledge acquisition methods, are developed in line with the firm's strategic choices. This argument is central to this study.

However, efforts are needed to understand the relationship between capabilities and performance, which have been described as complex. This is in part because internal processes through which open innovation activities influence performance are not well understood (Cheng \& Shiu 2015). Many of the studies that have set out to examine this relationship have focused primarily on the direct impact of activities on performance (Faems et al. 2010; Laursen \& Salter 2006), whereas recent studies have highlighted the indirect impact of organizational capabilities on performance (Lichtenthaler \& Lichtenthaler 2009; Wang \& Ahmed 2007). Lin and Wu (2014) in their study of the 
relationship between a firm's resources (i.e., internally owned resources and resources sourced externally) and firm performance found that dynamic capabilities serve to mediate this relationship. $\mathrm{Wu}$ (2007) also refers to dynamic capabilities as a mediating variable, which can be considered as a transformer for converting resources into enhanced performance. Dynamic capabilities are believed to achieve performance outcomes by "extracting the competitive combinations" through their ability to recombine VRIN resources into a form optimized for the changing competitive environment (Lin \& Wu 2014). Firms using dynamic capabilities to reconfigure resources and to learn through external alliances illustrate this potential (Jiang et al. 2010).

The nature of individual dynamic capabilities has also been broadly studied in the DC literature. Following Teece et al.'s (1997) seminal study on dynamic capabilities, Lin and $\mathrm{Wu}$ (2014) examined three types of dynamic capabilities: dynamic integration, dynamic learning, and dynamic reconfiguration capabilities. They emphasize the importance of integrating internal and external resources towards improved firm performance (Lin \& Wu 2014). These learning capabilities increase the probability of success in new market exploration by helping organizations to benefit from their experience (King \& Tucci 2002), and in integrating industry related technologies for NPD (Deeds et al. 2000). Mody (1993) and Otto (2012) examined learning and development of expertise through external cooperative alliances to enhance performance. They found that the role of knowledge learning and organizational capabilities are the mediating factors on collaborative open innovation activities. Explorative versus exploitative dynamic capabilities have also been examined as they relate to the information they provide in improving radical or incremental innovation performance respectively (Cheng \& Shiu 
2015). Atuahene-Gima (2005) reported on the importance of technical learning in diversifying knowledge inputs towards delivering radical innovation. Cheng and Shiu (2015) found that in addition to the mediating characteristics of these dynamic capabilities, firms that focus on inbound activities enhance radical innovation performance, but hinder incremental innovation performance.

VRIN resources have also been studied for their ability to improve firm performance (Barney 1986; Dierickx \& Cool 1989; Grant 1996), and their ability to influence the development of integration, learning, and reconfiguration of dynamic capabilities. Cohen and Levinthal (1990) in their work on absorptive capacity reported how firms should develop dynamic learning capabilities by creating mechanisms that support their ability to absorb information and develop new knowledge through iterative business practices. This was also supported by Ahn et al. (2016) who confirmed the relationship between integrative and search capabilities and firm performance. This link between the resource-based view of the firm and dynamic capabilities was also examined by Lin and $\mathrm{Wu}$ (2014). They further emphasized the importance of a firm understanding that competitive advantage is beyond simply the accumulation of resources, and the need to determine which dynamic capabilities effectively mediate the resources-performance relationship within the context of the competitive environments (Lin and Wu 2014). Search and integrative capacities have also been found to help enable firms in increasing their inventory of knowledge and to exploit specialized external information access and complementary assets (Levinthal \& March 1993), resulting in a direct impact on firm performance (Ahn et al. 2016). 


\section{Portfolio, Product and Project Initiative Capabilities}

Most studies examining performance and dynamic capabilities have focused at the firm-level. An area that has not been extensively examined is the role of dynamic capabilities at the level of new product, project initiatives and portfolio execution. Several studies have presented early findings of the dynamic capabilities-performance relationship below the firm-level. Deeds et al. (2000) propose that NPD capabilities are related to a firm's scientific, technological, and managerial skills. Their study on performance highlighted the role of location and access to skilled technical personnel and knowledge, scientific expertise that is recognized in the community, and the experience of senior executives (i.e., CEO) in managing commercial research (Deeds et al. 2000).

Marsh and Stock (2003) argue that long-term competitive advantage through NPD processes requires the development of dynamic integration capabilities. These capabilities build on experience to establish a platform for future product development. They propose the inter-temporal integration framework (ITI) which presents the processes of collecting, interpreting, internalizing and marketing capabilities from past offerings and incorporating that knowledge in the development of new future products. Specifically, they argue the need for developing a system view of knowledge-centric capabilities focused on activities related to knowledge acquisition, distribution, interpretation, retention, and application. Their framework speaks to the complex and integrated nature of NPD and the need for a systematic approach to developing dynamic capabilities. As with Deeds et al. (2000), Marsh and Stock (2003) also found a direct relationship between dynamic capabilities and project-level performance, through both a direct and indirect relationship with productlevel performance, and the resulting impact on firm-level performance. 
Finally, Daniel et al. (2014) claiming the first study to examine second-order dynamic capabilities in the information systems domain, presenting a new dynamic capability centred on project portfolio management. This dynamic capability focuses on activities related to strategic project-level investment decision-making, including the capabilities of business objective driven projects, multiple and dynamic prioritizations, the dynamic balancing of risk and reward, and the cancellation or reconfiguration of in-flight projects.

DCV researchers have noted that while the dynamic capabilities research has expanded into areas such as NPD and open innovation (Deeds et al. 2000; Petroni 1998), there is opportunity to more fully develop an understanding of the various types of capabilities and their contributions to performance (Lin \& Wu 2014).

\section{Context}

The literature has also examined the role of context and its impact on performance. Some have studied the impact of firm size and industry on the open innovationperformance relationship, highlighting the characteristics that influence available resources to effectively deploy open innovation strategies (Ahn et al. 2016). The impact of culture on the receptiveness of open innovation strategies (Ahn et al. 2016) and the impact of internal resistance due to "not invented here" syndrome (Katz \& Allen 1982). Consideration of these broader dynamics is necessary due to the impact of every aspect of a firm's strategy and innovation on its overall performance when an open innovation strategy is adopted (Ahn et al. 2016). 
Open innovation and co-creation of value are dependent upon inter-firm collaboration processes. These inter-firm engagements operate within the context of the firm's internal processes. While this relationship is intuitive, there has been little research to date examining the inter-firm/intra-firm dynamics of co-creation and NPD initiatives (Schleimer \& Faems 2015). In a recent study, Schleimer and Faems (2015) examined the moderating impact of intra-firm collaboration engagement on the relationship between inter-firm collaboration projects and the related NPD project performance. They reported a negative interaction effect between inter-firm and intra-firm collaboration engagements in radical inter-firm projects, and no significant relationship being found in the case of incremental innovation. They suggest that this is the first evidence that intra-firm relationship processes can impact the partner's ability to realize the value in inter-firm projects. They also highlight that the negative relationship between inter-firm and intrafirm collaboration engagements suggests trade-offs in the firm's ability to effectively acquire knowledge external to the firm (Schleimer \& Faems 2015).

Finally, Prieto et al. (2009) provided support for the relationship between context, dynamic capability development, and product development. They argue that the contextual elements of the economy, performance management, support, and trust facilitate dynamic capabilities in continuous product development processes, and help in shaping firm competencies. These findings provide support for the relationship between context and dynamic capabilities, reporting a positive relationship between complexity, support, and autonomy to dynamic capabilities, and a negative relationship between performance management and dynamic capabilities. 
It is clear from the growing literature in the areas of open and collaborative innovation, dynamic capabilities and knowledge management that these domains continue to converge on the topic of co-creation of innovation and knowledge acquisitions methods. The research presented serves as the theoretical foundation for this study. The following chapter will present the research objectives, research questions and conceptual framework used in this study. 


\section{Chapter 3 \\ STUDY OBJECTIVES, RESEARCH QUESTIONS \\ AND THE CONCEPTUAL FRAMEWORK}

Research in the areas of open innovation, co-creation of value, knowledge management, and dynamic capabilities continue to draw the interest of academics and practitioners. These streams of study provide the promise of greater insight into the mechanisms at play in establishing a competitive advantage through processes and organizational capacities aimed at exploiting external sources of knowledge, and effectively incorporating them into the firm's innovation process. The challenge that has been put before the research community is to undertake new study that provides greater insight into the mechanisms and relationships between the elements of these complex sets of interactions and activities. This includes greater understanding of the relationships between the elements of knowledge acquisition capabilities and practices, and achieving superior firm performance through the effective execution of new offering initiatives.

Three things are evident from a review of the literature. First, there is a convergence of the research in the areas of inbound open innovation strategies, co-creation of value, knowledge management, and dynamic capabilities (Lichtenthaler \& Lichtenthaler 2009; Wang \& Ahmed 2007). One goal of this convergence has been towards gaining a better understanding of the capacities a firm must develop to achieve competitive advantage in this new model of innovation development and superior firm performance (Lichtenthaler \& Lichtenthaler 2009). Secondly, while there have been advances in the empirical contributions towards understanding the relationships between firm-level innovation strategies, processes, resources, dynamic capabilities, and performance, there remain significant opportunity for researchers to contribute to advancing emerging theories in 
these individual and combined research streams. Finally, the consistent message from earlier research has been that the interaction of open innovation strategies, co-creation processes, and the necessary dynamic capabilities are complex, and there is an opportunity for research providing insight into "how" the various elements of these systems interact, and influence performance (Wang \& Ahmed 2007; Zahra et al. 2006). This study contributes to addressing these three research gaps.

This study explores the role of a knowledge acquisition method used by a firm in acquiring external knowledge as part of its innovation process. Specifically, it examines whether the knowledge acquisition method serves as a higher-order integrative dynamic capability aimed at enhancing inbound OI innovation strategies, and its impact on achieving superior initiative-level performance in co-creation engagements with external stakeholders (e.g., clients). This chapter will provide an overview of the objectives and informing theory for this study, the research questions that will be examined, and the conceptual framework designed to guide this research.

\subsection{Background for the Study}

Inbound open innovation, which represents activities sourcing external knowledge and expertise, expands the boundaries of the organization to bring in new resources and knowledge assets that can help a firm's pursuit for their competitive advantage (Piller et al. 2011). Central to inbound open innovation activity is the engagement of the firm with external stakeholders that possess complementary assets and knowledge, which help to fill gaps in the organization's current resource base (Piller et al. 2011). The processes and activities associated with open innovation strategies have been noted for their complexity, 
and as such, their need for the necessary resources and skills to execute effectively (Chesbrough \& Appleyard 2007). The primary objective in the adoption of inbound open innovation strategies is towards establishing a competitive advantage for the firm, in dynamic environments, towards enhancing overall firm performance. To date, the research on open innovation has focused primarily on inbound open innovation processes (Chesbrough and Crowther 2006; Huizingh 2011), and the relationship to performance has been primarily studied at the firm-level.

Inbound open innovation activities involve co-creation of value through collaboration with external stakeholders. Co-creation initiatives focus on knowledge acquisition by the firm and exploiting this information towards the development of new offerings that can be commercialized (Zahra \& George 2002). As seen in the knowledge management and learning literature and the concept of absorptive capacity, it is insufficient for firms to simply acquire knowledge. It is their ability to integrate and exploit this knowledge within the innovation process that provides a potential for translation into superior performance. Research has shown that there is a relationship between the integration of inter-organizational engagements and intra-organizational processes, suggesting that these two sets of activities are intertwined and must be viewed as a system when pursuing co-creation and knowledge acquisition activities towards creating compelling new innovations (Schleimer \& Faems 2015).

For this study, a knowledge acquisition method represents the set of processes used by the firm to identify, engage, acquire, integrate, and exploit external sources of knowledge and expertise that organizations can benefit from in their innovation process, and contribute in filling knowledge gaps. As in this research, a knowledge acquisition 
method may take the form of a formal program and is defined by a distinct set of objectives, scope and resources. The NPD, open innovation, and knowledge management literature have highlighted the role of knowledge acquisition methods in acquiring external knowledge and expertise through collaborative relationships with external stakeholders, including customers and users (Piller et al. 2011). Extant literature has described knowledge acquisition methods by various traits including the degree of openness of the method (Piller et al. 2011), the level of engagement required by the firm (Brockhoff 2003; Piller et al. 2011), the fit of the method for the type of innovation being pursued (i.e., incremental, radical) (Brockhoff 2003; Zogaj \& Bretschneider 2012), the method of governance employed (Pisano \& Verganti 2008), complexity of the type of knowledge being acquired (i.e., tacit versus explicit knowledge) (Piller et al. 2011), and where collaboration occurs in the innovation process (Brockhoff 2003; Piller et al. 2011; Zogaj \& Bretschneider 2012).

Decisions as to the appropriateness of a knowledge acquisition method has been expressed as a strategic management decision that must weigh the potential benefits of new external knowledge on performance against the associated costs and risks (Enkel et al. 2005; Pisano \& Verganti 2008). This includes evaluating traditional knowledge acquisition methods that are more controlled and closed in nature versus adopting open innovation methods that introduce the need for new skills and present new costs and risks to the firm. The literature on NPD, innovation management, and dynamic capabilities suggest that organizations consider knowledge acquisition methods as part of their innovation strategy based on the perceived net benefits of the knowledge being acquired, the channels through which knowledge is being sourced (i.e., open versus closed), and the nature of engagement 
between the firm and external partners in exchanging the knowledge. This further suggests that the combination of the openness of the knowledge acquisition method and the nature of the engagement between the firm and the external source of knowledge (Piller et al. 2011) will determine the nature of dynamic capabilities needed by the firm to establish and maintain a long-term competitive advantage.

\section{A Knowledge Acquisition Methods Framework}

A review of the literature presents a number of alternative frameworks examining the varied perspectives of open and collaborative innovation strategies and the tactical approach to engagement at the level of the knowledge acquisition process (Brockhoff 2003; Piller et al. 2011; Zogaj \& Bretschneider 2012).

However, as it has been argued, there is an inseparable link between the firm's innovation strategy, knowledge management strategy, and its effective execution of a knowledge acquisition method (Daniel et al. 2014; Wang \& Ahmed 2007). Of primary concern to organizations is the assessment of trade-offs between the value of the knowledge the firm is looking to acquire in pursuit of strengthening its competitive advantage and the cost of time and skills that must be invested to realize these benefits (Pisano \& Verganti 2008).

To illustrate knowledge acquisition methods through a combined lens of openness and engagement, this study presents an alternative framework which describes four classes of knowledge acquisition methods (see Figure 3.1). These classes are characterized along two dimensions: the degree of openness of the innovation engagement method used and the level of firm-user engagement intensity during the collaboration process. 
Figure 3.1 Knowledge Acquisition Methods Framework

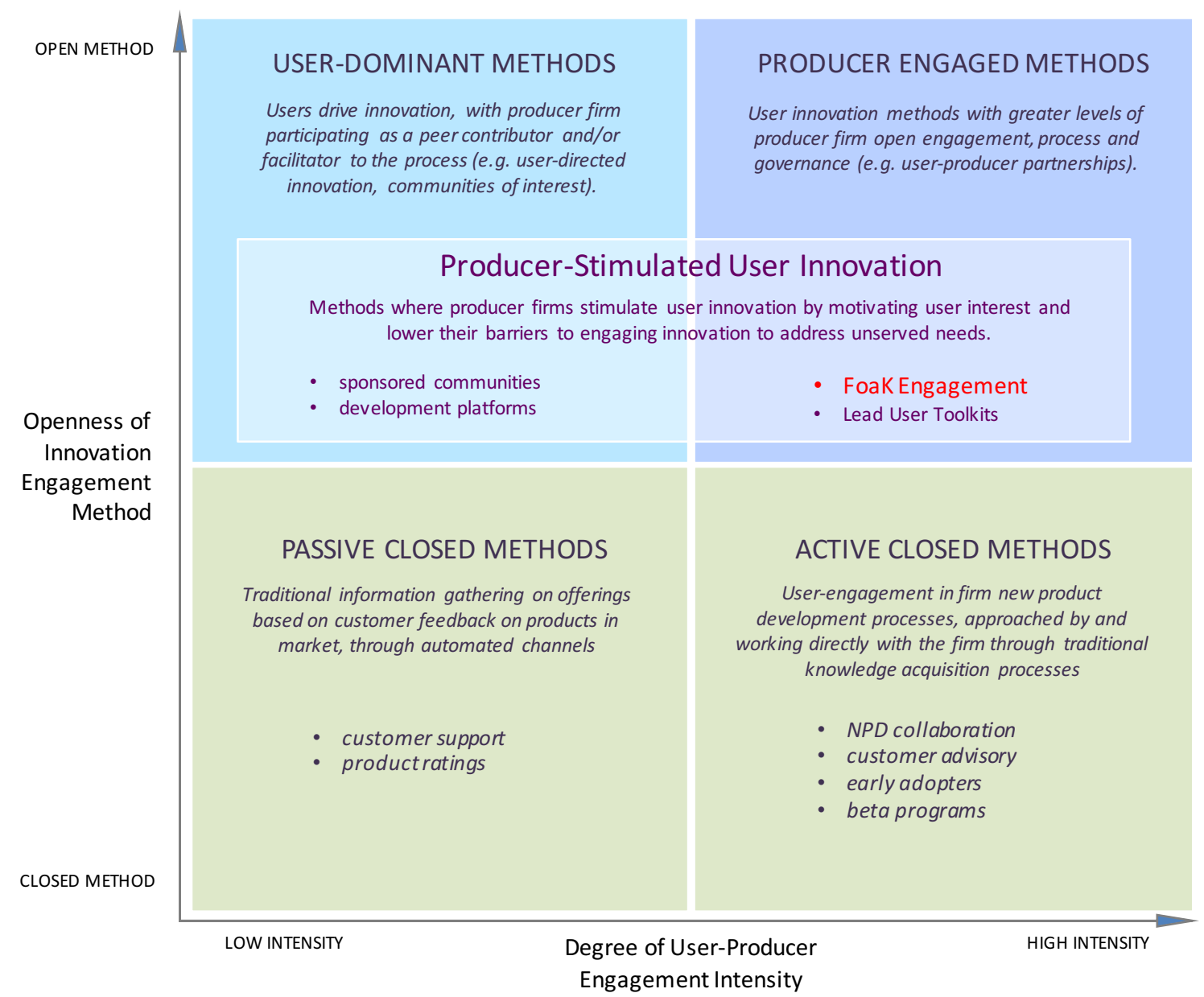

Source: Robert Helal, Thesis Proposal 2016

Informed by: Brockhoff (2003), Dahlander \& Gann (2010), Frederich \&Andrews (2009), Kristensson et al. (2008), Piller et al. (2011), Zogaj \& Bretschneider (2012)

Two classes of knowledge acquisition methods represent traditional closed engagement (i.e., passive closed and active closed methods). "Passive close methods" represent knowledge acquisition through well-established traditional channels and processes that are focused on collecting user volunteered feedback on firm offerings and typically require low levels of engagement between the firm and the user (e.g., customer support, product ratings). "Active closed methods" represent traditional firm controlled methods of engagement with a focus on more direct collaboration with external users and 
customers. These methods are characterized by varying degrees of firm-user engagement and collaboration through the innovation process.

The framework also presents two classes of knowledge acquisition methods that are prevalent in inbound open innovation models, described in this framework as "user dominant methods" and "producer engaged methods." These method types represent the broader firm boundaries for collaborative innovation and new forms of governance and interaction needed to effectively engage with external stakeholders in co-creation of value initiatives. "User-dominant methods" represent engagement where users drive innovation with the producer participating as a peer contributor and facilitating innovation process through the provision of programs or platforms that enable market-driven innovation activity (e.g., communities of interest, open source development). These methods are typically characterized by low levels of firm-user(s) engagement. "Producer engaged methods" represents more firm-directed user engagement methods, characterized by processes and governance models that enable greater levels of open knowledge sharing (e.g., firm-user innovation partnerships).

Research on open innovation also highlights that firms adopt innovation models that represent a hybrid approach to open and closed innovation. Firms combine both open and closed methods of innovation by selecting methods based on what is best suited for the type of innovation being pursued (i.e., incremental or radical), the objectives for the innovation, and the evaluation of the net benefit of a given method towards their strategic objectives. Studies have shown that while firms are expressing the importance of adopting open innovation methods, the majority are still slow in adopting more open methods in practice. Through the innovation management and dynamic capabilities lens, this would 
suggest that there is a middle ground in knowledge acquisition methods adopted by firms that serve as a bridge between traditional closed innovation cultures and the move towards a balance in favour of open knowledge acquisition methods.

A subclass of knowledge acquisition methods is represented in the framework as a type of innovation method that addresses a firm's objective to open the traditional boundaries of the firm for co-creation initiatives while looking to maintain more closed levels of governance and control of innovation assets and commercialization. These are referred to as "producer-stimulated user innovation" methods (see Figure 3.1). These methods represent the middle ground where firms look to stimulate market and user innovation activity and can involve varying levels of firm commitment and engagement with end users. One objective of producer-stimulated user innovation is to lower the traditional barriers to user engagement in the innovation process through the provision of some combination of programs, platforms, and knowledge sharing. These serve as a catalyst for external sources of knowledge (e.g., customers, users) to engage in innovation activities that ultimately contribute to the firm's new offerings strategy. Examples of such methods include von Hippel's work on lead user toolkits (Franke \& Piller 2004; von Hippell \& Katz 2002), open-source platforms (O’Hern \& Rindfleisch 2010), and innovation communities (O'Hern \& Rindfleisch 2010). This study will examine the First-of-a-Kind (FoaK) program as a co-creation of innovation knowledge acquisition method (Frederich \& Andrews 2009). Based on a review of the literature, this method of knowledge acquisition has yet to be examined and will serve as one point of contribution from this study to theory in co-creation, open innovation and NPD. 
Research across knowledge management, learning, and dynamic capabilities are consistent in the view that organizations engaged in co-creation of innovation and use of knowledge acquisition methods require the necessary skills and investment in the development of organizational and dynamic capabilities to effectively realize long-term gains to competitive advantage (Eisenhardt \& Martin 2000; Lichtenthaler \& Lichtenthaler 2009; Teece et al. 1997). Research in knowledge management theory has illustrated the important role that knowledge skills and capabilities play in identifying, acquiring, and integrating external sources of knowledge into the firm. Dynamic capabilities theory further supports this argument with its fundamental tenets stating that competitive advantage can only be achieved through the development of higher-order capabilities within the organization that allows for recombination of resources in response to the changing environment (Eisenhardt \& Martin 2000; Lichtenthaler \& Lichtenthaler 2009; Teece et al. 1997).

It can be argued that the emergence of open innovation and alternative knowledge acquisition methods reflects the changing competitive environment in which many industries exist today. This is reflected in studies that have started examining the dynamics of co-creation of innovation and knowledge acquisition methods from the combined theoretical perspectives of open innovation, knowledge management, and dynamic capabilities (Lichtenthaler \& Lichtenthaler 2009). However, it is clear that open innovation and dynamic capabilities research are still fields of study that present a significant opportunity for contribution from researchers (Lichtenthaler \& Lichtenthaler 2009; Wang \& Ahmed 2007). 


\subsection{Research Opportunities}

A common theme highlighted in the open innovation and dynamic capabilities literature is the complexity associated with co-creation activities, user engagement, and knowledge acquisition methods. Understanding the contributors to this complexity, the nature of collaborative relationships between entities and capabilities, and how these relationships influence performance has remained largely unexplored (Ahn et al. 2016; Cheng \& Shiu 2015; Lichtenthaler \& Lichtenthaler 2009; Lin \& Wu 2014).

\subsubsection{Interaction Between Activities in Open Innovation Initiatives}

Knowledge acquisition methods integrate activities across intra-firm processes and resources, with inter-firm engagements and knowledge exchange. Studies examining firm adoption of inbound open innovation practices have focused on the positive performance effects that new knowledge through external partners provides (Lichtenthaler \& Lichtenthaler 2009). However, the research on inbound open innovation activity tends not to investigate deeply into the specific nature of these activities (Cheng \& Shiu 2015). There has been a call for research to provide deeper insight and understanding into these complexities. While some research has examined open innovation as it relates to internal and external organizational factors (Dahlander \& Wallin 2006), Ahn et al. (2016) have asserted that little attention has been paid to the interaction between different capacities necessary to manage knowledge in the open innovation process. Cheng and Shiu (2015) state that organizations are expected to undertake multiple inbound and outbound open innovation activities simultaneously, which adds to the complexity in understanding the relationships impacting performance. While many previous studies have examined the 
benefits of open innovation activities, the focus has been primarily on the direct versus the indirect effects of activities on performance (Faems et al. 2010; Laursen \& Salter 2006). However, the interaction and potential counteraction of such activities and their impact is not well understood (Cheng \& Shiu 2015).

\subsubsection{Higher-Order Dynamic Capabilities, Capability Interaction and Performance}

A focus on dynamic capabilities theory is intended to explain how these types of capabilities can help enhance firm competitiveness and performance. It extends RBV by assisting in understanding why some firms succeed and others fail based on the same resource basket. However, some have warned that there is no guarantee that the promise of dynamic capabilities will be fully realized (Cheng \& Shiu 2015).

While some research has attempted to explain higher-order dynamic capabilities for managing other more basic capacities and sub-capacities (Brunswicker \& Van de Vrande 2014; Robertson et al. 2012), there have been few attempts to understand the possible relationships between the various levels of dynamic capability and organizational capacities (Ahn et al. 2016).

The literature has examined various organizational and dynamic capacities regarding exploration and exploitation of knowledge (Laursen et al. 2015; Lichtenthaler \& Lichtenthaler 2009). However, the understanding of open innovation related capacities remains fragmented. Lin and $\mathrm{Wu}(2014)$ state that while dynamic capabilities research has expanded into the areas of NPD (Deeds et al. 2000; Petroni 1998), they have yet to be fully considered when investigating the contribution of different types of dynamic capabilities. They state an urgent need for investigation to understand how dynamic learning 
capabilities mediate the impact of resources on firm performance. Similarly, Barreto (2010) argues that future research should draw significant attention towards the internal and external factors that enable or inhibit potential impact of dynamic capabilities on firm performance. This can be further extended to help understand the existence of intra-firm variance in knowledge acquisition method results.

As conceptualized by Lichtenthaler and Lichtenthaler (2009), open innovation knowledge management and dynamic capabilities can present a system view of integrated dynamic capabilities towards achieving competitive advantage and firm performance. To date, there has been little study found examining the integrated nature of second-order (i.e., higher order) dynamic capabilities and their interaction with other dynamic capabilities (Lichtenthaler \& Lichtenthaler 2009; Wang \& Ahmed 2007). Specifically, the literature provides little insight into higher-order dynamic capabilities that serve as an integrating mechanism across processes and capabilities, with a focus on extracting desired outcomes. Lichtenthaler and Lichtenthaler (2009) call for future research to help the validation of their knowledge capacity framework (KCF) to build out the role of knowledge and dynamic capabilities in an open innovation context.

Studies examining the interaction between open innovation activities, dynamic capabilities, strategy, and performance have for the most part been at the level of the firm. Little study has been conducted examining relationships at the level of the initiative, project or offering portfolio, which in turn also contribute to overall firm performance (Daniel et al. 2014; Schleimer \& Faems 2015). Research at this more focused unit of analysis would contribute to a deeper understanding linking firm operational execution to innovation strategy. 


\subsubsection{Relationship Between Context, Capabilities, and Performance}

Dynamic capability theory has also asked for additional research to help in understanding the role of context (e.g., industry scenarios) (Barreto 2010). With dynamic capabilities primarily focused on studies aimed at the understanding of firm adaptability, there is also a call for research examining the link between organizational strategy, context, and dynamic capabilities (Barreto 2010).

\subsection{Research Objectives and Questions}

The literature has established the increasing importance of the research agendas in open innovation, co-creation of innovation, knowledge management, and dynamic capabilities. The convergence of these streams of study promises to provide greater understanding in the areas of management strategy, competitive advantage, and managerial decision-making. A review of the literature has also highlighted that the theory emerging from these disciplines is still in its early stages. There remains significant opportunity for contribution examining the complexities of interaction, mechanisms, and the relationship to performance, both at and below the level of the firm. Also, open innovation theory can benefit from studies that provide greater insight into the knowledge acquisition method as a potential integrative dynamic capability, helping to further understand the role of higherorder capabilities in complex co-creation systems.

Of interest is the notion of higher-order dynamic capabilities that serve as an integrating function across lower level dynamic capabilities and operational capacities. As discussed in Chapter 2, Roser et al. (2009) represents all B2B co-creation activities as spanning organizational boundaries, engaging external users, and focusing on creating new 
value initiated by the firm (see Figure 2.1). It is believed that the nature of these interactions as expressed by Roser et al. (2009) extends to the concept of knowledge acquisition methods. By their nature, the linkage between a knowledge acquisition method's strategy and the integration between intra-firm and inter-firm processes require a systems perspective, as well as exploratory insight, to help understand the dynamics related to cocreation initiative performance.

A knowledge acquisition method represents mechanisms that connect and coordinate a range of processes and activities between external sources of expertise and internal functions that look to leverage the new knowledge as part of the innovation process. Intrinsic to a knowledge acquisition method is its role in managing the flow of information within a complex set of activities both within and outside the control of the firm. It is believed by this researcher, that knowledge acquisition methods can be examined as a higher-order integrative dynamic capability, consisting of a series of sub-capabilities. As a higher-order dynamic capability, it is believed that a knowledge acquisition method is a capacity that a firm must invest in developing, with the potential of enhancing initiatives and product-level performance through its function as a coordination and optimization set of capabilities.

The objective of this study is to examine a knowledge acquisition method and its impact on co-creation of innovation initiative performance, with a focus on gaining an exploratory perspective on the role of dynamic capabilities in achieving targeted performance. This includes taking both a system view of the phenomenon being studied and examining the role of knowledge acquisition methods as higher-order integrative dynamic capabilities. 
Maxwell (2012) describes that qualitative research questions should focus on the meaning of events that are being studied, the influence of context of these events, and about the processes of which the events and the related outcomes occur. This study will ask the following primary research question:

How does a knowledge acquisition method impact the performance of a co-creation collaborative new-to-the-firm innovation?

Insight into this question will be guided and gathered through an exploration of the following supporting questions:

What is the role of a knowledge acquisition method in creating a collaborative newto-the-firm innovation?

What are the attributes of a knowledge acquisition method that contribute to its effectiveness and its role as a capability in creating a collaborative new-to-the-firm innovation?

How are the attributes of a knowledge acquisition method related to the effective execution of a co-creation collaborative innovation initiative using the method?

How do the attributes of a knowledge acquisition method contribute to the performance of collaborative new-to-the-firm innovation?

How does the context of a collaborative innovation initiative impact the performance of a knowledge acquisition method in delivering new-to-the-firm innovation?

\subsection{Conceptual Framework}

A conceptual framework is intended to present a system of concepts, assumptions, expectations, beliefs, and theories that support the research (Maxwell 2012). Conceptual frameworks are expressed as visual or written artifacts that represent the factors, concepts, 
or variables, and the presumed relationships between them (Miles et al. 2013). It can be constructed based on existing theory and research, pilot or exploratory research, experiential knowledge, and may be assembled as a "bricolage" of conceptual frameworks used in previous studies (Maxwell 2012). A conceptual framework can be used as a tool for developing and clarifying theory and research study (Maxwell 2012), and plays a central role in the entire research process and final analysis.

A conceptual framework has been developed to guide this study, and is based on insights gathered from the literature and a preliminary exploration that was conducted in assessing the viability of the research study (see Figure 3.2). Several studies were referenced in the design of this conceptual framework, which provided alternate expressions of the relationship between dynamic capabilities, firm strategy, and performance (Daniel et. al. 2014; Heft \& Peteraf 2009; Lichtenthaler \& Lichtenthaler 2009; Prieto et al. 2009; Wang \& Ahmed 2007). Lichtenthaler and Lichtenthaler's (2009) conceptualization of the knowledge capacity framework (KCF), which represents dynamic capabilities and the concept of the higher-order integrative knowledge management capacity (KMC), is used as the dynamic capability component of the conceptual framework for this study.

Lichtenthaler and Lichtenthaler (2009) examined dynamic capabilities in the context of open innovation. Bringing together research in the areas of knowledge management, absorptive capacity, and dynamic capabilities, they introduced a conceptual integrated framework representing the range of firm capacities required in effective open innovation models. The framework identifies six "knowledge capacities" as critical capabilities that are characterized along two dimensions: their role for either intra-firm or 
inter-firm activities, and whether they are needed for knowledge exploration, knowledge retention, or knowledge exploitation processes (see Table 3.1). The firm's intra-firm knowledge capacities include their inventive capacity, transformative capacity, and innovative capacity. The inter-firm capacities include absorptive capacity, connective capacity, and desorptive capacity. Definitions for these dynamic capabilities as they pertain

Table 3.1 Knowledge Capacity Framework

\begin{tabular}{|c|c|c|c|}
\hline \multirow{3}{*}{$\begin{array}{l}\text { External } \\
\text { Knowledge } \\
\text { (KCe) }\end{array}$} & $\begin{array}{l}\text { Absorptive Capacity } \\
\text { (knowledge exploration) }\end{array}$ & ability to explore & $\begin{array}{l}\text { acquiring external knowledge } \\
\text { assimilating into firm's database }\end{array}$ \\
\hline & $\begin{array}{l}\text { Desorptive Capacity } \\
\text { (knowledge exploitation) } \\
\text { note: outside study scope }\end{array}$ & outward knowledge transfer & $\begin{array}{l}\text { identify external opportunities for knowledge exploitation } \\
\text { transfer knowledge to recipient }\end{array}$ \\
\hline & $\begin{array}{l}\text { Connective Capacity } \\
\text { (knowledge retention) }\end{array}$ & $\begin{array}{l}\text { retain knowledge in interfirm } \\
\text { relationships }\end{array}$ & $\begin{array}{l}\text { maintain interorganizational relationships } \\
\text { privileged access to external knowledge }\end{array}$ \\
\hline \multirow{3}{*}{$\begin{array}{l}\text { Internal } \\
\text { Knowledge } \\
\text { (KCi) }\end{array}$} & $\begin{array}{l}\text { Inventive Capacity } \\
\text { (knowledge exploration) }\end{array}$ & ability to explore new knowledge & $\begin{array}{l}\text { perceive new opportunities } \\
\text { set-up knowledge exploration processes } \\
\text { perception of particular opportunities }\end{array}$ \\
\hline & $\begin{array}{l}\text { Innovative Capacity } \\
\text { (knowledge exploitation) }\end{array}$ & $\begin{array}{l}\text { ability to internally } \\
\text { exploit knowledge }\end{array}$ & $\begin{array}{l}\text { transmuting knowledge } \\
\text { converting knowledge to products } \\
\text { or services } \\
\text { determine if commercialization opportunity exists }\end{array}$ \\
\hline & $\begin{array}{l}\text { Transformative Capacity } \\
\text { (knowledge retention) }\end{array}$ & $\begin{array}{l}\text { ability to internally retain } \\
\text { knowledge over time }\end{array}$ & $\begin{array}{l}\text { retaining knowledge in firm knowledge base } \\
\text { reactivating knowledge }\end{array}$ \\
\hline
\end{tabular}


to this study are presented in the chapter on research design and methodology (see Chapter 5). Finally, Lichtenthaler and Lichtenthaler (2009) highlight the importance of higher-order dynamic capabilities as integrative dynamic capabilities and present the concept of the "knowledge management capacity," which is used to reconfigure and realigned knowledge capacities (see Figure 3.2). Lichtenthaler and Lichtenthaler's (2009) framework informs part of the conceptual framework for this study, representing the dynamic capabilities in knowledge acquisition methods.

The co-creation of innovation initiative conceptual framework (see Figure 3.2) represents the knowledge acquisition method as a link between the internal knowledge transformation activities of the firm and user engagement activities between the firm and co-creation partner (i.e., client). It also represents a relationship between the firm's innovation strategy as it relates to the knowledge acquisition method, and that the knowledge acquisition method effectiveness will have an impact on co-creation innovation performance. Central to the model is the belief that there is a dynamic capabilities framework that represents the activities that span inter-firm and intra-firm processes as they relate to each type of knowledge acquisition method. The conceptual framework explores the existence of an overarching higher-order integrative dynamic capability (i.e., knowledge management capacity) that is believed to serve as a coordinating and optimizing mechanism across the system of dynamic capabilities and co-creation of innovation activities.

As this research represented an exploratory study, the conceptual framework served primarily in guiding data collection and in ensuring a comprehensive exploration of the observed phenomena. Learnings from the study were applied in testing and revising the 
conceptual framework accordingly. The following chapter details the research design used towards the study objectives and research questions discussed.

Figure 3.2 Co-Creation of Innovation Conceptual Framework

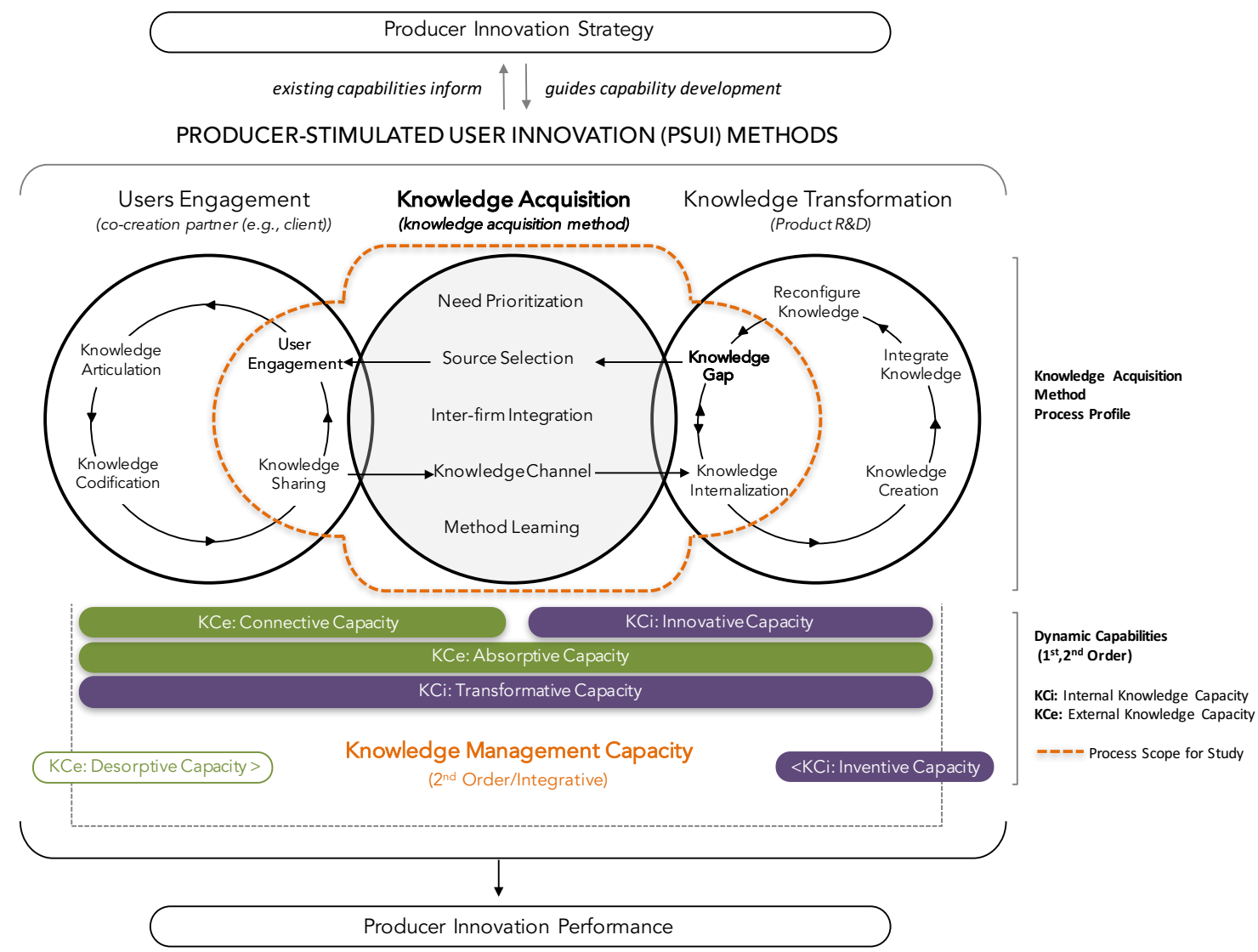

Source: Helal (2016)

Informed by Daniel et al. (2014), Frederich \& Andrews 2009, Helfat (2007), Lichtenthaler \& Lichtenthaler (2009), Nielsen (2013), Prieto et al. (1989), Wang (2007) 


\section{PART II \\ RESEARCH DESIGN AND METHODOLOGY}

This research presents an exploratory comparative multi-case study executed through a critical realist (CR) lens. The research design and methodology were focused towards building upon a sound foundation of research practices that reflect both case study methods and the CR philosophy. Part II discusses this study's research design and methodology. This section includes chapters presenting a discussion of the guiding principles from critical realism and case methodology that shaped the design decisions for the study, followed by the details of the research design and methodology used.

\section{Chapter 4 Guiding Principles for the Study Design}

Chapter 5 Research Design and Methodology 


\section{Chapter 4 GUIDING PRINCIPLES FOR THE STUDY DESIGN}

This study was designed to support a deep examination of the interactions and mechanisms involved in knowledge acquisition method processes, and their relationship to initiative performance. A critical realist informed case study method was followed, and an exploratory investigation was conducted using a comparative multi-case study design. The study design applies a two-tailed nested case study approach which includes a crosssectional retrospective examination, and multi-method data collection model.

This chapter discusses the philosophical and methodological foundation that informed the design of this study. It begins with an overview of the critical realist philosophy and case study strategy which are both fundamental to the study design. This includes a review of the guidance that informed the design principles for this research, including considerations towards case rigour, building theory and validity.

\subsection{A Critical Realist Lens}

This study is informed by a critical realist (CR) ontological lens. In this section, a brief background of critical realism will be discussed, outlining considerations that have served in guiding the research design for this study.

\subsubsection{The Critical Realist Paradigm}

Much of mainstream research is based on the positivist and interpretivist philosophical paradigms, which cover the two ends of the ontological continuum. Positivism is concerned with the testing, confirmation, and predictability of generalizable 
theories regarding research phenomena (Orlikowski \& Baroudi 1991). Interpretivism focuses on building theory based on exploring the subjective meanings that come from socially constructed perspectives of reality (Wynn \& Williams 2008). Critical realism is an emerging philosophy that provides an ontological alternative to these well-established postmodern traditions (Carlsson 2003; Wynn \& Williams 2008) - a middle-range realist theory (Layder 1996) that draws from both positivist and interpretivist beliefs. Critical realism has a growing following and is being applied more broadly across social research. Critical realism-based research is becoming more predominant across social science disciplines including economics (Lawson 1997), sociology (Easton 2010; Pawson \& Tilley 2009; Yin 2013) and business management (Reed 2009). There is also a call for the increased use of CR in addressing the complexities studied in information systems research (Wynn \& Williams 2012).

Critical realism is attributed to the seminal philosophical work of Roy Bhaskar (Bhaskar 1975a; Bhaskar 1978) who presented the philosophical underpinnings of CR for use in research. Bhaskar's writings present a form of realism that connects the scientific natural order with the social world (Carlsson 2003). Similarly, Andrew Sayer has been credited with the most significant impact on advancing the philosophy and its use in practice (Easton 2010; Sayer 1997). CR is a middle-range philosophy that argues a complexity of reality that exists well beyond what we can observe and comprehend. CR argues that to uncover the true meaning of reality, research must move beyond an emphasis on understanding a single reality through causality in nature (i.e., positivism) or on capturing the detailed description of our observations (i.e., interpretivism). CR focuses on deciphering reality with an emphasis on understanding the "why" and "how" of what is 
observed, asking the question, "what must reality be like to make what we (observe) possible?" (Collier 1994).

\subsubsection{Attributes of the Critical Realism Paradigm}

Wynn and Williams (2012) state that "the primary focus of critical realism is on the assumption that the theories which are generated by the conduct of scientific research must revolve around the objective reality which comprises the world, even though humans are often unable to understand fully or observe this reality." Critical realism is characterized by the belief in an independent reality, the existence of a stratified ontology, and that reality is an open system influenced by context. Sayer's statement of assumptions regarding critical realism argues the existence of a reality that is independent of what is perceived by observers that this reality is complex, and that it is not readily accessible (Carlsson 2003; Sayer 1997). CR in turn is characterized by its independent reality, stratified ontology and as an open system impacted by context.

An Independent Reality $\mathrm{CR}$ argues the existence of an independent reality, which is beyond our capacity as beings to fully understand (Wynn \& Williams 2012). The domain of the real is argued to exist independently of what is perceived (Carlsson 2003). While CR-based research infers the nature of reality, knowledge of the objective reality remains "fallible" (Wynn \& Williams 2008).

A Stratified Ontology Unlike the positivist and interpretivist single-layered view of reality (Wynn \& Williams 2012), CR is described as a stratified and nested three-tiered reality, linking the domains of the "real," the "actual," and the "empirical" (Bhaskar 1978; Carlsson 2003). It is the "real" that represents the structures, mechanisms, and relationships 
that hold the power to activate events and patterns that may or may not be observable in the real world (Bhaskar 1975b; Carlsson 2003; Wynn \& Williams 2012). Wynn and Williams (2012) state that it is "the primary objective of critical realism-based research ... to provide clear, concise and empirically supported statements about causation, specifically (the) how and why of (a) phenomena." This involves the study of phenomena in the "empirical" and inferring the nature of reality.

Open Systems and Context CR describes the reality of social phenomena as an open system which is complex and cannot be adequately explained through the use of controlled or closed system experiments (Sayer 1992; Wynn \& Williams 2012). Sayer $(1992,1997)$ emphasizes the importance of understanding the environmental context in which mechanisms are activated and observed, and that context is not static in a social setting. Observed phenomena are seen to affect the environment in which they exist, which in turn changes the social structure and the effects being examined (Archer 1995). Social systems are fluid, and as such, it cannot be assumed that observed patterns and mechanisms within a particular social structure and context will repeat in the future. It is for this reason that the CR philosophy emphasizes that research must focus on explanation rather than prediction, towards a deeper understanding of the meaning of "why?" (Wynn \& Williams 2008).

Critical realism presents a middle ground philosophy that connects both with the natural science positivist's view of reality, while acknowledging that there is a social constructivist element of reality that observes and interprets the structures, mechanisms, patterns that are believed to exist (Sayer 1992; Sayer 1997). Appendix 4.1 presents a comparison of the critical realist, positivist and interpretivist paradigms. 


\subsubsection{Critical Realism: Research Strategies and the Case Study Method}

A consideration in the research strategy for this study is that the phenomena to be examined are based within an information systems setting. Much of mainstream research in the area of management information systems (MIS) falls predominantly into the paradigms of positivism and interpretivism (Wynn \& Williams 2008). Longshore-Smith (2006) points out that these paradigms suffer from "persistent theory-practice inconsistencies" that have resulted in conflicting or inconclusive results across a range of information systems research that can be addressed through a broader application of a CR philosophy. It is believed that a CR approach can provide an alternative perspective for deep exploratory and explanatory research examining complex organizational phenomenon (Wynn \& Williams 2008).

Fundamental to critical realism is the belief that causal language can be used to describe the world we live in (Easton 2010). With its focus on understanding why causation occurs in complex open systems, critical realism as a philosophical foundation for research is emerging as a sound option in describing reality. Karlsson and Ackroyd (2014 p.26) state that "the issue for CR-guided researchers is always: what contents are required to understand the data available and to bring into focus the processes and mechanisms that are really at work?" This is achieved through the use of either abductive or retroductive logic of discovery (Edwards et al. 2014). Critical realists strive for the use of causal language with thinking, which provides strong support for case study research.

Karlsson and Ackroyd (2014) argue that there are eight alternative CR research strategies that are determined based on the nature of two dimensions of a study: whether research objectives are intensive or extensive in focus (i.e., breadth versus depth of the 
study), and the type of engagement the researcher will have in the study (i.e., detached versus engaged) (Edwards et al. 2014). A review of the Karlsson and Ackroyd (2014) framework (see Table 4.1) provides support for the choice of a comparative case analysis as the research strategy for this study, which will be discussed in more detail further in this chapter. This choice is further supported by growing research that recognizes the value of case studies as a method in the CR toolbox (Easton 2002; Easton 2010; Edwards et al. 2014; Morton 2006; Williams \& Karahanna 2013; Wynn \& Williams 2008; Wynn \& Williams 2012).

Table 4.1 Eight Designs Relevant to Critical Realist-Informed Research

\begin{tabular}{|c|c|c|c|c|}
\hline & \multicolumn{4}{|c|}{ Distinctive Research Strategies } \\
\hline & \multicolumn{4}{|c|}{ 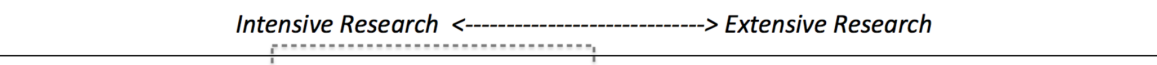 } \\
\hline & $\begin{array}{l}\text { What is the mechanism? } \\
\text { ("Context" as given) }\end{array}$ & $\begin{array}{c}\text { How does the context } \\
\text { and mechanism typically } \\
\text { interact? }\end{array}$ & $\begin{array}{c}\text { How does the context and } \\
\text { mechanism historically } \\
\text { interact? }\end{array}$ & $\begin{array}{l}\text { What is the context? } \\
\text { ("Mechanism" as given) }\end{array}$ \\
\hline \multicolumn{5}{|c|}{ Research Procedures: } \\
\hline Detached Study & $\begin{array}{l}\text { Case } \\
\text { Studies } \\
\text { (1) }\end{array}$ & $\begin{array}{l}\text { Comparative Case } \\
\text { Analysis } \\
\text { (2) }\end{array}$ & $\begin{array}{l}\text { Generative Institutional } \\
\text { Analysis } \\
\text { (3) }\end{array}$ & $\begin{array}{l}\text { Research Surveys and } \\
\text { Census Data } \\
\text { (4) }\end{array}$ \\
\hline Engaged Study & $\begin{array}{l}\text { Action } \\
\text { Research } \\
\text { (5) }\end{array}$ & $\begin{array}{l}\text { Intensive Realist } \\
\text { Evaluation } \\
\text { (6) }\end{array}$ & $\begin{array}{l}\text { Barefoot Historical } \\
\text { Research } \\
\text { (7) }\end{array}$ & $\begin{array}{l}\text { Extensive Realist } \\
\text { Evaluation } \\
\text { (8) }\end{array}$ \\
\hline $\begin{array}{l}\text { Dominant Logic } \\
\text { of Discovery }\end{array}$ & Abduction & Abduction & $\begin{array}{l}\text { Abduction/ } \\
\text { Retroduction }\end{array}$ & $\begin{array}{l}\text { Abduction/ } \\
\text { Retroduction }\end{array}$ \\
\hline
\end{tabular}

Adapted from Karlsson \& Ackroyd (2014) 


\subsubsection{Thick Description}

This study reflects "thick description" in qualitative research, a qualitative method most often attributed to the work of Clifford Geertz (1973). As this is an exploratory qualitative study aiming to bring new insight into a cross-disciplinary field of study early in its development, a thick description approach allows for an in-depth treatment of the subject. This description is also consistent with the objectives of research informed by the critical realist philosophy.

The definition of thick description remains a topic of debate. An integrative analysis by Ponterotto (2006) provides a working definition of a thick description which highlights the elements that define this form of qualitative study. Ponterotto (2006) states that thick description is characterized by five attributes: 1) a researcher describing and interpreting observed social action within a specific context, 2) context that can include either small or large entities, 3) an accurate description of social actions and assigning purpose and intention to those actions, 4) capturing the thoughts, feelings and interactions between participants, and 5) enabling thick interpretation and meaning. These elements of thick description are reflected in the study.

"Thick description" enables "thick interpretation" and "thick meaning," (Ponterotto \& Grieger 2007) which are essential in meeting the objectives of this exploratory study. A thick description approach in this study allows for a thorough examination of the dynamics of knowledge acquisition methods in co-creation of innovation and understanding the interaction between stakeholders, processes, capabilities, environment, and the impact on observed phenomena. 


\subsection{Case Study: Strategy and Approach}

To achieve the research objectives, this study was conducted using a two-tailed comparative case study approach to allow for detailed exploration of the research questions. To help better understand the fit of this method to the study, this section discusses the case study approach in research, highlighting the central themes in the definition of the method and associated strategies in its use.

\subsubsection{Case Study Definition}

The case study method is a common empirical research approach used to support a variety of epistemological orientations (Easton 2010; Yin 2013). It is typically associated with an in-depth examination of a phenomenon used in the development or testing of theory (Yin 2013). The literature presents many definitions of what a "case study" is.

An often cited definition is that presented by Yin (1989) who describes a case study as "an empirical inquiry that investigates a contemporary phenomenon within its real-life context; when the boundaries between phenomena and context are not clearly evident; and in which multiple sources of evidence are used." Yin (1994) further described case studies as "research situations where the number of variables far outstrips the number of data points."

Gerring (2004) defines a case study as "an intensive study of a single unit for the purpose of understanding a larger class of (similar) units," emphasizing the difference between the smaller number of units studied compared to statistically based methods. Easton (2010) highlights the relationship between case study and the diverse sources of evidence associated with case research, describing case studies as "investigating one or a 
smaller number of social entities or situations about which data are collected using multiple sources of data."

While the intention is not to provide an exhaustive collection of definitions, a review of the literature has demonstrated common themes in the description of the case study method. Academics consistently describe the case study method as an opportunity for the detailed study of complex and understudied contemporary phenomenon, often focusing on a small number of subjects. This study will adopt deWeerd-Nederhof's (2001) statement of a case study, a more detailed expression of the earlier definition from Yin (1989). DeWeerd-Nederhof (2001) defines the case study method as:

"1. ... an empirical inquiry that investigates a contemporary phenomenon (the case) - within its real-world contexts, especially when the boundaries between phenomenon and context may not be clearly evident (, and)

2 ...(an) inquiry (which) copes with the technically distinctive situation in which there will be many more variables of interest than data points, and other results relies on multiple sources of evidence, when data needing to converge that triangulating fashion, and as another result benefits from the prior development of theoretical propositions to guide data collection and analysis."

DeWeerd-Nederof's (2001) definition is noted both for its acknowledgment of the role of complexity and context when examining phenomena, which are central to considerations when research is informed through a CR ontological lens.

\subsubsection{Case Study Objectives}

Case studies are used when exploring contemporary events requiring in-depth examination. This method is ideal in understanding "how," "why," or "what" questions of 
observed phenomena (Yin 1989; Yin 2013). These types of questions move beyond the simple counting of the frequency of events, seeking to understand the operational links that are the result of what is being observed (Yin 1989; Yin 2013). Case studies are used when the objectives of the research require examination of phenomena within their real-life context, particularly when the boundary between what is being observed in this context is unclear (Yin 2013). Benbasat et al. (1987) emphasize the strengths of case studies as being three-fold: 1) they provide an opportunity to explore phenomena within their own environment, allowing for meaningful and relevant theory to be generated based on observing actual practice, 2) they enable a greater understanding of the related complexity surrounding the phenomena, and 3) they are a good fit for exploratory investigations in areas where phenomenon are still early in understanding.

The case study method is used when little is known about a phenomenon that is being explored, often as the first step in theory building (Yin 1994) at any level of knowledge development (Anderson et al. 2005). Yin (2013) and Eisenhardt (1989) agree that there are three types of case study research objectives: descriptive, explanatory, and exploratory investigation. Descriptive case studies are focused on capturing a phenomenon within its real-world context (Yin 2013) to help in describing a situation (Yin 1981). Exploratory case studies are used in situations where a phenomenon has not been examined in depth. The primary purpose of this type of study is to help in identifying the research questions or approach to be used in future research towards theory development (Yin 2013). Finally, explanatory case studies aim to gain insight and understanding into why or how a phenomenon has or has not occurred (Yin 2013). Explanatory case studies can, therefore, be used towards making causal inferences (Yin 1981). 
As the focus of this study is within a domain that has yet to be extensively studied, the research strategy includes an exploratory approach in the examination of knowledge acquisition methods and their role in co-creation of innovation performance.

\subsubsection{Case Study Reasoning - Deductive, Inductive, Abductive, Retroductive Theory}

Deductive, inductive, and abductive reasoning theory represents the three primary theories of research-based knowledge creation (Ong 2012). Deductive theory describes the most common perspective in examining the nature of the relationship between theory and research (Bryman \& Bell 2015). A deductive approach proposes hypotheses based on existing theory to explain observations of a phenomenon, and test the validity of the hypotheses (Tavory \& Timmermans 2014). Inductive theory focuses on understanding the connection between observed phenomena and theory (Bryman \& Bell 2015). The inductive approach is well established as a method for data analysis in theory development (Charmaz 2014; Glaser \& Strauss 1967), where data is collected and used to either reinforce or challenge well-established theories (Tavory \& Timmermans 2014). Inductive theory in case study research commonly involves the collection and analysis of qualitative data for use in exploring phenomena (Eisenhardt 1989). Finally, abductive theory describes the process of forming an explanatory hypothesis when examining a set of observations where the relationships are unclear (Tavory \& Timmermans 2014). Abduction observes phenomenon through the collection of data, and focuses on describing causation and generalized explanation through patterns identified in the events studied (Edwards et al. 2014). The abductive approach is commonly associated with a qualitative research method and CR-related methodologies (Edwards et al. 2014; Peirce \& Turrisi 1997). Similar to 
abduction is retroductive reasoning, which seeks to explain the higher-level causal reality through extended study (Edwards et al. 2014).

Abductive logic is distinctly different from an inductive reasoning approach. Induction begins with the collection of cases and proceeds to examine them to infer that there is a universal truth. An inductive approach provides no explanation of the relationship between entities studied to explain what is being observed (Edwards et al. 2014).

The CR philosophy argues a stratified reality with a focus on generalizing about theoretical propositions versus populations (Carlsson 2003; Easton 2010; Wynn \& Williams 2012). Through this layered approach of linking theory to data, abductive and retroductive logic can provide a richer perspective on reality than either inductive or deductive approaches (Edwards et al. 2014). As they are closely connected, many researchers view abduction and retroduction as a common school of logic. Abduction and retroduction help to provide theories for parts of reality that are unobservable, which serve as a basis for creating new knowledge (Dubois et al. 2014; Mingers 2006). Both approaches to creating explanation stress what emerges from qualitative data as the best theory explaining reality (Dubois et al. 2014; Mingers 2006).

A goal of CR researchers is to illuminate causal mechanisms at work (Edwards et al. 2014). Case studies provide the vehicle for the in-depth investigation required to achieve such insight, building on abductive and retroductive logic (Easton 2010). As this study examines phenomena within a complex open system through the use of a comparative case analysis, these positions in addition to Karlsson and Ackroyd's (2014) recommendations (see Figure 4.1) support the choice of abductive reasoning used in this research. 


\subsection{Case Rigour, Theory Building and Validity}

The research design for this study has been developed to ensure the needs for procedural rigour and validity of findings have been addressed. This section examines what rigour and validity entail in practice, and the considerations in building theory for this case study.

\subsubsection{Case Rigour}

Case research provides the potential for rich insight into phenomena for researchers and theory, through in-depth and rigorous study. Engagement at the field level presents a potential for the creation of new knowledge that would not be evident through traditional deductive methods (Voss et al. 2002). However, traditional case study research is not unchallenged in the face of other methodological choices, with criticisms ranging from "sloppy" execution, the lack of systematic processes, and the impact of various levels of bias on research conclusions (Yin 2013). Case research faces concerns regarding the degree of methodological rigour in studies as they relate to case reliability and validity (Daft \& Lewin 1990). Case study is also questioned on its actual role in developing theory and the generalizability of related findings (Noor 2008).

Arguments against case study often support a misconception that this method of research is limited in its ability to generalize findings to theory based on the typically small number of cases examined in studies. There is a tendency for researchers to "approach a case study as if it were a sample of one drawn from a wider universe of such cases" (Bryman \& Bell 2015). In fact, it is the approach of case research to sampling that is a unique feature of the method. Case study research, by its nature, is limited by small 
numbers in a study sample and in its role in delivering statistical representation that is not its objective (Easton 2010). Case research uses its detailed study approach towards achieving generalization to theory (i.e., analytical generalization) versus an extrapolation of probabilities to population (i.e., statistical generalization) (Yin 2013).

For research based on case study methods to be recognized for its contribution to the body of knowledge, accounting for case rigour is central to effective case study design. Gibbert, Ruigrok, and Wicki (2008) stress two primary concerns supporting the importance of designing for case rigour: 1) as case study is typically used in the early stages of theory development (Eisenhardt 1989; Yin 1994), the effects of any poorly executed research will potentially impact all related future study and research contributions, and that 2) case research is often conducted towards managerially related application (Leonard-Barton 1990), which when found to be based on inadequate methodological discipline could be deemed to have low relevance for practitioners.

To address such challenges, this study has applied rigorous case research to ensure the place of its research findings as "valid and relevant" (Voss et al. 2002). Chapter 5 details the research design and methodology.

\subsubsection{Case Validity and Reliability}

Research "validity is not a commodity that can be purchased with techniques," and is of such importance that it should be treated as its own topic, separated out from research methodology (Maxwell 2012 p.121). Maxwell (2012) argues that validity is achieved through a greater focus on research evidence over the methods used. Validity should be viewed as a context-dependent property of methods used and of the conclusions presented 
from research (Maxwell 2012). Positivist research has presented case rigour as consisting of external validity, internal validity, construct validity, and reliability (Cook \& Campbell 1979; Yin 1994), which were further developed for use in case study research (Eisenhardt 1989; Yin 1994)

External Validity ("generalizability"): External validity refers to the generalizability of research findings outside the context of the case(s) being studied (Maxwell 2012). As has been discussed, both single and multi-case studies, by design, are not intended to achieve statistical generalization towards a larger population (Yin 1994). Rather, case studies contribute to an analytical generalization of observations to theory (Yin 1994). As such, researchers should provide readers with clarity on the choices made regarding a study, including why particular cases have been chosen for the study and the context in which the research will be undertaken (Cook \& Campbell 1979).

The findings from this study present "analytical generalizability." The focus on achieving analytical generalizability is consistent with the nature of CR-based research (Yin 2013). From a CR perspective, the focus of the investigation is typically on examining the complexity of a phenomenon. As with the case study method, the goal of critical realism is not to achieve statistical inference for a population. The nature of complex phenomenon makes it unrealistic to expect similar outcomes for studies replicated in a different context (e.g., organization, industry, region, culture) (Wynn \& Williams 2012). Generalizability within a CR domain aims to use observations of causal mechanisms in one context, as a potential explanation for observations in other contexts (Wynn \& Williams 2012).

Internal Validity: Internal validity (or logical validity) refers to the relationship between variables in the study and the results presented (Yin 1994). Specifically, it asks 
the question of whether the research has given a strong argument in support of the conclusions presented (Gibbert et al. 2008; Gibbert \& Ruigrok 2010). Internal validity was achieved in this study through providing visibility of a "clear research process" (Gibbert et al. 2008), a strong analysis demonstrating the expected outcomes (Eisenhardt 1989; Yin 2013), and its demonstrated use of triangulation in the examination of the phenomenon being studied (Yin 1994). Chapter 5 outlines the research process developed and used in this study.

Construct Validity: Construct validity is a focus on research design and whether what is being studied is in fact what was intended, ultimately leading to accurate conclusions regarding the nature of reality (Denzin \& Lincoln 1994; Yin 1999). Often argued as being underdeveloped by many researchers, there are criticisms that constructs and measures are often attributed to researcher beliefs (Daniel et al. 2014; Flyvbjerg 2006). Yin $(1994,2013)$ guides researchers to address construct validity in their design by developing a clear "chain of evidence" that supports the reconstruction of a study by future researchers, and to triangulate using multiple methods of data collection and sources of data.

Reliability: Research reliability refers to researchers addressing random error by providing transparency and replicability in their design. Addressing reliability allows for future researchers to arrive at the similar conclusions if they were to conduct a study following the same steps outlined in the initial research (Denzin \& Lincoln 1994). Researchers should ensure that they operationalize as many steps as possible to help in increasing the reliability of their studies (Yin 2013). This was achieved in the study through the development of a case study protocol and the development of a database to manage and 
maintain all related study notes, documents, and artifacts for potential use in future research (Leonard-Barton 1990; Yin 2013).

Wynn and Williams (2008) propose Maxwell's (2012) validity typology for CRbased case study research, highlighting that while these are not the only criteria for evaluating validity, this set is most closely aligned with the CR philosophy (see Table 4.2). To address validity objectives, this typology was used in guiding the research design for this study.

Table 4.2 Validity Typology for Critical Realism-Based Case Research

\begin{tabular}{|l|l|l|}
\hline \multicolumn{1}{|c|}{ Validity Type } & \multicolumn{1}{|c|}{ Description } & \multicolumn{1}{c|}{ Addressing Threats } \\
\hline Descriptive & $\begin{array}{l}\text { Ensure accuracy of the researcher's } \\
\text { account of observable words and actions }\end{array}$ & $\begin{array}{l}\text { - triangulation } \\
\text {. interview recordings and transcripts } \\
\text {. participant checks } \\
\text {. multiple observers where feasible }\end{array}$ \\
\hline \multirow{2}{*}{ Interpretative } & $\begin{array}{l}\text { Researchers express and describe } \\
\text { meaning of participants' words and } \\
\text { actions }\end{array}$ & $\begin{array}{l}\text {. participant checks } \\
\text {. intercoder reliability tests } \\
\text {.participant review of findings } \\
\text {. multiple informants }\end{array}$ \\
\hline Theoretical & $\begin{array}{l}\text { Review validity of constructs and } \\
\text { relationships used as explanation }\end{array}$ & $\begin{array}{l}\text {. triangulation } \\
\text {. participants review of coding categories } \\
\text {. comparison to alternative theories } \\
\text {. causal evaluation of explanations }\end{array}$ \\
\hline Generalizability & $\begin{array}{l}\text { Extent that results can be applied to } \\
\text { settings other than those studied }\end{array}$ & $\begin{array}{l}\text {. purposive replication logic } \\
\text {. contextual analysis }\end{array}$ \\
\hline
\end{tabular}

Adapted from Wynn and Williams (2008)

\subsubsection{Researcher Impact on Validity}

Amongst the myriad of factors that can affect research validity, researchers must also consider their potential impact on either introducing bias or reactivity into a study (Maxwell 2012). Researcher bias refers to the possibility of researcher subjectivity being 
woven into their studies and conclusions, while reactivity describes the potential for them to influence the setting in which they engage with individuals being studied (Maxwell 2012). Researcher experience and context provide a valuable asset in case research, and the goal of research design should not be to eliminate the influence of the researcher, but rather to understand where there is a potential for impact and how it can be used productively in the study (Maxwell 2012).

\subsubsection{Triangulation and Multi-Methods in Case Research}

Triangulation refers to the use of different methods of data collection, each with their own benefits, that when brought together converge and support a single research conclusion (Eisenhardt 1989; Maxwell 2012; Stake \& Stake 2013). In case research, triangulation helps in addressing the challenges of research validity by applying a broad selection of information types available to a method of study (Yin 2013). It does so in drawing upon a range of theories and methods in conducting the research (Bryman \& Bell 2015; Maxwell 2012). The researcher's goal in the use of triangulation is to ensure that findings are not simplified or overplayed and that the conclusions being conveyed are supported by the information observed in the research (Stake \& Stake 2013). Triangulation and a multi-methods approach to data collection provide an important multi-directional view in support of case study analysis based on different sources, analytical methods, and theoretical perspectives (Wynn \& Williams 2012).

The most common triangulation strategy is the introduction of redundant data gathering and procedural challenges in the research design (Stake \& Stake 2013). Triangulation can be applied across data sources (“data triangulation"), different evaluators 
("investigator triangulation"), varying perspectives in the same data ("theoretical triangulation"), and across methods ("methodological triangulation") (Patton 2002). Data, theoretical and methodological triangulation were addressed in the research design. Evaluator triangulation does not apply as this study was conducted by a single research investigator. The choice of the types of triangulation used in this research study was determined by the research objectives, information sources, and context. Triangulation techniques are similar across both single and multi-case studies. The protocol used in single case analysis can be extended out to a group of cases in multi-case research by applying the same rigour and techniques. This applies whether the view from alternate sources of information support what has been observed either at the single or the cross-case level (Stake \& Stake 2013).

Triangulation is equally relevant and important in theory building informed by a CR philosophy. Critical realism is based on epistemological principles of mediated knowledge, observability, and the exploration of multiple possible mechanisms explaining the phenomenon being studied (Wynn \& Williams 2012). Critical realism embraces the value of triangulation, emphasizing that reality is complex and that knowledge is composed of many types structures requiring a variety of approaches to developing knowledge about the phenomenon (Wynn \& Williams 2012).

The design of this research study has included practices discussed to ensure the validity of research findings are well supported, and meet the needs of a critical realistinformed multi-case research process and analysis. The methods used in this study are detailed further in Chapter 5. 


\subsection{Single Versus Multi-Case Studies}

Case studies take the form of either a single or multi-case design (Eisenhardt 1989; Yin 2013), each approach serving different research objectives (Stake \& Stake 2013). Single case designs are the most common type of study (Yin 2009) that typically focus on either detailed exploration of unusual or extreme events, critical cases, or revelatory cases (Maxwell 2012; Yin 2013). Unlike the single case, multi-case designs are focused primarily on understanding observed phenomena and their context across a collection of cases (Stake \& Stake 2013). This study adopted a multi-case design approach.

Yin (2013) describes that single case studies are best used when research objectives are intended to perform either a critical test of existing theory, an examination of an extreme or unusual circumstance, or when there is a typical case serving a revelatory or longitudinal purpose. While single case design provides the opportunity for insight in the early stages of theory development, it is often challenged on the contribution of resultant findings and related generalizability of conclusions, models, or theory (Voss et al. 2002). Of primary concern is the study of a single event and exaggerating it across a population (Leonard-Barton 1990). This again is keeping in mind that the main goal of case research is towards analytical versus statistical generalizability (Eisenhardt \& Graebner 2007).

Multi-case studies consist of a number of single case investigations, in which each case is part of the overall study's "case quintain" (Stake \& Stake 2013). The role of the individual case in this design differs from a single case study in that the primary concern of researchers is in examining patterns, commonality, or differences across the cases (Stake \& Stake 2013). Stake and Stake (2013) stress that in multi-case studies researchers must manage the implicit tension which forms between the attention demanded from a single 
case investigation and its contribution to the overall objectives of the multi-case study. He argues that much of this pressure comes from a conflict between trying to achieve research analytical generalizability and applicability of findings.

A multi-case design is best suited for comparative multi-case studies (Stake \& Stake 2013; Yin 2013). Cases in a multi-case design should be selected either in support of examining similar results (i.e., a literal replication) or when considering contrasting results based on anticipatable reasons (i.e., a theoretical replication logic) (Yin 2013). Theoretical replication in a multi-case design allows for the examination of differences across cases. A two-tailed design can provide for cases from extremes on a particular condition of interest (e.g., initiative performance) (Yin 2013), which is the case in this study. This will provide for stronger findings compared to a single case study approach (Hanna 2005, Yin2013).

Multi-case designs are argued to be more useful in building theory than a single case approach due to the broader potential scope of research questions explored and their use in theory development (Eisenhardt \& Graebner 2007). Multi-case studies help to mitigate design-related risk, to some degree, by increasing external validity and minimizing the risk of observer bias (Voss et al. 2002). This is achieved by the often broader scope of study and comparative nature of multi-case design (Voss et al. 2002). While multi-case designs present distinct advantages over a single case approach, it must also be kept in mind that there are goals intended for single case methods that cannot be achieved using a multi-case approach (Maxwell 2012).

Eisenhardt and Graebner (2007) argue that while there are legitimate challenges to the use of case research and theory development, these can be mitigated using precise 
language, and thoughtful design that includes "careful justification of theory building, theoretical sampling of cases, interviews with limited...bias, rich presentation of evidence in tables and appendices, and a clear statement of theoretical arguments." For the reasons stated, this study will examine the impact of knowledge acquisition methods on new-tothe-firm innovation using a nested, comparative multi-case, two-tailed design. This strategy will allow for a cross-case comparison of successful to less successful initiatives, and the role of competencies and context on innovation performance. Eisenhardt and Graebner's (2007) sage advice to researchers provided a valuable compass in the design of this study.

\subsection{Time Horizon}

Case study design must also consider the time horizon that applies to the research. Specifically, the researcher must determine whether the study will examine phenomena at a point in time or over time, and whether the evidence is to be collected from events in the past or for those anticipated to occur in the future.

Cross-Sectional versus Longitudinal Study Case researchers must determine if their study is cross-sectional or longitudinal in nature. Cross-sectional design describes the study of multiple cases at a single point in time, through the collection of qualitative and quantitative information, which is examined to detect patterns and relationships (Bryman \& Bell 2015; Maxwell 2012). Longitudinal design aims at studying a phenomenon over time, with a focus on analyzing change (Bryman \& Bell 2015). This form of design helps in studying organizations and the drivers of change, including specific mechanisms and processes that underpin the structure (Bryman \& Bell 2015). Longitudinal studies typically 
involve the researcher collecting factual information on a regular basis in support of examining patterns (Maxwell 2012).

Retrospective versus Prospective Events Case studies must also be considered from the perspective of the timeframe or point of reference in which the study is exploring a phenomenon. Case studies can be retrospective or prospective in nature (Bryman \& Bell 2015). Retrospective studies are focused on examining events that occurred in the past and are conducted based on information that is available and from respondents' recollections (Bryman \& Bell 2015). Prospective studies, on the other hand, focus on the phenomenon of events that are likely to happen and attempt to establish what the outcome will be (Bryman \& Bell 2015).

This study represents a cross-sectional retrospective examination of multiple case studies that represent a collection of completed innovation initiatives. 


\section{Chapter 5 RESEARCH DESIGN AND METHODOLOGY}

Relative to traditional quantitative methods, case research provides for a less constrained approach, making it well suited for exploratory study of phenomena (Voss et al. 2002). This in part contributes to why some argue that building theory from cases is often the "most interesting" kind of research (Bartunek et al. 2006, Eisenhardt 1989). As with other research methods, the choice of a case study approach is dependent on its fit with the research problem and the nature of the phenomena being explored (Bryman \& Bell 2015). A recognition that cases studies are spatially and temporally bounded guides the best approach to be taken in case study design (Gerring 2004).

This chapter presents the research design and methodology employed in this research, the considerations for the comparative multi-case design, and the research roadmap for this study.

\subsection{Case Study Roadmap}

An examination of writings on research and case study methodology commonly recommend the development of a research roadmap to help guide research studies through successful execution (Bryman \& Bell 2015; Eisenhardt 1989; Kumar 2014; Maxwell 2012; Yin 2013). Research roadmaps in management research help guide investigations towards quality, relevant and dependable research findings (Eisenhardt 1989; Jaworski \& Kohli 1996).

There is significant guidance in the literature providing researchers with direction on designing and conducting case studies. These are typically from either a positivist 
(Eisenhardt 1989) or an interpretivist perspective (Walsham 2006). Wynn and Williams (2012) point out that this guidance is valuable and also serves in providing a rich source of important direction for CR researchers using case study methods.

This guidance on case study methodology presents several common themes. Wynn and Williams (2012) emphasize for the CR researcher the importance of the research question, case selection, and generalizability of findings. These areas are highlighted specifically to address the goals of CR research towards explaining causality in complex and open contexts. Stake and Stake (2013) underscore the importance of clarity in research questions and data collection, emphasizing the role of information that comes from individual cases in contrast to the analysis that must occur across all cases in their entirety. Researchers are encouraged to examine early findings and experiences with their research subjects to help shape their studies. Discussions with informants further contribute to shape research questions that are intended to explore the phenomena being studied and to assess the value of selected cases in achieving research objectives (Maxwell 2012).

Another important message to case study researchers is the emphasis placed on taking an adaptive approach in their design (Steak \& Stake 2013; Yin 2013) and adopting an integrated and interactive research model where research questions are at the centre of the investigation (Maxwell 2012). A common issue encountered in case research is maintaining a focus on the original set of problems and context. While case rigour needs to be maintained (Yin 2013), researchers must not fall into a trap of an overly restrictive design that may result in missing important evidence that emerges during the study (Steak \& Stake 2013). 
Case researchers must be ready to adjust when faced with potential issues that arise during the research process (Steak \& Stake 2013). Researchers should expect that case designs may change as new information is gathered during the data collection process. New data may provide valuable insights leading to a thoughtful decision to alter or modify an original research design (Yin 2013). In the end, the goal of good case study design should be towards maximizing the use of available resources over implementing a rigid approach to the research design (Maxwell 2012).

A roadmap has been developed for this study incorporating the guidance from Yin (2013) and Eisenhardt (1989) in their broadly cited works on case research design. This roadmap has also included direction from Wynn and Williams (2008) highlighting CR methodological principals and modifying stages in the process that are CR specific in the approach to data collection and analysis. Wynn and Williams (2008) guide CR researchers to observe six methodological practices in their research, that are intended to ensure: 1) there is a clear explanation of relationships between structure and context of the phenomenon over time, 2) they apply the use of abduction logic when linking events to the causal mechanisms, 3) the study focuses on a limited number of cases to gain insight into the phenomenon being examined, 4) there is support for causal explanations within observed empirical observations, 5) triangulation and multi-method research techniques are applied to address potential research bias, and 6) practices are put in place to incorporates the dimensions of research validity and generalizability. These principles (see Table 5.1) have been used as a foundation in guiding the design of this study.

Eisenhardt (1989) proposes an eight-step process for building theory from case study research (see Table 5.2). The process provides a systematic approach leading 
researchers from establishing a precise definition of their research goals, through determining research methods, case selection, field collection of data and its analysis, and reaching conclusions that are well supported, with contributions that are clearly articulated. These steps align closely with the guidance provided by Yin (2013) on executing case research.

Table 5.1 Methodological Principles of Critical Realism

\title{
Methodological Principles
}

\begin{abstract}
Explication of Structure and Context:

Identify and describe key components of the social structure and its contextual environment including components parts and their relationships, resources, rules and roles, significant actions (agency), and the cultural system of the target environment.
\end{abstract}

\begin{tabular}{|c|c|}
\hline $\begin{array}{l}\text { Abduction/Retroduction from } \\
\text { Events to Mechanism: }\end{array}$ & $\begin{array}{l}\text { A creative process which posits the existence of causal mechanisms that must exist } \\
\text { within a social structure to produce the phenomenon of interest. }\end{array}$ \\
\hline Idiographic Approach: & $\begin{array}{l}\text { Analyzing a particular case or limited number of cases in explicit detail to investigate } \\
\text { the causal processes in terms of individual, structural and contextual influences which } \\
\text { produced observed events. }\end{array}$ \\
\hline Empirical Corroboration: & $\begin{array}{l}\text { Use data from empirical observations to ensure proposed causal explanations are } \\
\text { clearly and accurately described in terms of generating outcomes within the given } \\
\text { context, and offer better explanatory power than other potential mechanisms that } \\
\text { have been identified. }\end{array}$ \\
\hline \multirow[t]{2}{*}{ Triangulation/Multi-Methods: } & $\begin{array}{l}\text { Using multiple perspectives in the form of multiple data sources, theories, } \\
\text { investigators and methods to avoid potential biases in the research process. }\end{array}$ \\
\hline & $\begin{array}{l}\text { Ensuring the quality of the hypothesized explanations is sufficient to allow readers to } \\
\text { accept the conclusions reached within a given research project. This incorporates four } \\
\text { dimensions including descriptive, interpretive, and theoretical validity and } \\
\text { generalizability. }\end{array}$ \\
\hline
\end{tabular}

Adapted from Wynn and Williams (2008)

The research roadmap for this case study draws upon and aggregates these broadly cited proven practices to support achieving the research objectives (see Figure 5.1). The roadmap reflects a comparative multi-case two-tailed study, which is retrospective and cross-sectional in nature. The study will be informed by the CR lens and will apply 
abductive reasoning in the forming and support of research conclusions. Note that each stage of the research roadmap has been highlighted within the Eisenhardt framework as well (see Table 5.2, "Research Roadmap Stage"). Each stage of the roadmap will be discussed in further detail in remaining sections of this chapter.

Table 5.2 Eisenhardt Process for Building Theory from Case Study Research

\begin{tabular}{|c|c|c|c|}
\hline Step & Activity & Reason & $\begin{array}{l}\text { Research } \\
\text { Roadmap } \\
\text { Stage } \\
\text { (see Fig 5.1) }\end{array}$ \\
\hline Getting Started & $\begin{array}{l}\text { Definition of research question ... } \\
\text { Possibly a priori constructs ... } \\
\text { Neither theory nor hypothesis ... }\end{array}$ & $\begin{array}{l}\text { focus efforts } \\
\text { improve grounding of constructs } \\
\text { retain theoretical flexibility }\end{array}$ & 1 \\
\hline $\begin{array}{l}\text { Crafting Instruments } \\
\text { \& Protocols }\end{array}$ & $\begin{array}{l}\text { Multiple data collection methods ... } \\
\text { Qualitative and quantitative data combined ... } \\
\text { Multiple investigators ... }\end{array}$ & $\begin{array}{l}\text { strengthen grouding of theory through triangulation } \\
\text { synergistic view of evidence } \\
\text { foster divergent perspectives/strengthen grounding }\end{array}$ & 2 \\
\hline Selecting Cases & $\begin{array}{l}\text { Specified population ... } \\
\text { Theoretical sampling vsrandom sampling ... }\end{array}$ & $\begin{array}{l}\text { constrain variation and sharpen external validity } \\
\text { focus efforts on theoretically useful cases }\end{array}$ & 3 \\
\hline Entering the Field & $\begin{array}{l}\text { Overlap data collection and analysis ... } \\
\text { Flexible/opportunistic data collection method ... }\end{array}$ & $\begin{array}{l}\text { speeds analysis / adjust data collection } \\
\text {. take advantage of emergent themes and case uniqueness }\end{array}$ & 4,5 \\
\hline Analyzing Data & $\begin{array}{l}\text { Within-case analysis ... } \\
\text { Cross-case pattern analysis ... }\end{array}$ & $\begin{array}{l}\text {. gain familiarity with data / prelim theory generation } \\
\text {. look beyond initial impressions / use multiple lens }\end{array}$ & 6 \\
\hline Shaping Hypothesis & $\begin{array}{l}\text { Context/mechanism/outcome mapping ... } \\
\text { Interative tabulation of evidence per construct ... } \\
\text { Replication logic across cases ... } \\
\text { Search relationship evidence for "how", "why"... }\end{array}$ & $\begin{array}{l}\text { critical realist explanation making } \\
\text { sharpens construct definition, validity, measurability } \\
\text {. confirms, extends, and sharpens theory } \\
\text {. builds internal validity }\end{array}$ & 7 \\
\hline Enfolding Literature & $\begin{array}{l}\text { Comparison with conflicting literature ... } \\
\text { Comparison with similar literature ... }\end{array}$ & $\begin{array}{l}\text { builds internal validity, raises theory, sharpens constructs } \\
\text { sharpens generalizability, improves constructs, raises } \\
\text { theorical level }\end{array}$ & 7 \\
\hline Reaching Closure & Theoretical saturation when possible ... & $\begin{array}{l}\text {. ends process when marginal improvement becomes } \\
\text { small }\end{array}$ & 7 \\
\hline
\end{tabular}

Adapted from Eisenhardt (1989)

\subsection{Case Research Site and Initiative Landscape}

First-of-a-Kind collaboration has been selected as the knowledge acquisition method to be studied. The case study examines IBM's First-of-a-Kind (FoaK) collaborative 
I

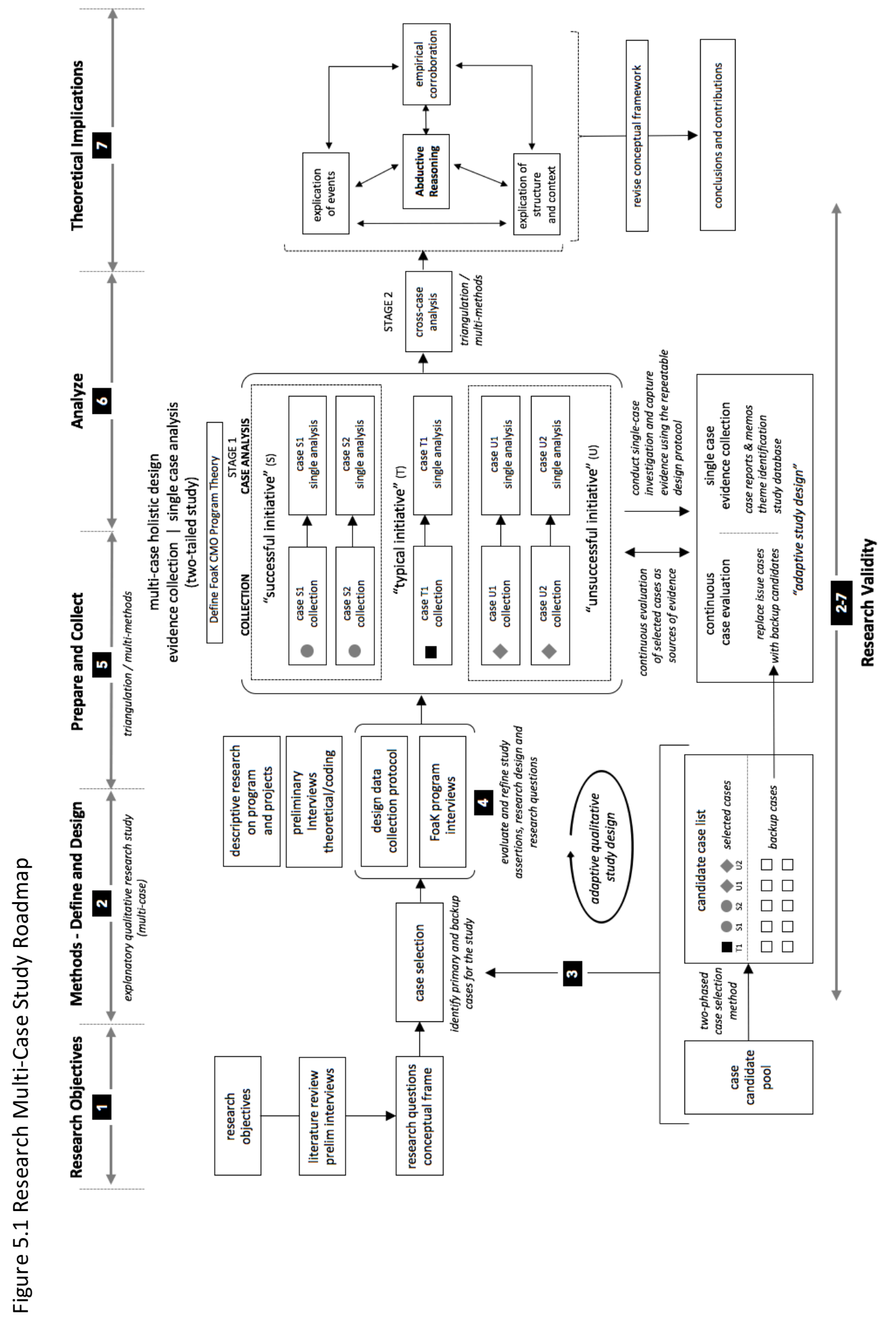


innovation program as a knowledge acquisition method. The FoaK method for the cocreation of innovation represents a "middle-ground" producer-stimulated user innovation method (see Figure 3.1 Knowledge Acquisition Method Framework). FoaK is characterized by the nature of it openness, complexity and the level of engagement between the firm and external stakeholders. For this study, an investigation of FoaK presents an opportunity for a rich source of information to explore the question of knowledge acquisition methods as a higher order integrative dynamic capability and to gain greater insight into inter-firm and intra-firm co-creation processes. It also offers an opportunity for contributions to the open innovation and NPD literature as an area of co-creation that has yet to be studied in detail.

This section discusses the study's research site and the landscape of individual case studies. Chapter 6 further builds on this overview with a detailed profile of IBM as the subject firm and a description of its FoaK program and related initiatives.

\section{The Research Site}

The research site for this study is the IBM Corporation. The study collected data directly from the FoaK program organization and its initiative teams. Informants in the study spanned across functions and divisions of the organization, as well as geographies. Internal IBM systems were used for all contact, collaboration, scheduling, and access to program artifacts for the study. This was found to be sufficient, and there was not further need for additional methods to be applied to ensure the level of access required. As a researcher, internal to the organization, there was access to the same IBM systems used by 
informants which allowed for a more efficient process of engaging with the study subjects, and other sources of evidence to be used in the multi-methods approach.

\section{Case Landscape}

As will be discussed in more detail further in this chapter, the unit of analysis in this study is the FoaK initiative. Multiple cases (i.e., FoaK initiatives) were examined in a comparative multi-case study design. Each of these cases shared common elements, which were considered in the design of data collection against the study's research questions.

Each case was guided by a common landscape (see Figure 5.2) and examined at multiple levels (i.e., macro, meso, micro) and across informant perspectives towards formulating the rival explanations of observed phenomena. This common case landscape includes the following four areas of examination: 1) the execution of the initiative and the link between the subject initiative and client firm, 2) the relationship between the initiative execution and its performance, 3) the relationship between FoaK program management and initiative governance, and 4) the relationship between firm context, the program and related initiatives.

\subsection{Unit of Analysis}

The unit of analysis in case study research is related to the research question(s) to be examined, and is defined as the "what" or the "thing" being studied (i.e., actors, objects) and measured (Myers \& Avison 1997; Yin 2013; Yurdusev 1993). At an analytical level, there are three types of units of analysis (Yurdusev 1993): 1) the individual person as an actor, 2) the society or group of individuals (agglomeration of actors), and 3) the universe 
or humanity (the all-inclusive actor). In case research, the unit of analysis is the case being examined (Yin 2013). It is the intended contribution of the case to theory, and previous studies in the area, which will dictate how the case will be defined and what the unit of analysis will be (Yin 2013). The unit of analysis must be distinguished from the unit of observation (i.e., the level at which data is collected), as the two are often not the same (Yurdusev 1993).

Figure 5.2 Individual Case Landscape

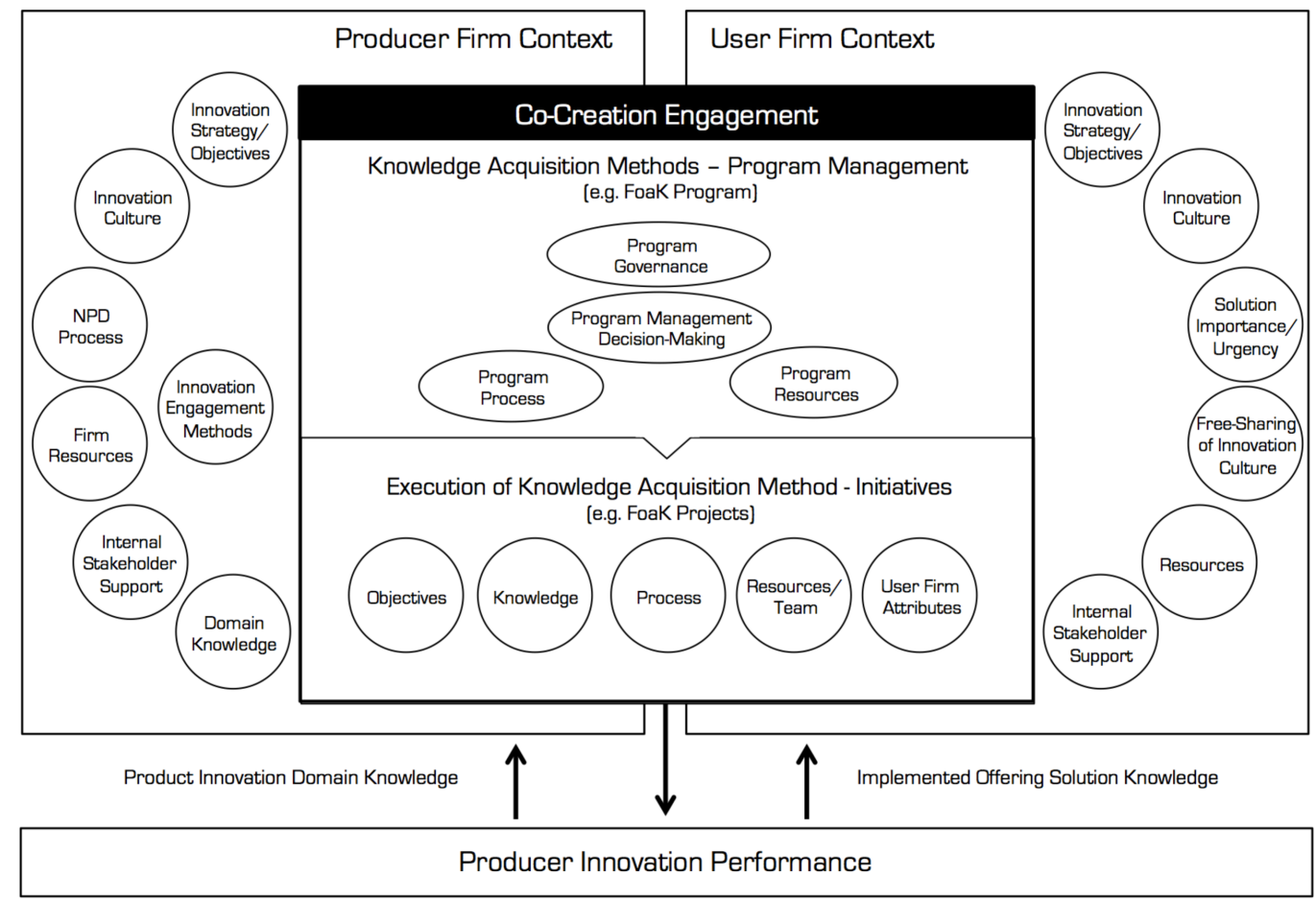

Source: Robert Helal (2017). Developed from program pre-study and interviews.

The unit of analysis in this study is the FoaK initiative (i.e., project), with each project representing a distinct case investigated. The research questions were all focused on understanding the mechanisms resulting in the observed performance of individual 
FoaK projects. This included the assessment of initiative performance, their execution, their management within the FoaK program (i.e., knowledge acquisition method), and the influence of context on FoaK innovation performance. The individual FoaK cases, in turn, formed the case quintain (Stake \& Stake 2013), representing the level of the comparative cross-case analysis performed. The choice of the FoaK project as the unit of analysis provided the necessary insight into the research questions and supported the research objectives for the study.

The unit of observation was primarily at the level of the individual informant, which is discussed further in the section in data collection section (see Section 5.5). This includes the collection of data through semi-structured interviews and the review of artifacts prepared by individuals throughout the process of their FoaK initiative.

\subsection{Case Sampling Strategy}

Case selection is a critical stage in case study research. This section will examine the role and principles of case study selection in research design and the theoretical sampling approach used in selecting the FoaK initiatives as cases for this study.

\subsubsection{The Role of Case Selection}

Case studies, by their nature, are intended to provide an in-depth analysis of a phenomenon being studied (Maxwell 2012; Stake \& Stake 2013; Yin 2013). They focus on achieving analytical generalizability to theory that is pursued through a detailed investigation of the phenomenon being examined (Yin 2013). Case selection must be focused on balancing this goal of gaining deeper understanding against the significant 
resource investment typically required in this method of research (Seawright \& Gerring 2008).

This research represents a retrospective study. Retrospective studies provide a source of reliable information when cases are recent and more control than can be found in real-time cases (Seawright \& Gerring 2008). Collecting and examining data that is historical in nature, whether through archives or interviews, can present researchers with challenges in accessing the most accurate explanation of informant observations. Karlsson and Ahlstrom (1997) describe a number of issues in retrospective studies that must be considered in design of the study. These include concerns such as informants not accurately recalling past events or their restating of history based on information that was not available at the time (i.e., post-rationalization), archival data that may represent a modified version of the whole truth to avoid controversial or disputed topics, and potentially only having limited access to the full set of the historical data required to provide a complete view of what is being examined.

Miles et al. (2013) state that when researchers are faced with the selection of cases to be studied, they must set boundaries that can be studied and create a simple framework that will help in shaping the processes and construct that will be the foundation of the study. Stake and Stake (2013) emphasize that researchers conducting multi-case studies should be guided by three criteria when selecting cases: 1) each case must be relevant to the group of cases (i.e., the case quintain), 2) those cases should provide diversity and contribute to the importance of the questions that led to the need for a multi-case study, and 3) the cases should provide a sufficient opportunity to learn about the complexity of the phenomena. 


\subsubsection{Case Selection Criteria}

Researchers that are not using either probability-based or convenience-based sampling are using a method that falls into the group known as "purposeful selection" (aka "purposive sampling" (Maxwell 2012)). Purposeful case selection is the method used in this study.

From a CR perspective, case study selection is focused on choosing cases that will provide the greatest insights into understanding the causal mechanisms that are influencing what is being observed (Wynn \& Williams 2012). Specifically, case selection through the $\mathrm{CR}$ lens is looking for cases that provide the greatest insight into causal mechanisms which produced a unique set of events and that have specific structural/contextual factors that came together to generate them (Wynn \& Williams 2012). The researcher is looking for cases that will provide the greatest insight towards the goal of building a theory that closely explains what is observed through the research (Sayer 1992). This approach helps the researcher to create "context-sensitive causal explanations of specific phenomena" (Wynn \& Williams 2012). Multiple-case selection can occur within a single structure or entity (e.g., company) (Bygstad 2010) or across a more complex composition such as multiple divisions (Morton 2006) or locations within a firm (Volkoff et al. 2007).

Voss et al. (2002) state the importance of cases being chosen based on a clearly defined set of criteria and reminding researchers that when examining candidate cases they should "have the courage to discard cases that do not fit the research design and sample structure." As such, it must be determined in advance which parameters of the population will be kept constant across the selection process to allow for some control in the design of the study (Voss 2002). Miles et al. (2013) propose a number of tests to guide researchers 
in determining which cases to include in their studies. They suggest consideration of the relevance of all case candidates to the conceptual framework and research questions, their richness related to the phenomenon being studied, their ability to enhance generalizability, and finally, the feasibility and ability of cases to address ethical concerns.

This study applied a selection framework based on Maxwell (2012) proposing a similar set of five goals for purposeful selection of informant cases. This guidance recommends that cases be selected based on their potential contribution to: 1) achieving representativeness or technicality of the settings, individuals, or activities selected, 2) adequately capturing the heterogeneity in the population when possible, 3) critical testing of theories that the study began with or have been subsequently developed, 4) establishing the best and most productive relationships towards gathering insight for research questions, and 5) exploring particular comparisons to illuminate the reasons for differences between settings and individuals. This last goal is often used in multi-case qualitative study research.

\subsubsection{Number of Cases}

There are varying opinions as to the number of cases that should be included in multi-case studies. It is argued the number of cases needed in a research study should be left to the judgment of the researcher as it is discretionary versus formulaic and is dependent on the level of certainty desired for the study results (Yin 2013).

Yin (2013) suggests between two and three literal case replications on theory that is straightforward with low certainty required and up to five or more cases when a higher degree of certainty is the goal. In two-tailed comparative case studies, as with this research, 2 to 3 cases per tail are recommended (Seawright \& Gerring 2008; Yin 2013). Eisenhardt 
(1989) recommends between four and ten cases. Stake and Stake (2013) also argue that fewer than four cases and more than ten will limit the benefits of a multi-case study. The argument is that too few studies do not show enough of the interactivity between constructs within context and too many cases will provide more uniqueness and interactivity than researchers or readers will be able to understand (Stake \& Stake 2013). With the various views on "how many," Stake and Stake (2013) remind the researcher that many multi-case studies have justified having the number of cases outside these boundaries.

The number of cases that are included in the study must be balanced against the available resources for the research (Seawright \& Gerring 2008). As case study research is labour intensive, researchers must consider what is feasible in successfully completing a quality study against the time and resources available (Yin 2013).

\subsubsection{Case Selection for this Study}

In this study, the unit of analysis is the FoaK initiative. This represents the knowledge acquisition method at the level of a specific funded project. A FoaK initiative is managed within a program that crosses organizations and divisions within the subject company (i.e., IBM). An individual case for the study is represented by an individual FoaK initiative.

Maxwell's (2012) guidance on the selection of cases was used for this study. This study applied a multi-case, comparative, two-tailed design, examining cases that represent successful and unsuccessful FoaK initiatives. It examined a total of five cases, which included two "successful" cases, two "unsuccessful" cases, and one "typical" case. This number of cases provided the necessary insight for this study, while meeting guidance for 
study validity (Eisenhardt 1989; Seawright \& Gerring 2008; Stake \& Stake 2013; Yin 2013). The cases included were drawn from a list of completed projects using theoretical sampling (Eisenhardt \& Graebner 2007) and a purposeful selection approach (Maxwell 2012). An overview of the cases studied is presented in Table 5.3. Profiles of each of these cases will be detailed in later chapters.

Table 5.3 Cases Studied Overview

\begin{tabular}{|c|c|c|c|c|c|}
\hline & \multirow{2}{*}{$\begin{array}{c}\text { "TYPICAL" INITIATIVE } \\
\text { Case } 1\end{array}$} & \multicolumn{2}{|c|}{ "SUCCESSFUL" INITIATIVE } & \multicolumn{2}{|c|}{ "UNSUCCESSFUL" INITIATIVE } \\
\hline & & Case 2 & Case 3 & Case 4 & Case 5 \\
\hline Solution Domain & Analytics Solution & Analytics Solution & Analytics Solution & Analytics Solution & Analytics Solution \\
\hline Industry & Industrial Products & Retail & Automotive & Travel \& Transportation & Healthcare \\
\hline Firm-Level Strategic Agenda & Yes & Yes & Yes & Yes & Yes \\
\hline Industry Co-Creation Partner & $\begin{array}{l}\text { Natural Resources } \\
\text { (Mining) }\end{array}$ & Retail & Automotive & $\begin{array}{l}\text { Travel \& Transportation } \\
\text { (Rail) }\end{array}$ & Healthcare \\
\hline Initiative Dates (start/end) & $2013.06 / 2014.12$ & $2013.10 / 2015.07$ & $2013.12 / 2016.01$ & $2011.08 / 2013.05$ & $2012.02 / 2013.06$ \\
\hline Client Region & South America & United States & Asia-Pac & Western Europe & Western Europe \\
\hline Firm Co-creation Investment & $5 \mathrm{FTE} / 12$ months & $5 \mathrm{FTE} / 12$ months & $6 \mathrm{FTE} / 12$ months & $4 \mathrm{FTE} / 12$ months & $5 \mathrm{FTE} / 12$ months \\
\hline Client Co-creation Investment & Yes & Yes & Yes & Yes & Yes \\
\hline Initiative Performance: & Moderate & High & High & Low & Low \\
\hline Client Success (Reference) & Yes & Yes & Yes & Yes & Yes \\
\hline Innovation Assets Transfer & No & Yes & Yes & No & No \\
\hline Follow-on Revenue & Active; TBD & Yes & Yes & No & No \\
\hline \multicolumn{6}{|l|}{ Case Study Content Summary } \\
\hline Informants (count) & 8 & 11 & 6 & 6 & 6 \\
\hline $\begin{array}{l}\text { Correspondence \& Transcripts } \\
\text { (pages) }\end{array}$ & 331 & 349 & 505 & 318 & 292 \\
\hline Supporting Artifacts (count) & 16 & 38 & 32 & 23 & 34 \\
\hline
\end{tabular}




\section{Case Selection Criteria}

A clear set of selection criteria was established for this study. Candidates are selected based on "diversity" and "most different" criteria for the two-tailed study, with a focus on initiative performance as the measure of comparison (Seawright \& Gerring 2008). Selection criteria for cases have been determined to ensure that cases are chosen that are most likely to provide the deepest insight into the research questions being explored, will be accessible, and are exemplary of the level of initiative performance they are intended to represent.

The cases chosen for the study all represented initiatives that were part of the FoaK program and completed the full cycle from selection to closure. Priority was given to cases that were no more than five years old to help address concerns of informants' recollection and availability. There were sufficient cases within this timeframe, so there was no requirement for older cases to be considered. Cases were selected within the domain of analytics-based projects. This was intended to control for the nature and the complexity of the projects by choosing initiatives of similar type. The available list of initiatives did not allow for all projects selected to be from within a common industry (e.g., banking, public sector). The selection criteria were not found to be too restrictive due to the number of available cases to select from.

\section{Initial Candidate Case Selection}

A three-stage process for selecting the initial list of cases was used. The process began with an initial review and filtering of the comprehensive list of available cases within the FoaK program archives. An initial list of candidate cases was created based on a review 
of initiative profiles, scope, and timeframe. This provided a preliminary list of interesting candidates. The second stage of the process focused on establishing a short list of candidate cases through discussions with FoaK program office, experts familiar with the details of individual initiatives. In this step, the process included a review of the initial list of candidates with the FoaK program manager. This individual is central to all initiatives that pass through the program and has the broadest perspective of initiatives and how they have performed. Her long tenure in the role extends beyond the time constraints being applied in the selection criteria, ensuring the consistent perspective over time across candidates considered. Through discussions with the program manager, the initial candidate list was once again reviewed and reduced to a focused list of candidates further categorized into the three groupings based on initiative performance. Other candidates that were not included on the initial list but that were recommended by the program manager, were reviewed and considered for inclusion. In the final stage of the process, the shortlist of candidates was assessed based on ability to support the research objectives of the study.

A profile of priority cases was prepared, and initial contact with project teams was made. Based on preliminary discussions regarding the study with these teams, the decision was made on the cases to be included and if any required replacement (Voss et al. 2002).

\section{Adaptive Design}

The research design was such that it anticipated risks associated with the final list of selected cases for the study (Stake \& Stake 2013). This was necessary due to the nature of the subject environment, in which the possibility existed that informant's involvement might become limited or that recollection of events present either significant bias or lack 
sufficient detail to provide the necessary insight into the study. As a contingency to such exceptional events, a pool of additional pre-evaluated replacement candidate cases was prepared. If it was found that a case must be dropped from the study, a replacement case would have been selected from this contingency pool, with any necessary adjustments to the study applied. This action was not required in the end.

\subsubsection{FoaK Initiative Performance Defined}

The FoaK program as a knowledge acquisition method focuses on funding initiatives to yield new-to-the-firm innovations that can be commercialized within one of the firm's mainstream product portfolios. The degree to which a FoaK initiative is deemed to have delivered either a successful, unsuccessful, or typical level of performance is based on the degree to which the initiative has performed against measures related to innovation quality and competitiveness. The FoaK program uses a three-part measure of initiative performance which are each monitored by the program. These measures include the cocreation client stakeholder satisfaction, the level of follow-on revenue from the FoaK innovation asset, and the successful transfer of the technology into a mainstream product portfolio for commercialization.

\section{Client Stakeholder Satisfaction}

Client stakeholder satisfaction represents the degree to which the partnering clients in the initiative found the resulting assets from the project to have delivered value and impact against their business problem. Co-creation client satisfaction is measured as the client's agreement to provide public endorsement of the resulting innovation asset, which 
can include a published reference, conference presentations, and participating in reference calls. It is believed by the program that client stakeholders that engage in co-creation partnerships and subsequently provide references on behalf of the FoaK initiative innovation are doing so as an indication of their satisfaction with the results from the initiative. Clients that are not satisfied with the value demonstrated from a FoaK initiative engagement would not agree to provide public endorsement. It is noted that some organizations do not allow for written published endorsement of any kind, regardless of their level of satisfaction. However, they do provide alternate public endorsement through other methods such as conference presentations and anonymous case studies.

\section{Follow-on Revenue}

The second measure of initiative performance is the degree to which the emergent asset from the co-creation initiative has stimulated follow-on revenue beyond the initial FoaK project. The initial source of client revenue during the initial FoaK initiative is limited to the client stakeholder providing funding for a project manager for the duration of the project. The funds provided for this resource typically represents less than $10 \%$ of the total FoaK resource investment required to deliver the initiative to completion. Followon revenue represents any new revenue that results from the current co-creation client stakeholder that decides to continue after the initial FoaK innovation asset is delivered. Alternatively, follow-on revenue may come from other clients interested in the results of the initiative and prepared to spend on partnering towards a services-based solution, or a similar second-of-a-kind project. Follow-on revenue in this case represents the quality of the FoaK innovation through its ability to generate monetary interest in the market. This 
represents market validation through the client's perception of the FoaK innovation's ability to address their business problem.

\section{FoaK Innovation Transfer}

The final measure of initiative performance for a FoaK initiative is whether the resulting innovation from a project has successfully transferred into a mainstream product stakeholder portfolio for commercialization. A condition for FoaK initiative funding requires that a product stakeholder sponsors and supports an initiative and expresses interest in any resulting FoaK innovations. A statement of interest does not represent a formal commitment, which is not a funding requirement. For each FoaK initiative, a product stakeholder is asked to provide their written support and confirmation that the innovation being explored would benefit their portfolio and the firm. If the FoaK initiative is successful in delivering a new-to-the-firm innovation, the sponsoring product stakeholder would be expected, but not required, to receive the innovation into their portfolio for further development and commercialization.

The successful transfer of a FoaK innovation into a product portfolio is described as aligned with the portfolio strategy, benefitting from the availability of development resources, and being properly timed with the portfolio's other priorities. As such, a product stakeholder accepting the FoaK innovation asset with the intention to commercialize is an indication of innovation quality. The FoaK innovation's quality in this case is represented through the innovation asset's ability to motivate the product stakeholder to invest a portion of their finite resources towards evolving the new FoaK asset to be market ready. Each product portfolio's performance is bounded in part by its limited resources and investment 
decisions. Since product stakeholders are not required to accept a FoaK initiative's innovation assets, regardless of their early-stage endorsement of the initiative, they are unlikely to adopt the resulting new-to-the-firm innovation asset unless it demonstrates merit and potential to contribute to the portfolio's overall performance.

\section{Classifying FoaK Initiatives by Performance}

For this study, it was necessary to distinguish between levels of FoaK initiative performance and assign a level of performance to candidate initiatives being considered as a case. This began with an initial review of all initiatives funded by the FoaK program, and filtering out initiatives that did not fall within the analytic applications domain. A review of the individual initiatives and their scope was conducted, including the potential for access to information and the availability of historical records. The three categories of performance were identified by the researcher for use in the study which included individual initiative performance being rated as either "successful," "unsuccessful," or "typical." The initial shortlist of initiative candidates was then each assigned one of the categories of performance based on program records representing their achievement on each of the three individual measures of performance tracked by the program.

Successful initiatives were defined as those that were found to have resulted in satisfied clients, new revenue after completion of the initiative, and whose co-creation innovation assets were successfully transferred into the mainstream products business. Unsuccessful initiatives were in turn represented by those that were clearly unsuccessful in delivering on two of the three performance measures tracked by the FoaK program. Finally, those that demonstrated a moderate level of success represented typical initiatives, 
potentially falling short in one of the three areas or which were continuing to evolve post the FoaK engagement with the client. The classification of each candidate initiative's performance was reviewed with the FoaK program manager. Any differences were discussed and adjustments made accordingly. A refined list of initiatives resulted, which was available for consideration in the study based on their level of performance. Overall, the evaluation model used was found to be effective and accurate in representing initiative performance and consistent with how the FoaK program management team evaluated performance across their portfolio.

A fourth input was used in determining which initiatives would be ideal as case studies. This was a list of exemplars for each level of initiative performance as identified by the FoaK program management office. After the initial sub-setting of initiatives was complete, the program manager was then asked, "When you are requested to highlight your best initiatives, which are the ones that you bring forward? Which are the ones that you point to when highlighting initiatives that did not go well?" With this information, all initiative candidates were then ranked and prioritized for the study and then further evaluated for fit and practicality as a source of rich information in examining the research questions. This resulted in the set of five initiatives selected for an individual case study and an additional pool of backup initiatives in the event of issues with the initial list. 


\subsection{Data Collection}

A CR-informed multi-method data collection process is used in this study. This section will describe the design of the data collection stage and the linkage between source evidence and this study's research objectives and questions.

\subsubsection{The Role of Data Collection}

Data collection is the process of gathering evidence from a variety of sources to provide insight into the phenomena being studied. Case studies are "eclectic in the variety of data that is collected and...used in the study of (a)... phenomenon." The focus of data collection in case research typically includes five types of questions. These include questions asked of: informants, individual case, the patterns in findings across multiple cases, the entire study, and normative questions about recommendations and conclusions once beyond the scope of the study (Yin 2013). The questions asked in research determine the appropriate data collection sources and methods to be used.

Sources of evidence should be considered as anything that will provide insight into the question being examined. The goal of case research, particularly through a CR lens, is not only to capture the information being provided by informants but also to understand how information is being shared and in what context (Maxwell 2012). Evidence for case study research typically comes from six common sources that include documentation, archival records, interviews, direct observations, informant's observation, and physical artifacts (Yin 2013). This study drew upon evidence primarily through interview, archival records, physical artifacts and documentation. 
It is evident that the detailed nature of case study research presents the potential for collecting vast amounts of information, which is why there is an emphasis placed on adopting strong research discipline in facilitating the definition of the research protocol and the choices of information sources (Voss et al. 2002). Yin (2013) emphasizes a number of guiding principles in data collection for each case study, which includes the use of multiple sources of evidence in support of triangulation, creating a case study database which would support reliability and readability of the research study, creating a chain of evidence for increased information reliability, and exercising care when using electronic sources of data. These guiding principles were applied in the design of the data collection strategy for this study.

While there are challenges associated with researcher bias in case study (Maxwell 2012), there is support for the use of a researcher's experiential knowledge as a source of information. Strauss (1987) argues that experiential data should not be ignored because of the usual arguments made governing research regarding the potential for personal experience impacting research findings. Mining the researcher's technical knowledge, research background, and personal experiences provide a potential source of additional insight which should be considered as part of the research process (Denzin \& Lincoln 1994).

Similarly, there is also an argument for going beyond the description of events from informants towards an understanding of the theories that they also hold related to the observed phenomena being examined. These insights are believed to be beneficial in that informant theories are based on a real phenomenon which likely informed their actions, are typically based on a deeper experience than the researcher will possess, and may provide 
valuable insights that could be missed if these theories are not considered (Maxwell 2012).

This can also be seen more formally in CR-driven research in the form of "program theories," which represent what is believed to be the causal model by program designers, and tested during the study.

This study used a multi-method data collection design, primarily sourcing evidence through a multi-tiered semi-structured interview process and archival data sources. The design of the data collection process adopted the guidelines and practices outlined by Yin (2013), in support of increasing case rigour and validity. In addition, a CR-based program theory is developed to guide the case study analysis (see Section 5.7.3). This will be further described in the following sections.

\subsubsection{Dynamic Capabilities: Defining and Identifying}

The study design has taken a two-step approach to identifying which dynamic capabilities are relevant in the co-creation of innovation initiatives examined in this study, and the role that knowledge capacities play. Dynamic capabilities in this study are represented by "knowledge capacities" in the knowledge capacity framework (KCF) (Lichtenthaler \& Lichtenthaler 2009). This section presents the definition of these knowledge-based dynamic capabilities and describes the two-step process used in mapping dynamic capabilities to the observed phenomena.

\subsubsection{Knowledge Capacities: Defining Dynamic Capabilities}

As part of this study, one objective is to gain a better understanding of the role of knowledge-based dynamic capabilities in the co-creation of innovation initiative process. 
Lichtenthaler and Lichtenthaler's (2009) Knowledge Capacity Framework (KCF) has been used as a baseline set of dynamic capabilities that will be explored. This framework provides a theoretical representation of interconnected knowledge-based dynamic capabilities identified as relevant to open innovation engagements.

The KCF represents six knowledge capacities as two sets of dynamic capabilities: external knowledge capacities (KCe) and internal knowledge capacities (KCi) (see Table 5.4). Each dynamic capability in the framework is described as focused either on knowledge exploration, exploitation, or retention. The external knowledge capacities represent dynamic capabilities required in open innovation activities that span the boundaries of the firm. These include absorptive capacity, desorptive capacity, and connective capacity. Internal knowledge capacities represent open innovation activities related to activity within the boundaries of the firm. These include a firm's inventive capacity, innovative capacity, and transformative capacity. These knowledge capacities represent first-order dynamic capabilities (see Section 2.1.2).

Table 5.4 Knowledge Capacity Framework

\begin{tabular}{|c|c|c|c|}
\hline & $\begin{array}{c}\text { Knowledge } \\
\text { exploration }\end{array}$ & $\begin{array}{c}\text { Knowledge } \\
\text { retention }\end{array}$ & $\begin{array}{c}\text { Knowledge } \\
\text { exploitation }\end{array}$ \\
\hline $\begin{array}{c}\text { Internal } \\
\text { (Intrafirm) }\end{array}$ & $\begin{array}{c}\text { Inventive } \\
\text { capacity }\end{array}$ & $\begin{array}{c}\text { Transformative } \\
\text { capacity }\end{array}$ & $\begin{array}{c}\text { Innovative } \\
\text { capacity }\end{array}$ \\
\hline $\begin{array}{c}\text { External } \\
\text { (Interfirm) }\end{array}$ & $\begin{array}{c}\text { Absorptive } \\
\text { capacity }\end{array}$ & $\begin{array}{c}\text { Connective } \\
\text { capacity }\end{array}$ & $\begin{array}{c}\text { Desorptive } \\
\text { capacity }\end{array}$ \\
\hline
\end{tabular}

Source: Lichtenthaler \& Lichtenthaler (2009) 
These dynamic capabilities, except for desorptive knowledge capacity, are within the scope of the study. Desorptive knowledge capacity is relevant to an outbound open innovation context, putting it outside the scope of the inbound open innovation focus in this research.

\section{External Knowledge Capacity}

\section{Absorptive Capacity (knowledge exploration)}

Absorptive capacity is a well-known based on the work of Cohen and Levinthal (1990) where they originally defined this knowledge capacity as the ability for a firm to recognize, assimilate, and apply external knowledge. Over time this was further extended to differentiate between potential and unrealized capacity (Zahra \& George 2002), and between exploratory, transformative, and exploitative learning processes (Lane et al. 2006). As this study is focused on knowledge acquisition (i.e., exploratory, potential), absorptive capacity is defined as the firm's ability to explore external knowledge with a focus on processes that acquire external knowledge and assimilate it into the organization knowledge base (Lane et al. 2006; Zahra \& George 2002).

\section{Desorptive Capacity (knowledge exploitation)}

Desorptive capacity focuses on the firm's ability to exploit knowledge it possesses within opportunities outside the boundaries of the firm and can be viewed as complementary to absorptive capacity (Lichtenthaler 2007). Desorptive capacity is defined as the process stages where the firm identifies external opportunities to exploit their 
knowledge and subsequently transfer that knowledge to an external recipient (Lichtenthaler 2007).

\section{Connective Capacity (knowledge retention)}

Connective capacity is defined as the firm's ability to retain knowledge about its relationships between firms (i.e., client co-creation partner). This includes the ability to gain access to the client and their resources (Luhmann 1995) and the ability to reactivate knowledge maintained by the organization about those relationships in the future (Grant and Baden-Fuller 2004). It is expected that a firm's connective capacity increases with higher levels of prior knowledge.

\section{Internal Knowledge Capacity}

\section{Inventive Capacity (knowledge exploration)}

Inventive capacity refers to the firm's ability to explore existing knowledge and generate new knowledge within the organization (Lichtenthaler and Lichtenthaler 2009). This is driven by a firm's perception of new potential opportunities (Shane 2000) and in turn investing in knowledge processes to explore them (Smith et al., 2005). Newly generated knowledge is integrated into the firm's knowledge bases (Garud and Nayyar 1994). It is also recognized that a firm's ability to generate new knowledge requires time (Khilji et al. 2006). The level of firm's inventive capacity is also affected by its prior knowledge in a domain, with previous knowledge facilitating generation and use of new knowledge (Khiliji et al. 2006). 


\section{Innovative Capacity (knowledge exploitation)}

Innovative capacity reflects the remaining aspects of absorptive capacity, mainly the exploitative characteristics as defined by Zahra and George (2002). It represents the firm's ability to match inventions with opportunities in the target market (Cohen and Levinthal 1990). This highlights the notion that while firms may be effective in creating large amounts of new knowledge, some may be ineffective in exploiting it (Lane et al. 2006). Similarly, other firms may be very effective in translating small amounts of new knowledge into many innovations that reach the marketplace. As such, innovative capacity is defined as the firm's ability to exploit knowledge internally (Lichtenthaler and Lichtenthaler 2007).

\section{Transformative Capacity (knowledge retention)}

Transformative capacity refers to the firm's ability to retain knowledge over time within the organization and subsequently reactivating it when needed (Garud and Nayyar 1994). It is expected that knowledge for this purpose needs to be managed to keep knowledge "alive" (Lane et al. 2006), and that the more previous knowledge a firm possesses, the easier it becomes to maintain and reactivate new knowledge (Garud and Nayyar 1994).

\subsubsection{Identifying Dynamic Capabilities in Case Studies}

The nature of these knowledge-based dynamic capabilities (i.e., knowledge capacities) as they have been defined, would suggest that each knowledge capacity can be expected to be reflected across a range of co-creation of innovation processes. As such, a 
two-step process was used in the study design allowing for data collection related to the co-creation initiative processes observed, followed by a mapping of processes to knowledge capacities.

This approach allowed for a focus in the data collection stage using terms that would be found in the archives and familiar with informants, namely, FoaK process and outcome-centric. It was expected that informants would not be familiar with terminology related to knowledge capacities and introducing these terms in the interview process would result in complexity that would negatively impact the data collection process. It was decided that focusing data collection efforts through a process lens would yield the greatest relevant information regarding case studies and their performance. This process approach also allowed for a direct linking of the multi-method inputs collected and assessed against the program theory mechanisms (see Chapter 7).

It is in the analysis stage where observed behaviour related to the program theory mechanisms were mapped to knowledge capacities (see Chapter 10). This level of analysis and mapping was intended to provide two forms of insight. First, the mapping helped to confirm which knowledge capacities were found to exist in the co-creation of innovation initiatives examined (i.e., relevant). Second, an analysis of the linkages of the knowledge capacities to program theory mechanisms would illuminate at which points and to which extent in the co-creation of innovation process (i.e., firm, program, initiative), that each knowledge capacity was observed (i.e., importance). This would help in understanding at which stages knowledge capacities were interactive and which were dominant in the cases studied. This two-step approach was found to serve both the data collection and data analysis objectives that influenced the design decision. 


\subsubsection{Archival Data}

Archival records, as a form of primary research, seek to draw information from original sources to provide documented evidence in support of research questions (Layder 1993, Singleton \& Straights 2010). This form of evidence often provides historical perspective related to a phenomenon and should be included as part of the data collection plan.

Archival records are typically maintained by an organization as a matter of record and represent a stable, specific and precise account regarding the phenomenon, as well as a view of events over time (Yin 2013). There are several related challenges associated with the use of archival records which include potential difficulties in finding and retrieving records, gaining access to records that may be deliberately withheld, or not having accessibility for privacy reasons (Seawright \& Gerring 2008; Yin 2013). There are also concerns of potential bias that may arise from the selective use of available documentation or biases associated with the reporting of facts within archives that are not evident upon review (Yin 2013).

A rich source of archival records was maintained and available from the FoaK program office. The primary source of archival records in this study came through the FoaK program office. The FoaK program's comprehensive repository of documentation for each of its funded initiatives provided insight into the intended expectations on the scope, stakeholder delivery, and performance for each collaborative innovation project. It also included historical documentation and email correspondence.

The FoaK archival database includes a detailed profile of initiatives from their point of initial introduction as candidates for the program, through their implementation, up to 
the final delivery of the innovation. The repository provides details related to innovation concepts, funding proposals, resourcing, collaboration client's details, status updates, and any relevant and related correspondence. The evidence maintained for each initiative includes artifacts that are required as part of the FoaK process, as well as any other ancillary artifacts of importance at the time of the initiative's execution. It was found that due to the methodology required by the FoaK program for each of the initiatives, there was a level of consistency in the types of artifacts and level of detail captured for each initiative examined.

Finally, it was understood that there may be additional relevant artifacts that had not been added to the database. During the interview process, informants were asked if there are any artifacts that were not included as part of the repository and that could provide additional insight into understanding the program or an initiative. All additional artifacts received were carefully reviewed for relevance.

\subsubsection{Semi-Structured Interview}

CR-driven case studies strive to uncover the possible causal explanations of observed phenomena (Edwards et al. 2014). This requires an adaptive and interactive approach to collect the broad range of information needed to provide the insight for shaping theory (Stake \& Stake 2013). Semi-structured interviews are central to this process and were the primary source of data collection for this case study. This part of the data collection design consists of a multi-tiered interview strategy designed to collect evidence from informants at different levels of the organization, as well as examining cases from the 
perspectives of initiative execution, the FoaK program, governance and the initiative context.

\subsubsection{Interview Strategy and Triangulation}

A multi-tiered approach was designed for the semi-structured interview data. Interviews for each initiative were conducted across a variety of informant types aimed at providing various perspectives on specific attributes of a phenomenon. This approach of applying redundant data collection as a method of theoretical triangulation supports the goal of contributing to research validity (Patton 2002).

One form of triangulation recommends the gathering of evidence from multiple sources and perspectives converging on a shared conclusion regarding a single event (Patton 2002; Stake \& Stake 2013). This study drew upon a variety of informant types that were common to each FoaK initiative and the FoaK program (see Figure 5.3). They provided differing perspectives at the levels of initiative execution, program management, program governance executive, or as program stakeholders and observers. Evidence was collected from across informant types along four principal themes: initiative execution, knowledge acquisition method management and bridging (program), method governance (program), and stakeholder context.

Pawson and Tilley (2009) advise that not all informants are equal, each possessing differing levels of knowledge based on their roles, degree of involvement and context. They describe three types of informants based on the level and types of input they can provide to a study: "practitioners" who are primarily involved in shaping programs and policies, "subjects" who exist within those programs and policies, and "evaluators" who bring an 
Figure 5.3 Study Informant Types (Initiative-Level)

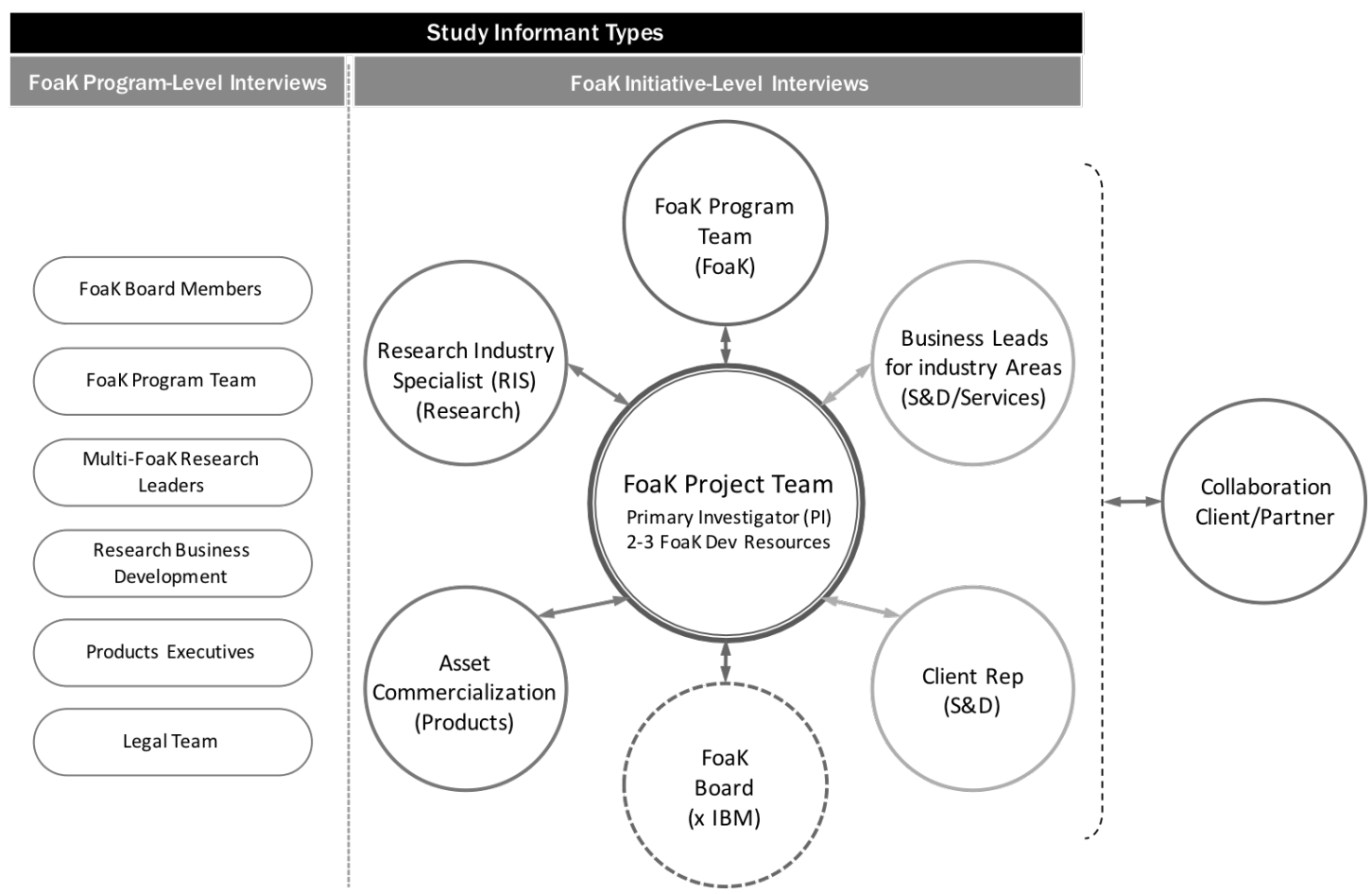

informed perspective from the outside looking in. Theoretical triangulation suggests a given phenomenon being studied can be described from the perspectives of multiple informants across various informant types (Patton 2002). Table 5.5 presents a profile of the range of informant types interviewed as part of this study.

In CR-informed research, an interview process should be both adaptive and iterative by design (Edwards et al. 2014). In this study, an effort was made to schedule individual interviews starting with more senior informants offering a broader perspective on initiatives and the FoaK method, proceeding then to more specific, detailed and narrower interviews. This was done where possible based on informant availability. Where needed, additional time was scheduled to revisit previous questions for clarification or 
$\sqrt{n}$

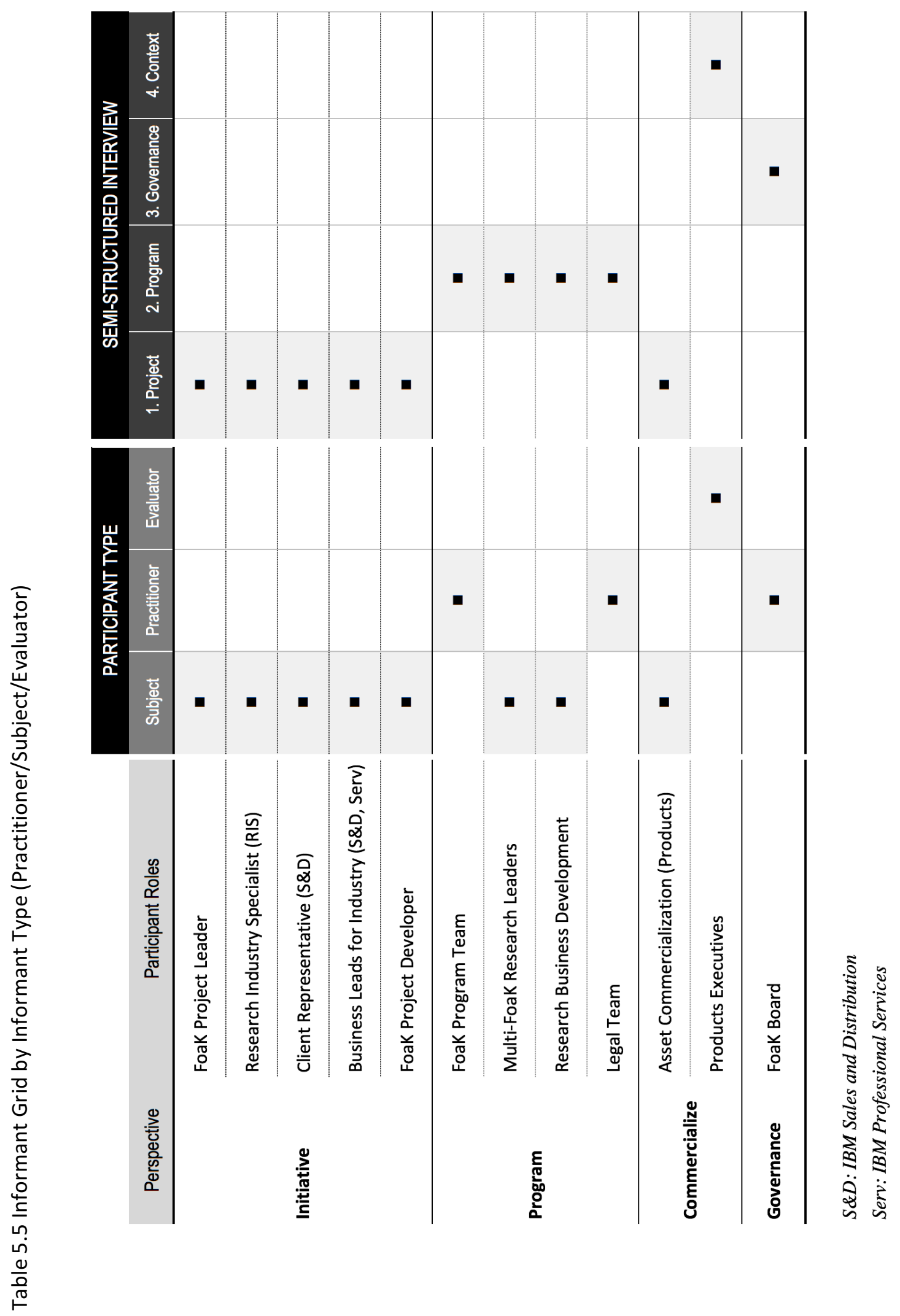


address any new questions that emerged later in the data collection process. Overall, informants were found to be interested and available to participate and share their experience with the study.

\subsubsection{Developing the Interviews}

Semi-structured interviews were developed for use in collecting evidence from various informant types covering different aspects of each case study. Two semi-structured interview types were included in the study, one for each of program perspective and initiative perspective (Appendix 5.1). Each semi-structured interview type was guided by its own script, all scripts covering shared themes regarding FoaK as a knowledge acquisition method. Differences between scripts were used to address a shift in emphasis for each type of conversation and to reflect the areas and depth of expertise that an informant holds on a given topic (i.e., "practitioner," "subject" or "evaluator" informant types) (Pawson \& Tilley 2009).

The design of the interview scripts was intended to be adaptive in nature (Edwards et al. 2014; Stake \& Stake 2013), allowing for clear direction in guiding the conversation with informants through a series of themes and predefined questions. Interviews were scripted, by design to provide for flexible conversation with informants and were targeted to require between 60 minutes to complete. Each interview included a series of open-ended questions and, where appropriate, prompts for additional areas to probe further. All questions included in interviews focused on gaining exploratory and explanatory insight into the research questions guiding this study and were aimed at uncovering causal mechanisms and interconnections. 
Initial interview scripts were treated as guidelines for conversations with informants and were refined in the early stages with feedback from the field. This study was designed to take a multi-stage approach to improving the interviews to be used with the key informants (see Figure 5.4). All interviews were approached with an interview plan, pre-interview preparation, and script-based guidance. With the adaptive design, this approach allowed flexibility during the interviews to explore new topics that emerged at the time of the discussion. However, the interview plan was found to adequately cover the intended scope of discussions with informants.

Figure 5.4 Data Collection - Process for Creating Questions

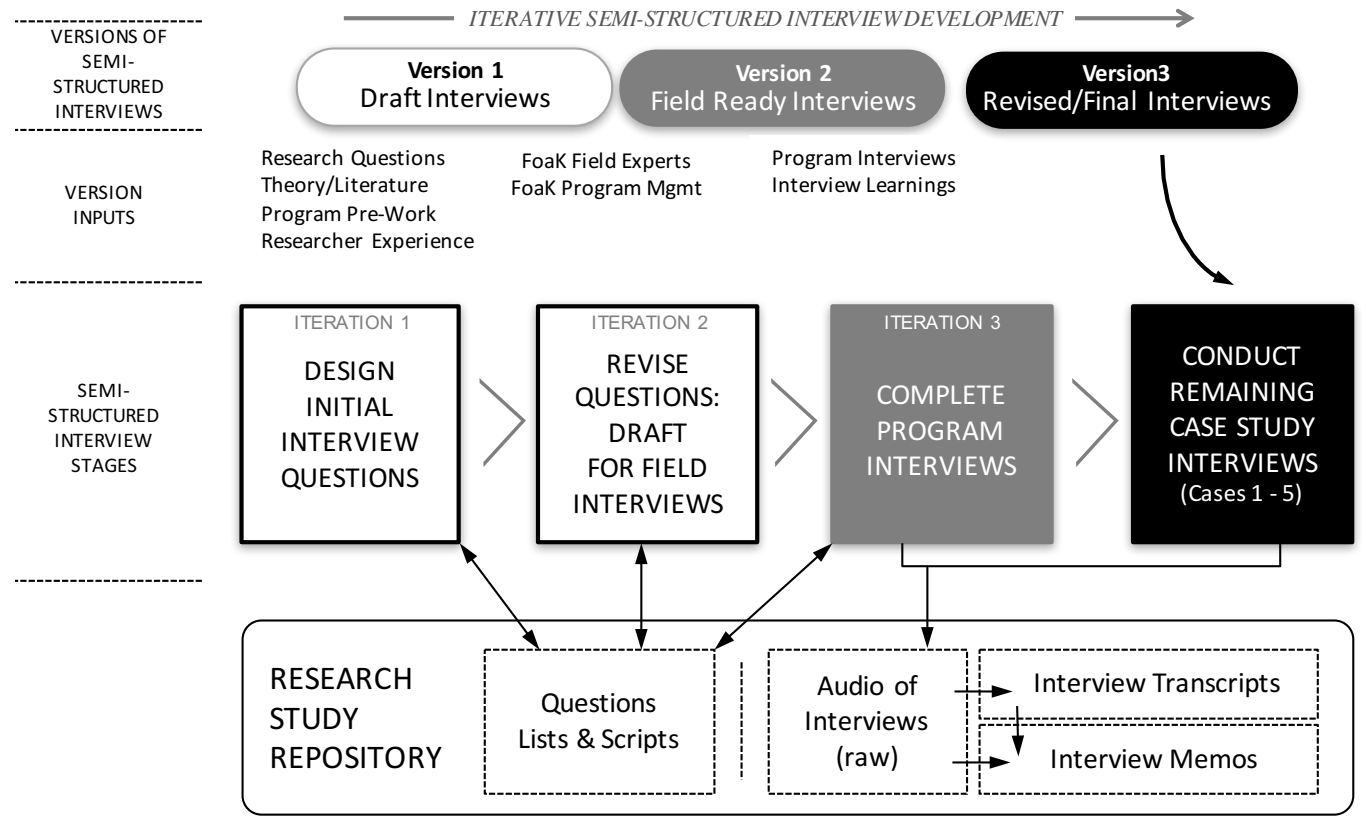

\subsubsection{Conducting Interviews}

Case study informants were identified for each case initiative, as well as informants at the level of the FoaK program and other stakeholders external to the individual 
initiatives. The list of respondent candidates for each case was determined through a review of the initiative artifacts captured by the FoaK program and discussions with the FoaK program management team. From the five cases studies, a total of 25 to 30 semi-structured interviews were initially targeted. As there was an opportunity for further informant interviews in support of triangulation, the total number of interviews successfully conducted during the study was expanded to a total of 47 . The summary of informant interviews is provided in Table 5.6, and developed further in Chapter 6.

Table 5.6 Informant Interview Summary

\begin{tabular}{|c|c|c|c|c|c|}
\hline \multirow{2}{*}{$\begin{array}{l}\text { INTERVIEWS BY CASE } \\
\text { Program - Level } \\
\end{array}$} & \multirow[b]{2}{*}{10} & \multicolumn{2}{|c|}{ INTERVIEWS BY INFORMANT ROLE } & \multicolumn{2}{|c|}{ INTERVIEWS BY INFORMANT LEVEL } \\
\hline & & Stage 1 Program-Level Informants & & Vice-President & 6 \\
\hline Typical Initiative & 8 & Program Management & 4 & Director/Executive/Legal/STSM/DE & 12 \\
\hline CASE 1 (Case Al.1) & 8 & Program Extended Team & 2 & Business Unit Executive & 17 \\
\hline Successful Initiative & 17 & Program Board & 2 & Manager & 6 \\
\hline CASE 2 (Case SI.1) & 11 & Stage 2 Initiative-Level Informants & & Developer/Consultant/Researcher & 6 \\
\hline CASE 3 (Case SI.2) & 6 & Program Management & 10 & Total Interviews & 47 \\
\hline Unsuccessful Initiative & 12 & Industry Leader/ RIS/ RRM & 7 & & \\
\hline CASE 4 (Case Ul.1) & 6 & Initiative Leader & 6 & & \\
\hline CASE 5 (Case UI.2) & 6 & Initiative Team & 3 & & \\
\hline \multirow[t]{3}{*}{ Total Interviews } & 47 & Products Interlock/Catcher & 7 & & \\
\hline & & Client Interlock & 6 & & \\
\hline & & Total Interviews & 47 & & \\
\hline
\end{tabular}

A first contact was made with the list of potential informants identified in the initial scoping of the cases in the study. This included a preliminary discussion with potential informants using internal site collaboration systems (e.g., IBM Sametime instant messaging, email) regarding the study. This was followed by a formal introduction email. It was anticipated that informants would be distributed across geographic locations, and 
their participation was not restricted by physical location. Informants were determined based on their ability to contribute insight into the study and availability to engage in the study. Appendix 5.2 presents the informant communication confirming participation in the study.

Individual interviews were targeted for a single telephone interview session, and only included one informant at a time. The focus of interviews was on achieving completeness of information in these discussions. Interviews were scheduled during the informant's business hours or an alternate time most convenient to them. Appendix 5.3 present pre-interview confirmation of participation and consent.

Each interview was recorded, transcribed, and summarized for individual and cross-case analysis. Interview recordings were digitally captured using both a primary digital recorder and backup recording device. Digital files from interviews were transcribed to digital text documents (i.e., Microsoft Word). Efforts were made for transcripts to be completed and cleansed within 72 hours of an interview to ensure accuracy in recall of both discussion details and tone. Interview memos were used to document highlights and key themes from interviews. Themes captured from the interview were reviewed with informants to confirm accuracy of interpretation. All interview artifacts were maintained within a research study repository, which included backup storage within a secure cloudbased file system.

\subsection{Researcher Experience}

Case study researchers require specific skills for the effective collection, analysis, and interpretation of evidence. Researchers should be good listeners that are not trapped in 
existing ideologies and inclinations, are adaptive, clearly understand the issues they are studying, and can conduct their research ethically (Yin 2013). A researcher's ability to build and maintain strong working relationships with informants is also a benefit that helps to ensure adequate access to information and support for the data collection process (Maxwell 2012; Weiss 1994).

Within a CR philosophy, it is believed that in the intransitive reality not all mechanisms are activated, some observable while others are not observable (Bhaskar 1975b). This suggests that the existence of mechanisms being examined will be based either on what can be observed (i.e., perceptual criteria) or on the researcher's ability to observe its effects (i.e., causal criteria) (Bhaskar 1975b). As such, researchers need to depend on "a rare blending of intellectual, practical-technical, and perceptual skills," allowing them to infer the existence of mechanisms based on what they have seen (Bhaskar 1975b p.47).

A researcher's previous experience should be embraced by the study, as it provides a level of context to help navigate the complexity of case research. There are benefits in the experiential knowledge of researchers, including their technical expertise, research background, and personal experiences (Maxwell 2012; Strauss \& Corbin 1994). This is further supported by others who promote the benefits of incorporating a researcher's identity and experience into their studies (Berg \& Smith 1988, Denzin \& Lincoln 2000, Jansen \& Peshkin 1992).

Multi-case studies are complex (e.g., range, volume, and depth of information collected) making it difficult to share between researchers what they see and accounting for perspectives they form through individual experience, interactivity, and context (Stake 
\& Stake 2013). Practices aimed at improving research validity suggest the use of investigator triangulation, the use of multiple researchers to help in balancing potential bias via data collection redundancy (Patton 2002). However, it has been argued that in multicase comparative research, at times, it is ideal to have the analysis conducted by a single researcher (Stake \& Stake 2013). Execution by an individual researcher allows for continuity and better pattern recognition across the evidence aggregated from the field (Stake \& Stake 2013). As a thesis study, this research by design is conducted by myself as the sole researcher, responsible for all stages of the research process.

Pre-work was completed to help further develop my background and experience in advance of the formal study. In combination with personal expertise, my relationship to the research site, and familiarity with the domain, the pre-work contributed to the necessary preparation to execute the fieldwork in this study.

As a researcher, I have previous experience with the subject organization and domain, having worked within the IBM products organization for over eight years as a program director. This provided an insider perspective on the organization, processes, and culture, necessary to build strong and credible working relationships with informants in the study, as well as helping gain access to information as part of the system. Previous professional experience has included close to 20 years in NPD and analytics solutions portfolios. This experience added to the level of credibility brought to discussions with informants in the study. In addition to other sources of input, this background contributed in part to formulating the initial list of interview questions in preparation for discussions with informants. The relationships both to the domain and the organization also introduced a level of interviewer flexibility during discussions with informants, allowing for follow- 
on meetings and sharing of artifacts in a timelier manner than if engagement with the organization was as an outsider.

Time was also invested in establishing relationships with the FoaK program team, executives, and key stakeholders to gain early support for this study. This included oneon-one overview sessions and participation in workshops and initiative checkpoint meetings, which revealed several recurring themes that were considered in shaping the data collection stage of the study.

\subsection{Data Analysis}

The final stage in the research process is conducting the data analysis and finalizing explanations and conclusions. Multi-case studies are complex and require analysis of large volumes of data, researchers needed to keep track of learnings from individual cases, and determining conclusions from patterns observed across all the cases studied (Stake \& Stake 2013). The primary goal in a CR-informed study, using an abductive reasoning approach, is to reach a theory of observed phenomena based on a process of identifying the most plausible explanation based on a well-supported argument for one alternative over a range of rival explanations (Wynn \& Williams 2012; Yin 2013).

Analysis of the broad and varied sources of evidence in case study analysis requires researchers to have a clear analytic strategy (Yin 2013). Miles et al. (2013) provide a recognized framework for the systematic analysis of case study data (Eisenhardt 1989; Yin 2013). This framework, informed by guidance on qualitative data coding (Saldaña 2015), the incorporation of principles for $\mathrm{CMO}$ (i.e., context, mechanisms, and outcomes) configuration (Pawson \& Tilley 2009), and guidelines for causal explanation in CR- 
informed study (Wynn \& Williams 2012) form the approach for data analysis in this study. Figure 5.5 provides an overview of the data analysis strategy used. It must be kept in mind that the data collection and analysis process are cyclical versus linear, with the two processes interwoven towards the goal of gaining insight (Saldaña 2015). In the end, the study must press for high-quality analysis. Yin (2013) states that quality is achieved by making sure the analysis highlights that all evidence has been considered, that there has been a broad set of plausible rival interpretations examined, that the analysis is focused on the most significant aspects of the study, and that the researcher should use their own expert knowledge during the case study.

The multi-method data collected from the multi-tiered unstructured interviews, archival records and documents were analyzed using qualitative data coding and analytical memo writing. The various sources of information, with the application of triangulation techniques, were translated using process mapping and a CMO program theory (see sections 5.7.2, 5.7.3) of the observed phenomenon, resulting in the formulation of rival explanations linked to findings and theory. Explanations from a cross-case analysis will be described at micro (i.e., initiative-level), meso (i.e., knowledge acquisition method), and macro levels (i.e., dynamic capabilities theory).

This section will describe the techniques used in analyzing the data collected, proposing theory, and presenting findings.

\subsubsection{Qualitative Data Coding and Analytical Memos}

Data coding is a common categorization strategy used in qualitative research (Maxwell 2012; Saldaña 2015). Saldana (2015) defines coding and qualitative data analysis 
as "a researcher-generated construct that symbolizes or translates data and thus attributes interpreted meaning to each individual data or later purposes of pattern detection, categorization, assertion or proposition development, theory building, and other analytic purposes." Coding in qualitative research is different from quantitative research, in that qualitative study uses a pre-established and unambiguous set of data categories, whereas qualitative research looks to fracture data and rearrange them into categories that help in the comparison of observations and development of theoretical concepts (Maxwell 2012). The process of data coding allows researchers to both use codes to assign and retrieve meaning from unstructured data and to help reduce the large volume typically associated with qualitative data (Miles et al. 2013).

Figure 5.5 Data Analysis Strategy

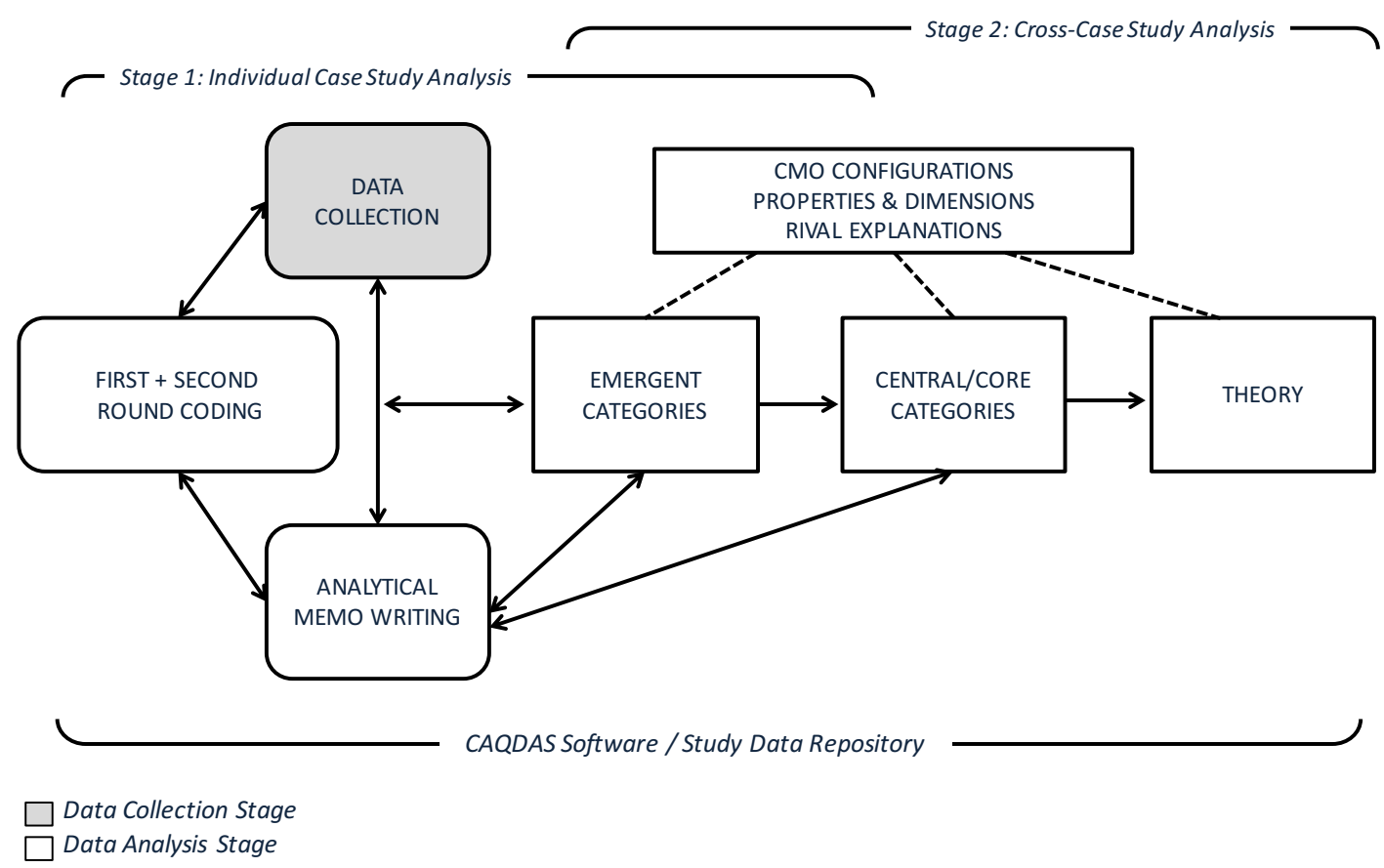

Adapted from Saldana (2015) 
The process of data coding involves translating the breadth of data collected during fieldwork into comparable codes, categories, and research themes. These themes in turn help in the shaping of assertions and theory (Maxwell 2012; Miles et al. 2013; Saldaña 2015) (see Figure 5.6). A two-cycle qualitative coding process was used in this study, completed through a software-based coding management application (Maxwell 2012; Yin 2013), and all artifacts were maintained within the study database. Analytic memo writing was further used to support this process. This analysis will be performed iteratively across individual case studies, and then as a comparative across all case studies. The focus will be on the use of codes, categories, and themes towards understanding phenomenon against the research questions.

Cycle 1 coding began with a pre-coding exercise (Layder 1993) to establish an initial list of codes from the study based on preliminary conversations with FoaK program and initiative experts. These codes were further refined through the process of programcentric interviews, which also contributed towards developing a program theory. A CAQDAS program (i.e., NVIVO ver. 10.2.2) was used to manage the coding process and coding was performed on semi-structured interview transcripts. The initial coding scheme was then being used in analyzing the evidence collected from the remaining case studies. A combination of evaluation (Patton 2002; Saldaña 2015) and causation (Maxwell 2012; Saldaña 2015) coding techniques were used as methods well suited for both exploratory and explanatory research objectives. These techniques focus on examining activities, characteristics, outcomes of programs, and understanding into the informants' rationale for observed phenomena (Saldaña 2015). 

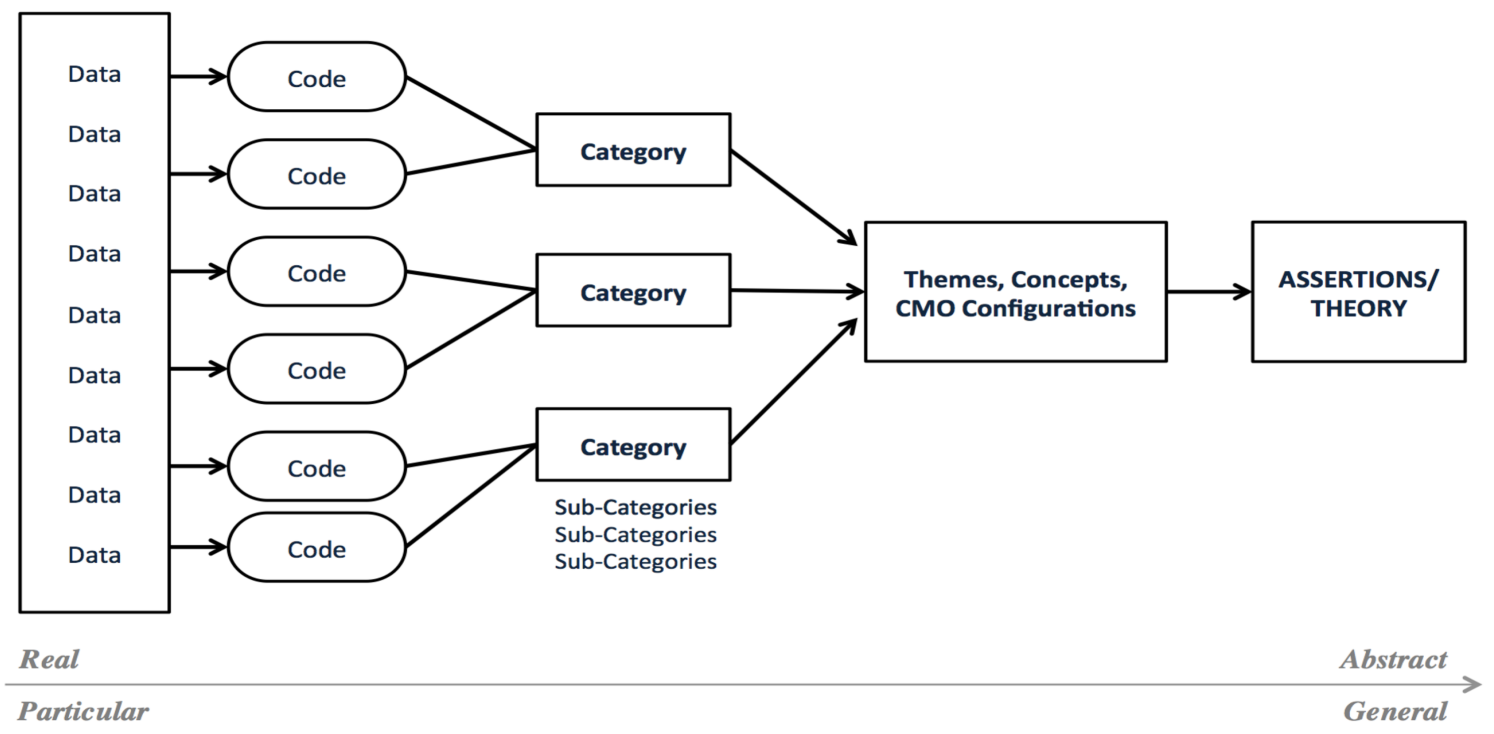

Adapted from Saldana (2015)

Upon completion of the first cycle and the collection of cross-case findings, a postcoding analysis was completed before moving to Cycle 2 analysis. The purpose of this stage was to provide an opportunity to reexamine and reshape the codes and resulting categories that emerged during the first cycle of analysis. The revised conclusions were used strategically in the second cycle to help ensure focus on outcomes aimed at the study objectives (Saldaña 2015). This stage used a combination of code mapping, landscaping, and operational model diagramming (Saldaña 2015).

The second cycle of coding is primarily focused on "develop(ing) a sense of categorical, thematic, conceptual, and/or theoretical organization from...first cycle codes" (Saldaña 2015 p.234). It is aimed at reorganizing and synthesizing the closing categories identified in the first cycle, to a focused list of "broader categories, themes, concepts, 
and/or assertions" (Saldaña 2015 p.234). Pattern coding and axial coding techniques were used for this cycle of analysis (Charmaz 2014; Saldaña 2015). These methods were to determine which codes in the research are dominant as well as identify emergent themes and explanation. These findings, in turn, were used in helping to formulate rival explanations for the observed phenomena in the study. These will contribute to the parallel process of CMO mapping, and program theory validation which uses a $\mathrm{CR}$ approach to describing observations as consisting of contexts, mechanisms, and outcomes (Pawson \& Tilley 2009).

\subsubsection{Anonymizing Data}

The preparation of this study has included anonymizing subject company data. All data regarding specific project names (i.e., cases), informants, subject company clients and specific performance results have been coded and presented as such throughout the study. Anonymizing the data did not limit access to the necessary information for the study and did not impact the completeness and rigour with which the study data collection was undertaken.

Individual cases have been consistently named and described throughout the document to ensure clarity for the reader. Similarly, informants and referenced stakeholders are identified throughout by their roles (e.g., client stakeholder, product stakeholder, sales stakeholder, program management, board members). A profile of the group of informants that have participated in the study is summarized by role, level and respective case study in Table 6.3. 
Informant quotations presented are in support of the analysis and findings. Maintaining anonymity in informant quotes was essential. Where quotes are presented, informant names, roles, and other potential identifying attributes have been represented by a study reference code that serves as the link back to the secured research database. All quotes inserted in the study are supportive of the context in which they are introduced, and informant types are reflected in the relevant section's narrative.

Finally, the study had full access to all case related content maintained by the program (e.g., internal memos, emails, analysis). All data were considered in the study analysis. Any confidential, sensitive or potentially identifying data were considered in the analysis and findings but were not included in the document as supporting quotes or in any other form.

\subsubsection{Context, Mechanisms and Outcomes (CMO) Mapping}

A case study method informed by critical realism seeks multiple explanations for the observed phenomenon as an outcome from the research study (Wynn \& Williams 2012). This is necessary to provide support for evaluating and comparing alternative explanations towards assertions and theory. This approach is referred to as judgmental reality, in which theory selection from alternatives presented is based primarily on their explanatory power in the transitive dimension of reality, rather than trying to compare them with entities of the intransitive dimension which may not be fully observed (Wynn \& Williams 2012). This process of selecting the most likely cause of observed phenomena is that which is believed to be the most accurate representation of the "real world" given knowledge to date (Wynn \& Williams 2012). 
Pawson and Tilley (2009) argue that program theories can be represented as a combination of observed "outcomes" $(\mathrm{O})$, the "mechanism" that trigger the behaviour $(\mathrm{M})$, and the "context" in which the phenomenon occurs (C). They present a model of mapping and linking $\mathrm{CMO}$ configurations and capturing a narrative in the form of causal matrices that are in turn used to represent rival explanations and arrive at conclusions believed to represent what is indeed being observed.

During the second cycle of qualitative coding of program interviews, early findings were used to start formulating the program theory. Explanation forming began with a focus on individual case findings, then extended to assertions and cross-case comparison.

\subsubsection{CMO - Program Theory Development}

The critical realist approach to data analysis focuses on understanding the underlying mechanisms that explain observed outcomes in an open system. This includes understanding the impact of supporting or opposing mechanisms related to the context within which a phenomenon is observed. The objective is to identify the mechanisms that have the strongest explanatory power of observed patterns.

In the study of programs, Pawson and Tilley (2004) described that the program at its essence is a theory developed by the creators of the program. It is the belief that the combination of a set of interventions will trigger predictable behaviour and outcomes. Programs are complex social systems (Pawson and Tilley 2004). The question is understanding why there is variation in program outcomes or where there is sufficient evidence in support of proposed program theory. However, the critical realist tradition also emphasizes that in the case of programs, the question is not "Does this program work?" 
but rather "What works for whom, and what circumstances, and in what respects, and how?" (Pawson and Tilley 2004). The goal of the analysis in this study is focused on understanding how the complex interactions and co-creation of innovation initiatives relate to initiative performance. In this study, there is a required understanding of shared context across initiatives with the FoaK program and the firm-level environment, which has been described in this section (i.e., Section 6.9). It also requires an analysis of each individual initiative at the level of execution and the specific contacts related to each initiative examined.

The approach used for the analysis of the case studies was guided by the Bygstad and Munkvold (2011) stepwise framework for critical realist analysis (see Figure 5.7). A two-stage analysis was conducted in this study. Stage I was focused on establishing a baseline program theory against which individual case studies would be evaluated. Stage I examined individual case studies against the program theory, using a two-tailed comparative case study approach, testing the proposed program theory.

Developing FoaK Program Theory The analysis began with establishing a theoretical description of FoaK initiatives and developing a program theory guided by the conceptual framework (see Section 3.4) and a series of program-level interviews and artifacts. This includes an analysis of FoaK program-level data. The FoaK program theory represents the FoaK system components and interactions that contributed to the design of the program. Chapter 7 details the method used in developing the FoaK program theory.

Multiple sources of information were used in developing the theory that represents the key components of the program, its stakeholders, processes, and interaction points contributing to FoaK initiative performance. Program artifacts, materials, and databases 
were reviewed, and an initial draft of a program theory created based on those findings. These preliminary findings were then used as a basis for a series of semi-structured interviews which were conducted at the program-level with informants independent of the individual executed cases to be studied. The FoaK program theory, in turn, was iterated and reviewed with the program management team until a level of confidence was reached that the formulated program theory provided a reliable base from which to examine individual case study initiatives.

Figure 5.7 Bygstad and Munkvold Stepwise Framework for Critical Realist Data Analysis

A Stepwise Framework for Critical Realist Data Analysis

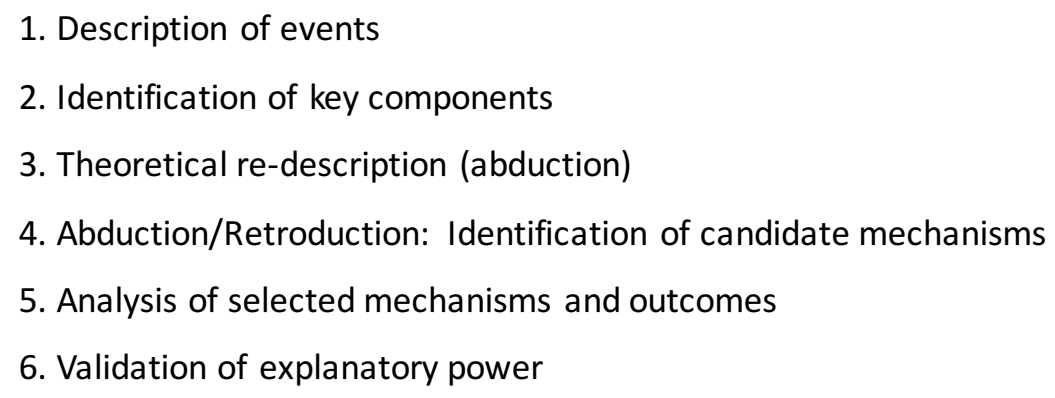

Source: Adapted from Bygstad and Munkvold (2011)

Stage 1 Findings Stage 1 examines the five individual case studies at varying levels of performance (i.e., typical performance ( 1 case), successful performance ( 2 cases), unsuccessful performance ( 2 cases)). All case studies were examined against the conceptual framework provided by the program theory established in Stage 1, exploring for variations of performance and the explanatory mechanisms (i.e., program interventions, initiative context). 
An analysis was conducted searching for explanatory mechanisms related to observed outcomes in each of the cases studied and across cases of differing performance. This included an assessment of micro-macro mechanisms as described by DeLanda (2006, p.34), who proposes examining the relative relationships of different program components to a FoaK initiative and the impact of those relationships on the emergent behaviour observed. By example, a micro-macro mechanism relationship can be the link between a lower-level mechanism, such as primary investigator (PI) engagement with product stakeholder teams during an initiative, and the subsequent impact on asset transfer to the product stakeholder portfolio. Similarly, an example of a macro-micro mechanism would be the relationship between a higher-level mechanism, such as FoaK program governance and the FoaK initiative leader communication of progress, and the subsequent impact on client stakeholder contract signing.

Individual case analysis (Chapter 8) and cross-case analysis (Chapter 9) were conducted to further evaluate the mechanisms identified and define context-mechanismoutcome patterns (Pawson and Tilley 2004). Further examination of these studies focused on validating program theory and identifying new candidate mechanisms. The goal was to understand the interplay of FoaK initiative elements and their contribution to observed performance outcomes.

Multi-method data collection was used in examining each of the individual cases, which included multi-tiered semi-structured interviews, an analysis of initiative artifacts, initiative history as tracked by the FoaK program office, and email logs (summarized in Table 6.2 Cases Studied Overview). Each initiative was examined at the level of its 
stakeholders, stakeholder interaction, the initiative process and execution, and initiative relevant context.

Stage 2 analysis was iterative in nature, beginning with individual case findings expressed using a CMO configuration approach based on the program theory initially developed. This was followed by identifying patterns between cases of similar levels of performance and concluding with comparative analysis and description of the primary themes observed across the FoaK co-creation initiatives. Based on this analysis, principal mechanisms were further evaluated for their explanatory power (Sayer 1992) and presented as the emergent themes for this study.

\subsubsection{Formulating Alternatives, Interpretation and Presentation}

Researchers should strive towards identifying rival explanations for observations emerging from their CR-based research study. Identifying and addressing as many potential competing alternatives possible further strengthens recommendations be made and supports the case for generalizability to theory (Edwards et al. 2014; Wynn \& Williams 2012; Yin 2013). The cross-case analysis must ensure that findings represent conclusions

across all cases examined. Any assertions that are made must be evidence-based, with significant attention put into presenting persuasive and credible logic as support for any statements made (Stake \& Stake 2013). In this stage of the data analysis, the combination of findings from the semi-structured interview coding, analysis, and CMO-based program theory definition contribute to defining rival explanations providing insight into the link between knowledge acquisition methods, dynamic capabilities, and new-to-the-firm innovation performance. 
The explanatory power of observed mechanisms, outcomes and context supported through the principles of triangulation, using the multi-tiered, multi-perspective findings related to each case-level FoaK initiative, at the FoaK program-level, and the initiative context. Additional was also provided through evidence acquired from archival and documentary data. As Yin (2013) emphasizes, the explanation-building process for the case study was iterative. 


\section{PART III \\ OVERVIEW OF THE CASE STUDIES AND FOAK PROGRAM THEORY}

A knowledge acquisition method represents the form of connection between the firm and its co-creation of innovation partners. In this study, the First-of-a-Kind (FoaK) program, as a knowledge acquisition method, is examined along with five FoaK initiatives at varying levels of performance. This section provides the necessary background to understand the program, its elements and what they reflect about program designer belief on causality. This along with an introduction to the case studies being explored provides a useful starting point for the reader heading into the study analysis.

Part III presents this overview in two chapters. Chapter 6 introduces the FoaK program and the case studies, with a discussion of the subject firm, FoaK program and its design, and the anatomy of individual FoaK initiatives. This includes a profile of each of the initiatives studied. Chapter 7 presents the critical realist informed program theory for the FoaK program. This context-mechanism-outcome (CMO) based definition contributes to the foundation in the analysis stage of the study.

Chapter 6 An Introduction to the First-of-a-Kind Program and Case Studies Chapter 7 Defining the FoaK Program Theory and Mechanisms 


\section{Chapter 6 \\ AN INTRODUCTION TO THE FIRST-OF-A-KIND PROGRAM AND CASE STUDIES}

To better understand the case findings from each of the individual FoaK initiatives studied, there is benefit to understanding the foundation upon which the FoaK program is based, and the background of co-creation initiatives executed within the FoaK knowledge acquisition method. This chapter describes FoaK program objectives, design, and management, as well as the context within which it operates. It will also discuss the details of a "typical" FoaK co-creation initiative, the execution process, and where interaction points between principal initiative stakeholders exist. This chapter will also profile the group of informant interviews in this study.

\subsection{IBM Research, Innovation and Funding}

The site organization for this study is the IBM Corporation, with the cases examined in this study drawn from IBM's FoaK program. IBM is reported amongst the largest companies in the world, ranking 81 on the Forbes Global 500 (2017). IBM generates approximately $\$ 80$ billion in annual revenue, operating in 175 countries, and employing close to 380,000 people globally (IBM 2016). The company, which was founded in 1910, operates primarily in business-to-business markets, providing offerings including hardware, software, solutions, and services to business customers across industries.

IBM states that central to its longevity has been its ability to transform with evolving markets and environment. In its most recent transformation, IBM has moved from divesting from hardware and global technology services (GTS) towards building leadership 
in the areas of cognitive business, analytics and cloud-based solutions. This has included an increase in R\&D investments to $35 \%$ of total revenue in the areas of analytics cloud, mobile, social and security (i.e., 2015 investment of \$29B) (IBM 2016). IBM has also directed over half (i.e., $\$ 13$ billion (54\%)) of its investment into strategic imperatives in these areas (IBM 2016). These strategic areas have grown in their contribution to the firm's overall performance as a result of redistributed investment across divisions, and an active acquisition strategy (IBM 2016). Acquisitions support IBM's strategic goals by accelerating the availability of required skills, necessary technologies, and entry into new markets (IBM 2016).

IBM is a recognized world leader in technology and innovation, which since 1993 has led industry in the number of patents held (IBM 2016). IBM highlights that a key competitive differentiator for its innovation is their level of investment in R\&D (i.e., $6 \%$ of revenue in 2015). This investment focuses on high-growth areas, where R\&D works with business units and clients in exploring and developing near and midterm innovations through global research laboratories (IBM 2016).

IBM is found to employ a hybrid innovation strategy in expanding its offerings portfolio. This includes combining closed organic product development methods with an acquisition and open collaborative innovation model. IBM is considered mature in its open innovation approach. Its open innovation strategy reaches outside the research organization, allowing the firm to draw upon innovations from its partner and customer ecosystem, and developers in the market (e.g., IBM developerWorks). This strategy includes IBM promoting innovation and knowledge acquisition through facilitating the 
process of the market converting ideas and inventions to prototypes (e.g., IBM OpenSource devWorx, FoaK program).

Competitive innovation is driven by the knowledge and expertise available to a firm, which either exists within the organization or requires that it be acquired from external sources. IBM promotes that it is rooted in a culture of collaboration with external experts and clients, enabled through the development of practices and skills within its new offering development process. IBM has formalized processes that integrate markets and product requirements (i.e., offering management discipline), product design (i.e., design thinking methodology), and an integrated cross-functional go to market strategy (IBM Annual Report 2015). IBM applies a product portfolio management model that can be perceived as a "survival of the fittest" philosophy. Budget and resources are distributed based on two annual planning cycles, the long-term strategic plan (i.e., Spring Plan), and annual budget plan (i.e., Fall Plan). These planning cycles are driven by IBM's strategic imperatives (IBM Annual Report 2015), influenced by changes in the external markets, the progress of individual division portfolios, and guidance that comes through $\mathrm{R} \& \mathrm{D}$ and $\mathrm{CEO}$ advisory boards.

Businesses within IBM compete for resources based on contribution and merit, and are client-driven in establishing their portfolio roadmaps. Internal business leaders look for opportunities to leverage expertise both inside and outside the organization, which includes identifying alternative sources of investment and resources from within the firm, all in support of bringing their portfolio ideas to the market. Towards this goal, there is crossbusiness collaboration in exploring new ideas and sharing of knowledge and resources (Frederich \& Andrews 2009). The IBM First-of-a-Kind (FoaK) program is an 
interdisciplinary R\&D function, which brings together IBM product teams, the IBM sales organization, IBM R\&D, and external client experts. The IBM FoaK program is one method in IBM's innovation strategy to acquire external knowledge through collaboration with clients, towards the assessment and development of offerings new-to-IBM (i.e., newto-the-firm) and new to the market.

\subsection{Knowledge Acquisition Method: The First-of-A Kind (FoaK) Program}

Within many companies, there are multiple potential channels for the creation of new-to-the-firm innovation. New innovation often emerges from efforts and processes predominantly internal to the firm (i.e., closed innovation strategies (Chesbrough 2007)) or may come from collaborative efforts with experts external to the organization using open innovation strategies (Chesbrough 2007). Within IBM, one source of new innovation is through programs managed by its research organization.

From the 1970s, IBM's research organization transformed from a centrally funded organization to one that moved towards a more open and collaborative model for innovation development (Frederich \& Andrews 2009). This transition went from a model of being fully financed by IBM's planning process to one where a variable portion of the research budget was provided by the IBM brand divisions. As such, collaborative programs were introduced to drive new innovations focused specifically on early-stage market problems explored in partnership with various divisions of IBM. It was this trajectory that contributed to the introduction of the FoaK program as a method of open collaboration and knowledge acquisition through expert clients. 
The research organization represents a group of skilled researchers focused on future looking invention and innovation, distributed across research labs globally, specialized by technical expertise and organized across strategic research agendas. In addition to conducting new invention and product offering exploration, these researchers also engage in organizational and client engagements. One of the primary roles of the research organization is to examine "cutting-edge" technical innovation questions that look beyond near-term product portfolio pressures. IBM Research operates in a cost-recovery model and employs several research engagement methods, each supporting varying organizational funding objectives. One model is their First-of-a-Kind (FoaK) innovation program.

The IBM FoaK program is an "innovation creation" program that was developed to shape and deliver new innovation through collaboration with clients. The FoaK program named to represent the introduction of first instances of new technical assets and innovations to the firm, was first funded in 1995 as a way to differentiate IBM's solution offerings (Frederich \& Andrews 2009). FoaK's primary objective is to accelerate the delivery of new technologies to the market in the form of new offerings, services engagements, asset licensing, or enhancements to core technologies and existing offerings. Innovations from the FoaK program are intended to help link early stage research and strategic initiatives from IBM's research division to real client problems, as well as gain valuable experience through collaboration and acquisition of expertise in the market. FoaK related innovations are also intended to allow the organization to identify emerging market opportunities and start the process of gaining mindshare within a new innovation market (Frederich \& Andrews 2009). Historically, FoaK has delivered approximately 15 projects 
annually out of 20 to 25 that are approved for funding (Frederich \& Andrews 2009). It is claimed that an estimated $70 \%$ of innovations emerging from FoaK initiatives are successfully transferred into a mainstream product portfolio for commercialization (Frederich \& Andrews 2009). A review of the FoaK program initiatives up to 2016 finds that over 200 new innovation and collaborative projects were delivered, at varying levels of success. These initiatives were executed across strategic areas for the firm, spanning business domains, industries, and geographies.

The FoaK program centres on collaboration with expert clients in the marketplace. It is a hybrid approach to inbound open innovation with clients, in that while the firm reaches out for clients to actively engage in early stage innovation, the method maintains control of the process and intellectual property for IBM. The primary goal of the program is to use FoaK as a method of knowledge acquisition in helping to shape innovations through a partnership with multiple clients. The program stimulates the user involvement in the innovation process by promoting the benefits of collaboration which include having early access to "cutting-edge technologies," benefitting from the first-to-market advantage, and getting access to "world-renowned" researchers (Frederich \& Andrews 2009). In particular, the FoaK approach emphasizes to prospective collaborators the benefit to their organization of gaining new skills and knowledge transfer from their involvement, and being able to engage directly in influencing the innovation process through a funded vehicle that will minimize the resource impact on their organization (Frederich \& Andrews 2009).

The FoaK method is intended as a repeatable knowledge acquisition method, designed to help reduce innovation project failures through the application of proven 
practices across projects of various domains and scopes (see Figure 6.1). The FoaK program focuses its efforts and resources on a selection process that aligns initiative funding to IBM's solution strategy. It aims to ensure that there is a linkage of an initiative to strategic markets, and that there is integration between the knowledge acquired, collaborative innovation development, and effective adoption of the innovation into a mainstream product portfolio (i.e., "asset commercialization owner" (ACO)) for commercialization. The FoaK program relies on performance scorecards for tracking the performance of the funded portfolio, which plays an important role in ensuring ongoing funding of the method (Frederich \& Andrews 2009).

Figure 6.1 IBM FoaK Program Collaborative Innovation Process

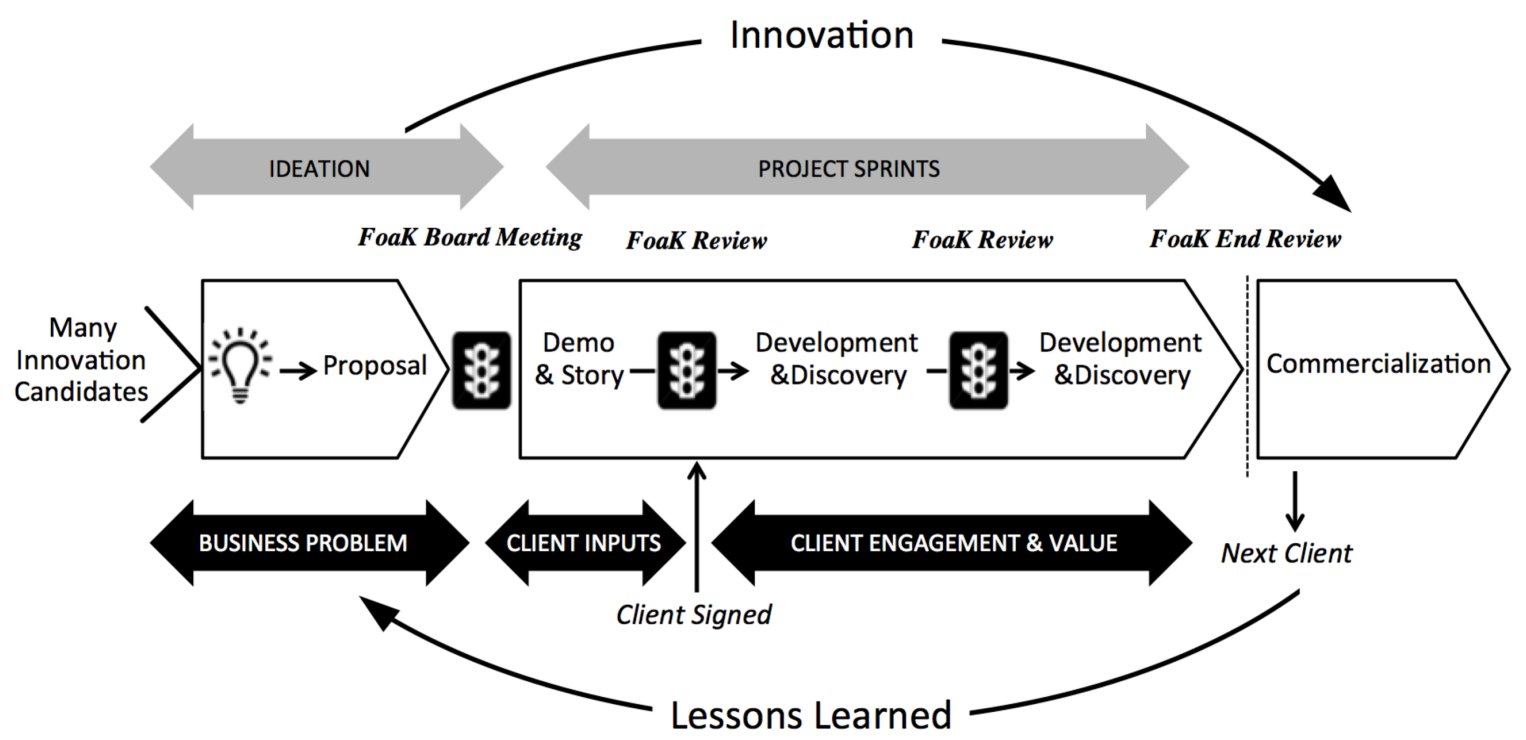

Adapted from Frederich \& Andrews (2009) 
The FoaK program provides a framework for researchers to examine new innovation with clients in the marketplace. This guiding framework emphasizes market validation resulting in early-stage assets that can be transferred to the products organization for commercialization.

"... one of the most valuable things to me in a First-of-a-Kind engagement, is the fact that you have direct, immediate contact with a client and you go and visit them and you stay and you do the work up there and they talk to you on a daily basis. You get to know them. You get to know their industry. You get to know their influence and discover these additional opportunities. I think it's part of our responsibility as researchers to try and open every door and see what happens." Int.08.1

The program provides funding to promising initiatives presented and approved by its governing FoaK board. Funded initiatives are selected based on their strategic fit to the firm's priorities, market potential, and technical merit. Successful FoaK initiative proposals are funded for a defined period with a dedicated number of researchers that will participate as part of the FoaK initiative team. The program evaluates and funds initiatives using a portfolio management discipline. This means that initiatives are selected and funded in part to ensure a balancing of investment across the firm's strategic innovation priorities. While there is a goal for all funded co-creation initiatives to succeed, it is recognized by the program that the nature of these early-stage explorations will result in some initiatives that yield little in the end. Overall, the program's goal for the portfolio is for the collective of invested initiatives to perform well.

"We need to be completely okay with failure because we have lots of new ideas and not all of them are going to stick. And if we become so conservative that every one of these has to be successful, then we won't be pushing the boundaries on the innovation. ... I think it has to be looked as a portfolio. Some of these will be very 
successful, just like any venture cap has a portfolio of start-ups which perform differently." Int.27.0

"I'm looking at the overall return of my whole portfolio. Sometime you make a bad bet. You move on. But the lesson that you learn for me is, 'Okay, what didn't go well?' And we write it down. And then who run the program?" Int.31.1.

The objective of the FoaK program as a method of innovation is to provide a repeatable and scalable model for directed co-creation research focused on funding radical innovation exploration. The achieve FoaK program objectives of repeatability and scaleability, the program looks to lower traditional barriers that impact co-creation initiative potential to deliver innovations that are new-to-the-firm and contribute to strengthening its portfolio (see Figure 6.2). The design of the program is aimed at achieving these objectives by directing its efforts on motivating shareholder engagement, focusing initiative activity on agreed outcomes and bridging knowledge gaps within initiative teams

\subsubsection{Research Funding and the FoaK Program}

The research organization funding recovery model receives a portion of research investment through corporate funding, with the remaining annual funding required achieved through initiatives secured by each of the strategic research units. The level of supplemental research-driven funding required varies across individual research units.

There are four primary alternatives for research funding. The first is an internal researcher-for-hire model where skilled researchers work with IBM organizations requiring their advanced expertise in product development or external projects. The second alternative is external funding through joint projects, where researchers enter client-funded 
engagements, working towards predefined objectives in an initiative led by the client, and supported by research. The third source of revenue is through participation in external research consortiums with funding coming from grants, where the scope of the research is led by the consortium's agenda. Finally, the fourth is the FoaK program which provides funding for researchers exploring new invention in a co-creation of innovation model, working with clients towards a defined project scope led by the firm, supported by the client.

Figure 6.2 FoaK Program Objectives

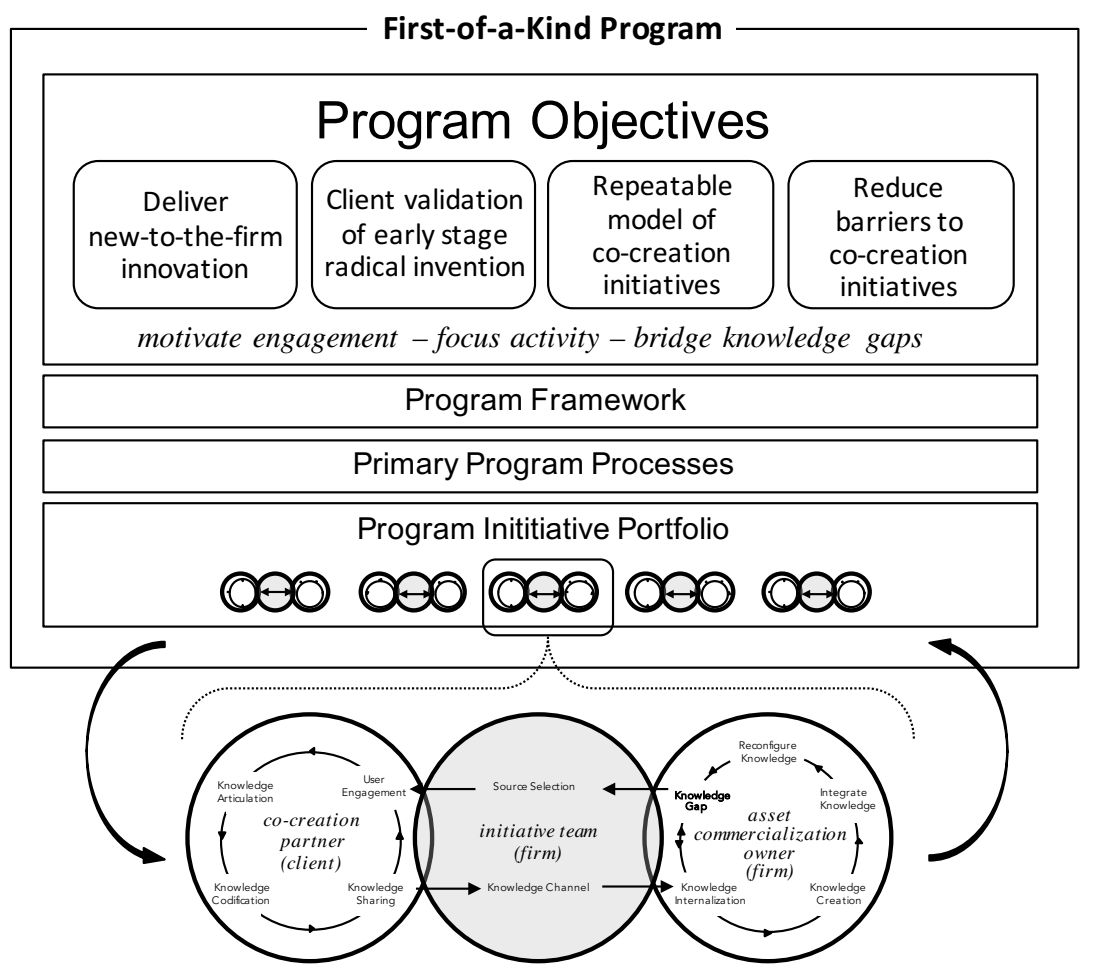

First-of-a-Kind Initiative

The FoaK model differs from the other funding recovery alternatives in its focus on the exploration of radical innovation through co-creation with clients, and led by 
researchers whose objectives are protected from the pressures of stakeholder funding agendas seeking predetermined outcomes. In addition to secured funding, informants highlight the benefit of the FoaK program model providing access to knowledgeable resources and experience from across a range of stakeholders associated with the program. Other benefits described include the program framework, guidance and practices from previous initiatives, and the opportunity to validate early research ideas or early-stage invention in a co-creative client setting.

“... the problem that (the FoaK program is) ...looking to tackle, which is support solutions that are a little bit more cutting-edge. That don't have a place in existing channels, or existing support, existing paths. Support those technologies with a lot of promise so that they can lead to revenue and opportunity ... down the road." Int. 47.0

“... when you think about (the) scenario of linking idea, to client, to products, and just a whole invention to innovation cycle, FoaK provides a platform in business terms, not technical terms and a framework." Int.18.0

"... one of the strengths of the program is that we are thinking beyond ... one engagement and we are thinking how we can scale what we are doing here and trying to plan out a path for that." Int.02.0

"Sometimes I found is that the customer says, 'I have a problem of A,' but the way you really discuss, and when you really understand the problem of $A$, the approach of actually handling that is a solution of B. But I think FoaK gives us a very good mechanism to be able to really step back a little bit to look at what are the customer needs and not only for this particular customer, that they are absolutely our anchor customer." Int.26.2

“... in terms of supporting investigation into totally new areas, (the FoaK program is) one of the best mechanisms available because you have direct funding from a parent group. Usually, the horizon or their requirements are much more specific to 
they're doing. So, that piece of really investigating new technologies and the fact that it has to be done with the (clients), not just some flight of fancy that research comes up with some topic and they are funded to do it, that the sooner you go work with a client, so that we do deal with a real problem and especially real data and validate with the client" Int.27.0

\subsubsection{Reducing Barriers to Initiative Realization}

As a formal program, the FoaK provides a framework by which its co-creation innovation initiatives can be guided through the complexities associated with early-stage innovation projects. A primary objective of the program is to help in reducing barriers often associated with these projects (Figure 6.2). Informants described this as being achieved through the program's provision of funding, credibility, a framework and proven practices. The funding provided to initiatives helps to motivate stakeholders to participate and support the FoaK initiative, with a perceived reduction in risk to their individual organizations. The formality of the program brings a level of credibility in discussions with stakeholders who are being requested to participate in initiatives, as well as setting the stage for the initiative team leaders (i.e., primary investigator (PI)) to pursue engagement and alignment with the key stakeholders needed in the execution of the initiative. Finally, the program's framework and proven practices provide an infrastructure that is leveraged by initiative teams in helping to accelerate and focus their resources on the exploratory engagements. This allows teams to benefit from the structure and packaged experience in the program, which otherwise would present additional overhead in standalone initiatives of this type.

“... one of the most obvious benefits (of FoaK) is the fact that (each initiative is) ... supported and funded ... as part of a formal program. And it's much easier for a team 
to approach a (co-creation) partner with new technology by virtue of saying that we're each going to work on pretty much our own dime, our own expense, and work together as a team to come up with the solution." Int.10.0

"The (FoaK) program enables all these things to come together, right, to drive and give innovation for the client. For (our company) and for the client." Int.19.1

"Where it works well is in providing a platform for delivering innovation that's relevant to clients." Int. 18.0

"I think the structure and experience and organization that we have set up is probably one of the best in the industry. I think the structure and the organization and the speed with which we engage in projects is probably the most positive." Int.10.0

"And the FoaK (program)... did a great job, I thought, in asking for all these things. Because if it's an afterthought, once you're halfway through the project-and then you have to figure out, 'Oh, wait a minute. So now, who's my asset commercialization owner and what's my client pipeline?' If that's something coming midway through the project, you're probably at a disadvantage as a PI (primary investigator), as opposed to having thought about this upfront and getting all the support upfront sure." Int.18.0

"If you don't have a program (like FoaK), and you are the only one who has to build a bridge on your own. Nobody will stop you from doing that. You can do it, but there are a lot of things that you have do by yourself. It's difficult. It's not that easy." Int.30.0

“... I know that for FoaK, (our initiative) never could have happened except for it and the reason it never could have happened except for it is that it was because it provided something. It provided visibility for the innovation to a broader set of people outside research into the brand." Int.46.2 


\subsubsection{FoaK Program Framework}

The FoaK program innovation is designed to bring together a repeatable disciplined approach to a class of co-creation of innovation initiatives within the firm. This is achieved by bringing together a governing board, program management function, a program framework, program knowledge and assets, guidance and extended team support (see Figure 6.3). The FoaK's packaged program approach contributes to reducing barriers for co-creation initiatives, with the intention to help improve the success rate of funded initiatives at the portfolio level.

\section{FoaK Governing Board}

Central to the FoaK program is its executive governing board. The board, which consists of senior executives from across IBM divisions, is responsible for the review, approval, and funding decisions for all FoaK proposals brought forward for program funding. Members of the board are executive level, typically vice presidents, who represent and link between corporate strategic priorities and program portfolio priorities. During the period studied, the board consisted of between 13 and 15 members, each serving voluntary and unrestricted term periods. During that time, board members were required to attend FoaK funding and review sessions four times a year, to participate in ad hoc FoaK initiative reviews as required, and in some cases, were assigned as mentors to initiative teams for the duration of a project. The board also plays a role as a source of experience and guidance for initiative teams, providing insight and connection into the broader organization, and into how initiatives may support or conflict with ongoing activity that a FoaK initiative team may not be aware of. The depth and range of board member experience, their roles in 
the firm, and their personal networks all contribute to helping improve the focus of candidate initiative proposals, and projects in execution.

Figure 6.3 FoaK Program Framework

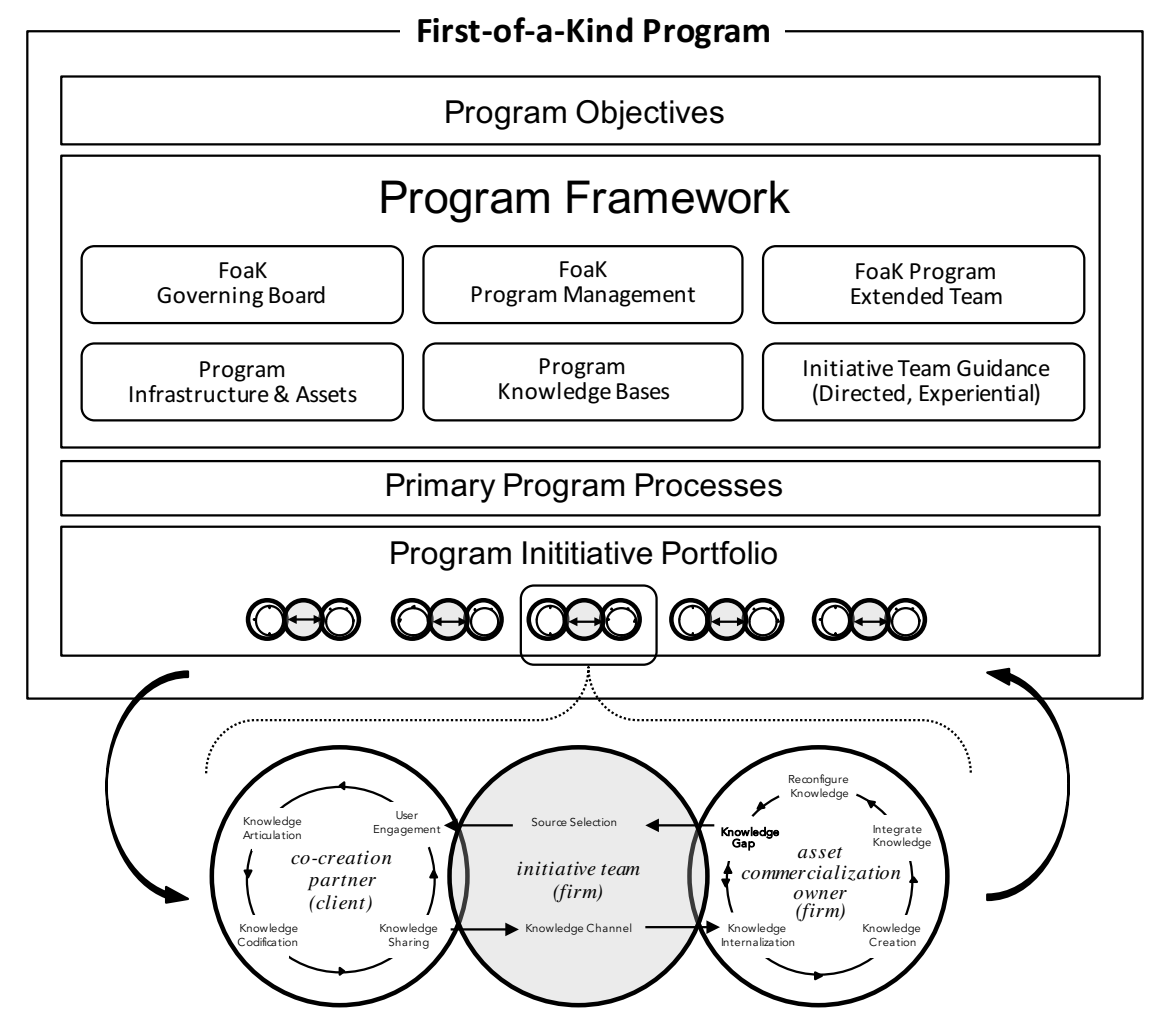

First-of-a-Kind Initiative

"Most of people on the board had been in the business for a while, so we might say, 'Okay. Let's just make sure that we have this services team aligned, because they may not have thought about it.' Or we might identify, 'We think that some other work that is going on in this product division that either can benefit from it or may become an issue." Int.01.0

"So, before funding decisions are made, (the board) might tell you, as a PI (primary investigator), 'We don't think this is the right area. Maybe you can change your proposal idea and direct it slightly differently.' So that kind of feedback I think is 
valuable. That then end(s) up with (a) much powerful and much more impactful solution." Int.18.0

"(The board members) are the decision makers of what gets funded, first and foremost. I think the board discussions are extremely healthy. ... We talk through the practicalities of the projects and then we may look at the merit of the project, we may look at how the project would fit into a business unit's portfolio, we could discuss the general strategic importance of the project." Int.45.0

\section{Program Management}

The FoaK program is managed by a core program team (Figure 6.3) that consists of the program director, program manager, program business development and support staff. The program manager is a dedicated resource assigned to managing the portfolio of initiatives and program operations, while the director, business development, legal and support staff operate on a part-time basis. The role of the program management team centres on identifying potential initiative candidates across the firm, requesting and reviewing proposals, helping FoaK initiative teams in preparation for business case presentations (i.e., "pitch") to the Board, and managing all operational aspects of funding approval and resource allocation, initiative monitoring, and issue resolution. The program management office is also responsible for tracking initiative performance and progress, organizing quarterly review sessions, creating and updating the knowledge repository and artifacts, tracking initiative history, and managing program templates and the program knowledge repository which is available to all initiative teams and stakeholders.

\section{Program Framework}

Central to the program is the FoaK framework that provides initiative teams looking for funding of their proposals (Figure 6.3). The FoaK program specifies the criteria for the 
initiatives to participate in the program, processes that guide initiative candidates and funded teams through the program, artifacts supporting stakeholder communication by an initiative team, and a knowledge repository where initiative teams can learn from previous initiatives and capture their own scope and progress in a predefined environment. Among the artifacts available, the framework includes legal agreements that have been designed specifically for engagement with co-creation clients in this context, and are used in setting the terms of engagement and IP ownership. The FoaK program framework provides a scalable approach to helping set the course for initiative teams from the point of submitting a proposal through to the transfer of a FoaK initiative asset to a mainstream product line.

"So, when you think about (a FoaK initiative, it is a) scenario of linking idea, to client, to products, and just a whole invention to innovation cycle. FoaK provides a platform in business terms, not technical terms and a framework." Int18.0

"I think the structure and experience and organization that we have set up (in our FoaK program framework) is probably one of the best in the industry. I think the structure and the organization and the speed with which we engage in projects is probably the most positive." Int.10.0

\section{Program Knowledge and Guidance}

The FoaK program also serves as a source of knowledge and guidance for initiative teams at each stage of the co-creation initiative process (Figure 6.3). This is provided as a combination of directed and experiential forms of guidance. Initiative teams have access to predefined templates and presentations for use with stakeholders that includes business plan development, client pitch presentations, and program overview content (i.e., directed guidance). Board members, program management, and extended team support of initiative teams provide both technical guidance and experience (i.e., experiential guidance). Teams 
have access to support on technical questions regarding aspects of program processes and requirements, IP protection and client engagements contracts. FoaK teams also require guidance with respect to strategic direction for their initiative, visibility into other areas of the organization that may help or hinder their efforts, guidance on developing and presenting their initiative business case, access to a broader internal network than is currently available to them, mentoring on initiative scope, client engagements and asset transfer to mainstream product portfolios. Initiative team experience, maturity, and leadership PI strength were found to impact the degree to which they reach out for the various forms of knowledge and guidance available to them.

\section{FoaK Program Extended Team}

One of the key elements of the FoaK program is the knowledge and guidance it can provide initiative teams of varying levels of experience and expertise. To this end, the FoaK framework has established a network of experts across various domains to support initiative teams through the stages of the co-creation initiative process (Figure 6.3). The FoaK extended team includes stakeholders from across functions including, the research, industry market development, sales, and products organizations. Legal support is provided in the client selection contracting stage as needed. Examples of this include geographic regions that are new to the FoaK process or where clients are looking to change the terms of the baseline engagement contract. In these cases, the FoaK legal team provides support and guidance in working through the process as needed. The research organization industry experts include research relationship managers (RRM) and research industry specialists (RIS) who help guide FoaK initiative teams in the areas of initiative definition, strategic relevance of the innovation being explored, client engagement, and securing product 
organization support for the eventual technology transfer of the FoaK initiative innovation. These research experts provide a deeper level of industry knowledge and experience and a broader internal network of potential contributors and supporters for the FoaK initiative concepts. Similarly, the sales organization also contributes support for initiative teams by providing guidance and access to potential co-creation clients candidates. As a condition to FoaK participation, certain stakeholders must demonstrate support and sponsorship for the initiative. It was found that individuals on the extended team that support FoaK initiative candidates at times also act as initiative sponsors, which are required to meet program conditions to participate.

\subsubsection{FoaK Initiative Investment Criteria, Funding, and Governance}

The FoaK program is tasked with identifying sources of potential new-to-the-firm innovation initiatives, and systematically determining which ones have the greatest potential for success and contribution to the firm. Those are the initiatives that receive funding. Towards this objective, the program uses a series of selection criteria within a predefined process to evaluate and select candidate initiatives in which the program will invest. Upon selection and approval, a level of funding is determined for the promising initiatives based on the resource needs anticipated for execution. The FoaK program in turn

monitors the initiative progress against objectives within its governance framework. These represent the primary FoaK program processes (see Figure 6.4). 


\section{FoaK Initiatives Pipeline}

Achieving a pipeline of attractive initiative candidates is a primary objective of the FoaK program and its program management team (Figure 6.4). The FoaK program is dependent upon having a pipeline of initiative candidates from which the board will select and fund. This pipeline is generated primarily using a notification process mechanism by which the FoaK program management team sends out a call for proposals four times a year. The themes of these calls reflect the strategic priorities and climate of the firm, and may focus on an agenda or industry problem. The research organization, and research industry specialists (RIS) in the main are familiar with the process and cadence for new FoaK funding sessions, and determine whether there are ideas or early-stage invention that will benefit from participating in a FoaK co-creation of innovation projects. These were proposals found to focus on ideas where clients can provide first validation and contribution to early stage ideas or invention. While the research organization is the primary source of candidates that enter the FoaK initiative pipeline, other organizations at times are also invited to contribute ideas in partnership with the research organization.

The program process was found to typically involve an informal pre-pipeline screening of candidates that emerge in response to the call for proposals. Researchers that believe they have a viable candidate for funding often work with their research team executive, research industry specialists (RIS), and their research relationship managers (RRM) to evaluate how their ideas for the FoaK program fit selection criteria. FoaK program management and FoaK business development teams then review ideas that pass this initial pre-screening as the next level of evaluation, prior to the developing the proposal business case for the FoaK board. 


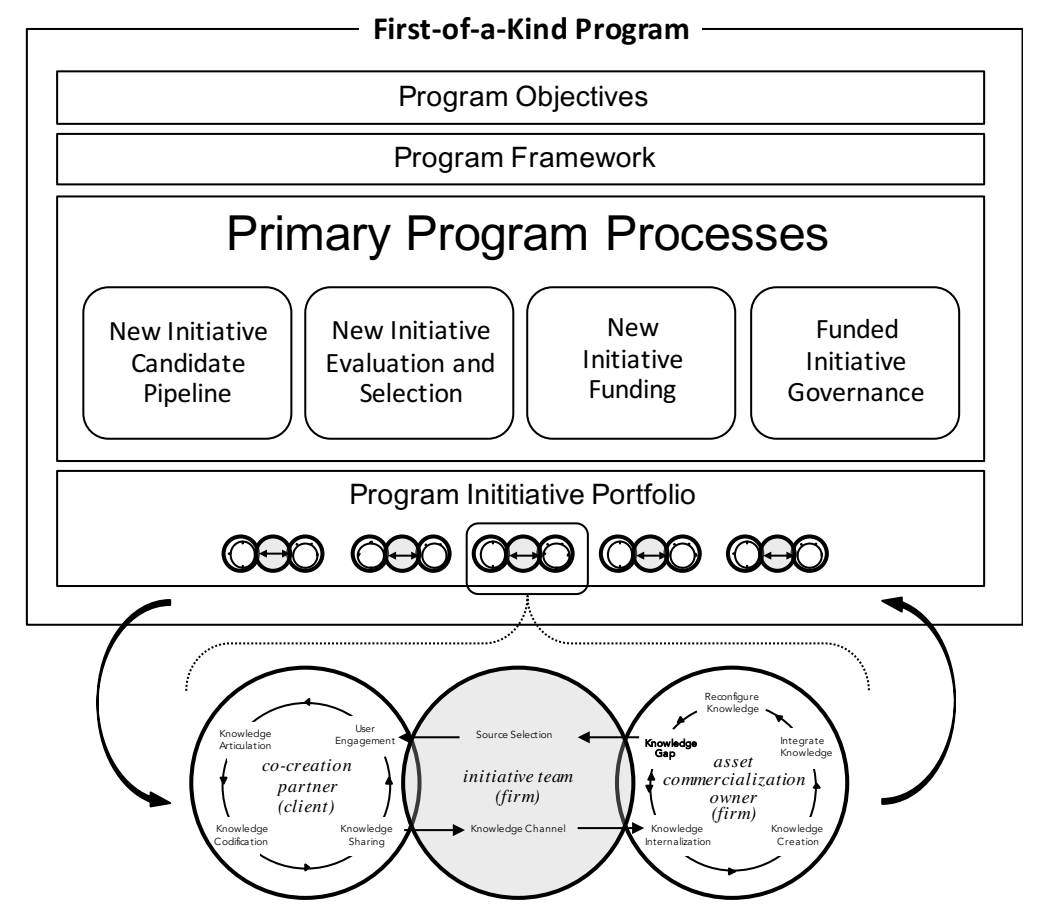

First-of-a-Kind Initiative

FoaK proposals are developed and shepherded by a primary investigator (PI) who is responsible for understanding the proposal process and requirements, the development of the proposal, securing proposal support and presenting (i.e., "pitching") the proposal at a scheduled quarterly board session. In addition to the support provided by domain experts within and associated with area of research, the FoaK program also provides additional support in the review and development of proposals to strengthen submissions requesting funding.

The FoaK program applies a set of selection criteria that guide initiative teams in assessing their fit and readiness for the program. These criteria help to shape and guide the proposals to be used in funding requests, and ultimately in determining which initiative candidates provide the greatest opportunities for success and impact on the firm. With the 
understanding that not all initiatives that are funded will result in successful assets, the selection criteria are intended to improve the success rate of initiatives, and the performance of the FoaK initiatives portfolio overall.

\section{Initiative Selection Criteria}

The business case proposal and presentation are central to an initiative team's "pitch" for funding (Figure 6.4). The selection criteria are a combination of requisite items to be demonstrated in the proposal process being presented, in addition to the overall perceived potential and merit of the innovation being explored (see Table 6.1). The selection criteria include a compelling case for a proposed FoaK innovation addressing a firm relevant user/market need, the fit of the proposal to the firm's strategic priorities, the viability and value of the scope proposed for the initiative, prerequisites alignment and support for the initiative by stakeholders in the sales, industry, research, and product organizations. Each of these stakeholder groups is expected to provide in writing, as well as in presentation, their expression of support for the value of the initiative to their organizations and the firm overall. A list of candidate co-creation clients is also required in the proposal process, and serves as a funding gating factor.

There are two gating criteria for all candidate initiatives. The program expects that all initiatives that are funded will work with a single client in the co-creation project, and that assets delivered from the FoaK initiative will, in turn, be transferred to a mainstream product portfolio within the firm for commercialization. Commercialization may be in the form of either a standalone product or component part contributing to a larger product offering. To this end, the first gate for FoaK initiative teams is identifying and confirming intention from a product organization within the firm (i.e., asset commercialization owner 
Table 6.1 FoaK Initiative Selection Criteria Categories

\section{FOAK INITIATIVE OVERVIEW}

Solution Description

Why is this a FOAK?

Target Industry for the FoaK Initiative

FoaK Initiative Team Leads (i.e., Primary Investigator)

FoaK Initiative Objectives

FoaK Initiative Stakeholder Sponsor Support (written): Sales, Industry, Asset Commercialization Owner(s) (ACO), Research Relationship Manager (RRM)

\section{INNOVATION VALUE}

Client Problem

- What gap (problem or opportunity) does this solution address within the Industry's Point of View/Solution Roadmap?

- Per the client profile for this FOAK, does this project provide an opportunity to gather data and refine the approach?

Business Value

- To the client: How does this solution solve a client problem or address a market opportunity?

- To IBM?

Technology:

- What Research innovation is being leveraged by this project?

- What differentiates this technology / solution from what is available today both internally and externally? Intellectual Capital:

- List and describe each asset created by this project How can the assets be reused within an Industry?

- Are these assets replicable across other Industries?

\section{INNOVATION STRATEGY AND MARKET OPPORTUNITY}

Strategy:

- How do the project's deliverables support the Industry's Point of View/Solution Map?

- What Research sub strategy area does this work represent?

Route to Market:

- What is the route to market? (e.g., services engagement, product, business partner, etc.)

Market Opportunity:

- What is the potential market opportunity for the solution area(s) over the next 3-5 years?

- Is this a worldwide opportunity?

- Is it an industry-wide problem or client-specific opportunity?

- What is the IBM opportunity for this solution within the solution area?

Competition:

- Describe the competitive landscape

\section{FOAK INITIATIVE STAKEHOLDERS AND EXECUTION}

Asset Commercialization Owner:

-What IBM Brand(s) or Business Partner(s) will be commercializing these assets?

- What is the asset commercialization strategy?

Source: Derived from IBM FoaK program archives. 
(ACO)) that they will receive the transferred technology. The second gate, which must be passed to release funds to the FoaK initiative team is a secured and signed agreement with a co-creation client within the first three months from initiative approval. Upon delivering the signed and aligned agreement for the FoaK initiative and its scope, funding is then released to the initiative team, and the project execution timeline begins.

During the proposal review process, the FoaK board provides guidance on the perceived fit of the proposed product stakeholder and initiative co-creation client stakeholder, which may require additional work from initiative teams to either demonstrate the value of the partners they are targeting or work towards securing different partners that are a better fit based on the feedback provided. Ultimately, the quality of fit of the selected initiative stakeholders resides with the initiative team.

"(The program tries) to assess whether the client really is a good client for us to invest because we are actually making a real monetary investment. I would say it's more about guiding in that sense, guiding in terms of the type of client that may not be a good fit and then assessing along the way in terms of maybe identifying some contacts who we think may be good clients." Int.01.0

\section{Initiative Funding}

The FoaK program is co-funded by the firm's sales and research organizations. Both organizations contribute internal budgets that in turn support the allocation of researchers to selected initiatives for a predefined period. Initiative funding is a competitive process where each FoaK initiative candidate vies for a portion of the available budget in the calendar year (Figure 6.4). The budget allocated to the FoaK program fluctuates annually, which in turn determines the number of projects that can be funded during a given year. During the period in which the cases studied were approved for the program (i.e., 
2011 to 2013), initiatives were typically funded with 4 to 6 researcher equivalents, for an active period of 12 months. In cases where initiatives were placed on hold, the remainder of their funded period would begin once the project resumed.

In addition to program funding, a budget is requested from client stakeholders that agree to participate. This is primarily to ensure a token stake in the engagement to cover the costs of hiring a project manager for the initiative. Additional resources may come from other groups within the firm, such as the services or products organizations that have a vested interest in the success of the initiative. The individual stakeholder units fund those resources from their own budgets. Other resources for projects are provided by the client stakeholder firm, which may include assigning members of its organization to participate in various roles supporting the initiative and in working with the FoaK initiative team

\section{Initiative Governance}

After an initiative, has been approved for the FoaK program, there are three forms of governance that apply in managing and monitoring the initiative's progress against its committed plan (Figure 6.4). These include successfully achieving "gating" criteria, threemonth formal status checkpoints with the FoaK board, and ad hoc meetings with the FoaK program management team or initiative mentors as required. As part of the proposal process, each FoaK initiative team submits a milestone plan highlighting timing on key events as prescribed by the program. The milestones are included in the initiatives 12month plan and are used by the program in monitoring initiative progress.

The primary role of initiative governance in the FoaK program is to ensure that the funded initiatives are progressing as expected against the plan. The checkpoints and ad hoc meetings are also used to identify potential issues early in the process that could benefit 
from program-level assistance. Initiative updates are provided by the initiative PI and are presented at the regularly scheduled board review meetings. These reviews are used to understand progress and are where questions are posed to initiative leaders to better understand the issues and remediation strategies when applicable.

The primary point of contact between the FoaK program management office and the initiative team is the FoaK initiative team's primary investigator (PI). The PI serves as the source of updates and other information required by the program during and after the initiative execution. Depending on the information requested, other members of the extended team (e.g., research industry specialists, legal counsel) were found to provide information on progress that is within their domain and responsibility.

Time constraints faced by the board members were highlighted as potentially impacting the effectiveness of new proposal reviews and assessment of initiative progress updates. During the period studied, board sessions were held four times a year, during which both new proposals and updates were covered in each session. As the amounts of time available to dedicate to updates is limited, it was found to potentially present a challenge in detecting potential issues that initiative teams either did not foresee or did not deem significant enough to raise in these review sessions. As such, detecting issues earlier in the cycle was at times found to be difficult.

\subsection{FoaK Initiative Teams}

FoaK initiative teams represent a group of cross-functional domain experts that are assigned to execute an approved and funded co-creation of innovation project with an external client. These are temporary transient teams that are assembled for the purpose of 
executing the project. These teams have varying degrees of experience with FoaK projects, client engagements, and interaction with other parts of the firm. A FoaK initiative team's leadership, members, team knowledge and experience all contribute to a team's effectiveness in executing a FoaK initiative.

\section{Initiative Team Participants}

FoaK proposals submitted to the program for funding, include a team of requested resources to staff the project. The initiative team is led by a primary investigator (PI), who serves as its focal point between the initiative with the program and stakeholders, and directs the initiative team priorities during the project. The PI plays a central role at each stage of the FoaK initiative. The PI is responsible for the development of the proposal submitted for program funding, negotiating the resources required, identifying and securing stakeholders that will support the initiative, and managing the project priorities and stakeholder alignments. It is the initiative team that is funded by the FoaK program.

The core FoaK initiative team typically consists of researchers with domain knowledge for a particular technology and application, and in some cases for a specific industry. Some describe researchers as having a focus primarily on "pure" research assignments, tending not to emphasize commercialization and other market considerations for their projects. PIs most often come from within the research organization. Effective PIs display a combination of technical knowledge, as well as a strategic vision for the initiative, and demonstrated business acumen.

An extended team of domain experts from across the firm supports the core initiative team. This support typically comes from the sales organization, the industry organizations, the legal team, and from the product organizations that are considering 
commercialization of the FoaK initiative asset. Extended team resource participation is not funded by the program, but rather by their respective functional organization.

\section{Initiative Team Attributes}

FoaK initiative teams comprised of resources from the research organization were found to share common characteristics. FoaK initiative teams were described as possessing a high-level of technical skill and expertise within their target domain. This in part is due primarily to the calibre of resources that reside within the firm's research organization.

Researchers experience in these cases is described by a focus on "pure research." "Pure research," in this case suggests that research projects done by this organization are focused primarily on exploratory work with less focus on achieving discrete outcomes and delivering within specific timelines. These are characteristics consistent with early stage radical innovation development.

FoaK initiative teams were also found to demonstrate varying levels of experience with the program. While some of the teams had previous projects approved and delivered within the program structure, there are other teams that confirmed having much less previous experience. In some of the cases studied, initiative team resources had no prior FoaK project experience with the program.

Also, due to the nature of work in the research organization, many researchers have limited exposure to direct client interaction, little involvement with senior-level executives within the firm, and modest personal networks within the firm. In some cases, this was found to limit the breadth of experience that individual researchers with less FoaK experience could bring to a FoaK initiative. 
"As you take research organizations from what I would call the pure science to research and development that is focused on client value and engagement - there might need to be a shift in the FoaKs that do that." Int.17.4

"There are some researchers that will focus on the technology and will say, "I'm developing a cool technology. Figure out where I could apply it. Let me find a solution. Let me find a problem for the solution that I have." And they'll focus on the technology which can be amazing. It can do what it's supposed to do, but they might, when they present to the board or when they talk to us, they might not have as good of a grasp of the bigger picture, but the business side of it, other elements." Int. 47.0

\section{Transient Nature of Initiative Teams}

The extended FoaK initiative team is characterized by the transient nature of the group of resources that comes together for the project. Initiative teams are assembled for delivery of the single FoaK initiative, which in some cases involves individuals that have not previously worked together within the firm. These initiative teams disband upon conclusion of the FoaK initiative. No pattern in previous team work experience was observed in some cases it was found that the core initiative teams come from a common research lab and have previous working experience together. However, in other instances multiple labs were found to combine resources for an initiative, resulting in researchers across geographies meeting and working together for the first time.

Effective initiative teams described their core teams as individuals who were flexible and prepared to take on a range tasks, often outside their formal role. It was found resources were commonly asked to take on roles they were not familiar with, and to acquire learning new skills necessary in delivering the project. Team members were expected to be collaborative in their approach to teamwork. In the execution of a FoaK initiative, team members often described that their primary focus was to deliver on the tasks assigned to 
the project, with all other alignment and integration activities between groups being managed by the PI.

FoaK initiative team members benefit from participation by gaining new experience and knowledge, which they carry forward to future projects. From a FoaK program perspective, previous initiative experience provides better understanding of the program and process, and better prepares them on what can be expected in future assignments. Initiative participation also allows FoaK team members the opportunity to expand their professional networks outside of their function, and develop their skills in interacting with the new stakeholder groups that their primary role may not provide. This includes the opportunity to interact with senior executives, sales professionals, as well as directly engaging with client stakeholders. This exposure contributes to the development of the individual researcher and benefits the firm by helping increase their potential effectiveness in both future FoaK and non-FoaK initiatives and responsibilities.

A risk noted with the transient nature of FoaK initiative and its teams is that of information and innovation leakage. FoaK innovations are developed through the combined knowledge of the initiative team with new knowledge creation, with each member contributing their expertise. As projects are completed, teams disband to other firm related priorities. While FoaK initiatives information is collected in the program systems regarding the specifics of the project and its innovation, there is also the tacit knowledge of participants that has not been captured. This non-codified knowledge related to a FoaK asset risks being lost within the firm. Similarly, as board members transfer in and out of the FoaK program, knowledge on the dynamics of the decision-making 
processes used at various times is also dependent upon the effectiveness of the FoaK program management team to capture and exploit.

This presents potential future challenges for the program and the firm in building a new knowledge competency in the given innovation domain where FoaK leaders and researcher expertise gained through the course of initiatives are overlooked or lost organizationally.

\section{The Role of the Primary Investigator}

Effective FoaK initiative teams were also described as those teams having strong leadership in their primary investigator (PI). Strong PIs were found to be more strategic in their approach to FoaK initiatives. They demonstrate strong communication skills and the ability to proactively work towards alignments across all stakeholders before, during, and after the delivery of their FoaK initiative. The most revered PIs were those found to approach their initiatives strategically, beyond typical project execution towards a new business entrepreneurship dynamic.

"Often the leaders of FoaK projects have no idea what it takes to turn an idea into a full-blown product within (the firm). And what it takes to navigate all the complexities of customer organization. They're pure technical people and that ends up causing most FoaK projects to have less than a stellar outcome. Because the technical people do their piece of work, but that's not guaranteed by any means that you're going to have business and backer in the customer or that you're going to be able to take that business impact and shape a product and convince a division to take it on." Int. 21.2

"FoaK team lead(ers)... are the ones that need to bridge the technical community and the business side. And so, since they are going to be the critical part of any of these engagements and understand the social and technical dynamics from the 
research perspective - those are the people that are the translators, the people in the middle... and that as a whole - to me, that's a very difficult person to find, and it's a rare person because they often have come up through the research organization, and that is based on their technical chops, their abilities to do that." Int.17.4

"The exceptional (FoaK) project leader is the guy that is not completely strict about the project itself...because when you start the project with a client, you have kind of a well-defined plan. But as you start to collect the data and you start to validate, a lot of new things happen. And an exceptional person would be one that was able to adapt and to seize the opportunity to even do more with the project to be very successful. And also, a person that has the skills, very good soft skills, because when you're dealing with situations like this with a client, you want also to bring the client to be passionate about the discovery and being kind of as a part of a journey. It's not only the project, the journey can become much more than FoaK itself." Int. 48.1

"I can think of on one hand the (four) researchers (that have demonstrated strong FoaK leadership ability). Those are four people that I've interacted with. And I probably have four times as many on the other side where brilliant people came up because of their brilliance, but their ability to interact with the client is lacking at some level." Int.17.4

\section{Initiative Team Knowledge Coverage}

Informants were consistent in expressing the importance of initiative team knowledge coverage in delivering FoaK projects. Based on informant inputs, initiative team "knowledge coverage" is described as requiring a combination of both an external knowledge perspective on where the FoaK innovation fits in creating market value, and an internal knowledge perspective that includes an understanding of how the initiative contributes to the firm's strategic direction and how to navigate the firm through the course of the initiative (see Figure 6.5).

External knowledge coverage was found to include three primary forms of knowledge: market knowledge, client knowledge, and technical knowledge. Market 
knowledge is needed to ensure that the innovation being proposed in the FoaK initiative creates value and delivers against a quantifiable need within the marketplace. Client knowledge reflects the initiative team's ability to identify the right client to engage with, have the skills to access, engage and build trust with those clients. This includes the ability to effectively navigate the complexities of client engagements and manage expectations. Technical knowledge reflects an understanding of how the FoaK innovation being explored compares to other options available in the market. This includes initiative teams having the technical skills needed to execute on the exploration process of the business use cases within the scope.

Figure 6.5 Initiative Team Knowledge Requirements

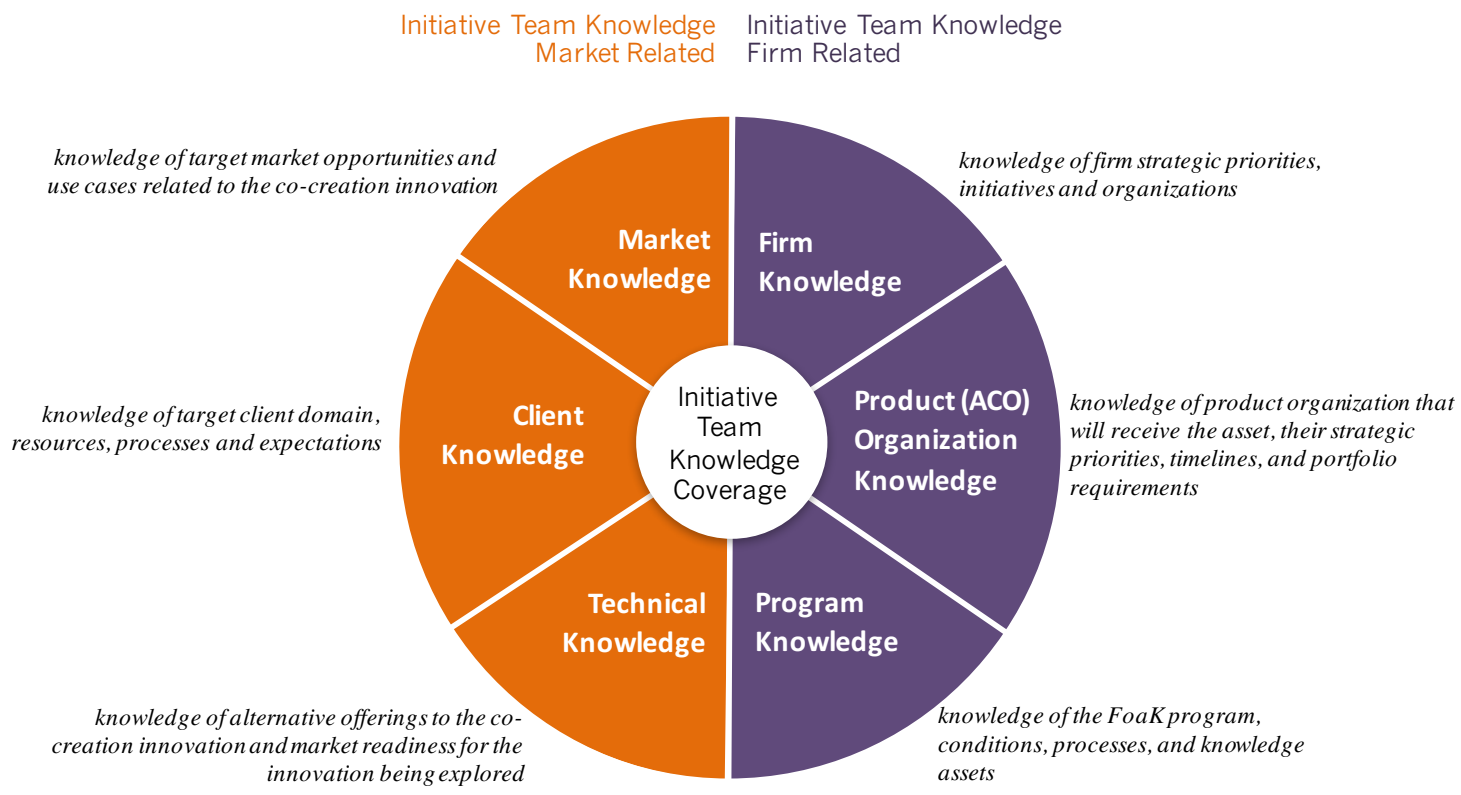

Source: Informant Interviews

The internal knowledge coverage required by a FoaK initiative team includes firm knowledge, product stakeholder knowledge, and FoaK program knowledge. These FoaK 
initiative team knowledge themes are all related to how effectively the initiative team can leverage the resources available within the firm, and the ability to navigate and gain alignments across the various internal stakeholder agendas. Firm knowledge speaks to an initiative team's understanding of what is important to the organization, and where the innovation being explored fits into those priorities. Similarly, product stakeholder knowledge reflects the initiative team's knowledge of the asset commercialization owner (ACO) target to receive the innovation asset from the initiative. This includes an understanding of the key players in the network, the ACO's priorities, how to establish committed alignment with those teams, and an awareness of any portfolio limitations which will either serve as a catalyst or an inhibitor to the transfer of the asset. Finally, program knowledge reflects an understanding of the conditions to participate in the FoaK program, the execution requirements, processes, and resources available to support the team.

Across all cases studied, it was found that the six knowledge themes each play a role in delivering a FoaK initiative, and that initiative teams have varying levels of knowledge development (i.e., experience and depth) across each one of these knowledge themes. The FoaK program framework recognizes the difference. As such, one role of the framework was observed to be the program helping to bridge initiative team knowledge gaps where necessary, to help ensure that each team has the information and support they need throughout the FoaK process. As individuals gather more domain and FoaK initiative experience, knowledge gaps across the themes decline resulting in less need for the supporting infrastructure put in place by the program bridges external and internal knowledge gaps. 


\subsection{Anatomy of a FoaK Initiative}

Co-creation of innovation initiatives are characterized by the nature of the problem they seek to address, and the breadth and complexity of inter-firm and intra-firm interactions between active contributors throughout the collaborative innovation process. In this study, the FoaK initiatives examined commonality in the class of innovation being explored (i.e., analytic applications) and in the relationships between the program, the FoaK initiative team and its contributing stakeholders. This section describes the nature of the analytic applications domain as it relates to FoaK initiatives, and discusses the principal stakeholder interaction types and where they occur across the co-creation process.

\subsubsection{Analytics Applications}

The FoaK initiatives examined in this study all focus on innovations in analytics. Analytic software solutions are a class of software offerings designed to improve decisionmaking effectiveness across industry and business specific domains. These offerings are designed to collect broad and varied forms of raw information from within a firm and from its environment, and transforming that data into insights that support decision-making processes at various levels of an organization.

Analyst firm International Data Corporation (IDC) classifies the market for analytics software as the big data and analytics (BDA) software market. They define it as "a collection of software tools and applications for data extraction, integration, governance, movement, duration, analysis, and visualization deployed to support or automate decisionmaking." (IDC 2017). IDC's big data and analytics taxonomy consists of three primary 
segments, which include BI and analytics tools and platforms, analytic data management and integration, and analytic and performance management applications (IDC 2017).

As described by IDC's taxonomy (2017), analytics software solutions support various forms of offering delivery to the end client. Innovations in this market are commercialized either as prepackaged offerings designed for implementation and configuration by clients, or in a form that allow for clients to custom build their own solutions. This includes using a collection of analytical platforms and tools available in the market for a custom client-centric offering.

Each of the innovations being studied share the common characteristics related to analytic and performance management segment offerings. IDC's taxonomy includes an analytic and performance management applications segment. IDC states that software in this segment meet three criteria (IDC 2017). First, the software in this segment demonstrate support for specific business processes, through the commercial application of software that is designed to "structure and automate a group of tasks pertaining to the review and optimization of business operations or the discovery and development of new business" (IDC 2017). Second, these offerings can operate independently from other transactional systems in the firm, may be dependent on those systems for source data, and potentially push data back to those systems (IDC 2017). Finally, these offerings represent both a time orientation and the integration of multiple sources of data. This requires that various sources and forms of data are brought together and transformed into analytics representing historical, current and future time perspectives (IDC 2017). All the FoaK initiatives selected for this study explore analytic innovations domains within this segment, either as 
standalone analytic and performance management applications, or contributing to larger offerings.

Analytic applications share common considerations regarding the architecture, skills, components, and processes required to innovate in this domain. These considerations are well understood by the analytics industry and their solution providers. A challenge in the creation of analytics offerings is the need for innovators to assemble the diverse set of team knowledge required to innovate. This includes solution expertise (i.e., business and technical knowledge), assets (e.g., software provider knowledge) and applying them against business use cases (e.g., client user knowledge). Co-creation of innovation relationships are often necessary in this domain to ensure that analytics solution providers understand the industry business domain they are developing for, the related decisionmaking priorities and challenges, where analytics can be applied in decision-making processes, and what sources of data are available to create new insights.

Each of the FoaK initiatives selected as case studies were found to share a common set of project elements which helped provide a comparative baseline of knowledge needed by each initiative team. The nature of these initiatives provides a level of understanding of the types of knowledge and skills required by the initiative teams to execute, complexity with the client engagement, and the types of resources required from the client during the execution of the initiative (e.g., client data). While each of the individual analytics solution initiatives varied on scope, complexity, and skills, the commonality across these initiatives based on the nature of the projects and innovation contributed to controlling this aspect initiative context. 


\subsubsection{Stakeholder Landscape}

An examination of FoaK initiatives reveals five principal stakeholder types: the firm, the partnering client (i.e., client stakeholder), the research organization (i.e., research stakeholder), the asset commercialization organization (i.e., products stakeholder (ACO)), and the industry development and sales organizations (i.e., industry and sales stakeholders). Each of these stakeholder types participate to some degree in each FoaK initiative. This in part is due to the program conditions that each FoaK initiative must secure the participation of certain stakeholder types if they are to be considered for funding. These stakeholders also represent the expected set of relationships and alignments necessary to promote a cocreation innovation asset from an early stage initiative deliverable between an initiative team and the client, towards a commercialized offering within a mainstream product portfolio. Each of these stakeholder types were found to participate in each FoaK initiative studied.

Achieving the necessary alignment across these FoaK initiative stakeholders requires an understanding of their functional motivations to participate, their desired benefits, and their expected contributions to the FoaK initiatives. Each of these areas varies across each of the stakeholder type. The engagement performance across each of these stakeholder groups is measured differently by design, in part due to each group operating against different time horizons. Thus, these differences between stakeholder groups introduce alignment tensions into the FoaK initiative system that require management in execution of a co-creation project (see Figure 6.6). 
Figure 6.6 FoaK Initiative Stakeholders

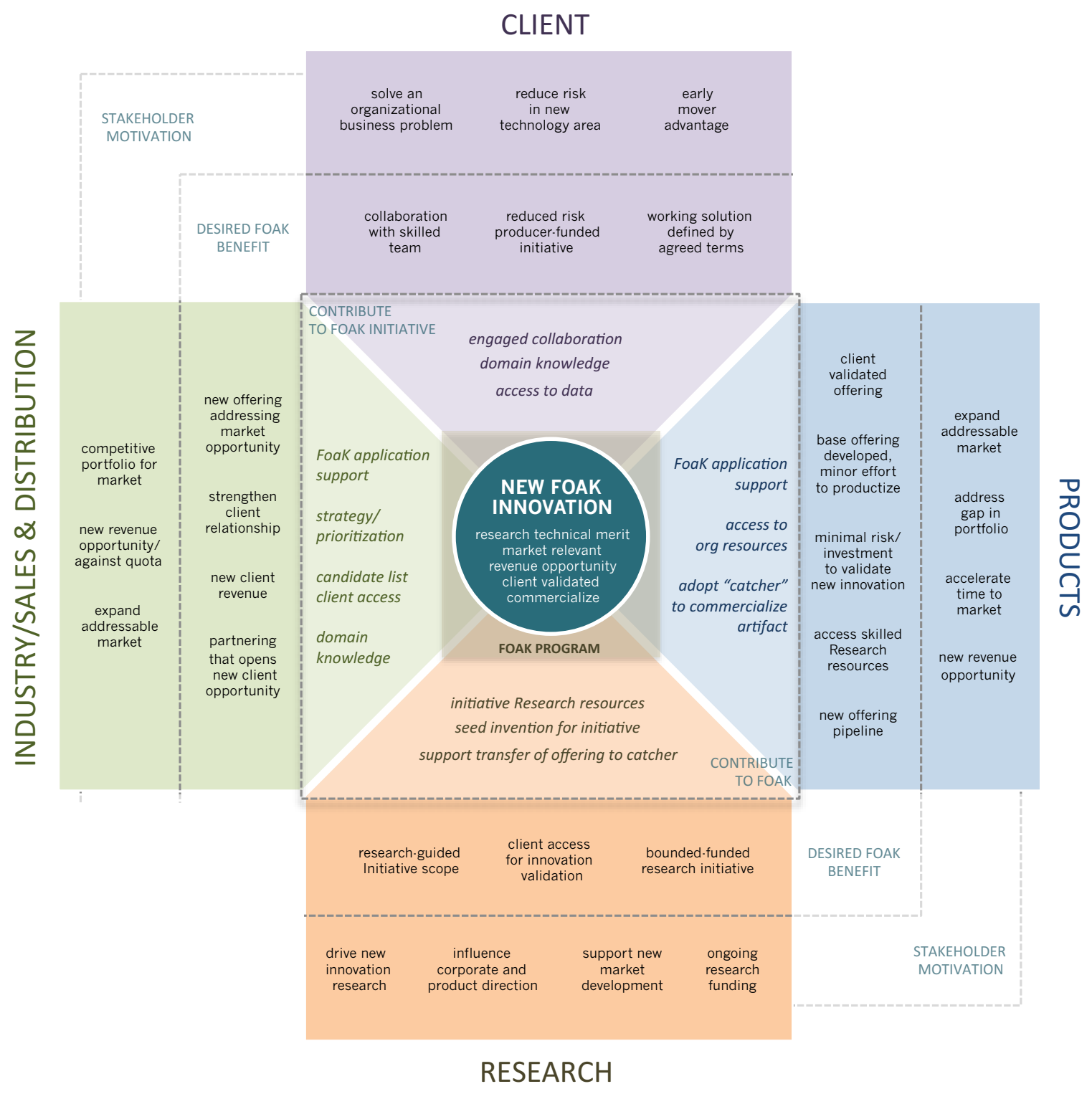

Firm Stakeholder (FoaK Program Board)

The firm is a principal stakeholder of the FoaK program and its initiative, and is represented by the FoaK board membership. The primary objective of the board is to ensure 
that a FoaK initiative has the potential to contribute to the firm's future competitive position through the delivery of new-to-the-firm innovations. The firm's motivation and benefit are reflected in the mandate of the FoaK program. Its contribution to FoaK initiatives is primarily based on the annual program funding budget. The firm views the FoaK program as one of multiple methods of generating new innovation, with its focus primarily on the co-creation of radical innovations. Radical innovations often span multiple product portfolios, which does not align with the focus of individual product portfolios on their own offerings and incremental innovation investments. A primary objective of the FoaK program for the firm is to provide a channel for potential innovations that otherwise would not receive sufficient consideration within an individual product organization's portfolio.

\section{Client Stakeholder}

All FoaK initiatives are funded co-creation of innovation projects engaging a primary co-creation client (i.e., client stakeholder). Clients participating in a FoaK program initiative are described as motivated primarily by the need to solve an organizational business problem, viewing a FoaK initiative as an opportunity to work with knowledgeable researchers from the program, a defined scope and investment. The FoaK co-creation method provides funding and expertise to the client stakeholder, presenting the potential for an early mover advantage with reduced risk to their organization. The client stakeholder time horizon operates within a near-term to midterm window for returns and outcomes from the investment in the initiative. The first major milestone for the client stakeholder is the delivery of a FoaK initiative asset, which influences their degree of future investment in the innovation asset and engagement with the firm beyond the project. 
"Each side is funding its own effort (in the FoaK). (Co-creation clients are) definitely doing it purely for the innovation and technology, as opposed to a more growthspecific goal in mind where payments involved. (Clients) definitely see an advantage over their competitors by teaming with (our firm) in going and developing something that hopefully will turn into a production use version of that same product that gives them, again, an advantage over their competition." Int. 10.0

"So we give to the customer a key competitive advantage because we are using things that are not available in the market as an existing product... I think many of our customers are happy to play that role because they know that going through (a FoaK initiative), they would have three months, six months - it would be three months, six months ahead of the market by editing that first" Int. 23.0

"Investment of the client is actually fairly minor in terms of the monitoring... It's an investment in their time and attention basically." Int.01.0

"There is minimal to no cost to the client other than paying for the project manager itself, and for the product itself. And this really depends on the client, so we see all sorts of things. Clients who have worked with research previously or bought into a FoaK program previously, it's much easier to get them over the hump and agree to sign to one because they see value in it. They're familiar with the process." Int. 27.0

As a FoaK initiative stakeholder, client stakeholders are expected to contribute to a project their domain knowledge, access to data, as well as commitment to "engaged" collaboration throughout the initiative. Client stakeholders are also asked to provide token financial contribution for a project management resource.

Research Stakeholder (Initiative Team and Research Organization)

Another primary stakeholder is the firm's research organization (i.e., research stakeholder). In the context of the FoaK program, research is represented within the initiative team that submits proposals for funding and exploration with clients, and as the 
research organization that supports those initiative teams through the process. The research organization, more than any other stakeholder group, works towards a long-time horizon. Research motivation is focused on exploring new early stage innovation that contributes to supporting future market development for the firm, and supporting mainstream product portfolio strategies. Their primary benefits in participating as a FoaK program stakeholder includes receiving directed research funding, and the opportunity to co-create with clients on radical inventions for validation and evolution of their inventions. Research's primary contribution to the program is the co-funding of FoaK initiatives, the assignment of researchers to participate in projects, and the provision of seed invention that form the basis of candidate proposals funded by the board.

\section{Products Stakeholder}

The products organization is also a stakeholder group within FoaK initiatives. Its primary role is to evaluate the potential for proposed innovations within their portfolios and provide support for FoaK initiative proposals. Product stakeholders that engage with do so with the intent of becoming recipients of the FoaK initiative assets at completion for commercialize as part of their portfolio.

The product stakeholder's primary motivation to participate in a FoaK initiative is its need to address gaps in its portfolio and the desire to expand their addressable market. Their relationship with the research organization is focused primarily on accessing and applying knowledgeable resources against the most difficult problems in their portfolio, contributing to offering differentiation and accelerating time-to-market. Product stakeholders seek benefit from participation in a FoaK initiative that provides access to skilled resources exploring business problems within a controlled engagement model, 
reducing their potential investment risk. Their expectation is that the resulting assets from a FoaK initiative will require less investment to commercialize and will provide an opportunity for new revenue, further supporting organizational goals and performance.

Product stakeholders work on a short-term to mid-term time horizon, planning incremental releases within a known release cycle cadence for their offerings. Product planning aligns to roadmaps with predetermined scope and timing to meet market needs. While product organizations may speak to the need to invest in longer-range development and research internal to their organization, this level of planning is often the first to suffer with budget pressures focusing on near-term revenue and releases.

\section{Industry/Sales and Distribution Stakeholder}

The final set of FoaK initiative stakeholders are the industry business developments (i.e., industry and sales organizations). Both groups are focused on developing new markets and growing revenue for the firm. Their primary motivations as a stakeholder are towards achieving a competitive portfolio for the marketplace that will help drive new revenue opportunities and achieve their sales plan commitments. The opportunity that sales and industry see in participating with the FoaK program is that it helps develop new assets that will address markets that the current portfolio does not support sufficiently. In addition to new revenue opportunities, the FoaK program offers a unique and differentiating opportunity for sales representatives by presenting strategic clients with a firm funded research effort. The program helps client-facing stakeholders by strengthening the relationship between the sales stakeholder and the client stakeholder firms. The co-creation partnering between the client stakeholder and the FoaK initiative team also presents the opportunity to open new opportunities within the client stakeholder organization aside from 
the FoaK initiative. This further helps the industry and sales organizations in achieving their performance objectives.

Industry and sales organizations are the primary boundary spanning client stakeholder interface for the firm. Their contribution to FoaK initiatives is in helping validate the strategy and prioritization of initiative proposals that will best address market needs, identify potential clients that will partner in a FoaK co-creation relationship, assist in managing client recruitment, and sharing domain knowledge in support of a credible offering conversation with the market. Sales and industry stakeholders operate on the shortest time horizon of all stakeholders. Their primary focus is on near-term results, including achieving sales and industry business development objectives.

\section{Stakeholder Expectations and Alignment}

A recurring theme with informants was the importance of stakeholder alignments and expectation setting. As part of a FoaK initiative each of the stakeholder groups is expected to contribute to the FoaK initiative team's successful delivery of their new-tothe-firm innovation asset. This requires an understanding of what is expected to be contributed, and alignments on those expectations.

While the FoaK program sets the stage for an initiative to perform by requiring participation of stakeholders believed essential for a co-creation initiative, FoaK initiative teams are also required to find a path towards achieving the necessary alignments across each of these stakeholder types. Stakeholder alignments are required across all engagement activities including the pre-FoaK pitch process, FoaK initiative execution, and the transfer of the FoaK innovation to a mainstream product portfolio. 


\subsubsection{Primary Stakeholders Relationships}

An examination of FoaK initiatives highlights a series of primary stakeholder relationships. These include FoaK initiative team relationships with the FoaK program, with their co-creation client, and with their asset commercialization owner within the products organization. There is also the relationship with the sales and industry organizations that support the initiative team in grounding FoaK initiative proposals in market opportunity, and in helping to manage the relationship with prospective clients as co-creation partners. An assessment of these points of engagement reveals seven primary stakeholder pairings, each representing a unique set of engagement dynamics (see Figure 6.7). These stakeholder relationships include: Initiative-FoaK program board (R1), Initiative-FoaK program management (R2), initiative-research (R3), initiativesales/industry (R4), initiative-client (R5), sales/industry-client (R6), and initiativeproducts (i.e., ACO) (R7). These stakeholder relationship pairings were found to exist across all FoaK initiatives, with varying degrees to which each was developed and effectively managed. An understanding of the dynamics of these stakeholder relationship pairings is essential in an analysis of observed phenomena across cases.

\section{(R1) FoaK Program Board - Initiative Team Relationship}

This relationship is primarily focused on two areas: the initiative selection process and initiative team mentoring by board members. The interaction between these two groups is typically focused on the evaluation of initiative ideas, guidance provided by the board to FoaK initiative teams on how to improve their proposals, addressing potential issues, and providing awareness of other potential opportunities in the broader firm 
Figure 6.7 FoaK Initiative Primary Stakeholder Relationships (Inter/Intra-firm Interlock Points)

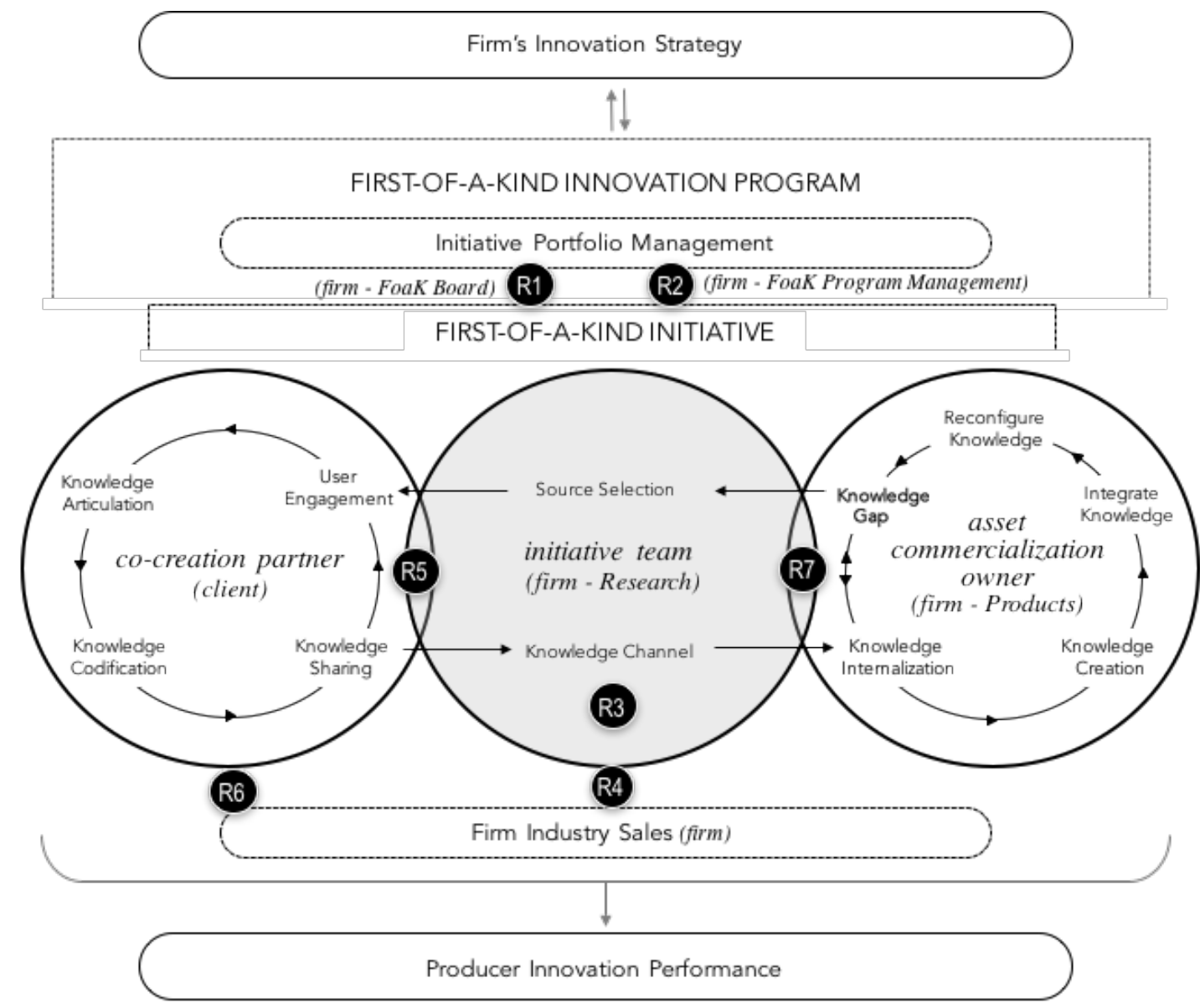

R1 FoaK Program Board - Initiative Team
R2 Initiative Team - FoaK Program Management
R3 Initiative Team - Research

R3 Initiative Team - Research
R4 Initiative Team - Sales/Industry Leaders
R5 Initiative Team - Co-Creation Client

R6 Sales/Industry Leaders - Co-Creation Client

R7 Initiative Team - Products Organization

landscape. This relationship provides an opportunity for initiative teams to gain exposure to senior executives throughout their FoaK process. It is also an opportunity for initiative teams to benefit from the guidance of senior executives, who can provide knowledge in areas that researchers typically have not developed in the context of their primary roles and responsibilities. After the selection process, the interaction between these groups was found to be limited to governance and monitoring activity, through the mandatory update 
meetings scheduled throughout the course of a FoaK initiative. FoaK initiative teams also benefit from an assigned board mentor who provide senior-level experience more frequently throughout the course of the initiative's execution.

As board members represent different divisions and functions, collectively, they serve as a proxy representation of the firm's strategy and priorities within the FoaK program. They help link the selection process and funding of FoaK initiatives to areas of strategic importance to the firm.

\section{(R2) Initiative Team (Research) - FoaK Program Management Relationship}

The primary connection point between the FoaK initiative team and the FoaK program is with the FoaK program management team. The FoaK program manager is responsible for the operationalization of program requirements as they relate to initiative candidates and funded FoaK initiatives. This relationship is one that ensures that initiative teams are clear on what they need to know as it relates to successfully applying for funding as part of the FoaK program, program expectations of all initiative teams, and awareness of the process and milestones that must be met to participate.

The FoaK program management team provides initiative teams with the support needed when overcoming operational issues in the execution of their initiatives. The intention is that aside from a clear and accurate representation of status during the regular governance meetings held throughout the execution of a FoaK initiative, that initiative teams will also proactively provide updates and request assistance when needed. The degree to which individual initiative teams proactively engage with the program has been found to vary. 
The FoaK program management team also supplements initiative team knowledge through formal and informal guidance included in the program framework. Formal directed guidance is provided by FoaK program management to initiative teams through predeveloped and curated artifacts, and a maintained program database. Program management also provides informal consultation-based guidance to initiative teams, sharing their experience from across the portfolio of initiatives which they have been engaged in overtime.

\section{(R3) Initiative Team - Research Relationship}

Initiative teams are primarily composed of research resources. The ongoing relationship between the research organization and FoaK initiative teams is close due to the functional relationship that exists with these resources outside of a FoaK initiative. The research organization supports initiative teams at multiple points throughout a FoaK initiative cycle. The research organization receives request for proposals from the FoaK program, which begins the process of evaluating whether there are any inventions underway that would benefit from involvement in the FoaK program. After identifying potential candidates, the research organization then helps to support the development of FoaK proposals for presentation to the board, and in establishing the necessary support from the other stakeholders needed for the initiative to be considered for funding.

The primary investigator (PI) of the initiative team is responsible for driving the proposal preparation, achieving alignments with the necessary stakeholders, and leading the initiative prioritization activities and execution. It is the research organization in the relevant domain area that provides the necessary functional support needed to demonstrate 
confidence in the invention being proposed for funding by the FoaK program. This support is often led by executive level researchers who help guide Initiative teams in improving their initiative proposal, towards a case with a higher potential for funding and initiative performance.

\section{(R4) Initiative Team - Sales Organization/Industry Leaders Relationship}

The relationship between the FoaK initiative team and the sales/industry organizations is the essential link between the market perspective, client validation, and the research idea. The sales organization and industry leaders provide the necessary support for industry teams in helping to ground their ideas in the realistic market opportunity and demand for the problem they are trying to solve, and help in identifying clients that would benefit as participants in a co-creation of innovation initiative. Aside from helping to supplement researcher knowledge with the market perspective, an area where researchers often are lacking, the sales and industry stakeholders serve in actively creating demand for participation in the initiative. This includes engaging both with FoaK potential client stakeholders and the asset commercialization organization (i.e., products organization). As described in the stakeholder profiles the dynamic between the research-based initiative team and sales and industry stakeholders are characterized by very different perspectives and time horizons. While researchers tend to focus primarily on "pure" research questions and learning through exploratory study, sales and industry leaders are focused primarily on sales and business development that are a near-term priority. This near-term focus presents the risk of sales and industry engagement with the client, setting expectations for near-term benefit that does not align with a long-term horizon for FoaK deliverables. 


\section{(R5) Initiative Team - Client (Co-Creation Partner) Relationship}

The initiative team-client relationship is a primary point of engagement within a FoaK initiative. It is within this relationship where the FoaK initiative team works to acquire the expert knowledge of the client to help validate and refine their invention across business use cases. There are three phases in the interaction between the FoaK initiative team and the client: the client selection and recruitment phase, the initiative execution phase, and the post-initiative execution phase.

The client selection and recruitment phase typically involve engagement with senior executives within the client stakeholder organization, the FoaK initiative team, and the support of the sales and industry stakeholders. The primary objective in this stage is to help convey the potential value to the client stakeholder in their participation, assessing the fit of the client stakeholder as an "ideal" partner for the initiative, and setting the expectations for participation in the initiative.

Upon successful recruitment of the client as a co-creation partner, the primary point of interaction with the client organization then moves to project leaders assigned by the client executives to participate in the initiative. These client contacts work directly with the initiative PI and the FoaK initiative team. Interactions at the initiative-level between the client and the initiative team are peer level interactions, and require effective communication and client management skills within the FoaK initiative team.

FoaK initiative teams use a variety of techniques to help identify the knowledge needed to validate and improve on their early-stage invention. These include the use of workshops and the development of prototypes that client stakeholder teams in turn can use to help focus their thinking and share their knowledge. There is also the linkage between a 
FoaK initiative team and functional experts within the client organization that can provide access to the necessary data needed to validate ideas within the developed innovation.

The final stage is the post-initiative relationship where the client stakeholder is focused on what will be implemented by their organization after the FoaK initiative is complete. At this stage, the client stakeholder's focus is on ensuring that their organization sees value beyond the initiative. This requires that the FoaK initiative team remains engaged both with the client stakeholder as well as the team that may implement a variation of the initiative for use by the client beyond completion of the FoaK initiative. As the FoaK initiative does not provide for long-term use of the emerging innovations by the client stakeholder, an additional effort is required between the firm and the client stakeholder organization to ensure a path beyond the completion of the initiative. This is to ensure that the firm is addressing the initial client problem that motivated their participation in the FoaK initiative initially.

The time horizon across these two stakeholders is typically focused around the duration of the FoaK initiative (i.e., 9-12 months), with the initiative scope remaining at the centre of the activity between the client and the FoaK initiative team. However, while the FoaK initiative team sees an end of their client engagement upon delivery of the assets from a FoaK initiative to the products organization, the co-creation client often looks beyond the FoaK initiative to how the findings from their engagements can then be applied within their organization moving forward. This requires a level of planning and expectation setting with clients so that they remain "whole" beyond the project. In cases where this has not been anticipated, a FoaK initiative client chasm has been described, where delivering on client expectations falls short due to the boundaries set by the FoaK initiative. 
At the end of a successful FoaK initiative, the client relationship is transferred between stakeholders, from the FoaK initiative team to the products organization receiving the FoaK innovation.

\section{(R6) Sales/Industry Leaders and Client Relationship}

The relationship between the client stakeholder and the firm's sales and industry leaders is also a central point of engagement in the FoaK initiative process. The dynamics of this engagement is influenced by two sides to the relationship.

First, clients that are recruited to participate in a FoaK initiative are typically existing and valued clients to the firm. Aside from the opportunity to co-create on new innovation, the firm also sees additional benefit in offering the FoaK research investment to strategic clients as a measure to further strengthen the existing inter-firm working relationship. As such, the sales and industry leaders see this as an opportunity to gain greater access or further build the relationship with the client stakeholder organization.

A second perspective on the relationship is the role of the sales and industry leaders in identifying the right clients to participate in a FoaK initiative. They serve the role of helping set the stage and the right expectations with the client stakeholder as a collaboration partner. Expectations of sales and industry leaders are such that their focus is on the goals of the FoaK initiative, and that they will help navigate and minimize the effects of any existing firm-client relationship (e.g., active sales cycle) outside of the context of the FoaK program. 


\section{(R7) Initiative Team - Products Organization (ACO) Relationship}

The final stakeholder pairing within a FoaK initiative is that between a FoaK initiative team and the products stakeholder (i.e., asset commercialization owner) that will assume the innovation asset resulting from a FoaK project. While the initial relationship between the initiative team and the product stakeholder may have previously been established by the research leadership or the industry leaders, the PI in the FoaK initiative team is responsible for engaging, recruiting, and securing the alignments of the product stakeholder organization and team.

The product stakeholder organization can be seen as an internal client, with their initiative team engagement bearing many similarities to the initiative-client relationship. The initial engagements between the FoaK initiative team and the product stakeholder organization is with product executives. The goal is securing support for the FoaK initiative and commitment to receive the transfer of the FoaK innovation asset into their portfolio for commercialization. The product stakeholder executive in turn assigns product and development management leaders to serve as the primary operational contact point. Their role is to work with the FoaK initiative team to learn about the initiative domain, and to share portfolio direction with initiative team. It is at this point that the FoaK initiative team can begin to consider how their innovation could fit into the product stakeholder portfolio prior to its transfer. This relationship is characterized by bidirectional knowledge sharing, where the initiative team shares with the product stakeholder organization details of the innovation being developed, the business use cases being explored within the FoaK initiative, details of the client's experience, and the potential technical considerations for capturing the invention and commercializing it. The product stakeholder organization in 
turn shares with the initiative team the priorities for their portfolio, and the technical considerations that they have when deciding on whether they can adopt innovation. The product stakeholder organization will also identify any priority areas that would help the FoaK initiative team in adjusting the scope of their work with clients, helping to prioritize any areas that would have the greatest impact for the firm and the product portfolio.

The FoaK initiative team and the product stakeholder organization work towards different time horizons. While the FoaK initiative teams focus on the delivery of an asset at the end of the 12-month project window, the product stakeholder organization is often working against a pre-established product roadmap. The effective transition of an asset from a FoaK initiative into a product organization requires that there is an understanding of the product portfolio's window of opportunity to capture the innovation being explored. This includes consideration of the investment required in the innovation's commercialization, while the product stakeholder organization continues to deliver against its pre-established commitments to the firm and the market.

\section{Stakeholder Landscape Takeaway}

When considering a FoaK initiative and its relationship to the FoaK program these stakeholder relationships and the engagements between them, the pattern of involvement between these pairings was found to be consistent across case studies. All pairings were found to exist and to be active in all cases. It is also evident that there are multiple intersection points across the FoaK process that connect these stakeholders, FoaK initiative related processes, and the individual functional processes related to each of the stakeholders. The intersection points across these processes also represent the knowledge 
flow between FoaK stakeholders and across these processes. This flow of knowledge contributes to the co-creation of innovation activity between the FoaK initiative team and the client stakeholder, as well as contributing to the performance of the FoaK innovation (i.e., client satisfaction, follow-on revenue, the successful transfer of the assets to a mainstream product organization).

These inter-firm and intra-firm stakeholder intersection points are presented in Figure 6.8, which illustrates the primary interlock points for FoaK co-creation of innovation initiatives. A review of this highlights the complexity and interdependence in co-creation of innovation activities brought forward in the literature. It also illustrates the need for internal and external knowledge types (see Figure 6.5) to effectively manage the dependencies across the FoaK process, both within the program and its initiative teams.

\subsection{Case Studies: Initiatives Profile, Informants and Program Context}

Initiatives selected as case studies were evaluated based on their potential for providing insight into the research questions being examined. Selection criteria limited candidate initiatives to those that were both representatives of the FoaK program, and were found to be rich sources of information in support of a critical realist approach. Efforts were made to select initiatives that represented a common context as related to their interaction with the FoaK program. Similarly, the critical realist philosophy advises that triangulation is essential to understanding the truth of the phenomena being studied (Yin 2013). To ensure triangulation, this study applied a multi-tiered informant interview strategy. A variety of informant perspectives were collected to contribute to a deeper understanding of what is being observed. This section describes the FoaK initiative profiles 
สิ




selected as case studies, summarizes the profile of contributing informants, and describes the program context.

\subsubsection{Case Studies - Shared Context}

The selection criteria used in determining which FoaK initiatives to include as case studies were focused on ensuring the richest sources of information that would provide the most clarity on understanding the research questions being examined. To this end, case study selection focused on controlling for potential variations in the FoaK program attributes that could influence the observed performance of individual initiatives. Selection criteria were guided in part by searching for initiatives that shared a common experience in FoaK program context including governance board structure, program management and funding processes, and the program strategic pressures as they relate to the firm at a point in time. All the cases selected shared a common context in these areas.

\section{Time Period}

All initiatives selected to be case studies in this research were approved for funding between the years of 2011 and 2013. It was found that during preliminary evaluation of initiatives from this period, there was sufficient information available, and informants that were available within the firm with sufficient recall of the initiative details. During this period, there was also stability in the FoaK program design, its processes, and its board participation. Also, the firm-level strategic imperatives were also stable during this period. 


\section{Firm Strategic Imperatives}

During the 2011 to 2013 period, the firm's strategic imperatives were focused on leadership in the analytics industry solutions domains and in developing its "Smarter" industries agenda. At the firm-level, investment was focused on bringing analytics and cognitive technology into industry-centric market offerings. This strategic agenda was supported both by the firm's acquisition strategy and shifts in individual portfolio direction, with an increased focus on analytics and industry solutions. The firm's strategic direction was in turn represented through the FoaK board members, as leaders from the various divisions across the organization.

As such, it is reasonable to state that all initiatives evaluated by the FoaK board during this period were in part assessed based on their ability to support the firm's higherlevel strategic imperatives for the firm. In addition, all the initiatives selected for this study fall within the analytics applications domain, aligning with the firm's strategic priorities during the period examined.

\section{FoaK Board Stability}

FoaK program board stability was found to be high during the period between 2011 and 2013. During that period, board membership remained steady at twelve members per year, including the chairperson. Each of the principal divisions of the firm was represented during each of those years, and the same chairperson led the board across all three years. In addition, the same program manager was responsible for operations and served as the focal point between the program and FoaK initiative teams. Turnover of members of the board during this period was minimal. Of the twelve board member positions, the same members held nine chairs during the 2011 to 2013 period. In addition, of the remaining six 
members that participated during the three-year period, three of those board members served for two years in that period. Appendix 6.1 provides a detailed representation of the FoaK board membership during the period studied.

Based on the stability of the board membership and leadership of the FoaK program during the period studied, it can be reasonably stated that initiatives presented for funding and those subsequently approved experienced a similar engagement process and decisionmaking dynamic with the governing FoaK board.

\section{FoaK Program Process Stability}

During the period studied, FoaK program management and processes were also found to be consistent for all initiatives presented and approved. The program was managed by the same program manager during each of the years in the period examined. In addition, the program manager has been the same since the launch of the program, and has served as the principal source of continuity across the program systems, initiatives, and processes. The program manager confirmed that the FoaK program and initiative selection process was unchanged during the period studied. The FoaK processes used, the board meeting structure and cadence, initiative candidate requirements, and funding levels all remained the same throughout this period.

\section{FoaK Funding Model}

A recurring theme with informants was the question of annual variations in FoaK funding available to new initiatives. As the co-sponsored program budget is determined annually, some years require that the program modify the maximum level of funding that can be awarded to any single approved initiative. This funding includes the number of 
researchers that can be assigned to a project, and for how long. It was confirmed by the FoaK program management office that for the period between 2011 and 2013, the funding model was similar for initiatives presented for approval during that time. On average, initiatives were funded for a twelve-month period with four to six research resources forming the core initiative team. This was consistent with the initiative selected to be case studies in this research.

\subsubsection{Case Study Profiles}

The findings for this study are based on a two-tailed comparative case analysis. A total of five cases (i.e., initiatives) were examined. All cases included in this study were initiatives executed to completion, and achieved either a "typical," "successful," or "unsuccessful" level of performance. For this study, the cases examined included one initiative of "typical" performance, two of "successful" performance, and two of "unsuccessful" performance (see Table 6.2).

Each of the initiatives explored innovations based in the analytic applications software domain. These included solutions examining the integration of multiple sources of business data, analytic algorithms and processes, hardware and software, all directed at improving data-driven decision-making. Each of the funded initiatives were also found to support a firm-level strategic agenda. The analytic application innovations being investigated in these co-creative innovation relationships addressed business use cases across five different industries. Co-creation partners were all existing clients of the firm, and all found to be leaders in their industry. Initiatives were conducted in the country of the co-creation client stakeholder team, which varied across the individual FoaK initiatives. 
Table 6.2 Cases Studied Overview

\begin{tabular}{|c|c|c|c|c|c|}
\hline & \multirow{2}{*}{$\begin{array}{c}\text { "TYPICAL" INITIATIVE } \\
\text { Case } 1\end{array}$} & \multicolumn{2}{|c|}{ "SUCCESSFUL" INITIATIVE } & \multicolumn{2}{|c|}{ "UNSUCCESSFUL" INITIATIVE } \\
\hline & & Case 2 & Case 3 & Case 4 & Case 5 \\
\hline Solution Domain & Analytics Solution & Analytics Solution & Analytics Solution & Analytics Solution & Analytics Solution \\
\hline Industry & Industrial Products & Retail & Automotive & Travel \& Transportation & Healthcare \\
\hline Firm-Level Strategic Agenda & Yes & Yes & Yes & Yes & Yes \\
\hline Industry Co-Creation Partner & $\begin{array}{l}\text { Natural Resources } \\
\quad \text { (Mining) }\end{array}$ & Retail & Automotive & $\begin{array}{c}\text { Travel \& Transportation } \\
\text { (Rail) }\end{array}$ & Healthcare \\
\hline Initiative Dates (start/end) & $2013.06 / 2014.12$ & $2013.10 / 2015.07$ & $2013.12 / 2016.01$ & $2011.08 / 2013.05$ & $2012.02 / 2013.06$ \\
\hline Client Region & South America & United States & Asia-Pac & Western Europe & Western Europe \\
\hline Firm Co-creation Investment & $5 \mathrm{FTE} / 12$ months & $5 \mathrm{FTE} / 12$ months & $6 \mathrm{FTE} / 12$ months & $4 \mathrm{FTE} / 12$ months & $5 \mathrm{FTE} / 12$ months \\
\hline Client Co-creation Investment & Yes & Yes & Yes & Yes & Yes \\
\hline Initiative Performance: & Moderate & High & High & Low & Low \\
\hline Client Success (Reference) & Yes & Yes & Yes & Yes & Yes \\
\hline Innovation Assets Transfer & No & Yes & Yes & No & No \\
\hline Follow-on Revenue & Active; TBD & Yes & Yes & No & No \\
\hline \multicolumn{6}{|l|}{ Case Study Content Summary } \\
\hline Informants (count) & 8 & 11 & 6 & 6 & 6 \\
\hline $\begin{array}{l}\text { Correspondence \& Transcripts } \\
\text { (pages) }\end{array}$ & 331 & 349 & 505 & 318 & 292 \\
\hline Supporting Artifacts (count) & 16 & 38 & 32 & 23 & 34 \\
\hline
\end{tabular}

Each FoaK initiative team had representation in the client stakeholder country, with two projects having their lead research team situated in another country.

All initiatives selected as case studies were approved for funding by the FoaK program within the three-year period examined (i.e., 2011 to 2013), representing a timeframe where the primary elements of the program including program management, governance board membership, and the program process remained consistent. The FoaK process used for each of these initiatives was the same, including the proposal evaluation, 
funding conditions, success criteria, selection, initiative execution support and monitoring. The initial resourcing approved for each of these FoaK initiatives were of similar scale, and involved the commitment of research staff to each initiative. Each of these initiatives involved one client stakeholder partner working with the FoaK initiative team, and included financial investment from the client for project management services. A detailed description of each of the individual cases is presented in Chapter 8 .

\subsubsection{Informant Interviews}

A multi-tier strategy was used in selecting informants for interviews both across the program-level analysis and the case study analyses (i.e., Stages 1 and 2). A total of 47 individual interviews were conducted across 37 informants. Several informants from the FoaK program management office contributed as both program-level informants and individual initiative-level informants due to their unique perspectives on both subjects. These interviews were separated to distinguish themes between program theory and observations on individual cases (see Table 6.3).

Multiple roles were interviewed to provide a range of perspectives on the program and each case initiative studied. Informants were of various levels of seniority, including executive (e.g., vice-president and director), management (e.g., business unit executives and managers) and initiative-level developers (e.g., developer, researcher, consultant). Informants were identified as being program-level or initiative-level stakeholders through the FoaK program database. A list of informants was prepared for each of FoaK program theory development and case study data collection and analysis, and reviewed with the FoaK program office for any potential additions to the list. There were no significant issues 
in securing informant interest and participation in the study, and the number and types of informants that participated provided for sufficient coverage of perspectives when examining the program and initiatives studied.

Table 6.3 Informant Interview Summary

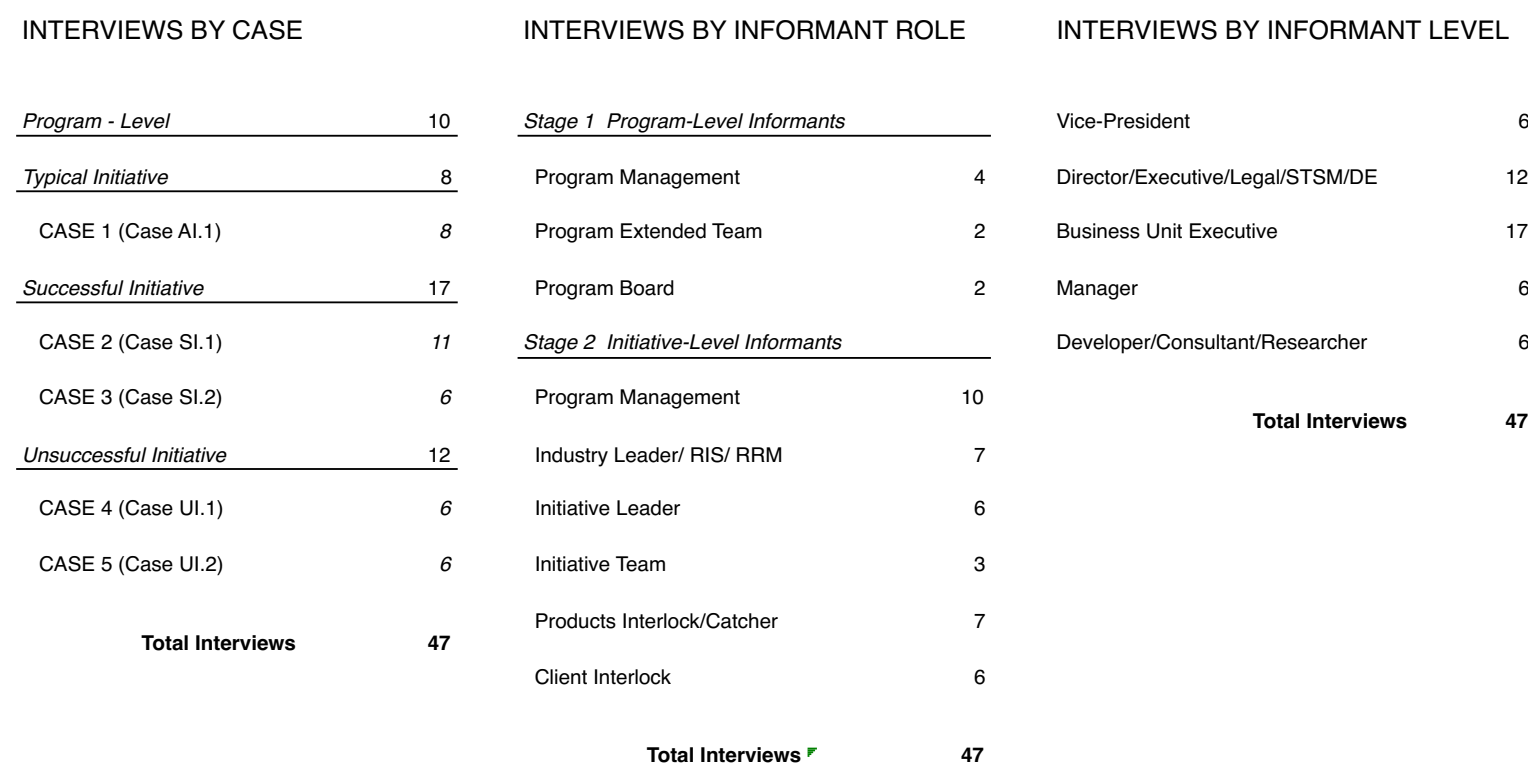

Interviews were scheduled and conducted by phone, after preliminary contact was made and the research objectives and interview objectives were shared with informants. A follow-up meeting invitation was sent to each informant with supporting details prior to the call. Interviews were scheduled for 60 minutes, and where necessary, shortened to accommodate the informant's availability. In several cases, follow-up interviews were also conducted to complete the informant interviews. All interviews were scheduled using the firm's internal systems, and using processes familiar to all informants as those used for internal discussions and consistent with the firm culture. Interview timing was set to accommodate informant availability, accounting for informant locations globally. All 
interviews were conducted in English, which did not result in a requirement to eliminate any candidate informants from participating in the study. All interviews were recorded with the informant's consent, and transcribed for analysis. During the findings analysis, analytic memos were prepared capturing primary themes and considerations for the final analysis. 


\section{Chapter 7 DEFINING THE FOAK PROGRAM THEORY AND MECHANISMS}

In case studies investigating programs, critical realism highlights the value of developing an initial program theory. Program theory serves as a representation of an organization's belief of causation, manifested in a program design (Pawson and Tilley 2004). Before entering case analysis, the findings stage of this study focused on defining a FoaK program theory believed to represent the primary causal mechanisms and interventions introduced in the FoaK program design. These program design elements represent the organization's intended impact on initiative team activity, stakeholder behaviour and initiative performance. Mechanisms were identified through an analysis of the FoaK program and initiative artifacts, co-creation of innovation theory, the conceptual model, and program-centric informant interviews. This chapter presents the initial FoaK program theory developed and used in guiding the analysis.

The FoaK program theory was found to consist of thirteen primary mechanisms (i.e., interventions) identified as contributing to a FoaK initiatives performance and outcomes. These mechanisms are believed to exist at three levels of the co-creation of innovation system: firm-level, program-level, and initiative-level (see Figure 7.1). The mechanisms identified represent FoaK program elements which includes the value of program-driven co-creation of innovation initiatives, the mechanisms related to the priorities and operation of the program in effectively setting the stage for FoaK initiative success, and the mechanisms that describe the criteria for an initiative to participate in the FoaK program. 
Figure 7.1 FoaK Process and Program-Initiative Mechanisms

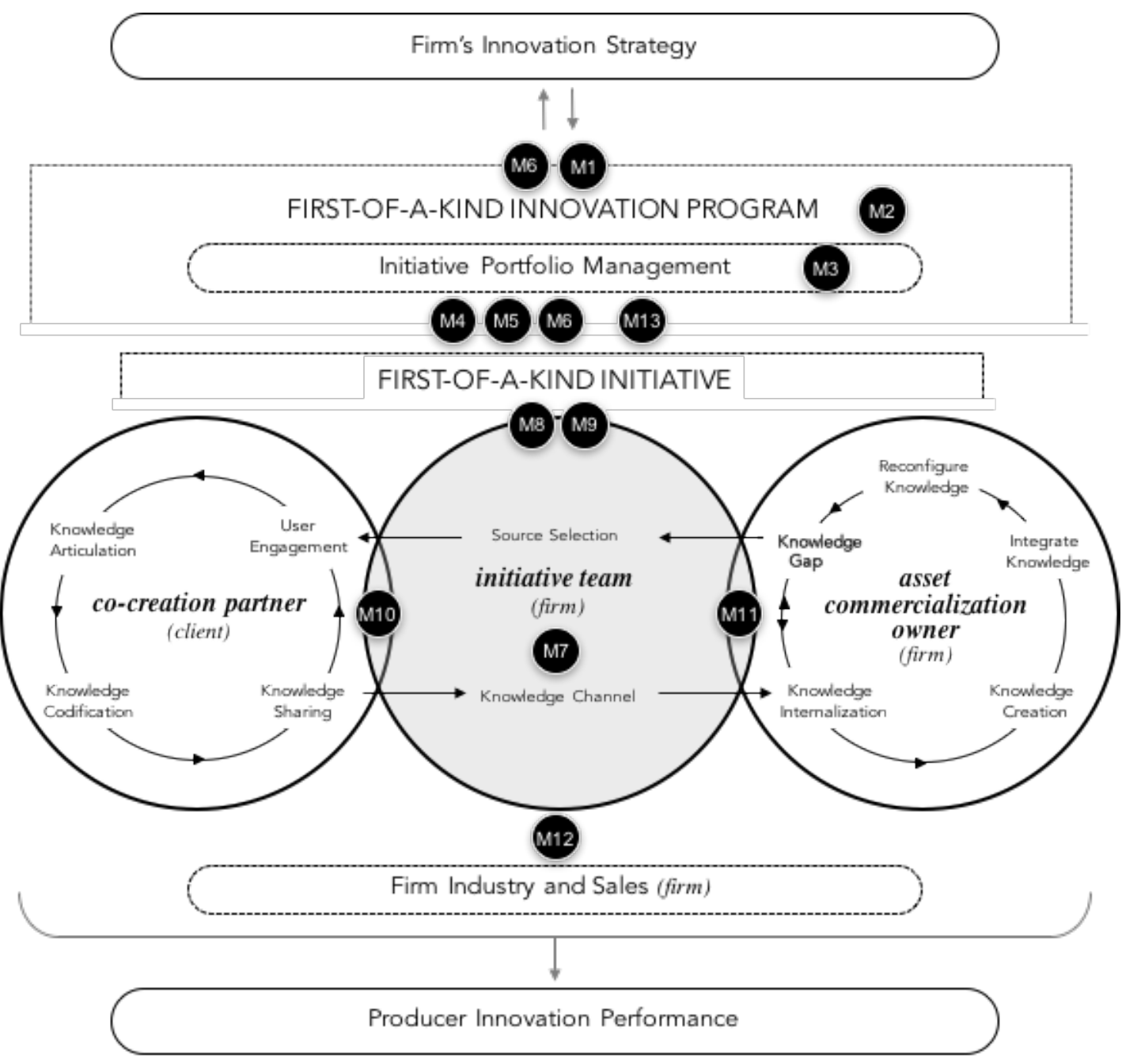

PRINCIPLE PROGRAM MECHANISMS

Firm Level:

M1 Innovate Through a Co-Creation Program

Program Level:

M2 Reduce Barriers to Co-Create Innovation

M3 Manage Initiative Candidates as a Portfolio

M4 Program as an Orchestrator

M5 Initiative Governance

M13 Gather Learnings and Refine Program
Initiative Level:

M6 Conditions to Participate for Initiative Candidates

M7 Initiative Team Ability to Execute

M8 Program Directed Guidance to Initiatives Teams

M9 Program Experiential Guidance to Initiative Teams

M10 Client Engagement

M11 Commercialization Partner Engagement

M12 Industry and Sales Engagement 
The FoaK program theory design presented assumes a common initiative execution context (i.e., FoaK program board and management, processes, funding levels), and a common firm-level context within which the program operates. All cases are exploratory co-creation initiatives, within an analytic application domain, focused on radical "new-tothe-firm" innovations requiring market validation. The initiatives are research-driven with an expectation that the resulting innovations will be transferred into a mainstream product stakeholder portfolio. All initiative-level mechanisms are understood to exist for each initiative executed within the FoaK program, while varying in the degree each mechanism contributes to observed outcomes. Each mechanism was also analyzed from the perspective of micro-macro relationships.

\section{M1 Innovate Through a Co-Creation Program (Firm-Level)}

The FoaK program represents a knowledge acquisition method for the firm, a cofunded exploratory innovation mechanism. The FoaK was established with the belief that a program approach to exploring radical innovations through a structured framework and repeatable model would lead to the delivery of market relevant and scalable innovation assets for commercialization by the firm. The innovations that emerge from the program would come through directed exploratory research working with client stakeholders, and introducing product management discipline to alternatively ad hoc initiatives. It is believed that the introduction of a formal program in these types of research efforts would be more effective in its return of new innovations to the firm.

"...the problem that (FoaK is) looking to tackle, which is to support solutions that are a little bit more cutting-edge. That doesn't have a place in existing channels, or 
existing support, existing paths. Support those technologies with a lot of promise so that they can lead to revenue and opportunity." Int.47.0

"FoaK was in a sense the only other primary mechanism when people wanted to work on a particular industry problem, both to have a venue to do this for a mechanism to work." Int.27.0

"We are thinking how we can scale what we are doing here (with the FoaK program) and trying to plan out a path for that." Int.02.0

“.... when you're going to scale, that means you need an entire organization to support that." Int.23.0

\section{M2 Reduce Barriers to Co-Create Innovation (Program-Level)}

Co-creation client partnerships developed outside the construct of a formal FoaK program, are pressured by funding, process, IP, and credibility challenges. It is believed that a FoaK initiative's performance is in part dependent on the ability to reduce barriers often faced in co-creation of innovation initiatives. The FoaK program in its design believes that providing funding for directed innovation research, a framework to guide initiative teams through a complex set of interactions, providing predefined assets (e.g., legal terms for engagement), and the credibility afforded by a firm supported program, all contribute to the effective execution of a FoaK Initiative. It is believed that an innovation program designed to reduce barriers in the co-creation of innovation process across all points of interaction, will motivate stakeholder engagement in the initiative.

"The First-of-a-Kind program provides enough support to not just create a minimally viable product, but also to test the technology to develop a solution that I wouldn't say necessarily is production-ready, but it's pretty close to being production-ready." Int.18.0 
"(FoaK initiative teams) really value that the structure of the process of engaging a client, going through the selling phase in terms of getting the client on board, and then going through the contract and negotiation phase. The fact that there are...structured processes is the way we've purposely made it is so as to not be bureaucratic, but at the same time, to prevent (FoaK initiative teams) from spinning their wheels by virtue of the fact that we've done this many times before and know what works and what doesn't work... (FoaK initiative teams) value the structure of the process and the relationships, because it does make it much easier for them to engage with clients." Int.10.0

So, when you think about that scenario of linking idea, to clients, to products, and just a whole invention to innovation cycle, FoaK provides a platform in business terms, not technical terms and a framework." Int.18.0

"The fact that we don't require huge investment from a client. It is a way for them to innovate, getting to dip their toe in the water, without having to invest huge amounts of money." Int.02.0

"There is a process in place makes it much more easy for (FoaK initiative teams) to go off and talk to three or four or five or ten clients over a period of a month or two or three and then get that client engaged." Int.10.0

\section{M3 Manage Initiative Candidates as a Portfolio (Program-Level)}

By design, the FoaK program enables individual initiative execution and performance within the context of an overall portfolio of FoaK initiatives. Program portfolio performance is determined by the proportion of initiative success across funded co-creation projects. A portfolio approach represents a competitive process for the funding of individual initiatives. As such the interaction and behaviour of the research organization and initiative teams is guided by program design, support, and predetermined conditions to participate. The program believes that managing initiative candidates as a portfolio 
improves the proportion of co-creation initiatives that succeed (e.g., shifting support to initiatives showing the most promise). Applying a competitive process that selects the strongest candidates increases the focus of an initiative team on what is viewed as most important to receive program support through funding and other resources.

"We're trying to ask (questions) like venture capital (firms) that would make investments, or seed funding" Int.01.0

"(The FoaK program is) a place where people bring new ideas, and then (FoaK as) an integrator who can try and make sure that the investment we are making in ... new ideas really builds fruit." Int.27.0

"'Guys, we are going to spend (significant) dollars with a (client) because even if we solve this, no one's going to care.' So, in that sense, we do (evaluate the investment). " Int.01.0

"So, I think that you have to think of the technology aspect, how deep it is, but the financial aspect and the market's impact are absolutely key." Int.23.0

"I see FoaK as being established, and there's a process to it, and it is democratic in the sense that you go through the specs, and if you do everything, if you put the effort in, you get to present, and then you get your day in the light. So, I think that there's an appeal there that it's in your control." Int.47.0

"We do report at the end of each year how we did on our metrics. So, we do give them more of an update on how the program is doing rather than the specific project. But they're definitely focused more on finding the right-- or deciding which areas to invest in and which projects look good." Int.02.0

"You have to weigh them, and then this is something that the board does do, and the board is weighing the proposals from a given day but also looking at previous things we've done. 'Why are we doing this? We've already done something similar, 'or, 'this group has done something before. It turned out well. It didn't turn out well." So, those are all board-level discussions. Int.45.0 
"We need to be completely okay with failure because we have lots of new ideas and not all of them are going to stick. And if we become so conservative that every one of these has to be successful, then we won't be pushing the boundaries... it has to be looked as a portfolio." Int.27.0

\section{M4 Program as an Orchestrator (Program-Level)}

The program's role in FoaK initiative performance is indirect, with its primary role being that of a co-creation of innovation orchestrator. The program design, requirements to participate, credibility of a firm sponsored program, and the influence of its governance board, all contribute to setting a stage for initiatives to focus in a given direction. The program serves as an orchestrator by bringing together both directly and indirectly the necessary elements believed needed for an initiative to succeed. FoaK initiative teams work within a framework that promotes the requirement for stakeholder recruitment and alignment, a signed client agreement for funding, mentoring, and other processes and resources available to support initiative teams in bridging knowledge gaps. Each of these elements is geared to focus an initiative team's efforts towards achieving on the program's performance measures. As such, it is believed that when the program is effective as an orchestrator, it sets a stage that will help motivate stakeholder participation, focus initiative team on performance outcomes, and bridge knowledge gaps with initiative teams.

"The FoaK program office is kind of the glue because they have been sort of touching all aspects of this project, not just the execution team, but also the full board which are executives across the organization, also the catching organization, also the industry leadership, etcetera." Int.36.0

"(The FoaK program) provide support for shepherding the product through, where for the first stage the product is supposed to try and build some prototypes and find 
a client. So, they help by meeting with usually the industry team, which is usually from (sales) - and (sales) is part of the board - to find a client to work with them and support for them finding the signed contract and things of that nature." Int.27.0

"As a FoaK program) the first thing we do on a project is approve-- which we have a kickoff call where all the members of the team upstart. That's key, too, because we- a lot of the members of the team have never worked on a collaborative FoaK project before. The client executive probably hasn't. The (services) project manager many times hasn't. Sometimes even the researcher, it's their first project. So, the first thing we do is to identify the players. That's very important. And we get them all on the call. Many times, they will all be scattered all over the world. And we take them through what the goals of the project are, what the process is, what the obligations of each one of them are, what their responsibilities are, and how we close the project within the limited period of time that we have so that everybody knows the big picture up front. Before we did that, we'd had much more problems." Int.10.0

\section{M5 Initiative Portfolio Governance (Program-Level)}

In addition to FoaK program structure and its support as an orchestrating role, the program also incorporates initiative governance to further help focus initiative teams on performance outcomes. Governance is applied through formal monitoring, ad hoc support for issue management, and mentoring of initiative teams. The program believes that providing a governance mechanism contributes to focusing initiative team execution to their agreed set of expectations and milestones. The governing role and its structure is also believed to help direct a co-creation initiative's efforts on performance outcomes through initiative team accountability against the terms of their participation in the program.

"That ends up being a lot of the question. 'Have you thought about this? How did you come up with that market protection?' Especially if it's an area where we have some personal knowledge, for something around banking I might say, 'Well that's just not going to work. We are just way late in the market because there are 20 other 
competitors that are very active.' So, things like that (the FoaK board) provides. That's where a lot of the questioning happens in terms of me." Int.01.0

"The FoaK board is more for reporting every month on progress to the FoaK board. The last final presentation where (the FoaK initiative team is) demonstrating all the success you've had or maybe even some road blocks that you've had as you step through the process as a project team. Once (the project is) approved that the FoaK board is directly, intimately, involved in the execution of the project, right? So, it's more of a reporting status back to the FoaK board. If you have difficulty finding a client, perhaps they connect you with some other executive sponsors in the industry or maybe some client execs in (services) that could connect you with opportunities. But it's not so much that they're trying to drive or influence the scientific agenda after funding is given." Int.18.0

\section{M6 Conditions to Participate for Initiative Candidates (Initiative-Level)}

The FoaK program provides a defined set of conditions required to be met by initiative candidates for their proposals to be eligible to participate and to receive funding. These conditions provide clarity on program expectations and direct initiative teams to act in a desired direction. This is achieved through program design, where it is believed that including the conditions to participate influences initiative team action towards the elements needed for FoaK initiatives to perform.

"The FoaK (program) did a great job, I thought, in asking for all these things (...setting the conditions). Because if it's an afterthought, once you're halfway through the project - and then you have to figure out, 'Oh, wait a minute. So now, who's my asset commercialization owner and what's my client pipeline?' If that's something coming midway through the project, you're probably at a disadvantage as a PI (primary investigator), as opposed to having thought about this up front and getting all the support up front sure." Int.18.0 
"Collaborating with the client who is involved, working with the asset commercialization team throughout the project, having a project that is a hot area so that the teams will invest resources (investing in writers to write it up). Having the attention of the research development group so they can help the team start selling following deals that certain areas of the focus on so if the project is in one of those areas it makes it easier to get follow-on sales. " Int.02.0

\section{M7 Initiative Team Ability to Execute (Initiative-Level)}

In the selection process of FoaK initiative candidates to be funded, it is also believed that consideration of an initiative team's ability to execute is required. Board members, program management, and product stakeholder organizations all express that they must have confidence in an initiative team if they are to fund and support an initiative. An initiative team's previous experience, track record, and familiarity with stakeholders were all mentioned as contributing to a more favourable view of initiative candidates seeking funding. Initiative teams with strong leadership in their primary investigator (PI) are also believed to perform better than teams with little to no previous FoaK experience or stakeholder engagement (i.e., clients, products organization).

"You can definitely tell the team that has gone through a FoaK process to understand all the potential pitfalls, but they can change FoaK to FoaK in terms of acquiring a client, or risk a team that they are working on. So, experience always helps, but there is a community in terms of people around them and the FoaK team itself. So, it helps but I don't think it's the critical success factor. " Int.27.0

"Teams that have been through it before, especially obviously, the research team, are much quicker to engage clients and much more successful in selling them on the project, and they work through the issues much more quickly and get the contract signed much more quickly. There's definitely a benefit to having worked on a previous project." Int.10.0 


\section{M8/M9 Program Guidance to Initiatives Teams (Initiative-Level)}

A program-driven approach to FoaK initiatives also looks to provide various forms of guidance to initiative teams to help bridge knowledge gaps in areas needed in the effective execution of the project. The program provides directed guidance as well as experiential guidance to FoaK initiative leaders and their team members. While guidance is available to all teams, the degree to which it is used is at the discretion of each FoaK initiative team.

Directed guidance (M8) is provided through the development, curating, maintenance and availability of artifacts, practices, and history from previous initiatives. The availability of this guidance to initiative teams is formally communicated, structured, and delivered through program systems, that allow for the support of multiple initiative teams in parallel. These knowledge bases and resources allow for repeatability and central access of relevant information to all FoaK teams.

In addition to directed guidance, the program also provides access to experiential guidance (M9) through its FoaK board, program management team, and extended FoaK team of stakeholders. This guidance is available to FoaK initiative teams at the various stages in the initiative lifecycle. For example, support may come in the form of helping clarify processes, addressing legal contract issues, or guidance on how to address stakeholder engagement challenges.

It is believed that the FoaK program's provision of guidance helps bridge FoaK initiative team knowledge gaps, which in turn help improve the execution of FoaK initiatives. 
"If you're coaching a start-up before they go pitch investors, you're going to give them tips like, all right, here's what they like to look for or not necessarily the content anymore, but make sure to dot your i's, cross your t's, do this, do that. So, that'll be with the teams that have less experience." Int.47.0

"So, before funding decisions are made, they might tell you, as a PI (primary investigator). 'We don't think this is the right area. Maybe you can change your proposal idea and direct it slightly differently.' So, that kind of feedback I think is valuable. I had a case in travel and transportation where a project team came in with one idea which was fairly solid. However, the FoaK board then said, 'You know what, if you change it from (A to B)...' . So, that then ended up with the much powerful and much more impactful solution right. So, that kind of feedback you get before the funding decisions are made. But once it's made, I think the FoaK board typically is taking a step back and just lets the research team as well as the industry, drive the proposal forward." Int.18.0

"You get some of those (teams) with less experience and even the ones there sometimes they might not have much guidance from people around them and so the less experience they have, the more that we will help them understand the process. (program management) does an amazing job of laying it all out. (They have) the steps, here's what you need to do. And some teams are usually really good about getting ahold of (them) but if they also ask us, 'Oh, how does this work, how does that work?', we'll also provide that help with these teams. Because some of them, a lot of them, have not presented before, don't know the details, don't know-some of them come in and say, 'Oh, for the presentation, I need this because it's in a template. How do I get this?" Int.47.0

"People that have a - not just know the industry, but also they have good linkages with different either clients or different organizations so that they can actually serve as some sort of mentor to help them. That may be fairly informal... So, that's one of the things that a board is supposed to do as well. If we are approving a project, then we try to assign a mentor to the project among the board members." Int.01.0 
M10 Client Engagement in the Initiative (Initiative-Level)

FoaK initiatives are co-creation of innovation projects between the firm and client stakeholders. All FoaK initiatives are required to identify co-creation client candidates for their project, and secure their engagement which in turn is governed by a FoaK legal agreement. FoaK initiative funding is released only upon securing a signed contractual commitment from the client stakeholder.

Clients are believed to play a critical role in validating early-stage invention from the firm's research organization, and are viewed in the program as an essential element in a FoaK initiative. This mechanism is related to the program's role as an orchestrator, and to the initiative team's ability to execute. The effectiveness of a FoaK initiative team with a client stakeholder is related not only to successfully extracting the necessary domain knowledge for the innovation, but also the ability to manage and maintain expectations and engagement throughout the project.

It is believed the client engagement provides asset validation, and helps improve a co-creation of innovation initiative's performance as measured by the program. This is central to the client satisfaction and follow on revenue performance measures of a FoaK initiative.

"Some of this is more client telling us what they need, some of it such identifying some ideas and then sharing with the client. So, in some instance, it may be something exactly they were looking for or maybe something completely new." Int.01.0

"(Clients) definitely see us as a collaborative partner and want to work with us. And some of (our) biggest clients are also collaborative partners, and they definitely see it as one of the perks of the relationship with (our firm). So, it definitely adds value 
to (clients) to be able to work with us on this cutting-edge technology quite frankly, that's why we do get some of them saying. 'I want you to agree not to give this to my competitors,' because they do see a competitive advantage to working with (us), to being the first to use the technology." Int.10.0

"Usually teams come to us already with something they have invented. Then they are trying it out with a client. So sometimes, not often, the products group will say 'wow we did not know we could do that,' or 'this is something outside that our group cannot do.' I guess, from that sense, it is important." Int.02.0

\section{M11 Commercialization Partner Engagement in the Initiative (Initiative-Level)}

As with client engagement, a principal objective for a FoaK initiative is the successful transfer of an innovation asset from the co-creation project to a mainstream product stakeholder portfolio for commercialization. A FoaK initiative team must meet the program requirement of securing a product stakeholder's support for the proposed initiative.

This requirement is related to the program orchestration role, also helping to guide initiative team behaviour towards securing funding. In addition to providing a channel for commercialization, a FoaK initiative team's engagement with a product stakeholder also provides an additional source of experiential guidance. Early engagement provides a FoaK initiative team with insight into the product stakeholder's criteria and considerations for receiving a FoaK innovation into their portfolio. It also presents an opportunity for the product stakeholder organization to participate in the initiative, gaining early knowledge on the innovation, business use cases being addressed, and familiarity with the client stakeholder organization prior to the technology transfer stage of the project. 
This mechanism is also related to an initiative team's ability to execute. FoaK initiative teams require the skills to demonstrate the value of their initiative to a potential product stakeholder organization, set expectations and reach alignment with that team, and manage their engagement throughout the course of the initiative. FoaK initiative teams with stronger leadership and experience are believed to be more successful in establishing effective stakeholder relationships with a product organization than teams that do not demonstrate those attributes.

It is believed that the requirement for a commercialization partner's engagement (e.g., product stakeholders) provides both a channel for commercialization of a FoaK innovation and helps to provide further asset validation from the FoaK initiative. This will contribute to improved initiative performance (i.e., asset transfer, follow-on revenue).

"So, this is a much stronger proposition because here, at the end of the FoaK, you already have a somewhat integrated solution where the asset commercialization owner would not just start from scratch at that point and say, 'Okay, so now how do I align all the resources and fold that into my portfolio? And what other infrastructure components do I have to put together?' The earlier this can be done in the project, the more successful these FoaK projects tend to be is my experience." Int.18.0

"I always try to align FoaK ideas with the industry roadmaps. (Product teams) always set out at the beginning of the year instead of big bets - so things that they're focusing on that would complement their solution blueprint - and if you can align-the big bets are usually things that are in progress. " Int.18.0

"We really we have trouble getting the catcher engaged up front. The catcher normally has a portfolio of assets that they're selling to clients, and they see this as something that's lower on their priority list than active, current services or products that they're selling. So, they look at it as, 'Well, we'll see how the project goes, and then we'll look at it in 6 to 12 months. ", Int.10.0 


\section{M12 Industry and Sales Engagement in the Initiative (Initiative-level)}

Industry and sales stakeholder engagement in a FoaK initiative is also a requirement imposed by the program on initiative teams. Engagement of the stakeholders provides market validation support of the strategic fit of an initiative candidate for the FoaK program, supporting the portfolio approach to initiative management. Industry and sales engagement also help in positioning an initiative with internal firm stakeholders, and in identifying external client interest in the initiative. These stakeholders interact across various processes related to a FoaK initiative, and ultimately serve in providing further validation of a proposed FoaK initiative and co-creation of innovation assets. The support of the sales and industry is believed to improve an initiative team's performance in executing the co-creation project.

"...the sales and the services teams see benefits to clients by doing these projects. Sometimes, they will try to combine an ongoing sales engagement with the FoaK project, and they'll tie them together. " Int.10.0

\section{M13 Gather Learnings and Refine Program (Program-level)}

The program also believes it is necessary to continually gather learnings and refine the program design based on its experience with previously funded initiatives, and also in reflecting changes in the program's context (e.g., changes in program funding, shifts in firm strategic priorities). Gathering and applying learnings will result in modifications to the program design that are expected to improve the proportion of funded FoaK initiatives that succeed as part of the program. 
"We asked the teams, although we don't always get it, we asked the teams for lessons learned especially in their final review meeting, so we can try to incorporate that into our guidance and share that for future teams. " Int.02.0

"I have periodic meetings with the research and with development executives, and (FoaK program management). They are the primary drivers. One area that I think we could improve on is if we engaged representatives from each of our research labs as part of that effort. ... We had a meeting of a FoaK symposium in the spring of this past year. We did get all the players together, and by the players I mean the research members of the different labs all over the world. ... That was very helpful." Int.10.0

These thirteen mechanisms form the basis of a FoaK program theory, representing the principal elements believed to explain observed outcomes related to the FoaK program, FoaK initiatives, and the relationship between both (see Table 7.1). The FoaK program theory presented served as the foundation for the case analysis findings in Stage 1 (i.e., individual case analysis) and Stage 2 (i.e., cross-case analysis). 
Table 7.1 Summary of FOAK Program Theory Mechanisms

MECHANISM

$>$

OUTCOME

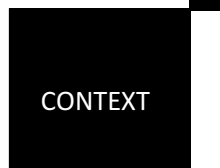

- exploratory co-creation initiatives

- radical "new-to-the-firm" (versus incremental) invention requiring market validation

- research-driven initiative to be transferred to mainstream business

- cross-functional sponsorship and funding

Firm Level Mechanism

\begin{tabular}{|c|c|c|c|}
\hline M1 & Innovate Through a Co-Creation Program & $>$ & $\begin{array}{l}\text { deliver market-relevant scalable innovation } \\
\text { assets for commercialization }\end{array}$ \\
\hline \multicolumn{4}{|c|}{ Program Level Mechanism } \\
\hline M2 & Reduce Barriers to Co-Create Innovation & $>$ & $\begin{array}{l}\text { motivates stakeholder engagement in the } \\
\text { co-creation initiative }\end{array}$ \\
\hline M3 & Manage Initiative Candidates as a Portfolio & $>$ & $\begin{array}{l}\text { improve the proportion of co-creation initiatives } \\
\text { that succeed }\end{array}$ \\
\hline M4 & Program as an Orchestrator & $>$ & $\begin{array}{l}\text { focuses a co-creation initiative's efforts on performance } \\
\text { outcomes }\end{array}$ \\
\hline M5 & Initiative Governance & $>$ & $\begin{array}{l}\text { focuses a co-creation initiative's efforts on performance } \\
\text { outcomes }\end{array}$ \\
\hline M13 & Gather Learnings and Refine Program & $>$ & $\begin{array}{l}\text { modify program design, improving the } \\
\text { proportion of co-creation initiatives that succeed }\end{array}$ \\
\hline \multicolumn{4}{|c|}{ Initiative Level Mechanism } \\
\hline M6 & $\begin{array}{l}\text { Conditions to Participate for } \\
\text { Initiative Candidates }\end{array}$ & $>$ & $\begin{array}{l}\text { focuses a co-creation initiative's efforts on } \\
\text { performance outcomes }\end{array}$ \\
\hline M7 & Initiative Team Ability to Execute & $>$ & improve initiative performance \\
\hline M8 & $\begin{array}{l}\text { Program Directed Guidance to } \\
\text { Initiatives Teams }\end{array}$ & $>$ & $\begin{array}{l}\text { bridges initiative team knowledge gaps improving } \\
\text { execution }\end{array}$ \\
\hline M9 & $\begin{array}{l}\text { Program Experiential Guidance to } \\
\text { Initiative Teams }\end{array}$ & $>$ & $\begin{array}{l}\text { bridges initiative team knowledge gaps improving } \\
\text { execution }\end{array}$ \\
\hline M10 & Client Engagement & $>$ & $\begin{array}{l}\text { provides asset validation, improving initiative } \\
\text { performance }\end{array}$ \\
\hline M11 & Commercialization Partner Engagement & $>$ & $\begin{array}{l}\text { provides asset validation, improving initiative } \\
\text { performance }\end{array}$ \\
\hline M12 & Industry Sales Engagement & $>$ & $\begin{array}{l}\text { provides asset validation, improving initiative } \\
\text { performance }\end{array}$ \\
\hline
\end{tabular}




\section{PART IV \\ RESEARCH FINDINGS AND ANALYSIS}

Part IV presents the analysis and findings for this study. The analysis is presented across four chapters, linking case analysis to knowledge-based dynamic capabilities. Chapter 8 introduces the individual case study analysis, followed by the comparative crosscase analysis in Chapter 9. As the data collection and initial analysis were based on the FoaK program theory, Chapter 10 presents the mapping of the program theory mechanisms to the knowledge-based dynamic capabilities in the knowledge capacity framework (KCF). This then concludes with a review of the principal research findings that emerged from the study. Figure IV.1 illustrates the research and findings roadmap followed in this study.

\section{Chapter 8}

Stage 1 Findings and Analysis - Individual Case Findings

\section{Chapter 9}

Stage 2 Findings and Analysis - Comparative Cross-Case Findings

\section{Chapter 10}

FoaK Program Theory and the Knowledge Capacity Framework

\section{Chapter 11}

Research Findings - Themes 
Figure IV.1- Research Findings and Analysis Roadmap

The findings and analysis for this study were conducted over eights steps, linking the initial conceptual framework and program theory to the final findings-driven updated conceptual framework and research themes. The following is a roadmap of the analysis steps.

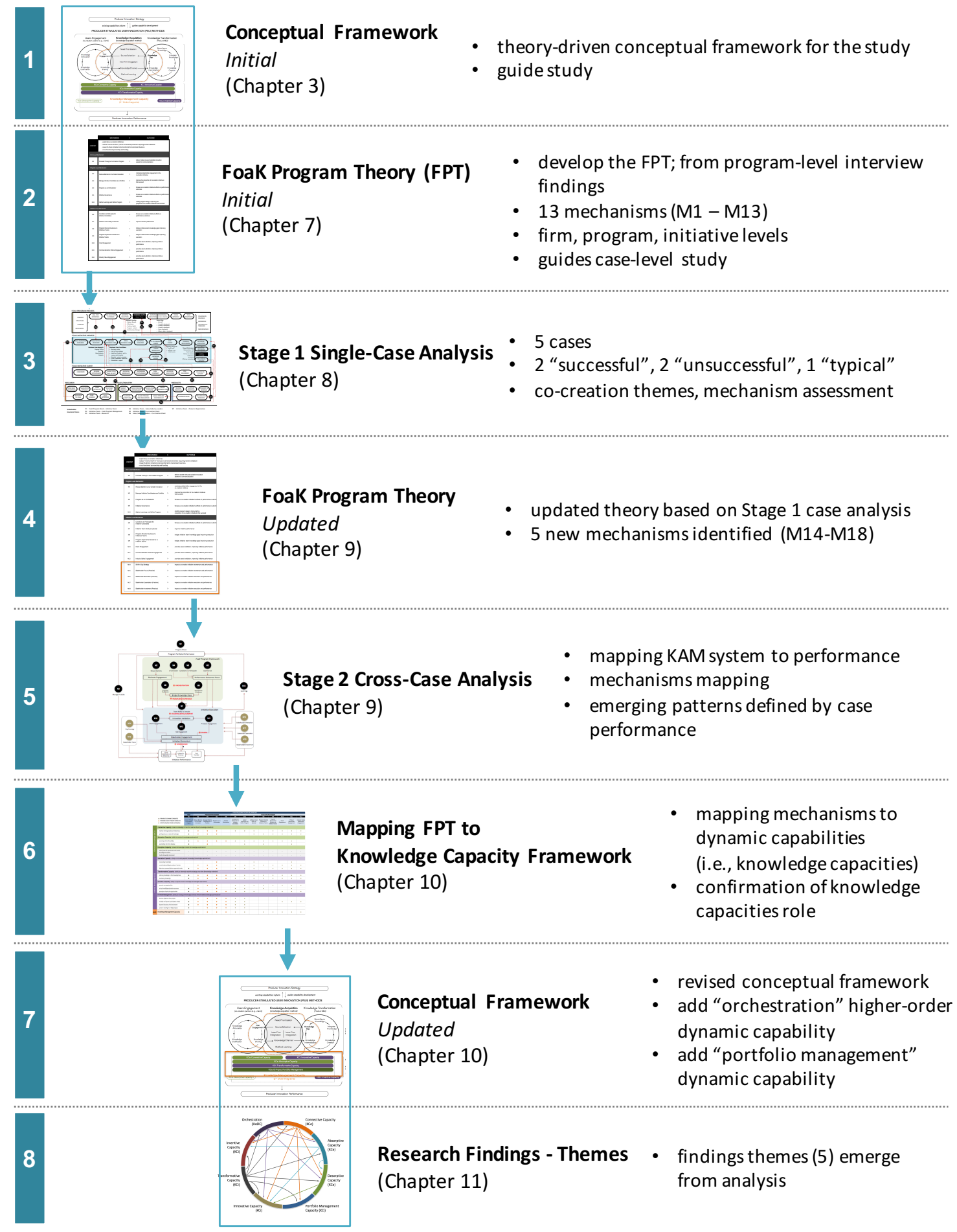




\section{Chapter 8 \\ STAGE 1 FINDINGS AND ANALYSIS \\ INDIVIDUAL CASE FINDINGS}

Case analysis and findings from the study were conducted in two stages. Stage 1

focuses on findings for each of the individual FoaK initiatives included in the study. Five cases (i.e., individual FoaK initiatives) were studied representing "successful," "unsuccessful" and "typical" levels of initiative performance. This section presents findings from each case study, including an examination of FoaK initiative innovation scope, the initiative team profile, the nature of stakeholder relationships observed, and a review of each initiative's execution and performance. Case profiles and a summary of findings is provided in Table 8.1.

\subsection{Case 1 - Analytics for Mining (“Typical” Performance)}

Case 1 in this study examined a FoaK initiative exploring an innovation for the mining industry, which resulted in a "typical" initiative performance. This co-creation initiative explored a visual analytics application aimed at the optimization of operations in the natural resources sector. This initiative was strategic for the firm both in its support of the analytics application agenda and in developing new solutions for the natural resources industry. The FoaK initiative proposal was first approved in May 2013, with the initiative completed in December 2014. The initiative was placed on hold during the process due to a delay in signing the co-creation client. The initiative resulted in a satisfied client who supported the resulting FoaK innovation through a written case study, presentations, and 
Table 8.1 Research Study Case Profiles

\begin{tabular}{|c|c|c|c|c|c|}
\hline & \multirow{2}{*}{$\begin{array}{c}\text { "TYPICAL" INITIATIVE } \\
\text { Case } 1\end{array}$} & \multicolumn{2}{|c|}{ "SUCCESSFUL" INITIATIVE } & \multicolumn{2}{|c|}{ "UNSUCCESSFUL" INITIATIVE } \\
\hline & & Case 2 & Case 3 & Case 4 & Case 5 \\
\hline \multicolumn{6}{|l|}{ FOAK INITIATIVE PROFILE } \\
\hline Industry & $\begin{array}{l}\text { Natural Resources } \\
\text { (Mining) }\end{array}$ & Retail & Automotive & $\begin{array}{c}\text { Travel \& Transportation } \\
\text { (Rail) }\end{array}$ & Healthcare \\
\hline Solution Domain & Analytic Applications & Analytic Applications & Analytic Applications & Analytic Applications & Analytic Applications \\
\hline Offering Summary & $\begin{array}{l}\text { visual analytics solution } \\
\text { developed for the } \\
\text { optimization of mining } \\
\text { operations (scheduling and } \\
\text { equipment preventative } \\
\text { maintenance) }\end{array}$ & $\begin{array}{l}\text { advanced cross-channel } \\
\text { analytics providing unified } \\
\text { key performance } \\
\text { indiciators across all } \\
\text { aspects of customer } \\
\text { interaction }\end{array}$ & $\begin{array}{l}\text { cogntive solution for } \\
\text { connected vehicles offering } \\
\text { advanced mobility services } \\
\text { (automated driving } \\
\text { assistance, accurate map } \\
\text { service) }\end{array}$ & $\begin{array}{l}\text { analytic solution to predict } \\
\text { optimal prices and } \\
\text { schedules for ticket } \\
\text { promotions in travel and } \\
\text { transportation }\end{array}$ & $\begin{array}{l}\text { analytic solution that } \\
\text { creates personalized } \\
\text { treatment plans for } \\
\text { patients, helping treat } \\
\text { cancer and other diseases } \\
\text { more effectively }\end{array}$ \\
\hline $\begin{array}{l}\text { Firm-Level Strategic } \\
\text { Relevance }\end{array}$ & $\begin{array}{c}\text { Yes } \\
\text { Natural Resources Sector } \\
\text { (Mining) } \\
\text { Analytics }\end{array}$ & $\begin{array}{c}\text { Yes } \\
\text { Smarter Commerce } \\
\text { Analytics } \\
\text { Signature Moment }\end{array}$ & $\begin{array}{c}\text { Yes } \\
\text { Internet of Things } \\
\text { (Connected Vehicles) } \\
\text { Analytics } \\
\text { Signature Moment }\end{array}$ & $\begin{array}{c}\text { Yes } \\
\text { Smarter Transportation } \\
\text { Analytics }\end{array}$ & $\begin{array}{c}\text { Yes } \\
\text { Smarter Healthcare } \\
\text { Analytics }\end{array}$ \\
\hline $\begin{array}{l}\text { Dates } \\
\text { (approve/start/complete) }\end{array}$ & $\begin{array}{l}\text { a } 2013.05 \\
\text { s } 2013.06 \\
\text { hold } 2013.09 \\
\text { c } 2014.12 \\
\text { Total: } 19 \text { months }\end{array}$ & $\begin{array}{c}\text { a } 2013.10 \\
\text { s } 2013.10 \\
\text { c } 2015.07 \\
\text { Total: } 22 \text { months }\end{array}$ & $\begin{array}{c}\text { a } 2013.11 \\
\text { s } 2013.12 \\
\text { hold } 2014.04 \\
\text { c } 2016.01 \\
\text { Total: } 26 \text { months }\end{array}$ & $\begin{array}{c}\text { a } 2011.05 \\
\text { s } 2011.08 \\
\text { hold } 2011.11 \\
\text { c } 2013.05 \\
\text { Total: } 24 \text { months }\end{array}$ & $\begin{array}{c}\text { a } 2012.01 \\
\text { s } 2012.02 \\
\text { hold } 2012.05 \\
\text { c } 2013.06 \\
\text { Total: } 18 \text { months }\end{array}$ \\
\hline Firm Co-Creation Investment & $5 \mathrm{FTE} / 12$ months & 5 FTE $/ 12$ months & $6 \mathrm{FTE} / 12$ months & $4 \mathrm{FTE} / 12$ months & $5 \mathrm{FTE} / 12$ months \\
\hline \multicolumn{6}{|l|}{ FOAK INITIATIVE TEAM } \\
\hline Initiative Team Profile & $\begin{array}{c}\text { Middle-East(lab)/ } \\
\text { South America (local) - } \\
\text { first time FoaK project }\end{array}$ & $\begin{array}{l}\text { United States; } \\
\text { experience with the } \\
\text { FoaK process }\end{array}$ & $\begin{array}{l}\text { Asia-Pac; } \\
\text { experienced with the } \\
\text { FoaK process }\end{array}$ & $\begin{array}{l}\text { West Europe (local); } \\
\text { not experienced with } \\
\text { FoaK process }\end{array}$ & $\begin{array}{c}\text { Middle-East(lab)/ } \\
\text { Western Europe (local) - } \\
\text { first time FoaK project } \\
\text { for local team }\end{array}$ \\
\hline $\begin{array}{l}\text { FoaK Initiative Team } \\
\text { Experience }\end{array}$ & Low - Moderate & $\begin{array}{c}\text { High } \\
\text { proactive, strategic }\end{array}$ & $\begin{array}{c}\text { High } \\
\text { proactive, strategic }\end{array}$ & $\begin{array}{l}\text { Low } \\
\text { guidance required }\end{array}$ & $\begin{array}{c}\text { Moderate } \\
\text { guidance required }\end{array}$ \\
\hline $\begin{array}{l}\text { Principal Investigator } \\
\text { FoaK Leadership Skills }\end{array}$ & Moderate & High & High & Low & Low \\
\hline \multicolumn{6}{|l|}{ INITIATIVE STAKEHOLDERS } \\
\hline $\begin{array}{l}\text { Program - Initiative } \\
\text { Engagement }\end{array}$ & $\begin{array}{c}\text { Moderate } \\
\text { (early stages }(\mathrm{H}) ; \\
\text { project/post (L)) }\end{array}$ & \begin{tabular}{|c|} 
High \\
proactive information \\
sharing; exemplar in sharing \\
best practices
\end{tabular} & $\begin{array}{c}\text { High } \\
\text { proactive information } \\
\text { sharing; program mentor } \\
\text { assigned }\end{array}$ & \begin{tabular}{|c|} 
Moderate-Low \\
early stages (M); \\
project/post (L) \\
program pulled information
\end{tabular} & $\begin{array}{l}\text { Moderate-Low } \\
\text { little proactive information; } \\
\text { program pulled information }\end{array}$ \\
\hline Co-Creation Client & $\begin{array}{l}\text { Industry Leader } \\
\text { (multi-national) } \\
\text { South America }\end{array}$ & $\begin{array}{l}\text { Industry Leader } \\
\text { (multi-national) } \\
\text { United States }\end{array}$ & $\begin{array}{c}\text { Industry Leader } \\
\text { (multi-national) } \\
\text { Asia-Pac }\end{array}$ & $\begin{array}{l}\text { National leader } \\
\text { Western Europe }\end{array}$ & $\begin{array}{l}\text { Industry Leader } \\
\text { Western Europe }\end{array}$ \\
\hline $\begin{array}{l}\text { Client Stakeholder } \\
\text { Commitment }\end{array}$ & Moderate - High & High & High & Moderate - Low & Moderate \\
\hline $\begin{array}{l}\text { Product Stakeholder } \\
\text { Commitment }\end{array}$ & Low & High & High & Low & Low \\
\hline $\begin{array}{l}\text { Sales/Industry Stakeholder } \\
\text { Commitment }\end{array}$ & Low & High & High & Low & Low \\
\hline INITIATIVE PERFORMANCE & Moderate & High & High & Low & Low \\
\hline Client Success (Reference) & Yes & Yes & Yes & Yes & Yes \\
\hline Innovation Transferred & No & Yes & Yes & No & No \\
\hline Follow-on Revenue & Active; TBD & Yes & Yes & No & No \\
\hline Program Mgmt Perspective & Typical Initiative & Successful Initiative & Successful Initiative & Unsuccessful Initiative & Unsuccessful Initiative \\
\hline \multicolumn{6}{|l|}{ INITIATIVE MOMENTUM } \\
\hline Post Initiative Momentum & $\begin{array}{l}\text { Low - Moderate } \\
\text { research team reassigned; } \\
\text { asset passed to new owner }\end{array}$ & $\begin{array}{c}\text { High } \\
\text { offering transferred to } \\
\text { business unit }\end{array}$ & $\begin{array}{c}\text { High } \\
\text { offering transferred to } \\
\text { business unit }\end{array}$ & $\begin{array}{c}\text { Low } \\
\text { not transferred to business; } \\
\text { pipeline not converting }\end{array}$ & $\begin{array}{l}\text { Low } \\
\text { not transferred to business; } \\
\text { no active pipeline }\end{array}$ \\
\hline
\end{tabular}


the use of their story in follow-on revenue activities. While transferred to a mainstream product organization upon completion of the initiative, the resulting innovation was found to be successful by both the client and the firm, no follow-on revenue was realized with the client. The innovation asset that emerged from the FoaK initiative was subsequently used in new revenue opportunities which remain active. The resulting innovation from the FoaK initiative was not successfully

\section{Initiative Overview/Offering}

This FoaK initiative explored an innovation for the use of analytics in increasing the safety and efficiency of large machinery operations in the mining industry. The initiative scope proposed a solution using big data and analytics to deliver visual analytic insights in identifying deficiency patterns and anomalies in the operation of large equipment. The innovation explored would present a new market solution relevant to both mining companies and mining equipment manufacturers globally, particularly in growth markets.

The research organization began with the belief that there was an opportunity for innovation that would facilitate organizational decision-making in this industry by providing an integrated view of operations in the planning, mining, and production of mined materials. The innovation that would emerge from this co-creation initiative would allow for real-time assessments of large equipment performance for the first time in an industry that is slow to innovate in these areas. It was believed that innovations providing data visualization, not available in the industry, could be expected to give advantage to adopters. 
The FoaK initiative proposal met all the criteria required by the program to participate. The proposed initiative was strategic in its support of both the analytics and industry agendas for natural resources, and the FoaK initiative team was successful in securing the support of a co-creation client stakeholder, as well as the required internal firm stakeholder groups (i.e., industry sales, product organization, research). The initiative team also secured project funding from the client stakeholder to fund the hiring of a project management resource. The initiative was resourced upon approval with the five full-time employee equivalents, for a project period of 12 months.

\section{Initiative Team Experience}

The FoaK initiative team was led by a research lab based in the Middle East. This team was responsible for the development of the initial invention targeting the business problem described. The primary investigator (PI) who led the FoaK initiative had previous FoaK experience, as did the rest of the initiative team based in the Middle-East lab. The primary investigator was found to demonstrate an understanding of FoaK initiative dynamics and processes, and a moderate level of leadership throughout the initiative. While the PI served the role as the focal point for the initiative, it was found that this role was also shared with the industry stakeholders. The PI was relatively proactive in communicating with the program and demonstrated a degree of strategic foresight in managing the initiative and its stakeholders.

This FoaK initiative project was executed at the client site based in South America. This resulted in the research lab in the Middle East partnering with a supporting research lab based locally in the client stakeholder's country. The firm's South American FoaK team 
members were new to the program, with this being the first FoaK initiative in the region. There was a learning period required by the South America research team to become familiar with the program requirements and processes. The primary role of the supporting research lab was to assist in bridging the language and cultural requirements with the client stakeholder, to help ensure engagement throughout the initiative.

Overall, the FoaK initiative team chemistry was described as strong throughout the project, including effective coordination and communication across geographies. In addition to the local representation of the FoaK initiative team with the client, the MiddleEast team also travelled to participate in face-to-face working sessions with the client. This further contributed to developing a strong working relationship with the client team.

"I think we have a very good team in place...They (were) motivated, willing to go to their minds and have their feet and hands dirty with the problem." Int.19.1

"Everyone was very... happy to work together. We enjoy the work... For me, it was a pleasure to work with this team. Nowadays, I think I have new friends, not colleagues." Int.13.1

\section{Stakeholder Relationships}

Stakeholder relationships across this FoaK initiative demonstrated varying levels of alignment and engagement. The cross-lab FoaK initiative team was found to be effective in their collaboration, as was their engagement with the client stakeholder, and the support of the industry stakeholder throughout the initiative. However, the engagement of the FoaK initiative team with the product stakeholder (i.e., FoaK asset commercialization owner) was not found to be well aligned, within minimal engagement noted during the project. The lower level of engagement between these two stakeholders was found to contribute to 
the FoaK innovation not being transferred into the product stakeholder portfolio for further development and commercialization.

Regarding product stakeholder engagement during the project: "They're always very busy, they always have their-- whether it's the offering or the product manager what used to be before and now Offering Managers. They never really-- they were never involved in the actual projects and this is I think a part of the reason that we had challenges later on doing technology transfers is because of that." Int.08.1

"We included them in the quarterly review that we had with the FoaK board. We invited them to the workshops. We shared information with them. I don't think there was any time where they asked us something that we didn't provide the information to them, but it's really more of a push than a pull." Int.08.1

Unclear expectations: "It turns out that the asset commercialization owner seemed to want a follow-on client before they really move into transferring the assets. So, it's kind of in this holding pattern now, as far as I know." Int03.1

\section{Program - Initiative Relationship (Guidance, Governance, Communication)}

The relationship between the FoaK initiative team and the FoaK program demonstrated a moderate level of engagement throughout the course of the project. Early in the FoaK initiative, the PI and lead research team worked closely with the FoaK program management team in developing the initiative proposal. The initiative team was found to leverage the assets and guidance provided within the FoaK framework. However, there were issues encountered in the client signing of the FoaK agreement, in part attributed to the inexperience of the local FoaK team. The FoaK initiative team worked closely with the FoaK program legal team to help navigate the process and resolve issues during the interchange with the client stakeholder. The FoaK program knowledge base was also 
described as having helped bridge the knowledge gap with the novice research team members working locally with the client.

The FoaK initiative team satisfied the standard governance checkpoint meetings throughout the project and provided information to the program team as requested throughout the project. In some instances, the industry stakeholders provided information to the program on behalf of the FoaK initiative team, on topics within their domain requiring status or clarifications. Although experienced and engaged, the FoaK initiative team was not found to be proactive in communicating progress or issues with the program. This required the FoaK program management to regularly ask for updates on the initiative. This was observed through a review of the communications between the program office and the initiative team over the course of the project.

\section{Initiative - Sales and Industry Strategy Relationship}

An examination of the sales and industry stakeholder teams were both found to be motivated in supporting this FoaK initiative. The innovation explored was in an area identified as strategic for the mining industry and natural resources sector, and these stakeholders had a vested interest in initiatives aimed at strengthening the firm's presence in these markets. This FoaK initiative was expected to contribute to establishing a stronger presence for the firm in the mining industry, and in supporting future sales revenue development for the firm.

"You know this is a hot area... Natural resources was one of the things (research) had identified in one of their [market trend reports], and so I think the board was excited to see something in that area." Int.03.1 
The initiative team and sales and industry stakeholders' relationship was found to be effective throughout the FoaK process. The industry team worked closely with the research organization in identifying candidate domain areas for innovation, supported the development of the FoaK proposal, and played a significant role in recruiting an internal product stakeholder to commit as the asset commercialization owner (ACO).

In addition to the industry stakeholder support, the firm's local South American sales and business development engagement also played an important role in the recruitment of a co-creation client stakeholder. The firm's business development focal point was new to the company and the FoaK program, requiring time to familiarize himself with the FoaK objectives and requirements.

\section{Initiative - Client Relationship}

The client stakeholder for this FoaK initiative was identified in collaboration with the sales and industry stakeholders from a group of global candidates. The selected client stakeholder represented leadership in their industry, with operations worldwide. In addition to their market position, the FoaK initiative team also believed that the client stakeholder represented the best option for access to the type business data that would be required in exploring the proposed innovation. The client's interest in participating as a co-creator was motivated by the firm's investment in the initiative, mitigating their own risk and investment. It was also stated that client interest in the opportunity was to work with the firm in solving a problem currently unaddressed in their organization and industry, the outcome of which they believed could improve their competitive position. 
The client stakeholder was also described as conservative in their approach to this type of partnering engagement. This was in part explained by the profile of companies in the mining industry, characterized as organizations that are slow to innovate.

"There are very few mining companies that have the innovative approach... They are mining companies that are very, I would say, operational...They would get innovation only when it is mature and proven in the market, so they are late adopters, not early adopters." Int.48.1

The client's conservative nature was experienced in the FoaK contract review of standard terms and the signing of the agreement. The FoaK program described that the client appeared unfamiliar with how to engage in these types of initiatives, which contributed to the delays. The FoaK contract process was drawn out, resulting in the initiative being placed on hold until the contract was signed, adding seven months to the original plan. Also, contributing to the delay was language differences between the South American and American legal teams, which required additional time to allow for translation and interpretation of the terms of the agreement.

"...and especially for mining companies, sometimes their own procurement do not know what the legal process is and how to structure innovation contracts." Int.48.1

FoaK initiative team effectiveness in setting client expectations was also found to contribute to project delays. Through the FoaK contract review process, it was apparent that the client's initial understanding of the FoaK initiative and the expectations of their organization was unclear. This was in part believed to be due to how the initiative was first positioned by the local FoaK sales team with the client. The learning curves on both sides of the discussions required additional effort in ensuring that expectations were clear. As a 
result, the FoaK program contract served as a common focal point to clarify scope and terms for this initiative.

Once the FoaK initiative contract was signed, the client was found to be very open and engaged with the initiative team throughout the duration of the project. The client assigned an internal sponsor who was motivated in participating in the co-creation initiative. The client was described as responsive, accessible, and engaged throughout the process. It was highlighted that the client relationship, as with the cross-lab research teams, demonstrated a positive "team chemistry" with the FoaK initiative team.

"We were very lucky to find a guy in the client that was very passionate about the business and had the quality that (to get his hands dirty)... He worked for the client (and was) motivated." Int.19.1

"in this particular case, the team had great chemistry, so everything worked well." Int.48.1

"I think we did a good job. I think really everything went well." Int.13.1

The FoaK initiative team worked towards providing the client with frequent milestones during the exploration, showing progress throughout the FoaK initiative. The client organization expressed the value of the innovation created through the FoaK initiative, demonstrating it in practice.

"(early proof points) in this is critical, both externally to the client and internally because the FoaK reviews are quarterly and you need to be able to go back to the FoaK board and say, 'This is what I achieved." Int.08.1

During the initiative, two additional challenges were experienced that were found to impact the adoption FoaK initiative asset by the client at the end of the project. First, it 
was reported that there was a level of internal conflict and misalignment within the client's business and IT organizations when examining whether to invest further in the initiative. The client stakeholder's IT organization expressed that next steps with the solution could be delivered by internally developing a similar offering to fit within a limited budget. The FoaK initiative and the client stakeholder also faced increasing market pressure as the mining industry continued to experience a market downturn. This transformation in the client's industry presented new competitive and budgetary pressures on their company.

"I think in this one the external economic climate was a big factor. I know that things are tough in the mining industry right now, and I just think that they had this momentum with (the client, and their) business got tougher and they couldn't really continue, and I think that just knocked it off the rail." Int.03.1

"There was some economic issues. Macroeconomic issues that I think prevailed that, I think, weren't present when we first started there. That probably really-- how the outcome turned out." Int 43.1

\section{Initiative - Products (ACO) Relationship}

The FoaK initiative team identified a product stakeholder organization that agreed to support the project proposal. However, the relationship with this product stakeholder was found to show their low commitment to the initiative. The product stakeholder was part of a newly formed division within the firm, focused specifically on the development of analytic solutions for industrial specific agendas in the market. As a new business, this product organization was in its early stages of development, both in defining their own product roadmap and securing resources for their organization. Of interest to this product 
stakeholder at this point in their evolution was the successful release of new products to the market and demonstrating revenue generating the potential for the firm.

The product stakeholder was approached both by the industry stakeholders and the FoaK initiative team to discuss their potential role as an ACO for the FoaK initiative asset. The product stakeholder acknowledged seeing potential fit of the initiative to their strategic direction. They also expressed their interest in gaining access to the co-creation client that was also on their target market list.

The product stakeholder involvement throughout the course of the initiative was limited. They provided the requisite support and endorsement needed by the FoaK initiative team in the proposal stage of the process, and attended the quarterly review meetings. There was no involvement during the FoaK initiative execution with the client stakeholder, and there was no engagement with the FoaK initiative team beyond the mandatory update checkpoints with the FoaK board.

"(The product team is) always very busy...They were never involved in the actual projects and this is I think a part of the reason that we had challenges later on doing technology transfers." Int.08.1

"If we had collaboration from the product team from day one, our lives would have been simpler. It would have been very valuable, I think for (them) to be there and listen to this. And so that the work that we had to do post-project, which was to figure out how to align the technical architecture and strategy, on top of the product would have been simpler." Int.08.1

"There was no relationship (between our team and the initiative)." Int.43.1

At the completion of the FoaK initiative, the product stakeholder organization did not accept the asset into their portfolio. They communicated to the FoaK initiative team 
that to accept the asset there would need to be further business justification, demonstrated revenue pipeline potential for the asset, and access to the client stakeholder. As the product stakeholder did not participate during the execution of the initiative, it did not gather any technical knowledge of the innovation asset or what would be required for the commercialization of the asset as part of their future portfolio. Thus, the assets did not successfully transfer to the product stakeholder portfolio, and subsequently were moved to be managed by the South American research lab moving forward.

"No (we were not clear on the criteria at the start of the project). The criteria they shared with us was exactly what they told me about. They told us that they need to have more accurate information (from) the marketing team." Int.13.1

"It turn(ed) out that the asset commercialization owner seemed to want a follow-on client before they would really move into transferring the assets." Int.03.1

\section{Initiative Execution and Performance}

Overall, the execution of the FoaK initiative was described by the FoaK program team as a "typical" initiative. The FoaK initiative team was relatively well engaged in seeking guidance and support in the creation of its FoaK proposal. They worked closely with their industry and sales stakeholders, as well as with the FoaK program management team, in refining their proposal for funding.

Based on the FoaK program measures of performance, this initiative was found to partially meet expectations. A review of the initiative demonstrates client satisfaction in the resulting innovation from the project. The initiative resulted in the client stakeholder endorsement of the innovation asset produced, and provided initial follow-on revenue for 
project management services. The innovation asset outcomes also demonstrated effectiveness in addressing the targeted business use case, which translated into new follow-on revenue opportunities for the sales of industry stakeholders. However, the FoaK initiative asset was not successful either in transferring into the product stakeholder portfolio for commercialization, or in proceeding with the co-creation client post the initiative.

The FoaK initiative team demonstrated diligence in the development of the proposal and meeting the criteria to participate. However, based on a review of the approach taken in managing the process, the FoaK initiative team's execution of the initiative can be described as "tactical" in nature. The team focus was found to be on what was needed to ensure program requirements were met. The FoaK team's execution was not found to place emphasis on anticipating what would be required to ensure successful asset transfer and commercialization or client stakeholder adoption of the FoaK initiative innovation. The combination of the tactical FoaK initiative leadership and the learning curve of the extended FoaK research team in South America contributed to delays in the FoaK process needed to sign the client and release funding. Similarly, the FoaK initiative team's inexperience and leadership approach, in part, contributed to the marginal relationship formed with the products stakeholder with whom expectations and commitment were not adequately secured.

The resulting impact on FoaK initiative performance was reflected in the lower levels of commitment both on the client side and the product side of the FoaK initiative. While the relationship with the co-creation client was positive and productive in developing the FoaK innovation asset, the client was not prepared to invest further in 
bringing the asset in-house or production use. The budgetary pressures resulted in the client declining a bid to continue working together outside of the scope of a FoaK initiative with the asset. Similarly, unclear expectations between the FoaK initiative team and the product organization resulted in the asset not being transferred upon completion of initiative and demonstrated value. This was considering the growing pipeline of new sales opportunities based on the value demonstrated by the asset during the FoaK initiative.

There were three contextual considerations that were also found to influence the FoaK performance measures for this initiative. First, the inexperience of the local FoaK team was believed to contribute in part to poorly set expectations with the co-creation client stakeholder initially, which resulted in execution delays. Second, the industry pressures faced by the co-creation client stakeholder appeared to have an impact on the potential for the client to generate follow-on revenue upon successful completion of the FoaK initiative. Finally, the product stakeholder that was recruited as the asset commercialization owner was itself in the early days of its own formation. Uncertainty in the product stakeholder's own roadmap contributed to shortened windows of opportunity to participate in their strategy. This presented an early warning on the viability of transferring assets from the FoaK initiative into the product stakeholder portfolio. Further complexity was introduced by the lack of clarity between the two teams on the conditions upon which the product stakeholder would assume the asset at the end of the initiative. There is also uncertainty on the degree to which the product stakeholder motivation was driven by the desire to generate new sales revenue with the client stakeholder versus support the new innovation being developed. This further highlights the gap between expectations and practice in the client stakeholder relationship. 


\subsection{Case 2 - Analytics for Retail ("Successful” Performance)}

Case 2 examines a "successful" FoaK initiative exploring an analytic application for the retail industry. This initiative focused on innovation in advanced cross-channel analytics providing a unified set of key performance indicators across customer interactions in a retail business. This initiative was first approved in October 2013, with its completion in July 2015 after two additional rounds of funding and development with the co-creation client. This initiative was characterized by the careful management of its stakeholder relationships, its effective execution, and steady development and maintenance of initiative momentum.

As a FoaK initiative, this project has been referred to as the "gold standard" in all aspects of its planning, stakeholder alignment, and execution. This FoaK initiative was successful across all of the program's measures of performance. It was successful in demonstrating client satisfaction, delivering a referenceable asset and follow-on revenue from the client stakeholder. Additional follow-on revenue was also secured in two new accounts adopting the FoaK initiative assets. The FoaK innovation asset was also successfully transferred to a mainstream product stakeholder portfolio for commercialization.

\section{Initiative Overview/Offering}

The increasing pressure on traditional retailers from online competitors was introducing pressures for change to business models that are more responsive to customer demand while managing operating costs and complexity. The FoaK initial proposal describes that the response from traditional retailers required solutions to help move 
towards an increasingly agile customer-centric response to fulfilling demand while managing costs more effectively. This FoaK initiative explored an innovation for effective decision-making in inventory management for the retail. The innovation would introduce the integration and enrichment of multiple sources of customer data to deliver insights from customer trends and preferences.

The proposed FoaK innovation introduced the application of advanced analytics that would allow traditional retailers to move away from marketing and merchandise into single channels, towards a cross-channel view of the business. The advanced analytics innovation would focus on addressing these business needs using various sources of customer-centric data, situational assessment, root cause analysis, demand analysis, merchandizing and marketing optimization. The FoaK initiative team presented that this innovation would be the first in the industry to deliver a cost-effective method to provide retailers with a complete view of promotional activity across multiple channels. In addition to the retail sector, the proposed innovation would also have applicability across industries including travel and transportation, banking, consumer products and other multi-channel promotion product optimization use cases.

The proposal for this FoaK initiative included a limited set of potential business use cases for the market problem to be explored. Their approach would include using components from the existing firm portfolio as building blocks for an innovation based on requirements definition with industry domain experts and the client stakeholder.

The initiative team was found to effectively manage the early stages of the FoaK proposal preparation. Significant effort was invested before the FoaK proposal in preliminary requirements gathering, identification of potential scope for the initiative, and 
in enlisting stakeholder engagement with the products organization, sales, and industry stakeholder leaders. The FoaK initiative team met all the FoaK criteria to participate in the program. They were found to be proactive in identifying a product stakeholder (i.e., asset commercialization owner) before submission of their proposal, and submitted a proposal that supported the firm's strategic agendas in analytics and "Smarter Commerce." Upon approval, the FoaK initiative was assigned five full-time employees equivalents for a project period of twelve months. The FoaK initiative's steady momentum and success during its execution resulted in two additional rounds of investment from the FoaK program, supporting further development of the assets with the client stakeholder.

\section{Initiative Team Experience}

A United States-based research lab working with a domestic client stakeholder executed this FoaK initiative. The FoaK initiative team included members with previous experience in FoaK projects, as well as members new to the team and FoaK. The primary investigator (PI) and the initiative development leader had previously worked as a team in successfully delivering earlier FoaK initiatives. At the time of interview, the PI stated success in previously delivering four FoaK initiatives.

The FoaK initiative delivered by this team was consistently described by its effective execution. Of note were comments highlighting the strategic approach taken by the PI in managing the initiative. The PI was described as proactive in anticipating the requirements and support needed for the initiative to be successful, and working to secure and align stakeholders across the sales, industry, products, and client organizations. This 
FoaK initiative team was also proactive in communicating progress with the FoaK program management team, and promoting milestones and momentum across its stakeholder group.

"(The FoaK initiative team was) I would say tightly knit, well-coordinated, well managed, tightly managed (by the FoaK initiative leaders) as well." Int.29.2

The PI and initiative development manager were found to demonstrate strong business acumen throughout the execution of the initiative, and leadership in managing the communication and agenda throughout the project. One informant described this team as positively "aggressive" in engaging senior executives in the process and keeping them informed throughout the initiative. The FoaK initiative PI's experience, leadership, and strategic perspective were highlighted as contributing to the strong alignment across stakeholders for this project.

"I think a lot of it had to do with personality. Some of the teams that are doing these things are more shy and some are more aggressive. I would say (the PI) leaned on the more aggressively engaged side of the spectrum, so he was definitely constantly engaging myself or engaging people in the (products) team to get feedback or to get help on different aspects if they might need some feedback or like you needed some technical assistance with." Int.42.2

Finally, this FoaK initiative team was found to consistently demonstrate the proactive management of both external and internal stakeholders throughout the course of defining use cases. In the early stages of the initiative, this team invested in use case definition with the products, sales, and industry stakeholders, and in early workshops with the client stakeholder. The use case definition was done in advance of any investment in the technical development of the proposed innovation, and before engaging the co-creation client stakeholder. As part of this early proposal preparatory work, the FoaK initiative team 
also invested in understanding the product organization's strategic direction, priorities, and technical considerations necessary for them to serve as the asset commercialization owner. This foresight in defining the use case value to the market and the FoaK initiative stakeholders, as well as examining considerations for successful technology transfer to the product stakeholder portfolio, both contributed to clarity in stakeholder alignment as observed for this initiative.

“(The PI) strength was really great in making this project a success, I would say. He went to a lot of, maybe most of, if not all of the customer meetings we had... He was really good at conveying the ideas to the business people or all the different parties. And keeping things on track, I would say. " Int.38.2

"(Our FoaK leadership team), especially in the early stages of (the initiative, spent) lots of time at the client site. So, that willingness to travel, stay away from the family. Because it's a lot of commitment, right? And spent a lot of time at the client site, to just get a few hours from (the client) to get their input. That was important I would say. And also, depending on how you look at it, is being able to get the agreement from multiple stakeholders." Int.16.2

\section{Stakeholder Relationships}

The strength of its stakeholder relationships, and the initiative team's management of stakeholder expectations, alignment, and engagement throughout the project characterize this FoaK initiative.

Program - Initiative Relationship (Guidance, Governance, Communication)

The FoaK initiative and program management teams were found to maintain an active working relationship throughout the project. The FoaK initiative team proactively 
worked with the program in setting the initial scope of their proposal, preparing their proposal for the board, and in meeting the conditions necessary for funding approval. Throughout the process, this FoaK initiative was clearly led by the PI and the development manager serving as the only focal points for the project. The FoaK initiative team sought guidance and provided progress updates to the board throughout the project. They also delivered the necessary progress updates and were responsive to requests for information by the program. The team was also found to maintain communication with the program team between formal checkpoints, to ensure awareness of progress in any milestones achieved along the way.

"FoaK is definitely the enabler of this, without FoaK program, we won't be here. I've always considered FoaK as the source of innovation, and have always appreciated the support of FoaK board." FoaK Initiative Team Lead Note to FoaK program

The FoaK initiative team established a working partnership with the program team. While working with the client stakeholder in defining use cases, the FoaK initiative team included a member of the FoaK program team to participate in the on-site sessions. This engagement with the program provided greater visibility into the initiative for the program management team. This level of access provided context for the FoaK program team, which was found to contribute to their confidence favourable support for the FoaK initiative team and its execution.

The FoaK initiative team leadership also demonstrated their expertise in setting expectations with the FoaK board for funding and timing. While other teams examined were found to comply with the guidelines as presented by the FoaK program, this team in the early stages insisted on flexibility for the signing window with the client stakeholder, 
and the funding period for the project. They argued to the board that securing clients for FoaK initiatives is a complex interaction and that three months would not be sufficient time to complete the process. While this team ultimately was required to align with the funding and timing conditions of the program, their early resistance was well founded and represented a recurring issue that was across all the remaining cases in the study. This FoaK initiative represents the only project of the five case studies where the client stakeholder signing was achieved within the time allotted by the program.

Initiative - Sales and Industry Strategy Relationship

The FoaK initiative team developed and maintained a strong working relationship with their sales and industry stakeholders for this project. The FoaK initiative leadership worked directly with sales and industry stakeholders in identifying candidate use cases for the proposal and developed a potential list of clients as co-creators of the initiative. The eventual selection of the co-creation client stakeholder in part was based on a pre-existing relationship with the sales stakeholder. The strong client stakeholder relationship contributed to accelerating the signing of the client within the allotted time window, and in providing the necessary access to the client organization for effective execution of the FoaK initiative. The sales and industry stakeholders helped in managing the client stakeholder relationship throughout the initiative.

"In this case (sales stakeholders) understood the program very well, and they know somehow the client's problems, "Hey, this client's perfect client. You should work this. Come to talk to my clients." So, without that call this wouldn't happen, I don't think. So, it was the right client and the right setting and call at the beginning." Int. 32.2 
As proof points began to emerge from the project, they then started building sales pipeline for the emerging FoaK innovation. Sales and industry stakeholder cultivation of the new sales opportunities was further supported by the FoaK initiative leadership team, who provided technical expertise on the FoaK innovation during sales cycles with potential clients.

Initiative - Client Relationship

This FoaK initiative was further characterized by the strength of its relationship with the co-creation client, which was described as the "right client" with a "flair for innovation." The FoaK initiative successfully secured client commitment for the signing of the FoaK contract within the three-month deadline, in part due to the strong relationship with the IBM research organization, pre-existing relationship with the sales stakeholder, and between the legal teams of the two firms.

"This signed very quickly because (the Client) and IBM research have great relationship." 3 Month Review Note

The client stakeholder played an active role in defining the scope for the co-creation initiative, which included prioritizing and developing market use cases previously captured by the FoaK initiative team. It was through collaborative working sessions with the client stakeholder that the candidate use cases were narrowed down to FoaK initiative scope.

The client stakeholder provided a high-level of access and engagement throughout the project, which included workspace and resources on their site for the FoaK initiative team. The client assigned a focal point within their organization to work directly with the FoaK initiative team. This individual helped to navigate the organization during the 
initiative and helped to manage client pressures on the initiative scope towards avoiding "scope creep" and maintaining focus with the project.

"(The client stakeholder) assigned somebody from his team to be more of the dayto-day, and he was actually great too. And was the rising star there as well, younger, and so he gave us somebody who was really good and did remain pretty consistently involved, which is nice." Int.29.2

The client had dedicated resources to the initiative, and client executives also invested in corporate visits to the IBM Labs for workshops reviewing the FoaK solution scope. The client stakeholder also worked with the initiative team in identifying major milestones that would help test and highlight the value of the FoaK initiative innovation, planning a test of the innovation during the American "Black Friday" sales season.

"The IBM Think workshop was fantastic, as has been the whole FoaK engagement. Our team came away completely energized by what has already been done as well as the possibilities for (our) business. Thanks for getting us into the program." Client Executive Stakeholder FoaK Workshop Note

The relationship between the FoaK initiative team and the client stakeholder was also characterized by the proactive management of client expectations throughout the project. The FoaK initiative team leadership was found to anticipate potential issues during the engagement with the client, and proactively set out to manage and remediate any potential issues that could negatively impact the forward momentum of the initiative.

"Our team has done a really good job of communicating ... and working through crises. (Our FoaK leaders) were really proactive about that, actually... So, we had an issue when we were trying to get (the client) to sign a contract, to try and get them to accept that it provided value. And at that point, it became a challenge because we had to convince not just the people we've been working with but other lines of 
business. (We had a crisis) situation for us, but (our leadership) were really proactive about going on-site and talking to the leadership in IT and also the leadership in logistics, and trying to figure out how to work things out. I think if they hadn't done that, it wouldn't have been successful. We wouldn't have gotten the contract signed." Int. 38.2

The productive relationship that was developed between the FoaK initiative team and the client stakeholder, combined with the demonstrated value of the FoaK co-creation innovation resulted in further investment by the client stakeholder in the implementation of the FoaK initiative scope as a production system within their organization. In addition to this follow-on revenue, the client stakeholder also served as a public reference for the FoaK partnership and the innovation that emerged from the initiative.

At the completion of the FoaK initiative, the transfer of the initiative assets to the product stakeholder portfolio also required the transition of the relationship with the client stakeholder from the project to the product organization. Two things were observed at this point in the process. First, the product stakeholder was not fully prepared to receive the asset for commercialization, requiring ongoing knowledge transfer support for the product's development team from the FoaK initiative team. Second, the client was not found to differentiate between the relationship with the FoaK initiative team and the firm. After transferring the FoaK innovation asset to the products organization, the client continued to contact the FoaK initiative development leader with any questions and issues, as this was the trust relationship established during the project. To help maintain client stakeholder confidence, additional time was required to manage the smooth transition of the client relationship between teams within the firm. 
"(After the transfer of the asset to our product team, the client stakeholder) still see it as the (FoaK) founders being in charge. I don't mind that so much right now, but that is the fact of the matter." Int.37.2

Initiative - Products (ACO) Relationship

The relationship between the FoaK initiative team and the products stakeholder was also found to be well managed. The FoaK initiative PI had an established relationship with the products stakeholder before the FoaK initiative. The PI was familiar with the organization, its leadership, and their strategic direction. As a starting point, the FoaK initiative team began the process of defining the potential scope for the initiative in conjunction with the products stakeholder.

"(Our product team was) pretty plugged in and really did a good job. I would say it was probably about monthly we would get an update of where (the FoaK initiative) were and often in the form of a demonstration. So, there was regular feedback from the (FoaK) research team, again, getting advice and feedback from us and also from the offering management team as we progressed more on what would we harvest and how would we harvest it? So, it was fairly rigorous feedback with the FoaK team to just make sure we not out of sync." Int.42.2

Time was invested by the PI early in the process in understanding the product stakeholder's portfolio priorities and technical considerations. Work was also done in the pre-proposal stage of the FoaK initiative in identifying potential use cases for the project, an assessing where candidate initiatives could fit strategically into the product organization's market and portfolio strategy.

The product stakeholder remained engaged throughout all stages of the FoaK initiative proposal. During the initiative, the FoaK team had "informal but full access" to 
the product stakeholder development organization. Their early and close involvement with the initiative allowed the product stakeholder to also act as an advisor to the FoaK initiative team on considerations related to the technology transfer at the end of the project. As such, the product stakeholder organization as the asset commercialization owner developed its knowledge in the offering scope and design. They also benefitted from direct exposure to the application of the FoaK innovation in the client environment.

"I think the (relationship between our product team and the FoaK initiative team) was fairly informal... I think the rigour was in reviews and regular communications. It was definitely contacts between the teams that were regularly exercised. I don't remember us assigning a specific team. I'm sure we did assign a full-time member to the (FoaK) project. But in a more informal level, they had pretty open access to the development team." Int.42.2

While the active product stakeholder engagement was found to contribute to the FoaK initiative's successful performance, it was also found that the product organization was not ready to receive the asset into their portfolio. At the time of transfer, further discussions were required to secure development resources that initially had been committed to the commercialization of the asset. This was resolved by resources being reassigned from another offering in the portfolio to the FoaK innovation asset. Similarly, the product stakeholder's development team did not have sufficient knowledge of the assets at the time of transfer. This required that the FoaK initiative researchers remain connected with the product development organization to further support knowledge transfer for the asset. This requirement did not appear to be identified as part of a technology transfer plan. It was stated that more involvement of the product stakeholder team during the initiative would have helped better prepare them to receive the assets at the end of the project. 
"I think a big part of (receiving the FoaK asset) is setting the right expectations... I think that is one of the things that's really challenging to a FoaK. Research does not typically produce product-level code, so the team catching this stuff really has to understand what they're going to get out of it. They're going to get out of it hopefully some really good thinking, good algorithms, and good approaches better understanding of the market, better understanding of the art of the possible. But they're going to still need to make a true investment, beyond some executive sponsoring it, to harvest this stuff." Int. 42.2

"It ends up taking a lot of our research team time in order to support (the products team) and help bring them up to speed on everything...As it is going now, we meant to hand it off earlier, and then we still had engagement with the customer, and that was supposed to be taken over by the product team...In the end, they needed to pull research in to help with all those things... We got tied into this maybe more than we expected or more than we wanted originally. It wasn't just like a clean hand-off or something like that." Int.38.2

"(The FoaK initiative has) produced a little tree that's growing kind of crooked and isn't properly watered. And now (our products team has) to go bring it a bunch of landscapers to feed it, and have it grow up into a proper tree." Int.37.2

Initiative Execution and Performance

The initiative delivered against all FoaK program requirements and achieved all its project plan milestones. Overall, the initiative was found to be a "successful" FoaK initiative, exceeding on all its performance measures. Its client stakeholder expressed satisfaction both through its feedback to the program and through the follow-on support and investment in the asset post-initiative. The success of the co-creation assets that emerged from the initiative resulted in follow-on signings with new client accounts. The successful execution of the initiative along with the momentum supporting the FoaK 
innovation resulted in two additional rounds of funding from the FoaK program to further develop the asset.

"Congratulations! I'm so happy to see all of your hard work paying off. Right from the start when (the client) signed this during their busy season in December, this project has consistently exceeded expectations -- and that's due to your amazing leadership and your outstanding team." FoaK program management to the FoaK Initiative Team, July 2016

The asset that emerged from the FoaK initiative was also successfully transferred to a mainstream products portfolio for commercialization. The FoaK initiative team described working towards fast turnaround on project milestones, recognizing the client window of opportunity and need to show progress to stakeholders within the client organization. The momentum and value demonstrated by the FoaK initiative, along with the involvement of the product stakeholder throughout the project, each supported the final transfer of the assets. It was also found that the successful performance of this initiative was attributed in part to the effective execution of the project by the FoaK initiative team leadership. This initiative's leadership was characterized by the level of engagement and alignment achieved with FoaK initiative stakeholders, and the management of initiative momentum.

"There is a lot more to do, naturally, after the launch of a new offering, but this is a big milestone, so please take a moment to appreciate the significance of the accomplishment you have all contributed to... This offering has gone from a First of a Kind engagement with a client to being a real SaaS offering with a strong product development team behind it." Product stakeholder Executive Transfer Announcement, September 2016 
"Awesome news, thank you to everyone for your vision and dedication. I strongly believe that we will look back at this moment in a few years and realize how it was truly the beginning of something very big. "Industry Stakeholder Executive on News of Asset Transfer Executive, September 2016

Leadership - The effectiveness of the FoaK initiative team was attributed to the strength of its leadership. The pairing of the primary investigator and the development manager for the initiative represented previous FoaK experience, both technical and business acumen, and a strategic approach to planning and executing the FoaK initiative. From the outset, attention was invested in understanding the market value and product stakeholder priorities when establishing the scope of the FoaK initiative proposal. The FoaK initiative team understood that for the initiative to be successful, they would need to understand the value sought by all their stakeholder groups. Throughout the initiative, the FoaK initiative leadership team demonstrated a strategic perspective guiding their priorities throughout the execution of the project. Towards the end of this FoaK initiative, the same leadership team had submitted a new proposal for funding by the FoaK program. As the FoaK program expressed interest in the new proposal and intent to fund, the initiative team negotiated that a portion of the new funds be reassigned to the Retail initiative to help further develop the assets while access to the co-creation client was still available.

Engagement - The FoaK initiative team demonstrated a high level of engagement with all FoaK stakeholders, beyond the technical execution of the project. The FoaK initiative team remained engaged closely with the FoaK program management office throughout the initiative. They leveraged the program framework and assets in engaging the co-creation client. The initiative team also worked directly across all stakeholder groups in assessing 
market opportunity for their FoaK initiative use cases, which they prioritized further in collaboration with their product and client stakeholders. All stakeholder relationships for this initiative were managed proactively by the FoaK initiative leadership team, with a focus on the strategic outcomes for the co-creation innovation.

Stakeholder Alignment - The FoaK initiative team was found to diligently pursue establishing clear expectations with its stakeholders and securing alignment for the project. They were strategic in their approach to these relationships, moving beyond tactically establishing relationships to meet the criteria set forth by the FoaK program, towards a position of establishing necessary steps towards getting the FoaK innovation to market. They worked with their product stakeholder starting from a position of what would be required for the transfer of the initiative asset into their portfolio. They work with their client stakeholder from a position highlighting the potential milestones and momentumbuilding events that would reinforce the value of the innovation being explored in the initiative. They work with the sales and industry stakeholders in identifying use cases that would have longer-range revenue potential for the firm. Finally, they worked with the FoaK program management team to ensure that they were embedded, aware of progress and positioned for possible follow-on funding if required.

Managing Momentum - The FoaK initiative team was also active in managing the momentum of their project. They demonstrated the importance of regular communication with their stakeholders, particularly in promoting the progress of their exploration and highlighting significant "proof points" emerging from their work with the co-creation client. For example, a major industry event was identified as a target goal where the FoaK 
innovation would be tested (i.e., Black Friday holiday shopping). The positive results from the test supported the value proposition presented by the FoaK innovation, with improvements in inventory management and optimization during the test period. This in turn translated into a significant proof point and major momentum building event, which the FoaK initiative team self-promoted. This was found to further strengthen stakeholder support for the FoaK initiative assets in the process towards commercialization.

Readiness (Technology Transfer versus Asset Commercialization) - Despite the strong execution of the FoaK initiative, in this case, issues were experienced in the transfer of the FoaK innovation to the product stakeholder. It was found that at the time of transfer, the product stakeholder was not ready to receive the asset, which some described as being "too early for the market." The assignment of resources was not complete, and the asset related knowledge needed by the new owners of the innovation was insufficient to adequately work with the assets at the time of transfer. This required ongoing support by the FoaK research developers to bridge the knowledge gap. There were also challenges in managing the transition of the client stakeholder relationship from the FoaK leadership to the product stakeholder.

The issues experienced at this point in the process suggested the lack of a technology transfer plan bridging the FoaK initiative to the FoaK innovation commercialization process. Other FoaK initiatives that were unsuccessful in transferring their innovation to a mainstream product portfolio speak to the notion of a "chasm" between a FoaK initiative and successful transfers to products. The current FoaK performance measure for the successful transfer of the technology to a products portfolio 
does not consider what is required to successfully commercialize the asset by the products organization.

A theme discussed regarding product portfolio stakeholders was the need for the FoaK program to extend its framework towards securing a greater commitment from product portfolios and consider transition requirements that must be accounted for in the planning stage (e.g., development resources, knowledge transfer to product teams, client stakeholder transition). Similarly, product stakeholders argue that the FoaK program must recognize there are different challenges when receiving a FoaK innovation into a portfolio. There is an internal competition for resources and prioritization, a necessary investment in development resources and commercialization activities, and the need for refining a preliminary concept that emerges from a FoaK initiative into in market ready offering that fits the target product portfolio. This suggests that if the goal for a FoaK initiative is the final commercialization of successful innovation, then there is a need for planning an alignment beyond the transfer of ownership from the initiative to the product organization.

\subsection{Case 3 - Analytics for Automotive ("Successful” Performance)}

Case 3 in this study represents a "successful" FoaK initiative exploring an innovation focused on cognitive analytic applications for the emerging "connected vehicles" market. This initiative examined innovations in advanced mobility services including automated driving assistance and high accuracy mapping services. The FoaK proposal was first accepted by the program in November 2013 and completed in January 2016. The FoaK initiative was placed on hold due to a delay in signing the co-creation client stakeholder, resulting in a 17 -month project delay. Upon securing the client 
stakeholder, the initiative delivered performance against all measures of success for the FoaK program. The initiative delivered significant success with the client stakeholder resulting in follow-on investment post-initiative, new clients were secured based on the success of the first initiative, and the innovation asset that emerged from the FoaK initiative was successfully transferred into a mainstream product organization for commercialization. As with Case 2, this initiative was characterized by its strong FoaK initiative leader, effective stakeholder engagement and alignment, close engagement with the FoaK program, leveraging the program framework, and an emphasis on a strategic approach to the co-creation of innovation with value for each of its stakeholders.

\section{Initiative Overview and Offering}

This FoaK initiative explored an innovation targeting the global automaker market and the emerging connected vehicles segment. The objective of this initiative was to deliver a next-generation analytic application that would assist in dynamic driving and provide analytic services for connected vehicles. The co-creation innovation included the development of a framework that would enable real-time alerts and mapping technology to support connected driving services. If successful, this framework would also support accelerated future development of similar innovative services for this emerging market.

The proposal was strategic to the firm in its support of the Internet of Things (IoT) agenda, as well as also supporting the analytics applications agenda. While the FoaK board was described as eager to fund proposals targeting the Internet of Things and the connected vehicle market, this initiative was first declined due to its initial scope. The FoaK initiative team was asked to rework their proposal, which subsequently met all the FoaK program 
conditions and was approved. After the proposal was accepted, early working sessions with the client stakeholder resulted in a re-evaluation of the priority of proposed use cases. The flexibility of the FoaK program initiative criteria allowed for a shift in the initiative focus towards a domain believed to be of higher value.

"We had two reviews. Initially, we put together a proposal. The board rejected the proposal. And then a few months later, we refined the proposal based on the board feedback and suggestion, and eventually they approved. I think-- from one side, the project got delayed from our perspective with additional few months, trying to answer the board question and put together some data point to support the proposal more strongly. But from the other side... a few extra months, we got a stronger partnership developed with the industry leader within the company. And also, the (products) catcher answered the challenge question from the board... I think that built the foundation of the potential success of the (FoaK) project already." Int.44.3

This FoaK initiative experienced a significant delay in identifying its co-creation client. The FoaK initiative team began recruiting the first choice on their candidate client list and worked towards an agreement in parallel with a sales opportunity that was in process with the account. After a significant delay, the FoaK team was unable to reach an agreement on terms for the FoaK project. The FoaK program agreed to extensions, with the guidance of a board assigned mentor who argued the significant strategic relevance of the proposed innovation, allowing for time to sign a new client stakeholder. In addition to the change in client stakeholders, the initial intended product stakeholder for asset commercialization also changed during the initiative. The formation of a new IoT business unit emerged as the product organization partner for the FoaK initiative. 


\section{Initiative Team Experience}

The FoaK initiative was executed by an initiative team based in two research labs located in the Asia-Pac region, the same region as the client stakeholder. The FoaK initiative team for this project consisted of a strong set of technical researchers with recognized expertise in IoT technology and were described as "active and efficient" in their domain. The FoaK initiative team resources were also part of the PI's functional team, with the assignment of their time under his discretion.

"I think the reason why the FoaK project was successful is related to the history of the China research labs... They were very much focusing on the IoT (Internet of Things) ... they had a lot of experience and developed a lot of assets for the future world of the IoT ... one of the use cases is a connected vehicle of IoT assets." Int.24.3

As with Case 2, the FoaK team primary investigator (PI) had previous experience and success with FoaK initiatives. The FoaK initiative team was distinguished by the proactive leadership of its PI. The PI served as the primary focal point across all stakeholders for the initiative and took a strategic approach to securing stakeholder alignment, setting and managing expectations throughout the project. In the early stages of the initiative, the FoaK team invested in working with stakeholders to define a scope for the initiative that would have the greatest strategic impact for the firm. As in Case 2, the PI for this FoaK initiative also had a previously established relationship with the product stakeholder organization, in addition to a well-established working relationship with the FoaK program management office. These stakeholders expressed their confidence in the PI's leadership of the FoaK. It was also highlighted that it was the persistence of the PI that 
ultimately established the FoaK board confidence and acceptance of the initiative proposal for program funding.

"The team involved, the lead was someone who had done prior FoaKs and had good results. So (the PI) on (this) FoaK ..., had a good track record so that always ... does add a little weight to whether or not a project is approved." Int.05.3

"(The FoaK PI) introduced a domain very well, right, so he has the right skill set to design it and he also had experience with many client relationships himself, and so the board say, 'Got it. You should have it.' And after that, it was smooth sailing, because of that aspect." Int.33.3

The FoaK initiative team demonstrated their ability to both form and develop new relationships across the firm in support of their FoaK initiative. The PI also leveraged previous relationships within his network to contribute to proposal development and building support for the initiative. As part of the proposal preparation phase, the FoaK initiative team also set expectations with the FoaK program management office that the client signing process would likely require more time than is allotted by the program due to the complexity of negotiations with clients of this magnitude. This further demonstrated the expertise of the PI in FoaK initiatives, and the foresight in setting expectations that reflect the reality of the initiative being proposed.

\section{Stakeholder Relationships}

This team was found to have successfully established a strong working relationship with all its stakeholder groups throughout the course of the FoaK initiative execution, and in the transfer of the innovation assets to the product stakeholder portfolio. 


\section{Program - Initiative Relationship (Guidance, Governance, Communication)}

The FoaK initiative team worked closely with the FoaK program management office during the project. They were found to leverage the assets provided from the program and to be proactive in providing progress updates during regular program checkpoints and on an ad hoc basis. The FoaK initiative team also included the program management office in client stakeholder workshops, providing further visibility to the program team of the innovation's value to the client.

"(The FoaK program and initiative teams) talked once per three days, at least, and we went to client visit together. I spent a whole day together. .. (The PI asks me) 'Do you want to go client meetings?' 'When are we going?'” Int.33.3

Due to the delays the team encountered in trying to secure their co-creation client stakeholder, further assistance was provided by a FoaK board mentor. The mentor was a senior strategy specialist familiar with the industry, and who guided the team on how to overcome the barriers they were encountering. In addition to his guidance, the mentor also supported a request for an extension of the FoaK program timeline on behalf of the initiative team. The case was made for the strategic importance to the firm of their FoaK innovation proposal, which was accepted and the time extension granted.

"There is significant value and opportunity associated with the assets and capabilities the team is building. These are a set of capabilities and opportunities we should continue to develop to provide differentiation to our auto industry clients." Board Mentor (VP), Note Requesting Extension, July 2014 


\section{Initiative - Sales and Industry Strategy Relationship}

The FoaK initiative team worked closely with the sales and industry stakeholder organizations in defining the scope of their proposal and identifying client candidates. The initial proposal submitted was tied to an active sales opportunity with the first client candidate. It was found that the sales activity underway, while the FoaK initiative team was trying to secure the client to participate in the project, introduced additional complexity to the process contributing to the signing delay. It was decided that the first candidate would be abandoned for the initiative, and the second qualified candidate was approached to participate. The sales stakeholder had an established relationship with the new client candidates, which helped successfully sign them to participate in the FoaK initiative. The sales and industry stakeholder relationships were found to be both supportive and active throughout the process for this initiative. Throughout the course of the initiative, the relationship between these teams resulted in the creation of a new revenue pipeline of clients interested in the offering.

"A lesson we already knew, but it's not always wise to tie a FoaK to a larger deal because you have to wait for that larger deal to get worked out, and usually, you don't have enough time for that. Sometimes the FoaK gets lost in a larger deal." Int.05.3

\section{Initiative - Client Relationship}

Both the FoaK initiative team and the co-creation client stakeholder were based in the Asia-Pac region. The combined initiative team (i.e., firm, client) working on the FoaK initiative shared a common language and culture, eliminating the need for communication bridging as was seen in Case 1. The initial co-creation client candidate was based in 
Western Europe, which would have required additional resources to help address the language and cultural gaps between the teams. As with the first client candidate, the eventual client stakeholder is also an industry leader, familiar with co-creation initiative partnerships.

The client stakeholder selected was chosen in part based on the previous relationship with the firm, and the deep access that was anticipated in the engagement. The client stakeholder assigned a focal point that helped the FoaK initiative team navigate their environment and manage expectations. A strong working relationship and level of trust in the expertise of the FoaK initiative with the client stakeholder.

Upon securing the replacement client stakeholder, the scope for the FoaK initiative was revisited with a focus on reprioritizing the use case options presented in the broader vision of the FoaK initiative team. This resulted in a new focus of the FoaK initiative scope to an alternative priority area. The client stakeholder was also described as being impressed with the potential impact of the innovation being explored in the FoaK initiative.

"We found the right person in the company from the customer side. And then we identify the right use case, which is meaningful to their interest, and then we deliver a quality of work impressing them." Int.44.3

"In general (the client) was very supportive. They are very supportive. There weren't any roadblocks at all. It was quite smooth, quite smooth. Because the FoaK is really new, they actually are learning as well, because there's innovation in the new space. They are very engaged. They were very engaged. So it was quite smooth in general." Int. 44.3

The working relationship between the FoaK initiative team and the client stakeholder was found to be strong. The client stakeholder was described as engaged and 
accessible throughout the project. They worked closely with the FoaK initiative team in identifying a major external event at which the FoaK innovation could be announced. This served as a compelling event to help focus activities of both organizations. The event announcement subsequently contributed to building momentum for the FoaK initiative across all stakeholder groups. As progress was made during the execution of the FoaK initiative, and proof points began to emerge, the client stakeholder team began to promote these milestones internally to their business users, promoting the potential of the new innovation for their organization. The level of engagement between the FoaK initiative and client teams, along with the support of the client sponsor in promoting the progress of the initiative within their organization, all contributed to the building of momentum for the FoaK initiative both inside and outside of the client organization.

"This First-of-a-Kind cognitive computing solution demonstrates our commitment to making driving safer and healthier. " Client CMO Announcement, July 2016

The FoaK initiative PI played an active role once again in building and maintaining expectations with the co-creation client stakeholder. As part of this working relationship, the PI also ensured that the products stakeholder was engaged throughout the project, providing the opportunity for knowledge to be transferred during the work with the client stakeholder.

\section{Initiative - Products (ACO) Relationship}

The products organization that would become the asset commercialization owner emerged during the FoaK initiative proposal process. During that time, there was a reorganization in the products organization resulting in the formation of a new business 
unit focused on the Internet of Things. This new business unit was mandated with creating an IoT portfolio of offerings, including solutions for the connected vehicles market. The FoaK initiative PI had a previously established relationship with the leadership in the new organization, which helped maintain momentum in the project. This development presented further support for the strategic relevance of the FoaK initiative proposed.

"Because we work together all the time not just across other projects, so they know us they know where technology pretty effectively and nicely so the decision to commercialize the asset was made quite smoothly and one of the other the reason why the decision was made pretty quickly." Int.44.3

With the formation of this IoT group, the initial commercialization team for the FoaK initiative proposal was transferred to the new organization. As the PI had already established a trusted relationship with these resources and understood the new business's strategy, he successfully leveraged the previous experience in positioning the participation of the new business unit as the new product stakeholder for the FoaK initiative. He was successful in securing their support for the funding proposal, as well as gaining their commitment to participate actively throughout the course of the FoaK project.

"(The products stakeholder) participated in the regular review meetings. They provide some coaching support during the FoaK execution phase. They understand the project and the progress we have made." Int.44.3

Both the growing profile of the IoT domain area, along with the commitment of the client stakeholder in the new business unit, contributed to building momentum for the FoaK initiative innovation. While the IoT organization was now a business unit working towards establishing a roadmap for their new mandate, it is reasonable to find that the potential 
contribution of the FoaK initiative innovation and the relationship with a high-profile client stakeholder both contributed to the ongoing support of the product stakeholder for the initiative. The FoaK initiative team and the product stakeholder were found to be collaborative and committed throughout the duration of the initiative. The subsequent outcomes from the FoaK initiative resulted in significant announcements for the new IoT business unit automotive markets. With the success of the FoaK initiative, the client stakeholder remained motivated and invested further into the development of the FoaK innovation as it was transferred to the product stakeholder portfolio. This included the transfer of the client relationship to the product stakeholder.

"The technologies developed from the scalable and accurate map analytic service FoaK has been included in the IoT business unit automotive industry platform offering which has become available for seller to sell on the market." FoaK PI Announcement of Commercialization

"This is a milestone for our combined team and will give us an excellent base to commercialize your team's excellent research work on an ongoing basis. " Product Leader (Director) Announcement on FoaK Asset Transfer

Initiative Execution and Performance

This FoaK initiative was found to be successful against all performance measures applied by the FoaK program. The outcomes from the initiative demonstrated client stakeholder satisfaction and their commitment both to the project and through follow-on investments related to the FoaK co-creation efforts. The outcomes from the FoaK initiative also resulted in new revenue opportunities emerging with other clients for the firm. The resultant innovation from the FoaK initiative was also successfully transferred for 
commercialization to a mainstream product portfolio within the firm. An examination of this FoaK initiative illuminated several highlights on the relationship between initiative execution and observed initiative performance.

Program Flexibility and Support - It was found that this FoaK initiative worked within the framework provided by the program, leveraging the assets and support provided. However, as complications were encountered in securing a co-creation client stakeholder that resulted in significant delays early in the initiative, there was a risk of cancellation of the project. It was the flexibility of the program and the assignment of a senior mentor that helped overcome the issues faced early in the FoaK initiative process. This demonstrated how the flexibility applied by the FoaK program management organization helped in identifying the true value of the opportunity for the firm. The result of the work between the FoaK programs in the FoaK initiative team was a successful initiative contributing to the firm's strategic agenda and revenue.

Leadership - As with Case 2, the benefit of the higher-level FoaK initiative team leadership was observed. Aside from the technical skill that the PI possessed, it was evident that a strategic approach to managing the FoaK initiative was also applied. Significant effort went into establishing relationships early in the process and setting expectations with key stakeholders. It was found that the PI served as the focal point for the FoaK initiative innovation, and effectively established trusting relationships across the client, product, industry, and sales stakeholders. It was also described that the PI's leadership contributed to stabilizing the FoaK initiative amongst the stakeholder changes in its environment. The FoaK initiative team was found to be effective in communicating with the FoaK program 
management team on progress and highlighting proof points throughout the FoaK process, further contributing to building and sustaining momentum for the initiative.

Stakeholder Engagement - This FoaK initiative was also exemplified by the level of stakeholder engagement through the project. Within the client stakeholder organization, the initiative sponsor worked at ensuring that the right resources were available throughout the FoaK initiative, and that demonstrated success was promoted internally. This helped build and maintain momentum with the right stakeholders within the client firm. Similarly, the FoaK initiative's internal clients, the product stakeholder, was engaged from the outset. While the formation of the new IoT business units occurred during the initiative, there was a risk of potentially losing the support for this project. However, it is reasonable to state that the relationship described between the FoaK initiative team and the product stakeholder in defining the initial scope for a FoaK initiative, and the perceived market value of that use case, both contributed to sustaining the support of the IoT business unit. The product stakeholder's involvement throughout the initiative contributed to their understanding of the offering and the FoaK initiative team's "coaching" on considerations that could impact the successful transfer of the assets at the end of the FoaK initiative.

Initiative Value and Momentum - It was also evident in this case that there was a relationship between the strategic fit of the FoaK initiative proposal and the impact of initiative momentum. The FoaK initiative team worked closely with the primary initiative stakeholders in establishing the proposal scope that would have the greatest strategic relevance for the firm within the target domain (i.e., IoT and connected vehicles). This early involvement helped to secure stakeholder interest and support throughout the process, 
which in turn focused efforts during the initiative. There is sufficient support for the belief that this early work, along with the additional refinement of the initiative scope with the client stakeholder, resulted in a FoaK research scope with greater resonance beyond the context of the project.

It was also found that the early FoaK recruitment of the first client candidate combined with an active sales cycle added complexity that negatively impacted the initiative's momentum. The intertwined objectives resulted in confusion for the client candidate and sales stakeholders, impacting both the FoaK signing process and the sales process. Once the decision was made to move to a second client stakeholder candidate, with the sole focus being on recruiting for the FoaK initiative, the process was understood to move forward without issue.

The strategic value of the FoaK initiative, in addition to the proof points and announcements related to the initiative, each contributed to building momentum for the cocreated innovation. The momentum for the initiative continued to sustain the interest of stakeholders beyond the program, helping to secure the necessary support and investment for commercialization. It is also believed that the momentum experienced for this initiative was magnified by stakeholder alignment efforts of the FoaK initiative leadership and the flexibility of the program design. The foresight of the FoaK program in granting the signing extension to the initial timeline provided an exchange of support for the potential of longterm value creation for the firm.

The formation of the IoT business unit presented the risk of losing support and momentum for the FoaK initiative. In this case, the timing of the new business unit and its strategic relevance to the firm worked in favour of the FoaK initiative team. It was the 
readiness of the FoaK initiative leadership to capitalize on this new organization that resulted in the further building of momentum in support of the project.

"Timing was right in the sense that this was technologies for connected vehicles with this market application were in high demand. So, coming with that type of solution and technologies was the right time." Int.09.3

Readiness (Technology Transfer versus Asset Commercialization) - As with Case 2, it was found that there were similar considerations concerning the need for planning of the asset from the FoaK initiative to the product stakeholder for commercialization. This includes consideration of the resources, skills, knowledge transfer, and transition of the client stakeholder from the FoaK initiative team to the product stakeholder team.

\subsection{Case 4 - Analytics for Travel (“Unsuccessful” Performance)}

Case 4 in this study examined a FoaK initiative exploring an analytic application innovation for the Travel and Transportation industry, which resulted in an "unsuccessful" performance. This FoaK initiative proposed an innovation delivering new optimization algorithms for pricing and scheduling that would transform current pricing techniques used in the industry. The initiative was first approved by the FoaK board in May 2011, with completion in May 2013. The initiative was placed on hold in November 2011 due to delays in signing the co-creation client stakeholder for the initiative. After the 15 months required to secure a client, the project resumed in August 2012. This initiative was led by an inexperienced FoaK initiative team and was characterized by a lower level of stakeholder alignment and client stakeholder engagement than is seen in the more successful FoaK initiatives. 
This FoaK initiative was unsuccessful in delivering against program performance measures. While it reached completion and delivered an innovation asset and sales reference, there was little client stakeholder related activity beyond the project. There has been no follow-on revenue reported since the completion of FoaK initiative, and the offering was not successfully transferred to a mainstream product portfolio in the firm.

\section{Initiative Overview/Offering}

This FoaK initiative explored analytics innovation with the potential to transform the current pricing methods used in the global transportation industry. At the time of the initiative, global transportation providers were moving away from single-mode static pricing models towards more advanced multimodal pricing methods. Achieving this transition would require innovations to pricing engine strategies not available in the market at the time. The FoaK innovation being proposed in this initiative would enable optimized pricing models through the management of thousands of pricing elements and rules, coordinated with the complexity of long-distance transportation service networks. It would apply sophisticated large-scale analytics and optimization techniques to help organizations in determining pricing within a complex set of user-specified constraints and criteria. In addition to targeting an emerging problem in this market, this proposed FoaK innovation was also strategic to the firm in support of its Smarter Transportation and analytics strategies.

As in Case 3, this FoaK initiative was initially proposed as part of a sales engagement, where the firm did not have a packaged solution to address the client business problem in this domain. To remain engaged with the client prospect, a sales strategy was 
proposed which included collaboration on a FoaK initiative. This would involve the prospect working with the firm on a new innovation addressing complex pricing optimization. If successful, it was believed that the FoaK initiative would produce a newto-the-market innovation to the travel and transportation industry, and would also contribute to further establishing the relationship between the firm and the client prospect.

The FoaK initiative initially presented for funding was not accepted by the FoaK board. The initial proposal was found to have a scope that was too narrow to be impactful, and the FoaK initiative leadership was guided to rework their proposal. The updated proposal was subsequently approved by the FoaK board, with the initiative being funded with four full-time resource equivalents for twelve months.

"It was an issue with the technology. They were focusing on, I think, just one area of the technology, it's supposed to be-- and the board wanted it to be more multimodal, I think. We were focused on just one element of the pricing and the board wanted them to make it more of a broader idea." Int.06.4

Upon approval of the FoaK initiative, engagement with the client stakeholder resulted in further adjustments to the initiative scope. However, while the FoaK initiative reached completion, it was found that the client stakeholder expectations were misaligned with the objectives of the FoaK initiative, resulting in a level of client dissatisfaction at the end of the initiative.

\section{Initiative Team Experience}

It was found that both the FoaK initiative team and its primary investigator (PI) were inexperienced with FoaK projects. For the PI and the participating team, this would 
be the first full FoaK project. The initiative team required guidance from the program and used the FoaK program assets and framework to help guide them through the initiative execution. The FoaK team was also described as having minimal previous client engagement experience, and a limited network outside of the research organization across the firm. This was found to have an impact on the pace of executing the FoaK initiative, and the degree of effectiveness in communication with stakeholders.

"It takes some experience. How you present very complex material to the client, for instance, how you listen. I mean, (the team) did a great job, but with more experience, I think one would have gotten to some things probably a bit faster... (The team was) new, joined us about a year or two with (the firm), and they didn't have the network and it was sometimes-- it took a while until they found out whom to ask. " Int.25.4

While the FoaK initiative team leadership successfully met the prerequisite requirements to participate in the program, including securing the necessary stakeholder alignments, the inexperience of the leadership was found to be reflected in the management of stakeholder relationships. It was found that the FoaK initiative leadership did not stand out as a clear focal point for the project, as was seen with more successful initiatives. The approach to managing the FoaK initiative was found to be tactical in nature, and business acumen was found to be lower than in successful FoaK initiative teams. These factors were believed to have contributed further to challenges observed in managing the execution of this FoaK initiative.

"One has to balance so many other things, so many other stakeholders, business units in the process. They all have their own agenda and their own targets, and their own goals of this project. If you're not able to even make sure that the basics work, then how are we supposed to juggle all of the rest?" Int.25.4 
Stability of the FoaK initiative team resources was also found to be an issue through the course of the project. The FoaK team was distributed across three countries (i.e., based in Western Europe, India and the United States), and was composed of research-centric resources working with the IBM services organization. While the research resources were initially committed to participate in the FoaK initiative, some of the team members were asked to share time on other assignments during the initiative, described as impacting the focus of the team.

In total, these factors are found to have impacted the time necessary to conduct the FoaK initiative as well as affecting the quality of stakeholder alignment during the project. It is reasonable to believe that the complexities that were introduced as part of this initiative, combined with the inexperience of the FoaK initiative team had a negative impact on the final performance of the project.

\section{Stakeholder Relationships}

A review of the key stakeholder relationships in this initiative did not find them to be developed to the same extent as observed in more successful FoaK initiatives. Engagement between the FoaK initiative team and its stakeholders were limited to an essential level required to secure funding and execute the scope of the project.

Program - Initiative Relationship (Guidance, Governance, Communication)

The relationship between the FoaK initiative team and the FoaK program was found to be tactical in nature with the level of engagement focused on satisfying basic program requirements. The FoaK initiative team used the program assets to prepare their quarterly 
updates, client proposals and presentations. There appeared to be little interaction with the FoaK program management office during the initiative. As the initiative team encountered issues in signing the client stakeholder, a FoaK board mentor was assigned to help coach the team through the challenges they were facing. There appeared to be little proactive engagement from the FoaK initiative team with the program team. A review of correspondence between the two teams highlighted that the FoaK program management team led communications, reaching out for updates directly from the FoaK initiative team throughout the project.

“(The initiative team didn't use the program artifacts) much I must say. I didn't have good previous examples. I saw a couple of presentations maybe or presentation texts of proposals, so I could get a little bit of an idea how to do it. But I think I was pretty much-- first time, and I was pretty much on my own there." Int.25.4

\section{Initiative - Sales and Industry Strategy Relationship}

The initial engagement of the FoaK initiative team with the sales and industry stakeholders was driven by a sales opportunity with the client stakeholder. The sales team was engaged in a sales cycle with the co-creation client stakeholder, and was not able to meet the need of the client with the existing portfolio of offerings. A strategy was taken that would have the FoaK initiative as part of the sales cycle, which subsequently was found to insert complexity into the engagement with the client stakeholder for both sales and FoaK initiative teams.

"So, the first and foremost motivation was that we wanted-so it all came out of the discussion of the travel and transportation industry team and sent out competencies that we were looking at a particular problem, industry problem, we didn't see any solution in the market. So, in that sense, it was really an idea to do a First-of-a- 
Kind. And at the same time we had a client opportunity and there was an upcoming tender, and so we positioned it. "Int.25.4

"So, the (sales team) were looking into this buy. Okay. We understand the client problem. We seem not to have a sort of solution which can easily map to this. So it seems to be a very complex solution, which not available ... out of the portfolio we run. And I think then we were trying to get together with research and look at customization of the solution with research capabilities. ... we do not seem to have anything which is tackling this business problem or this capability, so why not having a discussion with our research (about the FoaK)" Int.12.4

"(The client asks us) "What's behind research? Do you have really great people behind this?" and "What have you done before? Give me a proof of this. "So we were basically showcasing a couple of proof points, and then we started the discussion... We like this lab approach. Let's work on this." Int.12.4

It was then decided to separate the two streams of activity with the client, resulting in the signed engagement in the initiative. It was not found that there was significant engagement between the FoaK initiative team and the sales stakeholder through the execution of the project. The FoaK initiative team worked with sales and industry stakeholders to identify market use cases. Upon completion of the FoaK initiative, several potential follow-on revenue opportunities for the FoaK innovation asset were identified by the sales and industry teams. However, upon examination it was found that there was little activity regarding those new revenue opportunities, and no follow-on revenue reported.

\section{Initiative - Client Relationship}

The co-creation client stakeholder for this initiative is a national leader in rail transportation. Their engagement with the FoaK initiative was a top-down executive driven project, with the client executives assigning resources to the project. While executing the 
FoaK initiative, the client was described as engaged in the project with regular communication with the FoaK initiative team. However, the mode of engagement appeared to be more checkpoint meetings on the FoaK initiatives progress.

"In this case, it was the CIO's office, so it was technically the unit of the CIO in the company. It was moderated and orchestrated by the CIO, but we asked them to work also with a lot of business stakeholders from the client side." Int.12.4

"(The client was) quite involved. We also had regular - quite regular meetings. Now, I'm thinking of - it depended on the phase. I think in the main phase we even had sometimes a weekly - I would say a monthly meeting normally at least. Something between weekly and monthly. So, the project leader was more on the procedural side. So, setting up the project meeting and making sure that the project is internally communicated to their upper business management line. So, this was handled quite well." Int.25.4

It was described that from the outset, there was a misalignment in the expectation setting with the client on the objectives for the FoaK initiative, and that the client was conservative in their approach to the engagement. The client stakeholder appeared to have misunderstood the nature of the engagement and required commitment to the FoaK initiative. After committing to participate in the FoaK initiative, there were internal changes within the client organization that further impacted alignment on the project. Changes to the focus of the initiative were ongoing, and the FoaK initiative team appeared to struggle with the challenges of "scope creep." These issues with alignment further contributed to the client stakeholder questioning the value of the FoaK innovation to their organization, and increasing pressure to demonstrate return on investment against their budget. 
"The main challenge was that once the client signed and we sat together to determine the scope, it became very different from what we initially proposed. And so, we had to-- because, somehow-- in the end, we delivered two solutions. One that was developed before the clients got deeply involved and then another one with the client, and they were pretty disconnected. That process to define-- for them to understand what we're after and for us to understand what actually their pain points were and then to find a good compromise, that was challenging." Int.25.4

At the completion of the FoaK initiative, it was described that client expectations were such that the emerging innovation would be more refined in its usability and experience, indicating that there was a disconnect on the outcome of an early stage exploration. The client stakeholder also argued that the revised scope of the innovation was too narrow to benefit them, resulting in their loss of interest in further engaging or investing in the FoaK innovation. Although the client had access to the resulting innovation after the initiative, and the support of the FoaK initiative team, they were not interested in further evaluating the FoaK innovation within their own environment. In addition, the client was not prepared to promote the resulting innovation to their market in the belief that the culture of their market would not respond positively to any efforts potentially interpreted as managing pricing to maximize profits.

"... this is a flexible solution, whatever a new product they define in the principle underlined principle - is the same of the analytics we are building in this solution. But they didn't, somehow, they didn't want to go ahead with it. They said, "No, it's too volatile. We do not want to do this and we need a longer time to validate it." ... now, this is more on the functionality discussion, I would say. But looking back, what I think was one of the main problems is that we built didn't look nice. We focused a lot on the underlying-- on the analytics under the hood, and we had a very poor, I would say, user interface. The solution was not-- didn't look nice, wasn't nice to use. And even though we went into a, I would say, nine-month evaluation after the FoaK project where we gave them an evaluation licence-- they could use the tool. They 
could plan their portfolio. I think they hardly ever did it because they were not-- the usability aspect was not there." Int.25.4

"So, what (the client) said that based on the data, they said that they do not trust fully the results because it's a prediction into the future and we need to validate them. ... 'In two, three years, our whole product portfolio looks so much different--- so, what you are building today is for the old portfolio would not be applicable to the new portfolio. ," Int.25.4

An examination of the characteristics of the client stakeholder engagement in this case reveals potential issues with the fit of the client for this initiative, and the effectiveness of the FoaK initiative leadership in managing the expectations and engagement of the client during the project. The inexperience of the FoaK initiative leadership combined with the conservative nature of the client stakeholder were both found to contribute to the misalignment between the two teams and the lack of clarity on scope, investment, and expectations required at the start of the project.

\section{Initiative - Products (ACO) Relationship}

For this FoaK initiative, the emerging innovation asset was to be commercialized as part of a services-based analytic solutions portfolio. This portfolio represented a new asset commercialization model that would be based in the firm's services organization versus a traditional products portfolio. A primary objective of this commercialization model was to accelerate market availability of early-stage analytics applications targeting industry specific use cases. The FoaK innovation asset once delivered from the initiative would be incubated in the services portfolio, and used in supporting sales cycles related to the industry domain (i.e., pricing optimization for travel providers). 
"I think the way that being a very industry-focused solution the (Services) Solution Center for that particular industry it's an easier setup for being a catching organization compared to a software group with a more horizontal frame.

"Int.25.4

The FoaK initiative team worked directly with the services portfolio owners in the early stages of the FoaK initiative to set the scope and expectations for the project. During this FoaK initiative, a reorganization occurred resulting in the intended product stakeholder portfolio being disbanded, and reassigned against new strategic priorities. This resulted in the FoaK initiative asset being stranded upon completion of the project. Minimal effort was made to identify a new potential portfolio for the assets, and the FoaK initiative team was left to resolve where the assets could be transferred. It was found that the inexperience of the FoaK initiative team and their limited internal network impacted their ability to find a replacement organization that would receive the asset for commercialization.

"We kicked off (the FoaK project) but then suddenly there were some changes in maybe a few structures, and then we had to stop it abruptly. And then it was supposed to be taken over by another team, and there was a lot of restructuring which happened because most of the things moved to the development centre at that time. ... I'm not sure what happened after-- from that time. Then we were not in that loop." Int.39.4

"(The FoaK initiative team) had technical discussions with (a potential new product owner's) technical team, but also there I think what prevented them from not getting so excited about it is also-- yeah, the missing client excitement. So, they were only ready to put, to my understanding, resources into catching if they saw complete client opportunities." Int.25.4

"The (asset from the FoaK initiative) was then sort of parked, and nothing is archived since two years or three years. So, it has not been used actively with other clients, other than we had similar client situation... It's available, but it's not well 
maintained because we simply do not have the funding to maintain it further." Int.12.4

"Which is a pity for other FoaK projects... If these things are aging and not being up to the mark anymore, then I think the chance of reselling them is even lower, right?" Int.12.4

Based on this progression, the question of product stakeholder fit for this FoaK initiative should be examined. As a new business model and an alternative approach to commercialization, further planning in the technology transfer requirements did not appear to be in place. The suddenness of the reorganization also indicates that the relationship between the FoaK initiative team and the product stakeholder did not provide early indication that an alternative plan would be required. It is also recognized that the lack of a plan to redirect the asset with the knowledge that the reorganization would occur, as well as the challenges in finding a new product portfolio post the FoaK initiative, suggest that the strategic relevance of the FoaK initiative may have been lower than reflected in the initial approval of the proposal by the FoaK board.

\section{Initiative Execution and Performance}

During this FoaK initiative, there were changes in the initiative's context which contributed to the complexity of its execution. It is found that the inexperience of the FoaK initiative team, combined with the lack of stakeholder alignment and changing context all contributed to the observed FoaK initiative performance.

Program Flexibility and Support - The FoaK initiative experienced delays in securing its co-creation client. The flexibility of the program provided the initiative with additional 
time to signing client stakeholder contract, due to its perceived strategic potential for the firm and in support of the new solutions portfolio commercialization model. However, while the FoaK program framework and guidance were available to the initiative team, they did not appear to proactively engage for assistance during the project.

Leadership - The inexperience of the FoaK initiative team PI and researchers was reflected in the execution of the project, and in the quality of the stakeholder alignment observed. It was evident that this initiative experienced challenges in setting expectations across the client, product, and sales stakeholders, requiring additional effort from the initiative leadership to manage the issues that continued to emerge throughout the project. As seen in Case 2 and Case 3 experienced FoaK leadership is found to anticipate what is required by the initiative in advance of engaging with stakeholders. These leaders also anticipate potential future issues that they proactively set a course to counter or remediate with their stakeholders. There is sufficient support to argue that the level of experience of the initiative team and its leadership play a role in a FoaK team's ability to manage the complexity and unexpected issues encountered while conducting their initiative.

Stakeholder Engagement - Stakeholder engagement was found to be limited across this FoaK initiative. The sales stakeholders were primarily driven by the revenue opportunity at the start of the FoaK initiative process. As it was decided to separate the FoaK initiative from the sales cycle, engagement from these stakeholders appears to decrease. Similarly, client stakeholder expectations continued to be a struggle throughout the engagement for the FoaK initiative team. The client organization was described as unclear on the scope and purpose of the engagement once funding had been issued and execution of the initiative 
had begun. This resulted in an impact on the pace, scope and effectiveness in the execution of the initiative with the client stakeholder. In addition, interest and engagement decreased with the client during the initiative. These were all found to contribute to the client stakeholder being unsatisfied with the resultant innovation, arguing that the scope delivered was too narrow to be of value to their organization and that innovation were expected to be more refined as a final deliverable. Finally, the FoaK initiative engaged a product stakeholder organization that was part of a new and developing business model. The subsequent elimination of the committed product stakeholder portfolio during the execution of the FoaK initiative did not plan a path forward for the FoaK initiative innovation. This sudden end to the commercialization plan was an indication of a lower level of commitment and engagement between the FoaK initiative team and the products stakeholder.

Initiative Value and Momentum - It was found that the scope of the FoaK initiative was unable to generate a sustained level of momentum with the client, sales and product stakeholders after funding was provided for the project. The instability of the product stakeholder portfolio, the expectations and level of engagement of with the client stakeholder, and the multiple intertwined agendas between the sale stakeholder and the FoaK initiative team were all found to negatively impact the momentum of the FoaK innovation. As such, with no further engagement of the client, sales or potential new product stakeholders at the completion of the initiative, there is a question regarding the strategic value of the FoaK initiative that was the basis for this project. 


\subsection{Case 5 - Analytics for Healthcare (“Unsuccessful” Performance)}

Case 5 examines a FoaK initiative which explored an analytic innovation for the healthcare industry. This project, which is described as an "unsuccessful" FoaK initiative performance, applied analytics innovation designed to help create personalized patient treatment plans for cancer and other diseases. The solution brought together integrated advanced statistical techniques for analysis of multiple sources of case history that would provide physicians with evidence-based medical insights at a patient's bedside. The initiative was first approved in January 2012, with the project completed in June 2013. Due to challenges in securing a co-creation client, the initiative was placed on hold for five months until September 2012. While the FoaK initiative resulted in a satisfied client, external references, patents, and peer-reviewed journal articles, it was not successful in delivering follow-on revenue or in transferring the innovation assets into a mainstream products portfolio. As with Case 4, this FoaK initiative struggled in achieving stakeholder alignment and engagement which was found to impact its overall performance.

\section{Initiative Overview/Offering}

In healthcare practices, the oncology care process requires monitoring of guideline adherence, clinical outcomes, and resource-benefit ratios. Healthcare professionals are challenged in identifying deviations from guidelines and assessing the clinical outcomes related to those deviations. The innovation proposed for this FoaK initiative was targeted at providing state-of-the-art analytics-based clinical evidence to help in the tailoring of individual treatment plans towards improving clinical care and optimizing current cost 
analysis. This FoaK initiative was strategic in its support of the firm's Smarter Healthcare and analytics agendas.

This FoaK initiative was based on an early-stage invention developed within the firm's research organization. The product stakeholder for the FoaK innovation was intended to be the IBM service organization's emerging analytic solutions portfolio, a new commercialization model approach intended to incubate and accelerate the delivery of new industry-based solutions to the market. The FoaK initiative proposal was first rejected by the board with a request to the initiative team to refine the proposal further and consider clients related to the firm's newly formed Watson Health business unit. This business unit was created specifically to develop analytic solutions for the healthcare industry. The updated FoaK initiative proposal met the requirements for board approval. The revised proposal reverted to the initial co-creation client candidate, which was not related to the Watson health unit as recommended by the FoaK board. The FoaK initiative was funded with five full-time researcher equivalents for twelve months.

\section{Initiative Team Experience}

The FoaK initiative team for this project includes the lead research team that developed the base invention (i.e., Middle-East research lab) and an extended team based in the client stakeholder country (i.e., Western Europe). The local services resources served as the primary contact with the client in the execution of the initiative. The FoaK program team based in the United States further supported the FoaK initiative team. The FoaK initiative team was found to have a moderate level of previous FoaK experience. The FoaK 
initiative primary investigator (PI) had previously worked on three FoaK projects, none of which were successfully transferred to the mainstream product portfolio.

"Yeah, this team-- the good teams know how to work with the other groups in IBM. I don't think team had that same ability or just didn't seem to be as connected" Int.07.5

"I think they had some connection, but I think the fact that it kept changing probably made it difficult for them. But I don't think they were as connected as (other teams)." Int.07.5

It was also found that the FoaK initiative PI exhibited a tactical approach to managing the project, with less investment placed in establishing early stakeholder alignment than was seen in more successful initiatives (e.g., Case 2, Case 3). Management of this initiative was found to be limited to delivering the essentials required for the FoaK program. The resulting support for the initiative and the related stakeholder relationships appeared to be moderately effective through the course of the project. The FoaK initiative PI appeared challenged in managing the complexities of this FoaK engagement. A strategic outlook for the management of the initiative was not observed in this case. Correspondence from the FoaK initiative highlighted that the program team led communications, and pulled information regarding progress from the FoaK initiative team. It was found that there was a lower level of visibility into this project than was seen in more successful FoaK initiatives.

"It's not easy to deliver a system to a client in three or four people in half a year or a year. I think it is a naive assumption - at least from my experience. I am really familiar with few FoaKs, but all of them was in similar domain, similar difficulties. But it's very naive to think you can push a system to a client with such a short time and effort." Int.11.5 
"I think that it was a quite challenging experience, very complex and complicated in terms of requirements because at the time, there were several layers of information that we had to convey to (FoaK) board, but the same time, it was somehow helping us also to be very focused on innovation, to be very engaged with the customers, and the committee there as well" Int.28.5

The FoaK initiative research member in the client country was the primary point of contact with the client stakeholder. As a team member local to the client, this individual could partially bridge the communication and cultural differences with the core research team. This FoaK initiative team focal point had no previous FoaK experience and had less than one-year experience with the firm. This individual also conveyed previous domain experience and enthusiasm for the objectives of the FoaK program in working with clients.

A changing environment can impact junior resources - "It's not a comfort zone (for the FoaK team) because you give up some control, especially when (project) train is moving so fast that more junior people can hesitate. Because it exposes them to a lot of risk that they can't control, especially if you're in a European time zone and all the stuff that goes with that, right?" Int.07.5

\section{Stakeholder Relationships}

As seen in Case 4, a review of the key stakeholder relationships in this initiative was not found to be developed to the same extent as observed in successful FoaK initiatives. Engagement between the FoaK initiative team and its stakeholders were limited to an essential level required to secure funding and execute the scope of the project. 


\section{Program - Initiative Relationship (Guidance, Governance, Communication)}

The relationship between the FoaK program and the initiative team was found to be limited to essential communications. The FoaK initiative team leveraged program assets necessary to support the proposal process and client presentations. They also met the program requirements in providing quarterly FoaK board updates. It was found that there were no significant communications on progress or issues from the FoaK initiative team to the program management office between updates. The FoaK program management team made requests for information throughout the initiative.

FoaK initiative team uses program framework to onboard new team members - "I had to study several documentation from the FoaK perspective. And it was also quite challenging for me because I was already quite busy in working with the technicians. But it was a very interesting to me in the sense that I was able to understand how (the firm) was evaluating innovation and how was evaluating most of the strength of ideas and application in that respect." Int.28.5

"I think at least the program puts some boundaries around what's possible and what's really kind of out of bounds. It also gives us some collateral. We can go and say these are some examples of things we've done previously under this rubric. I think that does help, at least to reassure the client that there is some formal piece of IBM behind this. It's not just a bunch of propeller heads coming and saying we want to play with you." Int.22.5

While program support was available, it was also found that the FoaK initiative team did not draw upon the experience and guidance provided. There were no requests noted from the FoaK initiative team asking for guidance or assistance as issues were encountered through the course of the project. This was consistent with the FoaK initiative team's decision not to follow the guidance from the board to reconsider their choice for the 
client stakeholder, for one more closely aligned to the newly formed healthcare business unit.

"The board did give them a lot of guidance to align with Watson and choose a Watson client. So, we probably should've done a better job making sure that that was followed throughout the project." Int.07.5

"They came back in with this whole list of (client stakeholder options), all the ones we work with on Watson. And the board thought, 'Okay. They've broadened their list of their pipeline.' But then they ended up almost immediately going back to (their initial choice) and signing with them." Int.07.5

\section{Initiative - Sales and Industry Strategy Relationship}

During this FoaK initiative, the initiative team established a strong working relationship with the sales stakeholder that helped in the early stages of identifying a cocreation client for the project. The FoaK team described working closely with this individual and the dependency on that relationship as the primary contact and access to the client organization. During the FoaK initiative execution, the sales stakeholder that served as the primary contact for the initiative left the firm. This impacted the effectiveness of the interaction between the FoaK initiative team and the client in managing expectations, and maintaining access to the client organization through the project.

"So, we had some relations, we knew a lot of them, people (sales organization) in Europe. Specifically, these (healthcare) clients, I was in a meeting not related to (the FoaK) -- doing some visits for another FoaK, and we were introduced to these clients... and they were interested in the general idea." Int.11.5

"So (the sales stakeholder) was kind of the ring leader. He was the guy who wanted to do the project. He had some technology in mind that he thought would be valuable, 
and he and his team also found the client.... So, he found the client. Then he and I worked together to get support from the industry (stakeholder). "Int.22.5

FoaK initiative team dependency on sales stakeholders - "I wish I could not (have the dependency on the sales team), but there were so many bureaucracies, and things that you need to handle, and contracts, and regulations, which we could not do without them, I guess. " Int.11.5

"(The sales stakeholder) introduced us to this (client) and from there on we continued. By the way, he left (the firm) in the middle (of the initiative) and then also his successor left (the firm), and this was very challenging for us to continue and manage the relationship with the client with all these changes." Int.11.5

\section{Initiative - Client Relationship}

The co-creation client for this FoaK initiative is a leader in its industry. In the early stages of the initiative, the client was initially described as being very engaged and aligned with FoaK initiative team and objectives. There was a client executive sponsor that led the initiative and was responsible for identifying and assigning resources to participate from within their organization. It was described that as the FoaK initiative experienced internal delays in its execution, the initiative began losing momentum within the client stakeholder organization. The primary sponsor from the client organization was seen to be "losing interest" as time elapsed and the window of opportunity within their organization passed.

"The (client) were really having good faith in us but from time to time our documents were so dry and so confining in terms of content that they were limiting their understanding of how we were interacting with them." Int.28.5

"I think during the initiative it was too long and also there were a lot of problems on their side managing... And those are the kinds of things, I think, why these things got worse. (The client sponsor) actually lost interest in his baby which was this (a 
broader implementation of the initiative), and he moved to something a bit different." Int.11.5

The client internal sponsor subsequently left the project, and a new client contact was assigned to the initiative. As a result, the FoaK initiative appeared to "lack an owner" within the client sponsor organization, presenting a challenge for the FoaK initiative team in keeping the client engaged throughout the project. Expectation management was described as an issue in working with this stakeholder, in addition to low visibility of the FoaK initiative within the client organization. The FoaK initiative was found to lose momentum as it progressed, to the point where the client was not prepared to invest further at the end of the FoaK initiative.

"The (replacement) physician that we work with, was interested to some extent, but it was always a burden on his side to help us and work with us, so we could not get a lot of her time. So, yeah. It was challenging, but eventually there was support." Int.11.5

The engagement with the client stakeholder did result in the FoaK initiative team acquiring site and domain experience. There was also market visibility for the resulting FoaK innovation assets through industry accolades, published journal articles and patents that emerged from the initiative. In describing the execution and result from the FoaK initiative, the question of whether the co-creation client was the "right client" for this initiative was expressed in informant interviews.

"I think from a client perspective it was extremely successful. It won some national award... the actual solution turned out to be somewhat different than (the FoaK initiative) had originally envisioned, and that frequently happens in FoaK." Int.22.5 
"We learned a lot about (the domain), in this case, we really wanted to know about how it operates from a patient treatment perspective. We developed some relatively unique analytics that can be and are being reused. We got some pretty good press, at least locally. "Int.22.5

"There were some leading people there and they had data. We found good collaborators there and (our sales stakeholder said) that they were interested. So, altogether this made that to decide. Eventually, I don't think they were ideal clients, but from what we have that was the best that we could find." Int.11.5

Initiative - Products (ACO) Relationship

The emerging assets from the FoaK initiative were planned to be commercialized by the IBM services organizations, within their new analytic solutions portfolio. The relationship between the FoaK initiative team and the products stakeholder portfolio team appeared to be active, with an intention to adopt the innovation that emerged from the initiative for future refinement and support of sales opportunities in the healthcare market.

"There was no real product and real strategy for (the firm) to act in a specific industry (healthcare) market. And it's kind of itself a contradiction to my mind in the FoaK program where they wanted to work with clients." Int.11.5

"(This FoaK) was done before the (healthcare) Centers of Excellence were really put in place. And the healthcare (services) team at the time was also in quite a bit of flux." Int.22.5

During the FoaK initiative, this portfolio was eliminated and replaced with a new business unit (i.e., Watson Health) that would represent market offerings for the healthcare industry. The reorganization required that the FoaK initiative team identify a new product stakeholder portfolio for the commercialization of the asset. 
"It just seems like there was so much going on in the healthcare area and this asset commercialization owner kept moving as (the firm) restructured. They also tried to talk to the Watson people and that didn't go anywhere." Int.07.5

"Actually, when we started (a major strategic research initiative) was exactly on-when the FOAK won... And then what the (firm) started to do is (invest in the new initiative). So, there was a lot of things that happening parallel - which I think create a lot of clutter." Int.11.5

The FoaK initiative team approached the Watson Health business unit to examine the opportunity for them to serve as the new product stakeholder. The FoaK innovation was not found to be a fit for their portfolio due to technical considerations. It was also found that the FoaK initiative team was not effective in engaging and communicating with the new product portfolio team, resulting in early investigations ending between the two groups. With the increased focus on the Watson Health organization and other research initiatives, there was emerging competition for research resources to support these new strategic initiatives within the firm. As such, the potential for further support of the FoaK initiative began to decrease in part due to the low visibility and decreasing momentum. This contrasted with the increasing profile of the new business units emerging in the research organization.

"We had several discussions with (the new potential product stakeholder). We tried to understand from them the architecture. We got some points from them, who should we talk to and when? But they keep delaying us to the next release and the following release and eventually the projects were closed. It sounds like they just deprioritized the FoaK every round. Yeah. It's because they have so many things to do on their side." Int.11.5

"Quite frankly the research team never really seemed to have their (plan) together and never really followed up with any of the things that we asked them to. We tried 
to understand how it would fit in what we were doing. We asked them for additional information about what they were doing, how they were doing certain things. And we never got it, and we went through a very frustrating time with them ... they never did get us answers" Int.40.5

"I think it was really that we didn't have an analytics platform that matched the needs of the healthcare industry (product stakeholder). " Int.22.5

Timing was out of sync... "We were focused on getting our stuff done, and we didn't really have a whole lot of time to look at-- to investigate new things that-- there was a timing mismatch. They were looking for money and funding within a certain time period, and we were not-- we were more focused on executing the previous set of decisions. " Int.40.5

FoaK asset needed further incubation... "I think intellectually and conceptually (the FoaK innovation is) a great idea. I think operationally it's gotten a little bit flaky lately. I would like to see it get itself sorted out so that it's either going to be something valuable going forward or it just ends. Because I think right now it's just confusing the heck out of a lot of people." Int.22.5

Initiative Execution and Performance

During this FoaK initiative, there were changes in the context of the project that were found to impact the initiative's performance. In addition to changes with the FoaK initiatives stakeholders, there is support for an argument that the lower effectiveness of this FoaK initiative team in managing stakeholder engagement also impacted the resulting performance.

Program Flexibility and Support - The FoaK initiative team was found to maintain minimal engagement with the FoaK program team during the project. The inexperience of the FoaK initiative team and their limited network may have benefitted from the additional support 
available through the program. The FoaK initiative team was not found to draw upon the experience and network of the FoaK board or the program management team in helping to resolve issues (i.e., identify new product organization for the asset).

Leadership - The FoaK initiative leadership was also found to be limited in their effort and ability to establish "strategic" stakeholder relationships, as compared to more successful FoaK initiative teams. While this FoaK initiative team was effective in meeting the base requirements of the FoaK program, they were found to be challenged in establishing new relationships with primary stakeholder contacts (i.e., new project leader within the cocreation client, Watson Health as the new product stakeholder). One informant described that the engagement of the FoaK initiative team with this new product organization was a "frustrating process," and that the team "did not appear to be interested in commercializing" their asset.

Stakeholder Engagement - A review of the initiative highlights that there was limited stakeholder engagement throughout the initiative. Co-creation client engagements decreased as the initiative progressed, with a lack of clear ownership on the client side. Engagement with the products portfolio team did not include participation at the client level, and once the unit was disbanded, no progression path was provided for the FoaK initiative team. This raises the question of the strategic value of the initiative initially proposed. Engagement with the sales team was limited to a single sales representative, who upon leaving the organization, impacted co-creation client access for the initiative. Each of these observations suggests that the FoaK initiative leadership was not invested in 
establishing direct relationships with the essential stakeholders linked to the successful execution of the FoaK initiative.

Initiative Value and Momentum - An examination of this initiative highlights challenges in establishing and sustaining the momentum needed to move the project forward. Delays in the initiative execution impacted momentum from within the client stakeholder organization. Thus, the emerging proof points from the initiative appeared insufficient in gaining the attention of stakeholders within the firm when the FoaK initiative team required a new product stakeholder portfolio for the innovation. The FoaK initiative outcomes appeared to demonstrate market value based on the industry and academic acknowledgments received by the innovation. However, no further sales revenues resulted after the completion of this FoaK initiative.

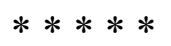

This review of the individual FoaK initiatives reveals several key observations regarding the FoaK initiative process, stakeholder interaction, and the FoaK initiative performance.

All cases studied were found to share the same processes as guided by the FoaK program, and a common set of active stakeholder relationships observed in the execution of each initiative. The profile of primary stakeholder relationships was found to include the FoaK program, the FoaK initiative team, and the initiative stakeholders (i.e., sales, industry, research and products organizations). While these relationships were active, the degree to which stakeholder relationships were aligned and engaged against pre- 
established expectations was found to vary across initiatives. The extent to which these relationships were developed into productive pairings was also found to reflect the initiative performance.

All cases were also found to display comparable process profiles and complexity in their intra- and inter-process interactions. An examination of the effectiveness of each initiative team in executing at various points across their processes and within their stakeholder relationships revealed patterns that distinguish cases of differing performance levels (i.e., "successful" versus "unsuccessful" FoaK initiatives). Also observed is the role of initiative proof points and momentum as a nexus between a FoaK initiative and its related processes.

These findings are illustrated in Figure 8.1, which provides a mapping of process and stakeholder interlock points, and a comparative view of individual FoaK initiative execution effectiveness. These observations will be further discussed in Stage 2 comparative cross-case findings (see Chapter 9). 
m

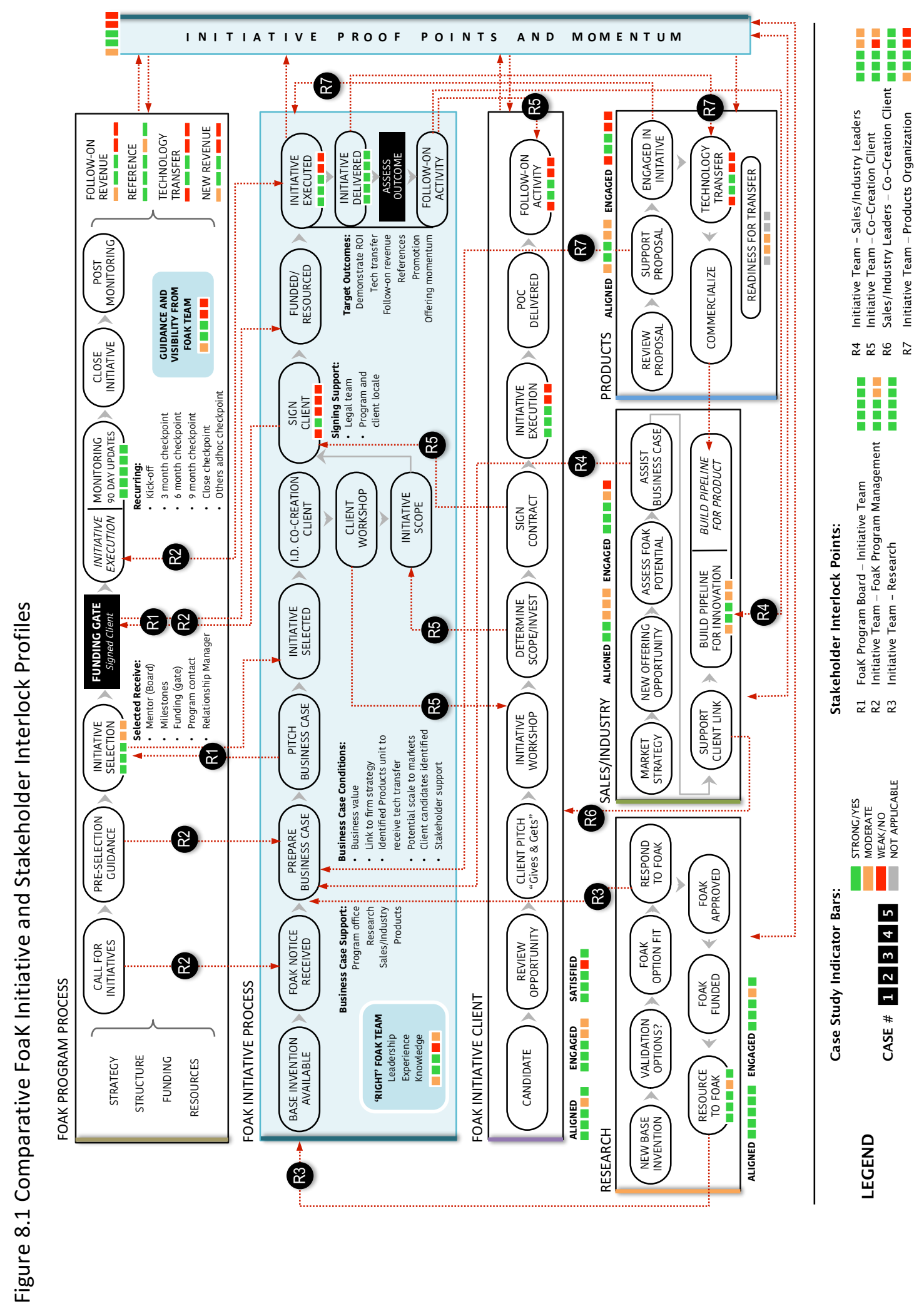




\section{Chapter 9 \\ STAGE 2 FINDINGS AND ANALYSIS COMPARATIVE CROSS-CASE FINDINGS}

Stage 1 analysis examined individual case studies across issues including initiative objectives, stakeholder relationships, initiative execution, and program support. This section presents findings from a cross-case comparative analysis using the FoaK program theory as a guide in examining the mechanisms and outcomes across initiatives of "successful," "unsuccessful," and "typical" levels of performance. This analysis includes discussion of case context and presents an updated program theory reflecting additional relevant mechanisms identified in the course of the analysis.

This chapter begins with an examination of newly identified case mechanisms from the analysis, and a discussion of case context as it relates to co-creation of innovation initiative performance. This is followed by a review of the FoaK firm and program-level mechanisms, which were found to be common across the cases studied. The cross-case comparative analysis is then presented, examining initiatives types based on performance, and an evaluation of initiatives against the program mechanisms. This stage of analysis concludes with a summary mapping of the mechanisms and their place in the knowledge acquisition method system, the linkages and outcomes, and the themes emerging as impacting initiative performance (Section 9.6).

\subsection{Program Theory: Additional Case Mechanisms (M14-M18)}

Critical realist-based analysis of case findings seeks to identify the principal mechanisms that describe the phenomenon being observed within the context in which they 
exist. The definition of a program theory and its mechanisms also suggests that in the analysis of the cases being studied, additional mechanisms will be identified that help better explain what is being observed, may impact other mechanisms, or may reflect the impact of the context on observed phenomena (Pawson and Tilley 2004).

In addition to the thirteen mechanisms that were included in the program theory describing the FoaK program (see Chapter 7), five additional mechanisms (i.e., M14-M18) were identified, each found to contribute to the outcomes observed in the study. These additional mechanisms describe the effect of changes in the organizational structure and the role of stakeholder behaviour in practice on the initiative, relative to their stated intentions (i.e., stakeholder focus, motivation, expectation, and investment). Stakeholders, in this case, refer to each of the principal groups engaged in the FoaK initiative along with the focused initiative team, including the client stakeholder, sales and industry stakeholders, research stakeholders, and the product organization stakeholders. This section will describe these five mechanisms, which become extensions to the initial program theory.

\section{M14 Shifting Organizational Strategy}

An examination of the cases in the study reveals the impact of changes in the environment within which the FoaK program and its initiatives operate. In several cases, we saw changes in the organizational strategy resulting in product portfolios being reorganized, and new business units being created to support a new firm strategy. These changes in organizational strategy were found to have impacts on investment focus and stakeholder focus. For example, in Case 4, the formation of a new Internet of Things 
business units represented an increased focus by the firm supporting the initiative that was underway. The FoaK initiative team was successful in transferring their work from the disbanded product team to this new organization, which ultimately accepted the transfer of the FoaK assets into their portfolio. In Case 5, the change in organizational strategy resulted in a reorganization of its healthcare portfolio, which in turn was eliminated and replaced by a new business unit focused on assets supporting these markets. In this case, the FoaK initiative team was unsuccessful in securing a new product commercialization owner for its FoaK innovation. It was found that shifts in the organizational strategy, in turn, impact outcomes through their influence on initiative momentum and performance.

Impact of reorganization: "We were, I'd say, in the middle of a transition as you can expect... So, in other words, the catch organizations, they're going through a major change." Int. 19.1

Momentum in new strategic area: "Yeah, it was very early. It was very early. At that time (the firm) didn't have a Internet of Things (IOT) yet. That's why you see many of the stakeholders had a new job moved around in the company. ... Another thing, at that time, is quite early but is already getting attentions at the corporate level corporate leadership level - so the company started to put together some technology capability around Internet of Things.... IoT started to get attention at that time. Definitely is becoming a major strategy (in the firm). " Int. 44.3

Impact of reorganization: "Initial plan was that the (services) solution centre for travel and transportation that was back then in India would be the catcher and we had the design architect that worked with us in the initial stage. But then during the project this entity in (the firm) disappeared so it was, I don't know, reorganized so it wasn't there anymore so the initial planned catcher was gone. And then we were struggling and looking for alternative catchers. " Int. 25.4

Impact of reorganization: "It just seems like there was so much going on in the healthcare area and this asset commercialization owner kept moving as (the firm) 
restructured. They also tried to talk to the (a new catcher organization) and that didn't go anywhere." Int. 07.5

Internal competition from acquisition strategy: "Yes, and if you look at overall (our firm) (the firm) has been shopping for innovation outside by acquiring companies." Int. 08.1

Internal partnering strategy: "Yeah, and so we contacted (the local research team) it started from a-- so you can think of it like a political reason. We wanted a joint project with (that South American lab). We wanted to open collaboration opportunities between the two labs, and this was a good use case for that." Int. 08.1

\section{M15 Stakeholder Focus in Practice}

While stakeholders may begin a FoaK initiative committed and with the intention to be engaged throughout the project, this intention may change at the time of execution. As seen in Case 5, the initiative began with an aligned and enthusiastic internal champion within the client stakeholder organization. However, as the project began to take longer than had been anticipated, the client stakeholder was found to become less focused on what was required to deliver the initiative, which included changing resources in and out of the project. This resulted in an increased effort required by the FoaK initiative team in progressing the project and keeping the client engaged. It is expected that as stakeholders lose focus in the practice of the initiative, there will be an impact on the co-creation initiatives momentum and performance.

Strong client focus on initiative: "The issues that always happen, right? That I think that make or break a project like that, in addition to what's already mentioned, is you have to have some sort of commitment from the client's side, right? So you need data, you need people inside that want to work to collect the data and provide data. You want to validate you have it, you have the commitment that people are going to be 
participating in the meetings. So there is an implicit time commitment from the partner's side that is very important to happening and this is not easily accomplished. Normally the project is not successful. In this case, we had that commitment that they think-- not only the commitment, but later it becomes kind of an excitement, because people, they see the value that you're bringing to the departments, and this becomes really a critical point for the success of this project. And I think in this project, they got that." Int. 48.1

Strong client focus on initiative: "Because with (our client), in balance, we found a great partner. That is they were willing to-- especially, a couple of people on their side were able to willing to provide data or connect us with different stakeholders. So that, definitely, was important." Int 16.2

Alternative agenda: "A lesson we already knew, but it's not always wise to tie a FoaK to a larger deal because you have to wait for that larger deal to get worked out, and usually, you don't have enough time for that. Sometimes the FoaK gets lost in a larger deal. I don't know if that was the case here, but the fact that there was this other deal that needed to be worked out just made it impossible for us." Int 05.3

Initiative focus: "The main challenge was that once the client signed and we sat together to determine the scope, it became very different from what we initially proposed. And so, we had to-- because, somehow-- in the end, we delivered two solutions. One that was developed before the clients got deeply involved and then another one with the client, and they were pretty disconnected. That process to define-- for them to understand what we're after and for us to understand what actually their paying points were and then to find a good compromise, that was challenging." Int 25.4

\section{M16 Stakeholder Motivation in Practice}

As with a change in stakeholder focus during an initiative, a change in the stakeholder's motivation in practice is also expected to have an effect on the co-creation initiatives momentum and performance. This describes a scenario where a stakeholder that has agreed to participate in a FoaK initiative and has demonstrated interest in co-creating 
the innovation, has displayed a level of motivation for the initiative that is different than what was anticipated by the FoaK initiative team at the outset. The level of motivation in practice may be greater or less than was expected, both of which potentially impact the initiative momentum and performance.

In Case 1 it was found that the product stakeholder was motivated by access to the client stakeholder and potential near-term revenue, which influenced their intention to participate. In Case 2, it was found that the client stakeholder's initiative leader was highly motivated in the success of the project, and worked towards ensuring all access and team requirements were met, as well as promoting the FoaK initiative's success within the client firm, contributing to the building and sustaining momentum. In three cases (i.e., Case 1, 3, 4), each of the FoaK initiatives was associated with a parallel sales cycle with the target client stakeholder. In all cases, the motivation to complete the sales transaction, impacted the execution of the FoaK proposal, resulting in delays.

Client stakeholder motivation: "The whole point of the optimization was to lower their overall costs. So if anybody in the company can lower there at all costs. I guess we are viewed positively by the organization. So, yeah, he absolutely saw that. He wasn't the most political-- not that he wasn't savvy, he's absolutely savvy, but he wasn't the most political guy on the planet. But he certainly did see the value of doing well by his organization." Int. 46.2

ACO motivation for collaboration: "Throughout the project (the ACO team) were involved. I can't remember, I think it was after the project officially ended but when we were (engaged in) some of the follow-on work. You know, we did a workshop and after the commercialization owner presented his plan. It wasn't just the research team thing. This is the plan. It was the owner saying, 'Here's what I'm doing here and here's-'So it was a very strong relationship." Int.04.2 
Motivation in maintaining an asset: "Which is a pity for other FoaK projects, as you probably know because if these things are aging and not being up to the mark anymore, then I think the chance of reselling them is even lower, right?" Int. 40.5

\section{M17 Stakeholder Expectations in Practice}

As new FoaK initiatives take shape, a FoaK initiative team's early engagement with their stakeholders (e.g., client, products, sales, industry) is centred on establishing their roles and expectations in the co-creation effort. However, it was found that in practice, the effectiveness of expectation setting at the beginning of a FoaK initiative may differ from the actual stakeholder expectations during the execution of the initiative. Differences between the expectations desired from stakeholders and the actual expectations that stakeholders are working with create a gap that must be managed by the FoaK initiative team. The effectiveness with which expectation gaps are managed with stakeholders is expected to impact initiative momentum and performance.

In Case 1, the product stakeholder expected that they would only transfer the asset to their portfolio once a sales pipeline had been established and the business case developed. The FoaK initiative team was not aware of this expectation until later in the process which resulted in the asset not being transferred. In Case 4, internal changes within the client stakeholder organization resulted in a shift of the FoaK initiative scope and participants in the initiative. At the completion of the prototype, the client stakeholder was not satisfied with the scope of the use case and the usability of the prototype, which they described as not being as refined as a final product. These expectations were different than what was set at the start of the initiative and prevented the asset being adopted further within the client stakeholder firm. 
Client stakeholder expectations: "All of this comes down to, in my mind, continuing to manage-- and manage sounds like you're in control, but you're not, right? But doing the best you can to help manage the relationship with the client because you can drive it towards success. And I think that all too often we acquiesce to a client demand, and I think that you have to make sure you've got a relationship where clients understand it's messy, understand that it could dead end, understand that it might require more." Int.17.4

Asset transfer expectations: "The criteria they shared with us was exactly what they told me about. They told us that they need to have more accurate information about the marketing team to plan that in the next phase of the offering." Int.13.1

Asset transfer expectations: "They were really counting on a sales deal with (the client) to help kind of-- I think to kind of fund some of the development for the hardening of the assets. And I think when that didn't happen, it really knocked them off track, and I don't believe they were able to find another client to do that work with." Int.03.1

Client stakeholder expectations: "Looking back, what I think was one of the main problems is that we built didn't look nice. We focused a lot on the underlying-- on the analytics under the hood, and we had a very poor, I would say, user interface. The solution was not-- didn't look nice, wasn't nice to use. And even though we went into a, I would say, nine-month evaluation after the FoaK project where we gave them an evaluation licence-- they could use the tool. ... I think they hardly ever did it because they were not-- the usability aspect was not there... In a way, they did not understand that this is a First of a Kind project and that this is coming out of research." Int.25.4

\section{M18 Stakeholder Investment in Practice}

In each FoaK initiative, there is also an expected level of stakeholder investment into the initiative, that is set at the start of the project. In cases where the actual investments contributed by a stakeholder (e.g., resources, time, data) differs from the expectations set at the start of the initiative, FoaK initiative teams may be required to adjust the execution 
of projects, impacting the initiative's momentum and performance. In Case 2, the client stakeholder organization demonstrated significant commitment and investment in the initiative. They provided office space and internal credentials for the FoaK initiative team and invested in their executives travelling to the IBM Think labs for workshops. This level of investment helped build momentum for the initiative and strengthen the working relationship and collaboration between the two firms. In Case 4, resources from research that were committed to the project were swapped out during the initiative due to internal priorities. This impacted the FoaK initiative team's efficiency in executing the project.

Client investment: "We were able to analyze data to some extent and to show (the client) some results, but I think when we showed this result, it was-- there was not enough interaction. There was not enough involvement from their side, and it requires also a lot of FoaKs from our side to adapt to their needs." Int.11.5

Research function resource investment: "The (FoaK program) are only asking for (resource)names, people working on the project and also... So as long as we put four names there-- if you got four PY (person years) advisors that's fine. But if internally, these four PYs are working only one day per week on the project, then this is not four PYS. So, that's what I mean with more strict reporting almost at an hourly basis because then they would have full clarity how much effort research is really putting into this project. And I think this clarity is needed." Int.25.4

Based on this analysis, the initial proposed FoaK program theory was extended to include these additional mechanisms for the cross-case analysis study (see Table 9.1). 
Table 9.1 Updated FOAK Program Theory

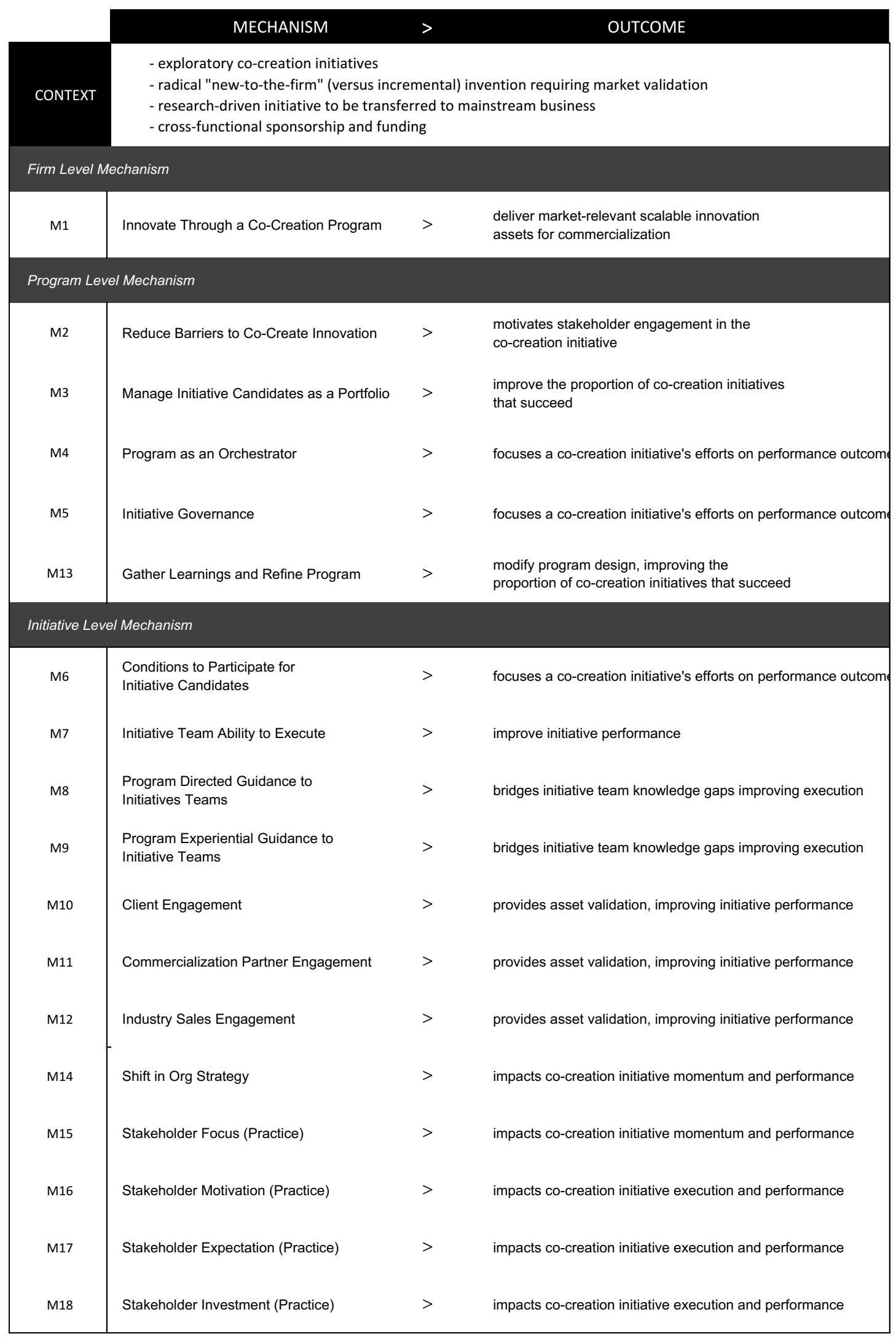




\subsection{Case Study Context}

The context in which each FoaK initiative is executed must be considered in the analysis of the observed phenomena. As supported by the critical realist tradition, the objective of the case analysis in this study is not to answer the question, "When does the program work?" Rather, it must be recognized that co-creation of innovation methods and programs are open systems that are complex, and that the context in which they operate must be considered when developing theory. The question to answer in this case is "What works when, under which conditions, for whom?" (Pawson and Tilley 2004).

For this study, two levels of context were considered in this cross-case analysis, common and initiative specific. The section will discuss the common context shared across all cases, and the case specific context variations which will be referenced in the analysis.

\subsubsection{Common Case Context}

To help better explore the research questions in this study, the research design controlled a number of FoaK initiative attributes to help focus the analysis. As such, there is a common shared context across all cases (i.e., initiatives) examined in this study.

All cases examined were approved for FoaK program funding between the years of 2011 and 2013. During this time there was stability in the program structure, board membership and roles, FoaK program processes, and funding parameters. All FoaK initiative candidates that came through the program and this time were subject to a similar decision-making dynamic and set of criteria. Also, each of the FoaK initiative teams worked directly with the US-based FoaK program management team, and operated in English. All of the FoaK 
initiatives studied were approved for funding, each met all the criteria to participate in the program, with some initiatives having required refinement in their initial proposal.

Each of the FoaK initiatives examined in this study focused on the analytic application domain and represented radical new-to-the-firm innovations. This introduced initiatives with similar levels of complexity and technical considerations for innovation development and transfer internally. Also, analytics was a primary strategic imperative for the firm, ensuring that each of these initiatives was considered to support a strategically relevant agenda at a minimum. Finally, each of these initiatives worked with client stakeholders that were leaders in their industries, either nationally or internationally. All client stakeholders are large enterprises that had the means to participate in a co-creation initiative.

\subsubsection{Case Specific Context}

Case specific context is described by four principal initiative attributes: the industry of the FoaK initiative use case and client stakeholders, the distribution of FoaKclient initiative teams geographically, the experience of the FoaK initiative team and leadership (i.e., PI), and the product stakeholder organization maturity. All of these contextual attributes are summarized for each case study (see Table 9.2), and are described as follows.

Industry Each case examined represented analytic application innovations for different industries. Each of these industries in turn represented varying levels of innovation maturity and stability external to the FoaK initiative, requiring consideration of the impact on the execution of the project by the FoaK initiative team. 
Initiative Location The client stakeholders within each innovation are also described by their geographic location relative to the lead research lab that forms the FoaK initiative team. Cases 2, 3, and 4 represented contexts where the primary FoaK initiative team was local to the client stakeholder initiative team. The remaining cases were

Table 9.2 Summary of Case-Specific Context

\begin{tabular}{|c|c|c|c|c|c|}
\hline & "TYPICAL" INITIATIVE & \multicolumn{2}{|c|}{ "SUCCESSFUL" INITIATIVE } & \multicolumn{2}{|c|}{ "UNSUCCESSFUL" INITIATIVE } \\
\hline & Case 1 & Case 2 & Case 3 & Case 4 & Case 5 \\
\hline \multicolumn{6}{|l|}{ Common Case Context } \\
\hline Initiative Approval Date & 2013.06 & 2013.1 & 2013.12 & 2011.08 & 2012.02 \\
\hline FoaK Initiative Status & \multicolumn{5}{|c|}{ Approved by FoaK Board; funded; FoaK initiative completed } \\
\hline FoaK Solution Domain & \multicolumn{5}{|c|}{ Analytics Application - similar solution complexity, architecture and process considerations in innovation activity } \\
\hline FoaK Program Stability & \multicolumn{5}{|c|}{ High - Common FoaK Program (i.e., framework, process, board construct, funding levels and criteria) } \\
\hline Firm-Level Strategic Relevance & \multicolumn{5}{|c|}{ Yes - period of common strategic priorities for the Firm (i.e., Analytic, Industry Solutions) } \\
\hline FoaK Innovation Type & \multicolumn{5}{|c|}{ radical new-to-the-firm innovation } \\
\hline \multicolumn{6}{|l|}{ Case-Specific Context } \\
\hline FoaK Initiative Industry & $\begin{array}{l}\text { Natural Resources } \\
\text { (Mining) }\end{array}$ & Retail & Automotive & $\begin{array}{c}\text { Travel \& Transportation } \\
\text { (Rail) }\end{array}$ & Healthcare \\
\hline FoaK Initiative Team Location & $\begin{array}{l}\text { Distributed FoaK Team } \\
\text { Client Team: } \\
\text { South America } \\
\text { Lead FoaK Team: } \\
\text { Middle-East } \\
\text { Supporting FoaK Team: } \\
\text { South America } \\
\text { FoaK Program Team: } \\
\text { United States }\end{array}$ & $\begin{array}{l}\text { Co-Located FoaK Team } \\
\text { Client Team: } \\
\text { United States } \\
\text { Lead FoaK Team: } \\
\text { United States } \\
\text { Supporting FoaK Team: } \\
\text { United States } \\
\text { FoaK Program Team: } \\
\text { United States }\end{array}$ & $\begin{array}{l}\text { Co-Located FoaK Team } \\
\text { Client Team: } \\
\text { Asia-Pac Country A } \\
\text { Lead FoaK Team: } \\
\text { Asia-Pac Country A } \\
\text { Supporting FoaK Team: } \\
\text { not applicable } \\
\text { FoaK Program Team: } \\
\text { United States }\end{array}$ & \begin{tabular}{|} 
Co-Located FoaK Team \\
Client Team: \\
West. Europe Country A \\
Lead FoaK Team: \\
West. Europe Country A \\
Supporting FoaK Team: \\
not applicable \\
FoaK Program Team: \\
United States
\end{tabular} & \begin{tabular}{|} 
Distributed FoaK Team \\
Client Team: \\
West. Europe Country A \\
Lead FoaK Team: \\
M iddle East \\
Supporting FoaK Team: \\
West. Europe Country A \\
FoaK Program Team: \\
United States
\end{tabular} \\
\hline FoaK Initiative Team Experience & $\begin{array}{c}\text { FoaK Leader: } \\
\text { moderate experience } \\
\text { tactical approach } \\
\text { FoaK Team: } \\
\text { moderate experience }\end{array}$ & $\begin{array}{c}\text { FoaK Leader: } \\
\text { very experienced } \\
\text { previous success } \\
\text { strategic approach } \\
\text { FoaK Team: } \\
\text { moderate experience }\end{array}$ & $\begin{array}{c}\text { FoaK Leader: } \\
\text { very experience } \\
\text { previous success } \\
\text { strategic approach } \\
\text { FoaK Team: } \\
\text { moderate experience }\end{array}$ & $\begin{array}{c}\text { FoaK Leader: } \\
\text { low experience } \\
\text { tactical approach } \\
\text { FoaK Team: } \\
\text { low experience }\end{array}$ & $\begin{array}{c}\text { FoaK Leader: } \\
\text { moderate experience } \\
\text { no previous success } \\
\text { tactical approach } \\
\text { FoaK Team: } \\
\text { low experience }\end{array}$ \\
\hline $\begin{array}{l}\text { Product Stakeholder Portfolio } \\
\text { Maturity } \\
\text { (early stage/mid stage/established) }\end{array}$ & $\begin{array}{l}\text { early stage portfolio } \\
\text { portfolio in development }\end{array}$ & mid-stage portfolio & $\begin{array}{l}\text { Portfolio 1: restructured } \\
\text { Portfolio 2: early stage } \\
\text { portfolio in development }\end{array}$ & $\begin{array}{l}\text { Portfolio 1: restructured } \\
\text { Portfolio 2: not applicable }\end{array}$ & $\begin{array}{l}\text { Portfolio 1: restructured } \\
\text { Portfolio 2: early stage }\end{array}$ \\
\hline
\end{tabular}


conducted by distributed FoaK initiative teams, where the lead researchers were located in a different country from the client stakeholder initiative team. Distributed teams introduce another level of complexity regarding language and culture that the FoaK initiative teams must manage.

FoaK Initiative Team Experience Each of the FoaK initiatives is conducted by teams with varying levels of experience. Experience, in this case, is described by both FoaK initiative team leaders skills and ability, and the previous FoaK project experience of each member of the team. The experience of a FoaK initiative's team leader and its researchers represents a level of base knowledge coverage coming into a project. The FoaK team's knowledge represents varying levels of understanding of the program requirements as well as what is needed to execute a FoaK initiative successfully. This includes the degree to which a FoaK initiative leader demonstrates business acumen and a strategic approach to project execution. Each of the initiative teams represent varying levels of knowledge coverage and gaps in the areas believed necessary for FoaK initiative teams to be effective. Product Stakeholder Organization Maturity A case initiative is also described by the maturity of the product stakeholder that has agreed to sponsoring and potentially commercializing the FoaK innovation asset. The cases in the study represent product organizations ranging from established and stable to newly formed product portfolios that are in the early stages of defining their organizational priorities. Contextually, product organizations maturity represents the stability of the relationship between the FoaK initiative team and the future asset commercialization owner, including their focus, motivation, and available resources. 


\subsection{Case Study Performance - FoaK Initiative Profiles}

Each of the cases included in this study are characterized by their level of performance, classified as either a "successful," "unsuccessful," or "typical" initiative (definitions in Section 5.4.5). Moreover, the objective of this study is to gain greater understanding into the FoaK program as a knowledge acquisition method, and how the attributes of the system interact and impact FoaK initiative performance. An examination of case studies by their class of performance reveals patterns related to initiative success. In this section, the patterns observed are presented as a precursor to the cross-case analysis of FoaK related mechanisms, outcomes, and context.

\subsubsection{Successful Case Performance}

Successful cases are defined as FoaK initiatives that have demonstrated customer satisfaction and referenceability, follow on revenue upon completion of the FoaK initiative and the successful transfer of the FoaK initiative innovation into a mainstream product portfolio. In this study, Case 2 (Retail) and Case 3 (Automotive) were defined as successful FoaK initiatives.

Successful cases were found to be executed by experienced FoaK initiative teams and led by strong initiative leaders (i.e.,primary investigators). Based on previous experience, these teams represented a good base of knowledge to execute their initiatives, and an understanding of what to expect through the course of their project. The initiative leaders were found to be proactive in the formation of stakeholder relationships, and strategic in their interaction with various stakeholders at all stages of the FoaK initiative process. In the course of executing a FoaK initiative, PIs were also found to shelter their 
initiative teams from the complexities of the FoaK program, having more junior team members focus on the specific initiative execution tasks.

These FoaK leaders were described as having strong business acumen and a focus on building trust with their stakeholders. They were also found to have broader internal networks than less experienced leaders, and were more proactive in their relationship with the FoaK program management team. These FoaK leaders demonstrated the importance they place in building and maintaining momentum throughout the project, and were also inclined in promoting progress and proof points as early and as frequently as possible throughout the course of their initiative. The FoaK initiative leaders in successful projects also invested early in the process to set the right expectations with their stakeholders. This included continually working towards ensuring that their stakeholders remained engaged throughout the project.

Successful cases were also characterized by client-FoaK initiative team relationships that were engaged, and that demonstrated confidence in the FoaK team. These cases included client stakeholders that were committed, invested, and motivated in participating in the co-creation of innovation initiative. The client stakeholders were found to assign strong resources within their organization to work as a liaison with the FoaK initiative team. Clients were also found to invest in the relationship and initiative beyond the initial request for funding of project management resources. These joint teams look to predetermine compelling events for the initiative to target, which helped build focus and momentum for initiative proof points. When success was seen during the initiative, the client stakeholder leads were found to promote the initiative within their own stakeholder community to help maintain the interest and engagement of their organization. Once again, 
the focus of PIs was proactive in nurturing the client stakeholder relationships at all levels, from the sponsor to the initiative lead.

It was also found that the proof points that emerge from the client engagement served as the fuel to further build momentum around the initiative and maintain the attention and interest of stakeholders including the sales, industry, and products organizations. The momentum that builds during the innovation exploration with the client, is found to trigger sales activity and pipeline development for follow-on revenue. These momentum building proof points include clients expressing confidence and achieving new innovation milestones. These contributors to momentum also serve as market validation for the product stakeholders that have committed to reviewing the innovation for their portfolio upon completion.

A characteristic of successful case initiatives was also how the relationship with product stakeholders was established and maintained, ultimately resulting in the transfer of the FoaK initiative asset into the product's portfolio. In both cases, the FoaK initiative PI was found to have a previous working relationship with the product stakeholder development organizations. They were active in engaging these product stakeholders early in the FoaK initiative process and negotiated the participation of these teams both at the proposal stage and throughout the FoaK initiative process. This was described as allowing for visibility into the product stakeholder organization early in the process, and presented the opportunity for bidirectional learning on the business use case. This included access to the FoaK initiative team into the technical considerations for successful transfer of their asset to the products organization. The close working relationship with the product stakeholder team helped to expose proof points early in the innovation process, which 
helped build their confidence in the innovation, and further contribute to the momentum for technology transfer.

A common issue observed in the successful case studies was the degree of readiness of the product stakeholder organization to accept the FoaK initiative assets into their portfolio. The recurring theme describes that FoaK innovation assets are not ready for market upon completion of a FoaK initiative. There is an incubation period required between the FoaK initiative and the commercialization process, suggesting the need for a technology transfer plan to improve the success rate at this stage in the cycle. However, the current boundary of the FoaK program does not extend beyond the successful transfer of a FoaK initiative asset into the business.

\subsubsection{Unsuccessful Case Performance}

Unsuccessful cases are defined as FoaK initiatives that did not demonstrate performance across all the measures considered by the FoaK program. They typically were successful in completing the project with some level of reference from the client stakeholder, but did not show any follow-on revenue or a transfer of the FoaK initiative asset into the product stakeholder portfolio. In this study, Case 4 (Travel/Rail) and Case 5 (Healthcare) were classified as unsuccessful FoaK initiatives.

The unsuccessful cases in this study were executed by FoaK initiative teams that were described as inexperienced in FoaK, and were led by FoaK leaders that were either inexperienced or unsuccessful in their previous FoaK experience. Less experienced teams were found to require more support during their FoaK initiatives, to bridge the knowledge gaps that they had related the FoaK program, its processes, engaging internal stakeholders, 
and in their work with the client stakeholder. The FoaK initiative leaders on these teams were found to be tactical in managing their stakeholder relationships. It was observed that these teams tended to work towards satisfying program requirements versus proactively guiding their stakeholder relationships towards the initiative's "end game" of success on all three performance measures. These teams were found to be less connected within their firm beyond the research organization and possessed less client engagement experience.

Although these teams could benefit from the FoaK program assets and guidance available to them as approved projects, these FoaK initiative teams were not found to proactively engage with the program management team to fully exploit the resources available to them. FoaK program management described these projects as providing less transparency to them, with their insights typically limited to the quarterly scheduled updates. These teams were not found to be effective in building and promoting momentum within their initiatives, which impacted the level of engagement with their stakeholders in sales, industry, and products organizations. These FoaK teams were also found to struggle to navigate changes in their FoaK initiatives during execution, which resulted in delays, loss of interest by stakeholders, and their ability to transfer the technology to the product organization. These teams were also challenged in their ability to establish clear expectations with their stakeholders (e.g., Case 4 client, Case 5 products), and keeping them engaged throughout the course of the initiative.

The unsuccessful FoaK initiatives were characterized by client stakeholder relationships that were less engaged with the FoaK initiative team, and appeared to suffer from changes in commitment and expectation throughout the course of their initiative, impacting initiative momentum. In both unsuccessful cases, clients initially demonstrated 
motivation and commitment early on in the process. However, both subsequently resulted in primary stakeholders transferring the initiative to someone else internal to their organization, resulting in less clarity and introducing new complexity to the initiative. Neither case identified critical milestones that would demonstrate value to the client stakeholder organization, a catalyst that was found to help build momentum for the more successful initiatives. At times, these cases were found to suffer from a level of disorientation during their execution. This in part could be attributed to the FoaK team's inexperience, tactical mindset, and the readiness of less experienced teams in overcoming the environmental challenges that were encountered during initiative execution.

The lack of momentum and clarity experienced by these FoaK initiatives was found to have an impact on the level of client stakeholder interest and commitment to the FoaK initiative after the completion of the FoaK project. In both cases, neither client stakeholders were found to make any follow-on investments in the innovation, as was seen with the successful FoaK initiatives. This in turn did not provide the necessary proof points needed to motivate the other stakeholders links to the initiative (i.e., sales, industry, and product) for the related follow-on activity after innovation delivery.

The sales and industry stakeholders were found to play a similar role as seen with the successful case studies. These groups helped the FoaK initiative in developing the initial FoaK initiative proposal, identify potential co-creation clients for the project, and in promoting the initiative with other stakeholder groups within the firm. However, unlike the successful initiatives, the stakeholders were found to be engaged but not as motivated in pipeline development. While both unsuccessful initiatives reported having sales pipelines for the FoaK initiative assets, neither reported any new sales opportunities or follow-on 
revenue for their assets. Both initiatives argue that the assets from their projects are available to the market for sale. However, they are also described as inactive and as having potentially lost their market relevance due to the lack of investment since the completion of their projects. It is believed that the absence of FoaK innovation momentum, the lack of compelling proof points emerging from the client stakeholders, and no path for the asset to be commercialized in a mainstream portfolio, have all contributed to the sales and industry stakeholders becoming disengaged with these FoaK initiatives.

Both of the unsuccessful cases were impacted by reorganizations in the products organization, resulting in the loss of their initial product stakeholder targeted for commercialization of the innovation asset. Part way through their initiatives, both initiative teams faced the need to identify a new product stakeholder organization to receive their asset. It was found that the inexperience and approach of the FoaK initiative leaders, the lack of internal networks, and the absence of compelling proof points for the initiative all contributed to an inability to find a replacement product stakeholder portfolio. It is believed that this part can be explained by the potential perception that the FoaK initiative and its innovation is not strategic enough based on the lack of momentum.

"(I feel) there was no real product and real strategy for (the firm) to act in our specific industry market. And it's kind of itself a contradiction to my mind in the FoaK program where they wanted to work with clients...there was no real product that was suitable for what we tried to do in research in healthcare." Int.11.5

As seen in Case 5, the FoaK initial leader was not effective in convincing the new healthcare business unit there was a compelling path for their FoaK initiative assets as part of their portfolio. This was in part due to the asset, the timing, and the effectiveness of the engagement with this product's replacement candidate. 


\subsubsection{Typical Case Performance}

Case 1 (Mining) represents a FoaK initiative of typical performance, having had success with the client stakeholder and an active pipeline with potential for future followon revenue. In this case, the resulting FoaK initiative asset was not successfully transferred into the mainstream portfolio. An examination of this case finds patterns consistent with those observed in either the successful or unsuccessful cases described, further supporting the findings presented.

The FoaK initiative team, in this case, was a distributed team across two research labs and two geographies. The primary FoaK team that was responsible for the base invention used in the initiative was located in the Middle East, whereas the client stakeholder and extended FoaK initiative team were based in South America. While the primary FoaK initiative team had previous experience with the program, this FoaK initiative would be a first for the client country. Although the team situated with the client stakeholder was inexperienced, they leveraged the program assets to ramp up and worked closely with the primary initiative team in the Middle East. The FoaK initiative leadership and investment in face-to-face interaction with the client contributed to a strong working relationship and effective execution of the project itself. However, the inexperience with the program and the tactical approach taken by the FoaK initiative leadership role were found to contribute to delays in the progress of the initiative. Both sides of these interactions were seen in some form across successful and unsuccessful FoaK initiatives described.

During the execution of this FoaK initiative, two things were observed. First, the FoaK initiative team was successful in establishing a strong working relationship with an 
engaged client team, which in turn resulted in a co-creation innovation reflecting the importance to both the firm and the client stakeholder. Second, even with this strong working relationship in place, no significant milestones were found to be identified or targeted as was seen in the successful case studies. While progress was made in this initiative, and the client expressed satisfaction with the innovation that emerged during the project, momentum was found to remain steady. The proof points from the FoaK asset and the momentum from the project presented other stakeholders inside the firm with the opportunity to build on the progress, as was seen with the active pipeline of new sales opportunities. However, there was not sufficient momentum and market validation of the asset to satisfy the product stakeholder, which resulted in a failed effort to transfer the innovation into their portfolio.

The tactical challenges of this FoaK initiative team were also reflected in the relationship with the product stakeholder engaged. While the initiative was successful in securing a product stakeholder, the interaction with this team was limited to early endorsements supporting the funding process. There was little to no engagement with this team beyond the formal checkpoints throughout the initiative. This low level of engagement was found to be a contributing factor to the misalignment in the expectations with the product stakeholder team. It was not until the end of the FoaK initiative that the program team was aware that they did not meet the product team's criteria for accepting the transfer of the asset into the products portfolio. This further supported the observations from successful cases where proactive FoaK initiative leadership that invested in setting expectations and maintaining engagement throughout a FoaK initiative were found to achieve better performance results than teams that did not do so. 
Similar to the unsuccessful cases examined, this initiative also experienced an external impact on the client's readiness to invest further in the FoaK initiative at the end of the project. A downturn of the mining industry resulted in the client's decision not to move forward with the FoaK innovations, despite the positive feedback on the asset benefit. In the end, the decision was ultimately made to examine implementing a homegrown solution for the same use case. This demonstrated the FoaK team's effectiveness in extracting the necessary knowledge from the client, which in turn was countered by an insufficient understanding of how to carry the initiative beyond the pressures introduced by the market downturn. This highlights the impact of the FoaK initiative team experience in successfully accessing the external knowledge necessary for the co-creation activity, but ineffectively navigating unforeseen complexities from the environment.

The next section will present the findings from the perspective of the program theory mechanisms, context, and outcomes across the case studies examined.

\subsection{FoaK Firm and Program Level Mechanism Findings (M1-M5, M13)}

An examination of the findings related to mechanisms at the firm and programlevel did not highlight any case specific differentiation from the observed outcomes. The mechanisms that were found to be common across all cases studied are described below.

\subsubsection{M1 Innovate Through a Co-Creation Program}

All cases in this study were approved from the same FoaK program, during a period of consistency and stability. As described in the discussion on common context, these cases were approved during a period of three years where the FoaK program framework, 
processes, funding criteria, and level of funding were consistent for all candidates presented to the FoaK board during that time. The FoaK board was stable with minor changes in its membership, including a consistent number of board members representing the functional areas of the firm. The FoaK program management team remained unchanged during the approval period, and the level of co-funding for the program by the research and sales organizations was relatively consistent over this time. In all cases, a new-to-the-firm radical innovation was delivered through the FoaK initiative, and available for transfer to the product organization for commercialization.

Findings from the cases studied highlighted that as FoaK initiative teams entered their projects with an initial invention at various levels of conceptualization, with Case 2 as an exception. The FoaK co-creation program provided a structure, credibility, and resources needed to explore the innovations in the market. The work with co-creation clients was found to result in changes of scope, priorities, definition of new use cases, and validation within a client environment, all delivering assets to the firm for commercialization.

“(The FoaK program) probably lent a, I don't know, I don't want to say a validity exactly to the research to the customers, but kind of that being able to say ... we're going to work with you as a part of this program and here's our checkpoints. And it makes it feel more real than just going to a client-- a researcher going to a client and saying, 'Hey, you want to do a couple things?' It lends a-- I'm not thinking of the right word for that.... Credibility. That's the word that was on the tip of my tongue" Int. 46.2 


\subsubsection{M2 Reduce Barriers to Co-Creation Innovation}

An examination of the FoaK program as it relates to each of the cases studied support the proposed mechanism that the FoaK co-creation of innovation program reducing barriers to the co-creation process motivates stakeholders to engage in the initiative. The use of a FoaK program to enable co-creation of innovation initiatives was found to reduce barriers to partnering initiatives often faced by collaborating teams including funding, initiative credibility, and a framework for initiative execution.

"The program enables all this thing to come together, right, to drive and give innovation for the client. For (our company) and for the client." Int.19.1

Inititiative funding was described as one of the primary reasons why many stakeholders were interested in participating in FoaK initiatives. The funding for client stakeholders represented a perceived reduction in risk while exploring solutions to business problems being faced by their organizations. For the research and product stakeholders, funding through the FoaK program provided a vehicle to explore new radical innovations, during a protected period of time, where alternative funding models would not support the necessary focus and uncertain outcomes.

"There is only one method, to be completely honest, to make a successful offering, right? And that one method is to work closely with a customer who understands what problem they're solving. Now, FoaK happens to be a funding mechanism that enables that easily and gives us some stability of funding for a year." Int.21.2

"(The program is) essentially like seed funding. It's a way of doing what the outside world does through with angel seed funding. It's a way of vet - doing a proof of concept - encouraging highly skilled, highly paid people to spend their time 
investigating whether a particular problem is solvable or not and to try to build some sort of prototype." Int.37.2

Initiatives being delivered under the umbrella of a FoaK program provided a level of credibility which also motivated participation by internal stakeholders as well as the external client stakeholder. The program was able to present itself as a firm sanctioned initiative, helping to build early momentum through its mandates and executive participation on its governance board.

"I deem (the program is) very relevant and very convincing for clients despite some of the obstacles of IP (intellectual property) and some specific contractual elements. I believe the program and such, or the very proposition of the FoaK is something which is very appealing. " Int.12.4

Finally, the framework provided by the FoaK program was found to help orchestrate the necessary elements needed by the FoaK initiatives. The FoaK program sets criteria for participation, provides proven practices for execution, includes pre-defined contracts protecting intellectual property, and prepared materials available to initiative teams to guide their proposal definition, recruit their stakeholders, and to both define and govern their milestones through the course of the project. Having the approach and experience of co-creation of innovation initiatives defined and available to focus teams help to reduce the perceived risk of participation, and motivating stakeholder engagement.

"...the overall (FoaK) process define(s) the model that we should work through. By working through, we get all the elements defining effective process so that we can execute with better chance to be successful. And the board members are experience, a leader getting very insightful suggestions and comments, leveraging those (feedback) at some time and so we can define or design the project more effectively, as well." Int. 44.3 
Two additional recurring themes in the study provide further support for the argument that reducing barriers to co-creation innovation motivate stakeholder engagement. The FoaK program in its current design limits its role in the engagement with the product stakeholder and the client stakeholder processes related specifically to the FoaK initiative. Both product stakeholders and FoaK initiative leaders discussed a "chasm" that exists between the FoaK initiative innovation and the successful technology transfer to the products organization. It was argued that many of the assets that do not successfully transfer from a FoaK initiative into a mainstream portfolio is due to the lack of funding to bridge the further incubation required to bring the assets to a level of maturity ready for a mainstream portfolio. These stakeholders state that an area for improvement in the program is to extend the boundaries of the program participation to include funding that supports product organization readiness to effectively receive the FoaK innovation asset. With the stated desire for this barrier to be addressed by the FoaK program, is reasonable to state that this turn would make participation in a FoaK initiative more attractive to product stakeholders.

Similarly, sales and industry stakeholders argue that the FoaK program must extend its borders beyond a project to consider what is required to keep the client stakeholder whole after the FoaK initiative is delivered. The argument here is that a client stakeholder is motivated in participating to the degree to which they can see how participation benefits their organization, which does necessarily end with the FoaK initiative. The current program does not require a plan for the client experience beyond the completion of the initiative, which stakeholders feel would benefit the client and the firm. 
"So I think the commercial side, in terms of how managing the FoaK from the commercial and from the organizational perspective, is probably the challenge for people who have not done this before. And also coming to sort of an end-to-end agreement after the FoaK, and not closing the FoaK when the FoaK is closed. And we have not really won anything. " Int.12.4

\subsubsection{M3 Managing Initiatives Candidates as a Portfolio}

The FoaK program was found to manage its initiative candidates as a portfolio. This included determining initial levels of funding based on a competitive process, and modifying funding and resources while initiatives were in execution. It was found that the program's use of a portfolio approach to managing its initiatives supports the argument that in doing so, it improves the proportion of initiatives that succeed.

All cases were evaluated by a common process and set of criteria for approval. Each of the FoaK initiatives examined were compared against other proposals, competing for available funding. Upon review, four of the initiatives (i.e., Cases 1, 3, 4, 5) were initially rejected by the FoaK board and required to rework their proposals for further consideration. Each of these initiatives was also granted extensions due to delays in the client signing process. The extensions were evaluated against other initiatives that were being proposed to the board, and granted due to the strategic relevance of the initiatives to the firm's portfolio. Teams were motivated to improve their business cases, resulting in greater clarity of their innovation vision and proposed collaboration with co-creation clients. Only Case 4 was found to deliver a scope that did not represent value to the client's stakeholder.

"so I think in the first round we were actually rejected. We had to rework, we came back and then it was accepted, and then you get the funding, you can start working 
for up to three months. And within three months you have to find the client, you have to find and sign the client" Int.25.4

Through the governance and monitoring of the FoaK initiatives being studied, the program also granted follow-on rounds of funding for two initiatives (i.e., Case 2, 3) that were demonstrating momentum and early proof points. The program was proactive in presenting the additional funds to help further fuel the momentum and refinement of these two initiatives, which in turn both delivered success against the program's three performance criteria, including their transfer into the mainstream portfolio for commercialization.

A recurring theme in the study was the argument that the FoaK program could improve its process by remaining more engaged with the individual initiatives during the execution process. It was found that in cases where there is little proactive communication by the FoaK initiative team that issues sometimes are not visible until they are too far along. In doing so, the FoaK program would be expected to adjust its funding and support accordingly to initiatives in progress. This observation from informants identifies an area of improvement from current practices that further supports the argument that the use of a portfolio management approach with FoaK initiatives would be expected to improve FoaK initiative performance overall.

"I can tell you that the FoaK program has been helpful to us in the execution of the project, quite frankly. Other than providing them with the quarterly report that never got into any kind of details or were very logistical in nature, 'Have you completed the technical review, and prepared that demo, and prepared that page for the website, 'and these kinds of things. We were pretty much on our own once the project was started." Int.08.1 


\subsubsection{M4 Program as an Orchestrator}

Findings also support the argument that the FoaK program serves as an orchestrator for its initiatives which results in a greater focus of Initiative teams on the performance outcomes of their project. The FoaK program through its design was found to direct the innovation activities of each of the initiatives and to motivate specific behaviour from its FoaK initiative teams. This was achieved primarily through the combination of conditions to participate in the program (e.g., stakeholder support, initiative proposal), supported guidance provided to enable initiative teams (e.g., directed guidance, experiential guidance and mentoring), and its actions aimed at motivating the innovation activity (e.g.,funding, co-creation client engagement and signing).

"Very important part of (the FoaK) that says you need this kind of a structure to protect the innovation spark. But again, if you want it to catch flame, you need to have this process, this structure, this methodology, the screening criteria, so that at the end of it you're creating a bonfire and not just a set of fireflies because all you're doing is creating sparks and you're not thinking about how this will go forward. So, I think there's a very important point that says having a ring fence instead of resources available to manage those funds, whatever, having some strict guidelines that will hopefully give you a better odds of achieving success from a scale business perspective, and then having the ability with enough skills and support to help guide the teams along that journey, of applying the methodology, I think those are all important elements of the process." Int.15.3

With funding being a primary motivator for FoaK initiative teams, these teams were motivated to focus at a minimum on whatever was required to secure funding for their initiatives, and achieve success in their initiative as defined by the program. The FoaK criteria to participate were such that initiative teams were directed towards identifying 
opportunities that were strategic to the firm, engaging and securing cross-functional stakeholders from within the firm, securing a co-creation client for their initiative, and reporting progress against their objectives quarterly to the governing board. This "orchestration" by the program help to ensure that the right elements were being brought together for each initiative based on the experience and expertise of the FoaK program leadership. FoaK initiative team commitment and accountability to the plan of resourcing provided were found to direct FoaK Initiative team behaviour in all cases.

"Again, formality is the mechanism. The power of the FoaK is, again, the way that it engage some kind of (opportunity)...that say, look, (our company) is willing to invest in your client that is important to us, who wants to help you. You have a business problem, now let's work together, and (we) will finance this part. This is a good fit and, again, this is a main value for us. So again, to get commitment for the different stakeholder for (sales) and literally you can go to the FoaK which are the good connections and good relationship with the (sales function)." Int.41.1

"In order to really engage with software group and help (sales function) we needed a way to formalize it and this is what the FoaK brought; a way to formalize it, to engage all the stakeholders, to give commitment that someone is take care until the technology will be delivered." Int 41.1

It was also noted that program orchestration of an initiative in and of itself does not necessarily translate into successful initiatives. The program orchestration sets the stage for successful exploration by initiative teams by providing a map, a compass, resources, and guidance. Initiative teams ultimately were found to own the final performance of their FoaK initiatives and to have varied in the degree to which they use the orchestration put in place by the program. While all FoaK initiative teams demonstrated behaviour in the execution of their projects that was guided by the program design (i.e., "orchestration"), 
teams with strategic leadership demonstrated a level of execution on the program elements that contributed to stronger initiative performance than those that did not.

"What I found is in any project that was successful, the leader - for whatever sort of prior experiences - had an appreciation that this was not just about technology. They were not necessarily pure kind of techies. They did have some flavor of integration in their background. They were willing to go and flex, and bend, and then do something that they didn't originally plan as part of the project." Int.21.2

\subsubsection{M5 Initiative Governance}

All cases studied were found to be governed using the same mechanisms including funding gates (i.e., client signing), formal quarterly updates to the FoaK board, and ad hoc meetings as required. The FoaK governance framework was found to focus initiative team activities on their committed plan for the project, and reporting on progress against the performance measures as defined by the FoaK program.

All FoaK initiative teams discussed the desire for their projects to perform well, and the requirement to report progress to senior executives on the regular cadence. It was expected that the FoaK program inclusion of governance-driven accountability with visibility to executive management would focus the initiative team activity on performance.

\subsubsection{M13 Gathering Learnings}

The study also examined the degree to which the FoaK program gathered learnings from its initiatives and applied those learnings to the program to modified design and practices to improve the proportion of co-creation initiatives that succeed. 
It was found that the FoaK program was not as effective as it hoped to be in gathering learnings from its initiatives. During the study one effort was observed where a workshop was held on best practices, where the FoaK leaders from Cases 2 and 3 presented their experience and guidance to the FoaK board and teams and how they can improve the execution of projects.

"Yeah, I think probably we could have gotten better lessons learned. Even though this was a great project and this owner was involved it still took a really big effort to get the asset transferred, and the solution announced. I don't know that I understand completely why it was that much of an effort so I would like to understand that a little better." Int.4.2

"Absolutely (no experience capture from the project by the program). I volunteered to the board on many occasions to mentor teams, but I guess just the process and the really right-- they just don't have a construct, to do that, right? They did conduct some kind of a workshop awhile back and asked me and (another researcher) and others to speak, and we did. I'm not sure if anything came out of that as being used to mentor teams." Int.21.2

The FoaK program management team confirmed that in the past they have gathered experiences in an ad hoc manner, and modified the program accordingly. While they believe that the changes resulted in better outcomes for their funded initiatives, their monitoring of the impact was not formalized. In Case 2, the FoaK initiative leadership had gathered their proven practices and prepared their own set of assets which they use for onboarding their project teams. It is reasonable to state that extending this approach to the program-level could see positive results in overall initiative performance.

"Best practice...at some point of time, I put a lot of these (practices) into a white paper" Int.21.2 
"I think at the beginning, I had this really brief description of what FoaK was and really, I think subsequent talks or in discussions with my manager, I gained more information about what FoaK was. It was never really the focus because the focus was on the project itself and getting things done for it. I never really thought about it much from a FoaK perspective so much as this is the task we have to accomplish and what do I need to know to do that and how do we do it?" Int.38.2

All informants interviewed were asked where they see opportunities for improvements in the FoaK program. A number of common themes emerged, including the expanding the role of the program in addressing the "chasm" between the FoaK program and the products organization, revisiting the funding model for initiatives, the potential role for the FoaK program in identifying co-creation client candidates as part of the process, and improving on-boarding methods for improving the skills for researchers new to FoaK. This pattern of feedback provides further support that gathering learnings from FoaK participants and applying them to advancing the program will result in improved performance. It is believed that this is sufficient support for the role of gathering learnings on the knowledge acquisition method and its role on initiative success.

\subsection{Cross-Case Comparative Findings (M6-M12, M14-M18)}

Further analysis of the initiative specific mechanisms, context, and outcomes provided additional support for the updated program theory. The findings for each of these mechanisms support the relationship to initiative outcomes, and linkages between mechanisms. 


\subsubsection{M6 Conditions to Participate for Initiative Candidates}

The conditions to participate in FoaK initiatives represent FoaK program defined criteria that initiative teams are required to meet, and are evaluated against for funding approval. As all the cases in this research were approved for funding, they were therefore also successful in meeting all the minimum criteria necessary. It was found that the difference between successful and unsuccessful initiatives is in how the FoaK initiative teams went about meeting their program requirements. Specifically, successful cases were found to take a more strategic approach versus a more tactical execution in delivering on the program requirements necessary for funding.

The FoaK initiative teams in successful cases were found to have previous FoaK experience and strong FoaK initiative leadership. Each of the teams in these cases was described as understanding the criteria needed by the program, and how the performance of their initiative would be measured. They were proactive in managing the process of securing and setting expectations with stakeholders, working with the program management team in defining their proposal, and in working closely with the sales and industry stakeholders in identifying the "right" client stakeholder for their initiative. These teams were also found to recognize the interdependencies between the various stakeholder groups, and the role of initiative momentum points across all stakeholders. These FoaK initiative teams were found to invest early in the process of setting the right tone with each of their initiative's stakeholder groups, and engaging each group throughout the process. They have demonstrated the importance of communicating across these groups and promoting the progress of their initiative during execution. 
"Yeah. Yeah, we actually, in all of my FoaK project I got the product team involved, keep them update at early stage and try to get their input, and their feedback as early as possible. Of course, it's not easy, right? Because everybody's busy and travel and all that, it's difficult. But I think that will be--in my mind that's a very important piece as the project team, we need to do." Int.26.2

A "typical performance" case display part of this behaviour. In Case 1, the FoaK initiative team was distributed across two geographies, with the lead team being remote from the client stakeholder country. The extended FoaK initiative team, which was local to the client stakeholder, did not have previous FoaK experience. In this initiative, the lead team was found to work diligently with the client stakeholders, and the sales and industry stakeholder teams. As with the successful cases, this effort was reflected in the quality of the working relationship with the client and the active participation of the sales and industry stakeholders both in the proposal stage and in the new client pipeline development. These efforts resulted in the FoaK initiative asset being delivered, the referenceable client, and an active pipeline of new sales opportunities.

The FoaK initiative teams in the unsuccessful cases were described as having less FoaK experience than more successful initiatives, and FoaK team leadership that is more tactical in their approach to satisfying program requirements. These teams were found to limit their activities in satisfying these requirements and were less proactive in the areas of expectation setting and stakeholder engagement. This tactical approach results in less established relationships during the execution of the initiative (e.g., Case 5 dependency on sales for client access), and issues arising due to gaps in expectation setting (e.g., Case 4 client user issues with usability). 
In the typical case, an examination of the relationships that were less developed in the initiative further support these findings. While this team was successful in meeting the product stakeholder requirements, this relationship was not developed beyond the initial introductory presentations resulting in support for the proposal. Upon completion of the FoaK initiative, the gap in the expectations of the product stakeholder resulted in a failed effort to transfer the technology into that product portfolio.

Outcomes The FoaK program introducing conditions to participate in the FoaK initiative is found to focus FoaK initiative team's efforts on performance outcomes. The criteria set out what is necessary for the teams to be considered for funding, how performance is measured, and has put in place the monitoring mechanisms to help govern the progress of each funded initiative. While all teams were successful in meeting the criteria to participate, the variation in the level of performance across cases indicates the impact of both the program's criteria and the FoaK initiative team execution of the initiative. While the program criteria set expectations with the FoaK initiative team, and helps orchestrate what is believed to be the essential elements for a successful initiative, how the FoaK initiative team executes the project must be considered when examining a FoaK initiatives performance.

\subsubsection{M7 Initiative Team Ability to Execute}

The program theory argues that FoaK initiatives that are led by teams with previous FoaK experience and demonstrated ability to execute projects of this type will demonstrate improved initiative performance. This mechanism was found to be supported by the cases studied. 
The successful cases were found to be executed by FoaK initiative teams with previous FoaK experience, and led by strong initiative leaders. These teams demonstrated a deeper knowledge set regarding the program requirements and expectations of what would be required in working with stakeholders. This includes an understanding of the interdependencies between stakeholders and what is required to achieve initiative performance as defined by the FoaK program. The FoaK initiative leaders for these teams demonstrated a strategic approach to managing their initiatives and their stakeholder relationships, investing in achieving early proof points that could contribute to the momentum of the initiative. These FoaK initiative leaders also demonstrate business acumen in addition to technical skills, and establish personal networks beyond the research organization within the firm.

"Oh, the thing I should have said also about the other project is, and this one, is the team involved, the lead was someone who had done prior FoaKs and had good results. (Previous projects) and had a good track record so that always... does add a little weight to whether or not a project is approved." Int.05.3

"Well the current thing that it's very important is that you have a technical leader (PI) that is experienced. If your leader doesn't have, it should be experience in the FoaK itself, but the head should be a leader that has experience in leading project(s)." Int.48.1

The unsuccessful cases were characterized by initiative teams with little to no previous FoaK program experience, both at the leader and team member levels. The FoaK initiative leaders in these cases were found to be less experienced, with lower levels of business acumen than was seen in the more successful cases. These teams were more tactical in their approach to managing their initiatives, particularly in the management of 
stakeholder expectations and engagement. In Cases 4 and 5, it was found that the expectation setting and engagement with the client stakeholders resulted in lost momentum and mindshare with clients which impacted their follow-on investment beyond the initiative.

"Yeah. So, it was here, the local team, and they were even less experienced than I was. None of them had worked in a FoaK project before. Most of them have, actually, not worked with a client before. So, all of this set-up scenario with the FoaK set-up was new to the team." Int.25.4

"It takes some experience (working with clients), so how you present very complex material to the client, for instance, how you listen. I mean, they did a great job, but with more experience, I think one would have gotten to some things probably a bit faster. And the second part of the experience maybe now thinking about it, was getting some things where you need (the firm's) internal support, for instance, tooling, IBM software that they're using, for people who just-- all of them are just new, joined us about a year or two with (the firm), and they didn't have the network and it was sometimes-- it took a while until they found out whom to ask." Int.25.4

When examining the resources that make up a FoaK initiative team, it was found that the focus of the initiative leader has the greatest impact on improving initiative performance. Teams across initiatives were found to have varying levels of experience. In all cases, the team members were asked to focus primarily on the tasks assigned to them to develop the innovation. The successful teams are found to emphasize the importance of flexible team members, prepared to take on new tasks and develop new skills during the course of the initiative. FoaK team members were managed by their initiative leaders, and were found to be sheltered from the complexities of the FoaK program itself. The primary for focus in this case was for the teams to ensure they had the right skills needed for the creation of innovation work with the client stakeholder. 
"So the way we've been working is most team members are involved somewhat with everything, and so (as a team member) I've sort of been involved with all that, different aspects of (the FoaK project)." Int.38.2

"All the members of the team that worked on (the FoaK initiative) and that made it so great as that. Each person is definitely a team player, and that's obviously there. But they are very much willing to pick up new skills, and also skills-- we don't think of our skills as isolated, for example, I know machine learning means I will just do machine learning and somebody, I would kind of demand saying, 'Oh, somebody has to give me all this clean data in this format.'"Int.16.2

The FoaK initiative leader served as the focal point for each FoaK initiative from the proposal stage through execution and delivery of the project. The FoaK initiative leaders were found to be responsible for managing each stakeholder relationship, addressing initiative issues, and set priorities during the execution of the project. It was found that the strength of the FoaK initiative leader was reflected in the effectiveness of their FoaK teams in managing the complexity of their projects and any issues encountered.

Outcomes This study supports the FoaK program theory that initiatives led by teams with a demonstrated ability to effectively execute their FoaK projects will result in improved initiative performance. FoaK teams led by stronger more experienced initiative leaders will benefit from their previous knowledge in managing the complexities of the FoaK projects and building the effective stakeholder relationships needed to achieve successful performance.

\subsubsection{M8/M9 Program Guidance (Directed, Experiential) to Initiatives Teams}

The program theory states that a FoaK program providing directed and experiencebased guidance to initiative teams will contribute to bridging knowledge gaps that will 
result in improving the execution of their projects. These mechanisms were supported by the findings across the cases studied.

FoaK initiatives are characterized by their complexity and requirement for managing multiple parallel activities, stakeholders, and priorities. Initiative teams were found to have varying levels of knowledge and experience across themes necessary for the effective execution of their projects. This includes external knowledge regarding the marketplace for their innovation domain, background about their client stakeholder and client management, and the technical innovation landscape in their marketplace. Similarly, FoaK teams also require a knowledge base regarding internal aspects related to their initiative including an understanding of firm-level priorities, products stakeholder knowledge, and experience regarding the FoaK program and its operation.

The FoaK program provided multiple sources of knowledge to supplement FoaK initiative team experience coming into the project. A comparative examination of initiative team knowledge found varying levels of knowledge across all six of these areas, giving each team a unique knowledge profile. Initiative teams in the successful cases were found to have deeper experience regarding the firm, their product stakeholder, and the FoaK program elements for their initiative. They were also found to have greater experience and skills of client engagement and management. The successful teams were also found to proactively supplement their knowledge gaps through their work with stakeholders to better understand the product organization priorities, market opportunity, client dynamics, and FoaK program expectations. Teams on the successful FoaK initiatives were found to be less reliant upon the artifacts provided by the program, likely due to their previous experience and awareness of what is available. However, they were also more proactive 
and engaged with the program management team in review cycles and discussing any issues they were encountering.

"Some (initiative teams) did (use FoaK guidance) and some didn't... I would have to say the ones, who's probably more just a characteristic of the team itself, but those who were more proactive in terms of coming forward, asking for help, etcetera tended-- from my recollection, they tended to do better because they just had that mentality of identifying obstacles and getting whatever help. They didn't feel like they needed to do it all themselves, and they tended to be able to extend their reach more broadly and get additional assistance as needed." Int.15.3

Less successful initiative teams were found to leverage the essential materials made available to them within the FoaK program repository. However, these teams were not found to invest heavily in supplementing their internal systems knowledge through their stakeholders, being more tactical in their approach to key stakeholder relationships. Similarly, these teams did not engage heavily with the FoaK program management team after approval of their initiatives, and in both cases, when provided guidance regarding their proposed client stakeholder resorted to their initial candidate.

All teams were found to be knowledgeable on their offering domain from the technical perspective, with the goal to validate their understanding of business use cases through the interaction with the client stakeholder.

Outcome Findings support the argument that a FoaK program providing guidance in various forms to initiative teams, helps to bridge their knowledge gaps that in turn will help improve execution of the project. Successful teams were found to be more inclined to recognize where knowledge is lacking and proactively search to fill those gaps. The successful teams were less dependent on baseline materials provided by the program and were more focused on engaging with more stakeholders, earlier, to more fully understand 
the context of their initiative. Less successful initiatives were found to leverage base level materials provided by the program. The effort by successful teams to fill out their knowledge profile earlier, better position themselves in executing their initiatives moving forward.

\subsubsection{M10 Client Engagement}

The program theory also proposes that client stakeholder engagement by FoaK initiative teams will help provide validation of the innovation, improving the initiative's performance. This was supported by findings from this study.

An examination of the cases highlights that effective client engagement requires that initiative teams work with client stakeholders at multiple levels. This includes a FoaK initiative team's ability to set expectations and secure engagement with the client stakeholder, manage the relationship throughout the initiative, effectively execute the knowledge acquisition process with the clients, and understand the client's perspective on success and how that will be achieved.

Successful cases were found to be effective on all levels of client engagement. Their focus initiative teams engaged with client stakeholders early in their relationships with sales and industry stakeholders. FoaK initiative team leaders served as the focal point for the project and invested in establishing strong working relationships through various levels of engagement. These teams worked closely with their client stakeholder counterparts in delivering early proof points to demonstrate the progress of the initiative. FoaK initiative teams were proactive in identifying potential oncoming issues, which they set out to address early to maintain progress for the projects. These relationships were characterized 
by the level of trust and "chemistry" established with the client stakeholder throughout the course of the initiative. In these cases, clients were found to be motivated, engaged, invested, and satisfied with FoaK initiative outcomes. The successful initiatives were also characterized by the momentum and proof points that developed because of the execution of the initiatives, further contributing to the performance of the initiative moving forward. These included follow-on revenues from both clients in these cases.

"And above all that, they tried to pushing kind of a regional network of hospitals. This was the thing that was attractive for us. (The client sponsor) was interested when we started with, but I think during the (project) it was too long and also there were a lot of problems on their side... And those are the kinds of things, I think, why these things got worse. (The sponsor) actually (lost) interest in his baby which was this regional network, and he moved to something a bit different." Int.11.5

The "typical performance" case was also found to display the same client engagement characteristics as the successful cases. In this case, the lead research team was in a different geography than the client stakeholder, and the local research team that was inexperienced with FoaK. To ensure a strong client relationship was established for the initiative, the lead research team invested face-to-face activity, travelling for workshops directly with their client stakeholder. The sales stakeholder in managing the client relationship further supported this effort.

"Having the buy-in of the account, of the (firm's) account, is critical. And so we, in all of the projects that we were involved with, we tried to bring in could be the (sales executive) of the account, or the client technical advisor, and whomever had that role. Bring them into the thinking, get them into the process as early as possible, always before going to the FoaK board. So with the early thoughts that we had on wouldn't it be great if we tried that, we'd go and find or try and find people from the different (client) accounts that could help us that what we're saying makes sense, and 
help us shape the way we-- eventually help us shape how we present this to the client." Int.08.1

"At the very beginning, it's just the idea of (FoaK funding) grant. If the client exec says, 'I'm going to bring the best of (our firm), and they're going to work with you," that's-- obviously, the client exec needs to have (an offer) for the customer. Otherwise, none of this is possible. If the client exec didn't have the relationship with the right people, they wouldn't even be able to open the door." Int.21.2

A review of unsuccessful case studies found that once engaged in the FoaK initiative development, these teams were effective in working with the client stakeholders in validating the business use cases being explored, and refining the co-creation innovation. However, these teams were also found to be more challenged in managing expectations and momentum within their client environments. These observations in part are attributed to the changing environment within the client sites. Both cases experienced losses in initiative momentum and client interest due to delays in the execution of their projects. It was also seen that a gap in the client expectations in Case 4, resulted in the users no longer seeing value in the scope or level of usability of the prototype developed during the initiative. In this case, expectation and scope management resulted in an asset of questionable value to the market. FoaK initiative team experience and leadership were also found to contribute to a less effective level of client engagements resulting in the issues experienced during these initiatives.

Outcomes All cases were found to benefit from their engagement with their client stakeholders. The FoaK initiative innovations that emerged from the co-creation activities were all found to reflect client input, refining the use cases and priorities represented in the final innovations. All teams reported having learned more about the business domain 
related to their innovation. The resulting asset from their initiatives were improved as a result of access to the client environment, working with the client on innovation, and the availability of "real-world data" to test the innovation. Successful teams were also found to further benefit from the momentum and proof points that emerged from their initiatives, which helped to further support relationships with sales and industry stakeholders in pipeline development, and product stakeholders for commercialization of the emerging asset.

\subsubsection{M11 Commercialization Partner Engagement}

The FoaK program theory also states that FoaK initiatives that engage with a commercialization partners (i.e., product stakeholders) will benefit from validation of their innovation assets, contributing to improve initiative performance. The findings from this study provide support for this mechanism, demonstrating that early and continued engagement with product stakeholders contribute to the effectiveness of FoaK initiative teams not only in transferring the technology at the end of the project, but also in their work with client stakeholders.

The successful FoaK initiatives were both characterized by the effectiveness of their initiative leaders in engaging product stakeholders early in the FoaK initiative cycle. Both FoaK initiative teams had previous relationships with their respective product stakeholder organizations. They proactively engaged the product teams in the initiative definition stage, and set expectations for their participation throughout the FoaK initiative. These working relationships were exemplified by information sharing between the initiative team and the product stakeholder, access to product organization resources, and 
guidance being provided by the products stakeholder on strategic priorities and technical considerations. These FoaK initiatives demonstrated an understanding of the product organization's priorities, and incorporated this information in the execution of their initiatives.

"Yeah, (the product stakeholder) participated in the regular review meeting (with our FoaK initiative team). They provide some coaching support during the FoaK execution phase. They understand the project and the progress we have made. They track the majority of the technology we develop. Then, eventually they come in, when we have a relatively mature technology verified with the (client stakeholder), and then they start to take over." Int.44.3

In both cases, the product stakeholder organization benefitted from early access to the FoaK initiative teams and the client stakeholder engagement in understanding the business use cases being explored, and the innovations being developed. The results of this insight facilitated the successful transfer of the FoaK initiative assets into the mainstream product portfolios in each case. However, there were found to be challenges in product organization readiness to receive the FoaK initiative assets for commercialization activity. These included issues with funding, resources, and timing. These issues were referenced as "a chasm" between a FoaK initiative and asset commercialization, which sits outside the boundaries of the FoaK program definition in its current form.

"The biggest issue is that (the transfer of the FoaK asset to a product organization) ends up taking a lot of our (FoaK) research team time in order to support them and help bring them up to speed on everything. I feel, yeah, that's probably been the biggest obstacle is how much time we put into the knowledge transfer and the support... So (our FoaK team) got tied into this maybe more than we expected or more than we wanted originally, it wasn't just like a clean hand-off or something like that." Int.38.2 
Unsuccessful FoaK initiatives in this case both experienced challenges in their projects with the reorganization of their product stakeholder organizations. The result was that their initial product stakeholder organizations were disbanded, and in Case 5, there was the formation of a new business unit for healthcare. Based on the observed dynamic between these FoaK initiative teams and their product stakeholders, it was found that the sudden changes were unexpected by the FoaK initiative teams, which reflects on the depth of relationship between themselves and the product organization.

Unsuccessfully replace ACO: "During the project this (the asset catcher) disappeared so it was, I don't know, reorganized so it wasn't there anymore so the initial planned catcher was gone. And then we were struggling and looking for alternative catchers... We had technical discussions with (a potential replacement catcher's) technical team, but also there I think what let them not to get so excited about it is also-- yeah, the missing client excitement (momentum). So, they were only ready to put, to my understanding, resources into catching if they saw complete client opportunities." Int.25.4

Case 5 was unsuccessful in securing interest with their potential replacement product stakeholder, which is attributed both to their method of engagement with this team and the relevance of the asset scope of the product portfolio. This was further indication that the effective engagement is beyond having access to the product stakeholder. It must consider the degree to which the FoaK initiative team incorporates expectation setting, and information sharing towards establishing a trusting relationship between the two teams. This is further supported by observations in Case 1 and Case 3. In Case 1, the relationship between the FoaK initiative and the product stakeholder was established as part of the proposal development process. However, there was little interaction and knowledge sharing between these teams during the initiative, which subsequently resulted in the 
product stakeholder setting conditions for the transfer of the technology into their portfolio at the end of the project. This raised question as to whether this was the "right" product portfolio for this initiative. In Case 3, as with the unsuccessful FoaK initiatives, the product stakeholder was reorganized and a new business unit was formed. However, the FoaK initiative leadership was effective in building the relationship and securing the new product organization, resulting in the successful transfer of the assets to that portfolio.

Successfully replace ACO: "So once an IOT business union was formed in the middle of the First of a Kind project, so when we executed the-- when we start to execute the project the IoT unit wasn't formed. During the execution of the project, the business unit was formed.... So, we got engaged with the new IoT solution team who's leading for the automotive work... And we share a very common understanding about the industry needs or the industry trend, and at that time they didn't have any product, but they are in the phase to start to define a new product." Int.44.3

Outcomes These finding support that FoaK initiative teams engaging with their commercialization product stakeholders benefit from new knowledge that helps validate their offerings, both for the market and within the product's portfolio priorities. This in turn ensures that FoaK initiative teams are considering not only market factors, but also internal client requirements (i.e., product stakeholder). These findings suggest the need for FoaK initiatives to take a strategic view with their innovation clients, both external and internal to the firm. 


\subsubsection{M12 Industry Sales Engagement}

Findings from the study also support the program theory that FoaK initiative engagement with industry and sales stakeholders contributes to FoaK innovation asset validation and in improving initiative performance.

In all cases, FoaK initiative teams had established relationships with their industry and sales stakeholder. This was in part attributed to the dependency of both industry and sales functional performance on the availability of new assets addressing market use cases not currently satisfied by the firm's portfolio of offerings. As such, these stakeholders were motivated to support initiatives that help address these gaps.

"FoaK was the main criteria, or the main vehicle, to which we could actually start new projects for our industry." Int.36.0

The FoaK initiative teams worked with these stakeholders in FoaK proposal development and in identifying co-creation client candidates. In all cases, the client sales executive served as the primary contact between the client and the FoaK initiative team, opening access for the FoaK project. In all cases, the method of engagement with these stakeholders was found to be consistent, and all FoaK initiatives were found to benefit from the support of these teams. However, the level of sales and industry stakeholder motivation was found to vary across initiatives.

These stakeholders contributed to FoaK initiative performance by helping to validate market opportunities and gaps in the current portfolio, and identifying priority use cases. As momentum and proof points emerged from the initiatives, the stakeholders transitioned into developing new sales opportunities for the innovation in the market. The 
degree of sales and industry stakeholder engagement was found to be related to the level of momentum that the FoaK initiative had developed. Momentum, in part, was related to the proof points emerging from the project, as well as the intentions of commercializing the offering for market.

FoaK initiative teams are also required to manage the expectations of their sales of industry stakeholders to ensure focus and alignment on the FoaK objectives. In particular, cases where stakeholder involvement was focused on the initiative versus on near-term sales revenue resulted in more effective execution. In contrast, cases where sale stakeholders combined their FoaK initiatives with an active sales cycle (i.e., Cases 3, 4), were found to introduce additional complexity into the initiative, negatively impacting the effectiveness of its execution. In both cases, the decision was made to separate the two streams of activity, to focus on the FoaK initiative.

While all the cases in this study reported sales pipelines for follow-on revenue, only the successful initiatives were found to convert sales opportunities into new business for the firm. Sales pipelines for unsuccessful cases were described as inactive with not resulting follow-on revenue achieved.

“...every time we had a meeting with them, they had a big pipeline of opportunities, but apparently, nothing ever came of that." Int.06.4

Outcome Findings support that FoaK initiative team engagement with industry and sales stakeholders contributes additional knowledge on both the market and the client stakeholder for the FoaK innovation being explored. These stakeholders serve boundaryspanning roles for the firm, and their insights provide further asset validation for the FoaK initiative team, both in the proposal definition and in the FoaK innovation delivery stages. 
In all cases, engagement with these stakeholders provided the necessary access to an eventual client stakeholder, and the development of a new sales pipeline. Both outcomes impact initiative performance.

\subsubsection{M14 - M18 Stakeholder Focus, Motivation, Expectation, and Investment}

\section{(Practice) and Shift in Organization Strategy}

The final mechanisms introduced into the program theory reflect findings regarding the impact of unanticipated changes in a FoaK initiatives environment or changes to the expected levels of stakeholder focus, motivation, expectations and investments in the initiative. The findings support the suggestion that unexpected changes in these areas have the potential to impact a FoaK initiatives momentum and its final performance.

In all cases studied, FoaK initiative teams moved forward into the execution of their projects based on pre-established relationships with their stakeholders including efforts to align on expectations, the required investment, and anticipated stakeholder focus and motivation in their participation. Each of the FoaK initiatives was also approved by the program based on the strategic relevance of the initiative to the firm priorities. While each FoaK initiative presented proposals based on a reasonable level of stability in the environments where they would be executing their projects, each case faced the potential of changes in those assumptions.

As was seen, four of the five cases studied experienced a change in their base assumption regarding their FoaK initiative environment, which provided the insights resulting in the extension of the FoaK program theory framework. The five mechanisms observed in the analysis were added to the program theory (i.e., M14-M18), and are 
elaborated earlier in Section 9.4. These mechanisms were added to the framework based on their observed impact within the cases studied, and will not be repeated here. However, in considering the findings it is important to note that each of these mechanisms has the potential to impact other mechanisms observed in the FoaK program system.

In the cases of stakeholder focus, motivation, expectations, and investment, the impact of stakeholder behaviour and practice can have either an accelerating or decelerating impact on the initiative's momentum and its resulting performance. As described, in Cases 2 and 3, the client stakeholders were significantly engaged in the execution process and invested beyond the required project management resource. This level of focus, motivation, and investment helped to fuel the momentum for each of these initiatives. This momentum was found to carry forward to other stakeholders including the sales and industry stakeholders in building a sales pipeline, and for the product stakeholders in receiving the asset into their portfolios.

Changes in organizational strategy saw three cases impacted by product portfolio reorganizations. Each resulted in planned product stakeholders no longer existing, and the need for the FoaK initiative teams to identify new product portfolios for their innovation assets. In all cases, the changes inserted disruption and additional complexity for each of the FoaK initiative teams. In Case 3, the FoaK initiative leadership was effective in securing a new business unit as its new product stakeholder and leveraged the momentum of this new business in helping to build further momentum for the offering. In Cases 4 and 5, FoaK initiative leadership was unable to overcome the new obstacle, with neither of the FoaK initiative assets transferred to a mainstream product portfolio. 


\subsection{Findings Summary}

The findings from this analysis concludes with a relative mapping of the program mechanisms and their place in the knowledge acquisition method system, the linkages and outcomes, and the themes emerging as impacting initiative performance.

The findings from this study support the revised FoaK program theory presented in Section 9.1 (see Table 9.1). The FoaK co-creation of innovation initiatives examined demonstrated their complexity both in the nature of stakeholder relationships and in the inter-process dependencies necessary to move and innovation from proposal to commercialization or the market (see Figure 6.8). The differences in FoaK initiative performance were found to be explained in part by the interaction between the FoaK initiative team execution of their project and the FoaK program framework within which their initiative exists. The combination of directed research by the program and effective execution by the FoaK initiative team contribute to explaining observed FoaK initiative performance outcomes. An illustrated summary of the findings and relationship of mechanisms is represented in Figure 9.1.

\subsubsection{FoaK Program}

The FoaK program approach to the co-creation of innovation looks to provide a framework that enables both scaleability and repeatability of this method of knowledge acquisition for innovation (M1). The influence of a FoaK program on the performance of its funded initiatives is indirect (M1). The program's primary role in initiative success is in 

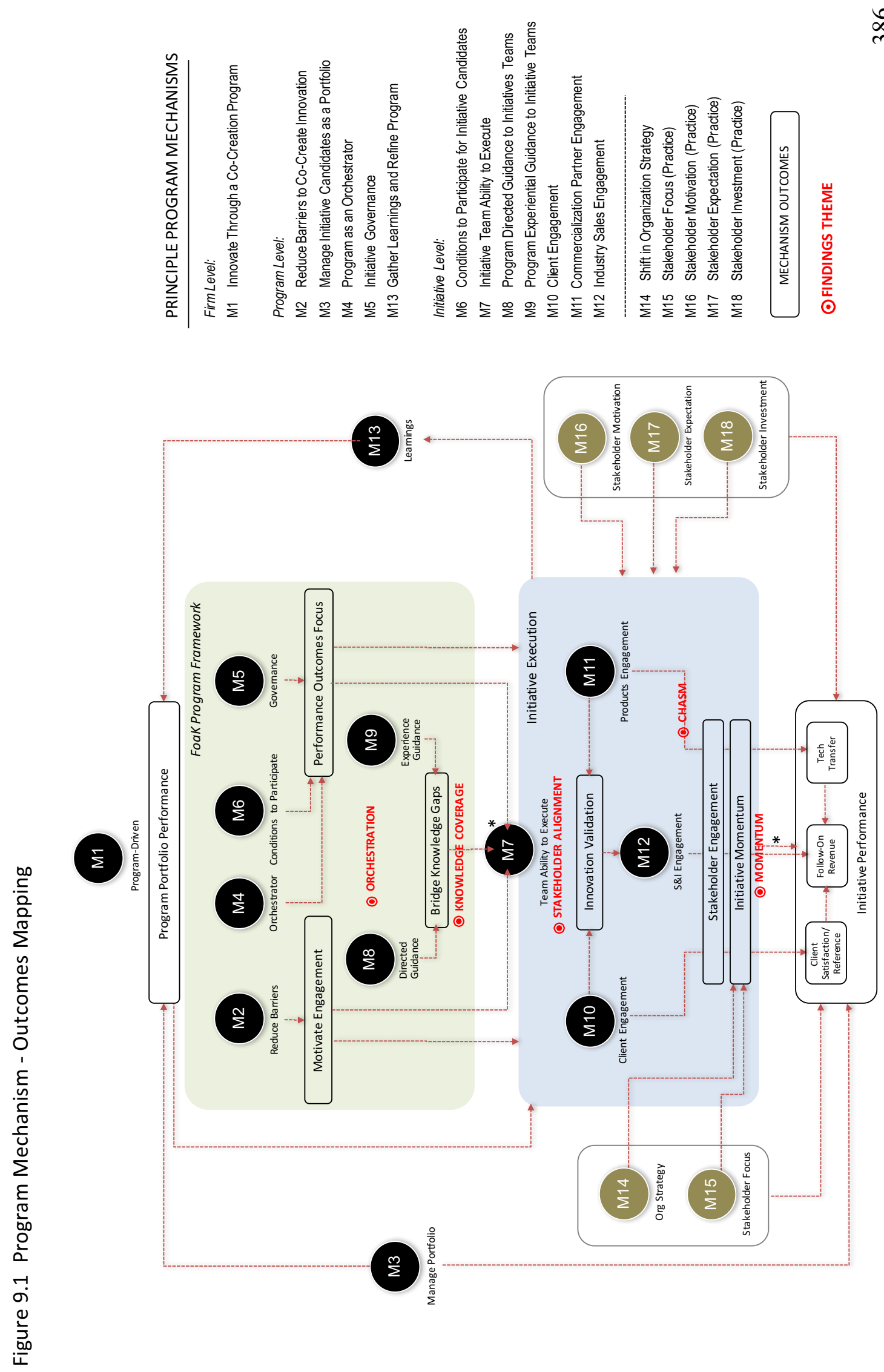
setting the stage to ensure that initiative teams are working with a focus on addressing the necessary elements needed for their FoaK project to be successful (M1).

This program-based guidance is established on the aggregated experience of past FoaK initiatives (M13) manifested through a repeatable framework. Each FoaK initiative success contributes to the program's overall goal of delivering new-to-the-firm innovation resulting in mainstream products that support the firm's competitiveness in the marketplace. As such, the program takes a portfolio management approach (M3) to selecting, funding, and supporting FoaK initiatives for the program. This relationship between the FoaK portfolio management's model and its initiatives directly impact individual FoaK initiative performance through an ongoing evaluation progress and merit against further support and resources.

The FoaK program serves as an orchestrator (M4) for initiative teams. The program outcomes and performance goals are supported through a combination of focusing initiative teams on the performance outcomes for their projects (outcome), putting in place conditions that motivate stakeholder engagement (outcome), and through enabling the bridging knowledge gaps within their initiative teams as necessary (outcome). The FoaK program brings together resources (M2) knowledge (M8, M9), and credibility (M2) to help lower barriers (M2) with the potential of preventing the realization of co-creation of innovation projects.

The FoaK program also puts in place the conditions that initiative teams must meet to participate in the program (M6). These conditions to participate further guide initiative team behaviour towards the use of these rules of engagement. FoaK initiatives teams are also guided by the program's governance mechanisms (M5) that monitor initiative progress 
against their committed milestones. Overall, this orchestration function was found to motivate behaviour in the FoaK initiative teams engagement with stakeholders believed necessary for co-creation initiatives to be successful (M13).

\subsubsection{FoaK Initiatives}

FoaK initiative performance is directly related to the ability of a FoaK initiative team to execute its project (M7). This is found to be a combination of both the team's skills and its people, and the strength of its initiative leader. FoaK initiative leaders that are proactive with strong business acumen were found to have a direct impact on its FoaK team's effectiveness in identifying, recruiting, and aligning the necessary stakeholders for their initiative. The effectiveness of the FoaK initiative teams was also found to be related to the nature of the relationship with the FoaK program, and the degree to which they are focused on performance outcomes (outcomes) for their initiative and its stakeholders. FoaK initiative team effectiveness was also found to be related to the degree which they leveraged the program framework and resources to help secure and engage their stakeholders (outcome), and then taking advantage of the guidance available to help bridge knowledge gaps within their team (outcome).

Successful FoaK initiatives are characterized by the degree to which initiative stakeholders were engaged (M10, M11, M12), focused (M15), motivated (M16), and invested (M18) in the initiative. Alignment of stakeholder expectations (M17) with the FoaK initiative goals was also found to positively contribute to successful initiative performance. 
Effective FoaK initiative teams were also found to recognize the role of building and sustaining initiative momentum through the course of a project (outcome). Successful initiatives were found to be those that leveraged their momentum in strengthening stakeholder relationships. FoaK initiative proof points and momentum were found to begin during the engagement with the client stakeholder, with subsequent impacts on products and sales stakeholder behaviour moving forward. This, in turn, impacted the initiative final performance (i.e., client success, following revenue, and technology transfer).

\subsubsection{FoaK Initiative Context}

The FoaK initiative context was also found to impact initiative execution. It was found that changes in organizational strategy (M14) and in primary stakeholder focus (M15) was found to impact FoaK initiative momentum. Similarly, any gaps in stakeholder motivation (M16), expectations (M17) or investment (M18), arising after the point of initial engagement with the FoaK initiative, each potentially affects initiative execution and performance. The gaps may be either in favour or hindrance of the FoaK initiative execution.

A further examination of these findings reveals five themes that will further contribute to the discussion on theory, and will be further developed in the following section. These include the role of the program as an orchestrator, knowledge coverage, FoaK initiative stakeholder alignment, momentum, and FoaK initiative chasms. 


\section{Chapter 10 \\ FOAK PROGRAM THEORY AND THE KNOWLEDGE CAPACITY FRAMEWORK}

This study set out to examine the role that knowledge acquisition method plays in the co-creation of innovation initiatives, from a dynamic capabilities perspective. Lichtenthaler and Lichtenthaler's (2009) knowledge capacity framework (KCF) was used as the baseline set of knowledge-centric dynamic capabilities identified as relevant for open innovation initiatives. To examine these dynamic capabilities within the context of the study's First-of-a-Kind (FoaK) initiatives, findings and analysis began with the definition of a FoaK program theory (FPT) that identified the relevant mechanisms, context, and outcomes for the initiatives examined (see Table 9.1).

The FPT represents the elements of the co-creation of innovation system, including the interaction between the knowledge acquisition method (KAM) and its collaborative innovation projects. The system elements include the collection of KAM strategy, processes, resources, skills and knowledge used in the creation of new-to-the-firm innovations. These system elements also translate to the concepts in the knowledge-based dynamic capabilities (i.e., KCF), in this case being knowledge capacities theorized as part of open innovation collaborative initiatives (Lichtenthaler \& Lichtenthaler 2009).

The use of the two-step approach (i.e., FPT to KCF) allowed for a more productive collection of evidence using terms relevant to informants and consistent with artifacts related to each case. This chapter describes the translated dynamic capabilities perspective on the analysis, including a findings-driven revision of the knowledge capacities framework. 
To note, the discussion on knowledge management capacities will reference desorptive capacity (i.e., an external knowledge capacity). This knowledge capacity is characteristic of outbound open innovation initiatives, which is outside the scope of this study. However, it will be included in parts of the discussion as continuity related to the knowledge capacity framework and broader implications on findings from the study.

\subsection{Mapping of Knowledge Capacities}

The FoaK program theory (FPT) was developed based on program-centric interviews and then evaluated against the five cases studied. The FPT represents the principal mechanisms across the firm, program, and initiative-level elements of the cocreation system. The revised FoaK program theory was found to be supported by the evidence collected from the cases studied.

The FoaK program studied represents a knowledge acquisition method (KAM) used in engaging external sources of expertise as part of a firm's innovation process. The FoaK program theory represented various knowledge types required by the FoaK program and the initiative teams that engage directly in the co-creation of innovation process with its stakeholders. Knowledge types required in the effective execution of FoaK initiatives was described as spanning both market (i.e., external) and firm (i.e., internal) related domains (see Figure 6.5), representing the various dimensions of these collaborative initiatives. External market-centric knowledge included understanding of target market opportunities, the co-creation client domain and priorities, and an understanding of the proposed co-creation innovation's competitive landscape and strategic market relevance. 
In addition to market-related knowledge, the FoaK program and its initiatives also require internal firm-related knowledge regarding the firm's strategic priorities, understanding of the commercializing product organization and its strategic priorities, and an essential understanding of the FoaK program and its conditions for participation.

The various dimensions of knowledge suggest the need of knowledge acquisition methods and that initiative teams understand the strategic objectives of the firm and cocreation project, the role of innovation initiative experience and organizational memory for repeatable scalable programs, the processes for effective stakeholder engagement, and the management and integration of co-creation innovation initiative complexity. The findings demonstrate that an initiative team's previous knowledge and capabilities across each of these domains are connected to their co-creation innovation initiative performance. The findings reflect the need for programs and initiative teams to have developed a range of knowledge types and capabilities, and highlight the relevance for the firm in developing various types of knowledge to support the co-creation of innovation process.

The research findings and the revised FoaK program theory (FPT) were found to support the role of the dynamic capabilities described in the knowledge capacity framework (KCF) and proposed two revisions. The first revision to the KCF includes adding portfolio management capacity as a new KAM-level internal knowledge capacity for optimization. The second revision introduced clarity on Lichtenthaler and Lichtenthaler's (2009) knowledge management capacity (KMC), developing the concept further as a higher-order dynamic capability representing knowledge acquisition methods as orchestrators in the co-creation of innovation initiatives. Each of the mechanism examined in the study (i.e., FPT) were found to align with a set of knowledge capacities to 
execution the co-creation process. Table 10.1 represents the mapping of the firm, program, and initiative mechanisms to their related knowledge capacities as represented in the KCF.

\subsection{External Knowledge Capacities}

\section{Connective Capacity (KCF external knowledge; knowledge retention)}

Connective capacity refers to an external knowledge dynamic capability that references the firm's ability to retain knowledge with respect to inter-firm relationships. This includes the ability to both maintain inter-organizational relationships with sources of external knowledge in the open innovation process, as well as having privileged access to the right sources of knowledge. In the context of the FoaK program theory, this refers to co-creation client access as the innovation engagement partner for these initiatives.

"we need the clients help in one, articulating their challenges, especially their technical challenges and two, providing us with their resources to help us help them implement what we are trying to customize for them." Int.36.0

"(FoaK initiatives need) a client that's willing to invest their time and sometimes money in it so we can get some market validation. " Int.01.0

"In general, I like to make sure that they are dealing with the right people in the company because sometimes our sales team is used to approaching a certain division or job responsibilities within the other side, and they're not as used to working with the technology side of it because that's kind of more behind the scenes in many companies." Int.10.0

The relevance of connective capacity as a knowledge acquisition method dynamic capability was demonstrated in the study at both the program and initiative-level. At the 
하

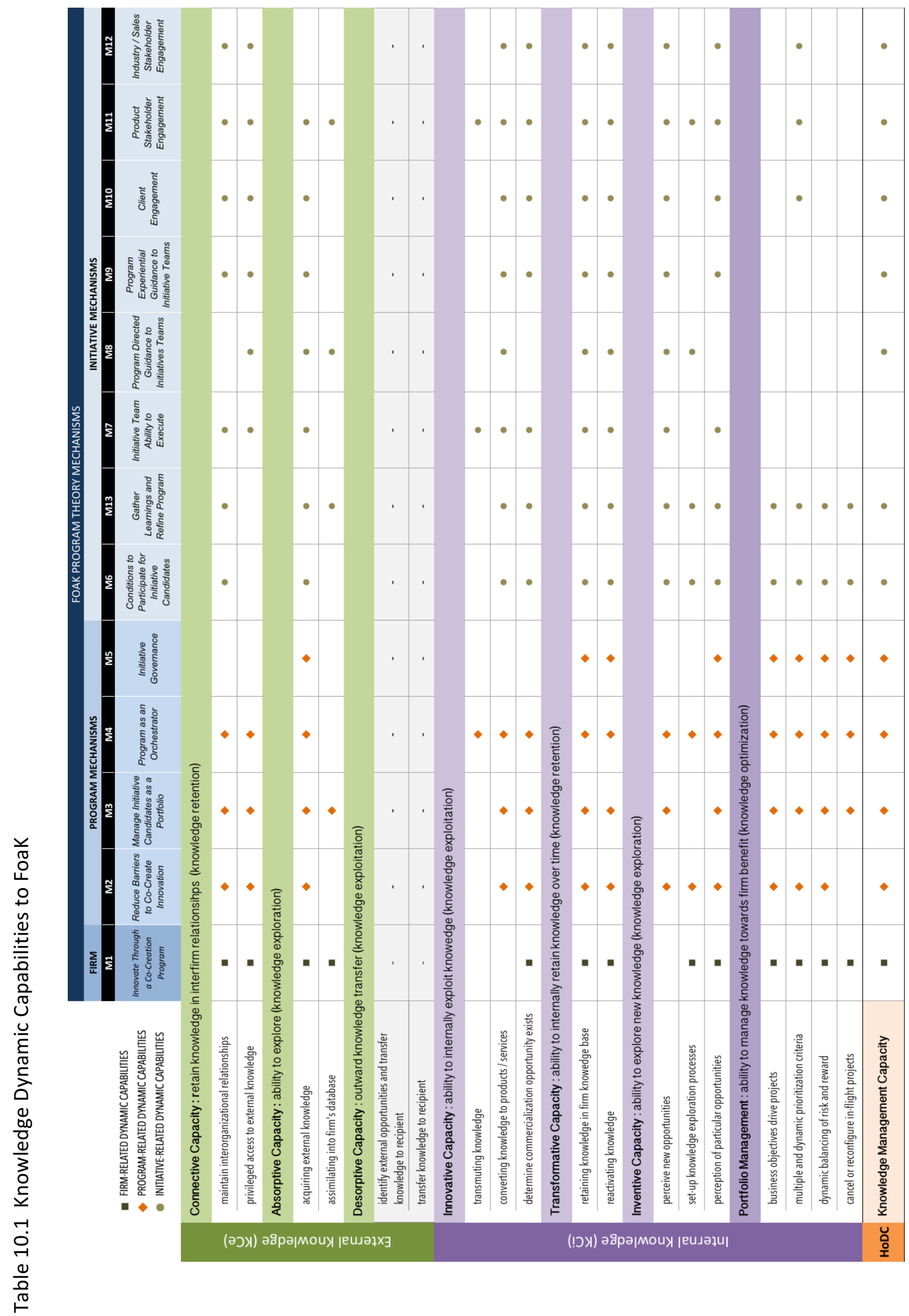


firm-level, connective capacity was demonstrated as previous relationships between the firm and existing strategic clients that are potential collaboration partners for FoaK initiatives. The FoaK program presented credibility and resources aimed at reducing barriers to participation for client involvement, and provided processes representing practices from past initiatives. These practices are viewed as exemplifying engagement methods that have proven effective in engaging client participation and access. Mentor experience with firm clients also serves as an access point for initiative teams, leveraging previously established inter-firm relationships. In addition, the program acting as an orchestrator for its initiatives requires that engage industry teams and sales stakeholders, who in turn provide access to their client knowledge and relationships.

Initiative-level connective capacity was observed as an initiative team's effectiveness in leveraging their own client knowledge, in addition to proactively accessing the program's developed knowledge within the program framework and the experience embodied within the supporting stakeholder participants (i.e., sales and industry stakeholders, product stakeholder).

"Yeah (the client stakeholder) definitely see us as a collaborative partner and want to work with us. And some of (the firm's) biggest clients are also collaborative partners, and they definitely see it as one of the perks of the relationship... So, it definitely adds value to them to be able to work with us on this cutting-edge technology quite frankly, that's why we do get some of them saying, "I want you to agree not to give this to my competitors," because they do see a competitive advantage to working with (with our firm), to being the first to use the technology." Int.10.0

"There is no way for the research team to know the potential pool of clients. So, the team that sponsors the work, sometimes they do have the right client relationships, 
and they can get them, but in other cases, unfortunately, we get (no client candidates)

for the research team. Then, that becomes really challenging." Int.27.0

"The fact that we don't require huge investment from a client. It is a way for them to innovate, getting to dip their toe in the water, without having to invest huge amounts of money." Int.02.0

Connective capacity was observed to be more effective in initiatives with greater experience in execution. Teams demonstrating previous client and FoaK experience were more skillful in anticipating and managing complexity and change. It was found that the more experience developed within a knowledge acquisition method and the individuals that execute on its initiatives contribute to better performance of co-creation projects relative to teams with less experience.

"The only person who can tell us where the data is, is the client. So, a lot of client workshop is back and forth. So, they understand some degree of analytics. Sometimes is really good to have a team or another team located in the client so they understand-- they speak our language, right?... A lot of times, if the client doesn't have the team, we need somebody to understand some degree what we try to do. Otherwise it's so difficult, we have two teams that talk different languages from each other it sounds like a big part of this is not only the fact that we're technically competent, but we also need to be able to identify the right people to communicate on the client's side their knowledge?" Int.27.0

Absorptive Capacity (KCF external knowledge; knowledge exploration)

In the KCF, absorptive capacity refers to the firm's ability to explore new innovations outside of the firm. Exploration in this case does not necessarily result in commercialization of new knowledge. Rather, absorptive capacity in this study is described as the ability to acquire external knowledge and assimilate that new knowledge into the firm's database. 
The absorptive capacity dynamic capability was also observed across the cocreation of innovation initiatives studied. At a firm-level, FoaK as a knowledge acquisition method (KAM) is put in place as a focused program with the intention to acquire and assimilate external knowledge for creating new-to-the-firm innovation. This direct investment demonstrates the intention to develop the absorptive capacity of the organization at the program-level. The knowledge acquisition method was found to invest in lowering barriers to motivate stakeholder engagement (i.e., products organization, research, services) to secure their role acquiring new knowledge. The KAM portfolio management practices motivate exploration and assimilation of new knowledge by focusing stakeholder behaviour through its funding criteria and assessment of the readiness of initiative teams and their stakeholders in assimilating new co-creation innovations. This focus is reinforced by the KAM governance processes. The program as orchestrator also serves to ensure that the right elements and conditions are in place to engage with external stakeholders and in bringing new knowledge into the organization.

At the initiative-level, absorptive capacity is observed as an initiative team's ability to engage with both the external (e.g., client) and internal stakeholders (e.g., products, services) required to convert knowledge into an internal asset. This ability is reflected as the team's readiness to execute the initiative, which includes their capacity to leverage program knowledge to help in capturing and converting new innovation, the selection of the right partners, and in overcoming process roadblocks.

"Previously FoaK was, a particular project was approved, that was that and then any follow-up would be through the business unit or otherwise. And sometimes with new technology requires more incubation time that six-month proof of concept. Especially when it has a chance of becoming a full-fledged new offering, new 
solution offering, and so the FoaK board now does have the flexibility where they look for areas which are either becoming important for (our company) or where they see a greater chance of success and increase the investment over there." Int.27.0

"At the end of the FoaK (initiative), you already have a somewhat integrated solution where the asset commercialization owner would not just start from scratch at that point and say, 'Okay, so now how do I align all the resources and fold that into my portfolio? And what other infrastructure components do I have to put together?' The earlier this can be done in the project, the more successful these FoaK projects tend to be is my experience." Int.18.0

Findings from the study argue that absorptive capacity as a dynamic capability is strengthened by previous experience (i.e., program-level, initiative team) in engaging external and internal stakeholders in the co-creation of innovation process and the technology transfer to the commercialization partner. Absorptive capacity was found to be related to the level of stakeholder motivation to participate in a co-creation initiative, the credible support of the program, and the effectiveness of initiative teams in the managing expectations and engagements of stakeholders. Managing stakeholders was found to be related to exploring new knowledge external to the firm, and receiving that knowledge into the organization.

"Quite frankly, (the FoaK initiative asset) just kind of sat because there was no one promoting it or no advocates for so I think naming a catcher has been important, but many times the catcher doesn't engage in - this is more my opinion now at this point - but doesn't engage quickly enough when the project is over. So, we still struggle with moving from client number one to in between client number one and a commercial offering." Int.10.0 
Desorptive Capacity (KCF external knowledge; knowledge exploitation)

In the KCF, desorptive capacity refers to the firm's ability to exploit knowledge through the transfer of its knowledge to stakeholders outside of the organization. Desorptive capacity is relevant to outbound open innovation processes, which are outside the scope of this study. However, findings highlight significant parallels in how initiative teams manage both external client stakeholders and internal product stakeholders. It is believed from the observed dynamics of the client-initiative team engagement processes in the FoaK program (i.e., inbound open innovation) knowledge acquisition method would also translate into the outbound open innovation model.

A primary theme from the findings was that the successful transfer of knowledge between stakeholders is dependent upon identifying the right stakeholders, engaging them effectively, and managing expectations in the open innovation process. Stakeholder engagement in turn is guided by program objectives and firm strategic priorities. The role of stakeholder expectations and engagement on initiative performance was found relevant for both stakeholders internal and external to the firm. It is believed that these primary elements would also be observed in open innovation initiatives with a firm's focus on exporting versus importing knowledge. This presents an opportunity for future research.

\subsection{Internal Knowledge Capacities}

Innovative Capacity (KCF internal knowledge; knowledge exploitation)

The firm's innovative capacity refers to an internal knowledge capability focused on the organization's ability to internally exploit knowledge in the innovation process. This includes the ability to gather information from various sources and transform it for the 
firm's strategic purposes, determining whether commercialization opportunities exist, and converting that new knowledge into products and services. As a dynamic capability, innovative capacity was clearly observed in this study, as well as its role on initiative performance.

"So, in some instance, (a FoaK initiative) may be something exactly (a client was) looking for or maybe something completely new. And so, we are providing something they had not even thought about or they didn't even know they needed. And then if we have a good understanding and now good technology end up as a (new offering) - through the First of a Kind. Then your goal is that-- now we have to now figure out how to repeat that into a reputable solution, whether that becomes part of something that (our) services can deliver, or it becomes part of our product portfolio." Int.01.0

Conversion of knowledge into new innovation was observed at several points in the FoaK initiative process. The program's role in orchestration set initiative conditions to ensure client and stakeholder engagement in actively validating new ideas and invention, and exploring these ideas in the form of initiatives and commercialization opportunities. The FoaK program as a knowledge acquisition method serves as a catalyst in bringing the right elements together, with individual initiatives being described as the environment where the initial ideas (i.e., invention) resulted in new ideas through engagement with stakeholders. Client engagement helps focus and validate innovation use cases relevant for the market. Product stakeholders viewed outcomes from FoaK initiatives as potential portfolio innovations that could continue through further transformation and enhancement for commercialization to the market.

The determination of commercialization opportunity was observed across the FoaK program processes. Initiative potential for commercialization was set as an objective of the knowledge acquisition method at the firm-level and program design level. The program's 
use of portfolio management practices and co-creation initiative orchestration assess new opportunities for their potential and investment. They also guide execution of these initiatives to focus on commercialization as an ultimate performance outcome. At the initiative-level, determining commercialization opportunity was found to begin with the initiative team's ability to demonstrate market potential of their innovation idea for the purposes of funding, stakeholder engagement with this outcome in mind, and continually evaluating the initiatives deliverables for the highest probability of commercialization.

"So it's all about knowing my market and knowing precisely what is my target for the asset that I'm developing...You have to think of the technology aspect, how deep it is, but the financial aspect and the market's impact are absolutely key (to investing as an ACO in the initiative)." Int.23.0

Similarly, the firm and the initiative team's ability to convert knowledge to new products or services was observed in the program design, stakeholder orchestration, and successful transfer of innovation between the initiative and the product stakeholder organization. The conversion of new knowledge into market offerings was found to be dependent both on successful transfer of the new innovation into the product organization, as well as the ability of that organization to receive and convert that information into a new market offering. The study demonstrated the existence of challenges in both of those processes, with a dependency on the effective management of stakeholder expectations and engagement throughout the process.

"I think we struggle with that many other companies do too... I don't want to say black hole, but kind of a gap many times between the period when the FoaK project is over and it moves into some sort of next stage of either commercialization, or becoming a service asset, or just kind of put aside. There's kind of a no-man's land 
in between where we need to do a better job of moving through that phase. The phase of going from a single customer and a single FoaK project to either a commercial offering, or a function, or a feature in the commercial offering that already exists." Int.10.0

"Once the funding ends after nine months you have an asset but it's not yet a commercial asset. It could be another transitional period where you start working with a second and third client and at the same time work with the product team to transfer the asset into the ACO organization. That process, we're just hanging out in thin air there." Int.18.0

The innovative capacity was also found to impact co-creation of innovation initiative performance. Specifically, initiatives where stakeholder expectations and engagement were managed strategically by initiative leaders, showed that when knowledge was shared early in the process, organizational readiness to innovate was better than initiatives where this was not the case. The firm's innovative capacity was also found to be related to the initiative's ability to build and exploit momentum for the innovation, which contributes to securing client and product stakeholders committed throughout the innovation process. Ineffective management of these stakeholder relationships was found to result in the commercialization "chasm" identified in the study. This chasm is found to reflect the innovative capacity at KAM initiative-level execution.

"Before a FoaK (asset) is announced as a business unit new solution offering which is the ultimate thing, but before that you'd have to have collaboration to work towards that. And the way we're set up iffunding were not an issue here, I'd be willing to put as many resources on this as necessary to make this successful but we do have recovery targets (i.e., research revenue generation targets). So, I think if there were a more formal process that would allow us to make this hand off easier that would ultimately result in more successful FoaK projects from the perspective of delivering new commercial products." Int.18.0 


\section{Transformative Capacity (KCF internal knowledge; knowledge retention)}

Transformative capacity is an internal knowledge dynamic capability in the KCF. It represents the organization's ability to retain knowledge over time (e.g., within a knowledge base), and reactivate that knowledge as needed. Transformative capacity was found to be central to the achieving the knowledge acquisition method objectives of creating a new innovation through repeatable and scalable co-creation relationships.

"Sometimes they don't, but the fact that there is a process in place makes it much more easy for them to go off and talk to three or four or five or ten clients over a period of a month or two or three and then get that client engaged." Int.10.0

"The programmatic knowledge is good, it has been accumulated, and is leveraged. But, when the fact of the matter is that the each proponent is so unique in terms of what it's developing, how it will be implemented, who will catch it, how it will be used, etcetera, that it's kind of difficult to actually create a knowledge base of technical dos and don'ts. One can do it in general terms, but the specifics become very difficult." Int.36.0

The role of the transformative capacity was observed throughout the study and the FoaK program theory. At the firm-level, the co-creation program was developed in part to establish a focused approach to guiding new research. This was based on proven practices learned through the execution of co-creation initiatives across the organization, and building a competency within a specific knowledge acquisition method (e.g., FoaK). At the program-level, the effectiveness of the FoaK program orchestration, conditions to participate, guidance and governance of initiative teams were each found to be related to the program's ability to gather, retain, and use knowledge relevant to initiative execution. This knowledge was demonstrated both as explicit codified artifacts available for FoaK initiative participants and stakeholders, and in the form of tacit knowledge retained within 
the FoaK program management office and its board members. Transformative capacity was also observed in FoaK initiative team members and stakeholder experience, and in their ability to leverage the knowledge and the guidance provided by the program. The findings demonstrated that extended initiative teams (i.e., initiative teams and their stakeholders) with previous FoaK experience and the ability to draw upon the program knowledge, were found to have initiatives that performed better the teams that did not have this same level of experience. Similarly, teams able to identify gaps in their knowledge, and that were capable of drawing upon the greater collective knowledge within the program were also found to perform better.

"You get some of those (FoaK initiative teams) with less experience and even the ones where sometimes they might not have much guidance from people around them and so the less experience they have, the more that (the FoaK program team) will help them understand the process. (Our program manager) does an amazing job of laying it all out. (The program) has the steps, here's what you need to do. And some teams are usually really good about getting ahold of (the office) but if they also ask us, 'Oh, how does this work, how does that work?' we'll also provide that help with these teams. Because some of them, a lot of them, have not presented before, don't know the details, don't know-- some of them come in and say, "Oh, for the (FoaK pitch) presentation, Ineed this because it's in a template. How do I get this?" Int.47.0

"(As an ACO, deciding on working with a FoaK initiative team is) something which is very pragmatic... (there are initiative team) people I know and the people I don't know. And there are people I know, and I've already done some First-of-a-Kind (with), and I know exactly how to encapsulate what they tell me, and I know to transcribe that into time to market, quality of the technology, and so on, and I know how I'm going to engage with them. Int.23.0

The transformative capacity for the FoaK as a knowledge acquisition method was found to be a combination and connection between the available knowledge retained by 
the program and its individual initiative-stakeholder teams. This knowledge bridge represents a bidirectional sharing of experience between the two entities. The program focuses on providing a knowledge base built on previous experience along with the current view of the firm's strategic priorities, which help address knowledge gaps within initiative teams. Initiative teams in turn contribute new knowledge from their projects into the program, and it is the program's ability to capture and reactivate knowledge of these experiences in future engagements that contribute to the degree to which the method will be successful for the firm.

"(The FoaK program and board may say) 'Okay. Let's just make sure that we have this services team aligned, because (the initiative team) may not have thought about it.' Or we might identify, 'We think there is some other work that's going on in this product division that (the FoaK initiative team) either can benefit from it or may become part of.", Int.01.0

"(The FoaK program team) asked the teams, although we don't always get it, we asked the teams for lessons learned especially in their final review meeting, so we can try to incorporate that into our guidance and share that for future teams." Int.02.0

The most apparent link between the transformative capacity and a co-creation of innovation initiative was found between initiative teams and their leader's (i.e., primary investigator) previous FoaK experience. Teams that have participated in previous FoaK projects, particularly initiatives that were successful, were found to have developed and retained knowledge related to the effective execution of these types of initiatives. These teams demonstrated greater efficacy in engaging stakeholders towards a common outcome. These initiative teams were also found to leverage the broader knowledge available to them from the program and their stakeholders. As such, their initiatives were found to perform 
better than those led by teams with less experience and less effective in drawing upon the knowledge available.

"Ifound that the teams that have been through (a FoaK initiative) before, especially obviously, the research team, are much quicker to engage clients and much more successful in selling them on the project, and they work through the issues much more quickly and get the contract signed much more quickly. There's definitely a benefit to having worked on a previous project. I found that all you need is really one person with the overlapped experience and that really helps... Quite frankly, if we have issues that come up, it's usually because of someone who has not worked on a project before and has stepped outside of (the FoaK program guidance) parameters, doing something they thought was good, but it ends up harming our relationship with the client." Int.10.0

"You can definitely tell the team that has gone through a FoaK process to understand all the potential pitfalls, but they can change FoaK to FoaK in terms of acquiring a client, or risk a team that they are working on. So, experience always helps, but there is a community in terms of people around them and the FoaK team itself." Int.27.0

Inventive Capacity (KCF internal knowledge; knowledge exploration)

Inventive capacity in the KCF refers to an internal knowledge dynamic capability reflecting an organization's ability to explore new knowledge. As with the innovation knowledge capacity, inventive capacity is central to open innovation and knowledge acquisition methods. Specifically, inventive capacity refers to the firm's ability to perceive new opportunities for invention, set up knowledge exploration processes and the approach to assessing and perceiving the new opportunity potential for the firm. The study observed the role of inventive capacity across all stages of the FoaK program theory.

"(Purpose of the FoaK program), we do need a mechanism to be able to invest in the early development of the solution, in many different areas because we serve every industry, basically. And if we only rely on the product team-- on our current products, 
the products, by and large, are not industry-specific such that the new one we're putting together. We just want new, whatever the needs, and different things we need to create." Int.01.0

"(ACO participation in a FoaK)... it's a kind of win-win deal where we learn more about how these assets can be used by a customer. How (our product organization) can integrate that better in our solution, so that's all benefit, and for the customer it's having the ability to touch to a specific (solution)." Int.23.0

At the firm-level, inventive capacity was seen both in the organization's strategy for invention (e.g., research organization) as well as the funding for new invention through knowledge acquisition methods and programs. At the program-level, program design and execution look to both stimulate invention within the organization before program funding, as well as providing a framework and channel for inventive exploration within a cocreation context. The study found that sources of invention (e.g., research function) within the organization would direct their efforts towards new strategic opportunities investing in early stage invention with the hope of benefitting from funding mechanisms provided by a method (e.g., FoaK program).

"The problem that (the FoaK program is) looking to tackle, which is support solutions that are a little bit more cutting-edge. That don't have a place in existing channels, or existing support, existing paths. Support those technologies with a lot of promise so that they can lead to revenue and opportunity for (our company) down the road." Int.47.0

The FoaK program builds on previous knowledge through a prescribed exploration process that is orchestrated and governed to help focus initiative team efforts towards innovations of value to the firm strategy. The ongoing review and evaluation processes, through both the program and stakeholders, assess the ongoing value of various 
opportunities. Applying a portfolio management discipline determines where investment would best be served based on the perceived benefit across the range of initiatives available. At the initiative-level, FoaK initiative teams continually focus their efforts on identifying new opportunities, validating the potential impact of those opportunities to the market and the firm, and adjusting the course of the invention process with their co-creation client and stakeholders towards outcomes that address market needs. While working within the framework imposed by the knowledge acquisition method, initiative teams were also found to design their execution process to fit their specific scenario and co-creation client.

"FoaK was in a sense the only other primary mechanism when people wanted to work on a particular industry problem, both to have a venue to do this for a mechanism to work with our industry, for the business, and is primarily in sales and distribution...to get engaged with the client to follow a real industry problem that then could work to what we're offering." Int.27.0

"I always try to align FoaK ideas with the industry roadmaps. So, in my case, travel and transportation, ...they always set out at the beginning of the year a set of big bets - so things that they're focusing on that would complement their solution blueprint - and if you can align-- the big bets are usually things that are in progress." Int.18.0

Inventive capacity as a dynamic capability is found to positively impact co-creation of innovation performance. Inventive capacity at the level of the firm and knowledge acquisition method supports repeatability of co-creation engagement. This was observed through the program's ability to identify new opportunities, guide and fund co-creation initiatives. This contributes to repeatability both in the selection of the right initiatives and in the ongoing support of those initiatives through execution. Inventive capacity was found to positively impact the performance of initiatives led by teams that were more strategic in 
their approach to creating new invention, before and during a FoaK initiative, and in their work with co-creation clients in prioritizing innovation exploration focus.

"(Research and FoaK initiative teams) are the ones who actually come up with the idea... and to submit it. We, (the FoaK program) don't come up with the idea and then say that we need these researchers to do it. These researchers are coming with the idea. Occasionally an industry (team) will compensate we need a solution for the problem in the space. And then (they will) go to their research industry specialists and say 'Hey, can you help put together a proposal in this area and get us the right researchers to work on it?",'. Int.02.0

\subsection{Revision of the Conceptual Framework}

The analysis and mapping of the FoaK program theory (FPT) to the knowledgebased dynamic capabilities that informed the initial conceptual framework (Figure 3.2) identified opportunities for revision to the knowledge capacity framework (KCF). These revisions are believed necessary to reflect the integration points between a knowledge acquisition method and its initiatives. Specifically, the analysis has provided clarity on the role of the knowledge acquisition method as an "orchestration" higher-order dynamic capability, and has identified the portfolio management capacity as an "optimization" dynamic capability connecting program to project. This section discusses these points of revision, and presents the revised conceptual framework.

\subsubsection{Portfolio Management Capacity (internal knowledge; optimization)}

A central theme in this study has been the role of the knowledge acquisition method in managing initiatives and the co-creation of innovation. The range of knowledge capacities including the original knowledge capacity framework describes their role in the 
exploration, retention, and exploitation of new knowledge and innovation process both within and between firms. However, the KCF was not found to adequately address the role of the knowledge acquisition method, in this case an innovation program, in effectively managing their innovation opportunities against available resources and priorities. The study found that the program's role as a portfolio manager contributes to the notion of dynamic capability orchestration at the initiative-level execution. Specifically, portfolio management capacity serves as the link between the knowledge acquisition method intention and actual innovation initiative execution.

"(The FoaK program) role is to evaluate the project, both in terms of the viability, the impact, and also we're trying to balance it, right?...We're trying to ask that like venture capital that would make investments, or seed funding" Int.01.0

"One of our biggest challenges, especially after the great recession of 2009, before then, we had a lot of companies that were happy to work with us on kind of research, collaborative level. After the great recession, companies became much more concerned with financial payback for all investments. And even though (collaboration clients) weren't paying money for a good part of the effort, they were still dedicating their time and resources to work with us on the project. And as a result of that, they really had very little interest on working on it from a research kind of level only. They had to show within their company some sort of financial payback in the end Int.10.0

The "portfolio management capacity" proposed leverages the portfolio management dynamic capability for information systems introduced by Daniel et al. (2014). The IS portfolio management dynamic capability represents the firm's ability to manage investments in initiatives based on business objectives, investment criteria, the dynamic balancing of risk and reward during the execution of initiatives, and determination of ongoing investment for in-flight projects. The knowledge capacity framework 
categorizes knowledge capacities as either contributing to knowledge exploration, retention, or exploitation. The portfolio management capacity introduces knowledge optimization as fourth classification to the KCF (see Figure 10.1).

"Let's say if we know that we, as a corporation, are going to put a lot offocus on IoT (Internet of Things), then we had a special (FoaK) session on IoT. So, we had a call for proposals where all the ideas has to be around IoT. When we were launching the (Company A) partnership three years ago, we did a call for proposal for anything related to (Company A) products. So, in that sense, it's more of a-- not so much there's a pressure from the business to do things differently... We are trying to align what we do from a First-of-a-Kind with where the business wants to go." Int.01.0

"So one of the things people should realize that - and that's why I gave that analogy of start-ups - that (these initiatives are) First-of-a-Kind. We need to be completely okay with failure because we've kind of lots of new ideas and not all of them are going to stick. And if we become so conservative that every one of these has to be successful, then we won't be pushing the boundaries .... I think (the FoaK program) has to be looked as a portfolio." Int.27.0

The portfolio management capacity within the KAM was found to exist as a nexus between the firm's strategic objectives, the FoaK program board's innovation priorities, and the initiatives seeking funding for their co-creation projects. The portfolio management approach in conjunction with the program management function fulfill the objectives of the knowledge acquisition method in identifying candidate initiatives with the greatest potential for the firm. Portfolio management in turn funds and guides those initiatives during their execution, and monitors their performance to determine whether ongoing investment benefits the firm. As was seen in two of the cases (i.e., Case 2, Case 3), cocreation initiatives demonstrating strong innovation potential received additional rounds of investment resulting in positive performance outcomes. 
"You want a client that is interested in collaborating, and interested in innovation, and is really excited about the (FoaK) project. They continue to work closely with the team throughout the project. I think it is also when there's collaboration with the asset commercialization owner. Where they are involved throughout the project and guiding how the project goes and are aware of what the team is creating and how they're creating it." Int.02.0

"I see FoaK as being established, and there's a process to it, and it is democratic in the sense that you go through the (FoaK initiative proposal) specs, and if you do everything, if you put the effort in, you get to present (to the board), and then you get your day in the light. So, I think that there's an appeal there that it's in your control. So, I think that (FoaK) teams like it and apply to it for that reason." Int.47.0

\subsubsection{FoaK as a Higher-Order Dynamic Capability - "Orchestration"}

One of the objectives of this study was to evaluate the dynamic capabilities that are relevant for open innovation initiatives, and whether higher-order dynamic capabilities play a role in the knowledge acquisition method (e.g., knowledge management capacity). Higher-order capabilities have been described as possessing the potential to integrate and coordinate the dynamic capabilities of the firm (Lichtenthaler \& Lichtenthaler 2009; Wang \& Ahmed 2007), in this case, in co-creation of innovation initiatives. The study provides first evidence for the notion of knowledge acquisition methods serving as a higher-order dynamic capability, and introduces of this capability as that of "orchestration" of the elements of co-creation of innovation initiative success.

As has been described, the FoaK program as a knowledge acquisition method was found to have an indirect impact on co-creation initiative performance. Recall that FoaK initiative teams and the execution of their projects were found to have the most direct impact on initiative performance. It was found that the role played by the program was that 
of an orchestrator for initiative and portfolio success. The program served in determining the elements and practices for co-creation success based on past knowledge and experience. This knowledge was used to set a framework aimed at motivating stakeholder behaviour, bridging knowledge gaps in initiative teams, and focusing initiative team activity on the co-creation innovation initiative performance. Orchestration as a higher-order dynamic capability is developed further in Chapter 11.

"(The FoaK program is), I think (of) as a place where people bring new ideas, and then (as) an integrator who can try and make sure that the investment we are making in... new idea(s) really builds fruit." Int.27.0

"So when you think about that scenario of linking idea, to client, to products, and just a whole invention to innovation cycle, FoaK provides a platform in business terms, not technical terms and a framework." Int.18.0

"The importance of the FoaK program office as book end or book ends, that they played an important role in the beginning and now they have to again play an important role in the end. ... the FoaK program offers that the asset is not developed and now the FoaK program office is kind of the glue because they have been sort of touching all aspects of this project, not just the execution team, but also the FoaK board which is executives across the organization, also the (products) catching organization, also the industry leadership, etcetera." Int.36.0

"(The FoaK program) provide support for shepherding the product through." Int.27.0

\subsubsection{Updated Conceptual Framework}

The initial conceptual framework for this study was developed based on research in the areas of knowledge management, dynamic capabilities, and open innovation (Daniel et al. 2014; Frederich \& Andrews 2009; Helfat 2007; Lichtenthaler \& Lichtenthaler 2009; 
Nielsen 2013; Prieto et al. 1989; Wang 2007). The conceptual framework combined the theoretical knowledge capacity framework (KCF) proposed by Lichtenthaler and Lichtenthaler (2009) with the model for co-creation of innovation linking firm strategy, the knowledge acquisition method and its stakeholders, and initiative performance.

The revised conceptual framework represents validation of what began as a literature-based dynamic capabilities perspective of co-creation of innovation initiatives. The findings have provided evidence in support of this framework based on the case studies examined. This includes clear support for the linkage between strategy to knowledge acquisition methods and initiatives. There was also new insight into the existence and interaction of knowledge capacities (i.e., dynamic capabilities) in the co-creation of innovation examined at the initiative-level. These findings also highlighted the role of primary stakeholders in the co-creation of innovation process, as well as the role of the knowledge acquisition method and initiative as a bridge across firm boundaries between co-creation clients and the firm (i.e., mainstream product portfolio stakeholders).

In addition to the study's support for theoretical knowledge capacity framework, findings also proposed revisions to the KCF and this study's conceptual framework. Three changes to the conceptual model are proposed based on the study findings (see Figure 10.1). The first change corresponds to an extension of the knowledge management capacities framework to include a new dynamic capability representing the portfolio management knowledge capacity (i.e., internal knowledge capacity for knowledge optimization). This revision to the knowledge capacity framework reflects the execution linkage between the knowledge acquisition method (i.e., FoaK program) and co-creation of innovation initiatives. The second change to the conceptual framework includes 
updating of the "knowledge management capacity" (Lichtenthaler \& Lichtenthaler 2009) as a higher order dynamic capability in the co-creation process, to specify its role as an "orchestration" dynamic capability. Finally, the co-creation initiative was initially represented as including inter-firm integration as a primary process. The study revealed that co-creation initiatives are characterized by managing both internal (i.e., product stakeholders) and external "client" stakeholders. It was found that initiative teams were less of an extension of internal stakeholder requirements for new innovation, and more of a bridge between the firm and external co-creation partners. Initiative team management of internal and external "client" stakeholders were found to require very similar considerations to successfully secure engagement and maintain alignment during the project. As such, the conceptual framework is updated to represent both inter-firm and intra-firm integration processes. These revisions are reflected in the updated conceptual framework in Figure 10.1.

The conceptual framework developed for this study was found to be effective and clear in its representation of the primary elements and linkages in inbound open innovation initiatives. While this conceptual representation of these types of collaborative innovation initiatives was validated in the context of a FoaK, the principal elements represented in the framework are consistent with other forms of knowledge acquisition methods which a firm may use in inbound open innovation engagements. It is believed that this conceptual framework provides future benefit as a guiding model for researchers studying co-creation of innovation methods and knowledge-based dynamic capabilities. 


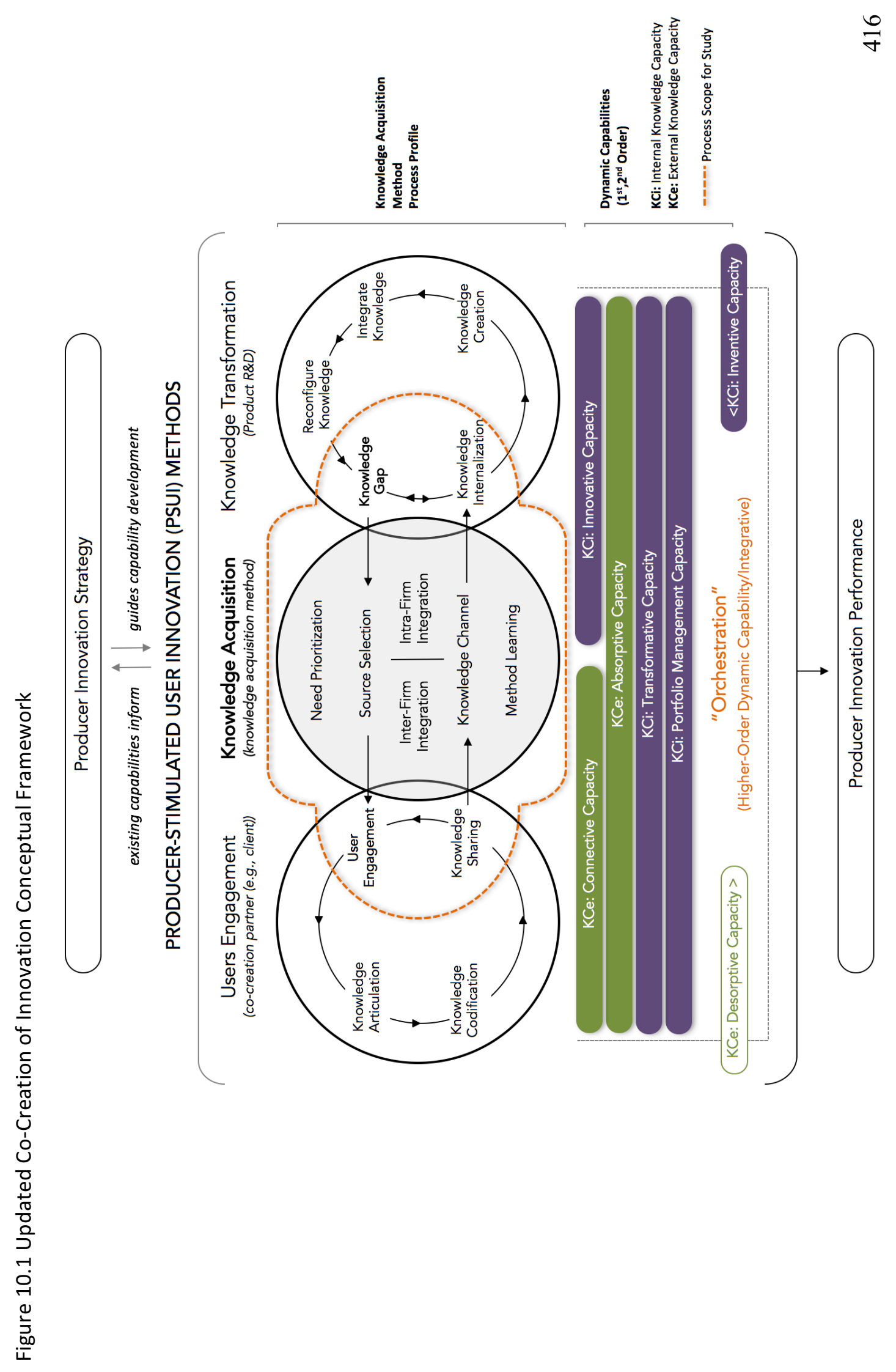




\section{Chapter 11 \\ RESEARCH FINDINGS - THEMES}

This chapter presents the key themes that have emerged from the findings and analysis. It begins with an examination of the range of knowledge types observed in the co-creation of innovation, and the implications of a multi-tier view of knowledge capacity development below the level of the firm (i.e., KAM level, co-creation initiative-level). This is followed by a discussion of the role of a knowledge acquisition method as a higher-order dynamic capability (i.e., orchestrator), and the inclusion of a KAM-level portfolio management dynamic capacity (i.e., internal knowledge capacity optimization) in inbound open innovation co-creation initiatives. Findings regarding the interaction between these knowledge-based dynamic capabilities will be discussed, providing insight into the complexity of interactions within co-creation initiatives. These findings will then be brought together through a conceptualization of the multi-tiered nature of knowledge capacity dynamic capability development and interaction across knowledge acquisition methods initiatives, and resources.

\subsection{Knowledge in Co-Creation of Innovation Initiatives}

A principal theme that emerged from the study is the range of knowledge types required to effectively execute co-creation of innovation initiatives. Knowledge types extend beyond the direct interaction between the firm and the client innovation interactions, to include understanding of internal and external environments, co-creation stakeholders and processes. This suggests that the knowledge of initiative teams goes beyond the domain of technical nature of use cases, and must include knowledge in the areas of strategic 
agendas, stakeholder engagement, expectation setting and management, and momentum building. This range of knowledge types was found to translate directly into knowledge capacities (i.e., dynamic capabilities) within open innovation practices.

When considering the effectiveness of FoaK initiative teams, it was found that teams with strong leaders and previous experience delivered FoaK initiatives that were more successful. A closer analysis of these teams revealed that there is a range of knowledge coverage that these teams must possess both of their leaders (i.e., primary investigators) and their researchers to effectively execute their FoaK initiatives. Successful teams were found to share similar knowledge profiles and knowledge coverage. It was also found that part of the orchestration conducted by the FoaK program was to ensure that the necessary linkages were in place to help focus initiative teams, bridging any knowledge gaps some teams may possess.

FoaK initiative teams were found to require knowledge coverage that was both market related and firm related, across six knowledge types. From a market perspective, teams are required to understand the value of the innovation they are exploring as it relates to their target market. This includes an understanding of target market opportunities, client knowledge regarding the co-creation partner's domain, resources and processes, and technical knowledge providing an understanding of alternative competitive offerings to their innovation. From an internal perspective, FoaK initiative teams require firm-level knowledge of strategic priorities initiatives and organizational activity, knowledge of the product stakeholder and an understanding of their priorities (i.e., timelines, portfolio requirements) and KAM program-level knowledge of requirements and processes. 
Each case initiative team was found to have varying degrees of knowledge along these knowledge themes, formed by their previous experience and access to sources of information to help supplement the gaps they perceive. A comparison of initiative team knowledge profiles found that the knowledge profile of successful teams are more balanced across the knowledge themes than less successful teams (see Figure 11.1). These teams were observed as having more FoaK experience than the less successful cases studied, and were found to have broader and deeper knowledge in each of the areas resulting from deep and active stakeholder relationships established across functions. Unsuccessful cases were found to have less active relationships with their stakeholders, and were not found to be proactive in accessing the assets available to them through the FoaK program.

Figure 11.1 Cross-Case Comparative Knowledge Type Profiles
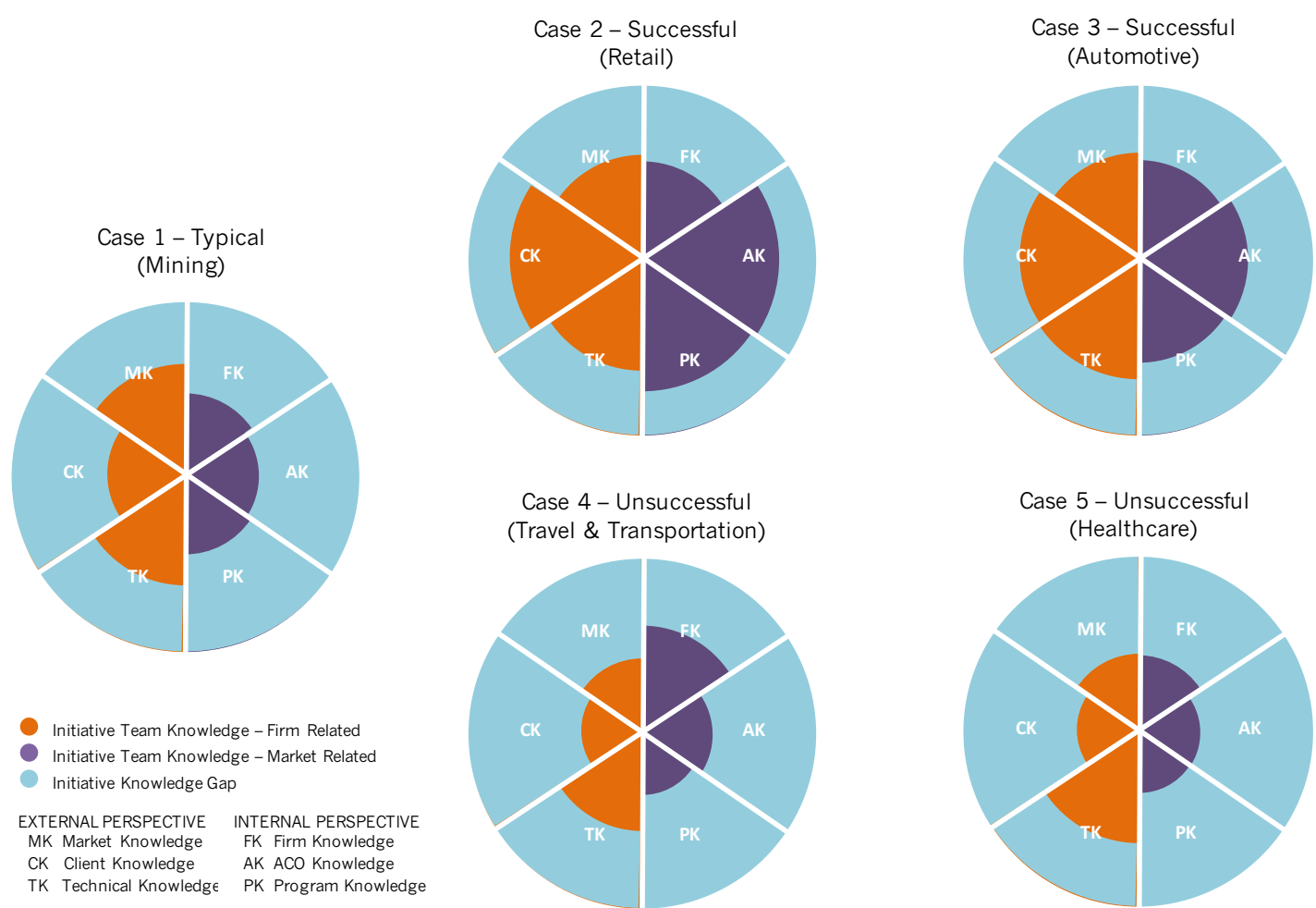

Case 4 - Unsuccessful (Travel \& Transportation)

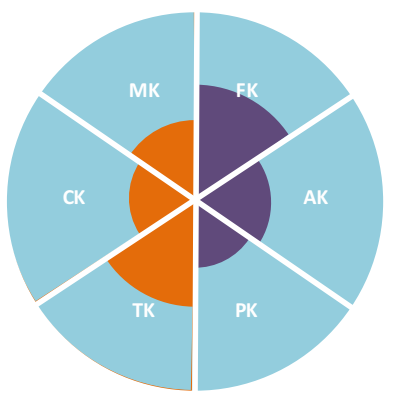

Case 5 - Unsuccessful (Healthcare)

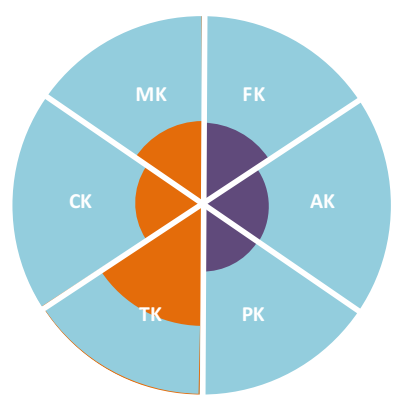


The design of the FoaK program and framework is such that it leads initiative teams to form the necessary relationships with cross functional stakeholders, and provides access to mentors and knowledge assets. This approach speaks to the role of bridging knowledge gaps as part of a co-creation initiative program design to develop new researchers, and to work towards levelling knowledge variations across initiative teams for greater repeatability.

Each of the six knowledge areas described as contributing to the FoaK initiative team's ability to execute can also be translated into knowledge about co-creation processes, engagement, stakeholders, and innovation domain. As such, each of these knowledge types is characterized by the knowledge capacities to which they contribute to. Table 11.1 illustrates a mapping of knowledge types to the knowledge capacities in the KCF. Each intersection point reflects either a knowledge type's participation as part of the knowledge capacity or the role that it plays in the development of that knowledge capacity.

For example, the client knowledge type contributes to knowledge capacities including connective capacity (i.e., external knowledge capacity) and innovative capacity (i.e., internal knowledge capacity). In the case of connective capacity, an initiative uses client knowledge contributes to an understanding of client engagement processes for the activities needed to both secure client participation and successfully transfer knowledge from the client to the initiative team. The client knowledge at the engagement level helps to ensure that the initiative team understands the stakeholders true motivation and expectations as well as the client environment. This helps the initiative team be more effective in how they approach the client and manage the relationship, beyond the primary technical innovation level of interaction. Finally, client knowledge contributes to the 
connective capacity through insights from initiative stakeholders (i.e., sales and industry experts) who provide their perspective into client potential, needs, and expectations. Similarly, client knowledge also helps in the connective capacity of the products stakeholder organization once the asset is transferred within the firm for commercialization. Product stakeholders assume ownership of the client relationship upon transfer of the co-creation initiative's innovation asset for further development. The client knowledge acquired through the process further strengthens the connective capacity of the firm both next stage of innovation engagements, as well as in the practices needed to successfully transfer the relationship and knowledge between organizations.

Similarly, client knowledge also contributes to the innovative knowledge capacity of a knowledge acquisition method and its initiative teams. In the case of this knowledge capacity, client knowledge contributes understanding the innovation process insights by working with clients and transforming initial invention use cases into new knowledge that emerges from the client-initiative team interaction. Client knowledge on the engagement process contributes to use case review and input activity addressed by the innovative knowledge capacity of an initiative team, with client validation contributing to the creation of new products and services. Finally, client knowledge contributes to an understanding of the client as a stakeholder and which of its resources are required in the process to access the information needed in the innovation process.

These examples illustrate how a knowledge type found in the analysis (i.e., client knowledge) contributes across multiple knowledge capacities that exist in inbound open innovation engagements. The relationships between FoaK knowledge types observed and knowledge capacities were drawn from the earlier findings and summarized in Table 11.1. 
These observations were well developed in earlier chapters, and do not require reiteration

at this stage.

Table 11.1 Mapping Dynamic Capabilities to FoaK Initiative Knowledge Types

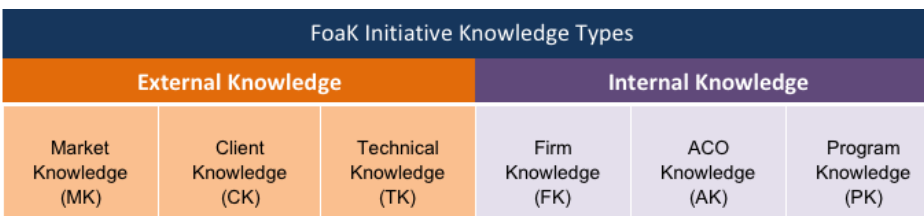

Connective Capacity : retain knowledge in interfirm relationsihps (knowledge retention)

\begin{tabular}{|c|c|c|c|c|}
\hline maintain interorganizational relationships & $\bullet$ & $\bullet$ & $\bullet$ & \\
\hline privileged access to external knowledge & $\bullet$ & $\bullet$ & $\bullet$ & \\
\hline orptive Capacity : ability to expl & & & & \\
\hline acquiring external knowledge & $\bullet$ & $\bullet$ & $\bullet$ & $\bullet$ \\
\hline assimilating into firm's database & $\bullet$ & $\bullet$ & $\bullet$ & $\bullet$ \\
\hline
\end{tabular}

Desorptive Capacity : outward knowledge transfer (knowledge exploitation)

Innovative Capacity : ability to internally exploit knowedge (knowledge exploitation)

\begin{tabular}{l|c|c|c|c|c} 
transmuting knowledge & $\bullet$ & $\bullet$ & $\bullet$ & $\bullet$ \\
\hline converting knowledge to products / services & $\bullet$ & $\bullet$ & $\bullet$ & $\bullet$ \\
\hline determine commercialization opportunity exists & $\bullet$ & $\bullet$ & $\bullet$ & $\bullet$ & $\bullet$
\end{tabular}

Transformative Capacity : ability to internally retain knowledge over time (knowledge retention)

\begin{tabular}{|c|c|c|c|c|c|c|}
\hline retaining knowledge in firm knowledge base & $\bullet$ & $\bullet$ & $\bullet$ & $\bullet$ & $\bullet$ & $\bullet$ \\
\hline reactivating knowledge & $\bullet$ & $\bullet$ & $\bullet$ & $\bullet$ & $\bullet$ & $\bullet$ \\
\hline perceive new opportunities & $\bullet$ & & $\bullet$ & $\bullet$ & $\bullet$ & $\bullet$ \\
\hline set-up knowledge exploration processes & & $\bullet$ & $\bullet$ & $\bullet$ & $\bullet$ & $\bullet$ \\
\hline perception of particular opportunities & $\bullet$ & & $\bullet$ & $\bullet$ & $\bullet$ & $\bullet$ \\
\hline
\end{tabular}

Portfolio Management : ability to manage knowledge towards firm benefit (knowledge optimization)

\begin{tabular}{|l|l|l|l|l|}
\hline business objectives drive projects & - & $\bullet$ & $\bullet$ & $\bullet$ \\
\hline multiple and dynamic prioritization criteria & $\bullet$ & $\bullet$ & $\bullet$ & $\bullet$ \\
\hline dynamic balancing of risk and reward & & $\bullet$ & $\bullet$ & $\bullet$ \\
\hline cancel or reconfigure in-flight projects & & $\bullet$ & $\bullet$ & $\bullet$ \\
\hline HoDC Knowledge Management Capacity & - & $\bullet$ & $\bullet$ & $\bullet$ \\
\hline
\end{tabular}




\subsection{Knowledge Capacities - Initiative-Level Perspective}

The discussion of knowledge in co-creation of innovation initiatives (section 11.1) suggests that knowledge capacity exist both at the level of the knowledge acquisition method (e.g., FoaK program) and at the level of the initiative team and its stakeholders.

The knowledge acquisition method represents a collection of knowledge consisting of program experience, initiative history, and the domain experience of its participants (e.g., FoaK board). This knowledge spans both internal and external knowledge capacities, and how they interact in the exploration, retention, and exploitation of new knowledge use in the creation of new-to-the-firm innovation. The knowledge acquisition method as an orchestrator of dynamic capabilities sets the stage for co-creation initiative execution, which is dependent upon the initiative team's ability to execute (see Figure 11.2).

It is found that initiative teams also possess the combination of knowledge management capabilities that represent the collection of knowledge across the initiative team's leadership, its team members, and the associated initiative stakeholders. The profile of knowledge management capacity development was found to vary across teams, reflecting the different levels of experience in these transient groupings of resources. As such, it is also expected that there will be varying levels of dynamic capability development between the knowledge acquisition method and the individual teams that are charged with executing co-creation initiatives.

It was found that knowledge acquisition method design is intended to bridge these dynamic capability differences through the combination of its orchestration role and its portfolio management capacity. The orchestration role works to bring together what is believed as the right elements needed for the innovation process with external stakeholders. 
It sets the stage that will inform the selection and guidance of initiatives supported by the method. It is the portfolio management knowledge capacity that represents the link between the strategic intent of the knowledge acquisition method with the tactical execution by individual initiative teams.

As the knowledge acquisition method experience grows, so does the development of its knowledge management capacity profile where relevant for the method. The portfolio management capability, in turn, represents the current adjusted direction and requirements of the knowledge acquisition method, which has been described as the proxy for the firm's strategic objectives. A well-developed portfolio management capacity is manifested through its evaluation of initiatives with the greatest potential to impact the firm, the assessment of the innovation's merits as a contributor to the firm's competitiveness, and a determination of an initiative team's ability to execute based on their level of knowledge and capability. The portfolio management's capability forms the link between the initiatives mandate and the knowledge acquisition methods performance objectives.

This suggests that organizations may look to developing dynamic capabilities both at the level of their knowledge acquisition methods as well as in participant resources over time. As experience grows at both levels, the knowledge capacity gap between the knowledge acquisition method's intended knowledge requirements and the actual knowledge within initiative teams participating in innovation initiatives decreases over time. The effectiveness of the knowledge acquisition method will in part be determined by its ability to bridge the knowledge capacity gap in practice using program-level mechanisms (e.g., mentoring, codified artifacts, proven practices). 
Figure 11.2 Co-Creation of Innovation Tiers (Strategy, KAM, Initiative)

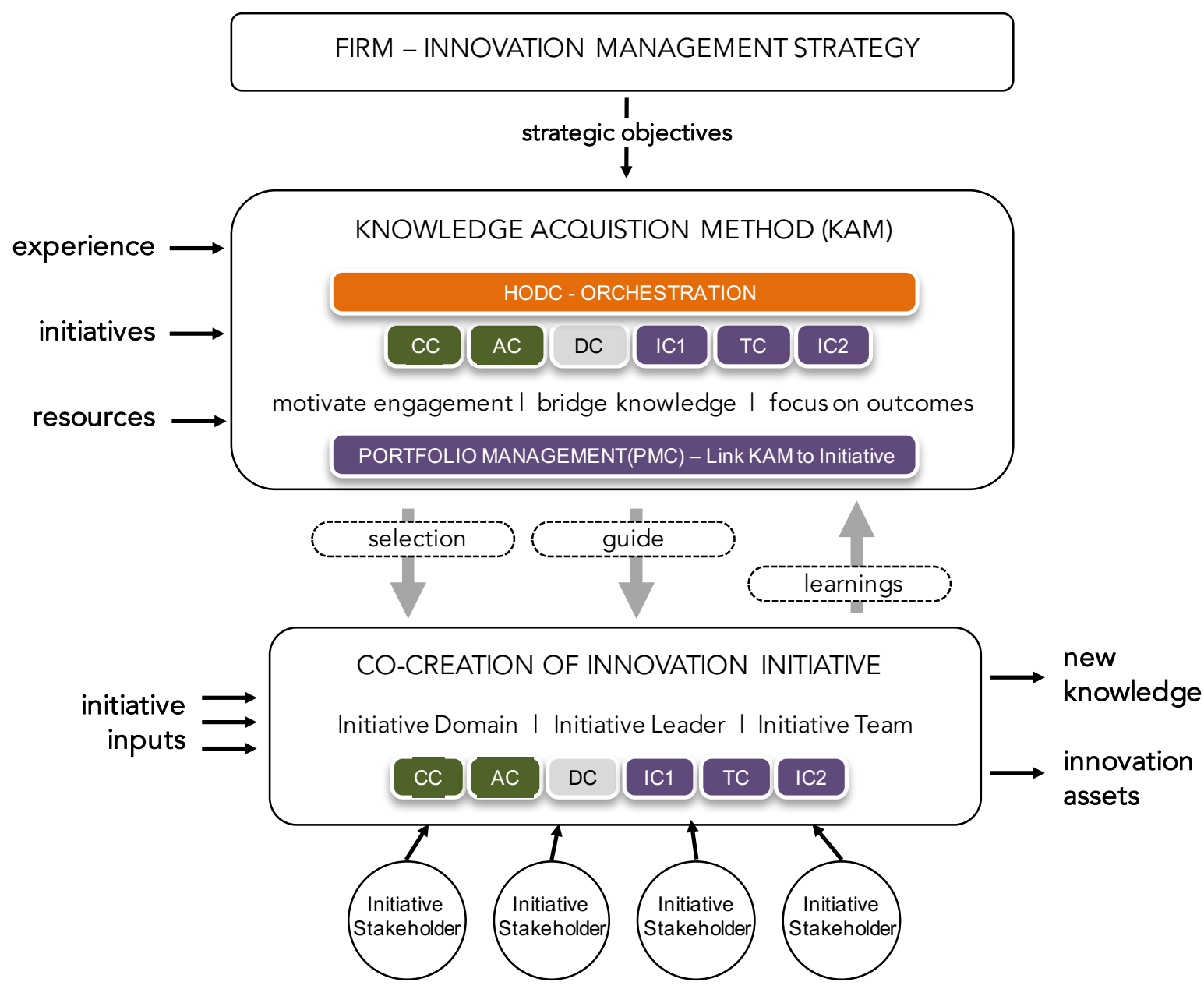

KNOWLEDGE MANAGEMENT CAPACITIES

Higher-Order Dynamic Capability (HoDC): External Knowledge Capacities:

Orchestration
CC Connective Capacity

AC Absorptive Capacity

DC Desorptive Capacity
Internal Knowledge Capacities:

IC1 Innovative Capacity

TC Transformative Capacity

IC2 Inventive Capacity

PMC Portfolio Management 


\section{Knowledge Capacity Gap and Intra-Firm Performance Variance}

The multi-tiered perspective of knowledge capacity development also provides insight into the intra-firm initiative performance variance observed in the study. Much of the research in the areas of dynamic capabilities and open innovation has been conducted at the level of the firm. The firm-level perspective on knowledge capacities suggests that dynamic capabilities are developed at the firm-level, and that similar initiatives should be expected to result in similar performance. As has been seen, this is not the case. Initiative performance was found to be driven by the interaction between the knowledge acquisition method and the initiative execution. As all the cases studied in this research were conducted within a common knowledge acquisition method context, it is argued that the difference in case performance is attributed in part to the corresponding knowledge capacity profiles of the teams conducting initiatives.

In this study, program context was limited to a three-year period where there was continuity in the program management team, the FoaK board membership, the firm strategic imperatives, the initiative approval process, the project domain (i.e., analytics), and the level of investment provided to approved initiatives in the study. An analysis of each of the KAM knowledge capacity profiles against cases of differing performance levels highlights the differences in knowledge capacity development across initiative teams. Table 11.2 presents a comparative mapping of knowledge capacity development at the level of the knowledge acquisition method (i.e., FoaK program), and each of the five cases studied. A review of the mappings shows different levels of knowledge capacity development across "successful," "unsuccessful," and "typical" initiative types, and how their knowledge capacity development compares during the period studied. 
Table 11.2 Comparative Dynamic Capability Development (KAM versus Cross-Case)

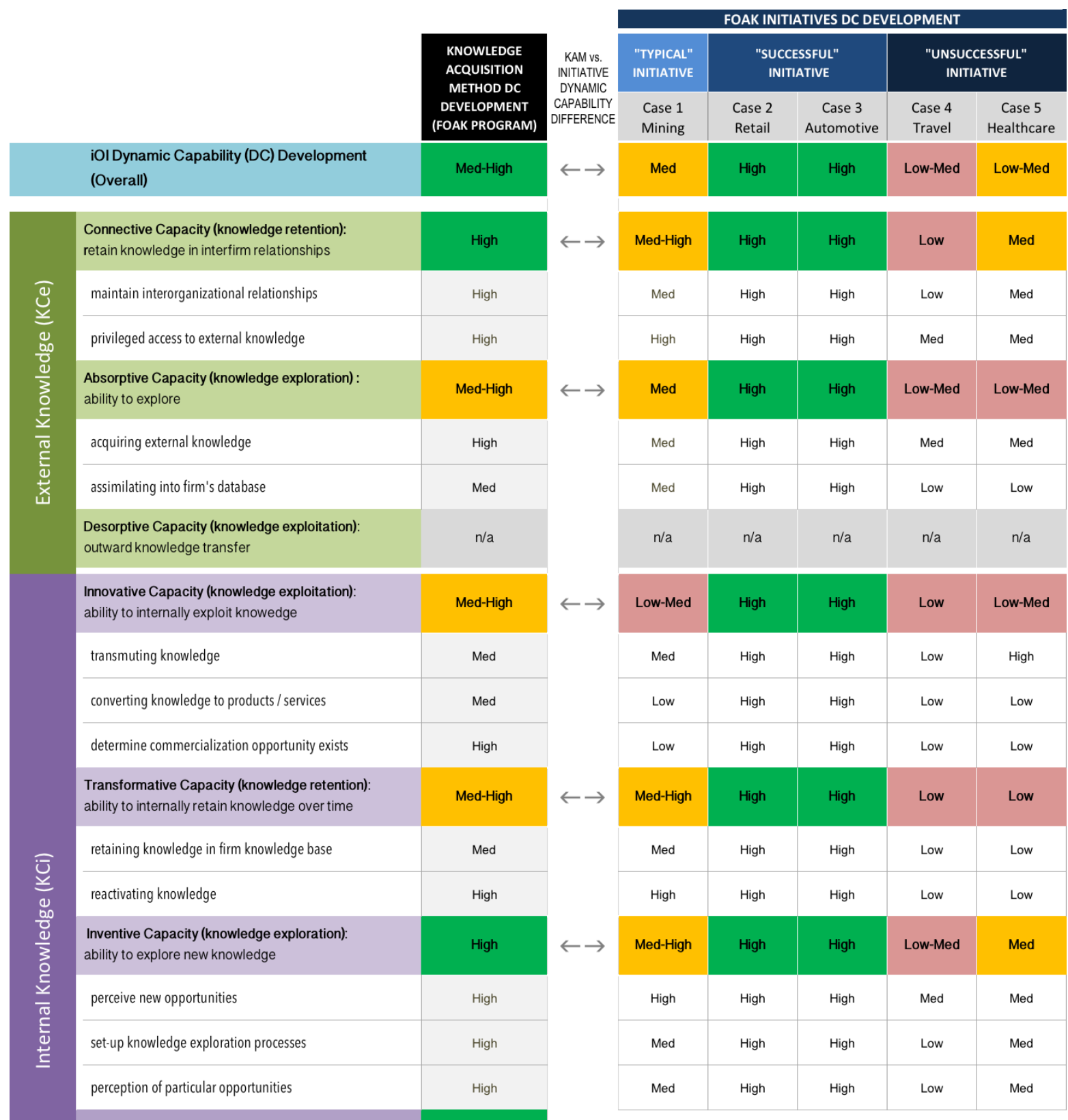

INTIATIVE LEGEND:

HIGH - well developed; high readiness fo co-creation activity

MED - sufficiently developed; moderate support required from KAM

LOW - low readiness; KAM support necessary to bridge knowledge gap 
The examination of knowledge capacity development at the program-level represents a period in which overall, knowledge capacities were well developed for this knowledge acquisition method (KAM). This was not surprising considering the close to 20-year history of the program in operation. The program was found to have welldeveloped knowledge capacities across its collective capacity, inventive capacity, portfolio management and higher-order orchestration knowledge. While also well-developed, transformative capacity, innovation capacity, and absorptive capacity were found to be less developed than the other knowledge capacities at the level of the KAM.

It was found that while the program made efforts to gather knowledge formally from its initiatives and "post-mortem" reviews, it was acknowledged by the program that there was room for improvement in retaining knowledge learned from its projects (i.e., transformative capacity). Similarly, this KAM was found to struggle with the FoaK innovation transfer between initiatives and the products organization, impacting the effectiveness of the knowledge acquisition method in converting new innovations into products and services (i.e., innovation capacity). While the program states that it is achieving its target conversion rates for funded initiatives, other viewpoints consistently highlight the existence of a "chasm" between the FoaK initiative and successful transfer to product stakeholders. It is believed that this chasm is, for the most part, attributed to the scope boundaries of the FoaK initiative. It is suggested that either expanding the boundaries of the program or creating an interlocking knowledge acquisition method for the incubation of FoaK innovations that emerge from initiatives would increase adoption by the product stakeholders. The notion of this innovation "chasm" will be discussed further in the chapter. Finally, absorptive capacity also appears to require additional development, 
observing that the level of readiness of product stakeholders to receive new technologies varied across cases. This in part was attributed to the effectiveness of initiative teams and their ability to engage product stakeholders earlier in the process, readying them to receive new innovations.

A study of the knowledge acquisition method and its knowledge capacity development suggests that while all knowledge capacities are relevant at the program-level, the knowledge capacities of primary focus for the knowledge acquisition method centred on the internal capacities (i.e., innovative, transformative, inventive, portfolio management). The relationship between the knowledge acquisition method and external knowledge capacities (i.e., connective, absorptive capacity) was found to focus primarily on supporting the effectiveness of these capacities for initiative teams, as a catalyst orchestrator. Specifically, the knowledge acquisition method works to bring the necessary stakeholders together as part of their FoaK initiatives (i.e., need for client interaction in their innovation development, need for the product stakeholder sponsorship as the commercialization partner).

The profile of the knowledge acquisition method and its knowledge capacities development represents a near steady state of readiness for initiative support (e.g., cases studied). Taking the perspective that knowledge capacities at the firm-level, or programlevel represents the right unit of study to explain co-creation initiative performance, then one would expect that the initiatives conducted during the period studied would have presented similar levels of performance and behaviour. This was not the case.

Each of the cases studied presented varying levels of initiative performance. An examination of the knowledge capacity development for each of these cases (see Table 
11.2) similarly showed varying levels of knowledge capacity development. This further supports the conclusion suggesting that knowledge capacity must be examined and understood both at the level of the knowledge acquisition method, as well as at the level of the initiative. It also supports the earlier findings arguing the need for understanding knowledge capacities at both levels in the KAM co-creation system to explain innovation initiative performance.

\subsection{Knowledge Acquisition Method as a Co-Creation Orchestrator (HoDC)}

The primary focus in this study is an examination of the role that the FoaK program plays in influencing a FoaK initiative performance. In all cases, the FoaK program was found to have an indirect relationship to the performance of a FoaK initiatives. The effectiveness of a FoaK initiative team in the execution of their initiative was found to represent the direct relationship to initiative performance. Experienced teams and wellexecuted initiatives resulted in better performance than initiatives led by less experienced teams, less effectively. The FoaK program, alongside a FoaK initiative team, serves as an orchestrator that sets the stage with the elements believed needed for co-creation initiative success.

Successful FoaK initiatives were characterized by an initiative team with a range of knowledge and experience, a set of stakeholders that are aligned and engaged to participate in the project, funding and resources needed to execute, and the directed plan that would guide the team to successful completion. The benefit of the FoaK program to FoaK initiatives was found to be the orchestration of the required stakeholders and all the required initiative elements, reducing complexity, and helping to guide teams of various 
levels of experience using proven practices and directed research. Central to the program design is the requirement on FoaK initiative teams to engage and secure the support of stakeholders needed for the successful execution of the co-creation effort. This includes aligning all stakeholders on the goals of the FoaK initiative and the expectations on their role and contribution. The program complements this requirement with initiative funding and formal program credibility to help lower potential barriers to participation with these stakeholders (see Figure 11.3).

As was observed, the FoaK program is structured such that it combines the mandatory conditions to be met to be eligible for funding, guiding initiative team behaviour and priorities. These design tensions intended to motivate the desired level of focus and engagement with stakeholders. The KAM program design ensures that funding is only provided based on strategic merit (e.g., business proposal), and demonstrated support by cross-functional stakeholders required to execute the co-creation initiative.

It is the role as orchestrator where the program design and management both guide and support the strongest candidates along a path, with elements which have been previously proven to contribute to successful co-creation of innovation initiatives. In the end, it was found that the combination of a FoaK initiative team's execution effectiveness within this framework, operating between the "guard rails" set in place by the program, that contributes to more successful initiative outcomes. 
Figure 11.3 Knowledge Acquisition Method as an "Orchestrator" (Higher-Order DC)

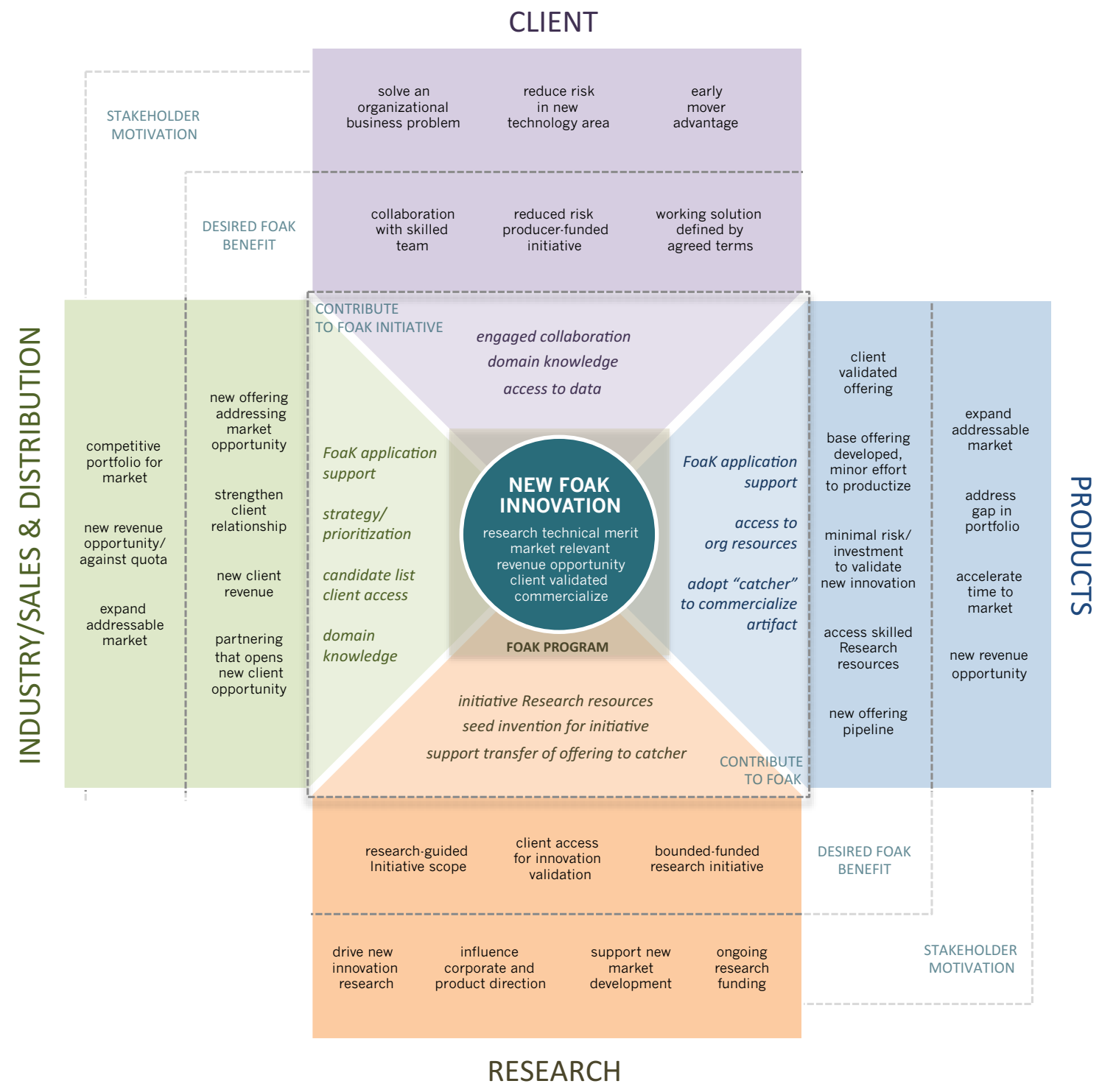




\subsection{Knowledge Acquisition Method and Portfolio Management Capacity (KCi)}

As presented in Chapter 10, study findings suggest extending the knowledge capacity framework to include an additional internal knowledge capacity for optimization, the portfolio management capacity. When considering the role of a knowledge acquisition method, the study has shown that the KAM's primary objectives focus on motivating stakeholder engagement, focusing initiative teams and stakeholders on initiative outcomes, and bridging knowledge gaps (i.e., knowledge capacities) between what is believed as needed by initiative teams to be effective in the innovation endeavours, and their actual level of development across each of the knowledge capacity types. The knowledge acquisition method represents a higher-order dynamic capability which orchestrates the elements believed necessary for co-creation of innovation initiatives to be successful. Orchestration sets the stage for the mission of success by setting the stage for co-creation initiatives (e.g., demonstrating strategic relevance, the requirement for stakeholder support before funding, signed clients for the release of funds).

Co-creation initiative teams, as described, have varying levels of knowledge development. The level of knowledge development is dependent on varied sourced experiences that individual members have acquired over time (e.g., previous knowledge acquisition methods experience, senior resources, previous stakeholder relationships, experience and stakeholder engagements). An initiative team's effectiveness in the execution of its projects will have a direct impact on the level of performance of their cocreation efforts. It is the initiative's link to the knowledge acquisition method (i.e., program) that serves as a guide and catalyst towards enhanced execution performance. 
The question that arises is how a knowledge acquisition method across both portfolio and individual initiative performance in a repeatable and scalable way. It was found that this link is achieved through the development of the portfolio management capacity (i.e., internal knowledge capacity optimization) of the knowledge acquisition method. The portfolio management capacity was found to serve as the link between the intentions of the knowledge acquisition methods, KAM orchestration and the execution of individual co-creation initiatives. The KAM's portfolio management capacity combines initiative evaluation, funding, support during execution of initiatives, and further support of momentum development in certain projects. Portfolio management capacity is also viewed as including program management processes to maintain the overarching knowledge acquisition method framework that supports individual initiatives. It is this dynamic capability that serves as the bridge between the strategic objectives of the KAM and the functional execution at the level of the funded project.

This dynamic capability is positioned such that it supports both the portfolio performance objectives by balancing the collection of initiatives strategically, and supports individual initiative objectives by prioritizing, guiding, funding, and governing initiative progress. This bidirectional influence on a knowledge acquisition methods portfolio and individual initiative performance was observed in the study. For example, it was found that four initiative proposals (e.g., Cases 1, 3, 4, 5) were initially rejected as they did not meet the strategic objectives and relevance for the program (i.e., proxy for the firm). Each required that teams rework their proposals for further consideration. Similarly, at the level of initiative performance, it was the portfolio management capacity that was responsible for the program proactively assessing two initiatives (i.e., Cases 2, 3) demonstrating 
positive momentum and potential, and then determining that additional funding would help fuel the further success of these projects. In both cases, each initiative performed successfully and were transferred to mainstream portfolios for commercialization.

\subsection{Knowledge Capacities in Co-Creation Initiative Interactions}

As has been described, co-creation of innovation initiatives are complex open systems that are characterized by a collection of knowledge capacities (i.e., dynamic capabilities) required in the delivery of new-to-the-firm innovation. This study has put forward a revised framework of knowledge capacities relevant to co-creation initiatives and has described orchestration as a higher-order dynamic capability that links knowledge acquisition methods with co-creation initiatives to its method. In addition to the gaining insight into which knowledge capacities are relevant in co-creation initiatives, the study also sought to understand how knowledge capacities interact within these efforts. The study findings further clearly illustrate the notion of complexity in co-creation initiatives and have highlighted that knowledge capacities do not operate as standalone dynamic capabilities. Rather, in the course of co-creation initiatives dynamic capabilities are found to be interrelated and to interact.

The role of knowledge capacities was found to be best represented at the intersection points between stakeholders in both intra-firm and inter-firm interactions. These intersection points reflect both process touchpoints and stakeholder interaction, each drawing on the knowledge represented from their knowledge capacities. A review of the earlier findings presented were analyzed, examining the primary co-creation interactions and the relevant knowledge capacities observed. These findings are summarized in Table 
11.3. Based on the earlier findings, these stakeholder interactions are described further in the following sections (i.e., 11.5.1, 11.5.2). Each of the primary sets of stakeholder interactions observed in the co-creation of innovation initiatives were found to demonstrate a set of knowledge capacities that serve either in a primary role affecting the dynamic of the stakeholder engagement or serving as a secondary role supporting the primary knowledge capacity. The study demonstrated that each of the main stakeholder interaction points in a co-creation initiative requires multiple dynamic capabilities for these points of engagement to be effective.

\subsubsection{Co-Creation Initiatives: Intra-Firm Interaction}

Intra-firm interactions represent engagement points within a co-creation initiative between stakeholders that are within the firm's boundaries. The main intra-firm interactions are described to be between the firm, the knowledge acquisition method (i.e., program), the initiative team, and the principal initiative stakeholders (i.e., product, sales, industry). The knowledge capacities found to be most prevalent in these interactions were found to focus primarily on the internal knowledge capacities, with external knowledge capacities serving primarily in support of the effectiveness of initiative execution related to those internal knowledge capacities.

Orchestration served a primary role in ensuring that the strategic objectives of the firm were represented in the KAM's priorities, in turn linking to initiative team selection and focus, and the conditions to participate. The impact of this orchestration was found to focus on the inventive and innovative capacities of the initiative as they relate to the 
Table 11.3 Mapping of Dynamic Capability Role across Co-Creation Relationships

\begin{tabular}{|c|c|c|c|c|c|c|c|c|c|c|}
\hline & & \multicolumn{9}{|c|}{ Knowledge Capacities in Knowledge Acquistion Method (KAM) Co-Creation Interactions } \\
\hline & & \multicolumn{5}{|c|}{ Intra-Firm Interactions } & \multicolumn{4}{|c|}{ Inter-Firm Interactions } \\
\hline & & $\begin{array}{l}\text { KAM } \\
\text { and } \\
\text { Firm }\end{array}$ & $\begin{array}{c}\text { KAM } \\
\text { and } \\
\text { Initiative } \\
\text { Team }\end{array}$ & $\begin{array}{l}\text { Initiative } \\
\text { Team } \\
\text { and } \\
\text { Product } \\
\text { Stakeholder }\end{array}$ & $\begin{array}{l}\text { Initiative } \\
\text { Team } \\
\text { and } \\
\text { Sales/Industry } \\
\text { Stakeholders }\end{array}$ & $\begin{array}{l}\quad \text { KAM } \\
\text { and } \\
\text { Sales/Industry } \\
\text { Stakeholders }\end{array}$ & $\begin{array}{c}\text { KAM } \\
\text { and } \\
\text { Client } \\
\text { Stakeholder }\end{array}$ & $\begin{array}{l}\text { Initiative } \\
\text { Team } \\
\text { and } \\
\text { Client } \\
\text { Stakeholder }\end{array}$ & $\begin{array}{l}\text { Product } \\
\text { Stakeholders } \\
\text { and } \\
\text { Client } \\
\text { Stakeholder }\end{array}$ & $\begin{array}{c}\text { Sales/Industry } \\
\text { Stakeholders } \\
\text { and } \\
\text { Client } \\
\text { Stakeholder }\end{array}$ \\
\hline$\widehat{\Xi}$ & $\begin{array}{l}\text { Connective Capacity: } \\
\text { retain knowledge in interfirm relationsihps } \\
\text { (knowledge retention) }\end{array}$ & $\mathbf{s}$ & $\mathbf{s}$ & $\mathbf{P}$ & $\mathbf{P}$ & $\mathbf{s}$ & $\mathbf{P}$ & $\mathbf{P}$ & $\mathbf{s}$ & $\mathbf{P}$ \\
\hline$\approx$ & maintain interorganizational relationships & $\mathrm{s}$ & $\mathrm{s}$ & $\mathrm{P}$ & $\mathrm{P}$ & $\mathrm{s}$ & $\mathrm{P}$ & $\mathrm{P}$ & $\mathrm{s}$ & $\mathrm{P}$ \\
\hline$\frac{\mathrm{d}}{7}$ & privileged access to external knowledge & & s & $\mathrm{P}$ & $\mathrm{P}$ & & $\mathrm{p}$ & $\mathrm{P}$ & & $\mathrm{P}$ \\
\hline$\frac{\underline{\underline{v}}}{\stackrel{\mathrm{C}}{c}}$ & $\begin{array}{l}\text { Absorptive Capacity : ability to explore (knowledge } \\
\text { exploration) }\end{array}$ & $\mathbf{s}$ & $\mathbf{P}$ & $\mathbf{P}$ & s & & s & $\mathbf{P}$ & $\mathbf{P}$ & $\mathbf{P}$ \\
\hline 希 & acquiring external knowledge & s & $\mathrm{P}$ & $\mathrm{P}$ & $\mathrm{P}$ & & s & $\mathrm{P}$ & $\mathrm{P}$ & $\mathrm{P}$ \\
\hline & assimilating into firm's database & $\mathrm{s}$ & $\mathrm{s}$ & $\mathrm{P}$ & $\mathrm{s}$ & & $\mathrm{s}$ & $\mathrm{P}$ & $\mathrm{P}$ & \\
\hline \multirow{16}{*}{ 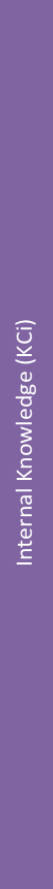 } & $\begin{array}{l}\text { Innovative Capacity: } \\
\text { ability to internally exploit knowedge } \\
\text { (knowledge exploitation) }\end{array}$ & & $\mathbf{s}$ & $\mathbf{P}$ & $\mathbf{P}$ & $\mathbf{P}$ & & $\mathbf{P}$ & $\mathbf{P}$ & $\mathbf{P}$ \\
\hline & transmuting knowledge & & s & $P$ & & & & $P$ & $\mathrm{P}$ & \\
\hline & converting knowledge to products / services & & $\mathrm{s}$ & $\mathrm{P}$ & & & & $\mathrm{P}$ & $\mathrm{p}$ & $\mathrm{P}$ \\
\hline & determine commercialization opportunity exists & & $\mathrm{s}$ & $\mathrm{P}$ & $\mathrm{P}$ & $\mathrm{P}$ & & $\mathrm{P}$ & $\mathrm{P}$ & $\mathrm{P}$ \\
\hline & $\begin{array}{l}\text { Transformative Capacity : } \\
\text { ability to internally retain knowledge over time } \\
\text { (knowledge retention) }\end{array}$ & $\mathbf{P}$ & $\mathbf{P}$ & $\mathbf{P}$ & $\mathbf{P}$ & $\mathbf{P}$ & $\mathbf{P}$ & $\mathbf{P}$ & $\mathbf{P}$ & $\mathbf{P}$ \\
\hline & retaining knowledge in firm knowledge base & $P$ & $\mathrm{P}$ & $P$ & $\mathrm{P}$ & $P$ & $P$ & $P$ & $P$ & $P$ \\
\hline & reactivating knowledge & $\mathrm{P}$ & $\mathrm{P}$ & $\mathrm{P}$ & $\mathrm{P}$ & $\mathrm{P}$ & $\mathrm{P}$ & $\mathrm{P}$ & $\mathrm{P}$ & $\mathrm{P}$ \\
\hline & $\begin{array}{l}\text { Inventive Capacity : } \\
\text { ability to explore new knowledge } \\
\text { (knowledge exploration) }\end{array}$ & $\mathbf{P}$ & $\mathbf{P}$ & $\mathbf{P}$ & $\mathbf{P}$ & $\mathbf{P}$ & $\mathbf{P}$ & $\mathbf{P}$ & $\mathbf{P}$ & $\mathbf{P}$ \\
\hline & perceive new opportunities & $\mathrm{P}$ & $\mathrm{P}$ & $\mathrm{P}$ & $\mathrm{P}$ & $\mathrm{P}$ & $\mathrm{P}$ & $\mathrm{P}$ & $\mathrm{P}$ & $\mathrm{P}$ \\
\hline & set-up knowledge exploration processes & $P$ & $P$ & $P$ & $\mathrm{P}$ & & $\mathrm{P}$ & $\mathrm{P}$ & $\mathrm{P}$ & \\
\hline & perception of particular opportunities & $\mathrm{P}$ & $\mathrm{P}$ & $\mathrm{P}$ & $\mathrm{P}$ & $\mathrm{P}$ & $\mathrm{P}$ & $\mathrm{P}$ & $\mathrm{P}$ & $\mathrm{P}$ \\
\hline & $\begin{array}{l}\text { Portfolio Management: } \\
\text { ability to manage knowledge towards firm benefit } \\
\text { (knowledge optimization) }\end{array}$ & $\mathbf{P}$ & $\mathbf{P}$ & $\mathbf{s}$ & s & $\mathbf{s}$ & $\mathbf{s}$ & $\mathbf{s}$ & & $\mathbf{s}$ \\
\hline & business objectives drive projects & $\mathrm{P}$ & $\mathrm{P}$ & $\mathrm{s}$ & $\mathrm{s}$ & $\mathrm{s}$ & $\mathrm{s}$ & $\mathrm{s}$ & & $\mathrm{s}$ \\
\hline & multiple and dynamic prioritization criteria & $\mathrm{s}$ & $\mathrm{P}$ & $\mathrm{s}$ & $\mathrm{s}$ & $\mathrm{s}$ & $\mathrm{s}$ & $\mathrm{s}$ & & $\mathrm{s}$ \\
\hline & dynamic balancing of risk and reward & $s$ & $\mathrm{P}$ & & & & & & & \\
\hline & cancel or reconfigure in-flight projects & $\mathrm{s}$ & $\mathrm{P}$ & & & & & & & \\
\hline HoDC & Orchestration & & $\mathbf{P}$ & $\mathbf{P}$ & & $\mathbf{P}$ & $\mathbf{s}$ & $\mathbf{P}$ & $\mathbf{s}$ & $\mathbf{P}$ \\
\hline
\end{tabular}

LEGEND:

$P$ (PRIMARY) - KC essential role in KAM co-creation interaction

$S$ (SECONDARY) - KC supportive role in KAM co-creatioN interaction 
co-creation innovation, and in setting conditions in support of the cross-boundary linkages between client knowledge and commercialization of initiative outcomes by the firm.

Firm - Knowledge Acquisition Method

Knowledge capacities in the interaction between the firm and the knowledge acquisition method focused primarily on the linkage between the firm's strategic objectives (i.e., inventive capacity) and the purpose for the inception of the knowledge acquisition method as a form of innovation creation. The firm invests in developing inventive capacity through the inception of the knowledge acquisition method that is funded with the distinct purpose of exploring new knowledge. The existence of the method as a firm sponsored endeavour lends credibility to discussions with existing clients (i.e., connective capacity) and internal stakeholders considering the opportunity to engage in a co-creation initiative (i.e., absorptive capacity). As such, this supports absorptive capacity and connective capacity as it relates to the KAM and the initiative's execution.

\section{Knowledge Acquisition Method - Initiative Team}

The relationship between the knowledge acquisition method and each of its initiatives is connected through the portfolio management capacity, which guides selection, funding, and governance. This point of interaction is characterized by the KAM providing a channel for knowledge exploration and bridging knowledge gaps within initiative teams. It is also centred on initiatives that are driven by the requirement for co-creation, and support for engaging clients as a source of external knowledge. This interaction is also characterized by its support of the innovative capacity by providing a focal point for 
stakeholders within the organization to participate in creating new innovations as part of a funded initiative. Similarly, the credibility that the program provides to the funded initiative also supports connective capacity by supplying additional credence for conversations in recruiting clients to participate.

\section{Initiative Team - Products Stakeholder}

The relationship between the initiative team and its product stakeholder represents the primary path for commercialization of innovation outcomes. This relationship is characterized by a need for knowledge supporting new opportunity identification (i.e., inventive capacity), the ability to gather new knowledge to transform them into offerings relevant to the firm and its strategy (i.e., innovative capacity), effective engagement with the co-creation client (i.e., connective capacity), and the readiness to explore and receive new knowledge through those engagements (i.e., absorptive capacity). The initiative team's effectiveness in engaging the product stakeholder identified early and in-depth in the co-creation process was found to improve the product stakeholder's readiness to receive the resulting innovation (i.e., absorptive capacity). Previous knowledge and experience of both sets of stakeholders further contributed to the effectiveness of the relationship and extracting client information and developing new iterations (i.e., transformative capacity).

Initiative Team - Initiative Stakeholders (Sales/Industry)

Relationships between initiative teams and its internal stakeholders in the cocreation of innovation process is characterized primarily by identifying new opportunities that are relevant to the firm's strategy (i.e., inventive capacity), identifying and accessing 
the right clients to participate in the co-creation process (i.e., connective capacity), and in bringing together multiple perspectives on how value will be defined in the innovation that will fit market needs (i.e., innovative capacity). This includes representing new innovation opportunities with internal stakeholders to help highlight where an innovation exploration can fit into the firm's mainstream portfolio (i.e., absorptive capacity). Alignment across the stakeholders (i.e., orchestration) ensures that there is a common focus on the innovation objectives, and in turn what is needed in the way of collaborators both outside the firm (i.e., client stakeholder) and inside the firm (i.e., product stakeholder). The more that the stakeholders are familiar with these types of engagements and working together, the more effective they are found to be in executing on co-creation of innovation initiatives (i.e., transformative capacity).

\section{Knowledge Acquistion Method - Initiative Stakeholders (Sales/Industry)}

The role between the knowledge acquisition methods and extended stakeholder support is focused primarily on ensuring that multiple stakeholders are contributing to the assessment of an opportunity's value to the market and the firm (i.e., inventive capacity). Stakeholder awareness of knowledge acquisition method priorities was found to stimulate activity in identifying potential co-creation clients (i.e., connective capacity). The knowledge acquisition method sets a framework in place that includes conditions for selection of initiatives to be funded, and which stakeholders must be involved in supporting the initiative as one of merit for the firm (i.e., portfolio management capacity). This includes identifying where potential emerging innovations will support market opportunities through commercialization (i.e., innovative capacity). As with the other intra- 
firm interactions associated with a co-creation initiative, the more experience that can be drawn upon supports better execution (e.g., market knowledge, firm and business level priorities, market opportunities, KAM co-creation process) (i.e., transformative capacity).

\subsubsection{Co-Creation Initiatives: Inter-Firm Interaction}

Inter-firm interactions were found between the knowledge acquisition method, initiative teams, initiative stakeholders (i.e., product stakeholders, sales and industry stakeholders) and the co-creation client stakeholder. These engagement points represent interaction across firm boundaries. Each of the knowledge capacities was found to exist in these interactions, with only portfolio management appearing as a secondary supportive dynamic capability. Considering that these inter-firm interactions represent activity after an initiative has been approved, portfolio management serves primarily to guide stakeholders and their focus on activity that links to the initial objectives of the initiative.

Like intra-firm interaction, orchestration served in ensuring the right elements were in place to support the identification of opportunities, engagement of the right stakeholders, and clarity on the value of the innovation being explored.

\section{Knowledge Acquistion Method - Client Stakeholder}

The relationship between the knowledge acquisition method and the co-creation client is primarily focused on supporting engagement clients in the co-creation process (i.e., connective capacity), evaluating the client as a fit for the proposed innovation exploration (i.e., portfolio), and presenting potential clients based on existing program knowledge and opportunities within the firm (i.e., inventive passively). The knowledge 
acquisition method learns from previous experience in engaging clients in the processes and methods that have been found most effective in extracting new knowledge. This includes participating in practices that engage internal stakeholders (i.e., product stakeholder) earlier in the process with the expectation of transferring the resulting innovation (i.e., transformative capacity). This assessment by the knowledge acquisition method of the client fit influences funding decisions and the ongoing monitoring of an initiative's progress towards delivering client satisfaction (i.e., portfolio).

\section{Initiative Team - Client Stakeholder}

The interaction between an initiative team is the client's principal relationship in the co-creation process. Knowledge capacities in this relationship are characterized by the ability to identify new opportunities with the client (i.e., inventive capacity), work through innovation ideas and transform them into validated use cases that have market relevance (i.e., innovative capacity), and maintaining productive working relationships with the client stakeholder (i.e., connective capacity). These relationships are also characterized by the degree to which product stakeholders are engaged throughout the process, identifying how knowledge extracted in these interactions can be used to prepare the firm to receive this new knowledge as part of its broader innovation objectives (i.e., absorptive capacity).

\section{Products Stakeholder - Client Stakeholder}

The product stakeholder and client stakeholder interaction are characterized by engagement at two points in the co-creation of innovation process. First engagement occurs in the exploration process, where product stakeholders can be introduced to work with the 
client and the initiative team which builds their working relationship with the client (i.e., connective) and provides early knowledge of the innovation being developed based on the use cases being explored (i.e., absorptive capacity). This early engagement helps to provide further validation of the market opportunity (i.e., inventive capacity) and introduces both stakeholders' perspectives in determining new commercialization opportunities (i.e., innovative capacity). The second point of engagement is at the completion of the cocreation initiative, where the client relationship is transferred from the initiative team to the products organization (i.e., connective capacity). Previous experience in managing the transfer of knowledge and relationship between groups within the firm is necessary to support the commercialization efforts of the innovation that emerged from the project (i.e., connective capacity).

\section{Initiative Stakeholders (Sales, Industry) - Client Stakeholder}

The initiative stakeholder and client stakeholder relationship are characterized primarily by knowledge capacities that help build on existing relationships and extending those relationships to co-creation activities. The initiative stakeholders are the primary source of client candidates for co-creation initiatives and serve as the initial contact point in recruiting new clients to participate in these projects (i.e., connective capacity). The initiative stakeholders take their understanding of the market and new innovation opportunities (i.e., inventive capacity) and use that to guide the identification of potential client's candidates to participate in these initiatives. The knowledge that they acquire in discussions with the client in addition to their market expertise is used with other internal stakeholders (i.e., product stakeholder) in presenting the market potential and business use 
cases that are believed to be strategic for the firm (i.e., absorptive capacity). This expertise, in turn, helps to focus information gathered from the client by the initiative stakeholders that in turn are shared with the initiative team and the firm. This helps guide prioritization for commercialization of use cases (i.e., innovative capacity). The initiative stakeholders that engage in the process are triggered by the knowledge acquisition methods orchestration activity.

\section{Cross-Knowledge Capacity Interaction}

The pattern of knowledge capacities observed across the knowledge acquisition method, and initiative stakeholder interaction points highlight the complexity in the cocreation of the innovation process. Each pairing of relationships represents a profile of internal and external knowledge capacities required to effectively manage that part of the innovation process. In examining the relationship between stakeholder interactions and knowledge capacities, interdependencies were also found to exist between knowledge capacities. Specifically, while knowledge capacities provide necessary information, skills, and experience required in the stage of the inbound open innovation engagement, it was also found that some capacities also had an impact on other capabilities within the process.

Transformative capacity is the essence of the knowledge acquisition method, representing the collection of experience, domain expertise, and knowledge needed to be drawn upon for a repeatable, scalable approach to co-creation activities. The stronger the transformative capacity is for knowledge acquisition method (e.g., the ability to draw upon previous experience), will impact other capacities effectiveness including the ability to identify new opportunities (i.e., inventive capacity), engage with client stakeholders (i.e., 
connective capacity), and the readiness of the firm to receive new knowledge into their portfolio (i.e., absorptive capacity). Similarly, it was found that the more effective and initiative team was in engaging with the client stakeholder (i.e., connective capacity) and including the product stakeholder early in the process, the more effective the innovation transfer to the product stakeholder was at the completion of the initiative (i.e., absorptive capacity).

Several themes emerged in examining the relationship of knowledge capacities to one another (see Figure 11.4). Transformative capacity was found to be essential in impacting the effectiveness of each of the other capacities in executing within their area of the co-creation process. This is not surprising considering the goal of repeatability and scaleability of the knowledge acquisition method. The more effective the organization is in retaining and drawing upon previous experience, both at the KAM and initiative team level, the more effective the execution of initiatives will be as knowledge grows. Orchestration was also found to impact several knowledge capacities, which is believed due to its primary role as a nexus point between the elements required for the co-creation initiative's execution. Connective capacity was also found to have broad effects on other knowledge capacities. This is explained by the fact that the entire co-creation initiative stems from engagement with the client stakeholder, and draws upon their knowledge in helping to drive new innovation. The effectiveness of the method and the initiative teams in engaging the right clients has a broad impact on the co-creation process overall.

This study provides early insight into observed relationships and interaction between knowledge capacities in the co-creation of innovation process. These findings 
suggest a significant opportunity for future research examining these interactions in more detail.

Figure 11.4 Dynamic Capability Relationships in Co-Creation Innovation Activity

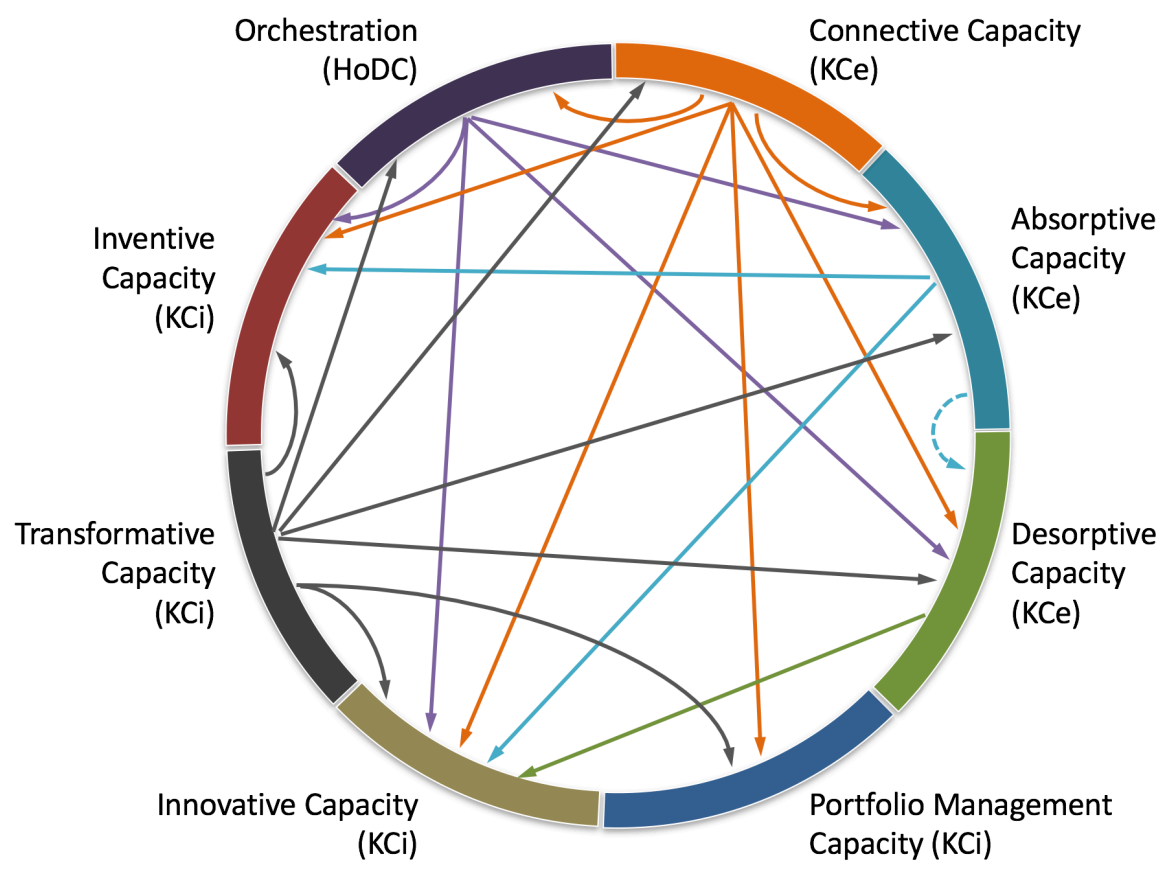

\subsection{KAM and Co-Creation Innovation Performance}

The study findings have provided insight into the role of the knowledge acquisition methods and the relationship to co-creation initiatives at the level of initiative execution. It has expanded on the relevance of knowledge-related dynamic capabilities (i.e., knowledge capacities) and has explained how these knowledge capacities exist, are developed, and interact in an inbound open innovation context. The study has also highlighted that due to the complexity of collaborative innovation, these capabilities must be examined below the level of the firm to understand co-creation initiative performance. The firm-level 
consideration of these dynamic capabilities is not sufficient to explain intra-firm initiative performance variance, as observed in the study.

This section will discuss the findings on co-creation initiative performance and how the knowledge acquisition method influences these efforts. It will discuss the observed contributors to intra-firm performance variance, and the role that knowledge profiles play in explaining performance differences. Three performance-related themes will also be discussed as they relate to their impact on co-creation of innovation: the knowledge acquisition method as an orchestrator, the role of initiative momentum on performance, and the impact of knowledge acquisition method boundaries on performance.

\section{Intra-Firm Performance Variance}

As described earlier in this chapter (i.e., Section 11.2), intra-firm performance variance represents observed differences in co-creation of innovation initiative outcomes based on a common stable knowledge acquisition method, program, and context. Of the five cases studied, initiatives executed within a common program setting were found to have initiative teams with different knowledge capacity profiles, which suggests that the development of knowledge capacities at the initiative team level contributes in part the performance variances observed.

It was found that there are several potential contributors to performance variance which include changes in firm and knowledge acquisition method strategy, differences in the innovation domain being explored, variations in initiative context, and the effectiveness of initiative team execution. 
When examining strategy, three cases (i.e., Case 3, Case 4, Case 5) experienced changes in their product stakeholder business strategy that impacted the transfer of their initiative innovation. While all three experienced a similar potential impact of strategic change on their execution, only two cases (i.e., Case 4, Case 5) were not ultimately successful as initiatives. In both these cases, the initiative team knowledge capacity profiles were found to be below what was expected as required to effectively execute on their cocreation initiative. The initiative team in Case 3, was found to possess a stronger knowledge capacity profile than the other two teams and was also considered to be a more successful co-creation initiative for this knowledge acquisition method. This difference in performance was primarily explained by the readiness of the stronger initiative team to respond to the changing environment. The initiative teams of the unsuccessful initiatives, in both cases, demonstrated uncertainty and lower skill levels in adapting to the changes in their environment.

When examining performance as it relates to individual case innovation domains and related industry use cases, it was not found that these were primary contributors to the variations in initiative performance. From a domain perspective, all the initiative shared use cases associated with analytic applications. In each of these cases, the scope and complexity of the use cases being innovative for required similar skill sets and architectures to deliver solutions. Each of the cases addressed different industries, and use cases specific to those industries. The industries had varying levels of adoption maturity for analytic solutions and as co-creation contributors (e.g., Case 4 rail, low maturity for analytics) versus Case 2 (retail, high maturity for analytics). The domain in the cases studied was not found to be a primary contributor to variations of initiative performance as explained by 
the common problem space being addressed in each. The industry for which the innovation was being explored may have contributed to performance variance based on the maturity level as co-creation client stakeholders, and reflecting the maturity of their industry. This presents an opportunity for future research.

The knowledge acquisition method processes, KAM portfolio agenda, initiative selection, and funding criteria were common across all initiatives studied. Conclusions highlighting the impact of variations within the knowledge acquisition method the scope of the study. The focus for this study examines the attributes of a single knowledge acquisition method (i.e., knowledge capacities and higher-order dynamic capability). Comparative KAM analysis presents a future research opportunity. This includes examining longitudinal examination of changes within the knowledge acquisition method and its impact on initiative performance, or in examining cross-firm initiative performance based on the common knowledge acquisition method approach. Due to the complexity of inbound open innovation systems, longitudinal studies may find richer results in understanding the detailed interaction between knowledge acquisition method changes on an initiative performance.

Finally, variance in initiative team knowledge capacity profiles as they relate to initiative execution and the KAM was found to provide the greatest insight into intra-firm co-creation initiative performance. The question is, "Is the observed variance in initiative performance explained?" This study provides insight into the observed variance in part through its examination of the difference in knowledge capacity profile patterns.

The study has shown that teams with less developed knowledge capacity profiles are intended to benefit from the knowledge acquisition method and its bridging of 
knowledge gaps. The purpose of the bridging role of the knowledge acquisition method was to ensure that teams that were new to co-creation initiatives and the knowledge acquisition methods had the support of expertise and codified knowledge to guide their planning and execution activities. However, the less developed teams were also found to be less engaged with the knowledge acquisition method (i.e., program). These teams were found to struggle more with unexpected issues during their projects, which in turn were found to impede initiative performance. While it is intuitive that teams with stronger knowledge capacity profiles relevant to inbound open innovation co-creation projects were found to do better, it is unclear whether successful teams began with similar knowledge capacity profiles when they first began, or if there was something else contributing to their success. While it is expected that all teams with previous experience in the knowledge acquisition method began with less developed knowledge capacity profiles early on, the development path of their competencies is unclear. However, each of the experienced focus initiative teams highlighted the value of previous experience in the knowledge acquisition method and co-creation initiative projects, which contributed to their effectiveness in later similar projects.

While the study did show that successful co-creation initiative teams had more previous experience with successful projects (i.e., Case 2, Case 3). Each of these teams was found to engage proactively and more frequently with the KAM program than less successful teams. Successful teams were found to be more positive on the role the knowledge acquisition method played in their success. In the early projects, the knowledge acquisition method program provided funding and a roadmap. In their later projects, the program became less about a guiding roadmap and more about collaborating in identifying 
strong innovation opportunities and working with initiative stakeholders more effectively. The study also highlighted that successful teams took a more strategic approach to executing their co-creation initiatives. This in part was attributed to previous experience and knowledge development that deepened the foresight of initiative teams early in the planning stages of their projects. The strategic nature of some teams was also attributed to the strength of their leaders, who were senior employees with deep domain experience. This was reflected in their well-developed knowledge capacity profiles. Successful initiative teams were also found to speak with greater authority on the contributors to their initiative performance. It was evident that unlike less successful teams, these initiative teams demonstrated the strength of their experience in evaluating what worked and what didn't in the execution of their projects. The role of knowledge development is further supported in this regard.

These observations support the argument that knowledge capacity profiles of cocreation initiative teams are a primary contributor to initiative success. These findings suggest that knowledge acquisition methods must consider how knowledge capacities can be proactively developed within initiative teams and their members, that can in turn, be strengthened over time. However, due to the transient nature of co-creation initiative projects and their teams, this requires that knowledge capacity profile development should extend to developing the knowledge at the level of the co-creation resource that can be brought forward over time into any number of future innovation initiatives.

The question is then "How is variance in performance explained between teams of moderate experience?" It was observed that cases with some experience and previous initiative success (i.e., Case 1) were found to perform better than teams with some 
experience and no previous initiative success (i.e., Case 5). It was found that in both cases, initiative teams were found to struggle with challenges encountered during the co-creation initiative process. Each of these teams spoke of the complexity of their co-creation initiatives and in engaging with the knowledge acquisition method. However, there was a marked difference in how each team viewed the role of their initiative team and the knowledge acquisition method. The more successful case (i.e., Case 1) was found to speak with greater confidence about their initiative, the potential for the opportunity, and the value of their contribution to the firm. They were also found to speak positively of the engagement with the knowledge acquisition method and the importance of the role that it played in helping to guide them through uncertainty and overcoming roadblocks. The less successful project (i.e., Case 5) was found to be more negative in their view of the initiative and its execution, and the value that the knowledge acquisition method provides for cocreation initiatives. In both these cases, each experienced a similar path in the initiative proposal process and both worked with distributed teams across geographies. These findings suggest that while knowledge capacity profiles contribute to initiative performance and the initiative team's approach to accessing required knowledge (e.g., KAM knowledge bridging), the strength of the initiative leader impacts a less experienced initiative team's ability to execute and overcome challenges experienced during the project.

The findings have shown that in all cases, initiative teams were found to be competent in engaging with the clients and extracting knowledge. As research, their knowledge competencies at this level of engagement were consistent and sufficient in executing this part of the innovation process. However, the variance in initiative performance would then suggest that strength in engaging the clients at the level of 
innovation creation (i.e., innovative capacity, absorptive capacity) is insufficient in explaining observed differences in project outcomes. The study suggests that effectiveness and co-creation initiatives require the supporting profile of knowledge capacities that reflect the complexities of these open systems. Knowledge capacity development must address activities ranging from opportunity identification and assessments, through stakeholder engagement and collaboration, to technology transfer and commercialization. It is argued that a well-developed knowledge capacity profile at the initiative team level, supported by a knowledge acquisition method's function as an orchestrator contributes to stronger initiative performance compared to projects with less developed profiles across each of these elements.

\section{KAM Orchestration, Alignment and Impact on Co-Creation Initiative Performance}

Another recurring theme when examining FoaK initiative performance, is the role of FoaK initiative teams in achieving congruence stakeholder alignments across all functions. The study found each FoaK initiative must establish the necessary stakeholder relationships to meet the program requirements. However the degree to which stakeholder relationships were established with proper expectations, motivation, engagement and investments, was dependent upon the effectiveness of the FoaK initiative team and its leader.

A comparison of the individual cases studied reveals that successful initiatives were more effective in developing and maintaining productive stakeholder relationships across functions, which was not seen with less successful initiatives. The findings of comparative stakeholder alignments across initiatives are summarized in Figure 11.5. It was also found 
that misalignments in stakeholder expectations and engagements were characteristic of the less successful initiatives, which resulted in introducing new levels of complexity and management efforts required by the FoaK initiative teams.

Figure 11.5 Orchestration, Alignment and Comparative Cross-Case Performance

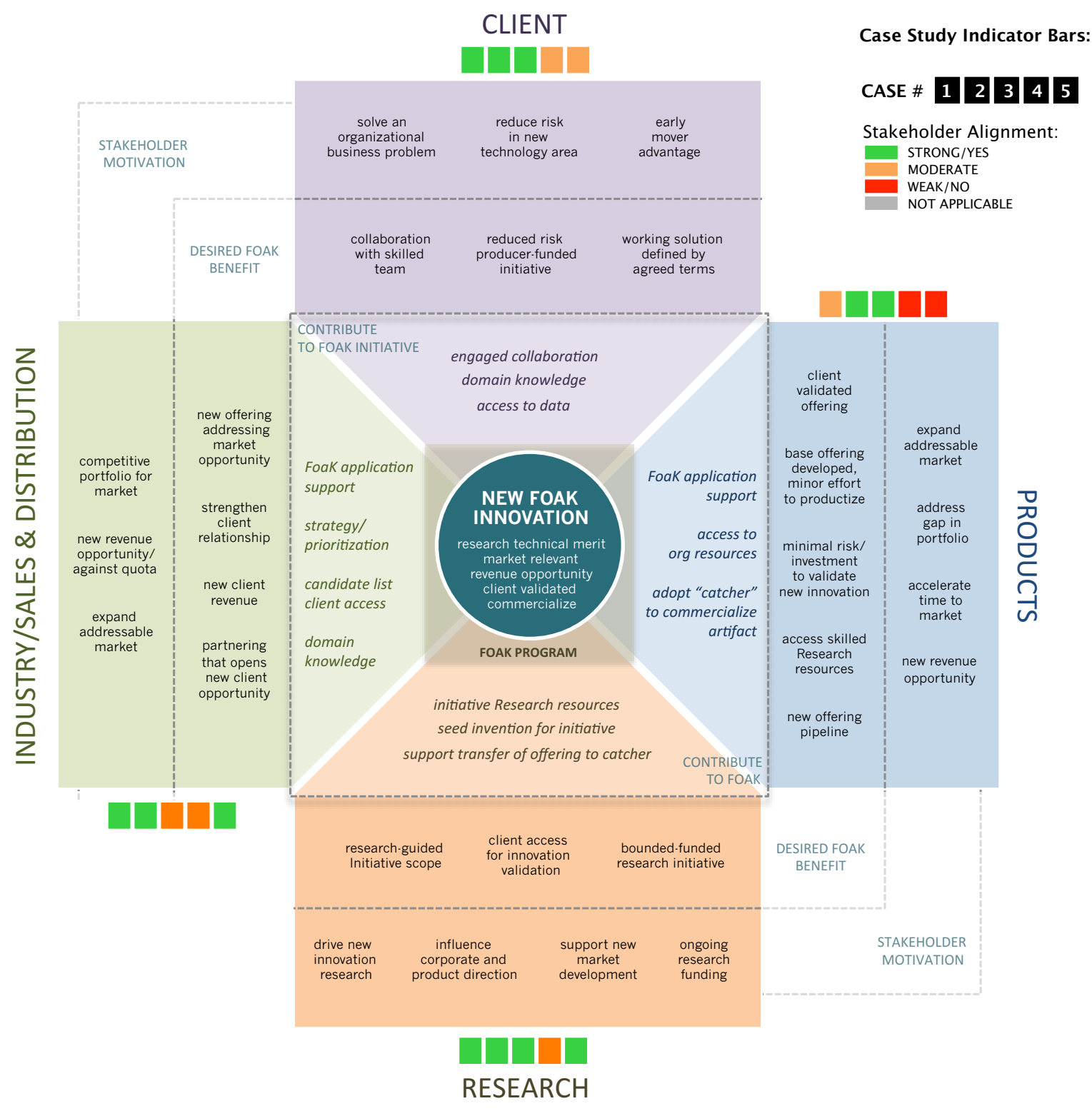




\section{Initiative Performance and Momentum}

In analyzing FoaK initiative performance, a contributing factor to the difference between successful and unsuccessful projects was found to be their ability to build, sustain, and leverage project momentum as the fuel to move initiatives forward. Momentum, in this case, is viewed as a FoaK initiative's display of proof of value (i.e., proof points), progress, and direction towards performance outcomes.

FoaK initiatives were found to focus on exploratory innovation, meaning there are many unknowns on the potential outcomes from the project. Stakeholders associated with the FoaK initiatives tend to work in a realm requiring greater certainty on results than the research organization before they invest. As such, initiative stakeholders look for evidence throughout a FoaK initiative that contributes to their confidence on how the initiative innovation may contribute to their functional goals, and by when. For example, sales stakeholders look for demonstrated value of the FoaK innovation against the client use case, that can be used to position new sales opportunities in the marketplace. While this is also important to the product stakeholder, they also require a greater level of demonstrated market validation in the form of client proof points and sales pipeline to increase their confidence before investing in the commercialization of the asset.

It was found that a FoaK initiative innovation follows a trajectory from the start of the initiative proposal process to its transfer for commercialization. A FoaK initiative's trajectory is impacted by the initiative's momentum and how it is developed and managed within innovation windows of opportunity. This includes initiatives successfully surpassing the necessary stakeholder thresholds of confidence in the innovation as it relates 
to their functional objectives. Initiative momentum is observed in the findings are illustrated in the comparative initiative momentum trajectory, represented in Figure 11.6. It is argued that as a FoaK initiative innovation gathers proof points from work with the client and other stakeholders, it builds momentum starting with support for researchdriven co-creation activity (i.e., FoaK funding). Early success with an initiative's innovation leads to passing the first threshold where the sales and services stakeholders see the opportunity to reproduce the work done within the Foak initiative, using the innovation, and generating new revenue through a services-based implementation model. Proof points can include demonstrated prototypes, client statements of value and support through further investment, and new sales. All cases in this study were found to successfully surpass the user value threshold, resulting in the development of sales pipeline for each innovation. As momentum continues to build, and confidence grows for the FoaK innovation as a scalable market offering, a second threshold is passed demonstrating scalable market value. Crossing this threshold moves interest for the innovation into the products-driven cocreation agenda. This is where the product stakeholder sees the value in investing their limited resources towards the new FoaK innovation as part of their portfolio. Only Case 2 (Retail) and Case 3 (Automotive) were successful in crossing this threshold, resulting in the innovation being successfully transferred to the product stakeholder portfolio.

The final element of the FoaK innovation trajectory is the effectiveness of the FoaK initiative team in delivering the innovation within "windows of opportunity" that align with its stakeholders. The timing of an initiative, the rate of progress, and availability of the innovation must be right, regardless of whether the innovation momentum thresholds have 


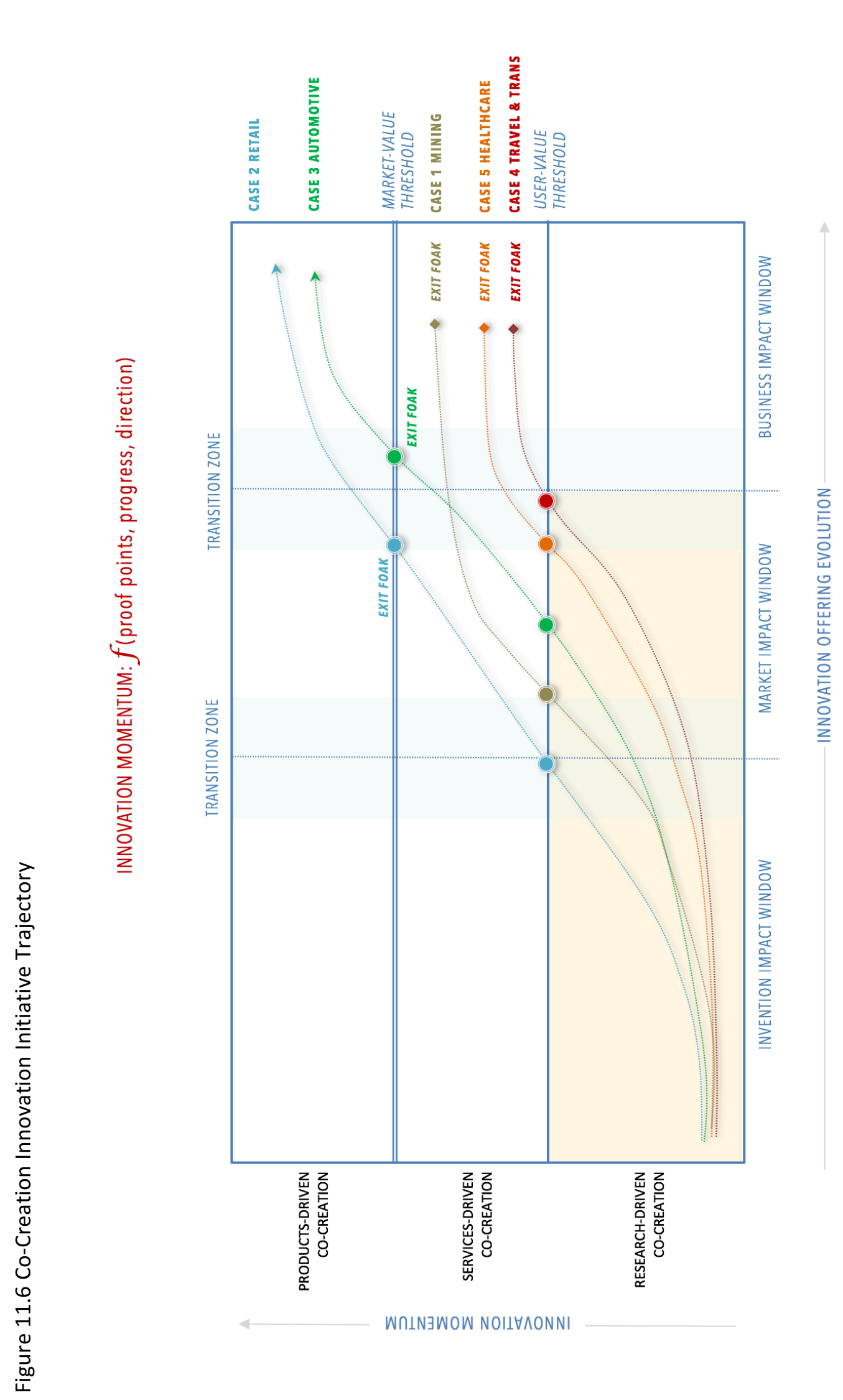


been reached. It was found that there are three windows of opportunity that each initiative must work within. A FoaK initiative must first demonstrate the potential of the invention to have value against a business use case. Further development of the innovation within the FoaK co-creation process results in additional market validation, but must be within a window where the potential for market impact is demonstrated. Finally, if the evolution of the FoaK innovation is moving forward at a pace where it is still relevant to the market, then it must finally demonstrate its fit to the firm's business objectives and timelines.

Findings from this study have demonstrated that FoaK initiative teams that consider and manage momentum as part of the co-creation of innovation process performed better than teams that do not manage this element effectively.

\section{Initiative Performance and KAM Boundaries (Interlocking Methods)}

The final theme that emerged from the study was regarding initiative success rates related to the transfer of a FoaK initiative to a product stakeholder portfolio, and client satisfaction after the completion of a FoaK initiative (see Figure 11.7).

It was found that many FoaK initiatives while successfully executed still fail to transfer to a product stakeholder portfolio for commercialization. This was commonly attributed to a lack of readiness of a FoaK innovation asset for a product portfolio at the end of a project. Many were found to speak to the notion that from successful FoaK initiative emerges assets that are "near ready" for the market. However, it was also acknowledged that the reality is that significant work remains in the product development of that asset. Often, the asset requires being redeveloped with the project learnings as the 


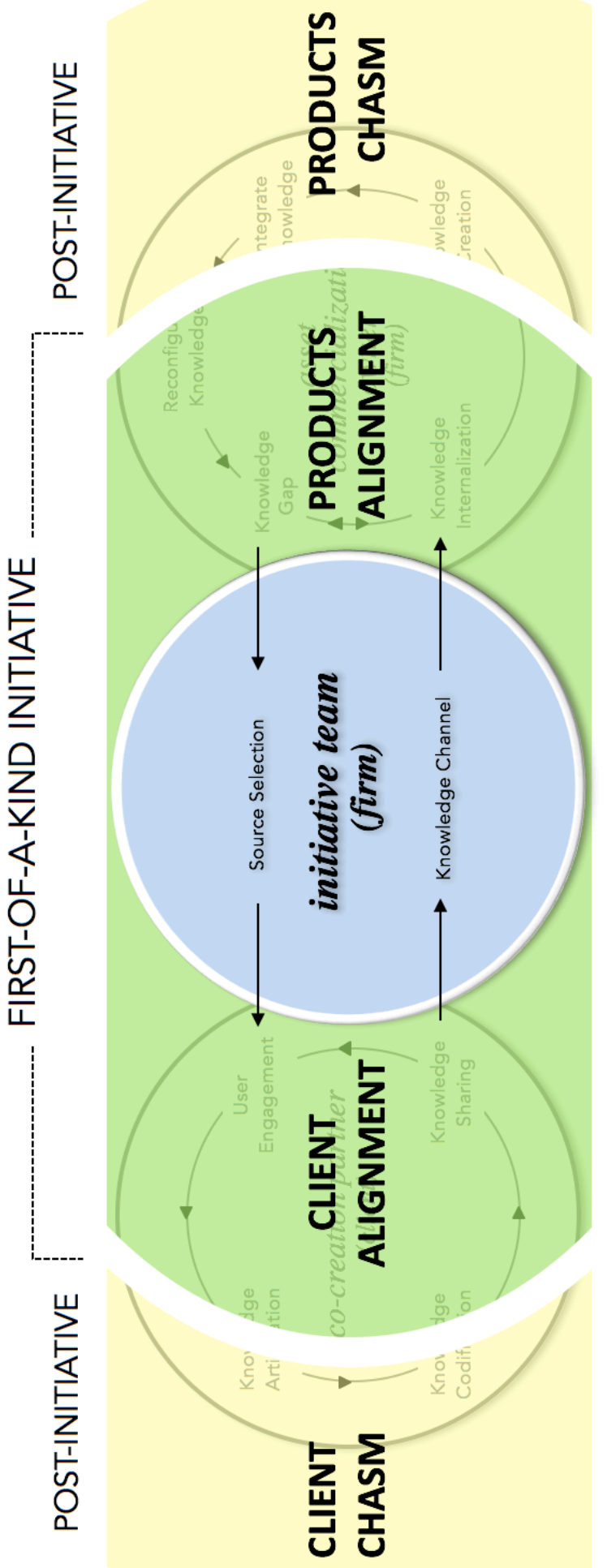


primary benefit to a product development organization. The development investment can be due to the need to bring the innovation to a form that aligns with the product portfolio architecture, expanding the innovation to address the specific aspects of the business use cases in their market, or refining usability of the asset to market standards.

As a result, there is a common gap observed between the FoaK innovation asset being delivered from the initiative, and what is required to be developed for the innovation to be market ready. This "product chasm" can be attributed to a gap in strategic fit, questions regarding the degree of value creation, misalignment in the expectations between the innovation team and the product stakeholder, a requirement for resources to finalize the asset development, or a need for a formal plan to transfer the technology to a product portfolio. It was found that the primary challenge faced by product organizations expected to receive and commercialize a FoaK innovation is inadequate consideration of the resources, funding, and timing necessary to complete the transition of the FoaK innovation asset.

Similar to the "product chasm," is a gap in the creation of value for the end client after completion of a FoaK initiative. This "client chasm" was described as a gap in planning final success for the co-creation clients after completion of the project. The essence of this chasm is that at the end of a FoaK initiative, the firm has collected the knowledge needed to move its innovation forward. However, the client does not distinguish between the FoaK initiative and its relationship with the firm. Their objective and expectation is that they will realize value from the time invested in the co-creation activity. However, FoaK initiatives are often not planned beyond the completion of the project. The result of this being that in cases where the client is interested in using what was learned 
from the initiative, there is no plan in place that guides the next steps to deliver on their expectations. As a mirror image of the "product chasm," it is suggested that a client transfer plan is required to ensure next steps are in place.

Two things are suggested from these findings. First, both scenarios are the result of activities occurring outside the boundaries of the knowledge acquisition method's scope. This would suggest that program design could be revisited to consider expanding its boundaries to account for the resources and transfer plans needed to ensure product and client stakeholder readiness within the scope of a FoaK project. The second suggests support for multiple interlocking KAMs. The FoaK is considered as one method of knowledge acquisition for the co-creation of innovation that by design does not complete all the activity required in the co-creation innovation process. It can be argued that the gap between the FoaK initiative and the stakeholder portfolio is supplemented by a second knowledge acquisition method defined by its own objectives and resource funding requirements to support the remaining incubation activity. The result is that the firm defines, manages and funds a series of interconnected KAMs (e.g., programs) that cover the full set of activities required to realize the desired outcomes for the firm. This would involve strategic planning of the steps from the idea and invention to market, and the cocreation of innovation activity are along the way. 


\section{PART V \\ RESEARCH DISCUSSION AND IMPLICATIONS}

This study has presented new contributions to the emerging cross-discipline literature on open collaborative innovation and the role of knowledge acquisition methods in co-creation of innovation performance. This initiative-level exploration has provided first empirical support and revision to Lichtenthaler and Lichtenthaler's knowledge capacity framework (2009). "Orchestration" has been identified as a higher-order dynamic capability governing the knowledge-based dynamic capabilities in co-creation of innovation activity, and the portfolio management optimization dynamic capability is included as the link between orchestration's strategic intent with co-creation initiative execution. The knowledge acquisition method was found to have an indirect relationship to co-creation performance. The findings suggest that knowledge-based dynamic capability development is managed across tiers in a KAM system, which in turn impacts KAM repeatability and scaleability in delivering collaborative innovation to the firm. Part V presents the discussion of research findings, and the implications for research and practitioners.

\section{Chapter 12 Discussion and Research Implications}

\section{Chapter 13 Managerial Implications}




\section{Chapter 12 \\ DISCUSSION AND RESEARCH IMPLICATIONS}

Research into open innovation (OI) and co-creation of innovation models continues to grow across multiple fields of study (e.g., open and collaborative innovation, dynamic capabilities, knowledge management). Studies have highlighted the contribution of external sources of expert knowledge to the creation of new innovation and firm performance (Chesbrough 2006; Cooper \& Kleinschmidt 1987; Lin \& Wu 2014; MontoyaWeiss \& Calantone 1994). However, the literature has not provided necessary insight into understanding the complexity of OI processes and the interaction of collaborative innovation processes that affect performance (Macpherson et al. 2004; Schleimer \& Faems 2015). This in part has been attributed to the firm-centric nature of research in this domain (Ahn et al. 2016). As such, the relationship between OI mechanisms and capabilities to innovation performance has been unclear (Cheng \& Shiu 2015). This study set out to bring new initiative-level insight into understanding the role that knowledge acquisition methods play in co-creation of innovation performance, from a dynamic capabilities perspective.

The findings push forward research in co-creation of innovation through the contribution of new insight into the inner workings of co-creation initiatives that highlight the role of the knowledge acquisition method (KAM), stakeholder engagement, and the interaction of collaborative processes within and across the boundaries of the firm. The study highlights that a KAM influences co-creation of innovation performance indirectly through its influence on an initiative team's ability to execute. The research findings suggest that the development of knowledge capacities is multi-tiered across levels of the 
KAM co-creation system (i.e., program, initiative, and the resource pool). This study presents evidence that the knowledge acquisition method serves as a higher-order dynamic capability with its primary role being that of co-creation "orchestration." In addition to this contribution, the study also presents first empirical support for the Lichtenthaler and Lichtenthaler (2009) open innovation knowledge capacity framework with revision, and the First-of-a-Kind knowledge acquisition method to the literature. As a guide for future study, researchers are also presented with a new conceptual framework for co-creation of innovation investigation, and a representation of the KAM multi-tiered framework for dynamic capability development.

This chapter presents a discussion of the research findings, their implications and contributions to literature, and the opportunities for future study. As this is deep exploratory research, effort has been put into developing each of the elements of the study that contribute to the conclusions presented on the central research question. Table 12.1 provides an overview of the key themes discussed.

\subsection{Discussion of Findings}

This section discusses the principal findings from this study. The discussion begins with findings on the role of KAM in co-creation initiatives, followed by a review of the observed contributors to co-creation complexity. Insights into the elements of knowledge management and dynamic capabilities as they contribute to co-creation initiatives are then presented. These findings lead into a discussion of the central research question regarding the KAM role in co-creation of innovation performance. The section ends with a synthesis of the discussion on KAM and competitive advantage, presenting a framework that 


\section{Table 12.1 Discussion of Findings - Overview}

Discussion of Findings

KAM operates with set of objectives, boundaries and resources

KAM focus on lowering barriers to execute co-creation initiative, focus activities on outcomes, bridge initiativ

KAM and the Role in knowledge gaps

Co-Creation Initiatives $\quad$ KAM provides direct support of KAM initiatives

KAM orchestrates elements desired for co-creation initiative execution

KAM strives for repeatability and scaleability across initiatives

\begin{tabular}{|c|c|}
\hline \multirow{16}{*}{$\begin{array}{l}\text { KAM and } \\
\text { Co-Creation Complexity }\end{array}$} & Stakeholders: \\
\hline & Complexity of stakeholder types and relationships (inter/intra-firm) \\
\hline & Range of stakeholders with varying agendas and motivations \\
\hline & Managing stakeholder complexity impacts momentum and performance \\
\hline & Initiative-Client relationship is primary influence on other stakeholder confidence (momentum) \\
\hline & Processes: \\
\hline & Intertwined nature of co-creation processes \\
\hline & Range of processes spanning outside of core exploration process \\
\hline & Initiative co-creation processes should be managed as a system \\
\hline & Develop knowledge and skills to manage processes - impacts initiative performance \\
\hline & Changes in Initiative Context: \\
\hline & KAM as an orchestrator of co-creation influences KAM initiative team ability to execute \\
\hline & Ability of initiative team to respond to changes in context impacts initiative performance \\
\hline & KAM adjusts to changes in context for the program \\
\hline & KAM Boundaries: \\
\hline & KAM boundaries overlap; successful KAM may result in unsuccessful innovation outcomes post initiative \\
\hline \multirow{7}{*}{$\begin{array}{l}\text { KAM and Knowledge } \\
\text { Development }\end{array}$} & KAM program is central stable store of method knowledge \\
\hline & KAM program knowledge bridges initiative team gaps \\
\hline & Knowledge capacity profile reflection of initiative team readiness \\
\hline & Knowledge types represent as dynamic capabilities (i.e., knowledge capacities) \\
\hline & Knowledge profile variance across initiative teams and between teams and program \\
\hline & Knowledge transience challenges knowledge retention for future use \\
\hline & Knowledge capacity framework (iOI) empirically supported \\
\hline \multirow{7}{*}{$\begin{array}{l}\text { KAM and Co-Creation } \\
\text { Dynamic Capabilities }(D C)\end{array}$} & Support for Knowledge Capacity Framework (KCF-iOI) (Lichtenthaler and Lichtenthaler 2009) \\
\hline & KAM program represents higher-order dynamic capability - "orchestration" \\
\hline & KCF revision to include Portfolio Management (optimization) - program-level \\
\hline & Knowledge capacities are developed across tiers in KAM system \\
\hline & DC interaction - effectiveness of one DC impacts others in KCF; "connective capacity" is dominant \\
\hline & DC development varies across program and initiatives \\
\hline & DC development must be viewed through tiered system \\
\hline \multirow{3}{*}{$\begin{array}{l}\text { KAM Role in Co-Creation } \\
\text { Innovation Performance }\end{array}$} & KAM impact on performance is indirect; direct influence on initiative team effectiveness \\
\hline & Initiative performance reflection of initiative team DC profile development \\
\hline & Performance variance linked to initiative team ability to execute \\
\hline \multirow{3}{*}{$\begin{array}{l}\text { KAM and } \\
\text { Competitive Advantage }\end{array}$} & KAM KCF tiered system introduced \\
\hline & Synthesis of findings into framework \\
\hline & Initiative-level insight into co-creation interactions and complexity \\
\hline \multirow[t]{2}{*}{ Additional Contributions } & Introduce FoaK as KAM \\
\hline & Revised Conceptual Framework \\
\hline
\end{tabular}


represents the KAM multi-tiered view to knowledge-centric dynamic capability development.

\subsubsection{KAM and its Role in Co-Creation Initiatives}

The essence of this study is the examination of a knowledge acquisition method and its role in co-creation of innovation initiative performance. Co-creation of innovation remains an emerging field of research requiring further study as a developing managerial paradigm (Hoyer et al. 2010; Piller et al. 2011). Earlier research has highlighted that existing studies in open innovation only partially explains observed relationships between open innovation activities and innovation performance (Cheng \& Shiu 2015). The interaction of activities in collaborative innovation is not well understood (Becker et al. 2014; Enkel \& Gassmann 2005). Moreover, it is unclear how the inter-firm and intra-firm processes contribute to new-to-the-firm innovation (Macpherson et al. 2004; Schleimer \& Faems 2015). This study has introduced to the literature a first empirical examination of the First-of-a-Kind program, a producer-stimulated co-creation of innovation method (i.e., knowledge acquisition method). The initiative-level exploration conducted in this study has contributed much needed detailed understanding into the role of the KAM (e.g., objectives, repeatability, and scaleability) and the inner workings of co-creation initiatives (e.g., KAM program-initiative interlock, processes, stakeholders).

\section{Clear KAM Objectives}

As with other knowledge acquisition methods represented in the literature (Brockhoff 2003; Piller et al. 2011), it was found that the FoaK method represents a distinct 
collaborative innovation profile of objectives, level of openness, degree of client engagement and innovation focal point for this method. The FoaK is characterized as an early-stage knowledge acquisition method, with the producer firm as the driver and focal point of innovation for this method. As with other KAMs, the FoaK represents clear objectives, boundaries and measures of performance, which in this case reflect its early stage collaborative innovation focus. This study describes the FoaK method as a producerstimulated knowledge acquisition method, supporting the earlier theorized position as a "producer-engaged" method (see Figure 3.1). These findings add further support to earlier research stating that firms operate multiple KAMs in parallel, each with their own purpose and attributes (Roser et al. 2013).

Cheng and Shiu (2015) state that organizations are expected to undertake multiple collaborative innovation activities in parallel (e.g., KAMs), which was observed in this study. A contribution to user collaboration research is provided through evidence supporting this study's knowledge acquisition method framework (Figure 3.1), proposing KAMs as both a collection of potentially complementary and competing methods available to the firm's stakeholder community. Each KAM is evaluated based on the resources required, the power dynamic between the firm and the collaboration partner (i.e., client stakeholder), the firm's degree of openness and the objectives of the co-creation efforts. These are consistent with the NPD and collaboration research (Brockhoff 2003; Dahlander \& Gann 2010; Kristensson et al. 2008; Piller et al. 2011; Zogaj \& Bretschneider 2012).

Findings from this study extend the notion of multiple KAMs coexisting within a firm, arguing that knowledge acquisition methods also compete within the firm. As was observed in this study, individual stakeholders evaluate KAMs based on their roles, the 
degree of fit to their objectives, resources, and motivation. It is found that much of the collaboration research focuses on managing risk through a client engagement lens (Enkel et al. 2005; Ogawa and Piller 2006). This study argues that when considering the role of a knowledge acquisition method in co-creation of innovation performance, the relationship between KAMs is a contributing factor to co-creation outcomes as they relate to overall functional and firm innovation objectives (e.g., commercialization). The findings suggest a portfolio-based perspective in balancing a methods role in the overarching intra-firm innovation chain will contribute to a clearer understanding of the firm's knowledge and dynamic capability development needs.

\section{Repeatability and Scaleability}

Each of the research domains considered in this study speak to the role of cocreation processes, knowledge, and capabilities contributing to competitiveness through repeatable and scalable models. However, details of the interactions of these systems are not well understood (Becker et al. 2014; Enkel \& Gassmann 2005). Powell et al. (1996) emphasize that organizations must develop practices (e.g., collaborative innovation models) that use knowledge more effectively towards building competencies. These competencies, in turn, can be used across co-creation engagements. Nielsen (2013) promotes that the use of formalized knowledge development activities (e.g., KAM projects and initiatives) supports creation of new knowledge. Similarly, Zahra et al. (2006) speak to achieving repeatability by evolving dynamic capabilities (e.g., knowledge capacities) by drawing upon a range of experiences (e.g., trial and error, improvisation, imitation of others). Chen et al. (2015) stress that successful collaborative innovation is dependent upon 
the ability to achieve the right balance of complementary knowledge resources and processes, by combining the right internal R\&D activities with external knowledge. Eisenhardt and Martin (2000) suggest that research must focus on improving understanding of repeatability and scaleability achieved through recurring practices, learning from past mistakes, and developing ways to accelerate learning.

This study presents a deeper insight into the KAM and its focus on developing an evolving co-creation system that is effective and displays potential for longevity. It was observed that a primary role of the KAM is that of developing the repeatability and scaleability of the method. The open innovation literature expresses systematic methodologies as desirable (Chesbrough 2003; Chesbrough \& Crowther 2006). The FoaK KAM adoption of a systematic program model reflected investment into developing a system that was increasingly more effective in identifying the best innovation opportunities and shepherding them. As a formal system and set of practices, the KAM defined the elements believed necessary for successful co-creation initiatives. This included developing and evolving a knowledge base built on the collective experience from previous initiatives, extended team expert knowledge, and networks, and codified knowledge in the form of process artifacts. As such, the KAM is a system consisting of the program, its initiatives and the set of primary stakeholders. In orchestrating the system, the KAM guides initiative focus and behaviour. The result is a direct impact on initiative team ability to execute, and an indirect impact on the co-creation of innovation initiative performance.

This study suggests that a formal program approach represents a firm's dedication to investing resources and focus on developing the method. It is argued that a KAM need not be instantiated as a formal standalone program, but may also appear as a developing 
competency within a function involved in co-creation efforts. For example, a KAM approach could be seen in smaller organizations who have adopted collaborative innovation models, but whose resources and portfolios are more limited. This presents an opportunity for future context-centric study examining the findings presented across firms of varying size.

Learning processes within the FoaK KAM were acknowledged as an important aspect of the method evolving, although it was also identified as an area of improvement for this subject organization. Learning in this context includes instantiating systems and processes for harvesting and managing knowledge from past initiative experience, and from varying sources of expertise across the firm. The collection of this knowledge, codifying practices where possible, providing guidance in areas characterized by tacit knowledge, all support the formalized and repeatable approach to co-creation initiative execution. This is consistent with the Zollo and Winter (2002) who describe the importance of capturing tacit knowledge into a form the firm can use, and Boisot (1998) who states that an organization requires some form of codification that allows for sharing of the knowledge across the firm.

\section{KAM Program and Initiative Interlock}

Earlier research has argued that there is an inseparable link between the firm's innovation strategy, knowledge management strategy, and its effective execution of knowledge acquisition methods (Daniel et al. 2014; Wang \& Ahmed 2007). However, while these relationships are intuitive, the literature states that more study is needed that 
examines the inter-firm/intra-firm dynamics of co-creation initiatives (Schleimer \& Faems 2015).

This study contributes to the literature by detailing the relationship between a knowledge acquisition method and the co-creation initiatives that employ the method. Findings highlight that the KAM serves as an orchestrator for its co-creation initiatives. It impacts directly upon the effectiveness of initiative teams and their ability to execute their innovation exploration. As such, these findings contribute to understanding previous research suggesting the impact of a knowledge acquisition method on innovation performance as being indirect (Wang \& Ahmed 2007; Zahra et al. 2006). The insight from these findings into the orchestration role of a knowledge acquisition method across its multiple initiatives also contributes new understanding into intra-firm variance in cocreation innovation performance (Barreto 2010).

The results show that the knowledge acquisition method operates primarily in setting the stage for the co-creation of innovation. Knowledge acquisition methods work with a co-creation initiative defining the method and boundaries by which a firm's cocreation initiative team engages its stakeholders, as per Owen et al. (2008). It is the individual initiative teams that are tasked with directly executing on co-creation activities, who were found to have their focus and scope guided by the KAM (Roser et al. 2013). Both the KAM and the initiative processes were seen to be linked throughout the cocreation process. As such, the initiative team is found to have a direct impact on co-creation innovation performance, with the KAM's influence being more indirect. This is consistent with Grant (1996) who reminds us that through a knowledge-based view of the firm, competitiveness rests in the firm's ability to apply knowledge (e.g., co-creation initiative 
execution), not simply create new knowledge (e.g., the knowledge acquisition method). The study also argues that this relationship may be seen in other co-creation paradigms. For example, the program method-initiative relationship described may be seen in situations where the KAM and initiatives exist within a single functional unit that is building its competency in co-creation innovation. In this case, the functional unit would distinguish between resources on the team that provide continuity and oversight, that in turn guide initiatives which their team subsequently executes.

The interdependence and linkage between the KAM and the initiative were also observed in the development of the skills and knowledge needed in collaborative innovation engagements. The KAM program was found to represent a stable and evolving base of knowledge and best practices, with individual initiative teams representing varying levels of knowledge and expertise development needed for their initiatives. As such, the KAM represents a stable, yet evolving, base of skills and practices that contribute to its development as a collaborative innovation competency over time. This is consistent with Dierickx and Cool (1989) who describe the role of knowledge management as being a focus on building and evolving the knowledge store for the organization, and all the activities associated with exploiting that knowledge. This translates into the role of the KAM as the evolving base of knowledge upon which future co-creation initiatives can draw.

The study also highlights that knowledge development is not limited to firm-level activity. Rather, knowledge development is observed along tiers in the KAM co-creation system (i.e., KAM program, initiative, resources). This suggests that organizations developing co-creation skills must consider knowledge requirements beyond the 
boundaries of the KAM program to include initiative teams and the pool of resources that staff these teams, the processes, and the stakeholders. This initiative-level study has contributed to a deeper understanding of knowledge development in the co-creation system.

\section{Co-Creation Processes and Stakeholders}

This study has contributed new insight into initiative-level interactions of stakeholders, and both inter-firm and intra-firm processes. Findings have contributed in addressing gaps in understanding the complexity of open innovation processes which have been identified as requiring further exploration (Becker et al. 2014; Enkel \& Gassmann 2005; Macpherson et al. 2004; Schleimer \& Faems 2015). They present insight into the FoaK KAM processes, points of dependency and overlap, and the observed stakeholder engagement dynamics.

The findings represent that KAM processes are not standalone, but rather are interconnected and dependent on processes and stakeholders from across the organizations (i.e., internal, external). The dynamic nature of the co-creation activities and interactions observed provide insight into earlier research statements that co-creation of innovation is characterized by the "intertwined" integration of inter- and intra-organizational processes (Schleimer \& Faems 2015). This study argues, beyond recognizing these interconnections, the KAM as an orchestrator must also approach these processes as part of a larger KAM system. This study has illustrated that the KAM incorporates a broader understanding of these processes and the critical stakeholder relationships into its design. A broader system 
view enables the method to more fully understand the elements that potentially impact performance of a KAM initiative team in their projects.

Complexity in the dynamics between a firm and its external stakeholder relationships has been highlighted in the open innovation literature (Szulanski 1996). These complexities were found to be reflected in both the interaction across a range of KAM stakeholders, and the processes that bring them together. From a dynamic capabilities perspective, Lin and $\mathrm{Wu}$ (2014) build on Teece et al. (1997) emphasizing the importance of integrating internal and external resources towards improving firm performance. They argue this is achieved by using dynamic integration, reconfiguration, and learning capabilities. This integration of resources helps firms benefit from their experience, contributing towards improving initiative performance (King \& Tucci 2002).

In the case of the FoaK as a KAM, the complexity of inter-firm and intra-firm processes and stakeholder interaction was characterized by the range of participating stakeholder types (i.e., internal, external) that the KAM engaged across the co-creation of innovation process. The initiatives studied were characterized by processes that extended beyond the actual exploration activity between the firm and the co-creation client. In addition to the innovation exploration, processes ranging from the initiative candidate evaluation to co-creation planning, onboarding, and stakeholder recruitment, highlighted the need for a broad set of skills and capabilities for initiatives to be identified, executed, and internalized for commercialization.

Open innovation and collaboration research have been consistent in expressing the value of KAMs acquiring new knowledge through strategic relationships with external sources of expertise (Chandler \& Hanks 1998; Chesbrough 2006; Tsoukas 1996). Also 
expressed are the challenges in knowledge acquisition due to the nature of tacit expert knowledge (Gils \& Zwart 2004). The results from this study contributed to initiative-level understanding of the range of stakeholders, their roles, and the nature of their influence on the co-creation process. Stakeholders were found to represent varying agendas and motivation, typically extending beyond the scope of the KAM initiative. These relationships were found to contribute to the initiative dependencies of which the cocreation team must anticipate, manage effectively, execute and perform. This study argues that effective KAM methods must anticipate and understand the range and nature of stakeholder engagement required for successful co-creation initiative execution. It was found that the ability to manage stakeholder expectations and engagement directly impacts the KAM initiative team's ability to execute. The KAM program as an orchestrator was found to anticipate which stakeholder types are relevant, and set conditions for initiative teams to engage. Extending beyond the scope of this study, there is opportunity for future research examining KAM-stakeholder relationships, drawing upon stakeholder theory and research in value creation through stakeholder engagement (Gould 2012).

\section{Role of the KAM}

The initiative-level analysis conducted in the study has contributed new insight that moves forward understanding into the dynamics of collaborative innovation interaction. These findings provide both necessary insight into the relationship between KAMs and their initiatives, and additional clarity into the role of the KAM as part of the co-creation

of innovation system (Becker et al. 2014; Enkel \& Gassmann 2005; Macpherson et al. 2004; Schleimer \& Faems 2015). 
Extant research examining co-creation methods describes collaboration as providing the building blocks to establish the necessary relationships needed in creating value through new innovation (Prahalad \& Ramaswamy 2004). This includes enabling the interaction between the firm and external sources of expertise, accessing specialized knowledge, and managing the risk in the collaboration (Prahalad \& Ramaswamy 2004). Dynamic capabilities research describes that co-creation of innovation is dependent upon well-established capabilities that reflect expertise in integration that allows for full exploitation of collaborative relationships (Croom 2001). Findings from this study provide new clarity on the role of the knowledge acquisition method as an orchestrator, and in developing capabilities that integrate processes, stakeholders, and resources within the firm, and with external collaboration clients.

The study findings support that a KAM serves primarily as a mechanism to manage the complexity of co-creation initiatives, and promotes the advancement of co-creation initiatives using the method. This is achieved through a focus on maintaining the right elements needed for an initiative to produce the best potential results with its innovation exploration. As observed, the knowledge acquisition method works within clear objectives and resources, bringing together cross-functional expertise and experience related to the attributes of the specific type of engagement (e.g., FoaK). The KAM program serves as a central hub of knowledge in evaluating and executing engagements using the method, with individual initiative teams being provided with the "blueprint." This supports the repeatability and scaleability of the method, versus individual projects having to develop all aspects of the complex system, and the necessary resources needed to effectively execute. The KAM presents co-creation initiative teams with a "beaten path" and guidance 
"through the forest," with the understanding that the initiative team still leads their journey through the complexity.

\subsubsection{KAM and Co-Creation Complexity}

Complexity is a common theme across the research disciplines considered in this study. Open innovation and co-creation literature speak to the challenges of accessing insight on customer needs (Brockhoff 2003; Leonard-Barton 1990). Teece et al. (1997) suggest the role that context plays in the effectiveness of dynamic capabilities within a collaborative innovation context. Due to the complexity of collaborative innovation initiatives, current research only partially explains observed performance (Cheng \& Shiu 2015). Much of these challenges are attributed to the fact that most open innovation researchers focus on understanding firm-level performance (Deeds et al. 2000; Marsh \& Stock 2003). As such, there is inadequate insight into the interaction of activities that can explain observed performance. Much of the complexity and open innovation processes are not well understood (Becker et al. 2014; Enkel \& Gassmann 2005; Macpherson et al. 2004; Schleimer \& Faems 2015).

This study provides a significant contribution in moving forward insight into the nature of the interaction between knowledge acquisition methods and their initiatives. The findings argue that understanding co-creation complexity at the initiative-level requires a view into the complexity as it relates to KAM stakeholders, processes, context, and acquisition method boundaries. This new insight has helped to further explain the role of knowledge-based dynamic capabilities in KAM systems. 


\section{Stakeholder Complexity}

This study contributes a deep analysis of stakeholder types and the nature of their interactions across the FoaK knowledge acquisition method. Complexity in the stakeholder landscape reflects the breadth of stakeholders required across the co-creation process, and the knowledge and skills needed in enlisting, engaging, and managing them against conflicting timelines and the desired benefits for each group.

Understanding of the stakeholder landscape and engagement in co-creation processes in the open innovation literature is not well understood (Cheng \& Shiu 2015). While the literature has spoken to stakeholder interaction as part of open innovation engagements, much of the study has focused on external stakeholder engagement (i.e., clients, suppliers) as a source of expert knowledge (Berthon et al. 2007). Nesheim (2001) spoke to the role of a firm's ability to build strong working relationships with external stakeholders and moving beyond standard transactions to strengthen the business core. Much of the collaborative innovation research has emphasized the potential for client stakeholder participation across a range of co-creation activities based on their level of expertise, skills, and motivation (Brockhoff 2003; Grabher et al. 2008; von Hippel 1986). Overall, limited research has been conducted examining the system of stakeholders engaged at the initiative-level in co-creation, in part because of the firm-level focus (Deeds et al. 2000; Marsh \& Stock 2003).

The dynamic capabilities and knowledge management research suggest the importance of understanding other stakeholder relationships beyond the client stakeholder. Studies have spoken to the importance of inter-firm relationships (Macpherson et al. 2004; Rothwell \& Dodgson 1991), the need to understand how knowledge is transferred in co- 
creation processes (Macpherson et al. 2004), and the benefit of encouraging knowledge sharing processes between entities (Dyer \& Singh 1998; Nonaka \& Takeuchi 1995). Other research has highlighted that open innovation collaboration contributes to a positive innovation climate through a systematic methodology engaging untapped sources of knowledge (Chesbrough 2003; Chesbrough \& Crowther 2006; Van der Meer 2007). This study argues that a positive climate of knowledge sharing extends beyond the firm-client relationship with internal stakeholders also serving as sources of knowledge.

This study contributes new insights into the nature of the internal and external stakeholder system as it relates to initiative-level co-creation of innovation, and complexity. The findings illustrate that complexity of the stakeholder landscape in cocreation initiatives is seen in a range of interdependent inter-firm and intra-firm stakeholder interactions (e.g., between KAM program management, board members, initiative team members, the client stakeholder, and internal organization functional stakeholders). Each set of relationships was found to serve a specific role in the overall co-creation system. The observed stakeholder interactions also contributed to further understanding the role of KAM as an orchestrator, the role of dynamic capabilities, and the challenges in managing stakeholder expectations and alignment to the initiative goals. As knowledge acquisition methods are found to share common functional connections (i.e., product commercialization, sales, research, client engagement), this study argues these findings on the nature of stakeholder relationships are equally relevant across other KAM types. It is believed that the objectives of a KAM will determine the nature of the relationships as expectations of each stakeholder may differ at various stages in the co-creation of innovation process. 
Extant literature in its focus on client stakeholder engagement with the firm, also speaks to the notion "right fit" of the client stakeholders as a rich source of ideas and opportunity (Berthon et al. 2007) and unique ideas (Chatterji \& Fabrizio 2007). Collaboration research also speaks to how user stakeholders are not all equal, with each bringing varying levels of knowledge and skills (Brockhoff 2003), and differing levels of motivation based on perceived benefits (Brockhoff 2003; von Hippel 1986). This study extends the notion of "right fit" clients across the system to all stakeholders, arguing that the alignment of KAMs co-creation stakeholders (i.e., internal, external) impacts initiative execution and performance.

Stakeholder-related complexity was observed in the management of stakeholder relationships, specifically in identifying the right stakeholders for an initiative, setting expectations, managing engagement, and maintaining alignment across participating stakeholders. The various stakeholders each come to the co-creation initiative with their own set of objectives, motivations, and time horizons. Managing this contributor to complexity further highlights arguments for dynamic capability development (Lichtenthaler \& Lichtenthaler 2009) in support of these processes. The study highlights that the KAM as an orchestrator provides a path to navigate complexities of stakeholder alignment using KAM specific mechanisms (e.g., program framework, conditions to participate, terms, resourcing). However, the initiative team's ability to effectively manage stakeholder relationships is found to have a direct impact on initiative momentum, and subsequent co-creation initiative performance.

This study further contributes to earlier research on stakeholders, arguing that the complexity observed in co-creation stakeholder relationships stems from the 
interdependencies. Earlier research speaks to the potential impact of the right client on innovation performance. Chen (2015) states that having a variety of user types may be helpful but is not likely to have equal impact on innovation performance. Brockhoff (2003) states that client users differ in their ability to contribute to delivering radical innovations. This study finds the initiative team-client stakeholder engagement is the primary stakeholder relationship. The effectiveness in managing this relationship impacts initiative momentum and subsequently other stakeholder relationships. Findings suggest the impact of initiative momentum is on stakeholder confidence in the innovation marketability. This study has presented a system-level insight view of stakeholder relationships in co-creation initiatives, with the results suggesting that understanding the complexity of co-creation of innovation and initiative performance requires understanding stakeholders beyond the client-initiative relationship.

\section{Process Complexity}

Open innovation research has highlighted the complexity in the co-creation of innovation processes, stating the linkage between inter- and intra-organizational processes (Macpherson et al. 2004; Schleimer \& Faems 2015). Research has discussed the relationship between inter-organizational engagements and intra-organizational processes, suggesting that these two sets of activities are "intertwined" as a system of knowledge acquisition activities for creating new innovations (Schleimer \& Faems 2015). Studies have examined firm-client interaction and processes (Deeds et al. 2000; Marsh \& Stock 2003), as well as the intra-firm processes reflected in work on absorptive capacity (Cohen \& Levinthal 1990; Zahra and George 2002). It is recognized that there is insufficient insight 
into the interactions and processes in open innovation activities that explain the performance outcomes observed (Cheng \& Shiu 2015).

In examining the FoaK KAM at the initiative-level, the study contributes new insight into the co-creation processes beyond the specific exploration between initiative team and client stakeholder. It argues that the interdependence between co-creation processes must be considered as a system, as these linkages impact overall innovation performance. The effectiveness of the KAM program initiative is dependent upon the development of distinct skills and knowledge required to manage the range of individual processes and their dependencies.

Examination of the processes related to the co-creation initiative highlighted that while the initiative team-client stakeholder exploration process presents complexities in extracting the expert knowledge from the client, additional layers of complexity are introduced by other necessary and interrelated processes beyond the direct exploration activity. These processes included program portfolio management activities, stakeholder identification and on-boarding, and integrating the co-creation initiative process into functional processes that feed the KAM initiative (e.g., research pipeline, co-creation innovation asset transfer, sales pipeline development, initiative governance and oversight). The degree to which these processes are understood and managed as part of the overarching initiative objectives impacts overall co-creation innovation performance. These findings move forward the understanding of the "intertwined" nature of co-creation processes (Schleimer \& Faems 2015), and the interdependence that exists in collaborative innovation engagements. 
This insight into co-creation initiative processes also contributes to understanding the role of various forms of knowledge and knowledge management activities discussed in the literature (Alavi \& Leidner 2001; O'Dell \& Grayson 1998; Teece 1998). A range of knowledge processes across a co-creation initiative (i.e., knowledge creation, acquisition, capture and articulation, knowledge assembly, knowledge sharing, knowledge integration

and recombination, and the leveraging of knowledge) were found to involve multiple stakeholders, adding to the complexity of the firm realizing benefit from the KAM's cocreation of innovation process. These findings from the study argue further for the need to develop a broad set of knowledge capacities that span the entire co-creation of innovation process. It was observed that the degree to which knowledge capacities are developed impacts the co-creation of innovation initiative outcomes. Development of these knowledge capacities were observed both at the KAM program and initiative level, and were found to be interrelated.

\section{Changes in Context}

In addition to examining the interaction of co-creation activities and processes, the study set out to examine the impact of contextual change on the KAM and co-creation of innovation performance at the initiative-level. Extant literature on open innovation, knowledge management and dynamic capabilities each examine context within their domains. However, insight into the role of context as it relates to co-creation of innovation activity is not well understood below the firm-level. This study argues that changes in KAM context in which a co-creation initiative is conducted impacts co-creation of innovation performance indirectly, by impacting the level of execution environment 
certainty. Co-creation of innovation context was found to impact program level priorities and to introduce initiative-level complexity through stakeholder related changes. The level of development of an initiative team's knowledge capacity profile determines their ability to anticipate and respond to context changes and optimizing the impact (i.e., positive or negative) that changes have on initiative performance.

Context has been examined in collaborative innovation across the domains of interest in this study. Open innovation research studied OI models across contexts, such as industry type (Christensen \& Bower 1996; Christensen et al. 2001; Van der Meer 2007) and levels of OI adoption (Chesbrough \& Crowther 2006). Rigby and Zook (2002) reported that open innovation models are characterized by higher levels of innovation intensity and in markets with significant volatility. In dynamic capability research, Teece et al. (1997) argue that context impacts the effectiveness of capabilities, and suggest they are best suited in highly dynamic environments. Eisenhardt \& Martin (2000) counter that dynamic capabilities are more broadly applicable. However, the role of context in collaborative innovation remains poorly understood. Barreto (2010) calls for new research to help in understanding the role of context, and its link to organizational strategy and dynamic capabilities. This study argues that to understand how context impacts co-creation initiative performance, it must be examined at the level of initiative execution. As this study focused on a single firm, single KAM program during a controlled period, the context was examined from the perspective of "contextual change" and its impact.

Findings from the study highlight a link between initiative context change, KAM initiative performance and KAM initiative team adaptability based on their knowledge capacity profiles. Initiative context change was found to introduce uncertainty to the KAM 
method and its initiatives. Changes in environmental context represent unanticipated factors in the co-creation initiative planning process. It was an initiative team's ability to anticipate and respond to changes in context (e.g., firm strategy, KAM strategy, economic environment) that was found to determine the degree to which context changes impact their KAM initiative performance. All the cases studied experienced some form of contextual change that impacted the course of the innovation exploration (e.g., economy, reorganizations, strategic focus). Initiative teams demonstrated differing levels of effectiveness in responding to the changes, which in turn were reflected in their ability to counter or exploit changes to the benefit of initiative performance. Teams that successfully adjusted to changing context were found to have stronger knowledge capacity profiles than less successful initiative teams.

Adjusting to changing context at the KAM program-level was found to be a primary role of the KAM, contributing to the firm's inventive and innovative capacities. The KAM program looks to achieve returns on investment in its initiatives which include focusing on initiatives that hold the greatest promise for the market and the direction of the firm. An understanding of the context in which the method operates shapes its focus, which in turn impacts its priorities and investment decisions.

\section{KAM Boundaries}

This study further highlights complexity introduced to collaborative innovation interactions and processes through interdependencies observed between knowledge acquisition methods. Open innovation research suggests that multiple open innovation models are used by a single firm in parallel. Firms consider blended strategies of open and 
closed innovation models (Chesbrough 2003; Jaworski et al. 2000). Studies also speak to the firm using multiple KAM types, each aligned to varying information needs (Zogaj \& Bretschneider 2012), with the selection of KAMs based on the strategic orientation and the co-creation goals (Roser et al. 2013). Research support for multiple KAM types has been presented across various taxonomies of KAM (Chesbrough \& Crowther 2006; Dahlander \& Gann 2010; Luthje et al. 2003). Collaborative innovation studies have also reported that not all KAMs are equally effective in accessing expert knowledge across a variety of information types (Brockhoff 2003; Leonard 2002). This study has contributed to the literature the knowledge acquisition methods framework (see Figure 3.1), a new taxonomy informed by the literature, representing knowledge acquisition methods that reflect a firm's potential set of methods based on the attributes of the engagement type they are pursuing (i.e., degree of openness of the method and the degree of firm-user engagement intensity). This model reflects the position in the OI research that organizations tend to adopt hybrid closed-open innovation strategies (Chesbrough 2003). This study argues that hybrid strategies suggest that firms not only use multiple methods of KAM in parallel, but that these methods are also interdependent, impacting the performance of co-creation initiative outcomes.

While there is significant literature examining individual knowledge acquisition methods (Brockhoff 2003; Grabher et al. 2008; von Hippel 1986), the research was not found to speak to the interrelationship between knowledge acquisition methods required between an invention idea and its commercialization. Findings from the study argue that while the individual knowledge acquisition method must align with the form of collaborative co-creation of innovation being undertaken, the boundaries of that KAM as 
it relates to the ultimate firm outcome objectives must be well understood. This relationship between KAMs contributes to co-creation initiative complexity as it relates to initiative innovation performance.

The knowledge acquisition method is defined, in part, by its objectives and the boundaries that it sets. In the cases studied, the innovation exploration focused within the boundaries of early-stage radical innovation, working with client collaborators, and ending at the transfer of the innovation to the product organization for commercialization. These boundaries determine how far an initiative team needs to go in delivering against KAM specific objectives. The level of clarity of the boundaries to stakeholders (i.e., KAM program-driven), and the effective planning and management of initiatives on both sides of the boundaries (i.e., KAM initiative team driven) insert complexity into initiative execution. This study argues that Piller et al.'s (2011) work on co-creation client modes of engagement can be extended to the notion of interlocking KAMs.

Modes of client engagement (e.g., listening into, asking, or build with customers) suggest that client stakeholders can play several potential roles based on the KAM process. This study suggests an alternative representation of the client role. As observed in this study, the client can indeed play multiple roles across various KAMs, and, these roles can occur in sequence across the innovation cycle. As observed in this study, the client begins in deep exploration in the FoaK KAM, and upon completion transfers to a validation role of clarifying requirements in the commercialization process (e.g., incubation KAM, validation KAM). This transfer of the asset to the mainstream business represents the next step in the chain of KAMs requiring further client collaboration in an alternative form. This 
hand-off point between KAMs was found to introduce additional complexity to the cocreation of innovation process (i.e., "the product chasm").

It was observed that while a KAM initiative may achieve sufficient levels of performance outcomes, the lack of clarity and alignment in the next step in the innovation cycle negatively impacts the desired innovation performance outcome (e.g., commercialization). The functional team (e.g., product portfolio) receiving the outcome innovation from the KAM was challenged by the lack of readiness of their organization (e.g., innovation asset knowledge, development skills, resources). This transfer issue was attributed, in part, to co-creation planning ending at the boundaries of the FoaK KAM, with little consideration given to the innovation path needed to reach commercialization. This additional complexity introduces a risk of losing knowledge and innovation that falls between the promise of the co-creation initiative (i.e., FoaK KAM), and the frustration of the receiving organization (i.e., incubation KAM).

What these findings suggest is that in addition to recognizing that firms can expect to use multiple KAMs in their innovation practices, they must also consider the chain of interdependent KAMs that forms the path for co-creation innovation opportunities from idea through to the ultimate firm objectives (e.g., commercialization). It is suggested that a more strategic approach to managing the range of KAMs used by an organization, and their boundaries, will positively impact innovation performance for the portfolio of funded co-creation initiatives. This does not suggest that a single KAM must cover all the steps in the journey, but that a firm can consider multiple KAMs and how they interconnect, providing clarity on KAM objectives, intended outcomes, and "what's next" for the innovation in the transfer of an innovation asset between KAMs. While it may be argued 
that this approach further adds to the complexity, is believed that a strategic perspective on managing KAMs in fact reduces complexity by the clarity that it provides. A strategic approach is also expected to present the organization with the opportunity to leverage program-level capacities across KAMs and reduce the potential knowledge lost through disconnected activity.

The findings from this study contribute new insight into the nature of complexity in co-creation of innovation processes, interactions and activities. A KAM system view illustrates that sources of complexity stem from beyond the direct exploration of new innovation with a collaboration client. Complexity in co-creation of innovation initiatives includes the broader set of stakeholder relationships that must be managed and the effective navigation of the co-creation related processes needed to fully exploit the investment in these initiatives. Findings suggest the need for a more strategic view of contextual implications and planning both within the KAM boundaries, as well as to the ultimate performance outcomes the organization is trying to achieve.

The ability for KAMs and their initiative teams to navigate complexity will impact the performance of a co-creation of innovation initiative. Navigating complexity includes both managing inherent complexity as well as the ability to anticipate contextual change. To navigate the complexity, a broad knowledge base is required both at the KAM programlevel and within its initiative teams. The knowledge capacity profiles reflect the aggregation of experience, program-level knowledge stores, and access to an extended network of expert knowledge. Co-creation initiative teams that led successful projects were 
found to manage complexity better than less successful teams who struggled with complexity of the various forms described. These more effective teams were characterized as having stronger knowledge capacity profiles than teams that delivered lower performing co-creation initiatives. The stronger knowledge capacity profiles serve as a reflection of knowledge-based dynamic capability development.

\subsubsection{KAM and Knowledge Development}

The role of external knowledge in co-creation of innovation is well developed in the literature. Research has examined the role of knowledge management processes, knowledge development, and the challenges in accessing tacit knowledge in collaborative innovation engagements. Much of these insights are at the firm-level, which has limited the understanding of the link between external knowledge and co-creation of innovation performance variance within a firm (Ahn et al. 2016; Barreto 2010; Lichtenthaler \& Lichtenthaler 2009). The literature is less developed in the study of knowledge across KAM types (e.g., FoaK), and in examining the development of knowledge at the level of knowledge acquisition method execution. This study has provided new insight into knowledge development in co-creation of innovation initiatives, the role of knowledge profiles, and their contribution in explaining the intra-firm co-creation innovation performance variance. Study findings also highlight the challenge of knowledge leakage due to the transient nature of KAM initiative teams.

The knowledge-based view of the firm states that a primary role of the organization is in building and evolving the knowledge stored in the enterprise (Dierickx \& Cool 1989). Organizational capabilities and knowledge learning are the fundamental 
capabilities that form bridges between open innovation activities and innovation performance (Cheng \& Shiu 2015). Knowledge is described as being at the centre of establishing a firm's competitive advantage, with the production and integration of all knowledge fundamental to organizational excellence (Macpherson et al. 2004). Macpherson et al. (2004) state that the firm depends on its ability to develop competencies in sourcing and exploiting the knowledge the organization needs. This also contributes to the KAM objective of achieving repeatability and scaleability in co-creation of innovation initiatives. Achieving this is dependent upon the firm's ability to acquire, exploit, and retain new knowledge.

The literature also highlights the need to invest in dynamic learning capabilities by creating mechanisms that support the firm's ability to absorb information and develop new knowledge through iterative business practices (Cohen \& Levinthal 1990). Lichtenthaler and Lichtenthaler (2009) in their knowledge capacity framework suggest investment in the development of six knowledge-based dynamic capabilities argued necessary in open innovation models.

The initiative-level findings from the study present deeper insight into knowledge profiles of the co-creation initiative teams and argue that knowledge development in the context of KAMs must be viewed as occurring across tiers in the knowledge acquisition method system (i.e., program, initiative, resources). The findings suggest that co-creation knowledge developed for a given KAM is used to bridge knowledge gaps within initiative teams. The benefit of the bridging knowledge is also dependent on the relevance of the knowledge store, and the KAM initiative team's willingness to draw upon it. Study findings 
highlight the impact of knowledge leakage on KAM knowledge retention because of the transient nature of initiative team resources and initiative-level tacit knowledge.

\section{Knowledge Profiles}

The literature speaks to the need for the firm to develop a broad set of skills to access external client knowledge in collaborative innovation engagements (e.g., learning capabilities, problem-solving skills) (Kim 1998; Lichtenthaler \& Lichtenthaler 2009). Other research speaks to knowledge management and the need for investing in integrative skills for knowledge development, (re)combination and use of knowledge (Nielsen 2013; Zahra \& George 2002). Ahn et al. (2016) argue that developing open innovation capacities, contributes to open innovation performance for the firm. As suggested by Nielsen (2013), regardless of the capacities and skills developed, the impact of knowledge in co-creation of innovation will be the degree to which it can flow in and out of the firm, spanning processes from the creation of new knowledge and its exploitation.

Lichtenthaler and Lichtenthaler (2009) propose in their knowledge capacity framework (KCF), a set of knowledge-based dynamic capabilities they argue as necessary by the firm and effectively executing open innovation activities. They suggest that firms that possess the capabilities identified in the framework benefit with a positive impact on innovation performance. The scope of this study focused on Lichtenthaler and Lichtenthaler's (2009) knowledge capacity framework for the knowledge capacities identified as necessary in inbound open innovation (iOI).

This study contributes to the role of knowledge capacity profiles in open innovation through its insight into knowledge within the FoaK KAM system, and the nature of 
knowledge profiles as they relate to initiative-level execution of co-creation projects. Findings from this study highlight knowledge capacity profiles of KAM initiative teams, reflecting the range of knowledge types needed to support the effective execution of a cocreation initiative. These profiles were found to vary across teams. Findings also support the notion that a primary role of the KAM program is to bridge knowledge gaps within initiative teams to help lower barriers to effective initiative execution. The study also answers the call for further research and empirical validation of the knowledge capacity framework (Lichtenthaler \& Lichtenthaler 2009) by exploring the framework and the interactions of the knowledge capacities as they relate to co-creation of innovation performance.

Range of Types: The study argues that there is a common set of knowledge types required both at the KAM program-level and the initiative-level that address the external and internal co-creation environments. The knowledge types identified were found to correspond to the knowledge capacities theorized by Lichtenthaler and Lichtenthaler (2009). These knowledge capacities exist at both the level of the KAM program and within individual initiative teams.

The KAM program was found to invest in building a common base of experience and practices that could be leveraged by initiative teams, and that helped in guiding prioritization and investment decisions made across initiatives. In addition to knowledge capacities identified in the $\mathrm{KCF}$, there were also KAM program specific knowledge types identified that support orchestration and portfolio management activities. These additional knowledge-based dynamic capabilities were identified as supporting the effective management of the KAM portfolio, and are argued to be applicable across KAM types. By 
design, the KAM program determines the perceived set of ideal knowledge types required by initiative teams for successful co-creation initiative execution. The knowledge capacity profile of individual teams, in turn, reflects the program conditions for participation in the KAM. Initiative teams, in part, were selected based on a minimum knowledge set and belief in their capability to execute. The ability to meet the selection criteria and successfully receive funding from the KAM program is a proxy acknowledgment that the initiative proposal has presented a knowledge profile that is believed to be sufficient to execute the initiative effectively. The program, in turn, bridges knowledge gaps with those teams. KAM initiative teams of successful co-creation initiatives were found to have stronger more balanced knowledge capacity profiles than initiative teams with less successful cocreation initiative performance.

Knowledge profile variance and bridging knowledge: Findings from the study also reported that the knowledge types observed for a KAM are consistent across both the program and its initiatives. However, while the program focuses on developing knowledge types in support of repeatability and scaleability, individual initiative teams look to assemble a baseline level of knowledge that supports initiative success. The level of development of initiative team knowledge capacity profiles was found to vary across teams. This is expected considering that co-creation initiatives and their teams are transient, with co-creation initiatives experiencing the frequent movement of participants by design. Co-creation initiative teams are assembled primarily for a specific project, consisting of internal and external stakeholders, many of whom have not worked together previously. The knowledge capacity profile of an initiative team, in turn, is the aggregate of individual 
member experience from their primary functional responsibilities and any previous cocreation initiative projects.

A key finding from the study is the role of the KAM program as a bridge of knowledge gaps across innovation teams. With its goal for repeatability and scaleability, the KAM program strives to level out any knowledge shortfalls within individual initiative teams towards an ideal knowledge base for the initiative, and at a minimum with sufficient knowledge so that innovation teams have a greater potential for delivering innovation to the firm. The KAM bridges knowledge across teams through of the formalization of processes, codifying common practices, provision of artifacts, and provide the support and guidance through an extended expert team and mentoring. This is consistent with Nielsen (2013) calls for the need to have formalized knowledge development processes. KAM initiative teams of successful co-creation initiatives were found to leverage program knowledge more effectively than initiative teams with less successful co-creation initiative performance. Also, knowledge bridging was found to benefit less experienced teams more than teams with previous FoaK KAM experience.

The study focused on a period of KAM program stability, allowing for an examination of knowledge variance across initiative teams. It was found that the level and substance of engagement between the KAM program of initiative teams varied based on individual initiative team experience. More experienced KAM initiative teams were found to focus on strategic guidance from the KAM program knowledge base, whereas the less experienced teams centred their program interaction on baseline guidance (e.g., navigating KAM program and processes). As expected, the impact of knowledge bridging by the program on an initiative team's ability to execute was found to be only as impactful as the 
degree to which initiative teams were prepared to exploit the knowledge and experience available to them.

The most influential factor relating initiative team knowledge capacity profiles to initiative performance was found to be previous co-creation experience. Initiative teams that had participated in previous co-creation of innovation projects were found to be better prepared for managing the complexity of new KAM initiatives than teams with little to no

previous experience. Initiative teams with a combination of previous KAM experience and related individual experience outside of these projects (e.g., executive presentation experience, client facing interaction) were found to have co-creation initiatives that perform better than teams with less experience. It was also found that the strength of the initiative team leader (i.e., PI) was significant in influencing initiative performance. However, it is unclear the extent to which the PI versus overall initiative team knowledge profiles contributes to initiative performance. This presents an opportunity for future study.

\section{Knowledge Acquisition and Creation}

The acquisition and creation of new knowledge are the cornerstone of the knowledge acquisition method and co-creation of innovation engagements, and have received attention in the literature. Open innovation and knowledge management research in this area have focused on firm-client relationships and methods for acquiring expert knowledge outside the firm. Research has highlighted the importance and challenges of acquiring expert knowledge due to this form of knowledge often being tacit in nature (von Hippel 1978). Day (1994) speaks to the need for new strategies (e.g., knowledge acquisition methods) to bridge information gaps and bring new thinking into the firm. Zollo 
and Winter (2002) speak to the potential of tacit knowledge as a source of innovation, whose value is only realized in the firm's ability to capture and convert it to a form that can be shared across the organization. Boisot (1998) speaks to the importance of codifying external knowledge if it is to be exploited in innovation. Kristensson et al. (2004) emphasizes that engagement with new expert users helps to create new knowledge by combining and reconfiguring insights from the co-creation process. Studies have also argued for the need to invest in knowledge acquisition methods that are effective in accessing and extracting tacit user knowledge in support of the innovation process (Leonard \& Rayport 1997; Leonard \& Sensiper 1998).

This study contributes to the literature through its examination of FoaK as a KAM, with the results confirming that this knowledge acquisition method was found to benefit the firm through the creation of new innovation ideas from collaboration with external experts. The FoaK KAM was found to centre on radical new-to-the-firm innovation. FoaK KAM was found to be consistent with other KAMs, in that skillful initiative teams found that their early stage innovation ideas were improved through their client engagement activity. The ideas with which clients engaged in co-creation initiatives were found to either be transformed based on expert input or new innovation ideas emerged from the collaboration. Positive outcomes from the knowledge acquisition and co-creation process were also found to contribute positively to co-creation initiative momentum, which in turn impacted innovation performance.

The study also contributed a deeper insight into the initiative-level dynamics in knowledge acquisition and co-creation activities. Specifically, results highlighted that there are parallel activities and interactions that surround the knowledge acquisition process 
between a KAM initiative team and the client stakeholder, which impacts knowledge acquisition effectiveness and the subsequent co-creation innovation performance. The results highlight that knowledge acquisition processes are interdependent, and that a shift in initiative objectives impacts the focus of those processes. It was found that the level of engagement and alignment across all co-creation stakeholders, in addition to shifting contextual factors (e.g., reorganization, changes in strategy) potentially impact client stakeholder motivation and engagement. These changes in turn have the potential to impact the ability to acquire the necessary knowledge from the client stakeholders. The ability to counter these potential knowledge acquisition challenges is in part influenced by the initiative team's knowledge capacity profile development. The ability to respond to changing context, once again, reflects initiative team knowledge capacity profile.

This study also argues that while new knowledge is created in the co-creation process, not all are suited to be codified. This suggests that there are limits to which a KAM program can capture the experience for future use towards scaleability. Some initiative specific knowledge was found to be specialized (i.e., industry-specific) and dynamic (i.e., short lifecycle) in nature, reducing the value in codifying for the KAM knowledge base.

It is also argued that due to the tacit nature of much of the knowledge acquired and developed, there is a benefit in combining stakeholders from across processes in engaging the client in the knowledge acquisition stage. A primary challenge observed was the transfer of knowledge between stages in the co-creation of innovation process. Specifically, knowledge transfer from the KAM initiative team to the commercialization product organization was found to be most effective when product organization resources participated in some form early in the innovation process. In the cases where active 
involvement was observed with product teams, there was development and sharing of knowledge early the co-creation process. This was found to reduce the potential for knowledge lost in transfer of knowledge related to the new innovation. Early engagement also allowed initiative team and product stakeholders to share a broader perspective of the program priorities, which further contributed to the shaping of new innovation and scope.

\section{Retaining and Transferring Knowledge - Transience}

The open innovation and knowledge management literature discuss the importance of transferability of knowledge both within an organization and between firms. Zahra and George (2002) state that in addition to knowledge acquisition by the firm, there must also be mechanisms in place to exploit this information for commercialization of new offerings. Retained knowledge has also been found to influence the interpretation of newly acquired information (Cohen \& Levinthal 1990), which in the case of the KAM suggests the evolution of the co-creation process. While research has argued that the impact of collective knowledge retention exceeds individual knowledge retention, the nature of knowledge retention and its influences are not sufficiently understood (Huber 1991). Similarly, there is limited research examining the challenge of co-creation knowledge retention at the level of the KAM and knowledge loss during the co-creation process. This study contributes to the understanding of knowledge retention in the co-creation process, highlighting the impact of KAM boundaries on knowledge transfer, and information loss due to co-creation initiative team transience.

KAM program members and initiative teams are not permanent to the KAM, which as a result has non-codified knowledge leaving with the individual. It was observed that in 
the cases where teams disband, accessing knowledge after the KAM initiative was a challenge for the organization, resulting in the offering losing visibility and value for the firm. These findings are similar to research examining the impact of downsizing on knowledge retention. Schmitt et al. (2011) found that resources lost due to downsizing negatively impact knowledge retention, leading to deterioration in quality, productivity, and effectiveness of organizations due to the loss of critical skills, capabilities, experience, and knowledge. While downsizing refers to a permanent loss of skills and knowledge, versus the redistribution in the case of disbanding teams, the net effect of low accessibility to the knowledge is the same to the firm.

The study suggests that the co-creation methods used in creating knowledge must consider how critical knowledge can be captured for future use. Where possible and of benefit, this may include codifying more knowledge than is currently done. Where not codifiable, knowledge retention may require instantiating processes where the broader set of resources are involved in primary knowledge gathering and creation. This includes the participation of stakeholders earlier in the co-creation process, and can also involve employee mentoring and shadowing models to develop knowledge across a broader set of resources.

The challenge regarding the transfer of the co-creation innovation asset to the product organization, where the product team was not involved early in the process, illustrates the negative performance impacts of poorly transferred or lost knowledge. It was observed that in initiatives where product commercialization teams were involved earlier in the process, there was a shared experience in understanding the new knowledge created with the client. As a result, early engagement contributed to joint stakeholder contribution 
to newly created knowledge, and at the point of transfer, there was less of a gap in understanding the asset specific knowledge between the initiative team and the product organization. However, in the cases studied, there remained significant knowledge transfer support required after the delivery of the co-creation initiative asset. In cases where product stakeholders were not involved in the co-creation process, the knowledge transfer curve to those teams was so steep that the potential partnership with new product organizations was abandoned. These findings further illustrate the complexity of the knowledge being shared, and the impact to the efficiency in its transfer when teams are not actively engaged throughout the process. This was further supported by the observed challenges in transferring the client stakeholder relationship from the KAM co-creation initiative to the innovation commercialization team (i.e., product stakeholder).

As observed, the impact of lost or poorly transferred knowledge will be experienced in initiative-level performance, as well as impacting KAM objectives to capture and share knowledge beyond a single initiative. This study has successfully highlighted the contributors to, and challenges of, knowledge transience in the co-creation of innovation process. This presents an opportunity for future study examining the nature and impact of knowledge transience in collaborative innovation initiatives and their performance.

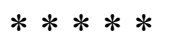

This study highlights that KAM initiative-level co-creation of innovation is dependent upon effective knowledge exploration, retention, and exploitation skills and execution. The study argues that knowledge development must be viewed at the level of 
the KAM system, considering both the KAM program-level, initiative-level and resourcelevel requirements separately. Program-level knowledge represents processes and practices (i.e., capacities) that are believed transferable between KAMs. This is primarily due to the KAM program focus on portfolio-level orchestration and prioritization of initiative investments, and practices that are domain independent. KAMs will improve with experience and gathering of more initiative-level knowledge, which in turn will help organizations in defining the ideal knowledge profile for initiative teams to be effective. This helps fulfill the role of the KAM as the orchestrator, providing guidance through proven practices. Knowledge profiles are expected to vary across teams, which would suggest that investing in resource-level knowledge development would reasonably be expected to help advance the base knowledge levels of the initiative resources over time. This in turn contributes to the evolution of the KAM method and its initiatives in support of portfolio-level competitive advantage.

\subsubsection{KAM and Co-Creation Dynamic Capabilities}

Each the streams of literature considered in this study highlight that organizational capabilities and knowledge learning form the foundation for competitive advantage, in turn, promoting superior firm performance (Grant 1996; Reinhardt \& Martin 2000; Teece et al. 1997). Dynamic capabilities theory speaks to the role of dynamic capabilities as contributors to a firm's adaptability to its changing environment, and to its long-term competitive advantage (Teece et al. 1997). Contributing to the focus of this study is the work of Lichtenthaler and Lichtenthaler (2009) who argue that there is a distinct set of knowledge-based dynamic capabilities needed to support open innovation engagement and 
activities effectively. Their theoretical knowledge capacity framework (KCF) represents both inbound and outbound open innovation (iOI, oOI) models. Lichtenthaler and Lichtenthaler (2009) highlight the shortcomings in dynamic capabilities literature explaining the integrative nature of dynamic capabilities and insight into the complexity of interaction between them. They put forward the call for research providing empirical support of the KCF.

This study contributes what is believed to be the first empirical examination and support of inbound open innovation elements of the knowledge capacity framework in cocreation of innovation initiatives. The findings present support for the iOI dynamic capabilities and a revision to the $\mathrm{KCF}$, with the introduction of a portfolio management "internal optimization" knowledge capacity. The study also introduces to the literature the role of the KAM as a higher-order dynamic capability, focused on "orchestration." The initiative-level examination has highlighted the role of dynamic capabilities in co-creation of innovation initiatives, answering the call for research into a system perspective of the integrative nature of dynamic capabilities (Lichtenthaler \& Lichtenthaler 2009, Wang \& Ahmed 2007). The findings also present to the literature that the development of dynamic capabilities in KAM systems occurs across individual tiers of the system, and must be examined within and between each level.

\section{Scope of Dynamic Capabilities}

All the iOI related dynamic capabilities examined from the KCF were found to exist in all cases examined. The existence of these dynamic capabilities and a collaborative innovation context would not be of surprise to those that study new product development 
and co-creation of innovation. A closer examination of each of the knowledge capacities in the framework represents primary processes in the innovation cycle. Inventive capacity focuses on the ability to identify the new opportunity, connective capacity speaks to the relevance of the client-firm relationship in these engagements, absorptive capacity and innovative capacity reflects the firm's ability to exploit and commercialize new innovations to the benefit of the firm's performance. Surrounding each of these dynamic capabilities is the transitive capacity which reflects the organization's ability to build, maintain, and trigger knowledge for the evolution, repeatability, and scaleability of collaborative innovation models.

The findings suggest that while all dynamic capabilities examined are expected to exist across KAMs, it is also believed that the relative emphasis and development of each of these knowledge capacities would vary across KAMs, as a reflection of the individual KAM's objectives and scope. In this study (i.e., FoaK KAM), the roles of connective capacity, inventive capacity, and absorptive capacity were found to be of greater importance in this early stage exploration of innovation. The capacities contribute to ensuring the ability to create new ideas, access to the right "engaged" client stakeholders, and a readiness of the commercializing organization to receive new knowledge was central. However, if the next step in the creation chain represents a hand-off to a beta testing KAM, where the focus is on innovation validation for commercialization versus creation, the KCF expected emphasis would be on absorptive and innovation dynamic capabilities. It is also argued that the level of dynamic capability development will also vary based on the type of innovation being explored in a co-creation context. Specifically, incremental innovations present fewer unknowns than radical innovation initiatives, requiring less 
emphasis on inventive capacities, and moderate focus on innovative and connective capacities.

Finally, dynamic capability research in collaborative innovation has mainly examined phenomena at the firm-level (Lichtenthaler \& Lichtenthaler 2009; Wang \& Ahmed 2007). This insight from this initiative-level study presents the argument that the role and development of each of the KCF dynamic capabilities must be considered across three tiers of the co-creation of innovation system: program-level, initiative-level, and resource-level. It is argued that these knowledge-based dynamic capabilities reflect an entity's ability to execute its activities. In other words, observations of dynamic capability development at the level of a program may differ from the level of development across the teams that execute initiatives within the program. This in turn may also differ in the individual resources that staff initiative teams.

The findings also highlight that initiative teams demonstrate a collection of the dynamic capabilities at various levels of development when comparing across initiatives. It was noted that the level of dynamic capability development within an initiative team was not at the same level as the KAM program. As well, each of the initiatives is staffed by resources that have various levels of experience and exposure, which impacted the degree to which an individual can contribute to a team's effectiveness. It was evident that part of the transitive capacity was the ability for teams to release knowledge they had within their members to make the unit as productive as possible. This is consistent with research on the benefits of collective knowledge retention exceeding individual knowledge retention (Huber 1991). 


\section{Revision of DCs - Portfolio Management and Optimization}

In considering dynamic capabilities within the $\mathrm{KCF}$, this study found that the $\mathrm{iOI}$ knowledge capacities did not account for the knowledge required in optimizing resources available across initiatives, a reflection of the internal competitive nature of innovation within the firm. This study proposes the extension of the first-order dynamic capabilities in the $\mathrm{KCF}$ to include the portfolio management dynamic capability as an additional internal knowledge capacity. This knowledge capacity was found to exist specifically within the KAM program tier, versus across each of the individual initiatives (i.e., KAM initiative tier).

Portfolio management is a dynamic capability that has been examined by Daniel et al. (2014) in the context of information system portfolio management, as a higher-order dynamic capability. However, it is argued in this case that the processes addressed by the portfolio management capacity in KAMs align more closely with the first-order dynamic capability definition. The portfolio management activities were viewed as standard operational processes, well-defined as a discipline for evaluating portfolio investments and management. While the implications are more semantic in nature, the inclusion of portfolio management as a dynamic capacity is supported by these findings.

Portfolio management as a dynamic capacity was found to link the strategic intention of orchestration as a higher-order dynamic capability for the KAM program to the execution of individual co-creation initiatives. The level of development of the portfolio management capacity was found to be reflected in how well the resources available for cocreation are managed and balanced across potential opportunities. There is also a direct linkage observed between the role of portfolio management as a knowledge capacity and 
KAM repeatability and scaleability. In this study, portfolio management was found to be mature in its development, and consistent in action across initiatives. The benefits of the KAM program framework supported by a portfolio management capacity were reflected in the evidence provided by informants. The portfolio management knowledge capacity was found to be central to knowledge creation, retention, and distribution across initiative teams. It represents practices that the KAM looks to improve over time, and uses in guiding individual teams through the "conditions to participate" for their projects.

\section{Higher-Order Dynamic Capability - KAM as Orchestrator}

Lichtenthaler and Lichtenthaler (2009) highlight the importance of higher-order dynamic capabilities as integrative dynamic capabilities. As part of their research on knowledge-driven dynamic capabilities for open innovation models, they present the concept of the "knowledge management capacity" (KMC), which is described as contributing to reconfiguration and realignment of knowledge capacities. This study's initiative-level exploration of dynamic capabilities in co-creation of innovation contributes significant new insight into the nature of KMC, arguing that the KAM as a method serves primarily as an "orchestrating" dynamic capability in the KAM system. The KAM role as orchestrator speaks directly to the method addressing initiative complexity by reducing barriers to initiative execution.

The KAM as an orchestrator was found to meet the literature's definition of higherorder dynamic capabilities. KAM "orchestration" was found to meet Winter's (2003) description, as a strategic activity and investment in organizational learning supporting strategic processes (i.e., KAM related) that facilitate the creation and modification of other 
dynamic capabilities. Consistent with the observed KAM "orchestration," higher-order capabilities generally have also been described by their potential to integrate and coordinate individual capabilities of the firm, which do not operate as standalone capabilities (Lichtenthaler \& Lichtenthaler 2009; Wang \& Ahmed 2007).

This study finds that the role of the KAM in collaborative innovation initiative performance is indirect. The KAM primarily serves in setting the course for success, whereas the attributes of the initiative execution and its team are directly linked to the performance outcomes. KAM was found to focus on managing the complexity of cocreation initiatives and guiding investment along a proven collaboration path for the method. It was found to do so through its orchestration of what the program has identified as the essential elements for an effective co-creation initiative: the strategic objectives of the program and the firm, enlisting the relevant stakeholders, guiding the co-creation process and resource towards a focused effort. This orchestration was achieved through a combination of prescriptive elements and guidance, which included the conditions to participate, lending, development of the knowledge base, provision of mentors and guidance, initiative oversight, and providing clarity on expectations from each of the initiative teams.

Three levels of orchestration were found to exist: strategic, program and tactical levels. Strategic orchestration represented the linkage between KAM and firm priorities. This orchestration focuses primarily on ensuring that the initiatives that were selected and funded were in line with what the firm would perceive as contributing value to its strategic direction. The scope of this focus was represented through the board and program framework. Program execution represented the managing of KAM resources towards the 
greatest impact across initiatives. The portfolio management dynamic capacity served as the link between the strategic orchestration and the selection process in execution. This involves managing KAM resources towards the co-creation of innovation initiative returns. Finally, tactical orchestration centres on managing knowledge and experience between the program and individual initiative success. This is observed through guidance and mentoring, and the provision of knowledge assets to help bridge knowledge gaps within individual teams.

The study identifies orchestration as residing at the program-level as a higher-order dynamic capability. There is some evidence from the study that part of the role of orchestration is in managing the development of knowledge across the KAM system. While the transitive dynamic capacity centres on retaining and exploiting knowledge, it is argued that orchestration complements this by developing knowledge across "people" resources that participate in the co-creation of innovation process. Support for this argument came as statements of a program shortcoming by informants. Specifically, informants suggest that the program must evolve to ensure that initiative teams and their resources are more effective in their projects. This presents an opportunity for deeper investigation.

The study also argues that the orchestration program-level higher-order dynamic capability develops it becomes a capability that can be transferred across the firm to other KAMs. Orchestration has demonstrated that it is domain independent, in that its focus is on assembling the right elements (e.g., people and resources), within a framework of proven practices, which evaluate information and make decisions regarding the domain being explored in the co-creation activity. 
It is believed that orchestration can be separated from the specifics of an individual co-creation initiative and applied as a developed competency across other KAMs. This in part is supported by the fact that orchestration activity within the FoaK studied presided over 200 initiatives across industries and innovation domains. The value of the orchestration in this context is in its knowledge of the co-creation process, above the details of the individual project execution. This extends the notion that as firms are expected to manage multiple KAMs in parallel as part of their innovation management activity, development of the orchestration capacity can be drawn upon across these methods, impacting performance across each of the stages of the full innovation cycle. Based on the findings, it is also believed that orchestration is relevant across organizations at varying levels of maturity and scale. Smaller organizations may not have the benefit of standalone program-driven KAMs, but the development of dynamic capabilities and the higher-order orchestration capacity could be reflected in how a function or team organize to best manage their co-creation initiatives.

\section{Dynamic Capability Interaction}

Dynamic capabilities research has been conducted examining dynamic and organizational capability hierarchies (Wang \& Ahmed 2007; Winter 2003), the cumulative nature of dynamic capability development and (Cohen and Levinthal 1990; Zollo \& Winter 2002), in the context plays a role in the effectiveness of dynamic capabilities (Teece et al. 1997). However, it is also identified that the literature has not adequately examined the integrative nature of dynamic capabilities and the complexity of interaction (e.g., cocreation of innovation initiatives) (Lichtenthaler \& Lichtenthaler 2009). The findings from 
this study contribute new insight into interactions and interdependencies of dynamic capabilities in the context of collaborative innovation engagements. The initiative-level findings highlight that in co-creation of innovation initiatives a range of knowledge-based dynamic capabilities are active in parallel and interact during the initiative. Interdependencies between dynamic capabilities were observed, with the effectiveness of one dynamic capability impacting the performance of other dynamic capabilities dependent upon it.

As has been presented, the dynamic capabilities identified in the study were found to exist across all the cases examined. The findings were found to support the argument that dynamic capabilities interact during the co-creation of innovation process, and new insight was gained into the nature of these interactions. Co-creation of innovation initiatives were found to represent a range of activities executed in parallel, that surround the core firm-client exploration process. The dynamic capabilities observed represent a series of distinct knowledge capacities necessary to effectively navigate collaborative initiative complexity towards the goal of creating new innovations for the firm. Each dynamic capability was found to serve a distinct role within an interlinked chain of processes observed in these co-creation efforts.

As described, the innovative and inventive capacities link to the essential ability of the firm to identify opportunities and create new ideas that support those opportunities. The connective capacity ensures the ability to access the right external sources of knowledge within client experts. The absorptive capacity, in this context, reflects the firm's ability to receive what has been cultivated with external co-creation clients, with the aim to commercialize. While these dynamic capabilities were found to serve distinct roles in the 
process, the linkages between dynamic capabilities across the co-creation process were also evident. Connective and transformative capacities were found to have the greatest impact on other dynamic capabilities in the process. Inventive and innovative capacities were found to be most influenced by other dynamic capacities. Absorptive capacity was observed to both impact and to be impacted by other dynamic capacities, all as part of the transfer of knowledge between the KAM and the commercialization process.

The question follows "why do certain dynamic capabilities interact in the cocreation process?" This study argues that the nature of dynamic capability interaction across the co-creation process reflects the elements that contribute to co-creation of innovation initiative performance. As will be developed in the next section, one of the contributors of performance was observed to be the ability to build and maintain momentum within the co-creation of the innovation process. Initiative momentum was found to be related to stakeholder motivation, engagement, and investment throughout the creation process. Momentum was found to stem primarily from the co-creation activity with the client (i.e., connective capacity). The ability to build up momentum and show promise was dependent on the initial appeal of the innovation concept presented to the client (i.e., inventive capacity), and the potential for impact of that idea as recognized by the program and the commercialization stakeholders (i.e., innovative capacity). The level of commitment from the product stakeholder as a channel for commercialization was found to impact the degree to which they were invested early in the process, improving their readiness to receive a new innovation (i.e., absorptive capacity), the crucial step in reaching the market. However, it was also observed that the product organization's interest was tied to the momentum and promise that came from innovation milestones from the client 
stakeholder engagement. Without the right access and availability of the necessary resources from the co-creation clients (i.e., connective capacity), momentum with the sales channel decreases (i.e., reducing revenue potential), ultimately impacting willingness to commercialize (i.e., absorptive capacity, innovative capacity). This scenario illustrates the interconnectedness of the dynamic capabilities and co-creation process, and further shines a light on the complexity and interdependencies between processes and dynamic capabilities in delivering against initiative objectives.

This study focused on a large multinational enterprise (LME), mature in its collaborative innovation experience. In this case, the knowledge acquisition method is represented as a standalone program. However, when considering the degree of complexity in co-creation initiatives that has been illustrated by these findings, it is expected that the nature of dynamic capability and process interaction will be observed in other contexts. While it may be questioned whether smaller firms would have the resources to manage the knowledge acquisition method as a standalone program, the findings support a position arguing that the elements of the co-creation process would remain the same. The ability to invent, engage the client, receive new innovation into the organization for commercialization, and effectively convert ideas into innovation, are all expected to be relevant regardless of firm size, maturity, or domain. The question is less about whether these dynamic capabilities are relevant and interact across contexts, but rather, the degree to which individual dynamic capabilities require development, and what the nature of their interaction would be. 


\section{Revised Conceptual Framework}

Throughout this study, the conceptual framework that emerged from the literature provided valuable guidance throughout the exploration. The findings resulted in a revised conceptual framework (see Figure 10.1), representing the expanded set of knowledge capacities observed (i.e., orchestration, portfolio management), and incorporating the notion of internal and external "client" stakeholders. Findings from this study were found to provide strong support for the conceptual framework, both in validating the literature contributing into the initial version, and introducing into the literature a revised guiding framework for future research. It is believed that the resulting conceptual framework will help shape future study aimed at advancing our understanding in this domain.

\subsubsection{KAM Role in Co-Creation Innovation Performance}

Much of the study that has been conducted on collaborative innovation from across research domains has focused on the impact of the various aspects of open innovation models on performance. Much of this study has centred on firm-level performance, with little research being conducted at the initiative-level (Deeds et al. 2000; Marsh \& Stock 2003). Open innovation literature has been consistent in its findings that there exists a positive relationship between external knowledge and innovation performance (Chesbrough 2006; Cooper \& Kleinschmidt 1987; Lin \& Wu 2014; Montoya-Weiss \& Calantone 1994).

Research in dynamic capabilities has identified an indirect relationship between capabilities and long-term firm performance (Wang \& Ahmed 2007; Zahra et al. 2006). Others have linked to dynamic capabilities to firm strategy, context and firm performance 
Ahn et al. 2016; Prieto et al. 2009). Dynamic capabilities research has also argued that observed performance is not dependent on the existence of capabilities, but rather how and what is done with those capabilities and knowledge in managing the firm (Eisenhardt \& Martin 2000; Grant 1996). Cheng and Shiu (2015) argue that dynamic capabilities serve as the bridge between inbound open innovation activities and innovation performance. However, as there has been little research at the initiative-level examining dynamic capabilities and collaborative innovation (Deeds et al. 2000; Marsh \& Stock 2003), open innovation research has only partially explained the complexity in observed performance (Cheng \& Shiu 2015). What is clear from the literature is that the link between open innovation mechanisms of performance (Cheng \& Shiu 2015), and dynamic capabilities interaction and performance (Lichtenthaler \& Lichtenthaler 2009), remains unclear.

This study has answered the call by providing new initiative-level insight into the role of knowledge acquisition methods in co-creation of innovation performance. Specifically, the study highlights execution level dynamics, dependencies, and dynamic capabilities in co-creation initiatives. The findings show that the KAM was found to impact co-creation innovation performance indirectly. This new insight into the indirect relationship between the KAM and co-creation of innovation initiative performance has also helped to understand the contributors to intra-firm initiative performance variance.

\section{KAM Role in Co-Creation of Innovation Performance - Indirect}

This study has been clear in illustrating the respective roles of the knowledge acquisition method and the co-creation of initiative teams in influencing collaborative innovation performance. While the KAM plays a key role in setting the stage for co- 
creation initiative realization, it is the initiative team and its ability to execute that ultimately impacts the final performance of a co-creation project. As described earlier in this discussion, the role of knowledge profiles, interlocking processes and dynamic capabilities were found to contribute at various stages of the co-creation process.

The findings from this research support the position that the knowledge acquisition method plays an indirect role in co-creation of innovation performance. The knowledge acquisition method contributes to co-creation of innovation performance through its impact on the co-creation initiative team's effectiveness (i.e., ability to execute). It has been argued that the KAM serves as an orchestrator, bringing together the key elements necessary to effectively execute on co-creation of innovation. The KAM builds on previous knowledge that spans the set of processes required from opportunity identification through to the transfer of the co-creation asset for commercialization.

The findings highlighted five principal themes that contribute to overall initiative performance. These included the role of the KAM in orchestration, the KAM bridging of knowledge gaps with initiative teams, the ability for initiative teams to achieve stakeholder alignments, the initiative team's ability to build and maintain initiative momentum, and the effectiveness of initiative teams in crossing the technology transfer chasm from successful completion of the KAM to the next step in the innovation process (e.g., commercialization).

From the findings, the KAM impact on performance is observed through its focus on three primary objectives: motivating stakeholder engagement, focusing initiative team activity on performance outcomes, and bridging knowledge gaps. The degree to which the KAM is effective in achieving these three objectives was observed to impact the effectiveness of initiative team execution. The KAM program design included reducing 
barriers to participation in an initiative that would motivate engagements (i.e., funding, credibility), acting as the initiative orchestrator, setting initiative conditions to participate in the program, and implementing a governance model to direct initiative team focus towards activities on agreed scope and measurements of success. These elements were found to contribute to strengthening an initiative team's ability to execute on their project, in addition to the KAM providing direct guidance and expert experience during the cocreation process.

\section{Intra-Firm Co-Creation Innovation Performance Variance}

The study also observed that while a knowledge acquisition method may be well developed and have proven success, this is not a guarantee that all initiatives conducted using the method, will be successful. This was illustrated clearly in the existence of cases under the same method with markedly different co-creation of innovation performance outcomes. This was found to suggest that understanding the level of development of dynamic capabilities at the firm-level, or program-level does not adequately explain the observed outcomes for individual projects.

Examining the effectiveness of initiative teams in executing their co-creation projects found that performance was dependent on their ability to build and maintain stakeholder alignments, create and sustain momentum for their initiative, and effectively manage the technology transfer of the co-creation innovation to the next step in the innovation cycle (i.e., avoiding the chasm). This ability reflects initiative team knowledge profiles, which were found to vary across teams. Central to this is the initiative team's effectiveness in achieving innovation validation that comes through client and product 
stakeholder engagement. It was found that the strength of the initiative team (i.e., knowledge capacity development) contributed to their ability to respond to environmental changes that may have a potentially counteracting impact on stakeholder alignment, initiative momentum, and the more prevalent risk of falling into the technology transfer chasm. It is the ability for the initiative team to anticipate and respond to changes in organizational strategy, stakeholder focus, and changes in stakeholder motivation, expectation, and investment, that directly impact innovation performance. Once again, this recognizes that there are factors outside of an initiative team's ability to execute that may negatively impact whether desired outcomes are achieved. This insight into the KAM and the co-creation of innovation as a system provides greater understanding into observed intra-firm performance variance.

These observations further support the argument that dynamic capability development must be viewed through a KAM tiered system lens, where development of dynamic capabilities should be examined at the level of the co-creation program, initiative, and resource-level of the firm.

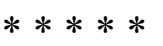

The initiative-level exploration in the study has provided deeper insight into the role of the knowledge acquisition method, and dynamic capabilities in co-creation of innovation initiatives. The findings have introduced further support that knowledge-based dynamic capabilities, and co-creation initiatives have an indirect impact on innovation performance. However, the findings emphasize that it is insufficient to limit the 
examination of dynamic capabilities the level of the firm. An understanding of firm-level innovation performance requires an understanding of the performance of initiative-level activity that contributes to the higher-level program and firm outcomes. This suggests that deeper co-creation system understanding is required of dynamic capabilities and their development at the level of the knowledge acquisition method, the co-creation initiative, and its team, and the resources that staff initiative teams.

\subsubsection{KAM and Competitive Advantage}

Research in the areas of dynamic capabilities, knowledge management, and open innovation all speaks to the firm focus on collaborative innovation towards developing competitive advantage. The discussion of the findings above has described the need for understanding co-creation dynamic capabilities across the KAM-system. Based on the findings from the study, it is argued that dynamic capability development occurs across various tiers in the KAM-system, and it is the strategic approach to developing dynamic capabilities from this perspective that ultimately contribute to the development of a firm's sustainable competitive advantage. This section contributes a synthesis and conceptualization of the KAM multi-tiered system and knowledge capacity development.

Findings from the study have provided insight into the role of knowledge acquisition methods in co-creation of innovation initiative performance. It has been argued that the role of the KAM is serving as an orchestrator of initiative success, bringing together elements believed necessary for effective execution co-creation endeavours. The study has argued that internal and external knowledge capacities (i.e., dynamic capabilities) represent the scope of knowledge required in inbound open innovation initiatives, and cover the 
range of insight required beyond the activity of working directly with a client stakeholder in innovation exploration. The study also demonstrates that these knowledge capacity profiles must be examined below the level of the firm, understanding their impact on cocreation of innovation initiative performance.

The findings present that knowledge capacities exist across multiple tiers of the cocreation system. The level of knowledge capacity profile development will vary within each of these tiers and across initiative teams. The role of the knowledge acquisition method, in part, is to bridge knowledge gaps in support of repeatable and scalable open innovation activity and its contribution to the firm. Figure 12.1 conceptualizes these findings and represents the interrelationship between tiers of knowledge. Co-creation of innovation related knowledge represents a profile of internal and external knowledge related to the exploration, retention, exploitation, and optimization of knowledge in collaborative co-creation of innovation endeavours. It is found that while these dynamic capabilities are often described at the level of the firm, understanding their relation to initiative performance requires that they are also examined at lower levels of the firm.

\section{Knowledge Capacities Exist Across Three-Tiers in KAM System}

The knowledge acquisition method, the co-creation of innovation initiative, and the internal expert resources are each drawn upon to execute on co-creation initiatives. The collection of knowledge capacities represents interconnected dynamic capabilities that portray a knowledge profile expressing the breadth of knowledge, experience, and expertise within each tier. 
Figure 12.1 Knowledge Acquisition Method System - Dynamic Capabilities Perspective

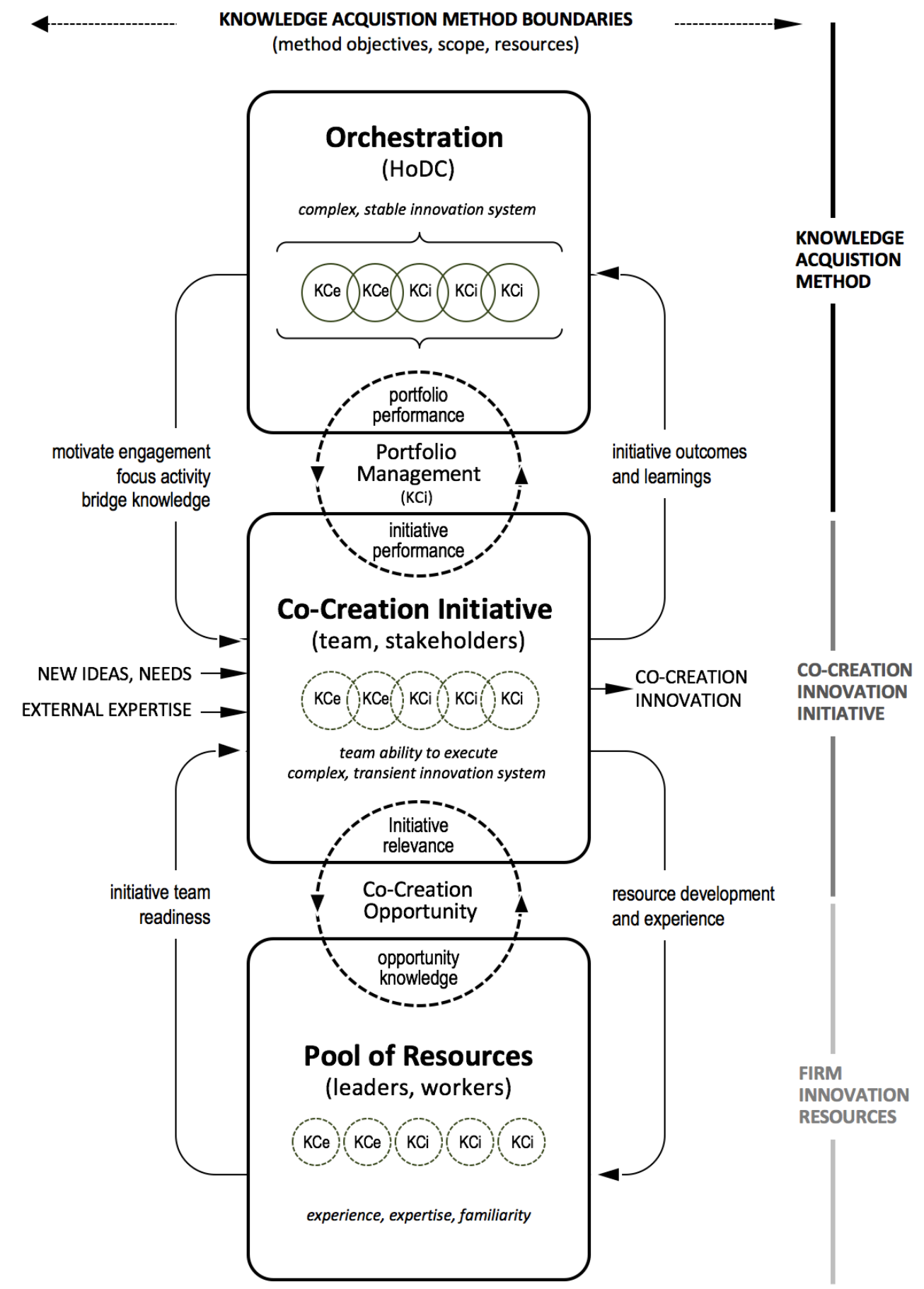


Knowledge acquisition methods represent a complex and stable innovation system that aspires to an ideal knowledge profile believed required for effective co-creation of innovation efforts. Knowledge acquisition methods are bound by their specific objectives, scope, and available resources. Individual KAMs may vary across each of these elements, and in turn, will work towards knowledge profiles that best support the method's specific objectives. In addition to the knowledge capacity profile, the knowledge acquisition method also serves as an orchestrator of co-creation initiative elements. This represents a higher-order dynamic capability that will manage the development and emphasis of knowledge management capacities towards the objectives of the method. Orchestration serves to help bring the right elements together in support of enhancing initiative performance by motivating engagement of stakeholders, focusing efforts on outcomes, and bridging knowledge gaps between what is required and what the reality is within initiative teams.

The co-creation initiative tier represents a complex and transient innovation system, where resources are brought together for the exploration and development of co-creation innovations with a focus on specific use cases. This tier represents transient teams that are composed of resources drawn from the broader organization and assembled towards a focused innovation scope and effort. This collection of resources represents a knowledge capacity profile based on the aggregated experience, expertise, and familiarity of those resources with their market, domain and the knowledge acquisition method. The knowledge capacity profile of co-creation initiatives will vary across teams.

The pool of resources tier represents potential co-creation initiative team members available to participate in these projects. These individuals represent initiative leaders and 
workers, that each brings their own unique knowledge capacity profile based on individual experience, expertise, and co-creation method participation.

\section{KAM System Tiers Are Interconnected}

The knowledge acquisition method, as an orchestrator, works towards selecting and supporting co-creation initiatives for effective execution of their projects, towards the delivery of new-to-the-firm co-creation innovation. The role of the knowledge acquisition method is primarily that of enabling success through the motivation of stakeholder engagement, the focus of initiative team activity, and bridging knowledge gaps. The cocreation initiative team works within the conditions and parameters outlined by the KAM, towards their co-creation of innovation. It does so by bringing together new ideas, with external expertise and the co-creation process.

The co-creation initiative operates between the guidance of the knowledge acquisition method and the available resources required for the co-creation process. The initiative balances available knowledge between the KAM and the pool of resources from which it draws internal experts. The knowledge acquisition method connects with its cocreation initiatives through its portfolio management capacity. The portfolio management capacity, as a dynamic capability, operationalizes the objectives of the knowledge acquisition method against its scope and resources. It balances activity with co-creation initiatives through the monitoring of initiative performance and its contribution to portfolio performance. The co-creation initiative, in turn, works towards satisfying KAM requirements as well as drawing upon its resources to achieve performance expectations. This influences the co-creation initiatives connection with its pool of resources. Co- 
creation initiatives draw upon those resources based upon the innovation opportunity's base of knowledge within the pool, and the relevance of the initiative for the firm. Initiative teams assembled from the resource pool result in initiative teams with varying levels of readiness based on their knowledge capacity profiles.

An objective of the knowledge acquisition method and its orchestration is to assess the readiness of a co-creation initiative team to execute and exploring new innovation opportunities. Achieving a scalable and repeatable level of performance requires predictability in the knowledge capacity profiles of initiative teams and the resources available to staff them.

\section{Development of the Knowledge Capacity Profile}

For firms to realize benefits from their KAMs, a strategic view on managing the development of knowledge capacity profiles will insert more predictability across the tiers of the system. It is expected that teams and resources that are inexperienced in co-creation activities will require time and exposure to develop their knowledge capacity profiles to levels viewed as ideal by the knowledge acquisition method. Similarly, it is expected that the KAM will also further evolve its own program-level knowledge profile and configuration over time.

Knowledge acquisition methods will mature based on learnings from co-creation initiatives that have been executed. These engagements provide new experiences that in turn contribute to strengthening the knowledge acquisition method profile of dynamic capabilities. Co-creation initiative execution also contributes to the development of the 
resource pool as each project exposes individual leaders and workers to new experiences strengthening personal knowledge profiles.

It is argued that the effectiveness of a KAM's impact on innovation performance requires that consideration be given to strengthening knowledge profiles beyond bridging knowledge for the co-creation initiative team. The KAM must move towards proactively developing the knowledge profiles of the resource pool. This suggests employing methods to help develop and sustain a higher level of experience within the pool. This approach contributes to reducing the gap between the co-creation initiative requirements and the level of knowledge available through their resources. The proactive development of knowledge within the resource pool is subsequently available to future initiatives and contributes to the development of knowledge capacity, both at the level of the method, and subsequently contributing at the firm-level.

\section{KAM Boundaries and Interlocking Methods}

KAMs adopting a strategic approach to managing initiatives and the development of a pool of resources suggests that, over time, the knowledge gaps between the tiers of knowledge acquisition method, co-creation initiative, and pool resources will decrease. This, in turn, inserts greater predictability in the KAM's co-creation innovation process.

Knowledge acquisition methods have been described as bound by the method's objectives, scope, and resources. This suggests that the firm may possess multiple knowledge acquisition methods focused on various forms of co-creation of innovation activities. Each one of these methods will be defined by its own objectives, scope, and resources, and will be characterized by the ideal knowledge capacity profile required to 
support those attributes. The findings support the argument for the existence of multiple knowledge acquisition methods and their interrelationship along the innovation process. For example, moving an innovation opportunity from idea to commercialization may be addressed by four interconnected knowledge acquisition methods: First-of-a-Kind exploration, new innovation incubation, new product development co-creation, new product beta testing.

Orchestration as a higher-order dynamic capability is argued to be transferable between knowledge acquisition methods. Firm-level development of their orchestration dynamic capability is expected to contribute to strengthening their effectiveness in inbound open innovation co-creation initiative performance across their portfolios, over time.

\subsection{Contributions to the Literature}

This exploratory study of knowledge acquisition methods (KAM) and their role in co-creation of innovation performance has presented rich new insight and contributions to the emerging cross-domain literature. As it relates to the primary research question, the study has presented evidence that the KAM impacts co-creation of innovation performance indirectly, through its impact on an initiative team's ability to execute. This was found to be achieved through the KAM's focus on reducing barriers to motivate stakeholder engagement and initiative momentum, focusing initiative team activities on performance outcomes, and bridging initiative team knowledge gaps. In addition to this principle finding, the study has also presented many contributions to the literature because of its "thick" exploratory study. This section will summarize the contributions presented throughout this thesis and in this discussion chapter. 
The nature of co-creation of innovation elements, the relationship between entities and capabilities, and how they influence collaborative innovation performance remains largely unexplored (Ahn et al. 2016; Cheng \& Shiu 2015; Lichtenthaler \& Lichtenthaler 2009; Lin \& Wu 2014). A review of the open innovation, dynamic capability, and knowledge management literature have revealed active and emerging streams of crossdisciplinary research drawing significant attention to these themes (Lichtenthaler \& Lichtenthaler 2009).

Evident has been the opportunity to contribute to theory development in helping better understand the nature of collaborative innovation complexity and its impact on cocreation innovation performance. Applying a cross-disciplinary lens reveals recurring themes in the literature examining the co-creation of innovation and user engagement (Schleimer \& Faems 2015), the role of knowledge acquisition methods and their link to strategy, competitive advantage, and firm performance (Ahn et al. 2016; Cheng \& Shiu 2015; Dahlander \& Gann 2010; Lin \& Wu 2014).

To date, research has reported on the complexity of these inbound open innovation activities and stakeholder engagement, and the role of developing knowledge-related operational and dynamic capabilities to realize firm performance objectives. There is a clear call from the research community to shine more light into the "black box" (Rosenberg 1982) and help unravel the complexity of the interactions between entities, capabilities, and performance, which to date has remained largely unexplored (Ahn et al. 2016; Cheng \& Shiu 2015; Lichtenthaler \& Lichtenthaler 2009). Three themes were evident in the literature as opportunities to contribute to theory development: 1) more research providing insight into the interaction between open innovation activities (Wang \& Ahmed 2007; 
Zahra et al. 2006), 2) the examination of the interaction between dynamic capabilities and the role of higher-order integrative dynamic capabilities in open innovation (Lichtenthaler \& Lichtenthaler 2009; Wang \& Ahmed 2007), and 3) investigation into the role of context in these interactions (Barreto 2010).

This study has answered the call by contributing new empirical insights from examining the question of collaborative innovation complexity through an initiative-level exploratory study of strategic co-creation of innovation initiatives and their use of knowledge acquisition methods. It is conducted from the perspective of dynamic capabilities theory, examining the mechanisms within these iOI engagements and their relationship to initiative-level performance. The study presents a system view of the phenomena being explored, examining knowledge acquisition methods as a dynamic integrative capability.

The findings from this study has provided contributions to theory development in the areas of open innovation, co-creation of innovation, dynamic capabilities, and new product development (NPD). Using Molina-Azorin's (2014) definition of the macro, meso and micro levels of theory, these contributions are described in this section (see Figure 12.2).

\subsubsection{Macro-Level Contributions}

Macro-level contribution to theory has been made to the dynamic capabilities and inbound open innovation (iOI) literature through this first empirical study examining the relationship between open innovation, dynamic capabilities, and knowledge management informed by Lichtenthaler and Lichtenthaler's (2009) knowledge capacity framework. 
Figure 12.2 Contributions to the Literature

\section{Macro-Level Contributions}

- Empirical validation and revision of iOI Knowledge Capacity Framework

- Knowledge Acquisition Method - "Orchestration as Higher-Order Dynamic Capability

- KAM Dynamic Capabilities - Portfolio Management Knowledge Capacity

- KAM System and Multi-Tiered Dynamic Capability Development

\section{Meso-Level Contributions}

- Portfolio-Initiative Level Analysis

- Conceptual Framework for Co-Creation Initiatives

- Intra-Firm Variance in Performance

\section{Micro-Level Contributions}

- FoaK Knowledge Acquisition Method Introduced to Literature

Previous research has highlighted the need for empirical study examining the role of dynamic capabilities and their interaction in co-creation of innovation initiatives (Lichtenthaler \& Lichtenthaler 2009; Wang \& Ahmed 2007), the nature of higher order dynamic capabilities (Lichtenthaler \& Lichtenthaler 2009; Wang \& Ahmed 2007) and their interaction with first-order dynamic capabilities (Ahn et al. 2016). These contributions provide empirical insights into the characteristics of integrative dynamic capabilities and their role in influencing performance in a co-creation of innovation and knowledge acquisition context.

\section{Knowledge Acquisition Method - “Orchestration” Higher-Order Dynamic Capability}

The study of first order dynamic capabilities and their role in OI and NPD continues to grow (Deeds et al. 2000; Petroni 1998). While the literature has expressed the importance of developing these capabilities in the context of open innovation towards 
competitive advantage (Macpherson et al. 2004), there is little understanding of the nature of capabilities as they relate to knowledge acquisition in the collaborative innovation context (Lichtenthaler \& Lichtenthaler 2009; Wang \& Ahmed 2007). In examining the $\mathrm{KAM}$, the findings describe the role of the knowledge acquisition method as that of a higher-order dynamic capability. In addition, the study highlights this capability as one of "orchestration" of co-creation initiative elements, processes, and activities.

The KAM program as an orchestration higher-order dynamic capability was found to serve the role as a momentum catalyst for co-creation initiatives using the method. The findings showed KAM as orchestration impacted innovation initiative performance indirectly by motivating stakeholder engagement in collaborative innovation, focusing engagement activities on outcomes, and bridging knowledge capability gaps for initiative teams leading co-creation initiatives.

This finding is a primary contribution to theory, providing new insight confirming the existence of higher-order dynamic capabilities in the co-creation of innovation initiatives, the orchestrating nature of that capability, and how this capability interacts with first order dynamic capabilities within open innovation engagements. While orchestration as a dynamic capability is beginning to appear in the literature (Brunswicker \& Van de Vrande 2014; Robertson et al. 2012), this study provides the first evidence of its role in open innovation. This study's initiative-level examination has delivered the deeper insight called for in understanding the complexity of co-creation of innovation. 


\section{Knowledge Capacity Framework Revision}

This study also referenced Lichtenthaler and Lichtenthaler's (2009) knowledge capacity framework $(\mathrm{KCF})$ as the starting basis for dynamic capability examination in the context of co-creation of innovation initiatives. This theoretical framework provided an initial guide to dynamic capabilities for this study, and an opportunity to contribute through empirical validation of the iOI knowledge capacities argued to contribute to open innovation initiatives (Lichtenthaler \& Lichtenthaler 2009).

Upon review of the literature, this study is found to be the first to provide empirical evidence for the iOI elements of the knowledge capacity framework, and the dynamic capabilities proposed. In addition to empirical support, this study proposes revision to the framework including the addition of the portfolio management capacity (Daniel et al. 2014) to the framework, and expanding to include orchestration as a higher-order dynamic capability that governs the open innovation knowledge capacities. Once again, the study's contribution is also at the level of the initiative, which has presented greater insight into the role of each of the dynamic capabilities and their interaction at the level of co-creation of innovation engagement.

\section{KAM Dynamic Capabilities - Portfolio Management Knowledge Capacity}

A further contribution of the study is the additional revision of the knowledge capacity framework to expand internal knowledge capacities (KCi) to include the Portfolio Management knowledge capacity. This optimization knowledge capacity is described as the link between the orchestration of the knowledge acquisition method and co-creation initiative execution. As with the orchestration higher-order dynamic capability, the 
portfolio management knowledge capacity is argued to be transferable across knowledge acquisition methods. It is also argued that the relevance the Daniel et al. (2014) IS PPM dynamic capability extends beyond traditional information systems in its applicability, across co-creation of innovation contexts where multiple initiatives are managed in parallel.

\section{Dynamic Capability Interactions - Multi-Tiered KAM System}

To date, there have been few attempts at study contributing to the understanding of the interaction between dynamic capabilities in the collaborative innovation context (Ahn et al. 2016). This study provides initial new insights into key stakeholder relationships as they relate to the co-creation of innovation, and the role of the interaction between dynamic capabilities in these collaborative engagements. A framework representing the KAM multi-tiered system for dynamic capability development was created based on the study, and introduced in support of future researchers.

Findings have demonstrated that the interaction between dynamic capabilities within co-creation initiatives and their role in contributing to co-creation of innovation initiative performance. Dynamic capability interaction has been presented as a multi-tiered system linking knowledge capacity development at the levels (i.e., tiers) of knowledge acquisition method, the initiative, and the resource pool drawn upon for co-creation of innovation initiatives.

The study has demonstrated that there are dominant dynamic capabilities in the creation of innovation initiatives (i.e., connective, transformative, absorptive) and that there are interdependencies and influences between dynamic capabilities in the effective 
execution of the creation of innovation initiatives. It is also found that the role of the "orchestration" higher-order dynamic capability in developing, managing, and emphasizing the various dynamic capabilities requires. This includes the linking function of the portfolio management knowledge capacity between knowledge acquisition method intent and co-creation initiative execution. Insights contributed in this study frame provided a path to continue the examination of dynamic capability interaction across other knowledge acquisition method types.

\subsubsection{Meso-Level Contributions}

At a meso-level, this study has contributed new insights into the role of knowledge acquisition methods in open innovation and co-creation of innovation practices, helping to better understand the relationships between the complex set of activities and mechanisms in collaborative engagements (Cheng \& Shiu 2015). The comparative case study approach has allowed this study to provide necessary insights into the impact of mechanisms on cocreation initiative performance (Becker et al. 2014; Enkel \& Gassmann 2005). This includes an additional contribution to theory examining open innovation and dynamic capabilities at the level of the offering initiative (Ahn et al. 2016). While previous literature has focused primarily on firm-level performance, this study presents new insight into the elements contributing to initiative-level performance, which ultimately impact firm-level performance. 


\section{Portfolio-Initiative Level Analysis}

The literature on open innovation and co-creation of innovation has focused primarily on firm-level investigation (Chiang \& Hung 2010; Chiesa et al. 2009; Christensen \& Bower 1996; Rohrbeck et al. 2009; Vega-Jurado et al. 2009). While this macro view is necessary for a cross-firm and cross-industry comparative, the nature of understanding open innovation collaboration at its essence requires investigation at the level of execution. It is studying below the level of the firm that provides the necessary insight into how the elements of open innovation, collaboration, and dynamic capabilities interact and contribute to initiative and portfolio performance, which in turn is reflected in overall firm performance. This gap in the literature has been identified by early researchers (Deeds et al. 2000; Marsh and Stock 2003).

This study's examination of individual initiatives within a single knowledge acquisition method (i.e., FoaK program) has contributed new insights into the multi-tiered nature of the co-creation of innovation system, and the relative roles of the knowledge acquisition method, to the initiative and resource pool. Once again, this is believed to be the first study examining dynamic capabilities in co-creation of innovation at this level of analysis, contributing necessary findings towards an understanding and unravelling the complexities of inter-and intra-firm processes (Lichtenthaler \& Lichtenthaler 2009).

\section{Conceptual Framework for Co-Creation Initiatives}

This study also introduces into the literature a conceptual framework for co-creation of innovation initiatives. A review of the literature for the study was unsuccessful in finding a conceptual framework that would guide the initiative-level investigation with a focus in 
co-creation initiatives and dynamic capabilities. In response, a conceptual framework for initiative-level co-creation was developed based on the literature (see Figure 3.2) (Daniel et al. 2014; Helfat 2007; Lichtenthaler \& Lichtenthaler 2009; Nielsen 2013; Prieto et al. 1989; Wang 2007).

An empirically validated and revised version of the conceptual framework (see Figure 10.1) contributes to the literature and researcher guidance for future initiative-level studies of collaborative innovation, further examining the connection between strategy, execution, and initiative performance, and linking inter- and intra-firm processes and dynamic capabilities.

\section{Intra-Firm Variance in Performance}

A further contribution to the literature of the study is the insight provided into the role of dynamic capabilities and collaborative processes in explaining the intra-firm variance of co-creation initiatives.

As has been highlighted, OI research has focused primarily on firm-level performance. Researchers have also emphasized that the existence of dynamic capabilities or their development that does not necessarily result in the expected performance impacts (Cheng \& Shiu 2015). While this relationship is intuitive, there has been little research examining the inter-firm/intra-firm dynamics of co-creation and NPD initiatives (Schleimer \& Faems 2015). The literature has not provided the necessary insights to understand why it is that similar the initiatives within a single firm can result in varying levels of performance.

This study has shown that the combination of the multi-tiered interaction between the knowledge acquisition method and its initiatives and resources, along with the varying 
levels of dynamic capability development, and initiative team ability to execute, all contribute to insight into why some initiatives succeed where others fail. The observed elements of the intra-firm variance in performance contribute to the call for study that explains how these initiatives operate and influence outcomes.

\subsubsection{Micro-Level Contributions - First-of-a Kind Knowledge Acquisition Method}

At the micro-level, the study has contributed to theory in open innovation and cocreation of innovation literature by examining the First-of-a-Kind (FoaK) approach to external stakeholder engagement, as a knowledge acquisition method. Previous studies in open innovation have presented a range of collaborative knowledge acquisition methods (Brockhoff 2003; Dahlander \& Gann 2010; Kristensson et al. 2008; Piller et al. 2011; Zogaj \& Bretschneider 2012), that each support specific objectives. The range of knowledge acquisition methods were presented in the Knowledge Acquisition Methods Framework (see Figure 3.1).

Based on the literature, this will be a first study examining the FoaK method as a systematic approach to sourcing external knowledge. FoaK is described as a product engaged knowledge acquisition method that supports producer-stimulated user innovation initiatives (Figure 3.1). As firms transition towards more open innovation-based strategies, a FoaK-style knowledge acquisition method provides a middle ground for firms. This study will present empirical insight that is expected to be of interest to researchers in this domain moving forward. 


\section{Chapter 13 \\ MANAGERIAL IMPLICATIONS}

The contributions of this study to academic theory extend to its managerial implications. The study has provided new insight into the relationship between knowledge acquisition methods (KAM) and co-creation initiative performance. It presents an argument that managers looking to optimize their co-creation innovation outcomes must look to the strategic development of knowledge profiles across their collaborative innovation system versus being transaction focused. This includes recognizing that the KAM has a program component that serves as an orchestrator across initiatives, which contributes to repeatability and scaleability for the firm. Similarly, practitioners should approach the management co-creation of innovation processes from an "end game" perspective, preparing for what happens with a KAM asset at the end of an engagement. A move from transaction to system focus in co-creation models is expected to yield more consistent performance outcomes for the firm. The following present managerial takeaways for consideration by practitioners of collaborative innovation models.

\section{KAMs - Repeatable and Scalable Co-Creation Initiative Performance}

Organizations that look to use knowledge acquisition methods as part of co-creation of innovation initiatives strive for repeatability and scaleability in their execution methods, in addition to achieving the targeted innovation performance. It is understood by practitioners that these types of engagements are complex. The nature of early-stage innovation and accessing knowledge for external stakeholders through collaboration often represents new and complex relationships and processes. Where possible, practitioners 
look to avoid variability in the execution of the processes, as a source of uncertainty and inefficiency into already resource strapped organizations. They look for opportunities where collaborative innovation variability can be reduced, and weigh those options against anticipated benefits from the collaboration.

The findings from the study highlight for practitioners the relative role of the KAM as an orchestrator of collaborative initiative execution that aims to motivate stakeholder engagement, focus initiative activities on outcomes, and bridge knowledge gaps with the initiative teams that are executing their projects. We must recognize that in the end, it is the initiative team's execution of the co-creation project that ultimately impacts its outcomes. In defining the knowledge acquisition methods, practitioners must be clear that each method type must be defined by clear objectives, mandates, resources, and boundaries of scope. These methods will evolve to represent the collection of experience that the organization holds from its co-creation engagements, and should be structured to guide initiatives based on proven practices from previous experience collected over time. As the firm's experience grows with a knowledge acquisition method, the knowledge profile, will also become stronger over time. In principle, the KAM should also see a stronger set of resources available to draw upon, with maturing knowledge capacity profiles that will improve initiative execution and performance.

This research highlights for practitioners that resources assembled for co-creation initiatives are often transient in nature. The knowledge that each of these resources possesses individually is brought together as part of a temporary team to explore an innovation of interest to the organization. Thus, individual teams will have varying levels of knowledge and ability to execute, which is expected to manifest itself in the form of 
varying levels of initiative performance across teams using the KAM. A strategic approach to knowledge acquisition will look to practitioners to develop resources proactively and to have systems in place to capture and codify experience for future teams.

Adopting a knowledge acquisition method approach allows organizations to explore a range of innovations and investigations continually, while developing new discipline in co-creation that strengthens over time. While it is understood that each collaborative innovation initiative will present its own unique challenges, there are also challenges that will appear across initiatives. It is in the cross-initiative view that the KAM program helps to focus efforts and investments while reducing variability in execution and outcomes.

\section{Takeaway 1:}

Organizations should look to guide co-creation of innovation initiatives using a knowledge acquisition method (KAM) model to reduce variability across co-creation initiatives of similar type.

\section{Be Strategic in Approach to Knowledge Acquisition Method Development}

An examination of the initiatives within a firm would find that not all share common goals and attributes. Each initiative could be described by the innovation being explored, stakeholders involved, type of innovation and strategic impact, the firm's expertise and dependence on external knowledge, and the resources available to develop the innovation. Variations occurring across each of these variables would suggest that a 
single knowledge acquisition method will not suit every co-creation initiative type. However, firms looking to develop knowledge acquisition methods should look to leverage what is common from successful methods into new areas, in addition to developing method specific elements needed for an initiative type. Ultimately, organizations are looking to ensure that their innovation management practices put in place programs that have the right elements to achieve the desired objectives while maximizing their return on investment.

The findings from the study support the notion of developing multiple knowledge acquisition methods, each serving a purpose along the innovation creation cycle. Organizations must look to developing their knowledge acquisition methods strategically, charting the course for new innovations from the point of idea to commercialization. This suggests determining the nature of methods needed and how they are interrelated along the course. A strategic approach to knowledge acquisition methods requires that firms understand the specific objectives of each method, their boundaries and how each of these methods will come together linking the stages of the innovation process.

In developing individual KAMs, the organization must emphasize developing the knowledge capacity profile across the multiple tiers in the KAM system. This means that organizations must develop the capabilities necessary for initiatives to be successful within the knowledge acquisition method, which becomes the proven practices for all initiatives. The method must also look to put together teams based on their ability to execute the initiative, which will be represented by their knowledge capacity profile. In parallel, the KAM program is also looking to opportunities to develop resources proactively, to serve in future initiatives. Resource development may use mechanisms such as shadowing and mentoring. The focus on knowledge capacity profile development at the initiative and 
resource-levels helps the organization to reduce the gap between the ideal knowledge capacity profile needed for co-creation initiatives and what is available within the resource pool.

The findings demonstrated that the lack of initiative planning outside the boundaries of the co-creation method results in "chasms." Chasms represent zones where, even with the successful execution of a co-creation initiative, the next steps for the initiative have not been adequately planned for. Ultimately, this results in a lower level of impact of the innovation for the firm. The existence of innovation chasms can result in innovation leakage within the firm, as well as lower levels of co-creation client satisfaction (i.e., internal, external).

\section{Takeaway 2:}

Approach KAM development as a series of methods that are interconnected, and that combine to bring new ideas and external knowledge together in delivering new-tothe-firm innovation to the market.

\section{Develop KAM Orchestration as a Core Competency}

Developing a knowledge acquisition method requires that a firm understands how the KAM will contribute to the performance of co-creation of innovation initiatives. In practice, the knowledge acquisition method works directly with individual initiatives exploring new innovations with expert sources of knowledge outside the firm. While 
execution happens at the level of the initiative, the knowledge acquisition method serves to motivate stakeholder engagement in the initiative, guides to focus initiative activities on performance outcomes, and helps bridge knowledge gaps with co-creation initiative teams. The KAM, as a higher-order dynamic capability, represents a core competency in cocreation of innovation related processes and engagement. This core competency has been identified as one of "orchestrating" the key elements that have proven successful in cocreation initiative performance, either for the firm or as proven industry practices.

Repeatability of co-creation initiatives is achievable at the level of the KAM. Developing the orchestration competency within the knowledge acquisition method helps to link the strategic intention of the method with the execution of individual initiatives. The KAM's primary role as an orchestrator is analogous to defining the playing field in setting up the boundaries within which initiative teams will execute their plays. The method represents what is believed to be needed for initiatives to be successful, and as the orchestrator guides all energy and investment in that direction. Orchestration is manifested through the structure of the knowledge acquisition method, the support provided to initiative teams, the processes and funding mechanisms. Ultimately, developing orchestration as a core competency results in the KAM contributing to the building of momentum for individual co-creation initiatives, ensuring strategic relevance of initiatives, and enabling the initiatives efforts through funding and support.

As orchestration is developed as a core competency, it is expected over time that the competency will be strengthened and modified with new experience and learning. Orchestration focuses on how elements of a co-creation initiative can be brought together in the right way to deliver the best possible performance outcome. As such, it is believed 
that orchestration as a competency is transferable across knowledge acquisition methods developed by a firm as part of its innovation management strategy. Therefore, firms taking a strategic view on developing their knowledge acquisition methods should consider that their investments in developing proficiency at the KAM and orchestration levels, is done so by looking forward to how these skills and processes can be applied in the development of future KAMs. The result is new methods starting with a stronger knowledge capacity profile than if it were to be developed from a blank page.

It is expected that investment in knowledge acquisition methods as an approach to co-creation initiative practices will be assessed based on the overall cost and the anticipated benefit of developing a formalized method. Practitioners should note that the guidance to invest in developing KAMs as competencies does not suggest that these investments need be significant by default. The approach may be simply that leaders are selected to participate and manage co-creation initiative projects, and consciously capturing learnings of what works and what doesn't for the firm. As co-creation initiatives grow in number, more investment is made towards formalizing as a program.

The findings from the study suggest to practitioners that taking the approach to developing their KAMs as an orchestration competency, will result in focusing efforts on initiatives that present the greatest opportunity for the organization, and guiding initiative teams with the level of support appropriate to their readiness to execute. The orchestration of conditions in stakeholder engagements helps to force alignment and focus in initiative teams. This results in setting initiative teams down a proven track for execution, with the support needed to allow them to focus on actual innovation execution. 


\section{Takeaway 3:}

Organizations developing KAM for co-creation of innovation should look to developing orchestration capability as a higher-order dynamic capability. This introduces a discipline in taking a strategic approach to initiative execution. Adopting this strategy acknowledges the complexity of co-creation initiatives and represents an opportunity to develop a capability that is transferable across knowledge acquisition methods.

\section{Portfolio Management - Linking KAMs and their Initiatives}

Organizations that develop their knowledge acquisition methods will still be faced with the question of how best to determine which initiatives should be pursued, and how best to guide them through their execution. As has been seen, initiative performance ultimately depends on the effectiveness of individual initiative teams in the execution of their projects. The linkage between the KAM and the individual initiatives is central to cocreation of innovation initiative outcomes. The KAM must look to motivate stakeholder participation in co-creation initiatives, focus initiative activity, and bridge knowledge gaps in the initiative team, all without restricting the innovation process.

The study found that the development of a portfolio management knowledge capacity within the knowledge acquisition method serves to determine that the critical elements are in place before funding individual initiatives. This capacity was found to govern progress against objectives and commitments, to stimulate momentum through 
increased funding where warranted, and to pull back on initiatives where monitoring suggests that outcomes are no longer achievable or relevant.

This suggests to practitioners that are developing knowledge acquisition methods that the development of a new portfolio management discipline serves as a link between the strategic intent of the KAM and the execution of individual initiatives. This portfolio management discipline applies checks and balances along the co-creation of innovation process from identifying new opportunities, through client engagements, to absorbing the innovation into the mainstream business.

The primary role of a knowledge acquisition method (e.g., program) is to identify the key elements of success, guide initiative direction, and bridge initiative team gaps to help increase the probability of achieving desired outcomes. The role of the initiative team is to deliver on its scope and objectives working within the boundaries of guidance set by the method, having the KAM serve as a catalyst for their co-creation activity. The role of the portfolio management capacity is to serve as a bridge between the method and the initiatives that use it.

As with the knowledge acquisition method, the portfolio management knowledge capacity develops over time, improving the effectiveness of methods for delivering innovation to the firm, and increasing predictability of initiative performance. Like orchestration, it is also believed that developing the portfolio management capacity within one method provides the potential for leveraging this competency in future KAM development. 


\section{Takeaway 4:}

The portfolio management approach to co-creation initiative management for KAMs improves the potential for repeatable and better initiative performance over time. This knowledge capacity helps the KAM optimize its resources against opportunities for greatest benefit to the firm.

\section{Competitive Advantage - Proactively Develop Knowledge Across the KAM System}

Co-creation of innovation initiatives require a range of knowledge types (i.e., knowledge capacities) to be successful in delivering innovation to the firm. This knowledge extends beyond the activity of working directly with the co-creation client within the innovation experiment interaction, to also understanding the dynamics between organizations, the mission of stakeholders, and the process of moving from idea to commercialization. Knowledge is found to exist at various levels of the co-creation system, including the method, the initiative team, across stakeholders, and with potential initiative team resources. It is expected that the level of knowledge and expertise will evolve at each of these levels over time. The study would suggest that organizations need to proactively manage the development of knowledge for these types of co-creation activities, to ensure that the organization's state of readiness is understood, that gaps that need to be bridged are identified, and the areas requiring the most development have been prioritized.

The study highlights that in cases where co-creation initiative teams are assembled for specific projects that individual teams will have different knowledge profiles. In part, the role of the knowledge acquisition method is to help in bridging knowledge gaps across 
these teams to ensure that there is sufficient readiness to be successful in executing the cocreation initiatives. Findings also demonstrate that as experience grows within team members and the team leader, initiative teams will be more effective in executing these complex projects.

Knowledge capacity development in the case of KAM is observed through refinements of the program and its processes. In the case of initiative team members, knowledge capacity development presents itself through increased familiarity with processes internal to the firm related to the knowledge acquisition program process, internal stakeholder priorities, and expectations of the technology transfer process. Initiative team members also develop new skills related to knowledge based on external experience including client engagement, industry and competitor familiarity, and development of technical expertise beyond the scope of their firm's portfolio. The most effective co-creation initiative teams were those that demonstrated well-developed knowledge capacities across the range of knowledge sites needed in the execution of their initiatives.

It was also found that the resources available to participate in co-creation of innovation initiatives often have limited experience with the knowledge acquisition method process. This includes little direct experience working with senior stakeholders and engaging directly with clients. In cases where the initiative team leaders were limited in their range of knowledge and experience, it was found to negatively impact the performance of their co-creation initiatives.

What these findings suggest is that organizations that adopt a more strategic approach to knowledge acquisition methods in co-creation activities, must include how 
they intend to manage knowledge development for each method. While a passive approach to addressing this need may result in successful performance from funded initiatives, organizations can expect to continually encounter knowledge gaps within the initiative teams, with minimal progress and development of the collective base of knowledge for cocreation initiatives using that method. The result is a slower development of the knowledge acquisition method within the firm, and lower levels of efficiency in executing initiatives over time. What this suggests is that the firm may achieve a moderate level of success with a KAM without actively developing system knowledge, but that without a strategic approach the method may not develop to the point where it contributes to competitive advantage for the firm.

This suggests that it is in the organization's best interest to strategically manage knowledge development not only at the method-level, but also within the pool of resources available to participate in future co-creation initiatives. At the method-level, the organization can look to processes that are more systematic in gathering learnings from individual initiatives to refine the program so that it performs best within its own changing context. At the level of initiative resources, the method can look to bridge knowledge gaps within their initiative teams in critical areas (i.e., internal networks, client engagement, domain expertise). In parallel, the method can look to identify resources that present the potential for participation in future initiatives, and begin developing their skills through efforts including mentoring and shadowing on projects. The method can also look to codify tacit knowledge from initiative experiences that can be used to bridge knowledge gaps within initiative teams, once again serving as a link between the ideal knowledge base required and the actual level of knowledge that exists within the team. 


\section{Takeaway 5:}

Strategically developing knowledge capacities occurs across multiple tiers of the KAM system. To achieve long-term objectives, the method must look to developing knowledge capacities and bridging knowledge gaps between the method itself, initiatives being executed, and within the pool of workers that will serve in future projects. The result is developing competencies that are transferable across initiatives over time. 


\section{Chapter 14 \\ CONCLUSIONS AND FUTURE RESEARCH}

"No company can act alone with the hope to innovate beyond the competition
without external sources as part of their overall strategy."

(Quinn 2000)

The study of open innovation continues to grow deep roots in both academic and practitioner arenas. As research has examined the role of external expert sources, knowledge management and dynamic capabilities in contributing to a firm's competitive advantage, there remains little study examining the initiative-level complexity that helps understand how the elements of open innovation engagements contribute to collaborative innovation performance. This study set out to contribute to the growing body of crossdisciplinary literature on open innovation by exploring the role of knowledge acquisition methods on collaborative new-to-the-firm innovation performance.

Using an exploratory comparative case study method, this study examined the Firstof-a-Kind (FoaK) knowledge acquisition method (KAM) across five case studies. The research focused on understanding the nature of the knowledge acquisition system, its initiatives, stakeholders, processes and engagement dynamics. Mindful of the link to themes in competitive strategy and advantage, the examination was conducted through a dynamic capabilities perspective. The results from this "thick description" study have contributed valuable initiative-level insight to the literature, by addressing gaps in understanding of open innovation complexity and the contributors to collaborative innovation initiative performance. 
This study has provided rich contributions to open collaborative innovation, dynamic capabilities, and knowledge management literature through its context of cocreation of innovation. Findings are presented that support that a knowledge acquisition method influences co-creation of innovation performance indirectly through its influence on an initiative team's ability to execute. The development of knowledge profiles (i.e., knowledge capacities) and the skills essential to executing co-creation initiatives are central to the KAM system. The findings describe that the development of knowledge capacities is multi-tiered across levels of a KAM co-creation system. Also, evidence is presented characterizing the knowledge acquisition method as a higher-order dynamic capability with its primary role being that of KAM "orchestration." In addition to these contributions, this study also presents first empirical support and revision for the Lichtenthaler and Lichtenthaler (2009) open innovation knowledge capacity framework, and introduces the First-of-a-Kind knowledge acquisition method to the literature. Finally, researchers are also presented with a new conceptual framework for co-creation of innovation study, and a KAM multi-tiered framework supporting dynamic capability development research in this domain.

\section{Research Limitations and Opportunities for Future Research}

As with all research that contributes to pushing a field of study forward, opportunities for future research also emerge. This study calls for researchers to continue to invest in understanding the inner workings of collaborative innovation initiatives. It is firmly believed that to truly understand the role of open innovation in impacting a firm's performance and competitive advantage, the dynamics of the interactions of these complex 
systems must be understood more fully. This study has recommended for future research building on its findings. These opportunities include further examining the evolution of KAMs, KAM interdependencies and management, the role of context across KAMs and further KAM stakeholder analysis. The study has also presented the research community with tools and conceptual frameworks representing a synthesis of cross-domain theory in the area of co-creation of innovation. It is hoped that these models are embraced and developed further by the academic community.

Theoretical contributions from this study have provided much needed insight into the dynamics of collaborative and open innovation at the initiative-level innovation performance. These contributions have included new understanding into open innovation and dynamic capabilities, the role of knowledge-based dynamic capabilities (i.e., knowledge capacities) across a knowledge acquisition method (KAM), their initiatives, and the resources drawn upon to execute within these multi-tiered integrated knowledge systems. This study has answered the call with empirically based contributions, and echoes forward the request for continued research that build further towards cross-discipline theory development in this open innovation domain. The following are presented to the research community as opportunities for future research and continued empirical study building on the contributions from this thesis.

\section{Evolving Knowledge Acquisition Methods}

This study examined a snapshot of a single knowledge acquisition method (i.e., FoaK) with context controlled to allow for a comparative study of co-creation initiatives delivered using that method. The focus of the study was to examine a KAM in a stable 
state, and examine the link between methods and performance, and provide explanatory insight into intra-firm variance in initiative performance. However, KAMs are expected to evolve with organizational learning (i.e., transitive knowledge capacity). There is an opportunity to examine the impact on innovation performance as knowledge acquisition methods evolve within the firm (i.e., initial launch versus mature program). This could be achieved through a longitudinal study within a single firm, examining the evolution of a method (e.g., program) over time and its impact on overall portfolio initiative performance. Alternatively, this can also be achieved through a cross-firm comparative study examining the same method across different organizations and at different levels of maturity. This direction of study would provide greater insight into the relative importance of dynamic capabilities at different stages of development across the method, initiative and resource tiers.

\section{Conceptual Frameworks}

Two conceptual frameworks have been introduced from this study. The co-creation of innovation conceptual framework (see Figure 10.1) was developed from cross-discipline research streams and revised through the course of this exploratory study. Findings also resulted in a second conceptual framework representing the knowledge acquisition method system (dynamic capabilities perspective) (see Figure 12.1), reflecting the collaborative innovation system and the interaction of knowledge-based dynamic capabilities across tiers. Both frameworks provide a foundation for future research across the range of collaborative innovation contexts. As these frameworks have resulted through the study of a single knowledge acquisition method (i.e., FoaK), it is believed that there is further 
benefit for the research community to apply these frameworks in the study of other knowledge acquisition methods and contexts.

These conceptual frameworks were also focused on inbound open innovation, as it is the primary method of open innovation being applied by organizations today (Chesbrough 2003). However, organizations must look at their open innovation activities as a portfolio within an overall innovation management strategy. It is believed that the findings from this study suggest a parallel role for these conceptual frameworks in examining dynamic capabilities and the complexity of interaction in the outbound collaborative innovation context. There is an opportunity to build on the findings from this study (i.e., conceptual frameworks, dynamic capabilities, and the roles of the higher order “orchestration” dynamic capability and portfolio management dynamic capability) from the perspective of the producer firm as the client stakeholder. This perspective would contribute to an examination of the desorptive capacity dynamic capability, which was outside the scope of this study. It will also provide an opportunity to explore the transferability of a firm's dynamic capabilities across knowledge acquisition methods and outbound open innovation initiatives.

\section{Strategic Management of Knowledge Acquisition Methods - Interlocking Methods}

This study highlights the impact of knowledge acquisition method boundaries and the existence of multiple knowledge acquisition methods within the firm. The notion of innovation initiative "chasms" were also presented. It was argued that chasms exist in part due to the lack of a planned path for innovation from idea to market, across a series of interlocked knowledge acquisition methods, each with their own distinct focus, objectives, 
and parameters. It is believed that the existence of these chasms in the co-creation process within firms results in innovation leakage (i.e., collaborative innovation created but not exploited by the firm). There is an opportunity for future research that explores the strategic management of a knowledge acquisition method portfolio, and the concept of interlocking knowledge acquisition methods. Specifically, studies examining the impact of managing knowledge acquisition methods across the co-creation of innovation development lifecycle and the transferability of dynamic capabilities across methods, would provide further understanding into the contributors of firm-level performance from open innovation strategies.

\section{Knowledge Acquisition Method Context}

This study examined knowledge acquisition methods within a large multinational enterprises (LME) subject organization, and examined co-creation of radical innovation initiatives. It is believed that the findings from this study extend across knowledge acquisition methods, firm types, and innovation types. There is a call for future research to further build on these findings through examination of dynamic capabilities at the initiative-level across contexts including small and medium-sized firms, and different levels of innovation (i.e., incremental versus radical). Also, while this study examined cocreation initiatives across five industries, a detailed assessment of industry context on performance was outside the scope of this study. There is an opportunity to examine the impact of industry maturity in co-creation initiatives on innovation performance, contributing greater insight into knowledge acquisition methods and initiative context and its role. 


\section{Stakeholder Research}

This study has highlighted the dynamics of stakeholder interactions at various stages in the co-creation of innovation process, representing a complex matching of interfirm, intra-firm, and cross-functional pairings. The study has revealed the role of orchestration and the impact of stakeholder alignment and engagement on initiative performance. There is an opportunity for researchers to examine KAM-stakeholder relationships, drawing upon stakeholder theory and research in stakeholder engagement (Gould 2012). Future research can explore the relative impacts of stakeholder motivation and management on co-creation initiative effectiveness, which in turn provides greater insight into initiative and firm-level performance in open innovation endeavours.

The opportunity for researchers to continue to build and contribute to theory development and industry practice remains significant in this domain. It is this researcher's hope that the contributions from this study and the suggested opportunities for future empirical research help in shaping an aggressive research agenda. 


\section{REFERENCES}

Afuah, A., 2001. Dynamic boundaries of the firm: are firms better off being vertically integrated in the face of a technological change? Academy of Management Journal, 44(6), pp.1211-1228.

Ahn, J.M. et al., 2016. Beyond absorptive capacity in open innovation process: the relationships between openness, capacities and firm performance. Technology Analysis \& Strategic Management, pp.1-20.

Ahuja, G. \& Katila, R., 2001. Technological acquisitions and the innovation performance of acquiring firms: a longitudinal study. Strategic Management Journal, 22(3), pp.197220.

Alavi, M. \& Leidner, D.E., 2001. Review: Knowledge management and knowledge management systems: Conceptual foundations and research issues. MIS quarterly, 25(1), pp.107-136.

Amit, R. \& Schoemaker, P., 1993. Strategic assets and organizational rent. Strategic Management Journal, 14(1), pp.33-46.

Anbardan, Y.Z. \& Raeyat, M., 2014. Open Innovation: Creating Value Through CoCreation. In Proceedings of the 7th World Conference on Mass Customization, Personalization, and Co-Creation (MCPC 2014), Aalborg, Denmark, February 4th - 7th, 2014. Lecture Notes in Production Engineering. Cham: Springer International Publishing, pp. 437-447.

Anderson, R.A. et al., 2005. Case study research: The view from complexity science. Qualitative Health Research, 15(5), pp.669-685.

Anfara, V.A., 2008. Theoretical frameworks. The Sage Encyclopedia of.

Archer, M.S., 1995. Realist social theory: The morphogenetic approach,

Atuahene-Gima, K., 2005. Resolving the capability — rigidity paradox in new product innovation. Journal of Marketing, 69(4), pp.61-83.

Atuahene-Gima, K., 1995. An exploratory analysis of the impact of market orientation on new product performance. Journal of product innovation management, 12(4), pp.275-293. Baldwin, C., Hienerth, C. \& Hippel, von, E., 2006. How user innovations become commercial products: A theoretical investigation and case study. Research Policy, 35(9), pp.1291-1313.

Barney, J., 1991. Firm resources and sustained competitive advantage. Journal of Management, 17(1), pp.99-120. 
Barney, J.B., 1986. Strategic factor markets: Expectations, luck, and business strategy. Management science, 32(10), pp.1231-1241.

Barney, J.B., 1996. The resource-based theory of the firm. Organization science, 7(5), pp.469-469.

Barreto, I., 2010. Dynamic capabilities: A review of past research and an agenda for the future. Journal of management, 36(1), pp.256-280.

Becker, M., Errichiello, L. \& Zirpoli, F., 2014. A Project-Based Perspective on Complex Product Development. In S. Bonesso, A. Comacchio, \& C. Pizzi, eds. Project-Based Knowledge in Organizing Open Innovation. London: Springer London, pp. 51-67.

Becker, W. \& Dietz, J., 2004. R\&D cooperation and innovation activities of firmsevidence for the German manufacturing industry. Research Policy, 33(2), pp.209-223.

Benbasat, I., Goldstein, D.K. \& Mead, M., 1987. The case research strategy in studies of information systems. MIS quarterly, pp.369-386.

Benner, M.J. et al., 2003. Exploitation, exploration, and process management: The productivity dilemma revisited. Academy of Management Review, 28(2), pp.238-256.

Bergen, M., Dutta, S. \& Walker, O.C., Jr, 1992. Agency relationships in marketing: a review of the implications and applications of agency and related theories. The Journal of Marketing, pp.1-24.

Berthon, P.R. et al., 2007. When customers get clever: Managerial approaches to dealing with creative consumers. Business Horizons, 50(1), pp.39-47.

Bhaskar, R., 1975a. A Realist, Theory of Science.

Bhaskar, R., 1975b. Forms of realism.

Bhaskar, R., 1978. On the Possibility of Social Scientific Knowledge and the Limits of Naturalism. Journal for the Theory of Social Behaviour, 8(1), pp.1-28.

Bogers, M., 2013. The open innovation paradox: knowledge sharing and protection in R\&D collaborations. European Journal of Innovation Management, 14(1), pp.93-117.

Boisot, M.H., 1998. Knowledge assets: Securing competitive advantage in the information economy. OUP Oxford.

Brettel, M. \& Cleven, N.J., 2011. Innovation Culture, Collaboration with External Partners and NPD Performance. Creativity and innovation management, 20(4), pp.253-272. 
Brockhoff, K., 2003. Customers' perspectives of involvement in new product development. International Journal of Technology Management, 26(5), pp.464-481.

Bruce, M. et al., 1995. Success factors for collaborative product development: a study of suppliers of information and communication technology. R\&d Management, 25(1), pp.3344.

Brunswicker, S. \& van de Vrande, V., 2014. Exploring open innovation in small-and medium-sized enterprises. New frontiers in open innovation, pp.135-156.

Brusoni, S., Prencipe, A. \& Pavitt, K., 2001. Knowledge specialization, organizational coupling, and the boundaries of the firm: Why do firms know more than they make? Administrative science quarterly, 46(4), pp.597-621.

Bryman, A. \& Bell, E., 2015. Business research methods. Oxford University Press, USA.

Buur, J. \& Matthews, B., 2008. Participatory innovation. International Journal of Innovation Management, 12(03), pp.255-273.

Bygstad, B., 2010. Generative mechanisms for innovation in information infrastructures. Information and Organization, 20(3), pp.156-168.

Carlsson, S.A., 2003, June. Critical realism: a way forward in IS research. In ECIS (pp. 348-362).

Chandler, G.N. \& Hanks, S.H., 1998. An examination of the substitutability of founders human and financial capital in emerging business ventures. Journal of Business venturing, 13(5), pp.353-369.

Charmaz, K., 2014. Constructing grounded theory. Sage.

Chatterji, A.K. \& Fabrizio, K., 2007. Professional users as a source of innovation: The role of physician innovation in the medical device industry. Working paper.

Chen, Y., Vanhaverbeke, W. \& Du, J., 2015. The interaction between internal R\&D and different types of external knowledge sourcing: an empirical study of Chinese innovative firms. $R \& D$ Management.

Cheng, C.C.J. \& Huizingh, E.K.R.E., 2014. When Is Open Innovation Beneficial? The Role of Strategic Orientation. Journal of Product Innovation Management, 31(6), pp.1235-1253.

Cheng, C.C.J. \& Shiu, E.C., 2015. The inconvenient truth of the relationship between open innovation activities and innovation performance. Management Decision, 53(3), pp.625647. 
Chesbrough, H., 2006. Open innovation: a new paradigm for understanding industrial innovation. Open innovation: Researching a new paradigm.

Chesbrough, H., 2010. Business Model Innovation: Opportunities and Barriers. Long Range Planning, 43(2-3), pp.354-363.

Chesbrough, H. \& Crowther, A.K., 2006. Beyond high tech: early adopters of open innovation in other industries. $R \& d$ Management, 36(3), pp.229-236.

Chesbrough, H., 2006. The era of open innovation. Managing innovation and change, 127(3), pp.34-41.

Chesbrough, H.W. \& Appleyard, M.M., 2007. Open Innovation and Strategy. California Management Review, 50(1), pp.57-76.

Chesbrough, H. \& Crowther, A.K., 2006. Beyond high tech: early adopters of open innovation in other industries. R\&d Management, 36(3), pp.229-236.

Chiang, Y.H. \& Hung, K.P., 2010. Exploring open search strategies and perceived innovation performance from the perspective of inter-organizational knowledge flows. $R \& d$ Management, 40(3), pp.292-299.

Chiesa, V. et al., 2009. Performance measurement in R\&amp;D: exploring the interplay between measurement objectives, dimensions of performance and contextual factors. $R \& d$ Management, 39(5), pp.487-519.

Christensen, C., Craig, T. \& Hart, S., 2001. The great disruption. Foreign Affairs, pp.8095.

Christensen, C. \& Bower, J.L., 1996. Customer power, strategic investment, and the failure of leading firms. Strategic Management Journal, 17(3), pp.197-218.

Clifford, G., 1973. Thick description: Toward an interpretive theory of culture. The interpretation of cultures, pp.3-30.

Cohen, W.M. \& Levinthal, D.A., 1990. Absorptive Capacity: A New Perspective on Learning and Innovation. Administrative science quarterly, 35(1), p.128-152

Collier, A., 1994. Critical realism: an introduction to Roy Bhaskar's philosophy.

Collis, D.J., 1994. Research Note: How Valuable are Organizational Capabilities? Strategic Management Journal, 15(S1), pp.143-152.

Conway, S. \& Steward, F., 1998. Mapping innovation networks. International Journal of Innovation ..., 02(02), pp.223-254. 
Cook, S., 2008. The contribution revolution: Letting volunteers build your business. Harvard Business Review, 86(10), pp.60-69.

Cook, T.D. \& Campbell, D.T., 1979. Design and analysis of quasi-experiments for field settings. Skokie, IL: Rand McNally.

Cooper, R.G., 1980. Project NewProd: factors in new product success. European Journal of Marketing, 14(5/6), pp.277-292.

Cooper, R.G., 1996. Overhauling the new product process. Industrial Marketing Management, 25(6), pp.465-482.

Cooper, R.G. \& Kleinschmidt, E.J., 1987. Success factors in product innovation. Industrial Marketing Management, 16(3), pp.215-223.

Corso, M. et al., 2001. Knowledge management in product innovation: an interpretative review. International Journal of Management Reviews, 3(4), pp.341-352.

Croom, S.R., 2001. The dyadic capabilities concept: examining the processes of key supplier involvement in collaborative product development. European Journal of Purchasing \& Supply Management, 7(1), pp.29-37.

Cui, A.S. \& Wu, F., 2016. Utilizing customer knowledge in innovation: antecedents and impact of customer involvement on new product performance. Journal of the Academy of Marketing Science, 44(4), pp.516-538.

Daft, R.L. \& Lewin, A.Y., 1990. Can organization studies begin to break out of the normal science straitjacket? An editorial essay. Organization Science, 1(1), pp.1-9.

Dahlander, L. \& Gann, D.M., 2010. How open is innovation? Research Policy, 39(6), pp.699-709.

Dahlander, L. \& Wallin, M.W., 2006. A man on the inside: Unlocking communities as complementary assets. Research Policy, 35(8), pp.1243-1259.

Daniel, E.M., Ward, J.M. \& Franken, A., 2014. A dynamic capabilities perspective of IS project portfolio management. The Journal of Strategic Information Systems, 23(2), pp.95111.

Day, G.S., 1994. The capabilities of market-driven organizations. The Journal of Marketing, pp.37-52.

de Weerd-Nederhof, P.C., 2001. Qualitative case study research. The case of a Ph.D. research project on organising and managing new product development systems. Management Decision, 39(7), pp.513-538. 
Deeds, D.L., Decarolis, D. \& Coombs, J., 2000. Dynamic capabilities and new product development in high technology ventures. Journal of Business venturing, 15(3), pp.211229.

Denzin, N.K. \& Lincoln, Y.S., 1994. Handbook of qualitative research, Handbook of qualitative research.

Deszca, G., Munro, H. \& Noori, H., 1999. Developing breakthrough products: challenges and options for market assessment. Journal of Operations Management, 17(6), pp.613630 .

Diener, K. \& Piller, F.T., 2010. The Market for Open Innovation: Increasing the efficiency and effectiveness of the innovation process. RWTH Aachen University, Technology \& Innovation Management Group.

Dierickx, I. \& Cool, K., 1989. Asset Stock Accumulation and Sustainability of Competitive Advantage. Management Science, 35(12), pp.1504-1511.

Douthwaite, B., Keatinge, J. \& Park, J.R., 2001. Why promising technologies fail: the neglected role of user innovation during adoption. Research Policy, 30(5), pp.819-836.

Doz, Y.L. \& Hamel, G., 1998. Alliance advantage: The art of creating value through partnering. Harvard Business Press.

Dubois, L.E., Le Masson, P., Weil, B. \& Cohendet, P., 2014. From organizing for innovation to innovating for organization: how co-design fosters change in organizations. In AIMS 2014.

Dyer, J.H. \& Singh, H., 1998. The Relational View: Cooperative Strategy and Sources of Interorganizational Competitive Advantage. Academy of Management Review, 23(4), pp.660-679.

Easterby Smith, M., Lyles, M.A. \& Peteraf, M.A., 2009a. Dynamic Capabilities: Current Debates and Future Directions. British Journal of Management, 20(s1), pp.S1-S8.

Easton, G., 2002. Marketing: a critical realist approach. Journal of Business Research, 55(2), pp.103-109.

Easton, G., 2010. Critical realism in case study research. Industrial Marketing Management, 39(1), pp.118-128.

Edwards, P.K., O'Mahoney, J. \& Vincent, S. eds., 2014. Studying organizations using critical realism: A practical guide (Vol. 17). OUP Oxford.

Eisenhardt, K.M., 1989. Building theories from case study research. Academy of Management Review, 14(4), pp.532-550. 
Eisenhardt, K.M. \& Graebner, M.E., 2007. Theory building from cases: Opportunities and challenges. Academy of management journal, 50(1), pp.25-32.

Eisenhardt, K.M. \& Martin, J.A., 2000. Dynamic capabilities: what are they?. Strategic management journal, 21(10-11), pp.1105-1121.

Enkel, E., Kausch, C. \& Gassmann, O., 2005. Managing the risk of customer integration. European Management Journal, 23(2), pp.203-213.

Enkel, E., Perez Freije, J. \& Gassmann, O., 2005b. Minimizing market risks through customer integration in new product development: learning from bad practice. Creativity and innovation management, 14(4), pp.425-437.

Faems, D. et al., 2010. Technology Alliance Portfolios and Financial Performance: ValueEnhancing and Cost-Increasing Effects of Open Innovation. Journal of Product Innovation Management, 27(6), pp.785-796.

Fjeldstad, Ø.D. et al., 2012. The architecture of collaboration R. Gulati, P. Puranam, \& M. Tushman, eds. Strategic Management Journal, 33(6), pp.734-750.

Flyvbjerg, B., 2006. Five misunderstandings about case-study research. Qualitative inquiry, 12(2), pp.219-245.

Fortune 2017, Fortune Global 500, accessed 18 November 2017, $<$ http://fortune.com/global500/>.

Franke, N. \& Piller, F., 2004. Value creation by toolkits for user innovation and design: The case of the watch market. Journal of product innovation management, 21(6), pp.401415.

Frederich, M.J. \& Andrews, P., 2009a. Innovation passport: the IBM First-of-a-Kind (FOAK) journey from research to reality, Pearson Education.

Füller, J. \& Matzler, K., 2007. Virtual product experience and customer participation-A chance for customer-centred, really new products. Technovation, 27(6), pp.378-387.

Gales, L. \& Mansour-Cole, D., 1991. User Involvement in Innovation Projects: A Reassessment Using Information Processing. In Academy of Management Proceedings. Academy of Management, pp. 347-351.

Garud, R. and Nayyar, P.R., 1994. Transformative capacity: Continual structuring by intertemporal technology transfer. Strategic management journal, 15(5), pp.365-385.

Gassmann, O., Kausch, C. \& Enkel, E., 2010. Negative side effects of customer integration. International Journal of Technology Management, 50(1), pp.43-63. 
Gerring, J., 2004. What is a case study and what is it good for? American political science review, 98(02), pp.341-354.

Geertz, C., 1994. Thick description: Toward an interpretive theory of culture. Readings in the philosophy of social science, pp.213-231.

Gibbert, M. \& Ruigrok, W., 2010. The" what" and" how" of case study rigor: Three strategies based on published research. Organizational Research Methods.

Gibbert, M., Ruigrok, W. \& Wicki, B., 2008. What passes as a rigorous case study? Strategic Management Journal, 29(13), pp.1465-1474.

Gils, A.V. \& Zwart, P., 2004. Knowledge Acquisition and Learning in Dutch and Belgian SMEs:. European Management Journal, 22(6), pp.685-692.

Glaser, B. \& Strauss, A., 1967. Applying grounded theory. The discovery of grounded theory: strategies of qualitative research. Hawthorne, NY: Aldine Publishing Company, pp.237-51.

Grabher, G., Ibert, O. \& Flohr, S., 2008. The Neglected King: The Customer in the New Knowledge Ecology of Innovation. Economic Geography, 84(3), pp.253-280.

Grant, R.M., 1996a. Prospering in Dynamically-Competitive Environments: Organizational Capability as Knowledge Integration. Organization science, 7(4), pp.375387.

Grant, R.M., 1996b. Toward a knowledge-based theory of the firm R. M. Grant \& J. C. Spender, eds. Strategic Management Journal, 17(S2), pp.109-122.

Grant, R.M., 1997. The knowledge-based view of the firm : implications for management practice. Long range planning, 30(3), pp.450-454.

Grant, R.M. and Baden-Fuller, C., 2004. A knowledge accessing theory of strategic alliances. Journal of management studies, 41(1), pp.61-84.

Grönroos, C., 2013. Service logic revisited: who creates value? And who co-creates? G. Svensson, ed. European business review, 20(4), pp.298-314.

Guba, E.G., \& Lincoln, Y.S., 2005. Paradigmatic controversies, contradictions, and emerging confluences.

Hamel, G. \& Prahalad, C.K., 1994. Competing for the future: Breakthrough strategies for seizing control of your industry and creating the markets of tomorrow (Harvard business school press). Cambridge, MA. 
Hansen, M.T., 1999. The search-transfer problem: The role of weak ties in sharing knowledge across organization subunits. Administrative science quarterly, 44(1), pp.82111.

Healy, M. \& Perry, C., 2000. Comprehensive criteria to judge validity and reliability of qualitative research within the realism paradigm. Qualitative Market Research: An International Journal, 3(3), pp.118-126.

Heide, J.B., 1994. Interorganizational governance in marketing channels. The Journal of Marketing, pp.71-85.

Helal, R.A., 2016. Knowledge Acquisition Methods and the Impact on Producer-Driven Collaborative Innovation Performance, unpublished presentation at: Research in Management: National Capital Forum, May 4, 2016, Ottawa, Canada

Helfat, C.E. \& Peteraf, M.A., 2009. Understanding dynamic capabilities: progress along a developmental path. Strategic Organization, 7(1), pp.91-102..

Helfat, C.E., 1997. Know-how and Asset Complementarity and Dynamic Capability Accumulation: The Case of R\&D. In Strategic Management Journal.

Helfat, C.E. \& Winter, S.G., 2011. Untangling Dynamic and Operational Capabilities: Strategy for the (N)ever-Changing World. Strategic Management Journal, 32(11), pp.1243-1250.

Hoyer, W.D. et al., 2010. Consumer cocreation in new product development. Journal of Service Research, 13(3), pp.283-296.

Huizingh, E.K.R.E., 2011. Open innovation: State of the art and future perspectives. Technovation, 31(1), pp.2-9.

IBM, 2016. 2015 IBM Annual Report. pp.1-156., accessed 18 November 2017, https://www.ibm.com/annualreport/2015/bin/assets/IBM-Annual-Report-2015.pdf>

IBM, IBM Acquisitions. http://www.ibm.com/cloud-computing/bluemix/. Available at: https://www.ibm.com/investor/strategy/acquisitions.html [Accessed June 15, 2016].

IDC, 2013. Worldwide Business Analytics Technology and Services 2013-2017 Forecast.

Jacobides, M.G. \& Billinger, S., 2006. Designing the boundaries of the firm : From "make, buy, or ally" to the dynamic benefits of vertical architecture. Organization science, 17(2), pp.249-261.

Jaworski, B., Kohli, A.K. \& Sahay, A., 2000. Market-driven versus driving markets. Journal of the Academy of Marketing Science, 28(1), pp.45-54. 
Jaworski, B.J. \& Kohli, A.K., 1996. Market orientation: review, refinement, and roadmap. Journal of Market-Focused Management, 1, pp.119-135.

Jiang, R.J., Tao, Q.T. \& Santoro, M.D., 2010. Alliance portfolio diversity and firm performance. Strategic Management Journal, 31(10), pp.1136-1144.

Joshi, A.W. \& Stump, R.L., 1999. Transaction cost analysis: Integration of recent refinements and an empirical test. Journal of Business-to-Business Marketing, 5(4), pp.3771.

Kang, K.H. \& Kang, J., 2009. How do firms source external knowledge for innovation? Analysing effects of different knowledge sourcing methods. International Journal of Innovation Management, 13(01), pp.1-17.

Karlsson, J.C. \& Ackroyd, S., 2014. Critical Realism, Research Techniques, and Research Designs. pp.21-45.

Karlsson, C. and Åhlstrom, P., 1997. Perspective: Changing product development strategy - A managerial challenge. Journal of Product Innovation Management, 14(6), pp.473-484.

Katz, R. \& Allen, T.J., 1982. Investigating the Not Invented Here (NIH) syndrome: A look at the performance, tenure, and communication patterns of $50 \mathrm{R} \& \mathrm{D}$ Project Groups. $R \& D$ Management, 12(1), pp.7-20.

Keil, T. et al., 2008. The effect of governance modes and relatedness of external business development activities on innovative performance. Strategic Management Journal, 29(8), pp.895-907.

Khilji, S.E., Mroczkowski, T. and Bernstein, B., 2006. From invention to innovation: toward developing an integrated innovation model for biotech firms. Journal of product innovation management, 23(6), pp.528-540.

Kim, L., 1998. Crisis Construction and Organizational Learning: Capability Building in Catching-up at Hyundai Motor. Organization science, 9(4), pp.506-521.

King, A.A. \& Tucci, C.L., 2002. Incumbent Entry into New Market Niches: The Role of Experience and Managerial Choice in the Creation of Dynamic Capabilities. Management Science, 48(2), pp.171-186.

Kogut, B. \& Zander, U., 1992. Knowledge of the Firm, Combinative Capabilities, and the Replication of Technology. Organization science, 3(3), pp.383-397.

Kohli, A.K. \& Jaworski, B.J., 1990. Market orientation: the construct, research propositions, and managerial implications. The Journal of Marketing, pp.1-18. 
Kristensson, P., Gustafsson, A. \& Archer, T., 2004. Harnessing the creative potential among users*. Journal of Product Innovation Management, 21(1), pp.4-14.

Kristensson, P., Matthing, J. \& Johansson, N., 2008. Key strategies for the successful involvement of customers in the co-creation of new technology-based services. International Journal of Service Industry Management, 19(4), pp.474-491.

Kumar, R., 2014. Research Methodology; A Step-by-Step Guide for Beginners, 4th Edition, SAGE Publications Ltd.

Lane, P.J., Koka, B.R. \& Pathak, S., 2006. The Reification of Absorptive Capacity: A Critical Review and Rejuvenation of the Construct. Academy of Management Review, 31(4), pp.833-863.

Laursen, K. \& Salter, A., 2006. Open for innovation: the role of openness in explaining innovation performance among UK manufacturing firms. Strategic Management Journal, 27(2), pp.131-150.

Layder, D., 1993. New strategies in social research: An introduction and guide. Polity Press.

Learned, E.P. et al., 1969. Business Policy: Text and Cases (Homewood, Illinois), Richard D. Irwin,

Leonard, D., 2002. The limitations of listening. Harvard Business Review, 80(1), p.93.

Leonard, D. \& Rayport, J.F., 1997. Spark innovation through empathic design. Harvard Business Review, 75, pp.102-115.

Leonard, D. \& Sensiper, S., 1998. The role of tacit knowledge in group innovation. California management review, 40(3), pp.112-132.

Leonard-Barton, D., 1990. A Dual Methodology for Case Studies: Synergistic Use of a Longitudinal Single Site with Replicated Multiple Sites. Organization science, 1(3), pp.248-266.

Leonard-Barton, D., 1992. Core capabilities and core rigidities: A paradox in managing new product development. Strategic Management Journal, 13(S1), pp.111-125.

Levinthal, D.A. \& March, J.G., 1993. The myopia of learning. Strategic Management Journal, 14(S2), pp.95-112.

Lichtenthaler, U., 2008. Open Innovation in Practice: An Analysis of Strategic Approaches to Technology Transactions. Engineering Management, IEEE Transactions on, 55(1), pp.148-157. 
Lichtenthaler, U. \& Lichtenthaler, E., 2009. A Capability-Based Framework for Open Innovation: Complementing Absorptive Capacity. Journal of Management Studies, 46(8), pp.1315-1338.

Lilien, G.L. et al., 2002. Performance Assessment of the Lead User Idea-Generation Process for New Product Development. Management Science, 48(8), pp.1042-1059.

Lin, Y. \& Wu, L.-Y., 2014. Exploring the role of dynamic capabilities in firm performance under the resource-based view framework. Journal of Business Research, 67(3), pp.407413.

Luhmann, N. (1995). Social Systems. Stanford, CA: Stanford University Press.

Lusch, R.F., Brown, S.W. \& Brunswick, G.J., 1992. A General Framework for Explaining Internal vs. External Exchange. Journal of the Academy of Marketing Science, 20(2), pp.119-134.

Luthje, C., Herstatt, C. \& von Hippel, E., 2002. The dominant role of "local" information in user innovation: The case of mountain biking.

Macdonald, E.K. et al., 2011. Assessing value-in-use: A conceptual framework and exploratory study. Industrial Marketing Management, 40(5), pp.671-682.

Macpherson, A., Jones, O. \& Zhang, M., 2004. Evolution or revolution? Dynamic capabilities in a knowledge-dependent firm. $R \& d$ Management, 34(2), pp.161-177.

Maklan, S., Knox, S. \& Ryals, L., 2008. New trends in innovation and customer relationship management: a challenge for market researchers. International Journal of Market Research, 50(2), p.240.

Marsh, S.J. \& Stock, G.N., 2003. Building Dynamic Capabilities in New Product Development through Intertemporal Integration. Journal of Product Innovation Management, 20(2), pp.136-148.

Maxwell, J.A., 2012. Qualitative Research Design: An Interactive Approach (Vol. 41). SAGE Publications.

Miles, M.B. \& Huberman, A.M., 1994. Qualitative data analysis. 1994. Beverly Hills: Sage Google Scholar.

Miles, M.B., Huberman, A.M. \& Saldaña, J., 2013. Qualitative Data Analysis, SAGE Publications, Incorporated.

Mingers, J., 2006. A critique of statistical modelling in management science from a critical realist perspective: its role within multimethodology. Journal of the Operational Research Society, 57(2), pp.202-219. 
Mody, A., 1993. Learning through alliances. Journal of economic behaviour \& organization, 20(2), pp.151-170.

Molina-Azorín, J.F., 2014. Microfoundations of strategic management: Toward micromacro research in the resource-based theory. BRQ Business Research Quarterly, 17(2), pp.102-114.

Montoya-Weiss, M.M. \& Calantone, R., 1994. Determinants of new product performance: A review and meta-analysis. Journal of Product Innovation Management, 11(5), pp.397417.

Moore, G., 2002. Crossing the chasm: Marketing and selling disruptive products to mainstream business customers., Harper Collins

Morton, P., 2006. Using critical realism to explain strategic information systems planning. JITTA: Journal of Information Technology Theory and Application, 8(1), p.1.

Murphy, S.A. \& Kumar, V., 1996. The role of predevelopment activities and firm attributes in new product success. Technovation, 16(8), pp.431-449.

Myers, M.D. \& Avison, D., 1997. Qualitative research in information systems. MIS quarterly, 21, pp.241-242.

Nambisan, S. \& Baron, R.A., 2007. Interactions in virtual customer environments: Implications for product support and customer relationship management. Journal of interactive marketing, 21(2), pp.42-62.

Nesheim, T., 2001. Externalization of the core: antecedents of collaborative relationships with suppliers. European Journal of Purchasing \& Supply Management, 7(4), pp.217-225.

Newey, L., 2010. Wearing different hats: How absorptive capacity differs in open innovation. International Journal of Innovation Management, 14(04), pp.703-731.

Nielsen, A.P., 2013. Understanding dynamic capabilities through knowledge management. Journal of Knowledge Management, 10(4), pp.59-71.

Nitzche, P., Wirtz, B.W. \& Gottel, V., 2016. Innovation Success in the Context of Inbound Open Innovation. International Journal of Innovation Management, 20(02), p.1650025.

Nonaka, I., 1994. A dynamic theory of organizational knowledge creation. Organization science, 5(1), pp.14-37.

Nonaka, I. \& Takeuchi, H., 1995. The knowledge-creating company: How Japanese companies create the dynamics of innovation. Oxford university press. 
Noor, K.B., 2008. Case study: A strategic research methodology. American journal of applied sciences, 5(11), pp.1602-1604.

O'Dell, C. \& Grayson, C.J., 1998. If Only We Knew What We Know: Identification and Transfer of Internal Best Practices. California Management Review, 40(3), pp.154-174.

Ogawa, S. \& Piller, F.T., 2006. Reducing the risks of new product development. MIT Sloan management review, 47(2), p.65.

O'Hern, M.S. \& Rindfleisch, A., 2010. Customer co-creation: a typology and research agenda. Review of marketing research, 6, pp.84-106.

Olson, E.L. \& Bakke, G., 2001. Implementing the lead user method in a high technology firm : A longitudinal study of intentions versus actions. Journal of Product Innovation Management, 18(6), pp.388-395.

Ong, B.K., 2012. Grounded Theory Method (GTM) and the Abductive Research Strategy (ARS): a critical analysis of their differences. International Journal of Social Research Methodology, 15(5), pp.417-432.

Orlikowski, W.J. \& Baroudi, J.J., 1991. Studying information technology in organizations: Research approaches and assumptions. Information systems research, 2(1), pp.1-28.

Otto, P., 2012. Dynamics in strategic alliances: A theory on interorganizational learning and knowledge development. International Journal of Information Technologies and Systems Approach (IJITSA), 5(1), pp.74-86.

Owen, L., Goldwasser, C. \& Choate, K., 2008. Collaborative innovation throughout the extended enterprise. Strategy \& ..., 36(1), pp.39-45.

Pals, N. et al., 2008. Three approaches to take the user perspective into account during new product design. International Journal of Innovation Management, 12(03), pp.275-294.

Patton, M.Q., 2002. Two decades of developments in qualitative inquiry a personal, experiential perspective. Qualitative Social Work, 1(3), pp.261-283.

Pawson, R. and Tilley, N., 1997. Realistic evaluation. Sage.

Pawson, R. \& Tilley, N., 2009. Realist Evaluation.

Payne, A.F., Storbacka, K. \& Frow, P., 2008. Managing the co-creation of value. Journal of the Academy of Marketing Science, 36(1), pp.83-96.

Peirce, C.S. \& Turrisi, P.A., 1997. Pragmatism as a Principle and Method of Right Thinking, SUNY Press. 
Penrose, E., 1959. The theory of the firm. NY: John Wiley \& Sons.

Petroni, A., 1998. The analysis of dynamic capabilities in a competence-oriented organization. Technovation, 18(3), pp.179-189.

Piller, F.T., Ihl, C. \& Vossen, A., 2011. A Typology of Customer Co-Creation in the Innovation Process. SSRN Working Paper Series.Available at SSRN 1732127.

Pisano, G.P., 2000. In search of dynamic capabilities: the origins of R\&D competence in biopharmaceuticals (pp. 129-154). Oxford University Press, Oxford, New York.

Pisano, G.P. \& Verganti, R., 2008. Which kind of collaboration is right for you. Harvard Business Review, 86(12), pp.78-86.

Ponterotto, J.G., 2006. Brief note on the origins, evolution, and meaning of the qualitative research concept thick description. The Qualitative Report, 11(3), pp.538-549.

Ponterotto, J.G. and Grieger, I., 2007. Effectively communicating qualitative research. The Counseling Psychologist, 35(3), pp.404-430.

Porter, M.E., 1980. Competitive strategy: Techniques for analyzing industries and companies. New York.

Porter, M.E., 1985. Competitive advantage: creating and sustaining superior performance. 1985. New York: FreePress.

Powell, W.W., Koput, K.W. \& Smith-Doerr, L., 1996. Interorganizational Collaboration and the Locus of Innovation: Networks of Learning in Biotechnology. Administrative science quarterly, 41(1), p.116.

Prahalad, C.K. \& Ramaswamy, V., 2004a. Co-creating unique value with customers. Strategy \& Leadership, 32(3), pp.4-9.

Prahalad, C.K. \& Ramaswamy, V., 2004b. Co-creation experiences: The next practice in value creation. Journal of interactive marketing, 18(3), pp.5-14.

Prieto, I.M., Revilla, E. \& Rodríguez-Prado, B., 2009. Building dynamic capabilities in product development: How do contextual antecedents matter? Scandinavian Journal of Management, 25(3), pp.313-326.

Quinn, J.B., 2000. Outsourcing Innovation: The New Engine of Growth. Sloan Management Review, 41(4), pp.13-28.

Ramakrishnan, R. et al., 2004. Mass collaboration: a case study [customer support system]. Database Engineering and Applications Symposium, 2004. IDEAS'04. Proceedings. International, pp.133-146. 
Reed, M.I., 2009. Critical realism in critical management studies,

Rigby, D. \& Zook, C., 2002. Open-market innovation. Harvard Business Review, 80(10), pp.80-93.

Rindfleisch, A. \& Heide, J.B., 1997. Transaction cost analysis: past, present, and future applications. The Journal of Marketing, pp.30-54.

Robertson, P.L., Casali, G.L. \& Jacobson, D., 2012. Managing open incremental process innovation: Absorptive Capacity and distributed learning. Research Policy, 41(5), pp.822832.

Roger, E.M., 1995. Diffusion of innovations, New York: Free Press.

Rohrbeck, R., Hölzle, K. \& Gemünden, H.G., 2009. Opening up for competitive advantage-How Deutsche Telekom creates an open innovation ecosystem. $R \& D$ Management, 39(4), pp.420-430.

Rosenberg, N., 1982. Inside the black box: technology and economics. Cambridge University Press.

Roser, T., DeFillippi, R. \& Samson, A., 2013. Managing your co-creation mix: co-creation ventures in distinctive contexts. European Business Review, 25(1), pp.20-41.

Roser, T., Samson, A., Humphreys, P. \& Cruz-Valdivieso, E., 2009a. Co-creation: new pathways to value: an overview, Promise \& LSE Enterprise.

Rothwell, R., 1977. The characteristics of successful innovators and technically progressive firms (with some comments on innovation research). $R \& D$ Management, $7(3)$, pp.191-206.

Rothwell, R. \& Dodgson, M., 1991. External linkages and innovation in small and mediumsized enterprises. $R \& D$ Management, 21(2), pp.125-138.

Saldana, J., 2015. The Coding Manual for Qualitative Researchers. SAGE.

Sanders, E.B.-N., 2002. From user-centred to participatory design approaches. Design and the social sciences: Making connections, pp.1-8.

Sapienza, H.J., Parhankangas, A. \& Autio, E., 2004. Knowledge relatedness and post-spinoff growth. Journal of Business venturing, 19(6), pp.809-829.

Sawhney, M., Verona, G. \& Prandelli, E., 2005. Collaborating to create: The Internet as a platform for customer engagement in product innovation. Journal of interactive marketing, 19(4), pp.4-17. 
Sayer, A., 1997. Critical Realism and the Limits to Critical Social Science. Journal for the Theory of Social Behaviour, 27(4), pp.473-488.

Sayer, A., 2010. Method in Social Science: Revised 2nd Edition. Routledge.

Sánchez-González, G., González-Álvarez, N. \& Nieto, M., 2009. Sticky information and heterogeneous needs as determining factors of R\&D cooperation with customers. Research Policy, 38(10), pp.1590-1603.

Schleimer, S.C. \& Faems, D., 2016. Connecting Interfirm and Intrafirm Collaboration in NPD Projects: Does Innovation Context Matter?. Journal of Product Innovation Management, 33(2), pp.154-165.

Schreier, M. \& Prügl, R., 2008. Extending Lead-User Theory: Antecedents and Consequences of Consumers' Lead Userness*. Journal of Product Innovation Management, 25(4), pp.331-346.

Schreier, M., Oberhauser, S. \& Prügl, R., 2007. Lead users and the adoption and diffusion of new products: Insights from two extreme sports communities. Marketing Letters, 18(12), pp.15-30.

Seawright, J. \& Gerring, J., 2008. Case selection techniques in case study research a menu of qualitative and quantitative options. Political Research Quarterly, 61(2), pp.294-308.

Selden, L. \& MacMillan, I.C., 2006. Manage customer-centric innovation-systematically. Harvard Business Review, 84(4), p.108.

Shane, S., 2000. Prior knowledge and the discovery of entrepreneurial opportunities. Organization science, 11(4), pp.448-469.

Sheth, J.N. \& Uslay, C., 2007. Implications of the revised definition of marketing: from exchange to value creation. Journal of Public Policy \& Marketing, 26(2), pp.302-307.

Smith, K.G., Collins, C.J. and Clark, K.D., 2005. Existing knowledge, knowledge creation capability, and the rate of new product introduction in high-technology firms. Academy of management journal, 48(2), pp.346-357.

Smith, M.L., 2006. Overcoming theory-practice inconsistencies: Critical realism and information systems research. Information and organization, 16(3), pp.191-211.

Sofianti, T.D., Suryadi, K., Govindaraju, R. \& Prihartono, B., 2010. Customer knowledge co-creation process in new product development. In Proceedings of the World Congress on Engineering (Vol. 1).

Stake, R.E., 2013. Multiple case study analysis. Guilford Press. 
Strauss, A. \& Corbin, J., 1994. Grounded theory methodology. Handbook of qualitative research, pp.273-285.

Stuart, T.E., 2000. Interorganizational alliances and the performance of firms: a study of growth and innovation rates in a high-technology industry. Strategic Management Journal, 21(8), pp.791-811.

Swink, M., 2006. Building collaborative innovation capability. Research technology management, 49(2), pp.37-47.

Szulanski, G., 1996. Exploring internal stickiness: Impediments to the transfer of best practice within the firm. Strategic Management Journal, 17, pp.27-43.

Tavory, I. \& Timmermans, S., 2014. Abductive Analysis: Theorizing Qualitative Research. University of Chicago Press.

Teece, D.J., 1980. Economies of scope and the scope of the enterprise. Journal of economic behaviour \& organization, 1(3), pp.223-247.

Teece, D.J., 1982. Towards an economic theory of the multiproduct firm. Journal of economic behaviour \& organization, 3(1), pp.39-63.

Teece, D.J., 1986. Profiting from technological innovation: Implications for integration, collaboration, licensing and public policy. Research Policy, 15(6), pp.285-305.

Teece, D.J., 1998. Capturing Value from Knowledge Assets: The New Economy, Markets for Know-How, and Intangible Assets. California Management Review, 40(3), pp.55-79.

Teece, D.J., 2000. Strategies for Managing Knowledge Assets: the Role of Firm Structure and Industrial Context. Long Range Planning, 33(1), pp.35-54.

Teece, D.J., 2007. Explicating dynamic capabilities: the nature and microfoundations of (sustainable) enterprise performance. Strategic Management Journal, 28(13), pp.13191350.

Teece, D.J. \& Pisano, G., 1994. The dynamic capabilities of firms: an introduction. Industrial and Corporate Change, 3(3), pp.537-556.

Teece, D.J., Pisano, G. \& Shuen, A., 1997. Dynamic Capabilities and Strategic Management. Strategic Management Journal, 18(7), pp.509-533.

Thomke, S. \& Hippel, Von, E., Customers as Innovators: A new way to create valueHarvard Business Review (April 2005), 74-81.

Trott, P. \& Hartmann, D., 2009. Why"open innovation"is old wine in new bottles. International Journal of Innovation Management, 13(04), pp.715-736. 
Tsoukas, H., 1996. The firm as a distributed knowledge system: A constructionist approach

R. M. Grant \& J. C. Spender, eds. Strategic Management Journal, 17(S2), pp.11-25.

Un, C.A., Cuervo Cazurra, A. \& Asakawa, K., 2010. R\&D Collaborations and Product Innovation*. Journal of Product Innovation Management, 27(5), pp.673-689.

Urban, G.L. \& Hippel, Von, E., 1988. Lead user analyses for the development of new industrial products. Management Science, 34(5), pp.569-582.

Van der Meer, H., 2007. Open innovation-the Dutch treat: challenges in thinking in business models. Creativity and innovation management, 16(2), pp.192-202.

Vanhaverbeke, W., Van de Vrande, V. \& Cloodt, M., 2008. Connecting absorptive capacity and open innovation. Available at SSRN 1091265.

Vargo, S.L. \& Lusch, R.F., 2004. Evolving to a new dominant logic for marketing. The Journal of Marketing, pp.1-17.

Vega-Jurado, J., Gutiérrez-Gracia, A. \& Fernández-de-Lucio, I., 2009. Does external knowledge sourcing matter for innovation? Evidence from the Spanish manufacturing industry. Industrial and corporate change, 18(4), pp.637-670.

Volkoff, O., Strong, D.M. \& Elmes, M.B., 2007. Technological Embeddedness and Organizational Change. Organization science, 18(5), pp.832-848.

von Hippel, E., 1978. A customer-active paradigm for industrial product idea generation. Research Policy, 7(3), pp.240-266.

von Hippel, E., 1986. Lead Users: A Source of Novel Product Concepts. Management Science, 32(7), pp.791-805.

von Hippel, E., 1976. The dominant role of users in the scientific instrument innovation process. Research Policy, 5(3), pp.212-239.

von Hippel, E., 1994. "Sticky information" and the locus of problem solving: implications for innovation. Management Science, 40(4), pp.429-439.

von Hippel, E. \& Katz, R., 2002. Shifting innovation to users via toolkits. Management Science, 48(7), pp.821-833.

Voss, C., Tsikriktsis, N. and Frohlich, M., 2002. Case research in operations management. International journal of operations \& production management, 22(2), pp.195-219. 
Voss, C.A., 1985. The role of users in the development of applications software. Journal of Product Innovation Management, 2(2), pp.113-121.

Walsham, G., 2006. Doing interpretive research. European Journal of information systems, 15(3), pp.320-330.

Wang, C.L. \& Ahmed, P.K., 2007. Dynamic capabilities: A review and research agenda. International Journal of Management Reviews, 9(1), pp.31-51.

Wang, C.L. \& Ahmed, P.K., 2004. Leveraging knowledge in the innovation and learning process at GKN. International Journal of Technology Management, 27(6-7), pp.674-688.

Wayne Gould, R., 2012. Open innovation and stakeholder engagement. Journal of technology management \& innovation, 7(3), pp.1-11.

West, J. \& Bogers, M., 2014. Leveraging external sources of innovation: a review of research on open innovation. Journal of Product Innovation Management, 31(4), pp.814831.

Williams, C.K. \& Karahanna, E., 2013. Causal explanation in the coordinating process: a critical realist case study of federated IT governance structures. MIS Quarterly, 37(3), pp.933-964.

Williamson, O.E., 1981. The Economics of Organization: The Transaction Cost Approachl. American Journal of Sociology, 87(3), pp.548-577.

Williamson, O.E., 1989. Transaction cost economics. Handbook of Industrial Organization, 1, pp.135-182.

Winter, S.G., 2000. The Satisficing Principle in Capability Learning. Strategic Management Journal, 21(10-11), pp.981-996.

Winter, S.G., 2003. Understanding dynamic capabilities. Strategic Management Journal.

Witell, L. et al., 2011. Idea generation: customer co-creation versus traditional market research techniques. Journal of Service Management, 22(2), pp.140-159.

Wu, L.-Y., 2007. Entrepreneurial resources, dynamic capabilities and start-up performance of Taiwan's high-tech firms. Journal of Business Research, 60(5), pp.549-555.

Wynn Jr, D.E. \& Williams, C.K., 2008. Critical realm-based explanatory case study research in information systems. ICIS 2008 Proceedings, p.202.

Wynn Jr, D.E. \& Williams, C.K., 2012. Principles for conducting critical realist case study research in information systems. MIS Quarterly, 36(3), pp.787-810. 
Yin, R.K., 1981. The case study crisis: Some answers. Administrative science quarterly, pp.58-65.

Yin, R.K., 1989. Case study research: Design and methods, revised edition. Applied Social Research Methods Series, 5.

Yin, R.K., 1994. Discovering the future of the case study method in evaluation research. Evaluation practice, 15(3), pp.283-290.

Yin, R.K., 1999. Enhancing the quality of case studies in health services research. Health services research, 34(5 Pt 2), p.1209.

Yin, R.K., 2013. Case study research: Design and methods. Sage publications.

Yurdusev, A.N., 1993. 'Level of Analysis' and "Unit of Analysis": A Case for Distinction. Millennium - Journal of International Studies, 22(1), pp.77-88.

Zahra, S.A. \& George, G., 2002. Absorptive Capacity: A Review, Reconceptualization, and Extension. Academy of Management Review, 27(2), pp.185-203.

Zahra, S.A., Sapienza, H.J. \& Davidsson, P., 2006. Entrepreneurship and Dynamic Capabilities: A Review, Model and Research Agenda*. Journal of Management Studies, 43(4), pp.917-955.

Zhou, K.Z.\& Li, C.B., 2012. How knowledge affects radical innovation: Knowledge base, market knowledge acquisition, and internal knowledge sharing. Strategic Management Journal, 33(9), pp.1090-1102.

Zogaj, S. \& Bretschneider, U., 2012. Customer integration in new product development: a literature review concerning the appropriateness of different customer integration methods to attain customer knowledge. SSRN Electronic Journal (SSRN 2485240).

Zollo, M., Reuer, J.J. \& Singh, H., 2002. Interorganizational Routines and Performance in Strategic Alliances. Organization science, 13(6), pp.701-713.

Zollo, M. \& Winter, S.G., 2002. Deliberate Learning and the Evolution of Dynamic Capabilities. Organization science, 13(3), pp.339-351. 


\section{APPENDICES}

\section{Appendix 4.1}

Comparison of Critical Realist, Positivist and Interpretivist Philosophical Paradigms

\section{Appendix 5.1}

Semi-Structured Interview Guides

\section{Appendix 5.2}

Informant Recruitment Communication

\section{Appendix 5.3}

Pre-Interview Informant Consent Form

\section{Appendix 6.1}

FoaK Board Membership Profile (between 2010 and 2014) 


\section{Appendix 4.1}

\section{Comparison of Critical Realist, Positivist and Interpretivist Philosophical Paradigms}

\begin{tabular}{|l|l|l|l|}
\cline { 2 - 4 } \multicolumn{1}{|c|}{ Positivism } & \multicolumn{1}{c|}{ Interpretivism } & \multicolumn{1}{c|}{ Critical Realism } \\
\hline Ontology & $\begin{array}{l}\text { Objective reality, } \\
\text { unproblematically apprehended. }\end{array}$ & $\begin{array}{l}\text { Reality socially constructed by } \\
\text { humans via subjective meanings, } \\
\text { symbolic action, and social } \\
\text { politics. }\end{array}$ & $\begin{array}{l}\text { Objective, stratified reality } \\
\text { (consisting of structures, } \\
\text { mechanisms and events), but } \\
\text { imperfectly and fallibly } \\
\text { apprehended. }\end{array}$ \\
\hline Epistemology & $\begin{array}{l}\text { Empirical testing and verification } \\
\text { of predictive theories via } \\
\text { hypothetical- deductive methods; } \\
\text { findings assumed true until } \\
\text { falsified. }\end{array}$ & $\begin{array}{l}\text { Knowledge generated by } \\
\text { understanding the meanings and } \\
\text { actions or subjects; findings } \\
\text { "created" by researcher based on } \\
\text { this understanding. }\end{array}$ & $\begin{array}{l}\text { Knowledge retroduced to } \\
\text { theories regarding underlying } \\
\text { reality which explain observable } \\
\text { events; findings probably true, } \\
\text { but mediated by humans. }\end{array}$ \\
\hline Methodology & $\begin{array}{l}\text { Researcher assumed to be } \\
\text { unbiased (value-free); } \\
\text { generalizability to target } \\
\text { population; primarily quantitative } \\
\text { methods, such as surveys, } \\
\text { experiments. }\end{array}$ & $\begin{array}{l}\text { Researcher's biases part of study } \\
\text { (value-laden); primarily } \\
\text { qualitative methods such as } \\
\text { ethnographies based on } \\
\text { hermeneutics, dialectics. }\end{array}$ & $\begin{array}{l}\text { Researcher controls for bias via } \\
\text { triangulation (value-aware); } \\
\text { multiple methods including case } \\
\text { studies (for idiographic studies) } \\
\text { and surveys or statistical } \\
\text { methods. }\end{array}$ \\
\hline
\end{tabular}

Source: Wynn and Williams 2008 


\section{APPENDIX 5.1 SEMI-STRUCTURED INTERVIEW GUIDES}

Interviewee:

Role:

Date: Time: Method: Duration (mins):

\section{INTERVIEW GUIDE 1: INITIATIVE MEMBER (FULL) [Version 4.0]}

\section{SECTION 1 - ORAL CONSENT SCRIPT / INTERVIEW PREAMBLE}

- THANKS: Thanks again for making the time

Some formality on protocol that need to cover before we begin:

- MY GOAL I am working towards completion of my Ph.D. in Management at Carleton University, under the supervision of Dr. Michel Rod. My area of focus is on open and collaborative innovation models.

- TOPIC Contributes to my doctoral thesis; examining FoaK as a collaborative method of innovation creation.

- DURATION Scheduled for 60 minutes; schedule if need more time; pending your availability

- APPROACH:

I have some questions for today's conversation - explore your experience with FoaK program

My goal to have a rich conversation about your experience; explore areas of interest as they come up

- AUDIO-RECORDING With your consent, the interview will be audio-recorded;

- ANONYMOUS Feedback will be kept anonymous in the transcript, and the final analysis will be at an aggregated level; study is being stored securely / cloud repository that will be password protected / only I will have access to the repository, with files being shared with my thesis supervisor as needed.

- RETAIN CONTENT Once the project is completed, all research data will be retained as I may use it for other follow-on research projects on this same topic. Once no longer needed - will be destroyed.

- WITHDRAW You may withdraw at any time, up to December 31, 20016 by letting me know. If you choose to no longer participate, all the information you provided will be destroyed. 


\section{APPENDIX 5.1 SEMI-STRUCTURED INTERVIEW GUIDES (CONT'D)}

\section{SECTION 2 - GENERAL VIEWS ON FOAK AS KNOWLEDGE ACQUISITION}

1. Please describe your current role and background with IBM? Your experience with FoaK program/project.

2. In general, please describe your FoaK project:

- What was the motivation/the objective of FoaK projects?

- Describe your role?

- A brief description of the initiative? Was it successful? Why?

3. There are multiple roads to new innovation, why do you believe the FoaK program chosen for initiatives?

4. What do you believe is the primary role of A Foak program? Initiative level.

5. How does the FoaK program set-up initiatives for success?

6. What is the role of A FoaK program in innovation quality to the company?

7. From your perspective, what does success look like? How is performance measured for a Foak program?

\section{SECTION 3 - STAGES IN THE FOAK PROCESS: PROCESS DETAILS}

8. Please provide an overview of your FoaK initiative? Each stage ....

Probe: . Link to strategy

- Team- Program/initiative skills

- Innovation/invention - already developed invention?

- Client relationship

- Catching/commercialization organization

- Project steps

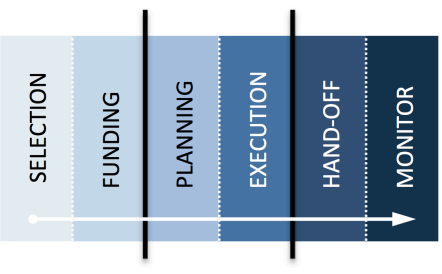

- Role of FoaK program office; integration role

- Role of Foak board

9. Describe what went well on the initiative?

10. Describe what went poorly on the initiative?

11. What dependencies? 


\section{APPENDIX 5.1 SEMI-STRUCTURED INTERVIEW GUIDES (CONT'D)}

\section{SECTION 4 - POSITION ON PERFORMANCE (Overall)}

1. Describe overall initiative performance? Why?

2. What elements of a project, program, the environment had the greatest positive/negative impact on the performance of an initiative? Why?

3. To what degree does the FoaK program impact performance of an initiative? How?

4. What do you believe are the key success factors when using FoaK as a method to innovate through collaboration with clients? Any key dependencies?

5. What role does previous experience play for FoaK decisions and projects? How is it gathered?

6. What could have been done differently to improve performance of the initiative?

\section{SECTION 4 - SUMMARY ON FOAK PROGRAM}

7. If advising another organization interested in developing a FoaK method, what are the critical elements need in a FoaK program in creating new innovation? Why?

- PROBE: What is the importance of the following elements ...

- As program: having FoaK as a program/ versus ad hoc projects

- Integrator: FoaK as an integrator/orchestrator of initiatives

- Client Access: client access and engagement and relationship

- Skills in Exploring: firm skills in exploring sources of new knowledge (program and team)

- Convert: firm's ability to convert new knowledge acquired to usable in offerings

- Resourcing: firm's ability to access and mobilize resources/experience as needed

- Innovate: firm's ability to innovate (commercialize the assets from FoaK projects)

- Invent: firm's ability to invent (build our own),

- Knowledge Base: role of knowledge and experience base: past and adding new

8. What do you believe the FoaK program does well? Some of the main challenges?

9. Which elements could be changed or added to the program to improve performance?

10. Any other comments on FoaK program and impact on initiatives?

Q. May I come back with more questions for clarification if needed?

Thank you! 


\section{APPENDIX 5.1 SEMI-STRUCTURED INTERVIEW GUIDES (CONT'D)}

Interviewee:

Role:

Date: Time: Method: Duration (mins):

\section{INTERVIEW GUIDE 2: INITIATIVE LEVEL - PROGRAM MANAGEMENT [Version 3.3]}

\section{SECTION 1 - ORAL CONSENT SCRIPT / INTERVIEW PREAMBLE}

- THANKS: Thanks again for making the time

Some formality on protocol that need to cover before we begin:

- MYGOAL I am working towards completion of my Ph.D. in Management at Carleton University, under the supervision of Dr. Michel Rod. My area of focus is on open and collaborative innovation models.

- TOPIC Contributes to my doctoral thesis; examining FoaK as a collaborative method of innovation creation.

- DURATION Scheduled for 60 minutes; schedule if need more time; pending your availability

- APPROACH: I have some questions for today's conversation - explore your experience with FoaK program

My goal to have a rich conversation about your experience; explore areas of interest as they come up

- AUDIO-RECORDING With your consent, the interview will be audio-recorded;

- ANONYMOUS Feedback will be kept anonymous in the transcript, and the final analysis will be at an aggregated level; study is being stored securely / cloud repository that will be password protected / only I will have access to the repository, with files being shared with my thesis supervisor as needed.

- RETAIN CONTENT Once the project is completed, all research data will be retained as I may use it for other follow-on research projects on this same topic. Once no longer needed - will be destroyed.

- WITHDRAW You may withdraw at any time, up to December 31, 20016 by letting me know. If you choose to no longer participate, all the information you provided will be destroyed. 


\section{APPENDIX 5.1 SEMI-STRUCTURED INTERVIEW GUIDES (CONT'D)}

CASE\# INITIATIVE NAME:

\section{SECTION 1 - ORAL CONSENT SCRIPT / INTERVIEW PREAMBLE}

\section{Initiative Background:}

1. Please describe your current role and background with IBM?

2. Describe your experience with the FoaK program/projects and role on the governance board?

\section{SECTION 2 - INTERVIEW IN CASE INITIATIVES}

3. Please provide an overview of the FoaK initiative?

Probe:

- Link to strategy

- Innovation/invention - already developed invention?

- Client relationship

- Role of FoaK program office; integration role

- Role of Foak board

- Catching/commercialization organization

- Program/initiative skills

4. Describe what went well on the initiative?

5. Describe what went poorly on the initiative?

6. What could have been done differently to improve performance of the initiative?

7. Describe overall initiative performance? Why?

8. What role did the FoaK program play in this initiative? What could have been done differently?

9. What was the role of the Foak board on this initiative?

10. What were any lessons learned? 
Role:

Date:

Time:

Method: Duration

(mins):

\section{INTERVIEW GUIDE 3: PROGRAM OVERVIEW (FULL) [Version 3.3]}

\section{SECTION 1 - ORAL CONSENT SCRIPT / INTERVIEW PREAMBLE}

- THANKS: Thanks again for making the time

Some formality on protocol that need to cover before we begin:

- MY GOAL I am working towards completion of my Ph.D. in Management at Carleton University, under the supervision of Dr. Michel Rod. My area of focus is on open and collaborative innovation models.

- TOPIC Contributes to my doctoral thesis; examining FoaK as a collaborative method of innovation creation.

- DURATION Scheduled for 60 minutes; schedule if need more time; pending your availability

- APPROACH: I have some questions for today's conversation - explore your experience with FoaK program

My goal to have a rich conversation about your experience; explore areas of interest as they come up

- AUDIO-RECORDING With your consent, the interview will be audio-recorded;

- ANONYMOUS Feedback will be kept anonymous in the transcript, and the final analysis will be at an aggregated level; study is being stored securely / cloud repository that will be password protected / only I will have access to the repository, with files being shared with my thesis supervisor as needed.

- RETAIN CONTENT Once the project is completed, all research data will be retained as I may use it for other follow-on research projects on this same topic. Once no longer needed - will be destroyed.

- WITHDRAW You may withdraw at any time, up to December 31, 20016 by letting me know. If you choose to no longer participate, all the information you provided will be destroyed.

- ETHICS PROTOCOL (IN MEETING INVITE) The ethics protocol for this project was reviewed by the Carleton University Research Ethics Board, which provided clearance to carry out 


\section{APPENDIX 5.1 SEMI-STRUCTURED INTERVIEW GUIDES (CONT’D)}

\section{SECTION 1 - ORAL CONSENT SCRIPT / INTERVIEW PREAMBLE}

Initiative Background:

1. Please describe your current role and background with IBM?

2. Describe your experience with the FoaK program/projects and role on the governance board?

\section{SECTION 2 - GENERAL VIEWS ON FOAK AS KNOWLEDGE ACQUISITION}

3. There are multiple roads to new innovation, why do you believe the FoaK program chosen for initiatives?

4. What do you believe is the primary role of A FoaK program ? Company level and initiative level.

5. Describe the FoaK governance board and it's role? \& office?

6. What drives FoaK decisions on investment? How do you identify high probability winners?

7. From your perspective, what does success look like? How is performance measured for a Foak program?

8. What do you believe are the key success factors when using FoaK as a method to innovate through collaboration with clients? Any key dependencies?

9. How does the FoaK program set-up initiatives for success?

10. What is the role of A FoaK program in acquiring new knowledge from outside the company?

11. What is the role of A FoaK program in innovation quality to the company?

12. Projects are complex does the FoaK program play a role as an integrator/orchestrator? Explain?

13. Are there external pressures that projects face? Explain? Do they impact execution and performance?

14. In hindsight, when a funded initiative doesn't perform in the end, why? Are there key contributors?

15. If advising another organization interested in developing a FoaK method, what are the critical elements need in a FoaK program in creating new innovation? Why? 


\section{APPENDIX 5.1 SEMI-STRUCTURED INTERVIEW GUIDES (CONT'D)}

\section{SECTION 3 - POSITION ON PERFORMANCE (Overall)}

1. What role does FoaK program play in impacting the performance of a FoaK initiative? To what degree?

- What elements of a project, program, the environment had the greatest positive impact on the performance of an initiative? Why?

2. Are there elements of the program that have a negative impact? Which and Why?

3. Which elements could be changed or added to the program to improve performance?

4. Any other comments on FoaK program and impact on initiatives?

Q. May I come back with more questions for clarification if needed?

Q. Anyone suggest I speak with?

\section{SECTION 4 - STAGES IN THE FOAK PROCESS: PROCESS DETAILS}

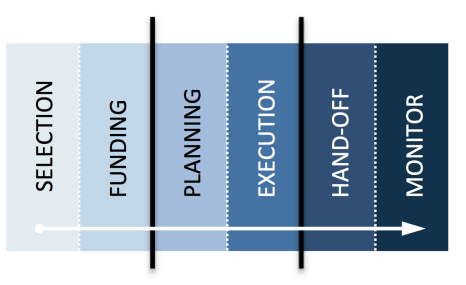

5. What is the primary role of the PROGRAM and the BOARD in each of these stages?

Probes for each of the following stages:

- What happens in this stage (objectives and activities)?

- Role of FoaK? How important is FoaK program (1 not - 10 very) and why?

- Role as an integrator?

- Key success factors/key failure factors

- How important is this stage in overall initiative performance?

- Dependencies for the stage that impacted performance

- External pressures

- The role of each $D C$, and why (integrative, connective, absorptive, innovative, transformative, desorptive, inventive)

- How does each of these contribute to performance? 


\section{APPENDIX 5.2 INFORMANT RECRUITMENT COMMUNICATION}

Artifact 1: Confirmation Email Invitation - Robert Helal Thesis Study

(follow-up email confirming discussion with participant next steps)

Subject: Details on the First-of-a-Kind (FoaK) study and scheduling time for our discussion

$\mathrm{Hi}<\mathrm{John}>$,

Thanks again for taking the time to talk today, and for agreeing to participate in my study of the FoaK program and its approach to innovation creation. As mentioned, as well as being an IBMer, I am also working towards completion of my Ph.D. at Carleton University in Ottawa, focusing on open collaborative innovation. This research I am conducting contributes to the completion of my doctoral thesis.

As discussed, I will be scheduling a 60-minute discussion by phone, based on availability in your calendar. With your consent, I will also be recording a conversation that I will transcribe later. This will allow us to have a free-flowing discussion, and ensure that I capture what we discuss. Also, all interviews and responses I collect are being kept as anonymous, where participants in the study will be identified by codes. All research data, including audio-recordings and any notes, will be securely maintained. Research data will only be accessible by me and my research supervisor. I will be maintaining all data securely for this study and for potential future follow-on studies.

The ethics protocol for this project was reviewed by the Carleton University Research Ethics Board, which provided clearance to carry out the research. Should you have questions or concerns related to your involvement in this research, please contact the REB Chair, Dr. Andy Adler, Chair, Carleton University Research Ethics Board, 613-5202600 ext. 2517, ethics@carleton.ca.

I know we are all running on shorter cycles these days, and am respectful of the time you are providing. I will ensure that we get the most out of our discussion. Also, if for any reason you decide you are no longer interested or able to participate in the interview just let me know, and I will adjust accordingly. Also, if you choose to participate, and are longer able to by $<$ date $>$, all the information you provided will be destroyed. I am very much looking forward to hearing your thoughts and experience. Thanks again for agreeing to discuss your experience with me.

Speak with you soon, Robert Helal 
Artifact 2: Oral Consent Script Interview Preamble - Reviewed prior to interview

Date: Participant's name/Pseudonym/Initials:

$\mathrm{Hi}<\mathrm{John}>$,

Thanks again for making the time to speak today and sharing your experience about the First-of-a-Kind (FoaK) program as a method of creating innovation. Before we get started, I would like to take a couple of minutes to recap on a few things regarding the discussion and how it will be used.

As mentioned earlier, I am working towards completion of my Ph.D. in Management at Carleton University, under the supervision of Dr. Michel Rod. My area of focus is on open and collaborative innovation models. This study contributes to my doctoral thesis and is aimed at examining FoaK as a collaborative method of innovation creation.

Our discussion today is scheduled for 60 minutes. If we find that we need a bit more time in the end, and pending your availability, we will schedule a brief follow on if needed. With your consent, the interview will be audio-recorded.

I have some questions for today's conversation, which are intended to explore your experience with FoaK program. They will help to ensure that we cover the necessary areas for the study, but I also want to emphasize that my goal is for us to have a rich conversation about your experience, and would like us to explore areas of interest as they come up during the discussion.

I also want to mention that your feedback will be kept anonymous in the transcript, and the final analysis will be at an aggregated level. All the data collected during the study is being stored securely. Electronic files will be stored in a cloud repository that will be password protected at the level of the repository, folder and file. Only I will have access to the repository, with files being shared with my thesis supervisor as needed. Once the project is completed, all research data will be retained as I may use it for other follow-on research projects on this same topic. Once no longer needed, all research data will be destroyed.

You may withdraw at any time, up to <insert date of data collection deadline>, by letting me know. If you choose to no longer participate, all the information you provided will be destroyed.

The ethics protocol for this project was reviewed by the Carleton University Research Ethics Board, which provided clearance to carry out the research. Should you have questions or concerns related to your involvement in this research, please contact Dr. Andy Adler, Chair, Carleton University Research Ethics Board, 613-520-2600 ext. 2517, 
ethics@carleton.ca. You can also reach me at the coordinates I provided in my meeting invitation.

Do you have any questions or need clarification?

Do I have your permission to begin: __ Yes___ No (thanks!)

Do you agree to be audio-recorded: ___ Yes__ N N No

Date:

Participant's name/Pseudonym/Initials:

Researcher's Signature: 


\section{Appendix 6.1 FoaK Board Membership Profile (between 2010 and 2014)}

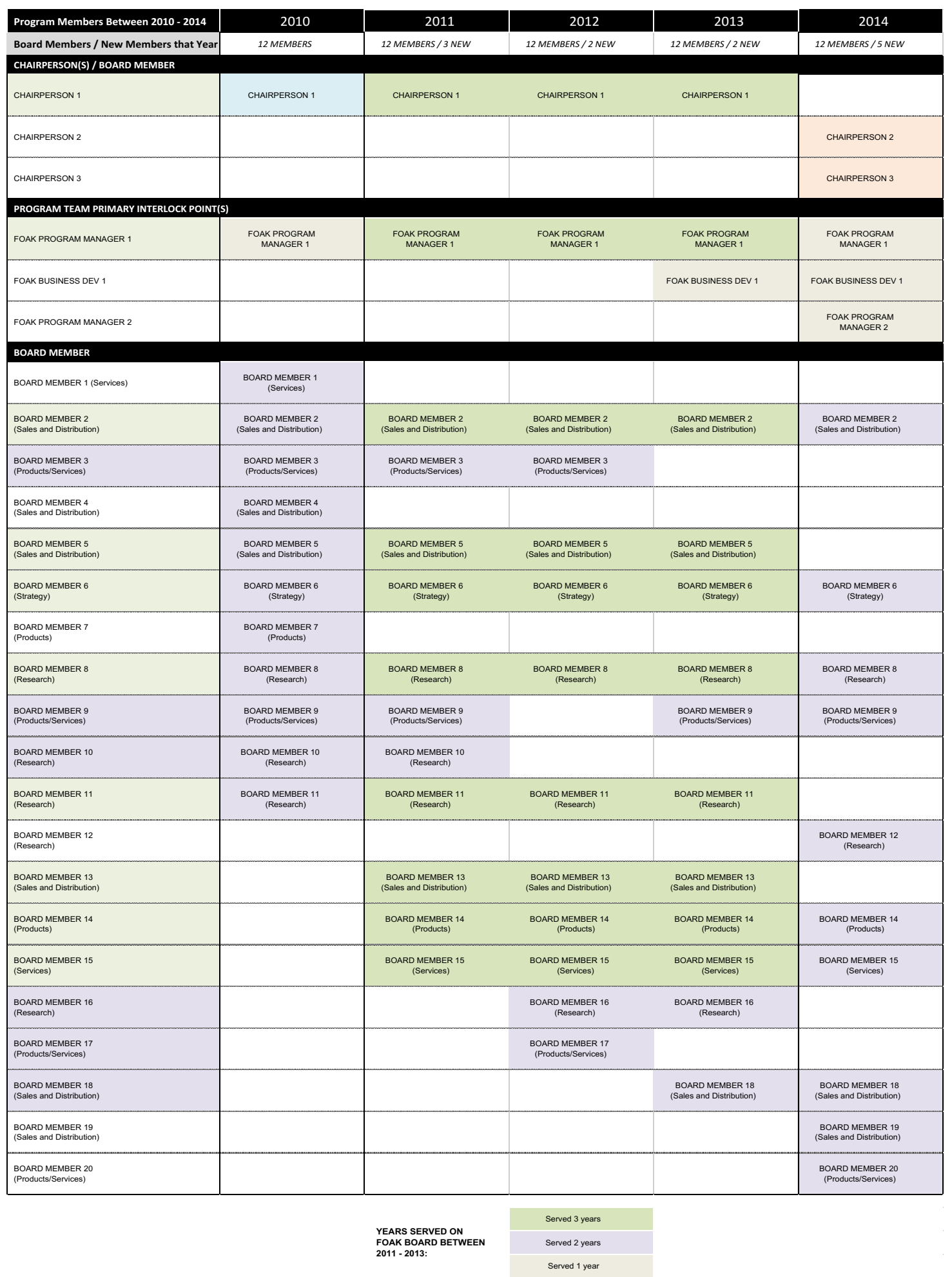

